\title{
New methods for the ab-initio simulation of correlated systems
}

\section{Dissertation}

zur Erlangung des mathematisch-naturwissenschaftlichen Doktorgrades „Doctor rerum naturalium” der Georg-August-Universität Göttingen

im Promotionsprogramm ProPhys

der Georg-August University School of Science (GAUSS)

\author{
vorgelegt von \\ Robert Schade \\ aus Mühlhausen
}

Göttingen, 2018 


\section{Betreuungsausschuss:}

Prof. Dr. Peter E. Blöchl

Institut für Theoretische Physik

Technische Universität Clausthal

PD. Dr. Salvatore R. Manmana

Institut für Theoretische Physik

Georg-August-Universität Göttingen

\section{Mitglieder der Prüfungskommission:}

Referent: $\quad$ Prof. Dr. Peter E. Blöchl Institut für Theoretische Physik Technische Universität Clausthal

Korreferent: PD. Dr. Salvatore R. Manmana Institut für Theoretische Physik Georg-August-Universität Göttingen

Weitere Mitglieder der Prüfungskommission:

Prof. Dr. Jörg Behler

Institut für Physikalische Chemie

Georg-August-Universität Göttingen

Prof. Dr. Christian Jooß

Institut für Materialphysik

Georg-August-Universität Göttingen

Prof. Dr. Stefan Kehrein

Institut für Theoretische Physik

Georg-August-Universität Göttingen

PD. Dr. Martin Wenderoth

IV. Physikalisches Institut

Georg-August-Universität Göttingen

Tag der mündlichen Prüfung: 29.01.2019 
At some point, everything's gonna go south on you... everything's going to go south and you're going to say, this is it.

This is how I end.

Now you can either accept that, or you can get to work.

That's all it is.

You just begin. You do the math. You solve one problem... and you solve the next one... and then the next.

And if you solve enough problems, you get to come home.

- Mark Watney, "The Martian" by Andy Weir 



\section{Abstract}

Strong electronic correlations are at the heart of many interesting phenomena. For the theoretical description of these materials, a proper treatment of the local atomic physics is required. We propose a novel approach combining functionals of the electron density and functionals of the one-particle reduced density matrix to improve this description. The proposed method has a solid foundation in reduced density-matrix functional theory and no double-counting problem arises. It employs a decomposition of the electron-electron interaction in real space. The interaction close to the correlated orbitals, for example, the partially filled 3d-orbitals of transition-metal ions, is described with a density-matrix functional and otherwise with a local or semi-local density functional.

We propose to evaluate the density-matrix functional from Levy's constrained search problem, i.e., via a constrained minimization over an ensemble of many-particle wave functions. In contrast to approximate parametrized density-matrix functionals, this evaluation allows us to systematically improve the functional towards the exact result. In situations where the one-particle basis is too large to evaluate the density-matrix functional from Levy's constrained minimization problem, we apply a series of approximations that each can be converged to the exact result: the first approximation step is the local approximation of the density-matrix functional proposed by Blöchl, Walther and Pruschke. For the density-matrix functionals within the local interactions, we propose the adaptive cluster approximation (ACA) that systematically truncates non-interacting one-particle states and drastically reduces the computational effort. The resulting densitymatrix functional for a local interaction and a small number of non-interacting one-particle states is then evaluated with the constrained minimization problem. We explore different parametrizations of many-particle wave functions in this constrained minimization problem. A parametrization based on a configuration-interaction-like ansatz is shown to converge rapidly if suitable selection criterion for the Slater determinants is chosen. An impurity-bath-separation ansatz is shown to be suitable for single-impurity Anderson models. It is shown that the constrained minimization can be solved for matrix product states with a DMRG-like iterative minimization. Furthermore, we show that GutzwillerJastrow-correlated wave functions can be used with a quantum Monte Carlo procedure as many-particle wave functions. Finally, we formulate an algorithm for the evaluation of the density-matrix functional on near-term quantum computers. Results from the execution of the algorithm on an existing quantum computer with transmon qubits are presented.

The proposed approach combining density functionals and density-matrix functionals is implemented in the CP-PAW code based on the projector augmented-wave formalism. We present results for the dissociation curve of the hydrogen molecule as the prototypical case of strong static correlation and show that static correlation is well described with the new approach. We show results for the nonmagnetic state of the transition-metal oxide $\mathrm{NiO}$ that is described qualitatively wrong with the $\mathrm{DFT}+\mathrm{U}$ method. The proposed method properly describes the nonmagnetic state of $\mathrm{NiO}$ as an insulator and predicts a qualitatively correct spectral function. 



\section{Acknowledgments}

First of all, I would like to thank Peter E. Blöchl for his support, patience and the opportunity to pursue my Ph.D. research in his group. I will always fondly remember our fruitful and sometimes controversial discussions and his excellent advice. I would also like to thank Thomas Pruschke who co-supervised me during the first years of my Ph.D. project and was the reason for me to go in the direction of solid-state theory. In 2009, Thomas gave the lecture "Introduction to solid state theory" that motivated me to do my bachelor project in his group. Interestingly, this bachelor project put me in contact with Peter E. Blöchl and density-functional theory which resulted in a very fruitful collaboration during my Bachelor project, my Master thesis and, finally, my Ph.D. project. For facilitating this connection, Thomas will have my everlasting thanks. After Thomas passed away in 2016, Salvatore R. Manmana took over the co-supervision of my project, and I am very thankful for that.

Moreover, I would like to thank Thomas Köhler for our discussions about DMRG and matrix product states, for answering my beginner's questions in this field and for motivation to try matrix product states for the density-matrix functional. I want to thank Andreas Savin for our lengthy and inspiring discussion during the International Workshop on "New challenges in Reduced Density Matrix Functional Theory: Symmetries, timeevolution and entanglement" in Lausanne 2017. On the other hand, I also would like to thank Benedikt Fauseweh for our discussion on quantum Monte Carlo methods for Gutzwiller- and Jastrow-correlated wave functions. I want to thank Peter E. Blöchl and Axel Ehrich for the opportunity to further my experience in high-performance computing, hardware acquisition, computing-cluster maintenance and setup, code optimization as well as software engineering.

Furthermore, I would like to thank the German Research Foundation for their financial support through the research group FOR1346. I have always enjoyed the internal meetings of the research group and especially the autumn schools in Jülich.

Finally, I thank my family for my education and their support during my school time and physics studies.

Robert Schade, December 2018 



\section{List of publications}

Parts of this thesis have been published in peer-reviewed journals. The corresponding references are:

1. Ebad Kamil, Robert Schade, Thomas Pruschke, and Peter E. Blöchl. Reduced density-matrix functionals applied to the hubbard dimer. Phys. Rev. B, 93: 085141, Feb 2016. doi: 10.1103/PhysRevB.93.085141. URL https://link.aps.org/doi/ 10.1103/PhysRevB.93.085141.

2. Robert Schade, Ebad Kamil, and Peter Blöchl. Reduced density-matrix functionals from many-particle theory. The European Physical Journal Special Topics, 226 (11): 2677-2692, Jul 2017. ISSN 1951-6401. doi: 10.1140/epjst/e2017-70046-0. URL https://doi.org/10.1140/epjst/e2017-70046-0.

3. Robert Schade and Peter E. Blöchl. Adaptive cluster approximation for reduced density-matrix functional theory. Phys. Rev. B, 97: 245131, Jun 2018. doi: 10.1103/PhysRevB.97.245131. URL https://link.aps.org/doi/10.1103/ PhysRevB.97.245131. 



\section{Contents}

1. Introduction 1

2. The quantum many-particle problem 5

2.1. One-particle basis . . . . . . . . . . . . . . . . . . 5

2.2. Non-interacting many-particle states . . . . . . . . . . . . . . 5

2.3. Interacting many-particle states and ensembles . . . . . . . . . . . . 7

2.4. Thermodynamic quantities . . . . . . . . . . . . . . . . . . . . . 9

2.5. Ground-state energy and fundamental gap . . . . . . . . . . . . . . 11

2.6. Non-relativistic Hamiltonian of a solid . . . . . . . . . . . . . . . . . . 12

2.7. Born-Oppenheimer approximation . . . . . . . . . . . . . . . . . . 14

2.8. Beyond the non-relativistic Hamiltonian . . . . . . . . . . . . . . . 16

2.8.1. Dirac equation . . . . . . . . . . . . . . . . 16

2.8.2. Relativistic two-particle interactions . . . . . . . . . . . . . . 18

2.9. Reduced density matrices . . . . . . . . . . . . . . . 20

2.9.1. Basic definitions . . . . . . . . . . . . . . . 20

2.9.2. Natural orbitals and occupations . . . . . . . . . . . . . . 23

2.9.3. The N-representability problem . . . . . . . . . . . . . . . . . 24

2.10. Metals and insulators . . . . . . . . . . . . . . . 26

2.10.1. Distinction between metals and insulators . . . . . . . . . . 26

2.10.2. Classes of insulators . . . . . . . . . . . . . . . . 29

3. Models for many-particle systems $\quad 31$

3.1. Hubbard model . . . . . . . . . . . . . . . . . . . . . . 31

3.2. Single-impurity Anderson models . . . . . . . . . . . . . . . . 33

3.2.1. Characteristic parameter regimes . . . . . . . . . . . . 33

3.2.2. One-particle reduced density matrix of the bath . . . . . . . . . 36

4. Density functional theory 41

4.1. Hohenberg-Kohn theorems . . . . . . . . . . . . . . . . . 41

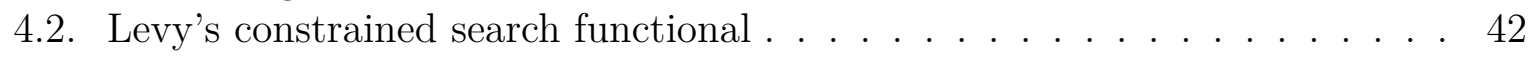

4.3. Lieb's convex-conjugate functional . . . . . . . . . . . . . . . . . 43

4.4. The exchange-correlation functional . . . . . . . . . . . . . . . . . 44

4.4.1. From the density functional to the exchange-correlation functional . 44

4.4.2. Kinetic contribution to the exchange-correlation functional . . . . . 47

4.5. Kohn-Sham system . . . . . . . . . . . . . . . . . . . 47

4.6. Minimization of the total energy . . . . . . . . . . . . . . . . 49

4.7. Extensions . . . . . . . . . . . . . . . . . . 52

4.7.1. Spin-polarized systems . . . . . . . . . . . . . . . 52

4.7.2. Finite temperatures . . . . . . . . . . . . . . . . . 52

4.8. Approximate local functionals . . . . . . . . . . . . . . . . . 53 
4.9. The symmetry-breaking dilemma . . . . . . . . . . . . . . . . 54

4.9.1. The hydrogen molecule and static correlation . . . . . . . . . . . 54

4.9.2. The Hubbard dimer as a minimal model . . . . . . . . . . . . . 55

4.9.3. Broken-symmetry HF-Slater determinants . . . . . . . . . . . . . . 56

4.9.4. Interpretation of the symmetry-breaking dilemma . . . . . . . . . . 59

5. Reduced density-matrix functional theory 63

5.1. Introduction . . . . . . . . . . . . . . . . . . . . . . 63

5.2. Basic formalism . . . . . . . . . . . . . . . . 63

5.3. Properties of the RDMF . . . . . . . . . . . . . . 65

5.4. Parametrized approximations of the density-matrix functional . . . . . 68

5.5. Local approximation . . . . . . . . . . . . . . . . . 68

5.6. Gap and spectral function . . . . . . . . . . . . . . . . . . . . . 69

5.6.1. Local spectral function . . . . . . . . . . . . . . . . . . 70

5.6.2. Kohn-Sham-like spectral function . . . . . . . . . . . . . 71

6. DF+RDMF approaches $\mathbf{7 3}$

6.1. Hybrid functionals, DFT $+\mathrm{U}$ and DFT+DMFT . . . . . . . . . 73

6.2. General DF+RDMF approach . . . . . . . . . . . . . 76

6.3. Orbital-based DF+RDMF approach . . . . . . . . . . . . . . 77

6.3.1. Foundation . . . . . . . . . . . . . . . . . . 77

6.3.2. Local approximation . . . . . . . . . . . . . . . . . . . . . . . . . . . . . . . . 78

6.3.3. Limits . . . . . . . . . . . . . . . . . . . . . 79

6.3.4. Example results for $\mathrm{H}_{2} \ldots \ldots \ldots \ldots$. . . . . . . . . . 80

6.4. Real-space-decomposition DF+RDMFT . . . . . . . . . . . . 83

6.4.1. Foundation . . . . . . . . . . . . . . 83

6.4.2. Local approximation . . . . . . . . . . . . . . . 84

6.4.3. Limits . . . . . . . . . . . . . . . . . . . . 85

6.4.4. Availability of the hole function . . . . . . . . . . . . . 86

6.4.5. Practical considerations for the choice of $f(r) \ldots \ldots$. . . . . . . 90

6.5. Screening and effective Coulomb interactions . . . . . . . . . . . . . . 92

6.5.1. Different aspects of screening . . . . . . . . . . . . . . . 92

6.5.2. Effective Coulomb interactions in the DF+RDMF approach . . . . 93

\begin{tabular}{l} 
7. Projector augmented-wave formalism 97 \\
\hline
\end{tabular}

7.1. Transformation theory . . . . . . . . . . . . . . . . . 97

7.2. Energy minimization . . . . . . . . . . . . . . . . . . 101

7.3. One-particle reduced density matrix . . . . . . . . . . . . . . 101

7.3.1. New one-particle basis . . . . . . . . . . . . . . . . . 101

7.3.2. Projection construction . . . . . . . . . . . . . . . 103

7.3.3. Local one-particle reduced density matrix . . . . . . . . . . . . 107

7.3.4. N-representability . . . . . . . . . . . . . . . . . . . . 107

$\begin{array}{ll}\text { 8. Adaptive cluster approximation } & 109\end{array}$

8.1. Introduction . . . . . . . . . . . . . . . . . . . . . . 109

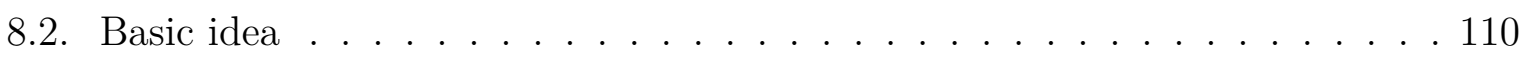

8.3. Related approaches . . . . . . . . . . . . . . . . . . . . 113 
8.4. Exact limits . . . . . . . . . . . . . . . . . . . . . 113

8.5. Correction using parametrized functionals . . . . . . . . . . . . . 114

8.6. Application to Anderson models . . . . . . . . . . . . . . . . . . . . . . . . 114

8.7. Application to Hubbard models . . . . . . . . . . . . . . . . . . . . . . . . 119

8.8. Adaptive-cluster transformation for reduction of entanglement . . . . . . . 123

8.8.1. Matrix product states and correlation functions . . . . . . . . . . . 123

8.8.2. Enhancing the spatial decay of correlation functions . . . . . . . . . 128

8.8.3. Operations with matrix product states . . . . . . . . . . . . . 130

8.8.4. Minimization over matrix product states . . . . . . . . . . . . . . 132

8.8.5. ACA-transformation for ground-state calculations . . . . . . . . . . 136

8.8.6. ACA-transformation for time-evolutions . . . . . . . . . . . . 138

9. Wave-function based approach for the RDMF 145

9.1. Introduction . . . . . . . . . . . . . . . . . 145

9.2. Simplifications . . . . . . . . . . . . . . . . . . . 147

9.3. Solution of the minimization problem . . . . . . . . . . . . . . . . 149

9.3.1. Lagrange function . . . . . . . . . . . . . . . . . . . . . . . . 149

9.3.2. Conceptual challenges . . . . . . . . . . . . . . . . . . . 150

9.3.3. Powell-Hestenes augmented Lagrangian . . . . . . . . . . . . . . . . 152

9.4. Configuration-interaction ansatz . . . . . . . . . . . . . . 154

9.4.1. Ansatz for the many-particle wave function . . . . . . . . . . . . . . 154

9.4.2. Numerical challenges . . . . . . . . . . . . . . . . . . . . 155

9.4.3. Selection of one-particle basis and Slater determinants . . . . . . . 157

9.5. Impurity-bath separation ansatz . . . . . . . . . . . . . . . . 162

9.5.1. Wave-function ansatz . . . . . . . . . . . . . . 162

9.5.2. Example results . . . . . . . . . . . . . . . . . . . 164

9.5.3. Conclusion and outlook . . . . . . . . . . . . . . . 167

9.6. Matrix product states . . . . . . . . . . . . . . . . . . . 170

9.6.1. Constrained optimization over MPS . . . . . . . . . . . . . . . . 170

9.6.2. Example results . . . . . . . . . . . . . . . . . . . . 172

9.7. Gutzwiller-and Jastrow-correlated wave functions . . . . . . . . . . . . 175

9.7.1. Context . . . . . . . . . . . . . . . . . 175

9.7.2. General considerations . . . . . . . . . . . . . . 175

9.7.3. Variational wave function . . . . . . . . . . . . . . . 176

9.7.4. Monte-Carlo sampling of expectation values . . . . . . . . . . . . 180

9.7.5. Simultaneous perturbation stochastic approximation . . . . . . . . 184

9.7.6. Reweighting . . . . . . . . . . . . . . . . 186

9.7.7. Example results . . . . . . . . . . . . . . . . . 188

9.7.8. Outlook . . . . . . . . . . . . . . . . . 189

9.8. Density-matrix functional on quantum computers . . . . . . . . . . . . 194

9.8.1. Context . . . . . . . . . . . . . . . . . . . . 194

9.8.2. Quantum Turing machines and quantum circuit models . . . . . . . 194

9.8.3. Physical realization of quantum computers: transmon qubit . . . . 201

9.8.4. Representing fermionic wave functions on quantum computers . . . 209

9.8.5. Measurement of observables . . . . . . . . . . . . . . . . . 210

9.8.6. Ab-initio quantum chemistry on quantum computers . . . . . . . . 212

9.8.7. RDMF from a VQE-like algorithm . . . . . . . . . . . . . . 217 
9.9. Comparison of approaches . . . . . . . . . . . . . . . 223

10. N-representability based approach to the RDMF 225

10.1. Introduction . . . . . . . . . . . . . . . . . . . . 225

10.2. One-index N-representability conditions . . . . . . . . . . . . . . . 226

10.3. Two-index N-representability conditions . . . . . . . . . . . . . . . . . 226

10.4. Three-index N-representability conditions . . . . . . . . . . . . . . . 228

10.5. Numerical scheme . . . . . . . . . . . . . . . . . . . . . . . . 229

10.6. Boundary-point semidefinite programming algorithm for energy minimization 229

10.7. Boundary-point semidefinite programming algorithm for the densitymatrix functional . . . . . . . . . . . . . . . . . 231

10.7.1. Explicit restriction of the two-particle reduced density matrix . . . 231

10.7.2. Enforcing of constraints with the augmented Lagrangian . . . . . . 233

10.8. Example results . . . . . . . . . . . . . . . . . . . 234

11. Application of the DF+RDMF scheme to real systems 239

11.1. General implementation details . . . . . . . . . . . . . . . . 239

11.2. Hydrogen molecule . . . . . . . . . . . . . . . . . . . . . 241

11.3. $\mathrm{NiO} \ldots \ldots \ldots \ldots \ldots \ldots \ldots$

11.3.1. Properties of $\mathrm{NiO} \ldots \ldots . \ldots . \ldots . \ldots 246$

11.3.2. DFT ground state . . . . . . . . . . . . . . . . . . 247

11.3.3. Local one-particle states and decomposition function $f(r) \ldots . .250$

11.3.4. Evaluation of the density-matrix functional . . . . . . . . . . 256

11.3.5. Results for nickel-only cluster $(N N=0) \ldots \ldots . . .261$

11.3.6. Results for nickel-and-oxygen cluster $(N N=1) \ldots 266$

$\begin{array}{ll}\text { 12.Summary and conclusion } & 277\end{array}$

A. Supplemental information for quantum computer calculations 281

A.1. Hardware-efficient trial state . . . . . . . . . . . . . . . . . 281

A.2. Measurements . . . . . . . . . . . . . . . . . . . . . . . 282

B. Proofs related to the density-matrix functional 287

B.1. Separation property . . . . . . . . . . . . . . . 287

B.2. Proof of existence of banded form . . . . . . . . . . . . . . . . . 289

B.2.1. One transformation step . . . . . . . . . . . . . . . . . . 290

B.2.2. Iterated transformation . . . . . . . . . . . . . . . . . . 292

B.3. Proof of relation of eigenvalue spectrum and block-diagonality . . . . . . . 293

C. Construction of an initial $\mathrm{Cl}$-state for the density-matrix functional 295

$\begin{array}{ll}\text { D. References } & 297\end{array}$

$\begin{array}{ll}\text { E. Curriculum vitae } & 333\end{array}$ 


\section{Introduction}

Density-functional theory (DFT, [Hohenberg and Kohn, 1964; Kohn and Sham, 1965; Levy, 1979; Lieb, 1983a; Kohn, 1999]) with approximate exchange-correlation functionals has become the standard method for the ab-initio description of molecules, solids and surfaces in solid-state theory, material science and quantum chemistry [Jones, 2015]. The main reason for this success is a favorable computational scaling with the number of atoms and a good description of the ground-state properties of many materials. However, even though density-functional theory is in principle an exact theory, approximations have to be introduced in practice for the exchange-correlation functional. The reason for the astonishingly good performance of the widespread local or semi-local approximations of the exchange-correlation functional like LDA- and GGA-functionals is the good description of the spherical average of the exchange-correlation hole [Perdew and Zunger, 1981; Jones and Gunnarsson, 1989].

Density-functional theory with local or semi-local functionals is challenged by the description of static (non-dynamical) electronic correlation, i.e., in situations with degenerate or near-degenerate states. The simplest case of strong static correlation is the dissociation limit of the hydrogen molecule. In the physically correct non-spin-polarized description the total energy is greatly overestimated. In contrast, if the spin-symmetry is broken and a magnetic extension of a local density functional is used, the total energy agrees very well with the exact ground state energy in the dissociation limit. This situation is known as the symmetry-breaking dilemma. Perdew, Savin and Burke [Perdew et al., 1995] have proposed a reinterpretation of spin-polarized density functional theory where instead of the magnetization density the on-top two-electron density (on-top pair density) is predicted. This reinterpretation circumvents the symmetry-breaking dilemma. However, we consider the necessity to break the spin symmetry in order to obtain predictions in agreement with experimental results in situations with strong static correlation as a shortcoming of the approximations of the approximate local of semi-local exchangecorrelation functionals. Investigations by Hollet and Gill [Hollett and Gill, 2011] have shown that, for example, for Be-like ions, there are contributions to the correlation energy from static correlation that cannot be recovered by a broken-symmetry solution. Other shortcomings than the static correlation error, like the delocalization error [Cohen et al., 2012], are not cured by breaking the spin-symmetry and reinterpreting the results with the ideas of Perdew et al.

Thus, approaches are required to improve the description of strong electronic correlations. DFT + U methods [Anisimov et al., 1991, 1993; Solovyev et al., 1994; Liechtenstein et al., 1995; Anisimov et al., 1997b; Dudarev et al., 1998; Cococcioni, 2012] or hybrid functionals [Perdew et al., 1996c; Adamo and Barone, 1999] try to improve upon the DFT results by correcting the exchange-correlation functional with a Hartree-Fock-like energy contribution. Although this correction leads to an improvement of the results in many cases, it does not cure the symmetry-breaking dilemma. For example, the band gap of the transition-metal oxide $\mathrm{NiO}$ in the antiferromagnetic ground state can be described 


\section{Introduction}

in good agreement with experimental results with $\mathrm{DFT}+\mathrm{U}$ or local hybrid functionals. The nonmagnetic state of $\mathrm{NiO}$ is predicted as a metal by $\mathrm{DFT}+\mathrm{U}$ or hybrid functionals.

The DFT+DMFT method [Anisimov et al., 1997a; Lichtenstein and Katsnelson, 1998; Held, 2007; Vollhardt, 2011], that combines density-functional theory with dynamical mean-field theory (DMFT, [Georges and Kotliar, 1992; Anisimov et al., 1997a; Lichtenstein and Katsnelson, 1998; Kotliar et al., 2006]), improves on the DFT+U method by treating a frequency-dependent self-energy instead of an frequency-independent selfenergy as in DFT $+\mathrm{U}$. The local atomic physics of the strongly correlated orbitals, for example, the partially filled 3d-states of transition metal ions, are treated with dynamical mean-field theory and the remaining states with the density functional. This combination of DFT and DMFT results in a physically correct description of the insulating behavior of $\mathrm{NiO}$ without antiferromagnetic order [Ren et al., 2006]. DFT+DMFT is suffering from the double-counting problem because dynamical mean-field theory is formulated with a diagrammatic expansion whereas the existing local or semi-local density functional cannot be written in this way. Hence, it is not clear how to remove the contribution of the interaction from the exchange-correlation functional that is captured by DMFT.

Static correlation is more easily described in terms of the one-particle reduced density matrix than with the electron density. The orbital occupations available in the one-particle reduced density matrix are more natural for the description of electronic correlations than the density. Reduced density-matrix functional theory (RDMFT, [Gilbert, 1975b; Levy, 1979; Valone, 1980; Lieb, 1983b]) is a relative of density-functional theory and uses the one-particle reduced density matrix as the basic variable. Even though RDMFT has been first proposed shortly after the development of DFT, it has only recently emerged as a viable option to describe strong electronic calculations.

The basic definitions of the quantum many-particle problem, reduced density matrices and the N-representability problem are given in chapter 2. Chapter 3 covers the discussion of the Hubbard model [Pariser and Parr, 1953a,b; Pople, 1953; Gutzwiller, 1963b; Hubbard, 1963; Kanamori, 1963] and single-impurity Anderson models [Anderson, 1961], that are used as benchmark systems within this thesis. The ideas of density-functional theory and the static-correlation problem are discussed in chapter 4 . The foundations of reduced density-matrix functional theory are introduced in chapter 5 .

Hybrid approaches combining DFT and RDMFT have been proposed by Pernal [Pernal, 2010], Rohr et al. [Rohr et al., 2010] as well as Blöchl, Walther and Pruschke [Blöchl et al., 2011]. While the former two methods proposed to use approximate parametrized functionals, Blöchl et al. proposed to evaluate the density-matrix functional from Levy's definition as a constrained minimization problem [Levy, 1979].

In this thesis, we follow the approach of Blöchl et al. and propose a novel method combining density functionals and reduced density-matrix functionals. We will propose a method that avoids the double-counting problem and is tailored towards the description of the local atomic physics in strongly correlated solids with a real-space decomposition of the electron-electron interaction. This endeavor results in three important challenges that have to be solved: the main challenge is the formulation of a hybrid method that combines density-functionals and reduced density-matrix functional theory. We tackle this challenge in chapter 6 . The requirements are a consistent definition so that electron-electron interaction is not counted twice, the ability to treat local and non-local interactions and welldefined limits. Blöchl, Walther and Pruschke have proposed such a combination [Blöchl et al., 2011]. However, due to their orbital-based definition of the local interaction, there 
arises an issue in the double counting of the electron interaction. We discuss this theory in section 6.3. We propose an improved combination in section 6.4. The main difference is the definition of the local interaction in real space instead of orbital space, which allows us to evaluate the double counting of the electron interaction numerically exact in practice.

The second challenge is the definition of the local one-particle reduced density matrix. For advanced density-matrix functionals, the one-particle reduced density-matrix is required to be ensemble N-representable, that is, there has to exist an ensemble of normalized many-particle wave functions that generates this one-particle reduced density matrix. This condition is not required for local hybrid functionals or DFT+U-like approaches because the contribution of exact exchange or the DFT $+\mathrm{U}$-expression of the total energy can be evaluated for one-particle reduced density matrices that are not ensemble N-representable. Thus, no attention has been paid to the aspect of N-representability, and existing constructions of the local one-particle reduced density matrix can violate the N-representability condition in practical calculations. In chapter 7 we first discuss the projector augmented-wave method (PAW, [Blöchl, 1994]) and then introduce a new construction of the local one-particle reduced density matrix that is guaranteed to yield an ensemble N-representable local one-particle reduced density matrix.

After the local one-particle reduced density matrix has been obtained, we are faced with the third and most significant challenge: the density-matrix functional has to be evaluated for this one-particle reduced density matrix. We conclude from the pathologies that we found in a common class of approximate parametrized functionals [Kamil, Schade, Pruschke, and Blöchl, 2016], that instead of parametrized approximate functionals one should start from the exact definition of the density-matrix functional and introduce systematically improvable approximations.

The evaluation of the exact density-matrix functional has a computational complexity that scales exponentially with system size. The combination of approximate density functionals with local density-matrix functionals allows us to restrict the evaluation of the density-matrix functional to a rather small one-particle basis. However, for example for $\mathrm{NiO}$, the local approximation of the interaction gives only a small number of interacting one-particle states, but the one-particle reduced density matrix for a solid contains in principle an infinite number of one-particle states. By proposing the adaptive cluster approximation [Schade and Blöchl, 2018] in chapter 8, we show that in this situation the density-matrix functional can be well approximated with 24 or 36 spin-orbitals. We also show how the transformation of the one-particle basis constructed within the adaptive cluster approximation can be used to drastically reduce the computational cost of ground-state calculations and time evolutions of quantum dots or single-impurity Anderson models in the framework of matrix product states (MPS, [White, 1992, 1993; Östlund and Rommer, 1995; Schollwöck, 2005; Schollwöck, 2011]).

The adaptive cluster approximation (ACA) allows us to systematically approximate the density-matrix functional by a density-matrix functional for a smaller one-particle basis. The resulting density-matrix functional then has to be evaluated somehow. We propose to employ parametrizations of the many-particle wave function that have already been used with great success in solid-state theory or quantum chemistry. We have solved the challenge of the arising equality constrained minimization problem, that defines the density-matrix functional, with the Powell-Hestenes augmented Lagrangian [Powell, 1969; Hestenes, 1969]. In contrast to previous proposals for the solution of the constrained minimization problem, that required a numerically exact enforcing of the constraints in 


\section{Introduction}

every iteration step, the framework of the augmented Lagrangian allows us to employ parametrizations of many-particle wave functions for which no practical scheme is known to construct an initial guess that fulfills all constraints.

In chapter 9 we propose, implement and evaluate approaches to evaluate the densitymatrix functional with

1. a configuration-interaction-like approach with an iterative construction of the space of Slater determinants,

2. an impurity-bath separation ansatz,

3. matrix product states in a DMRG-like iterative solution [White, 1992, 1993; Östlund and Rommer, 1995; Schollwöck, 2005; Schollwöck, 2011],

4. Gutzwiller- and Jastrow-correlated many-particle wave functions [Jastrow, 1955; Clark and Feenberg, 1959; Gutzwiller, 1963a, 1965; Bünemann et al., 1998] within a Monte Carlo-based scheme,

5. and hardware-efficient trial states [Kandala et al., 2017] on existing and near-term gate-based quantum computers

as ansatzes for the many-particle wave functions. In chapter 10 we additionally show how the two-particle reduced density matrix instead of the many-particle wave function can be used for the evaluation of the density-matrix functional together with a subset of the N-representability constraints of the two-particle reduced density matrix. This approach is complementary to the wave-function based approaches because in contrast to the wave-function based approaches the two-particle reduced density matrix is not implicitly restricted but explicitly restricted by the N-representability conditions.

Finally, in chapter 11 we combine our solutions to the three challenges of DFT+RDMFT and apply the new approach to the hydrogen molecule as the simplest example of strong static correlation and the nonmagnetic ground state of the transition metal oxide $\mathrm{NiO}$ as a solid with strong local correlation. We discuss computational details and analyze the ground states of these systems within the proposed DF+RDMF approach. 


\section{The quantum many-particle problem}

\subsection{One-particle basis}

To set the stage for the quantum-mechanical many-particle problem, we start by introducing several quantities. We start from single-particle quantum mechanics. The dynamics of a single spin-less particle with mass $m$ in a potential $V$ is described by the time-dependent one-particle Schrödinger equation in position space [Schrödinger, 1926]

$$
i \hbar \partial_{t}|\psi(t)\rangle=\left(-\frac{\hbar^{2}}{2 m} \vec{\nabla}^{2}+V(\vec{r}, t)\right)|\psi(t)\rangle .
$$

Here $\hbar$ denotes the reduced Planck constant ${ }^{1}$ and the one-particle wave function $\psi(\vec{r}, t)=$ $\langle\vec{r} \mid \psi(t)\rangle$ is a complex-valued function in three-dimensional space. The one-particle state $|\psi(t)\rangle$ is an element of the Hilbert space $\mathcal{H}$ of square integrable functions $L^{2}$ and it must be continuous and continuously differentiable everywhere in order for it to be physically reasonable ${ }^{2}$.

A one-particle basis set $\left|\chi_{\alpha}\right\rangle$ is a linearly independent set of states in which any oneparticle state can be expanded, i.e., complete. A one-particle state can thus be written in the form

$$
|\psi(t)\rangle=\sum_{\alpha} a_{\alpha}(t)\left|\chi_{\alpha}\right\rangle
$$

with complex time-dependent expansion coefficients $a_{i}(t)$. A one-particle basis is a orthonormal basis if it fulfills

$$
\left\langle\chi_{\alpha} \mid \chi_{\beta}\right\rangle=\delta_{\alpha \beta} \quad \forall \alpha, \beta
$$

However, a basis does not have to be orthonormal. Later in this thesis, we will also deal with non-orthonormal one-particle basis sets. We also refer to a set of functions $\left|\chi_{\alpha}\right\rangle$ that is not complete usually as basis set as long as it spans the physically relevant subspace. For practical purposes, infinitely dimensional Hilbert spaces must be approximated by incomplete finite-dimensional Hilbert spaces.

\subsection{Non-interacting many-particle states}

For a system consisting of $N$ non-interacting particles, the many-particle wave function $\Psi\left(\vec{r}_{1}, \ldots, \vec{r}_{N}\right) \in \bigotimes_{n=1}^{N} \mathcal{H}$ can be written as a product of one-particle wave functions $\psi_{i}\left(\vec{r}_{i}\right) \in$ $\mathcal{H}$

$$
\Psi\left(\vec{r}_{1}, \ldots, \vec{r}_{N}\right)=\psi_{1}\left(\vec{r}_{1}\right) \cdot \ldots \cdot \psi_{N}\left(\vec{r}_{N}\right)
$$

${ }^{1} \hbar=h / 2 \pi$ with $h=6.626070015 \cdot 10^{-34}$ Js.

${ }^{2}$ Also not square-integrable functions such as plane waves can be physically interesting wave functions. 
We will use uppercase Greek letters for many-particle wave functions and lowercase Greek letters for one-particle wave functions because the distinction is essential in the context of density-functional and reduced density-matrix functional theories. The above product state, however, does not possess the required antisymmetry for fermions with respect to exchanges of particles. Thus, we antisymmetrize it with the antisymmetrization operator $\mathcal{A}$

$$
\Psi_{F}\left(\vec{r}_{1}, \ldots, \vec{r}_{N}\right)=\sqrt{N !} \mathcal{A}=\frac{1}{\sqrt{N !}} \sum_{\pi \in S_{N}}(-1)^{\sigma(\pi)} \hat{P}_{\pi} \Psi\left(\vec{r}_{1}, \ldots, \vec{r}_{N}\right)
$$

where the sum runs over all permutations $\pi$ of $N$ elements of the group of the permutations $S_{N} . \sigma(\pi)$ gives the number of neighboring transpositions in $\pi . \hat{P}_{\pi}$ is the corresponding operator that permutes the $N$ coordinates according to

$$
\hat{P}_{\pi} \Psi\left(\vec{r}_{1}, \ldots, \vec{r}_{N}\right)=\Psi\left(\vec{r}_{\pi(1)}, \ldots, \vec{r}_{\pi(N)}\right) .
$$

An antisymmetrized many-particle wave function $\left|\Psi_{F}\right\rangle \in \mathcal{A} \otimes_{n=1}^{N} \mathcal{H}$ for non-interacting fermions is thus a Slater determinant [Slater, 1929]

$$
\Psi_{F}\left(\vec{r}_{1}, \ldots, \vec{r}_{N}\right)=\frac{1}{\sqrt{N !}} \operatorname{det}\left(\begin{array}{ccc}
\psi_{1}\left(\vec{r}_{1}\right) & \ldots & \psi_{N}\left(\vec{r}_{1}\right) \\
\vdots & \vdots & \vdots \\
\psi_{1}\left(\vec{r}_{N}\right) & \ldots & \psi_{N}\left(\vec{r}_{N}\right)
\end{array}\right)=\frac{1}{\sqrt{N !}}\left|\psi_{1} \ldots \psi_{N}\right|
$$

As this thesis only deals with fermions, we will leave away the index 'F' for many-particle wave functions and all further mentions of many-particle wave functions imply antisymmetry with respect to exchange of particles. If the one-particle wave functions $\psi_{i}\left(\vec{r}_{i}\right)$ in the set are non-orthonormal, we call the corresponding Slater determinant also a nonorthonormal Slater determinant. If the functions in the set, however, are not complete we can add one-particle states $\left|\chi_{\alpha}\right\rangle$ such that they are orthonormal to the $\left|\psi_{i}\right\rangle$ as well as among each other and span the complete one-particle Hilbert space. For this one-particle basis consisting of $\left\{\left|\psi_{i}\right\rangle\right\}$ and $\left\{\left|\chi_{\alpha}\right\rangle\right\}$ we can write the Slater determinant in Eq. (2.7) in occupation number representation as

$$
|\Psi\rangle=|\underbrace{11 \ldots 1}_{\text {N-times }} 0 \ldots 0\rangle,
$$

because the first $N$ one-particle basis states are occupied and the remaining ones are unoccupied. A Slater determinant can be expressed with the fermionic creation operators $\hat{c}_{\alpha}^{\dagger}$ in the one-particle basis $\left\{\left|\psi_{1}\right\rangle, \ldots,\left|\psi_{N}\right\rangle,\left|\chi_{1}\right\rangle,\left|\chi_{2}\right\rangle, \ldots\right\}$ as

$$
|\Psi\rangle=\prod_{\alpha=1}^{N} \hat{c}_{\alpha}^{\dagger}|\mathcal{O}\rangle
$$

where $|\mathcal{O}\rangle=|0 \ldots 0\rangle$ is the vacuum state. The anticommutation relations for the fermionic creation and annihilations operators are

$$
\begin{aligned}
& \left\{\hat{c}_{\alpha}, \hat{c}_{\beta}\right\}=\left\{\hat{c}_{\alpha}^{\dagger}, \hat{c}_{\beta}^{\dagger}\right\}=0 \\
& \left\{\hat{c}_{\alpha}, \hat{c}_{\beta}^{\dagger}\right\}=\delta_{\alpha, \beta},
\end{aligned}
$$


where $\{\hat{a}, \hat{b}\}=\hat{a} \hat{b}+\hat{b} \hat{a}$ is the anticommutator. Thus, these operators act on states in the occupation number representations as

$$
\begin{aligned}
& \hat{c}_{\alpha}^{\dagger}\left|n_{1} \ldots\right\rangle=(-1)^{\sum_{i<\alpha} n_{i}} \cdot\left(1-n_{\alpha}\right)\left|n_{1} \ldots\left(n_{\alpha}+1\right) \ldots\right\rangle \\
& \hat{c}_{\alpha}\left|n_{1} \ldots\right\rangle=(-1)^{\sum_{i<\alpha} n_{i}} \cdot n_{\alpha}\left|n_{1} \ldots\left(n_{\alpha}-1\right) \ldots\right\rangle .
\end{aligned}
$$

Because density-functional theory discussed in chapter 4 relies on a position space treatment and the later considerations within reduced density-matrix functional theory use a finite countable basis set, we will give most quantities in the infinitely dimensional position basis $|\vec{r}\rangle$ as well as for a countable one-particle basis set $\left|\chi_{\alpha}\right\rangle$ of dimension $d<\infty$. The real-space field operators $\hat{\psi}(\vec{r})$ and $\hat{\psi}^{\dagger}(\vec{r})$ are related to the creation and annihilation operators, $\hat{c}_{\alpha}^{\dagger}$ and $\hat{c}_{\alpha}$, in the one-particle basis $\left|\chi_{\alpha}\right\rangle$ by the relations

$$
\begin{aligned}
\hat{\psi}^{\dagger}(\vec{r}) & =\sum_{\alpha} \hat{c}_{\alpha}^{\dagger}\left\langle\chi_{\alpha} \mid \vec{r}\right\rangle \\
\hat{\psi}(\vec{r}) & =\sum_{\alpha} \hat{c}_{\alpha}\left\langle\vec{r} \mid \chi_{\alpha}\right\rangle .
\end{aligned}
$$

This thesis deals with electrons and, thus, even in a non-relativistic treatment we consider them to be spin- $\frac{1}{2}$ fermions. Consequently, we extend the real space coordinate to the combined space-spin coordinate $\vec{x}=(\vec{r}, \sigma)$ with $\int d^{4} \vec{x}=\sum_{\sigma} \int d^{3} \vec{r}$. The anticommutation relations for the field operators read

$$
\begin{aligned}
\left\{\hat{\Psi}(\vec{x}), \hat{\Psi}\left(\vec{x}^{\prime}\right)\right\} & =\left\{\hat{\Psi}^{\dagger}(\vec{x}), \hat{\Psi}^{\dagger}\left(\vec{x}^{\prime}\right)\right\}=0 \\
\left\{\hat{\Psi}(\vec{x}), \hat{\Psi}^{\dagger}\left(\vec{x}^{\prime}\right)\right\} & =\delta_{\sigma, \sigma^{\prime}} \delta\left(\vec{r}-\vec{r}^{\prime}\right)
\end{aligned}
$$

\subsection{Interacting many-particle states and ensembles}

More general $N$-particle states $\left|\Psi^{N}\right\rangle$ that can also describe interacting fermions are superpositions of Slater determinants ${ }^{3}$ with $N=\sum_{j} n_{j, i} \forall i$ with complex amplitudes $c_{i}$ in the form

$$
\begin{aligned}
\left|\Psi^{N}\right\rangle & =\sum_{i} c_{i}\left|n_{1, i} n_{2, i} \ldots\right\rangle \\
\Psi^{N}\left(\vec{x}_{1}, \ldots, \vec{x}_{N}\right) & =\left\langle\vec{x}_{1}, \ldots, \vec{x}_{N} \mid \Psi^{N}\right\rangle .
\end{aligned}
$$

The wave function is normalized such that $\sum_{i}\left|c_{i}\right|^{2}=1$ or, equivalently, $\int d^{4} \vec{x}_{1} \ldots \int d^{4} \vec{x}_{N} \Psi^{N^{*}}\left(\vec{x}_{1}, \ldots, \vec{x}_{N}\right) \Psi^{N}\left(\vec{x}_{1}, \ldots, \vec{x}_{N}\right)=1$. The number of complex coefficients $c_{i}$ scales exponentially with the dimension of the one-particle basis.

The most general form of a many-particle wave function $|\Psi\rangle$ can contain Slater determinants of different particle numbers. In other words, a general many-particle wave function has the form

$$
|\Psi\rangle=\sum_{i} c_{i}\left|n_{1, i} n_{2, i} \ldots\right\rangle
$$

\footnotetext{
${ }^{3}$ The efficiency of this expansion in Slater determinants is discussed later in section 9.4.
} 
with $\sum_{i}\left|c_{i}\right|^{2}=1$. These many-particle states are elements of the Fock space $\mathcal{F}$ [Fock, 1932] corresponding to the one-particle Hilbert space $\mathcal{H}$,

$$
\mathcal{F}(\mathcal{H})=\bigoplus_{n=0}^{\infty}\left(\mathcal{A} \bigotimes_{i=1}^{n} \mathcal{H}\right)
$$

which is the direct sum of all antisymmetrized $n$-particle Hilbert spaces with $n \in\{0,1, \ldots\}$. The distinction between $N$-particle wave functions and general many-particle wave functions will become important for the discussion of the $N$-representability problem in section 2.9.3. Any normalized many-particle wave function $|\Psi\rangle$ can be written as a superposition of normalized $N$-particle wave functions $\left|\Psi^{N}\right\rangle$ in the form

$$
|\Psi\rangle=\sum_{N=0}^{\infty} C_{N}\left|\Psi^{N}\right\rangle
$$

with $\sum_{N=0}^{\infty}\left|C_{N}\right|^{2}=1$.

The many-particle states discussed till this point have been pure states in the sense that they can be described with a single many-particle wave function $|\Psi\rangle$. A statistical mixture, i.e., a mixed state, as arises for degenerate ground states or at finite temperatures, is an ensemble of more than one many-particle wave function. Such an ensemble can be described by wave functions $\left|\Psi_{n}\right\rangle$ and their corresponding statistical weights $P_{n} \geq 0$. The latter are also called ensemble probabilities and sum up to one.

The $N$-particle density operator $\hat{\rho}^{(N)}$ is defined as [Neumann, 1927]

$$
\hat{\rho}^{(N)}=\sum_{n} P_{n}\left|\Psi_{n}^{N}\right\rangle\left\langle\Psi_{n}^{N}\right|
$$

and can describe mixed states as well as pure states. The system is with probability $P_{n}$ in the state described by the $N$-particle wave function $\left|\psi_{n}^{N}\right\rangle$. Here, the many-particle wave functions $\left|\Psi_{n}^{N}\right\rangle$ have to be normalized, i.e. $\left\langle\Psi_{i}^{N} \mid \Psi_{i}^{N}\right\rangle=1 \forall i$, but not necessarily orthogonal. A density operator represents a pure state if and only if the density operator is idempotent, i.e. $\hat{\rho}^{(N)}=\left(\hat{\rho}^{(N)}\right)^{2}$. The density operator of a state can be used to calculate the expectation value of any operator $\hat{A}$ as

$$
\langle\hat{A}\rangle=\operatorname{Tr}\left(\hat{\rho}^{(N)} \hat{A}\right)=\sum_{n} P_{n}\left\langle\Psi_{n}^{N}|\hat{A}| \Psi_{n}^{N}\right\rangle .
$$

The $N$-particle density matrix $\rho^{(N)}$ is defined as the expectation values of the manyparticle density operator in a specific one-particle basis

$$
\begin{aligned}
\rho_{i j}^{(N)} & =\left\langle n_{1, i} n_{2, i} \ldots\left|\hat{\rho}^{(N)}\right| n_{1, j} n_{2, j} \ldots\right\rangle \\
\rho^{(N)}\left(\vec{x}_{1}, \ldots, \vec{x}_{N} ; \vec{x}_{1}^{\prime}, \ldots, \vec{x}_{N}^{\prime}\right) & =\left\langle\vec{x}_{1}^{\prime}, \ldots, \vec{x}_{N}^{\prime}\left|\hat{\rho}^{(N)}\right| \vec{x}_{1}, \ldots, \vec{x}_{N}\right\rangle \\
& =\sum_{n} P_{n} \Psi_{n}^{N^{*}}\left(\vec{x}_{1}^{\prime}, \ldots, \vec{x}_{N}^{\prime}\right) \Psi_{n}^{N}\left(\vec{x}_{1}, \ldots, \vec{x}_{N}\right) .
\end{aligned}
$$

The many-particle density operator $\hat{\rho}^{\text {(many) }}$ generalizes the $N$-particle density operator to arbitrary numbers of particles in the sense that the many-particle wave functions $\left|\Psi_{n}\right\rangle$ in

$$
\hat{\rho}^{\text {(many) }}=\sum_{n} P_{n}\left|\Psi_{n}\right\rangle\left\langle\Psi_{n}\right|
$$


are not necessarily $N$-particle states. The corresponding many-particle density matrix is defined as in Eq. (2.25). The $N$-particle density operator as well as the many-particle density operator are hermitian, positive semi-definite and have unit trace. However, as we will see in section 2.6, the many-particle Hamiltonian of a solid contains only oneparticle and two-particle operators and conserves the particle number. Thus, as will be discussed in section 2.9.3, the exponential amount of information contained in a manyparticle wave function or the many-particle density matrix is not necessary to determine the energy expectation value.

\subsection{Thermodynamic quantities}

Even though the focus of this thesis is the zero-temperature limit, finite temperatures are of interest, too. We define the inverse temperature $\beta$ as

$$
\beta=\frac{1}{k_{B} T}
$$

where $T$ is the temperature and $k_{B}=1.380649 \cdot 10^{-23} \mathrm{~J} \cdot \mathrm{K}^{-1}$ denotes the Boltzmann constant. We begin with the grand canonical ensemble: in this case, the system is in thermal equilibrium with a heat bath of temperature $T$ and in chemical equilibrium with a particle reservoir ${ }^{4}$. The grand potential $\Omega_{\beta, \mu}$ for a grand canonical ensemble with the Hamiltonian $\hat{H}$ and chemical potential $\mu$ in thermal equilibrium at inverse temperature $\beta$ is defined as

$$
\Omega_{\beta, \mu}=-\frac{1}{\beta} \ln \left(\operatorname{Tr} e^{-\beta(\hat{H}-\mu \hat{N})}\right)
$$

The grand potential is an extensive quantity and independent of the chosen one-particle basis set. It is concave ${ }^{5}$ with the matrix-elements $h_{\alpha, \beta}$ of the one-particle Hamiltonian defined by Eq. (2.118), i.e.,

$$
\Omega_{\beta, \mu}\left[(1-\lambda) h_{1}+\lambda h_{2}\right] \geq(1-\lambda) \Omega_{\beta, \mu}\left[h_{1}\right]+\lambda \Omega_{\beta, \mu}\left[h_{2}\right]
$$

holds for all hermitian matrices $h_{1}$ and $h_{2}$. Thus, the grand potential is a concave function of the chemical potential. It is strictly concave at finite temperature $T \geq 0$. The density operator $\hat{\rho}_{\beta, \mu}$ of the corresponding grand canonical ensemble is

$$
\hat{\rho}_{\beta, \mu}=\frac{1}{Z} e^{-\beta(\hat{H}-\mu \hat{N})},
$$

where $Z$ denotes the grand canonical partition function

$$
Z_{\beta, \mu}=\operatorname{Tr} e^{-\beta(\hat{H}-\mu \hat{N})}
$$

\footnotetext{
${ }^{4}$ We ignore mechanical variables such as the volume of the system for the discussion presented here.

${ }^{5}$ We distinguish convexity $\left(f\left((1-\lambda) x_{1}+\lambda x_{2}\right) \leq(1-\lambda) f\left(x_{1}\right)+\lambda f\left(x_{2}\right)\right)$ and concavity $\left(f\left((1-\lambda) x_{1}+\lambda x_{2}\right) \geq\right.$ $\left.(1-\lambda) f\left(x_{1}\right)+\lambda f\left(x_{2}\right)\right)$ as well as their strict variants, that exclude equality in the relations, the in this thesis.
} 
Expectation values $\langle\hat{A}\rangle$ of hermitian operators $\hat{A}$ in the grand canonical ensemble can be evaluated as

$$
\langle\hat{A}\rangle=\operatorname{Tr}\left(\hat{\rho}_{\beta, \mu} \hat{A}\right) .
$$

Because the Hamiltonian is assumed to conserve the particle number, the eigenstates $\left|\Psi_{n}\right\rangle$ to the energy $E_{n}$ can be chosen such that they are also eigenfunctions of the total particle number operator $\hat{N}$ with the eigenvalue $N_{n}$, i.e.

$$
\begin{aligned}
& \hat{H}\left|\Psi_{n}\right\rangle=E_{n}\left|\Psi_{n}\right\rangle \\
& \hat{N}\left|\Psi_{n}\right\rangle=N_{n}\left|\Psi_{n}\right\rangle .
\end{aligned}
$$

Thus, with the orthonormal eigenfunctions $\left|\Psi_{n}\right\rangle$, the corresponding eigenvalues $E_{n}$ and particle numbers $N_{n}$ of the Hamiltonian $\hat{H}$, the above quantities can be written as

$$
\begin{aligned}
\Omega_{\beta, \mu} & =-\frac{1}{\beta} \ln \left(\sum_{n} e^{-\beta\left(E_{n}-\mu N_{n}\right)}\right) \\
Z_{\beta, \mu} & =\sum_{n} e^{-\beta\left(E_{n}-\mu N_{n}\right)} \\
\langle\hat{A}\rangle & =\frac{1}{Z} \sum_{n}\left\langle\Psi_{n}|\hat{A}| \Psi_{n}\right\rangle e^{-\beta\left(E_{n}-\mu N_{n}\right)} .
\end{aligned}
$$

The density operator of Eq. (2.32) can be written as

$$
\hat{\rho}_{\beta, \mu}=\sum_{n} P_{n}\left|\Psi_{n}\right\rangle\left\langle\Psi_{n}\right|
$$

with the ensemble probabilities $P_{n}=\frac{1}{Z} e^{-\beta\left(E_{n}-\mu N_{n}\right)}$. The thermodynamic entropy $S_{\mu, \beta}$ is defined as

$$
S_{\mu, \beta}=-k_{B} \operatorname{Tr}\left(\hat{\rho}_{\beta, \mu} \ln \hat{\rho}_{\beta, \mu}\right)=-k_{B} \sum_{n} P_{n} \ln P_{n}
$$

Consequently, the grand potential can also be written as

$$
\Omega_{\beta, \mu}=\sum_{n} P_{n}\left\langle\Psi_{n}|\hat{H}-\mu \hat{N}| \Psi_{n}\right\rangle-T S_{\mu, \beta}
$$

or, if the eigenstates and eigenvalues of the Hamiltonian are not know, as the minimization problem

$$
\Omega_{\beta, \mu}=\min _{\left\{P_{n}\right\},\left\{\left|\Psi_{n}\right\rangle\right\}: P_{n} \geq 0, \sum_{n} P_{n}=1,\left\langle\Psi_{n} \mid \Psi_{m}\right\rangle=\delta_{m, n}}\left(\sum_{n} P_{n}\left\langle\Psi_{n}|\hat{H}-\mu \hat{N}| \Psi_{n}\right\rangle+\frac{1}{\beta} \sum_{n} P_{n} \ln P_{n}\right) .
$$

For a system that is only in contact with a large heat bath, i.e., the canonical ensemble, the thermodynamic potential is the Helmholtz potential

$$
H_{\beta, N}=\max _{\mu}\left[-\frac{1}{\beta} \ln \left(\operatorname{Tr} e^{-\beta(\hat{H}-\mu \hat{N})}\right)+\mu N\right] .
$$


It is defined as the result of the Legendre-Fenchel ${ }^{6}$ transformation [Legendre, 1787; Fenchel, 1949] of the grand potential with respect to the chemical potential. Thus, the Helmholtz potential is convex with respect to the particle number. Similarly to the grand potential in Eq. (2.43), the Helmholtz potential can be written as

$$
H_{\beta, N}=\min _{\substack{\left\{P_{n}\right\},\left\{\left|\Psi_{n}\right\rangle\right\}: \\ P_{n} \geq 0, \sum_{n} P_{n}=1,\left\langle\Psi_{n} \mid \Psi_{m}\right\rangle=\delta_{m, n}, N=\sum_{n} P_{n}\left\langle\Psi_{n}|\hat{N}| \Psi_{n}\right\rangle}}\left(\sum_{n} P_{n}\left\langle\Psi_{n}|\hat{H}| \Psi_{n}\right\rangle+\frac{1}{\beta} \sum_{n} P_{n} \ln P_{n}\right) .
$$

\subsection{Ground-state energy and fundamental gap}

We define the ground-state energy $E(N)$ for arbitrary particle number $N$ as the zerotemperature limit $\beta \rightarrow \infty$ of the Helmholtz potential

$$
E(N)=\lim _{\beta \rightarrow \infty} H_{\beta, N}
$$

If the many-particle Hamiltonian preservers the total particle number, the ground-state energy $E(N)$ consist of piecewise linear segments between integer particle numbers $N$ and is convex with the particle number. The slope of the ground-state energy is the chemical potential,

$$
\mu=\lim _{n \rightarrow N^{+}} \frac{d E(n)}{d n}
$$

There can be derivative discontinuities of $E(N)$ at integer particle numbers. Hence the chemical potential is composed of piecewise constant functions and can have points of discontinuity at integer particle numbers. The fundamental gap $E_{g}$ can be defined as the height of the jump of the chemical potential as

$$
E_{g}(N)=\lim _{\delta \rightarrow 0^{+}}\left(\left.\frac{d E(n)}{d n}\right|_{n=N+\delta}-\left.\frac{d E(n)}{d n}\right|_{n=N-\delta}\right) .
$$

Equivalently, the fundamental gap can be defined as the difference of the ionization potential $I(N)=E(N-1)-E(N)$ and the electron affinity $A(N)=E(N)-E(N+1)$ as

$$
E_{g}(N)=I(N)-A(N)=E(N+1)-2 E(N)+E(N-1) .
$$

For the exact ground state, where the ground-state energy consists of piecewise linear segments, the two definitions of Eq. (2.48) and Eq. (2.49) are equivalent. However, most

\footnotetext{
${ }^{6}$ As Legendre-Fenchel transformations or convex conjugates we denote in this thesis transformations of the kind $f(x) \rightarrow g(y)=\inf _{x} \pm[f(x)-x y]$. The upper sign is for convex $f(x)$ and the lower sign for concave $f(x)$. In both cases the resulting $g(y)$ is convex. We use the term Legendre-Fenchel transform here to distinguish this transformation from the Legendre transformation $f(x) \rightarrow g(y)=f(x)-y x_{y}$ with $y=\partial_{x} f\left(x_{y}\right)$, that is commonly used in thermodynamics or classical mechanics and only applies to differentiable and strictly convex or strictly concave functions. The Legendre-Fenchel transform is the extension of the Legendre transform to non-differentiable and not strictly convex or not strictly concave functions.
} 
approximations result in ground-state energy curves without derivative discontinuities even though the exact solution would have derivative discontinuities. Thus, the two definitions are not equivalent for most approximations anymore. In case the approximation used doesn't produce a derivative discontinuity, the gap can be approximated with Slater's [Slater, 1972; Liberman, 2000] transition rule as

$$
E_{g}(N)=\left.\frac{\partial E(n)}{\partial n}\right|_{n=N+1 / 2}-\left.\frac{\partial E(n)}{\partial n}\right|_{n=N-1 / 2}
$$

or with a tangent construction for the chemical potential [Helbig et al., 2007, 2009].

\subsection{Non-relativistic Hamiltonian of a solid}

The main challenge of theoretical solid-state theory or quantum chemistry is the solution of the quantum-mechanical many-particle problem. To formulate this problem, we have to set up a model of a solid or molecule as a set of $N_{N}$ nuclei with masses $M_{A}$ and $N_{e}$ electrons with mass $m_{e}=9.10938356 \cdot 10^{-31} \mathrm{~kg}$ each. For the energy scales of below a few electron volts, length scales above one picometer, and time scales of above one femtosecond, we can neglect the inner structure, shape, and spin of the nuclei. The nuclei are thus approximated by localized positive charge densities with a total charge equal to the atomic number $Z_{A}$ times the elementary charge $e=1.602176634 \cdot 10^{-19} \mathrm{C}$. Electrons are treated as point charges with a total charge of $-e$ and spin $\hbar / 2$, where $\hbar$ denotes the reduced Planck constant. When also neglecting relativistic effects, magnetic fields, and electrodynamics, we can describe the system with the non-relativistic many-particle Hamiltonian $\hat{H}$

$$
\hat{H}=\hat{T}_{e}+\hat{T}_{n}+\hat{V}_{e e}+\hat{V}_{n}+\hat{V}_{e n}
$$

The operator of the kinetic energy of the electrons

$$
\hat{T}_{e}=\sum_{i=1}^{N_{e}} \frac{\hat{\vec{p}}_{i}^{2}}{2 m_{e}}
$$

and the kinetic energy of the atoms

$$
\hat{T}_{n}=\sum_{A=1}^{N_{N}} \frac{\hat{\vec{P}}_{A}^{2}}{2 M_{A}}
$$

depend on the momentum operators $\hat{\vec{p}}_{i}=-i \hbar \vec{\nabla}_{\vec{r}_{i}}$ of the electrons and atoms $\hat{\vec{P}}_{A}=$ $-i \hbar \vec{\nabla}_{\vec{R}_{A}}$, respectively. $\vec{r}_{i}$ denotes the position of the $i$-th electron and $\vec{R}_{A}$ the position of the $A$-th nucleus.

The electron-electron interaction operator

$$
\hat{V}_{e e}=\frac{1}{2} \sum_{i \neq j} \frac{e^{2}}{4 \pi \epsilon_{0}\left|\hat{\vec{r}}_{i}-\hat{\vec{r}}_{j}\right|}
$$

represents the Coulomb interaction of the electrons and depends on the position operators $\hat{\vec{r}}_{i}$ of the electrons. $\epsilon_{0}=1 /\left(\mu_{0} c^{2}\right)$ denotes the absolute dielectric permittivity of vacuum, 
$c=299792458 \mathrm{~ms}^{-1}$ the speed of light in vacuum and $\mu_{0}=4 \pi \cdot 10^{-7} \mathrm{~m} \cdot \mathrm{kg} \cdot \mathrm{s}^{-2} \cdot \mathrm{A}^{-2}$ the permeability of vacuum. The nucleus-nucleus interaction

$$
\hat{V}_{n n}=\frac{1}{2} \sum_{A \neq B} \frac{e^{2} Z_{A} Z_{B}}{4 \pi \epsilon_{0}\left|\hat{\vec{R}}_{A}-\hat{\vec{R}}_{B}\right|}
$$

represents the Coulomb interaction among the nuclei and depends on the position operators $\hat{\vec{R}}_{A}$ of the nuclei. Moreover, the electron-nuclei interaction

$$
\hat{V}_{e n}=-\frac{1}{2} \sum_{A, i} \frac{e^{2} Z_{A}}{4 \pi \epsilon_{0}\left|\hat{\vec{R}}_{A}-\hat{\vec{r}}_{i}\right|}
$$

is the Coulomb interaction between the positively charged nuclei and the negatively charged electrons. The position operators $\hat{\vec{r}}_{i}=\left(\hat{r}_{i, 1}, \hat{r}_{i, 2}, \hat{r}_{i, 3}\right)$ and $\hat{\vec{R}}_{A}=\left(\hat{R}_{A, 1}, \hat{R}_{A, 2}, \hat{R}_{A, 3}\right)$ commute among each other. On the other hand the momentum operators $\hat{\vec{p}}_{i}=$ $\left(\hat{p}_{i, 1}, \hat{p}_{i, 2}, \hat{p}_{i, 3}\right)$ and $\hat{\vec{P}}_{A}=\left(\hat{P}_{A, 1}, \hat{P}_{A, 2}, \hat{P}_{A, 3}\right)$ also commute among each other. Position and momentum operators of different particles commute and for the same particle fulfill the commutations relations

$$
\begin{aligned}
{\left[\hat{r}_{i, a}, \hat{p}_{i, b}\right] } & =i \hbar \delta_{a, b} \\
{\left[\hat{R}_{A, a}, \hat{P}_{A, b}\right] } & =i \hbar \delta_{a, b} .
\end{aligned}
$$

The many-particle wave function $|\Psi\rangle$ depends on the positions $\vec{r}_{i}$ and spin quantum number $\sigma_{i} \in\{\uparrow, \downarrow\}$ in z-direction of the electrons as well as the positions of the nuclei $\vec{R}_{A}$

$$
\left\langle\vec{r}_{1}, \sigma_{1}, \ldots, \vec{r}_{N_{e}}, \sigma_{N_{e}}, \vec{R}_{1}, \ldots, \vec{R}_{N_{N}} \mid \Psi\right\rangle=\Psi\left(\vec{r}_{1}, \sigma_{1}, \ldots, \vec{r}_{N_{e}}, \sigma_{N_{e}}, \vec{R}_{1}, \ldots, \vec{R}_{N_{N}}\right) .
$$

This wave function is antisymmetric with respect to the interchange of two electrons and thus obeys the Pauli principle. The dynamics of the many-particle wave function is described by the time-dependent Schrödinger equation

$$
\hat{H}|\Psi(t)\rangle=i \hbar \partial_{t}|\Psi(t)\rangle \text {. }
$$

A solution of this equation can be obtained by inserting the eigenvectors $\left|\Psi_{n}\right\rangle$ of the many-particle Hamiltonian $\hat{H}$,

$$
\hat{H}\left|\Psi_{n}\right\rangle=E_{n}\left|\Psi_{n}\right\rangle,
$$

into the time-dependent Schrödinger equation in the form

$$
|\Psi(t)\rangle=\sum a_{n} e^{-i \frac{E_{n}}{\hbar} t}\left|\Psi_{n}\right\rangle
$$

with the complex parameters $a_{n}$ defined by the initial state

$$
|\Psi(t=0)\rangle=\sum_{n} a_{n}\left|\Psi_{n}\right\rangle
$$

Thus, with the set of energy eigenvalues $E_{n}$ and eigenstates $\left|\Psi_{n}\right\rangle$ of the Hamiltonian $\hat{H}$ the time-dependency can easily be solved. Also thermal expectations values of any observable are determined by the energies $E_{n}$ and the eigenstates. 


\subsection{Born-Oppenheimer approximation}

For practical investigations a separation of the electronic and nuclear degrees of freedom is useful. This is the first step in the derivation of the Born-Oppenheimer approximation [Born and Oppenheimer, 1927; Born and Huang, 1954]. For the purpose of the separation the total Hamiltonian in Eq. (2.51) is written as

$$
\hat{H}=\hat{T}_{e}+\hat{T}_{n}+\hat{U}
$$

with $\hat{U}=\hat{V}_{e e}+\hat{V}_{n}+\hat{V}_{e n}$ as the total Coulomb interaction of nuclei and electrons. Additionally we define

$$
\hat{H}^{\mathrm{BO}}\left(\vec{R}_{1}, \ldots, \vec{R}_{N_{N}}\right)=\hat{T}_{e}+\hat{U}=\hat{H}-\hat{T}_{n}
$$

as the Born-Oppenheimer (BO) Hamiltonian. It represents the Hamiltonian of the electrons for fixed nuclear coordinates. The BO-Hamiltonian does not contain the momentum operators of the nuclei $\hat{\vec{P}}_{A}$ and acts on electronic wave functions $\left|\Psi^{\mathrm{BO}}\right\rangle$ that are called the Born-Oppenheimer wave functions. The Schrödinger equation for the electronic degrees of freedom thus also depends parametrically on the nuclear coordinates and has the form

$$
\left[\hat{H}^{\mathrm{BO}}\left(\vec{R}_{1}, \ldots, \vec{R}_{N_{N}}\right)-\epsilon_{n}^{\mathrm{BO}}\left(\vec{R}_{1}, \ldots, \vec{R}_{N_{N}}\right)\right]\left|\Psi_{n}^{\mathrm{BO}}\left(\vec{R}_{1}, \ldots, \vec{R}_{N_{N}}\right)\right\rangle=0
$$

The eigenvalues $\epsilon_{n}^{\mathrm{BO}}\left(\vec{R}_{1}, \ldots, \vec{R}_{N_{N}}\right)$ are named the Born-Oppenheimer surfaces. Even though the solution of Eq. (2.66) is exponentially hard in most cases, we assume that it is solved for the purposes of the explanation of the Born-Oppenheimer approximation in this section. We use this knowledge to solve the Schrödinger equation Eq. (2.61) of the full Hamiltonian $\hat{H}$. Born and Huang have proposed the decomposition [Born and Huang, 1954]

$$
\begin{aligned}
& \Psi\left(\vec{r}_{1}, \sigma_{1}, \ldots, \vec{r}_{N_{e}}, \sigma_{N_{e}}, \vec{R}_{1}, \ldots, \vec{R}_{N_{N}}, t\right)= \\
& \quad \sum_{n} \Phi_{n}\left(\vec{R}_{1}, \ldots, \vec{R}_{N_{N}}, t\right)\left\langle\vec{r}_{1}, \sigma_{1}, \ldots, \vec{r}_{N_{e}}, \sigma_{N_{e}} \mid \Psi_{n}^{\mathrm{BO}}\left(\vec{R}_{1}, \ldots, \vec{R}_{N_{N}}\right)\right\rangle
\end{aligned}
$$

where $\Phi_{n}\left(\vec{R}_{1}, \ldots, \vec{R}_{N_{N}}, t\right)$ denote the time-dependent nuclear wave functions. Inserting the Born-Huang expansion into the time-dependent Schrödinger equation for the manyparticle wave functions, Eq. (2.60), yields the nuclear Schrödinger equation [Born and Huang, 1954]

$$
\begin{aligned}
i \hbar \partial_{t} \Phi_{n}\left(\vec{R}_{1}, \ldots, \vec{R}_{N_{N}}, t\right) & =\left[\hat{T}_{N}+\epsilon_{n}^{\mathrm{BO}}\left(\vec{R}_{1}, \ldots, \vec{R}_{N_{N}}\right)\right] \Phi_{n}\left(\vec{R}_{1}, \ldots, \vec{R}_{N_{N}}, t\right) \\
& +\sum_{n^{\prime}}\left[\sum_{A} M_{A}^{-1}\left(\vec{A}_{n n^{\prime}, A} \hat{\vec{P}}_{A}+B_{n n^{\prime}, A}\right)\right] \Phi_{n^{\prime}}\left(\vec{R}_{1}, \ldots, \vec{R}_{N_{N}}, t\right) .
\end{aligned}
$$

The matrix elements

$$
\begin{aligned}
\vec{A}_{n n^{\prime}, A} & =\left\langle\Psi_{n}^{\mathrm{BO}}\left(\vec{R}_{1}, \ldots, \vec{R}_{N_{N}}\right)\left|\hat{\vec{P}}_{A}\right| \Psi_{n^{\prime}}^{\mathrm{BO}}\left(\vec{R}_{1}, \ldots, \vec{R}_{N_{N}}\right)\right\rangle, \\
B_{n n^{\prime}, A} & =\frac{1}{2}\left\langle\Psi_{n}^{\mathrm{BO}}\left(\vec{R}_{1}, \ldots, \vec{R}_{N_{N}}\right)\left|\hat{\vec{P}}_{A}^{2}\right| \Psi_{n^{\prime}}^{\mathrm{BO}}\left(\vec{R}_{1}, \ldots, \vec{R}_{N_{N}}\right)\right\rangle
\end{aligned}
$$


determine the last term in Eq. (2.68), which is named the 'derivative couplings' because it couples different BO-surfaces $n$ and $n^{\prime}$ due to derivatives of the corresponding BOwave functions with respect to the nuclear coordinates. It should be noted that the decomposition of the many-particle wave function in Eq. (2.67) is exact and no other approximation has been introduced so far. As the second step in the derivation of the Born-Oppenheimer approximation, we neglect the derivative couplings in the nuclear Schrödinger equation, Eq.(2.68). We obtain an approximate Schrödinger equation for the dynamics of the nuclear wave functions

$$
i \hbar \partial_{t} \Phi_{n}\left(\vec{R}_{1}, \ldots, \vec{R}_{N_{N}}, t\right)=\left[\hat{T}_{N}+\epsilon_{n}^{\mathrm{BO}}\left(\vec{R}_{1}, \ldots, \vec{R}_{N_{N}}\right)\right] \Phi_{n}\left(\vec{R}_{1}, \ldots, \vec{R}_{N_{N}}, t\right) .
$$

The Born-Oppenheimer surfaces $\epsilon_{n}^{\mathrm{BO}}\left(\vec{R}_{1}, \ldots, \vec{R}_{N_{N}}\right)$ act as an effective potential surface for the motion of the nuclei. Born and Oppenheimer [Born and Oppenheimer, 1927] have shown with a perturbation expansion in the parameter $\kappa=\left(m_{e} / M\right)^{1 / 4}$, where $M$ is some mean value of the nuclear masses, that the term emerging as the fourth order term in the Hamiltonian couples the nuclear and electronic motion adiabatically. Up to this order of the perturbation expansion the many-particle wave function can be written as a product of a nuclear wave function $\Phi$ and a Born-Oppenheimer wave function $\Psi_{n}^{\mathrm{BO}}$ [Born and Huang, 1954] in the form ${ }^{7}$

$$
\begin{aligned}
& \Psi_{n}\left(\vec{r}_{1}, \sigma_{1}, \ldots, \vec{r}_{N_{e}}, \sigma_{N_{e}}, \vec{R}_{1}, \ldots, \vec{R}_{N_{N}}, t\right) \\
& \quad=\Phi\left(\vec{R}_{1}, \ldots, \vec{R}_{N_{N}}, t\right) \Psi_{n}^{\mathrm{BO}}\left(\vec{r}_{1}, \sigma_{1}, \ldots, \vec{r}_{N_{e}}, \sigma_{N_{e}}, \vec{R}_{1}, \ldots, \vec{R}_{N_{N}}\right)+\mathcal{O}\left(\kappa^{3}\right) .
\end{aligned}
$$

Thus electrons act as though the nuclei were fixed at the positions $\vec{R}_{1}, \ldots, \vec{R}_{N_{N}}$ and follow their motion adiabatically. This is a reasonable approximation for the common situation where the electron mass $m_{e}$ is much smaller than the nuclear masses $M_{A}$ and, thus, the electronic motion is much faster than the nuclear motion. Consequently, the adiabatic theorem [Born and Fock, 1928] can be used. It states for this case that if the atomic motion can be regarded as a small perturbation and the energy eigenvalue is isolated, the system remains in its instantaneous eigenstate. This adiabatic approximation is related to the full separation of the electronic and nuclear motion in the Born-Oppenheimer approximation above and is the reason why the term 'the adiabatic approximation' is often wrongly used as a synonym for the Born-Oppenheimer approximation. However, also the Born-Huang approximation [Born and Huang, 1954] ${ }^{8}$

$$
i \hbar \partial_{t} \Phi_{n}\left(\vec{R}_{1}, \ldots, \vec{R}_{N_{N}}, t\right)=\left[\hat{T}_{N}+\epsilon_{n}^{\mathrm{BO}}\left(\vec{R}_{1}, \ldots, \vec{R}_{N_{N}}\right)+\sum_{A} M_{A}^{-1} B_{n n, A}\right] \Phi_{n}\left(\vec{R}_{1}, \ldots, \vec{R}_{N_{N}}, t\right),
$$

which in contrast to the Born-Oppenheimer approximation does not neglect the diagonal terms of the derivative couplings, is an adiabatic approximation because it does not couple different Born-Oppenheimer surfaces. The adiabatic approximation breaks down when different Born-Oppenheimer surfaces cross or come close.

\footnotetext{
${ }^{7}$ In higher orders of the perturbation expansion in $\kappa$ the wave function cannot be written in the simple form of equation (2.72).

${ }^{8}$ The matrix elements $\vec{A}_{n n, A}$ vanish if the Born-Oppenheimer wave functions are normalized and can be chosen as real.
} 
When using the Born-Oppenheimer approximation, the nuclei are often approximated as classical particles in practice. This classical-particle approximation yields Newton's equations of motion for the nuclei

$$
M_{A} \partial_{t}^{2} \vec{R}_{A}=-\vec{\nabla}_{\vec{R}_{A}} \epsilon_{n}^{\mathrm{BO}}\left(\vec{R}_{1}, \ldots, \vec{R}_{N_{N}}\right) .
$$

Here, $n$ denotes Born-Oppenheimer surface the system is on. Thus, the nuclei move as classical particles in the effective potential $\epsilon_{n}^{\mathrm{BO}}\left(\vec{R}_{1}, \ldots, \vec{R}_{N_{N}}\right)$ that includes the electronic degrees of freedom. The nuclei will be considered on this level of theory, that is, the BornOppenheimer approximation and the classical-particle approximation, for the remainder of this thesis. The main focus will be on approximations of the Born-Oppenheimer surface $\epsilon_{0}^{\mathrm{BO}}\left(\vec{R}_{1}, \ldots, \vec{R}_{N_{N}}\right)$ of the ground state. Thus, we will leave away the superscript $\mathrm{BO}$ and refer to the Born-Oppenheimer Hamiltonian simply as the Hamiltonian and to Born-Oppenheimer wave functions simply as wave functions unless stated otherwise. Furthermore, we drop the explicit mention of the dependence of the Hamiltonian and wave functions on the coordinates of the atoms and shift all energies by the nucleus-nucleus interaction such that

$$
\hat{H} \mapsto \hat{H}-\sum_{A \neq B} \frac{e^{2} Z_{A} Z_{B}}{4 \pi \epsilon_{0}\left|\vec{R}_{A}-\vec{R}_{B}\right|} \mathbb{1}
$$

\subsection{Beyond the non-relativistic Hamiltonian}

\subsubsection{Dirac equation}

The non-relativistic model represented by the Hamiltonian in Eq. (2.51)-Eq. (2.56) can be extended to describe some of the neglected effects approximately. Relativistic effects caused by the finite speed of light $c$ can influence many properties of molecules and solids ${ }^{9}$.

Relativistic non-interacting electrons in an external vector potential $\vec{A}$ and an external scalar potential $V$ are described with the time-dependent Dirac equation [Dirac, 1928a,b]

$$
i \hbar \partial_{t} \psi=\left[c \vec{\alpha} \cdot(\hat{\vec{p}}-e \vec{A})+\beta m_{e} c^{2}+e V\right] \psi .
$$

The four-component Dirac matrices are defined as

$$
\begin{aligned}
& \gamma^{0}=\alpha^{0}=\beta=\left(\begin{array}{cc}
\mathbb{1}_{2} & 0 \\
0 & -\mathbb{1}_{2}
\end{array}\right) \\
& \gamma^{i}=\gamma^{0} \alpha^{i}=\gamma^{0}\left(\begin{array}{cc}
0 & \sigma_{i} \\
\sigma_{i} & 0
\end{array}\right)
\end{aligned}
$$

via with the Pauli matrices $\sigma_{i}$

$$
\begin{array}{rlrl}
\sigma_{0} & =\left(\begin{array}{ll}
1 & 0 \\
0 & 1
\end{array}\right), & \sigma_{1}=\left(\begin{array}{ll}
0 & 1 \\
1 & 0
\end{array}\right), \\
\sigma_{2}=\left(\begin{array}{cc}
0 & -i \\
i & 0
\end{array}\right), & \sigma_{3}=\left(\begin{array}{cc}
1 & 0 \\
0 & -1
\end{array}\right) .
\end{array}
$$

\footnotetext{
${ }^{9}$ For an overview we refer the reader to reviews by Pyykko et al. [Pyykko and Desclaux, 1979; Pyykko, 1988; Pyykkö, 2012] as well as [Schwerdtfeger, 2002, 2004] and references therein.
} 
The wave function $\psi$ is a four-component Dirac spinor that can be written as two twocomponent spinors

$$
\psi=\left(\begin{array}{l}
\psi^{L} \\
\psi^{S}
\end{array}\right)
$$

where $\psi^{L}$ is called the large component and $\psi^{S}$ the small component. This naming becomes clear when one observes the non-relativistic limit $c \rightarrow \infty$ : the large component converges to the solution of the non-relativistic Schrödinger equation and the small component vanishes.

The time-independent Dirac equation for a vanishing vector potential $\vec{A}$ can be written as

$$
\left(c \vec{\alpha} \cdot \hat{\vec{p}}+\beta m_{e} c^{2}+e V-E\right) \psi=\left(\hat{H}_{D, 0}+e V-E\right) \psi=0 .
$$

The free Dirac Hamiltonian $\hat{H}_{D, 0}$ has states with negative energy eigenvalues, that are named as positronic states. Positrons are the anti-particle of the electron with the same mass and spin but opposite charge and magnetic moment. The spectrum of the free Dirac Hamiltonian can be classified into four regions [Thaller, 1992]: the positronic scattering states for $E \leq m_{e} c^{2}$, the positronic bound states in $m_{e} c^{2}<E<0$, the electronic bound states in $0<E<m_{e} c^{2}$ and the electronic scattering states for $E>m_{e} c^{2}$. The energy ranges of scattering states belong to the essential spectrum, and are independent of boundary conditions and invariant under compact perturbations such as potentials with compact support. The energy region $\left(-m_{e} c^{2}, m_{e} c^{2}\right)$ of the bound states belongs to the discrete spectrum. Thus, there exist scattering states with energies below the electronic bound states.

The Dirac equation, Eq. (2.76), couples the large and small component of the spinor wave function, Eq.(2.81). However, the small component can be derived from the large component for $\vec{A}=0$ as

$$
\psi^{S}=\frac{c \vec{\sigma} \cdot \hat{\vec{p}}}{E+2 m_{e} c^{2}-e V} \psi^{L}
$$

and the equation for the large component is

$$
\left(\frac{1}{2} \vec{\sigma} \cdot \hat{\vec{p}}\left[1+\frac{E-e V}{2 m_{e} c^{2}}\right]^{-1} \vec{\sigma} \cdot \hat{\vec{p}}+e V-E\right) \psi^{L}=0 .
$$

The Pauli approximation [Schwerdtfeger, 2002] uses an expansion up to first order in $1 / m_{e} c^{2}$ of

$$
\left[1+\frac{E-e V}{2 m_{e} c^{2}}\right]^{-1}=1-\frac{E-e V}{2 m_{e} c^{2}}+\mathcal{O}\left(\left(m_{e} c^{2}\right)^{-2}\right)
$$

This expansion is only valid for $|E-e V| \ll m_{e} c^{2}$ and, hence, not near the nucleus where the Coulomb potential is strongly negative and variationally unstable [Schwerdtfeger, 2002]. The issues of the Pauli approximation can be avoided by regularization of the approximation. For that purpose, Eq. (2.85) is rewritten as

$$
\left[1+\frac{E-e V}{2 m_{e} c^{2}}\right]^{-1}=\frac{2 m_{e} c^{2}}{2 m_{e} c^{2}-e V}\left[1+\frac{E}{2 m_{e} c^{2}-e V}\right]^{-1}
$$


and expanded in orders of $E /\left(2 m_{e} c^{2}-e V\right)$. The expansion in lowest order yields the zeroth-order regular approximation (ZORA) of the Dirac Hamiltonian [Chang et al., 1986; van Lenthe et al., 1993, 1994; Faas et al., 1995; van Lenthe et al., 1996]

$$
\hat{H}_{\mathrm{ZORA}}=\vec{\sigma} \cdot \hat{\vec{p}} \frac{c^{2}}{2 m_{e} c^{2}-e V} \vec{\sigma} \cdot \hat{\vec{p}}+e V
$$

The regular approximation is well founded for Coulomb potential and variationally stable. However, the Pauli approximation has coined the distinction of relativistic effects into spin-orbit coupling effects that occur in the Pauli Hamiltonian due to the term [Schwerdtfeger, 2002]

$$
\hat{H}_{S O}=\frac{\hbar e}{4 m_{e}^{2} c^{2}} \vec{\sigma} \cdot[(\nabla V) \times \hat{\vec{p}}] .
$$

The remaining effects have been named scalar relativistic effects. The scalar relativistic effects cause a contraction and energetic stabilization of the s- and most p-states and due to the stronger screening of the nuclear charge and expansion and destabilization of the d- and f-states [Pyykkö, 2012]. The spin-orbit coupling effects introduce an additional quantum number $j$. For $2 l+2$ states of the $2 \cdot(2 l+1)$ degenerate spin-orbitals of the non-relativistic solution the spin and angular momentum are parallel $\left(j=l+\frac{1}{2}\right)$. These states have higher energy eigenvalues compared to the corresponding states in the nonrelativistic limit. The remaining $2 l$ states have anti-parallel spin and angular momentum $\left(j=l-\frac{1}{2}\right)$ and a lower energy than in the non-relativistic limit.

The ZORA-Hamiltonian in Eq. (2.87) can be split up into a scalar-relativistic contribution $\hat{H}_{\mathrm{ZORA}, \mathrm{SR}}$ and a spin-orbit coupling contribution $\hat{H}_{\mathrm{ZORA}, \mathrm{SO}}$

$$
\begin{aligned}
\hat{H}_{\mathrm{ZORA}} & =\hat{H}_{\mathrm{ZORA}, \mathrm{SR}}+\hat{H}_{\mathrm{ZORA}, \mathrm{SO}} \\
\hat{H}_{\mathrm{ZORA}, \mathrm{SR}} & =\hat{\vec{p}} \frac{c^{2}}{2 m_{e} c^{2}-e V} \hat{\vec{p}}+e V \\
\hat{H}_{\mathrm{ZORA}, \mathrm{SO}} & =\frac{\hbar e c^{2}}{\left(2 m_{e} c^{2}-e V\right)^{2}} \vec{\sigma} \cdot(\nabla V \times \hat{\vec{p}}) .
\end{aligned}
$$

Relativistic effects are treated in the CP-PAW code at the level of the scalar relativistic ZORA-Hamiltonian, i.e., $\hat{H}_{\mathrm{ZORA}, \mathrm{SR}}$. The partial waves and projectors of the projector augmented-wave method are constructed with the scalar ZORA-Hamiltonian. The projector augmented wave formalism is described in detail in section 7. In my Master thesis, I have developed and implemented an extension of the projector augmented-wave method from the scalar-relativistic ZORA-Hamiltonian to the four-component Dirac equation such that also spin-orbit coupling is included [Dal Corso, 2010; Schade, 2012]. However, since calculations that include spin-orbit coupling are naturally non-collinear spin-polarized calculations, they are much more numerically demanding than non-spin polarized or collinear spin-polarized calculations. Therefore, for the calculations presented in this thesis spinorbit coupling has been neglected, scalar-relativistic effects have been treated on the level of the ZORA-Hamiltonian for the augmentation.

\subsubsection{Relativistic two-particle interactions}

Many-particle Hamiltonians with two-particle interactions can be derived perturbatively from quantum electrodynamics. The first-order term in the perturbation expansion in $c^{-2}$ 
is the Dirac-Coulomb-Breit Hamiltonian (DCB, [Breit, 1929]) and can be written within the Born-Oppenheimer approximation as

$$
\hat{H}_{D C B}=\hat{H}_{D}+\frac{1}{2} \sum_{i \neq j} \hat{g}_{\text {Breit }}(i, j) .
$$

$\hat{H}_{D}$ is the one-particle Dirac Hamiltonian

$$
\hat{H}_{D}=c \sum_{i} \vec{\alpha}_{i} \cdot\left(\hat{\vec{p}}_{i}-e \vec{A}\right)+\beta m_{e} c^{2}+e V
$$

and acts on four-component Dirac spinors. The Dirac matrices with a subscript $i$, like $\alpha_{i}$, are the Dirac matrices in Eq. (2.77)-Eq. (2.80) corresponding to the electron $i$. The one-particle Dirac Hamiltonian depends on the external scalar potential

$$
V=-\frac{1}{2} \sum_{\beta, i} \frac{e Z_{\beta}}{4 \pi \epsilon_{0}\left|\hat{\vec{R}}_{\beta}-\hat{\vec{r}}_{i}\right|} \mathbb{1}_{4}
$$

and the external vector potential $\vec{A}$. The contributions to the two-particle Hamiltonian can be split up into

$$
\begin{aligned}
\hat{g}_{\text {Breit }}(i, j) & =\hat{g}_{\text {Coulomb }}(i, j)+\hat{g}_{\text {Gaunt }}(i, j)+\hat{g}_{\text {Gauge }}(i, j), \\
\hat{g}_{\text {Coulomb }}(i, j) & =\frac{e^{2}}{4 \pi \epsilon_{0}\left|\hat{\vec{r}}_{i}-\hat{\vec{r}}_{j}\right|}, \\
\hat{g}_{\text {Gaunt }}(i, j) & =-\hat{g}_{\text {Coulomb }}(i, j) \vec{\alpha}_{i} \cdot \vec{\alpha}_{j}, \\
\hat{g}_{\text {Gauge }}(i, j) & =-g_{\text {Coulomb }}(i, j)\left|\hat{\vec{r}}_{i}-\hat{\vec{r}}_{j}\right|\left(\vec{\alpha}_{i} \cdot \nabla_{i}\right)\left(\vec{\alpha}_{j} \cdot \nabla_{j}\right)\left|\hat{\vec{r}}_{i}-\hat{\vec{r}}_{j}\right| .
\end{aligned}
$$

The instantaneous Coulomb repulsion $\hat{g}_{\text {Coulomb }}(i, j)$ is often called the longitudinal part as it stems from the longitudinal part of the polarization function. All other contributions are usually referred to as transverse contributions and mainly contain magnetic interactions and retardation effects [Schwerdtfeger, 2004]. Table 2.1 shows results for the singleparticle energy levels of the neutral mercury atom from the exchange-only relativistic optimized effective potential method (x-only ROPM, x-only ROEP, [Engel et al., 1998]) for different relativistic approximations. The energy levels are given for the states $n l_{j}$, where $n$ denotes the principal quantum number $n, l$ the angular momentum quantum number and $j=l \pm 1 / 2$ and the total angular momentum quantum number. Comparing these eigenvalues from the fully relativistic potential, the Dirac-Coulomb-Breit approximation in Eq. (2.92) and the Dirac-Coulomb-approximation in Eq. (2.98) that the valence levels are practically unaffected by transverse contributions. Also, the effect of spin-orbit coupling, that is the difference between the energies of $n l_{l-\frac{1}{2}}$ and $n l_{l+\frac{1}{2}}$, is much more significant than the transversal effects for all states. Thus, the transversal effects are only considered for extremely heavy atoms [Engel and Dreizler, 2011] and for all calculations presented in this thesis we used only the instantaneous Coulomb repulsion. 
2. The quantum many-particle problem

\begin{tabular}{l|rrr|} 
state $n l_{l \pm \frac{1}{2}}$ & REXX & \\
& C+full transverse & $\mathrm{C}+$ Breit & $\mathrm{C}$ \\
\hline $1 \mathrm{~s}_{\frac{1}{2}}$ & -3036.871 & -3032.278 & -3047.431 \\
$2 \mathrm{p}_{\frac{1}{2}}$ & -516.198 & -515.546 & -518.062 \\
$2 \mathrm{p}_{\frac{3}{2}}$ & -445.422 & -445.013 & -446.683 \\
$4 \mathrm{f}_{\frac{5}{2}}$ & -3.766 & -3.770 & -3.757 \\
$4 \mathrm{f}_{\frac{7}{2}}$ & -3.613 & -3.616 & -3.603 \\
$5 \mathrm{~s}_{\frac{1}{2}}$ & -4.394 & -4.394 & -4.404 \\
$5 \mathrm{p}_{\frac{1}{2}}$ & -3.004 & -3.002 & -3.013 \\
$5 \mathrm{p}_{\frac{3}{2}}$ & -2.360 & -2.360 & -2.364 \\
$5 \mathrm{~d}_{\frac{3}{2}}$ & -0.507 & -0.507 & -0.506 \\
$5 \mathrm{~d}_{\frac{5}{2}}$ & -0.440 & -0.441 & -0.440 \\
$6 \mathrm{~s}_{\frac{1}{2}}$ & -0.330 & -0.330 & -0.330
\end{tabular}

Table 2.1.: Selected single-particle energy eigenvalues in Hartree from the relativistic optimized effective potential method [Engel et al., 1998] for the neutral mercury atom from an relativistic exchange-only (REXX) treatment of the complete relativistic exchange-only potential $(\mathrm{C}+$ full transverse), i.e. including the complete transverse contribution beyond the Breit approximation, the Dirac-Coulomb-Breit approximation (C+Breit) in Eq. (2.92) and the DiracCoulomb-approximation (C) using only $\hat{g}_{\text {Coulomb }}(i, j)$.

\subsection{Reduced density matrices}

\subsubsection{Basic definitions}

The non-relativistic many-particle Born-Oppenheimer-Hamiltonian $\hat{H}$ of Eq. (2.65) of a molecule or solid discussed in detail in section 2.6 only contains the kinetic energy of the electrons $\hat{T}_{e}$, a local one-particle potential ${ }^{10} \hat{V}_{e n}$ and the electron-electron interaction $\hat{V}_{e e}$. Thus, written with real-space field operators we get the form

$$
\begin{aligned}
\hat{H} & =\int d^{4} \vec{x} \hat{\Psi}^{\dagger}(\vec{x})\left(-\frac{\hbar^{2} \nabla^{2}}{2 m_{e}}-\frac{1}{2} \sum_{A} \frac{e^{2} Z_{A}}{4 \pi \epsilon_{0}\left|\vec{R}_{A}-\vec{r}\right|}\right) \hat{\Psi}(\vec{x}) \\
& +\frac{1}{2} \int d^{4} \vec{x} \int d^{4} \vec{x}^{\prime} \frac{e^{2} \hat{\Psi}^{\dagger}(\vec{x}) \hat{\Psi}^{\dagger}\left(\vec{x}^{\prime}\right) \hat{\Psi}\left(\vec{x}^{\prime}\right) \hat{\Psi}(\vec{x})}{4 \pi \epsilon_{0}\left|\vec{r}-\vec{r}^{\prime}\right|}
\end{aligned}
$$

Therefore, we can express the Hamiltonian with the one-particle reduced density operator

$$
\hat{\rho}^{(1)}\left(\vec{x} ; \vec{x}^{\prime}\right)=\hat{\Psi}^{\dagger}\left(\vec{x}^{\prime}\right) \hat{\Psi}(\vec{x})
$$

and the two-particle reduced density operator

$$
\hat{\rho}^{(2)}\left(\vec{x}_{1}, \vec{x}_{2} ; \vec{x}_{1}^{\prime}, \vec{x}_{2}^{\prime}\right)=\hat{\Psi}^{\dagger}\left(\vec{x}_{2}^{\prime}\right) \hat{\Psi}^{\dagger}\left(\vec{x}_{1}^{\prime}\right) \hat{\Psi}\left(\vec{x}_{1}\right) \hat{\Psi}_{\left(\vec{x}_{2}\right)}
$$

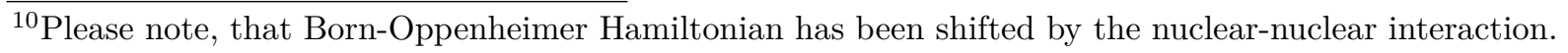


in the form

$$
\begin{aligned}
\hat{H} & =\int d^{4} \vec{x} \lim _{\vec{x}^{\prime} \rightarrow \vec{x}}\left(-\frac{\hbar^{2} \nabla^{2}}{2 m_{e}} \rho^{(1)}\left(\vec{x} ; \vec{x}^{\prime}\right)\right)-\frac{1}{2} \sum_{A} \frac{e^{2} Z_{A}}{4 \pi \epsilon_{0}\left|\vec{R}_{A}-\vec{r}\right|} \hat{\rho}^{(1)}(\vec{x}, \vec{x}) \\
& +\frac{1}{2} \int d^{4} \vec{x} \int d^{4} \vec{x}^{\prime} \frac{e^{2} \hat{\rho}^{(2)}\left(\vec{x}^{\prime}, \vec{x} ; \vec{x}^{\prime}, \vec{x}\right)}{4 \pi \epsilon_{0}\left|\vec{r}-\vec{r}^{\prime}\right|} .
\end{aligned}
$$

It can be further simplified by noting that the diagonal elements of the one-particle reduced density operator $\hat{\rho}^{(1)}(\vec{x} ; \vec{x})$ in real space are just the operators of the one-particle spin density

$$
\hat{n}(\vec{x})=\hat{n}_{\sigma}(\vec{r})=\hat{\rho}^{(1)}(\vec{x} ; \vec{x})
$$

and that the two-particle reduced density operator $\hat{\rho}^{(2)}\left(\vec{x}^{\prime}, \vec{x} ; \vec{x}^{\prime}, \vec{x}\right)$ represents the operator of the two-particle density

$$
\hat{n}^{(2)}\left(\vec{x}^{\prime}, \vec{x}\right)=\hat{\rho}^{(2)}\left(\vec{x}^{\prime}, \vec{x} ; \vec{x}^{\prime}, \vec{x}\right) .
$$

Thus, we can rewrite the Hamiltonian with these quantities as

$$
\begin{aligned}
\hat{H} & =\int d^{4} \vec{x} \lim _{\vec{x}^{\prime} \rightarrow \vec{x}}\left(-\frac{\hbar^{2} \nabla^{2}}{2 m_{e}} \rho^{(1)}\left(\vec{x} ; \vec{x}^{\prime}\right)\right)+\int d^{4} \vec{x} v_{\mathrm{ext}}(\vec{x}) \hat{n}(\vec{x}) \\
& +\frac{1}{2} \int d^{4} \vec{x} \int d^{4} \vec{x}^{\prime} \frac{e^{2} \hat{n}^{(2)}\left(\vec{x}, \vec{x}^{\prime}\right)}{4 \pi \epsilon_{0}\left|\vec{r}-\vec{r}^{\prime}\right|}
\end{aligned}
$$

with the definition of the external potential $v_{\text {ext }}(\vec{x})$ as

$$
v_{\text {ext }}(\vec{x})=-\frac{1}{2} \sum_{A} \frac{e^{2} Z_{A}}{4 \pi \epsilon_{0}\left|\vec{R}_{A}-\vec{r}\right|} .
$$

The more general form of the Hamiltonian

$$
\begin{aligned}
\hat{H} & =\int d^{4} \vec{x} \lim _{\vec{x}^{\prime} \rightarrow \vec{x}}\left(-\frac{\hbar^{2} \nabla^{2}}{2 m_{e}} \rho^{(1)}\left(\vec{x} ; \vec{x}^{\prime}\right)\right)+\int d^{4} \vec{x} \int d^{4} \vec{x}^{\prime} v_{\mathrm{ext}}\left(\vec{x}, \vec{x}^{\prime}\right) \hat{\rho}^{(1)}\left(\vec{x}^{\prime} ; \vec{x}\right) \\
& +\frac{1}{2} \int d^{4} \vec{x} \int d^{4} \vec{x}^{\prime} \frac{e^{2} \hat{n}^{(2)}\left(\vec{x}, \vec{x}^{\prime}\right)}{4 \pi \epsilon_{0}\left|\vec{r}-\vec{r}^{\prime}\right|}
\end{aligned}
$$

with a non-local external potential $v_{\text {ext }}\left(\vec{x}, \vec{x}^{\prime}\right)$ will be used later in the context of reduced density-matrix functional theory.

The definition of the one-particle and two-particle reduced density operator can be generalized to the $p$-particle reduced density operator

$$
\hat{\rho}^{(p)}\left(\vec{x}_{1}, \ldots, \vec{x}_{p} ; \vec{x}_{1}^{\prime}, \ldots, \vec{x}_{p}^{\prime}\right)=\hat{\Psi}^{\dagger}\left(\vec{x}_{p}^{\prime}\right) \ldots \hat{\Psi}^{\dagger}\left(\vec{x}_{1}^{\prime}\right) \hat{\Psi}\left(\vec{x}_{1}\right) \ldots \hat{\Psi}\left(\vec{x}_{p}\right) .
$$

Please note that there are different normalization conventions for the $p$-particle reduced density operators and $p$-particle reduced density matrices. We choose here the definition by Eq. (2.108) that is common in solid state theory and is for example also used in [Coleman and Yukalov, 2000] or [McWeeny, 1960]. With this definition the two-particle density $\hat{n}^{(2)}\left(\vec{x}_{1}, \vec{x}_{2}\right)$ describes the probability that a particle is at $\vec{x}_{1}$ and another particle at $\vec{x}_{2}$ 
irrespective of their order. However, it should be noted that with this definition the $p=N$-case of the $p$-particle reduced density operator in Eq. (2.108) is not identical to the $N$-particle density operator $\hat{\rho}^{(N)}$ defined in Eq. (2.23) but has an additional factor $N$ !. The alternative definition

$$
\hat{\tilde{\rho}}^{(p)}\left(\vec{x}_{1}, \ldots, \vec{x}_{p} ; \vec{x}_{1}^{\prime}, \ldots, \vec{x}_{p}^{\prime}\right)=\frac{1}{p !} \hat{\rho}^{(p)}\left(\vec{x}_{1}, \ldots, \vec{x}_{p} ; \vec{x}_{1}^{\prime}, \ldots, \vec{x}_{p}^{\prime}\right)
$$

is common in quantum chemistry and used for example in [Cohen and Frishberg, 1976].

The one-particle and two-particle density operators can be generalized to the $p$-particle density operator

$$
\hat{n}^{(p)}\left(\vec{x}_{1}, \ldots, \vec{x}_{p}\right)=\hat{\rho}^{(p)}\left(\vec{x}_{1}, \ldots, \vec{x}_{p} ; \vec{x}_{1}, \ldots, \vec{x}_{p}\right)=\hat{\Psi}^{\dagger}\left(\vec{x}_{p}\right) \ldots \hat{\Psi}^{\dagger}\left(\vec{x}_{1}\right) \hat{\Psi}\left(\vec{x}_{1}\right) \ldots \hat{\Psi}\left(\vec{x}_{p}\right) .
$$

The expectation value of the $p$-particle reduced density operator is the $p$-particle reduced density matrix

$$
\begin{aligned}
\rho^{(p)}\left(\vec{x}_{1}, \ldots, \vec{x}_{p} ; \vec{x}_{1}^{\prime}, \ldots, \vec{x}_{p}^{\prime}\right) & =\frac{N !}{(N-p) !} \int d^{4} \vec{x}_{p+1} \ldots \int d^{4} \vec{x}_{N} \\
& \rho^{(N)}\left(\vec{x}_{1}, \ldots, \vec{x}_{p}, \vec{x}_{p+1}, \ldots, \vec{x}_{N} ; \vec{x}_{1}^{\prime}, \ldots, \vec{x}_{p}^{\prime}, \vec{x}_{p+1}, \ldots, \vec{x}_{N}\right) .
\end{aligned}
$$

The trace of $p$-particle reduced density matrices is

$$
\operatorname{Tr} \rho^{(p)}=\frac{N !}{(N-p) !}
$$

and they follow the reduction property

$$
\rho^{(p-1)}\left(\vec{x}_{1}, \ldots, \vec{x}_{p-1} ; \vec{x}_{1}^{\prime}, \ldots, \vec{x}_{p-1}^{\prime}\right)=\frac{1}{N-p+1} \int d^{4} \vec{x}_{p} \rho^{(p)}\left(\vec{x}_{1}, \ldots, \vec{x}_{p} ; \vec{x}_{1}^{\prime}, \ldots, \vec{x}_{p}\right)
$$

if they corresponds to an $N$-particle state. Thus, the expectation value of the manyparticle Hamiltonian in Eq. (2.107) can be written as a function of the two-particle reduced density matrix in the form

$$
\left\langle\Psi^{N}|\hat{H}| \Psi^{N}\right\rangle=\int d^{4} \vec{x}_{1} \int d^{4} \vec{x}_{2} \int d^{4} \vec{x}_{3} \int d^{4} \vec{x}_{4} \hat{H}\left(\vec{x}_{1}, \vec{x}_{2}, \vec{x}_{3}, \vec{x}_{4}\right) \rho^{(2)}\left(\vec{x}_{1}, \vec{x}_{2} ; \vec{x}_{3}, \vec{x}_{4}\right),
$$

where the operator $\hat{H}\left(\vec{x}_{1}, \vec{x}_{2}, \vec{x}_{3}, \vec{x}_{4}\right)$ is defined implicitly by Eq. (2.107). In a finitedimensional one-particle basis $\left\{\left|\chi_{i}\right\rangle\right\}_{i=1, \ldots, d}$ of dimension $d$ the $p$-particle reduced density operator has the form

$$
\hat{\rho}_{\alpha_{1}, \ldots, \alpha_{p} ; \alpha_{1}^{\prime}, \ldots, \alpha_{p}^{\prime}}^{(p)}=\hat{c}_{\alpha_{p}^{\prime}}^{\dagger} \ldots \hat{c}_{\alpha_{1}^{\prime}}^{\dagger} \hat{c}_{\alpha_{1}} \ldots \hat{c}_{\alpha_{p}}
$$

and the $p$-particle reduced density matrix has the reduction property

$$
\rho_{\alpha_{1}, \ldots, \alpha_{p} ; \alpha_{1}^{\prime}, \ldots, \alpha_{p-1}^{\prime}}^{(p-1)}=\frac{1}{N-p+1} \sum_{\alpha_{p}} \rho_{\alpha_{1}, \ldots, \alpha_{p-1}, \alpha_{p} ; \alpha_{1}^{\prime}, \ldots, \alpha_{p-1}^{\prime}, \alpha_{p}}^{(p)}
$$


if it corresponds to an $N$-particle state. A general Hamiltonian $\hat{H}=\hat{h}+\hat{W}$ with a one-particle contribution $\hat{h}$ and a two-particle interaction $\hat{W}$ can be written in a finite one-particle basis as

$$
\begin{aligned}
\hat{H} & =\hat{h}+\hat{W} \\
& =\sum_{\alpha, \beta} h_{\alpha, \beta} \hat{c}_{\alpha}^{\dagger} \hat{c}_{\beta}+\frac{1}{2} \sum_{\alpha, \beta, \gamma, \delta} U_{\alpha, \beta, \gamma, \delta} \hat{c}_{\alpha}^{\dagger} \hat{c}_{\beta}^{\dagger} \hat{c}_{\delta} \hat{c}_{\gamma} .
\end{aligned}
$$

For the Born-Oppenheimer Hamiltonian, we have for the matrix elements in Eq. (2.117) the expressions

$$
\begin{aligned}
h_{\alpha, \beta} & =\left\langle\chi_{\alpha}\left|-\frac{\hbar^{2} \nabla^{2}}{2 m_{e}}-\frac{1}{2} \sum_{A} \frac{e^{2} Z_{A}}{4 \pi \epsilon_{0}\left|\vec{R}_{A}-\vec{r}\right|}\right| \chi_{\beta}\right\rangle \\
U_{\alpha, \beta, \gamma, \delta} & =\int d^{4} \vec{x} \int d^{4} \vec{x}^{\prime} \frac{e^{2}}{4 \pi \epsilon_{0}\left|\vec{r}-\vec{r}^{\prime}\right|} \chi_{\alpha}^{*}(\vec{x}) \chi_{\beta}^{*}\left(\vec{x}^{\prime}\right) \chi_{\gamma}(\vec{x}) \chi_{\delta}\left(\vec{x}^{\prime}\right) .
\end{aligned}
$$

Please note, that for the matrix elements of the interaction there are different conventions: the definition used in solid-state theory is also used in this thesis and is given in Eq. (2.119). On the other hand, the definition that is commonly used in quantum chemistry is

$$
U_{\text {chem }, \alpha, \beta, \gamma, \delta}=U_{\alpha, \gamma, \beta, \delta}=\int d^{4} \vec{x} \int d^{4} \vec{x}^{\prime} \frac{e^{2}}{4 \pi \epsilon_{0}\left|\vec{r}-\vec{r}^{\prime}\right|} \chi_{\alpha}^{*}(\vec{x}) \chi_{\beta}(\vec{x}) \chi_{\gamma}^{*}\left(\vec{x}^{\prime}\right) \chi_{\delta}\left(\vec{x}^{\prime}\right) .
$$

\subsubsection{Natural orbitals and occupations}

The one-particle reduced density matrix is a hermitian matrix. Thus, its eigenvalues are real. Löwdin has coined the term occupations for the eigenvalues $f_{i}$ and the term natural orbitals for the eigenvectors $|\phi\rangle$ of the one-particle reduced density matrix [Löwdin, 1955]. Thus, the one-particle reduced density matrix can be written in the spectral representation as

$$
\begin{aligned}
\rho^{(1)}\left(\vec{x} ; \vec{x}^{\prime}\right) & =\sum_{i} f_{i} \phi_{i}(\vec{x}) \phi_{i}^{*}\left(\vec{x}^{\prime}\right) \\
\rho_{\alpha, \beta}^{(1)} & =\sum_{i} f_{i} \phi_{i, \alpha} \phi_{i, \beta}^{*} .
\end{aligned}
$$

The natural orbitals are defined to be orthonormal

$$
\begin{aligned}
\int d^{4} \vec{x} \phi_{i}^{*}(\vec{x}) \phi_{j}(\vec{x}) & =\delta_{i, j} \\
\sum_{\alpha} \phi_{i, \alpha} \phi_{j, \beta}^{*} & =\delta_{i, j}
\end{aligned}
$$

and the occupations sum up to the trace of the one-particle reduced density matrix, i.e.,

$$
\operatorname{Tr} \rho^{(1)}=\sum_{i} f_{i}
$$

The natural orbitals and occupations in the spectral representation in Eq. (2.121) are uniquely defined up to phase factors if the spectrum of the one-particle reduced density 
matrix is non-degenerate. For a degenerate spectrum, the natural orbitals are defined up to unitary rotations in the degenerate sectors.

If the occupations are integers, i.e., if $f_{i} \in\{0,1\}$, then a many-particle wave function can be constructed, that only contains the single Slater determinant

$$
|\Psi\rangle=\left|f_{1}, f_{2}, \ldots\right\rangle
$$

in the one-particle basis of the natural orbitals.

\subsubsection{The $\mathrm{N}$-representability problem}

With the reduction relation of the two-particle reduced density matrix given in Eq. (2.116), we can write the energy for a $N$-particle state purely in terms of the two-particle reduced density matrix $\rho_{\alpha, \beta, \gamma, \delta}^{(2)}$ as $^{11}$

$$
\left\langle\Psi^{N}|\hat{H}| \Psi^{N}\right\rangle=\sum_{\alpha, \beta, \gamma, \delta} \rho_{\alpha, \beta, \gamma, \delta}^{(2)}\left(\frac{1}{N-1} h_{\gamma, \alpha} \delta_{\beta, \delta}+\frac{1}{2} U_{\delta, \gamma, \beta, \alpha}\right) .
$$

Thus, the energy expectation can be written in terms of the two-particle reduced density matrix, which is an element of $\mathbb{C}^{N \times N \times N \times N}$. If the ground-state energy could be evaluated exactly by solving the minimization problem

$$
\tilde{E}_{0}=\min _{\rho_{\alpha, \beta, \gamma, \delta}^{(2)}} \sum_{\alpha, \beta, \gamma, \delta} \rho_{\alpha, \beta, \gamma, \delta}^{(2)}\left(\frac{1}{N-1} h_{\gamma, \alpha} \delta_{\beta, \delta}+\frac{1}{2} U_{\delta, \gamma, \beta, \alpha}\right),
$$

we would have a solved the quantum many-body problem in polynomial runtime complexity. However, the energy $\tilde{E}_{0}$ from this minimization severely underestimates the exact ground-state energy. This is due to the fact, that the two-particle reduced density matrix at the minimum does not necessarily correspond to an $N$-particle wave function or an ensemble of $N$-particle wave functions. The condition that a $p$-particle reduced density matrix corresponds to an ensemble of $N$-particle wave functions $\left\{\left|\Psi_{i}^{N}\right\rangle\right\}$ and ensemble probabilities $P_{i}\left(P_{i}>0, \sum_{i} P_{i}=1\right)$ in the form

$$
\rho_{\alpha_{1}, \ldots, \alpha_{p}, \beta_{1}, \ldots, \beta_{p}}^{(p)}=\sum_{i} P_{i}\left\langle\Psi_{i}^{N}\left|\hat{c}_{\beta_{p}}^{\dagger} \ldots \hat{c}_{\beta_{1}}^{\dagger} \hat{c}_{\alpha_{1}} \ldots \hat{c}_{\alpha_{p}}\right| \Psi_{i}^{N}\right\rangle .
$$

has been termed the ensemble N-representability condition of the $p$-particle reduced density matrix [Coleman, 1963; Garrod and Percus, 1964; Kummer, 1967; Erdahl, 1978; Mazziotti and Erdahl, 2001; Mazziotti, 2012]. On the other hand, the condition that a $p$-particle reduced density matrix corresponds to an $N$-particle wave function $\left|\Psi^{N}\right\rangle$ via

$$
\rho_{\alpha_{1}, \ldots, \alpha_{p}, \beta_{1}, \ldots, \beta_{p}}^{(p)}=\left\langle\Psi^{N}\left|\hat{c}_{\beta_{p}}^{\dagger} \ldots \hat{c}_{\beta_{1}}^{\dagger} \hat{c}_{\alpha_{1}} \ldots \hat{c}_{\alpha_{p}}\right| \Psi^{N}\right\rangle
$$

has been termed the pure-state N-representability condition, that is also known as the generalized Pauli constraint, of the p-particle reduced density matrix [Müller, 1999; Altunbulak and Klyachko, 2008a; Chakraborty and Mazziotti, 2014; Theophilou et al., 2015; DePrince, 2016].

\footnotetext{
${ }^{11}$ We discuss the N-representability for the case of a finite-dimensional one-particle basis. The equations for the real-space representation are analogous.
} 
Either the ensemble or pure-state N-representability condition have to be enforced in Eq. (2.128) during the minimization. Thus, the minimization problem to be solved is

$$
\tilde{E}=\min _{\rho_{\alpha, \beta, \gamma, \delta}^{(2)}: \exists\left\{\left|\Psi_{i}^{N}\right\rangle, P_{i}\right\}} \sum_{\text {that fulfills Eq. } 2.129} \rho_{\alpha, \beta, \gamma, \delta}^{(2)} \rho_{\alpha, \beta, \gamma, \delta}\left(\frac{1}{N-1} h_{\gamma, \alpha} \delta_{\beta, \delta}+\frac{1}{2} U_{\delta, \gamma, \beta, \alpha}\right) .
$$

The minimum $\tilde{E}$ of this problem is identical to the exact ground-state energy $E_{0 \text {,exact }}$. The complete set of $N$-representability conditions for the two-particle reduced density matrix have been formulated by Maziotti [Mazziotti, 2012]. Approaches that employ the minimization in Eq. (2.131) and a subset of the $N$-particle representability constraints for the two-particle reduced density matrix belong to the class of non-perturbative variational two-particle reduced density-matrix functional theories (2RDMFT, [Erdahl, 1979; Mazziotti and Erdahl, 2001; Zhao et al., 2004; Hammond and Mazziotti, 2005; Nakata et al., 2001; Mazziotti, 2002; Verstichel et al., 2011; Mazziotti, 2011; Verstichel, 2012]). In chapter 10 we discuss the N-representability conditions for the two-particle reduced density matrix in detail and show how the reduced density-matrix functional of the oneparticle reduced density matrix can be obtained from the N-representability conditions of the two-particle reduced density matrix with a 2RDMFT-like procedure.

The ensemble N-representability conditions of the one-particle reduced density matrix have been given by Coleman [Coleman, 1963]. Coleman has shown that a hermitian matrix is ensemble N-representable if and only if the eigenvalues $f_{i}$ lie between zero and one, i.e., $0 \leq f_{i} \leq 1$. This is equivalent to the condition, that $\rho^{(1)}$ and $\mathbb{1}-\rho^{(1)}$ are positive semi-definite, in short $0 \leq \rho^{(1)} \leq \mathbb{1}$.

The pure-state N-representability conditions for the one-particle reduced density matrix are still an open issue. It is known that these pure-state N-representability conditions take the form of inequalities of weighted sums of eigenvalues of the one-particle reduced density matrix. Borland and Dennis [Borland and Dennis, 1972] have shown that for three particles in a one-particle basis of six one-particle states the conditions can be written as

$$
\begin{array}{r}
f_{1}+f_{6}=1 \\
f_{2}+f_{5}=1 \\
f_{3}+f_{4}=1 \\
f_{5}+f_{6}-f_{4} \geq 0 .
\end{array}
$$

Even though Altunbulak and Klyachko [Klyachko, 2006; Altunbulak and Klyachko, 2008b] have proposed a systematic way to construct the pure-state N-representability conditions, an efficient way to derive the condition for one-particle bases of practically relevant sizes has not been found yet. It should be noted here, that any ensemble N-representable one-particle reduced density matrix can be represented with only one many-particle wave function $|\Psi\rangle$ that is not necessarily an $N$-particle wave function as

$$
\rho_{\alpha, \beta}^{(1)}=\left\langle\Psi\left|\hat{c}_{\beta}^{\dagger} \hat{c}_{\alpha}\right| \Psi\right\rangle
$$

A simple scheme for the construction of this wave function is available [Walther, 2011] and described in appendix $\mathrm{C}$. 


\subsection{Metals and insulators}

\subsubsection{Distinction between metals and insulators}

We begin with the discussion of when a material or model is classified as an insulator or a metal. For this purpose, we follow the discussions by Scalapino, White and Zhang [Scalapino et al., 1993] as well as Scalettar [Scalettar, 2017], Resta [Resta, 2017], and Gebhard [Gebhard, 2003]. We assume a perfect crystal without impurities and neglect superconductors. The central quantity for the distinction of metals and insulators is the electrical conductivity $\sigma$. The electrical conductivity is a three-dimensional tensor that relates the current density $\vec{j}$ of electrons to the total electric field $\vec{E}(\vec{r}, t)=\vec{E}_{0} e^{i \vec{q} \cdot \vec{r}-i \omega t}$ in linear response for small wave vectors $\vec{q}$ as

$$
\vec{j}(\vec{r}, t)=\sigma(\vec{q}, \omega) \vec{E}_{0} e^{i \vec{q} \cdot \vec{r}-i \omega t}
$$

where $\omega$ denotes the frequency. For the following discussion, we assume a macroscopically homogeneous system and a homogeneous electric field, so that only the long-wavelength case $\vec{q} \rightarrow 0$ is of interest. A material is an insulator if the real part of its dc conductivity at zero temperature

$$
\sigma_{\mathrm{dc}, T=0, i, j}=\lim _{T \rightarrow 0} \lim _{\omega \rightarrow 0} \sigma_{i, j}(\vec{q}=0, \omega)
$$

vanishes. In linear-response theory a Kubo-formula for the conductivity [Louie and Cohen, 2006; Mahan, 2013]

$$
\sigma_{i, j}(\vec{q}, \omega)=\frac{i n e^{2}}{m \omega} \delta_{i, j}+\frac{1}{\hbar \omega V} \int_{0}^{\infty} d t e^{i \omega t}\left\langle\left[\hat{j}_{i}^{\dagger}(\vec{q}, t), \hat{j}_{j}(\vec{q}, 0)\right]\right\rangle_{\beta}
$$

can be derived, where $m$ denotes the electron mass, $n$ the density of charge-carrying electrons, $V$ the volume and $\beta$ the inverse temperature. The retarded current-current correlation function $\left\langle\left[\hat{j}_{i}^{\dagger}(\vec{q}, t), \hat{j}_{j}(\vec{q}, 0)\right]\right\rangle_{\beta}$ in Eq. (2.139) is evaluated with respect to the canonical ensemble at inverse temperature $\beta$. In the $\omega \rightarrow 0$-limit the first term in Eq. (2.139) diverges and this divergence is compensated by the second term. With a complete set of eigenfunctions $\left|\Psi_{n}\right\rangle$ and eigenvalues $E_{n}$ of the many-particle Hamiltonian $\hat{H}$ of the system, Eq. (2.139) can be written as [Louie and Cohen, 2006]

$$
\sigma_{i, j}(\vec{q}, \omega)=\frac{i n e^{2}}{m \omega} \delta_{i, j}+\lim _{\eta \rightarrow 0} \frac{i}{\omega V} \sum_{n, m} \frac{e^{-\beta E_{n}}-e^{-\beta E_{m}}}{Z} \frac{\left\langle\Psi_{n}\left|\hat{j}_{i}^{\dagger}(\vec{q})\right| \Psi_{m}\right\rangle\left\langle\Psi_{m}\left|\hat{j}_{j}(\vec{q})\right| \Psi_{n}\right\rangle}{\hbar(\omega+i \eta)-\left(E_{m}-E_{n}\right)} .
$$

In the $\vec{q}=0$-limit, one obtains for the real part

$$
\begin{aligned}
\operatorname{Re} \sigma_{i, j}(\vec{q}=0, \omega) & =\frac{\pi}{\omega V} \sum_{n, m} \frac{e^{-\beta E_{n}}-e^{-\beta E_{m}}}{Z} \\
& \cdot\left\langle\Psi_{n}\left|\hat{j}_{i}^{\dagger}(\vec{q}=0)\right| \Psi_{m}\right\rangle\left\langle\Psi_{m}\left|\hat{j}_{j}(\vec{q}=0)\right| \Psi_{n}\right\rangle \delta\left(\hbar \omega-\left(E_{m}-E_{n}\right)\right) .
\end{aligned}
$$

The real part of the conductivity in the $\vec{q}=0$-limit given in Eq. (2.141) obeys the sum rule

$$
\int_{0}^{\infty} d \omega \operatorname{Re} \sigma_{i, i}(\vec{q}=0, \omega)=\frac{\pi e^{2} n}{2 m}
$$


The conductivity for non-interacting free electrons is

$$
\operatorname{Re} \sigma_{i, i}(\vec{q}=0, \omega)=\frac{\pi e^{2} n}{m} \delta(\omega)
$$

The factor $\pi e^{2} n / m$ in known as the Drude weight $D_{\text {non-int,free }}$ of non-interacting free electrons. Thus, the sum rule in Eq. (2.142) can be expressed as

$$
\int_{0}^{\infty} d \omega \operatorname{Re} \sigma_{i, i}(\vec{q}=0, \omega)=\frac{D_{\text {non-int,free }}}{2}
$$

If electrons are in a periodic potential of ions or electron-electron interaction is added, the conductivity in the $\vec{q}=0$-limit can be written as a sum of two terms [Resta, 2017]

$$
\sigma_{i, j}(\vec{q}=0, \omega)=D_{i, j}\left[\delta(\omega)+\frac{i}{\pi \omega}\right]+\tilde{\sigma}_{i, j}(\omega)
$$

$D_{i, j}$ is now the system-specific Drude weight. Consequently, the first term is the Drudelike term and the second term contains the remaining effects. The sum rule obtains the form

$$
\int_{0}^{\infty} d \omega \operatorname{Re} \tilde{\sigma}_{i, i}(\vec{q}=0, \omega)=\frac{D_{\text {non-int,free }}-D_{i, i}}{2}
$$

For an insulator, the Drude-like weight $D_{i, j}$ is zero and the $\operatorname{Re} \tilde{\sigma}_{i, j}(\vec{q}=0, \omega)$ vanishes in the $\omega \rightarrow 0$-limit at zero temperature. The Drude weight $D_{i, j}$ can in principle be calculated from Eq. (2.139) and is hence related to the retarded current-current correlation function, which is a two-particle correlation function.

However, it would be advantageous to distinguish metals and insulators based on a criterion of a one-particle quantity. The main requirements for electron transport as formulated by Gebhard [Gebhard, 2003] are

1. the availability of states for particle-hole excitations with energies above but close to the energy of the ground state

2. and that these excitations correspond to delocalized charges that can contribute to transport on a macroscopic scale.

The first requirement is immediately clear from the form of the conductivity in Eq. (2.141): for a finite conductivity, some of the matrix elements $\left\langle\Psi_{n}\left|\hat{j}_{i}\right| \Psi_{m}\right\rangle$ have to be finite. The operator of the electron current-density

$$
\hat{\vec{j}}=-\frac{e}{m} \sum_{j} \hat{\vec{p}}_{j}
$$

contains the momentum operator of the $j$-th electron $\hat{\vec{p}}_{j}$ which in a finite one-particle basis can be written as

$$
\hat{\vec{p}}_{j}=\sum_{\alpha, \beta}\left\langle\chi_{\alpha}|-i \hbar \nabla| \chi_{\beta}\right\rangle \hat{c}_{\alpha}^{\dagger} \hat{c}_{\beta}
$$

and is hence related to particle-hole excitations. The second requirement excludes Anderson insulators from electron transport because even though states near the Fermi energy 
might be available, these states are localized and cannot contribute to a macroscopic conductivity. Thus, with the exception of Anderson insulators or superconducting ground states, the gap as defined by Eq. (2.48) or Eq. (2.49) is a good quantity to characterize insulators in practice: a material with a finite gap of charge-carrying excitations at zero temperature is an insulator, otherwise a metal. This characterization explains why estimates of the gap are of interest.

Another tool for the distinction of metals and insulators is the one-particle spectral function $A_{\alpha, \beta}(\epsilon)$. It describes the photoemission and inverse photoemission spectrum of a system. Consider a complete set of eigenfunctions $\left|\Psi_{n}\right\rangle$ and eigenenergies $E_{n}$ of a particle-number conserving many-particle Hamiltonian $\hat{H}$ that are chosen as simultaneous eigenfunctions of the operator $\hat{N}$ of the total particle number, i.e.,

$$
\begin{aligned}
& \hat{H}\left|\Psi_{n}\right\rangle=E_{n}\left|\Psi_{n}\right\rangle \\
& \hat{N}\left|\Psi_{n}\right\rangle=N_{n}\left|\Psi_{n}\right\rangle .
\end{aligned}
$$

The one-particle spectral function can be written in the Lehmann representation as

$$
A_{\alpha, \beta}(\epsilon)=\frac{1}{Z} \sum_{m, n}\left(e^{-\beta\left(E_{n}-\mu N_{n}\right)}+e^{-\beta\left(E_{m}-\mu N_{m}\right)}\right)\left\langle\Psi_{n}\left|\hat{c}_{\alpha}\right| \Psi_{m}\right\rangle\left\langle\Psi_{m}\left|\hat{c}_{\beta}^{\dagger}\right| \Psi_{n}\right\rangle \delta\left(\epsilon+E_{n}-E_{m}\right) .
$$

$Z$ denotes the grand canonical partition function. Thus, it can also be written as the imaginary path,

$$
A_{\alpha, \beta}(\epsilon)=-\frac{1}{\pi} \operatorname{Im} G_{\alpha, \beta}\left(\epsilon+i 0^{+}\right),
$$

of the Matsubara Green function [Matsubara, 1955]

$$
G_{\alpha, \beta}\left(i \omega_{\nu}\right)=\frac{1}{Z} \sum_{m, n} \frac{e^{-\beta\left(E_{n}-\mu N_{n}\right)}+e^{-\beta\left(E_{m}-\mu N_{m}\right)}}{i \omega_{\nu}+\mu-\left(E_{m}-E_{n}\right)}\left\langle\Psi_{n}\left|\hat{c}_{\alpha}\right| \Psi_{m}\right\rangle\left\langle\Psi_{m}\left|\hat{c}_{\beta}^{\dagger}\right| \Psi_{n}\right\rangle
$$

with the Matsubara frequencies $\omega_{\nu}=(2 \nu+1) \pi / \beta$. The one-particle spectral function obeys the sum rule

$$
\int_{-\infty}^{\infty} d \epsilon A_{\alpha, \beta}(\epsilon)=\delta_{\alpha, \beta}
$$

and defines the one-particle density of states as

$$
D(\epsilon)=\sum_{\alpha} A_{\alpha, \alpha}(\epsilon)
$$

The projected density of states

$$
D_{i, j}(\epsilon)=\sum_{\vec{k}} A_{(\vec{k}, i),(\vec{k}, j)}(\epsilon) .
$$

are the partial sum of the spectral function over all k-points. The remaining indexes $i$ and $j$ count some one-particle states in the unit cell.

In infinite dimensions [Pruschke et al., 1993] or if interactions of individual charge excitations can be neglected, the vertex corrections to the conductivity vanish and the 
static conductivity can be written as [Schweitzer and Czycholl, 1991; Pruschke et al., 1993]

$$
\left.\sigma_{i, i}(\vec{q}=0, \omega \rightarrow 0) \propto \sum_{\alpha, \beta} \int_{-\infty}^{\infty} d \epsilon A_{\alpha, \beta}^{2}(\epsilon) \frac{\partial f\left(\epsilon^{\prime}\right)}{\partial \epsilon^{\prime}}\right|_{\epsilon^{\prime}=\epsilon}
$$

$f(\epsilon)=1 /\left(e^{-\beta(\epsilon-\mu)}+1\right)$ denotes the Fermi-Dirac distribution. Thus, the static zerotemperature conductivity can be directly related to the one-particle spectral function $A_{\alpha, \beta}(\mu)$ at the chemical potential.

\subsubsection{Classes of insulators}

The electron-ion interaction or electron-electron interaction can be the reason of an isolating behavior in different ways. Insulators are classified by the main mechanism that causes them to be insulating either as band insulators, Peierls insulators, Anderson insulators, Mott insulators or charge-transfer insulators.

The first three types are insulating due to the electron-ion interaction and the remaining two because of electron-electron interaction. The main reason for the insulating behavior of band insulators is the finite gap in the one-particle band structure that exists even without electron-electron interaction, disorder or static deformations of the lattice. Peierls insulators are quasi-one-dimensional materials in which chains of atoms are responsible for the conductivity. At high temperatures the atoms are equidistant. If the temperature is lowered the so-called Peierls transition [Fro, 1954; Peierls et al., 1955] can occur. The atoms in the chain dimerize and hence double the chain periodicity. This doubling of the lattice vector in the direction of the chain can open a gap that makes the material insulating if the band was half-filled without the lattice deformation. In Anderson insulators [Anderson, 1958], impurities or other disorder of the atomic structure localize electronic states. These localized states cannot contribute to the conductivity. The insulating behavior of Mott and charge-transfer insulators is caused by electron-electron interaction. In 1937 de Boer and Verwey [de Boer and Verwey, 1937] pointed out that several transition-metal oxides such as $\mathrm{NiO}$ or $\mathrm{CuO}_{2}$ are insulators even though according to band theory they should be conductors because they have an odd number of electrons per unit cell. Mott and Peierls [Mott and Peierls, 1937] discussed this result and concluded that the Coulomb repulsion of electrons leads to a localization of the electrons in these materials. Mott [Mott, 1949] later showed how electron-electron interaction leads to an insulating ground state of $\mathrm{NiO}$. This localization can occur for example in transitionmetal oxides, where the partially filled and localized d-states of the transition-metal ion form a narrow band and the electrons in this band interact strongly. Figure 2.1 shows the schematic density of states of Mott insulators and charge-transfer insulators. The charge transfer energy $\Delta$ denotes the energy difference between the states of the anion (e.g. the p-states of oxygen) and the strongly interacting states of the cation (e.g. the d-states of the transition-metal ion). The main effect of the electron-electron interaction is the split-up of the strongly interacting and partially filled bands of the cations into two bands. The distance of the resulting fully occupied band and the empty band has been termed the Hubbard $U$. Thus, depending on the relation between the charge-transfer energy and the Hubbard $U$ the material is either classified as a Mott insulator for $\Delta>U$ or a charge-transfer insulator for $\Delta<U$ [Zaanen et al., 1985]. This classification includes 

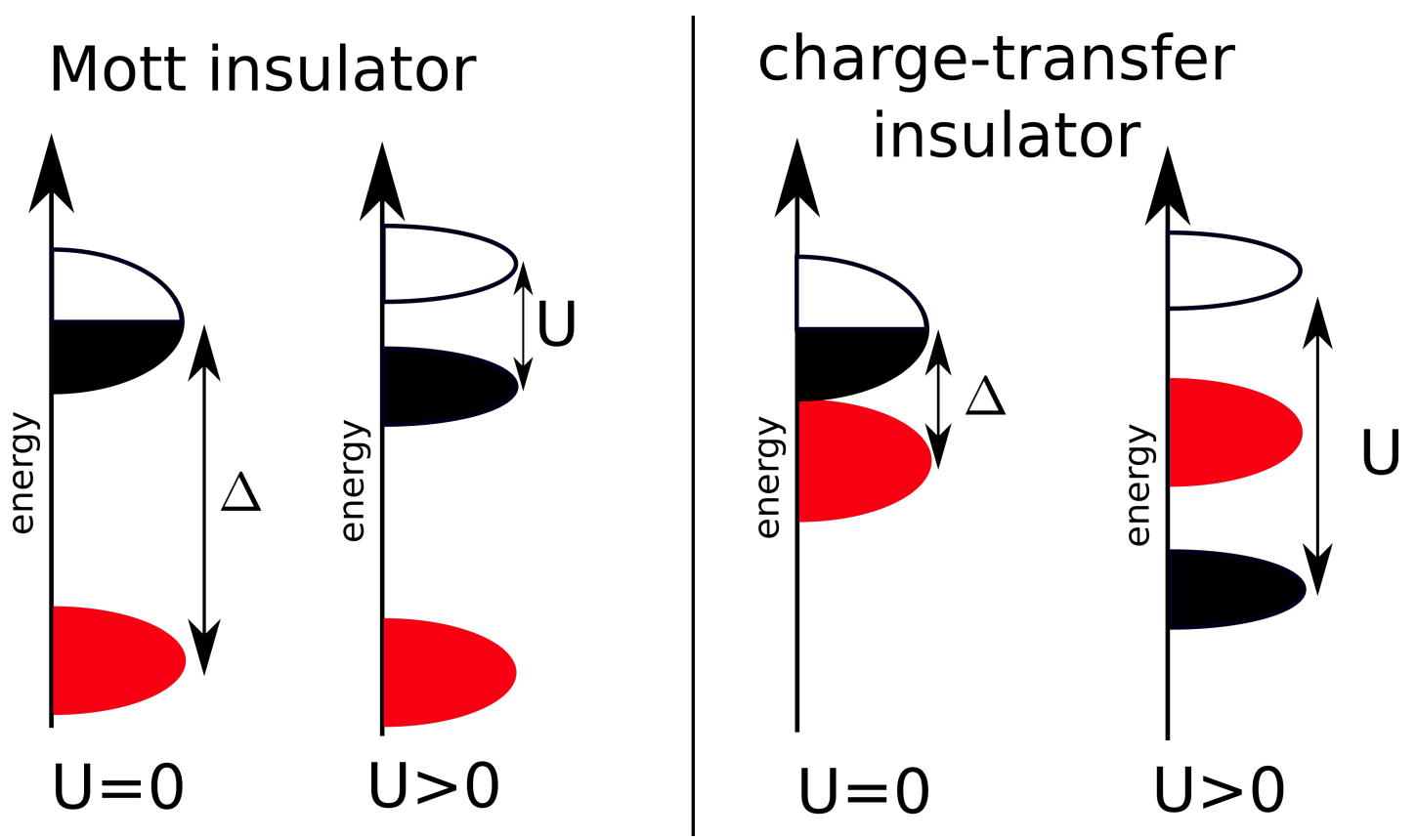

Figure 2.1.: Schematic density of states of Mott insulators (left figure) and charge-transfer insulators (right figure). Each case shows on the left side the situation without electron-electron interaction $(U=0)$ and on the right side the situation with electron-electron interaction $(U>0)$. The charge transfer energy $\Delta$ and the Hubbard $U$ are indicated in the figures. Red areas denote states of the anion, e.g., oxygen states, and black areas states of the cation, which is, for example, a transition-metal oxide.

information if the gap is between the filled and empty bands of the cation in case of a Mott insulator or between filled bands of the anion and the empty bands of the cation for a charge-transfer insulator. 


\section{Models for many-particle systems}

Models are required in solid-state theory to investigate and understand physical effects in well-controlled settings. The simplest model for the description of interacting electrons in a solid is the single-orbital Hubbard model [Pariser and Parr, 1953a,b; Pople, 1953; Gutzwiller, 1963b; Hubbard, 1963; Kanamori, 1963]. We describe the Hubbard model in section 3.1. The discussion focusses on half-filled, one-dimensional systems in the zerotemperature limit, because we use this well-understood situation as a simple benchmark case for the methods derived in this thesis. We also show how the Hubbard dimer, i.e., two-site Hubbard chain, is connected to the dissociation limit of the hydrogen molecule. We take the hydrogen molecule as the simplest case of the static correlation problem and discuss the implications of broken-symmetry solutions that are very relevant in the context of practical descriptions of strong electronic correlations in ab-initio calculations.

While the Hubbard model is a representative of a lattice model, also impurity models give interesting insights into the physics of correlated systems. We introduce the single-impurity Anderson model (SIAM, [Anderson, 1961]) in section 3.2 and discuss its properties in various parameter regions.

\subsection{Hubbard model}

The one-dimensional, single-orbital Hubbard model [Gutzwiller, 1963b; Hubbard, 1963; Kanamori, 1963] is defined by the Hamiltonian

$$
\hat{H}=-t \sum_{\langle i, j\rangle, \sigma} \hat{c}_{i \sigma}^{\dagger} \hat{c}_{j, \sigma}+U \sum_{i} \hat{n}_{i, \uparrow} \hat{n}_{i, \downarrow}
$$

Similar models have also been proposed early in quantum chemistry [Pariser and Parr, 1953a,b; Pople, 1953]. The simple form of the model is based on the observations that for the competition between itinerancy and localization only a single-site density-density interaction is required and that the two-particle interaction of electrons on two different sites is screened efficiently in many physical systems. Thus, the single-orbital Hubbard model usually describes one localized spin-degenerate one-particle state per lattice site. $\hat{c}_{i \sigma}^{(\dagger)}$ is the annihilator (creator) of an electron with spin $\sigma$ in the one-particle state at site $i$. The sites are arranged in some lattice. In this thesis, we only consider finite one-dimensional Hubbard models, where the sites are arranged in a finite chain (open boundary conditions) or as a ring (periodic boundary conditions. The one-particle terms of the Hamiltonian in Eq. (3.1) run over all neighboring sites. The interaction strength $U / t$, i.e., the fraction of the interaction parameter $U$ and the hopping parameter $t$, determines the physics of the model. An exact solution for the ground state of the one-dimensional Hubbard model can be obtained from the solution of the Bethe ansatz equations [Bethe; Lieb and $\mathrm{Wu}, 1968$ ]. Figure 3.1 shows the dependence of the ground-state energy $E=\langle\hat{H}\rangle$ and the interaction energy $W=U\left\langle\sum_{i} \hat{n}_{i, \uparrow} \hat{n}_{i, \downarrow}\right\rangle$ of a half-filled, 12-site Hubbard ring on the interaction 

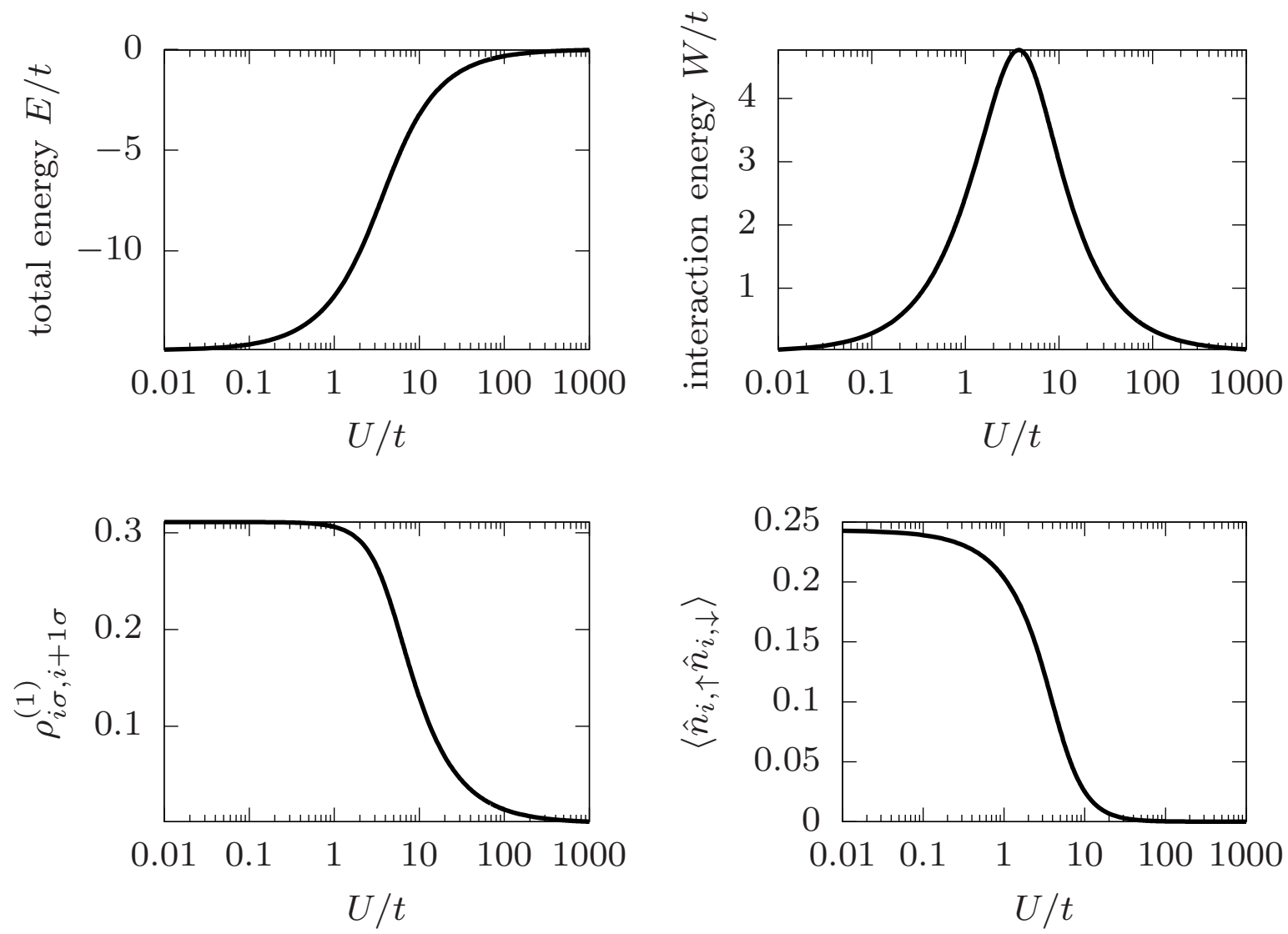

Figure 3.1.: Ground-state energy $E=\langle\hat{H}\rangle$ (upper left graph), interaction energy $W=U\left\langle\sum_{i} \hat{n}_{i, \uparrow} \hat{n}_{i, \downarrow}\right\rangle$ (upper right graph), next-neighbor elements $\rho_{i \sigma, i+1 \sigma}^{(1)}=$ $\left\langle\hat{c}_{i+1 \sigma}^{\dagger} \hat{c}_{i \sigma}\right\rangle$ of the one-particle reduced density matrix (lower left graph) and double occupancy $\left\langle\hat{n}_{i, \uparrow} \hat{n}_{i, \downarrow}\right\rangle$ (lower right graph) of a half-filled, 12-site Hubbard ring obtained from the numerical solution of the Bethe ansatz equations [Bethe; Lieb and $\mathrm{Wu}, 1968$ ].

strength $U / t$ obtained from the numerical solution of the Bethe ansatz equations [Bethe; Lieb and $\mathrm{Wu}, 1968]$. The interaction energy is maximal for intermediate interaction strengths and goes to zero for $U / t \rightarrow \infty$ because the double occupancy $\left\langle\hat{n}_{i, \uparrow} \hat{n}_{i, \downarrow}\right\rangle$ is suppressed for large interaction strengths. Figure 3.1 also shows the next-neighbor elements $\rho_{i \sigma, i+1 \sigma}^{(1)}=\left\langle\hat{c}_{i+1 \sigma}^{\dagger} \hat{c}_{i \sigma}\right\rangle$ of the one-particle reduced density matrix and the double occupancy for the same system. The next-neighbor elements of the one-particle reduced density matrix are a measure for the itineracy of the system. In the non-interaction limit $U / t \rightarrow 0$, the double occupancy and the next-neighbor elements of the one-particle reduced density matrix are maximal, which indicates a covalent bonding. Increasing $U / t$ from zero suppresses the double occupancies and localizes electrons. We will only use the onedimensional single-orbital Hubbard model for benchmarks of the proposed methods and, thus, will not discuss the plethora of exciting physics happening at finite temperature, in higher dimensions or at different fillings. 


\subsection{Single-impurity Anderson models}

An Anderson model [Anderson, 1958] describes a correlated impurity in a host lattice of weakly correlated or uncorrelated sites. The model was set up by Anderson to describe the local-moment behavior of correlated impurities in metals. The host lattice is usually referred to as the bath and described in k-space with the operators $\hat{c}_{\vec{k}, \sigma}^{(\dagger)}$. The impurity contains two degenerate one-particle states with the operators $\hat{f}_{\sigma}^{(\dagger)}$ with $\hat{n}_{f, \uparrow}=\hat{f}_{\sigma}^{\dagger} \hat{f}_{\sigma}$ in the case of the single-orbital Anderson model. The Hamiltonian of a generic single-impurity, single-orbital Anderon model reads

$$
\begin{aligned}
\hat{H} & =\hat{H}_{\mathrm{bath}}+\hat{H}_{\mathrm{imp}}+\hat{H}_{\mathrm{hyb}} \\
\hat{H}_{\mathrm{bath}} & =\sum_{\vec{k}, \sigma} \epsilon_{\vec{k}} \hat{c}_{\vec{k}, \sigma}^{\dagger} \hat{c}_{\vec{k}, \sigma} \\
\hat{H}_{\mathrm{imp}} & =\epsilon_{f} \sum_{\sigma} \hat{f}_{\sigma}^{\dagger} \hat{f}_{\sigma}+U \hat{n}_{f, \uparrow} \hat{n}_{f, \downarrow} \\
\hat{H}_{\mathrm{hyb}} & =\sum_{\vec{k}, \sigma}\left(V_{\vec{k}, f} \hat{c}_{\vec{k}, \sigma}^{\dagger} \hat{f}_{\sigma}+V_{\vec{k}, f}^{*} \hat{f}_{\sigma}^{\dagger} \hat{c}_{\vec{k}, \sigma}\right) .
\end{aligned}
$$

The Hamiltonian $\hat{H}_{\text {bath }}$ of the bath, i.e., the host lattice, describes a non-interacting system with the dispersion relation $\epsilon_{\vec{k}}$. The Hamiltonian $\hat{H}_{\mathrm{imp}}$ of the impurity contains an impurity on-site energy $\epsilon_{f}$ and a local two-particle interaction with the interaction parameter $U$. The hopping of electrons between the impurity and the bath is described by the hybridization Hamiltonian $\hat{H}_{\text {hyb }}$ with the hybridization parameters $V_{\vec{k}, f}$.

To set up a model that covers most of the essential physics of a single-impurity Anderson model and is only determined by a minimal number of parameters, we choose a finite non-interacting Hubbard chain with $L_{\text {bath }}$ sites as the bath and a k-independent real hybridization parameters $V_{\vec{k}, f}=V / \sqrt{L_{\text {bath }}}$. The choice of the Hubbard ring as a bath defines the dispersion relation

$$
\epsilon_{k}=-2 t \cos \left(\frac{2 \pi k}{L_{\mathrm{bath}}}\right)
$$

and the choice of the hybridization parameters defines the hybridization Hamiltonian as

$$
\hat{H}_{\text {hyb }}=\frac{V}{\sqrt{L_{\text {bath }}}} \sum_{k=0}^{L_{\text {bath }}} \sum_{\sigma}\left(\hat{c}_{k, \sigma}^{\dagger} \hat{f}_{\sigma}+\hat{f}_{\sigma}^{\dagger} \hat{c}_{k, \sigma}\right) .
$$

\subsubsection{Characteristic parameter regimes}

In this section, we discuss different parameter regimes of a single-impurity Anderson model with the Hamiltonian defined by Eq. (3.2)-Eq. (3.7). We choose $L_{\text {bath }}=11$ bath sites, a filling of $N_{e}=L_{\text {bath }}+1=12$ electrons, $t>0$ and the zero-temperature limit. The exact ground state of this system can be obtained with exact diagonalization. The chosen singleimpurity Anderson model is identical to the one studied in [Töws and Pastor, 2011]. The discussion and data presented in this section have been published in [Schade and Blöchl, 2018]. The energy levels of the non-interacting bath are given by the dispersion relation in Eq. (3.6). The Fermi energy $\epsilon_{F \text {,bath }}$ of the non-interacting bath is

$$
\epsilon_{F, \text { bath }}=\lim _{n \rightarrow N^{+}} \frac{\partial E_{\mathrm{bath}}(n)}{\partial n},
$$


where $E_{\text {bath }}(n)$ is the energy of the bath with $n$ electrons. Thus, for the given choice of parameters the Fermi energy is

$$
\epsilon_{F, \text { bath }}=-2 t \cos \left(\frac{6 \pi}{11}\right) \approx 0.28 t
$$

and larger than zero. We discuss the physics of the different parameter regimes here shortly, because in chapter 8 we will benchmark the adaptive cluster approximation for these parameter ranges.

\section{Interaction-strength dependence}

First, we investigate the dependence of the ground state on the interaction parameter $U$ of the impurity orbital. We choose $\epsilon_{f}=0, V / t=0.4$ and $U / t \in[0,8]$. Figure 3.2 shows the ground-state energy $E=\langle\hat{H}\rangle$, the interaction energy $W=U\left\langle\hat{n}_{f, \uparrow} \hat{n}_{f, \downarrow}\right\rangle$, the impurity occupation $n_{f}=\left\langle\hat{n}_{f, \uparrow}+\hat{n}_{f, \downarrow}\right\rangle$ and the impurity magnetization $m_{f}=\left\langle\hat{n}_{f, \uparrow}-\hat{n}_{f, \downarrow}\right\rangle$. The Fermi level of the bath is higher than the impurity level and hence the impurity is more than half-filled $\left(n_{f}>1\right)$ in the non-interacting limit $(U=0)$. For small interaction strengths, the Hartree-Fock approximation holds, and the interaction energy $W$ depends as

$$
W \approx \frac{U}{4} n_{f}^{2}
$$

on the impurity occupation $n_{f}$. The spin-unrestricted Hartree-Fock approximation is valid for small interaction strengths, but shows an unphysical transition to a state with a non-zero impurity magnetization $m_{f}=\left\langle\hat{n}_{f, \uparrow}-\hat{n}_{f, \downarrow}\right\rangle \neq 0$ at around $U \approx t$. A large impurity interaction $U$ penalizes the impurity occupation and for $U / t \rightarrow \infty$ the impurity is less than half occupied.

\section{Impurity on-site-energy dependence}

The on-site energy $\epsilon_{f}$ of the impurity directly influences the number of electrons on the impurity, because if shifts the energy level of the impurity relative to the energy levels of the bath. The left column of figure 3.3 shows the dependence of important observables of the single-impurity Anderson model defined by Eq. (3.2)-Eq. (3.7) with $L_{\text {bath }}=11$, $N_{e}=L_{\text {bath }}+1=12, t>0, U / t=8$ and $V / t=0.4$ in the range $\epsilon_{f} / t \in[-10,5]$. The impurity occupation $n_{f}$ defines three regimes of this model:

- The doubly-occupied region, i.e., $n_{f} \approx 2$, exists for $\epsilon_{f} \ll \epsilon_{F \text {,bath }}-U \approx-7.72 t$. Due to the double-occupation the interaction energy takes its maximal value of $W \approx U$ in this region.

- The singly-occupied region, i.e., $n_{f} \approx 1$, exists for $\epsilon_{f}<\epsilon_{F \text {,bath }} \approx 0.28 t$ and $\epsilon_{f}>$ $\epsilon_{F, \text { bath }}-U \approx-7.72 t$. The region is also known as the Kondo-regime [Kondo, 1964] and the ground state in this regime has only contributions from Slater determinants that are singly-occupied on the impurity. Thus, the impurity can be described by an effective local spin-1/2-moment coupled to the bath.

- The empty-impurity region exists for $\epsilon_{f}>\epsilon_{F, \text { bath }} \approx 0.28 t$. 

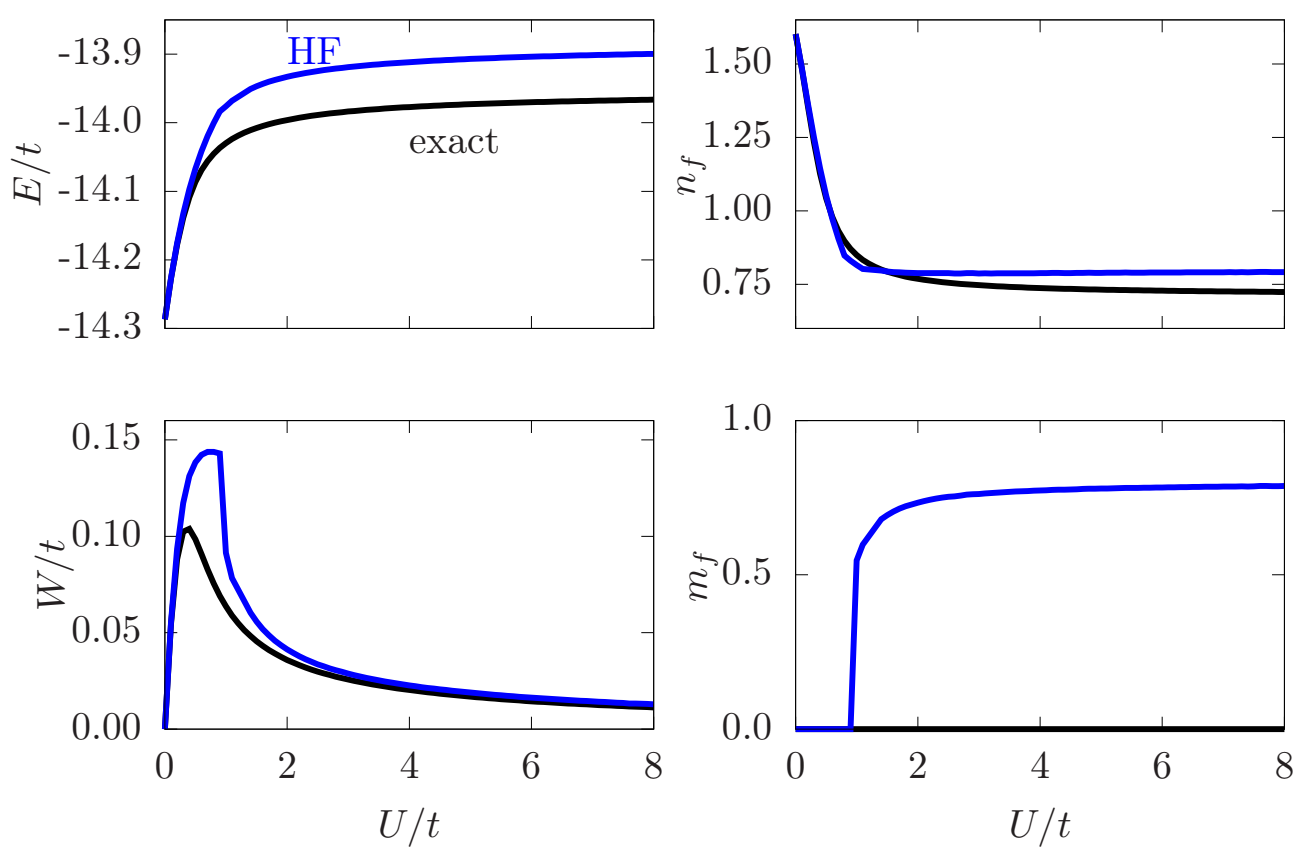

Figure 3.2.: Interaction-strength dependence of the ground-state of a half-filled singleimpurity Anderson model defined by Eq. (3.2)-Eq. (3.7) with $L_{\text {bath }}=11, N_{e}=$ $L_{\text {bath }}+1=12, \epsilon_{f}=0, V / t=0.4$ and $t>0$. Exact results (black lines) are compared to the spin-unrestricted Hartree-Fock approximation (blue lines).

At the boundaries of the three regions there are mixed-valence regions. The unrestricted Hartree-Fock approximation reproduces the separation into three regions qualitatively but shows an unphysical ground state with a finite impurity magnetization in the singlyoccupied region.

\section{Bandwidth dependence}

Finally, we investigate the dependence on the bandwidth of the bath. The bandwidth $w_{b}$ of the bath is related to the hopping as

$$
w_{b}=4 t
$$

Thus, we will use the bath hopping $t$ instead of the bath bandwidth $w_{b}$ in this section. The dependence of importand observables of the single-impurity Anderson model defined by Eq. (3.2)-Eq. (3.7) with $L_{\text {bath }}=11, N_{e}=L_{\text {bath }}+1=12, t>0, U / V=5, V>0$ and $\epsilon_{f} / V=-1$ on $t / V \in[0,20]$ is shown in the right column of figure 3.3. The behavior of the total energy $E$ is dominated by the hopping and hence the total energy is visually identical to a linear function in $t$. In limit of degenerate bath energy levels $t \rightarrow 0$, we have $\epsilon_{F, \text { bath }}=0$ and hence with the given choice of the remaining parameters $\epsilon_{f}+U \gg \epsilon_{F \text {,bath }}$ as well as $\epsilon_{f}<\epsilon_{F, \text { bath }}$. Thus, the system is close to the transition from the empty impurity to a singly occupied impurity. In the limit of widely separated bath energy levels $t \rightarrow \infty$, we have a doubly occupied impurity, and hence the interaction energy is maximal. The limit of degenerate bath energy levels and the limit of widely separated bath energy levels are 
particularly important because the adaptive cluster approximation with one effective bath level becomes exact in these limits due to the equivalence to the two-level approximation of Töws and Pastor [Töws and Pastor, 2011, 2012].

\subsubsection{One-particle reduced density matrix of the bath}

During our studies of single-impurity Anderson models, we found that models of the type defined in section 3.2.1 have a very interesting structure of the eigenvalue spectrum of the one-particle reduced density matrix of the bath states in the exact ground state at zero temperature. Numerical results show that the eigenvalue spectrum of the oneparticle reduced density matrix of the bath consists only of a few clusters of eigenvalues. The individual clusters have a tiny spread. Because of the fact that the number of unique eigenvalues can be related to the number of effective bath levels for which the adaptive cluster approximation in chapter 8, we discuss this feature of single-impurity Anderson models here in detail. The findings presented in this section have been published in [Schade and Blöchl, 2018].

We define the one-particle reduced density matrix $\rho_{k, \sigma, k^{\prime} \sigma^{\prime}}^{(1)}$ of the bath as

$$
\rho_{k, \sigma, k^{\prime} \sigma^{\prime}}^{(1)}=\left\langle\hat{c}_{k^{\prime} \sigma^{\prime}}^{\dagger} \hat{c}_{k \sigma}\right\rangle
$$

and the bath occupations $f_{\mathrm{bath}, i}$ as the eigenvalues of $\rho_{k, \sigma, k^{\prime} \sigma^{\prime}}^{(1)}$. Figure 3.4 shows the bath occupations for the three parameter regimes discussed in section 3.2.1. For all parameters, except $t=0$, there are at most three clusters of bath occupations, namely around $f_{\mathrm{bath}, 1}=$ $0, f_{\text {bath }, 2}=1$ and except in limiting cases also around an intermediate value $0<f_{\text {bath }, 3}<1$. Please note, that the bath occupations in a cluster are not necessarily equal, but the clusters can have a finite spread. However, the spread of values in a cluster is very small, so that all occupations in a cluster can be regarded as approximately equal for the purpose of new approximate solution methods for single-impurity Anderson models. Thus, the number of clusters should be regarded as a qualitative feature.

One might be tempted to ascribe the low number of clusters of bath occupations to the high symmetry of the single-impurity Anderson model studied so far. Especially the choice $V_{k, f}=V / \sqrt{L_{\text {bath }}}$ could be an issue. In the following, we show that this is not the case. To study a single-impurity Anderson model with a more physical bath density of states, we choose a different model with the Hamiltonian

$$
\begin{aligned}
\hat{H} & =\hat{H}_{\mathrm{bath}}+\hat{H}_{\mathrm{imp}}+\hat{H}_{\mathrm{hyb}} \\
\hat{H}_{\mathrm{bath}} & =\sum_{j=1}^{L_{\mathrm{bath}}} \epsilon_{b, j} \sum_{\sigma} \hat{c}_{j, \sigma}^{\dagger} \hat{c}_{j, \sigma} \\
\hat{H}_{\mathrm{imp}} & =\sum_{i=1}^{L_{\mathrm{imp}}} \epsilon_{f, i} \sum_{\sigma} \hat{f}_{i, \sigma}^{\dagger} \hat{f}_{i, \sigma}+\sum_{i=1}^{L_{\mathrm{imp}}} U_{i} \hat{n}_{f, i, \uparrow} \hat{n}_{f, i, \downarrow} \\
\hat{H}_{\mathrm{hyb}} & =\sum_{i=1}^{L_{\mathrm{imp}}} \sum_{j=1}^{L_{\mathrm{bath}}} V_{i, j} \sum_{\sigma}\left(\hat{c}_{j, \sigma}^{\dagger} \hat{f}_{i, \sigma}+\hat{f}_{i, \sigma}^{\dagger} \hat{c}_{j, \sigma}\right) .
\end{aligned}
$$

$L_{\text {imp }}$ denotes the number of interacting sites in the impurity, $L_{\text {bath }}$ the number of bath sites, $\epsilon_{f, i}$ the impurity on-site energies, $\epsilon_{b, j}$ the bath on-site energies, $U_{i}$ the on-site interaction parameter of the $i$-th impurity site and $V_{i, j}$ the hybridization parameters. Thus, 
this model can describe an arbitrary bath density of states and multi-orbital impurities. For large baths, i.e. $L_{\text {bath }} \gg 1$, this model cannot be solved in practice by exact diagonalization. We have obtained the numerically exact ground state of this model instead with matrix-product-state based density-matrix renormalization group theory implemented in ITensor $^{1}$ (MPS-DMRG, [White, 1992, 1993; Östlund and Rommer, 1995; Schollwöck, 2005; Schollwöck, 2011] ${ }^{2}$ ). To reduce the numerical effort of these calculations, we have proposed and employed a new entanglement reduction procedure that will be discussed in section 8.8.5 in detail.

The first question to be investigated is if the structure of the eigenvalue spectrum of the one-particle reduced density matrix of the bath persists in the limit of a continuous bath density of states. We choose a single-orbital single-impurity Anderson model, i.e. $L_{\text {imp }}=1$, with bath bandwidth $w_{b}=2 \mathrm{eV}$ and $\epsilon_{f, 1}=0$. We consider a model with a total number of $L_{\mathrm{imp}}+L_{\mathrm{bath}}=200$ sites and a filling with $N_{e}=200$ electrons. We distribute the on-site energies $\epsilon_{b, j}$ of the bath sites uniformly in $\left[\epsilon_{b}-w_{b} / 2, \epsilon_{b}+w_{b} / 2\right]$. This gives a rectangular density of states of the bath with a spacing of the bath energy levels of approx $0.01 \mathrm{eV}$. To avoid accidental symmetries, we chose the hybridization parameters $V_{i, j}$ randomly in $\left[0, w_{b} / L_{\text {bath }}\right]$. The bath occupations of the model are shown in figure 3.5. As for the small bath, there are three distinct clusters of bath occupations. Thus, we expect the clustering of the bath occupations to persist in the limit of a continuous bath density of states. A two-site impurity with $U_{i}>0$ and $\epsilon_{f, i}$ chosen randomly leads to four clusters of eigenvalues as shown in figure 3.5 for $L_{\mathrm{imp}}=2$ and $L_{\mathrm{imp}}+L_{\mathrm{bath}}=40$. On the other hand, a three-site impurity, $L_{\mathrm{imp}}=3$ leads to five clusters of bath occupations. Thus, we conjecture for a single-impurity Anderson model with a non-degenerate ground state and a spin-degenerate Hamiltonian the rule that the number of clusters $n_{\text {cluster }}$ of bath occupations is related to the number of impurity sites $L_{\text {imp }}$ by

$$
n_{\text {cluster }}=2+L_{\text {imp }}
$$

\footnotetext{
${ }^{1}$ ITensor C++ library (version 2.1.1), http://itensor.org/

${ }^{2} \mathrm{~A}$ more complete list of references can be found in the reviews [Schollwöck, 2005], [Verstraete et al., 2008] and [Schollwöck, 2011].
} 

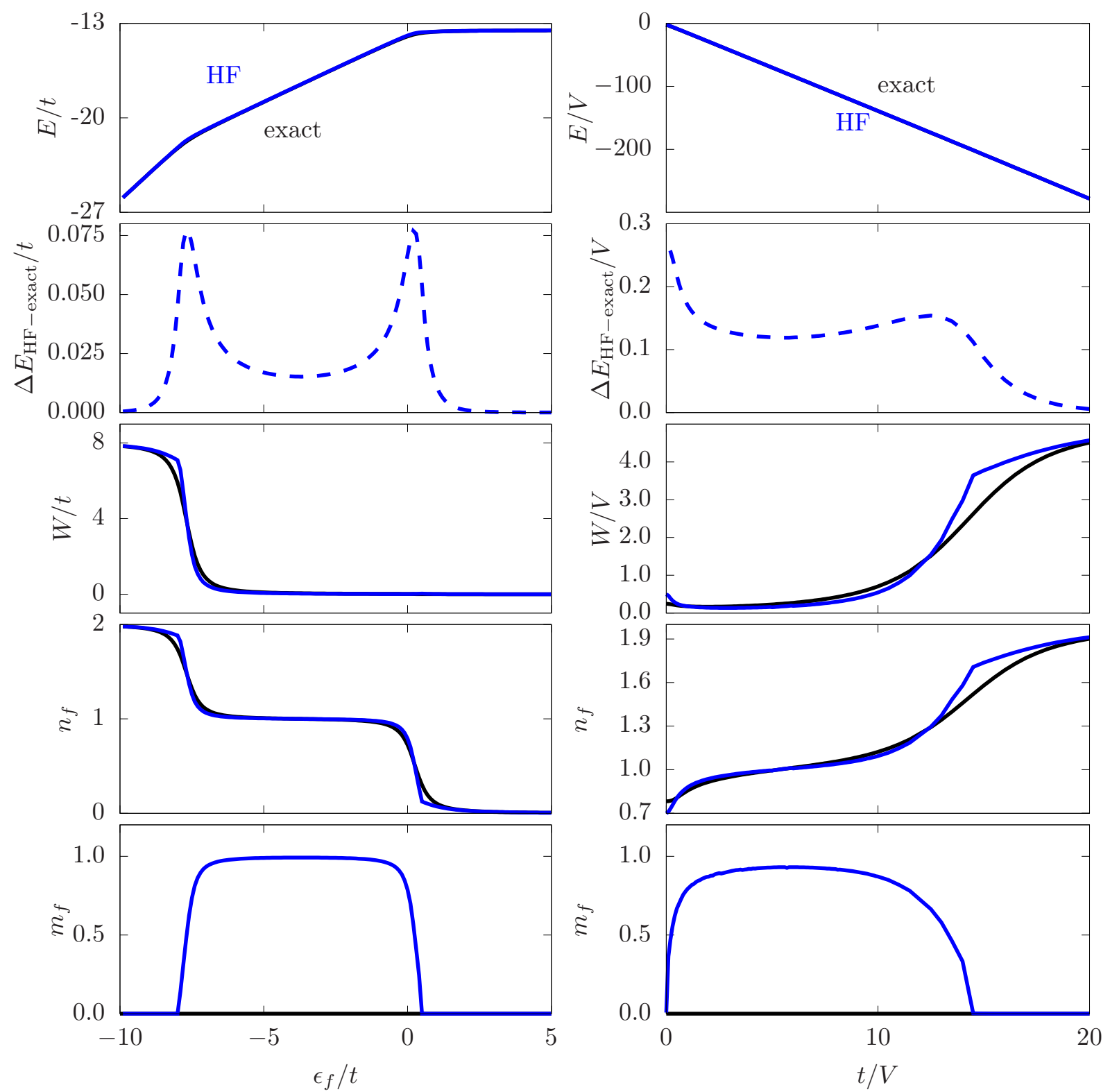

Figure 3.3.: Impurity on-site-energy dependence (left column) and bandwidth dependence (right column) of important observables of the single-impurity Anderson model defined by Eq. (3.2)-Eq. (3.7) with $L_{\text {bath }}=11, N_{e}=L_{\text {bath }}+1=12$ and $t>0$. For the impurity on-site-energy dependence $U / t=8$ and $V / t=0.4$ were chosen. For the dependence on the bandwidth $U / V=5, V>0$ and $\epsilon_{f} / V=-1$ were chosen. Exact results (black lines) are compared to the spin-unrestricted Hartree-Fock approximation (blue lines). 

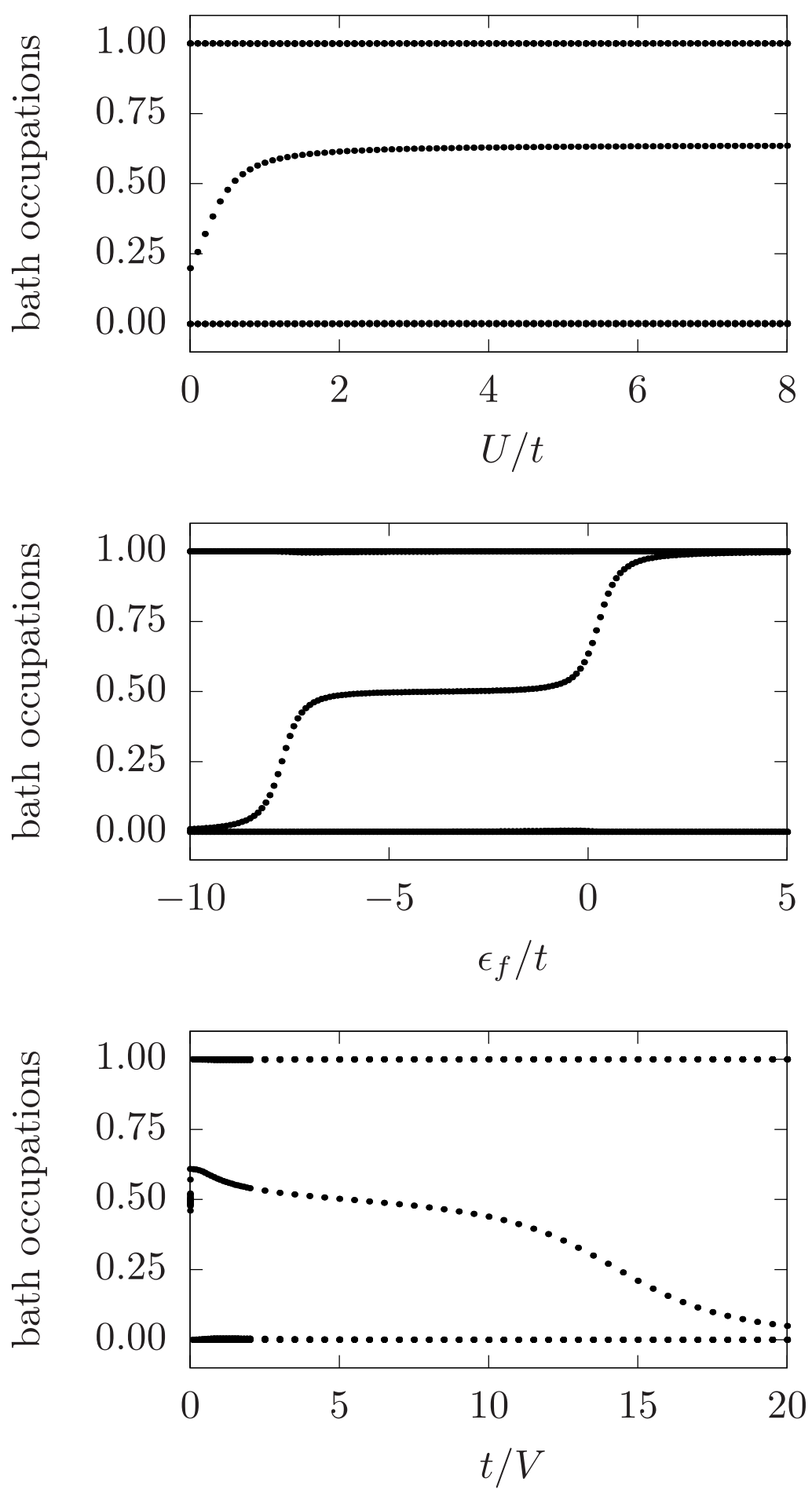

Figure 3.4.: Bath occupations, i.e., the eigenvalues of $\rho_{k, \sigma, k^{\prime} \sigma^{\prime}}^{(1)}=\left\langle\hat{c}_{k^{\prime} \sigma^{\prime}}^{\dagger} \hat{c}_{k \sigma}\right\rangle$, of a singleimpurity Anderson model for the three parameter regions discussed in section 3.2.1. 

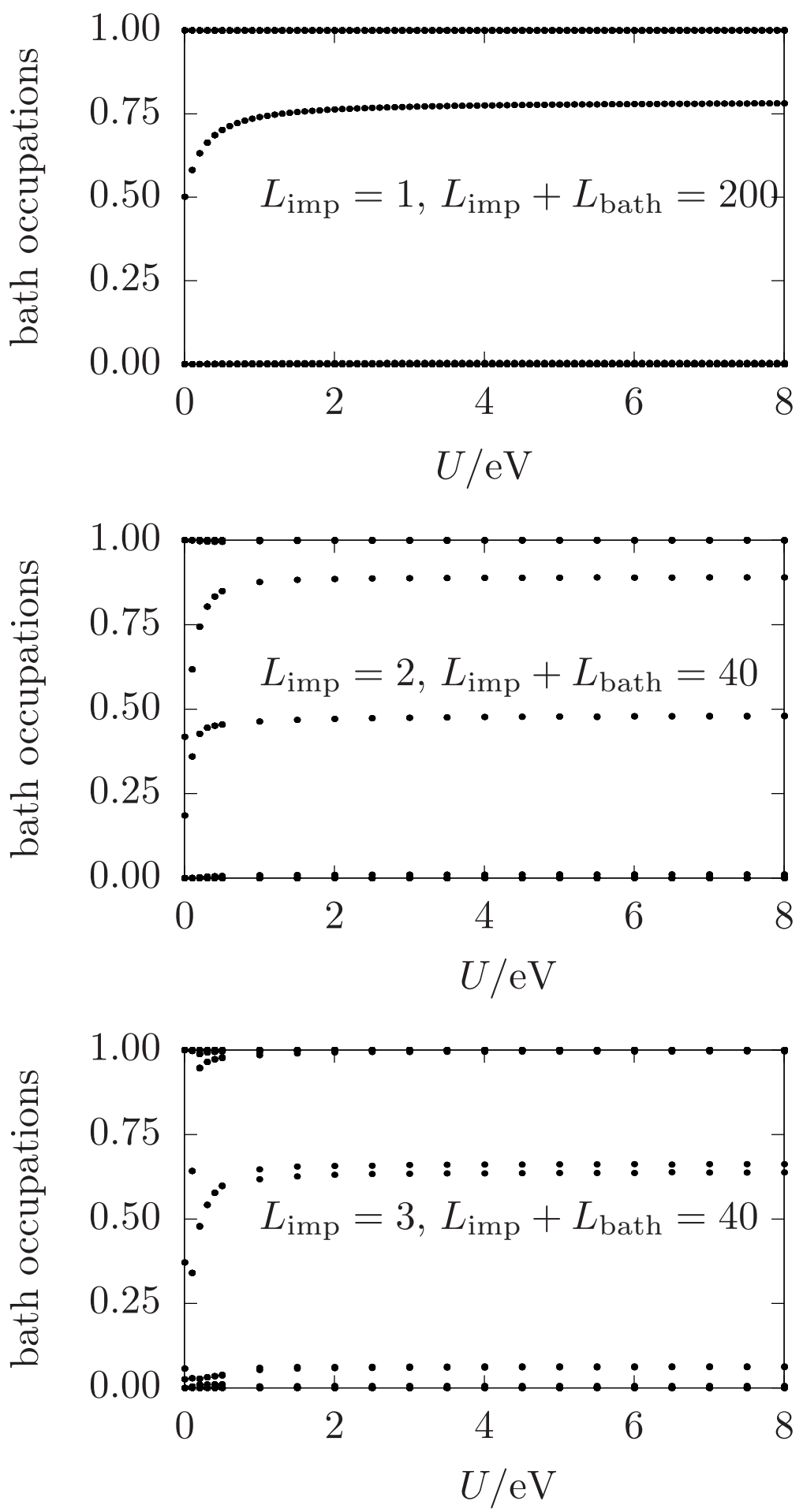

Figure 3.5.: Bath occupations, i.e., the eigenvalues of $\rho_{k, \sigma, k^{\prime} \sigma^{\prime}}^{(1)}=\left\langle\hat{c}_{k^{\prime} \sigma^{\prime}}^{\dagger} \hat{c}_{k \sigma}\right\rangle$, of a singleimpurity Anderson model for a single-site impurity (top figure), a two-site impurity (middle figure) and a three-site impurity (bottom figure). The remaining parameters are described in detail in section 3.2.2. 


\section{Density functional theory}

In this section, we will discuss the foundations of density functional theory starting with its historically first foundation in the form of the Hohenberg-Kohn theorems [Hohenberg and Kohn, 1964; Kohn and Sham, 1965; Kohn, 1999]. We will then point out some shortcomings of the Hohenberg-Kohn definitions, introduce Levy's constrained-search functional [Levy, 1979] and finally Lieb's generalization based on the Legendre-Fenchel transformation [Lieb, 1983a]. Furthermore, we will also discuss some practical approximations of the exchange-correlation functional as they are relevant for the combination of densityfunctional theory and reduced density-matrix functional theory. We restrict the main part of the discussion to the case of zero-temperature, integer particle numbers, and non-spinpolarized states to keep the notation concise. These restrictions are then subsequently dropped, and necessary extensions of the theory explained.

\subsection{Hohenberg-Kohn theorems}

Historically, the first foundation of density functional theory was set by the two Hohenberg-Kohn theorems [Hohenberg and Kohn, 1964; Kohn, 1999]. For a general discussion, we closely follow [Eschrig, 2003] and define the Hamiltonian as an operator-valued functional of the external potential $v(\vec{r})$ as

$$
\hat{H}_{\lambda}[v]=\hat{T}+\lambda \hat{W}+\sum_{i} v\left(\vec{r}_{i}\right)
$$

where $\hat{T}$ is the operator of the kinetic energy, $\hat{W}$ denotes the electron-electron interaction and $0 \leq \lambda \leq 1$ scales the interaction strength. The ground-state energy is also a functional of the potential and defined as

$$
E_{\lambda}[v]=\inf _{|\Psi\rangle \in \mathcal{W}_{N}}\left\langle\Psi\left|\hat{H}_{\lambda}[v]\right| \Psi\right\rangle
$$

with the class $\mathcal{W}_{N}$ of normalized antisymmetric $N$-particle wave functions with finite kinetic energy.

The first Hohenberg-Kohn theorem states that the electron density $n_{0}(\vec{r})$ of the ground state determines the external potential $v(\vec{r})$ for which the ground state was obtained up to an additive constant. This theorem holds for all potentials $v(\vec{r}) \in \mathcal{V}_{N}$. $\mathcal{V}_{N}$ is defined as the class of all potentials, that are $\mathrm{in}^{1} L^{p}$ for some $p$ and can bind $N$ electrons, i.e.,

$$
\mathcal{V}_{N}^{\lambda}=\left\{v \mid \exists p \in \mathbb{R}: 1 \leq p<\infty, v \in L^{p} \text { and } \hat{H}_{\lambda}[v] \text { has } \mathrm{N} \text { - particle ground state }\right\}
$$

\footnotetext{
${ }^{1} f \in L^{p}$ if and only if $\left[\int d x|f(x)|^{p}\right]^{1 / p}<\infty$.
} 


\section{Density functional theory}

and is also named the class of $n$-representable potentials. On the other hand, the first Hohenberg-Kohn theorem implicitly only applies to electron densities that come from an $N$-electron ground state and, thus, are elements of the class of pure-state $v$-representable densities

$\mathcal{A}_{N}^{\lambda}=\left\{n \mid \exists v \in \mathcal{V}_{N}^{\lambda}: n\right.$ is the density of an $\mathrm{N}-$ particle ground state of $\left.\hat{H}_{\lambda}[v]\right\}$.

The first Hohenberg-Kohn theorem closes the cycle of the conventional solution of the many-particle problem of $\hat{H}_{\lambda}[v]$ (in the absence of degeneracies)

$$
v \rightarrow\left|\Psi_{0}\right\rangle \rightarrow n_{0}
$$

by relating the electron density $n_{0} \in \mathcal{A}_{N}^{\lambda}$ to exactly one external potential $v \in \mathcal{V}_{N}^{\lambda}$. Thus, the potential $v=v_{\lambda}[n]$ and the ground-state wave function $\left|\Psi_{0}\right\rangle=\left|\Psi_{0}^{\lambda}[n]\right\rangle$ are functionals of the electron density. Consequently, instead of the many-particle wave function the electron density can be used as the basic quantity of a system.

The second Hohenberg-Kohn theorem states that a universal functional $F_{\mathrm{HK}}^{\lambda}[n]$ can be defined that yields the exact ground-state energy for the external potential $v_{\text {ext }} \in \mathcal{V}_{N}^{\lambda}$ as result of the minimization

$$
E_{\lambda}\left[v_{\mathrm{ext}}\right]=\min _{n \in \mathcal{A}_{N}^{\lambda}} F_{\mathrm{HK}}^{\lambda}[n]+\int d^{3} \vec{r} n(\vec{r}) v_{\mathrm{ext}}(\vec{r}) .
$$

The minimum is obtained for the density $n$ that fulfills $v_{\text {ext }}=v_{\lambda}[n]$. In the non-degenerate case, this functional can be written as

$$
F_{\mathrm{HK}}^{\lambda}[n]=\left\langle\Psi_{0}^{\lambda}[n]|\hat{T}+\lambda \hat{W}| \Psi_{0}^{\lambda}[n]\right\rangle
$$

or more generally also for degenerate cases as

$$
F_{\mathrm{HK}}^{\lambda}[n]=E_{\lambda}\left[v_{\lambda}[n]\right]-\int d^{3} \vec{r} v_{\lambda}[n](\vec{r}) n(\vec{r}) .
$$

The Hohenberg-Kohn functional $F_{\mathrm{HK}}^{\lambda}[n]$ is independent of the external potential. The explicit form of the exact functional $F_{\mathrm{HK}}^{\lambda}[n]$ is unknown and the computational complexity for it's evaluation is the same as for the solution of the many-particle problem up to differences of polynomial complexity [Liu et al., 2007; Schuch and Verstraete, 2009; Whitfield et al., 2014]. The main shortcomings of the Hohenberg-Kohn functional are that it is only defined for the class $\mathcal{A}_{N}^{\lambda}$ of densities. Neither the set $\mathcal{A}_{N}^{\lambda}$ nor $\mathcal{V}_{N}^{\lambda}$ are explicitly known. This has been termed the $v$-representability problem.

\subsection{Levy's constrained search functional}

The main step of solving the $v$-representability issue of the Hohenberg-Kohn theorems was performed by Levy [Levy, 1979]. He extended the definition of the universal kinetic-energy functional for non-interacting systems of Percus [Percus, 1978]

$$
T[n]=\min _{\rho^{(1)}\left(\vec{x}, \vec{x}^{\prime}\right): n(\vec{x})=\rho^{(1)}(\vec{x}, \vec{x})} \operatorname{Tr}\left[T \rho^{(1)}\right]
$$


to interacting systems

$$
F_{\text {Levy }}^{\lambda}[n]=\min _{|\Psi\rangle \in \mathcal{W}_{N}: n(x)=\langle\Psi|\hat{n}(x)| \Psi\rangle}\langle\Psi|\hat{T}+\lambda \hat{W}| \Psi\rangle .
$$

The Levy-functional can be used in place of the Hohenberg-Kohn functional in Eq. (4.6) because

$$
F_{\text {Levy }}^{\lambda}[n]=F_{\mathrm{HK}}^{\lambda}[n] \forall n \in \mathcal{A}_{N}^{\lambda}
$$

Furthermore, Levy's functional is defined also for densities that are not $v$-representable. The minimization over densities from the unknown class $\mathcal{A}_{N}^{\lambda}$ in Eq. (4.6) can be extended to the class of densities that come from any many-particle wave function not just from ground states. This class of densities can be defined as [Gilbert, 1975a; Harriman, 1981; Lieb, 1983a]

$$
\mathcal{I}_{N}=\left\{n \mid n(\vec{r}) \geq 0, n \in L^{1}, \nabla n^{1 / 2} \in L^{2}, \int d^{3} \vec{r} n(r)=N<\infty\right\}
$$

and contains the class $\mathcal{A}_{N}^{\lambda}$. Lieb has shown that any density from $\mathcal{I}_{N}$ can be represented by a single Slater determinant and that the density of every $N$-particle wave function from $\mathcal{W}_{N}$ is in the class $\mathcal{I}_{N}$ [Lieb, 1983a]. It can be shown that the class $\mathcal{I}_{N}$ is convex. That means that if $n_{1}, n_{2} \in \mathcal{I}_{N}$ then also $\eta n_{1}+(1-\eta) n_{2} \in \mathcal{I}_{N} \forall 0 \leq \eta \leq 1$. This is in sharp contrast to the non-convexity of $\mathcal{A}_{N}^{\lambda}$ which has been shown by Lieb [Lieb, 1983a]. Thus, Levy has generalized the Hohenberg-Kohn functional to a convex domain. But even though the domain $\mathcal{I}_{N}$ of Levy's functional is convex, the functional itself is not convex [Lieb, 1983a]. A convex functional was later introduced by Lieb and will be discussed in the subsequent section.

In conclusion, with Levy's constrained search functional we can generalize the minimization of Eq. (4.6) to

$$
E_{\lambda}\left[v_{\text {ext }}\right]=\inf _{n \in \mathcal{I}_{N}}\left(F_{\text {Levy }}^{\lambda}[n]+\int d^{3} \vec{r} n(\vec{r}) v_{\text {ext }}(\vec{r})\right)
$$

which, however, is still not a convex minimization problem.

\subsection{Lieb's convex-conjugate functional}

For Lieb's generalization of Levy's constrained search functional, we first note some important properties of the ground-state energy. The ground-state energy is concave ${ }^{2}$

$$
E_{\lambda}\left[\alpha v_{1}+(1-\alpha) v_{2}\right] \geq \alpha E_{\lambda}\left[v_{1}\right]+(1-\alpha) E_{\lambda}\left[v_{2}\right]
$$

for $v_{1}, v_{2} \in \mathcal{V}_{n}^{\lambda}$ and $0 \leq \alpha \leq 1$. On the other hand, the ground-state energy is a monotonically decreasing function of the potential, that is, if $v_{1}(\vec{r}) \leq v_{2}(\vec{r}) \forall \vec{r} \in \mathbb{R}^{3}$, then $E_{\lambda}\left[v_{1}\right] \leq E_{\lambda}\left[v_{2}\right]$.

\footnotetext{
${ }^{2}$ Please note, that we distinguish concavity $\left(f\left(\alpha x_{1}+(1-\alpha) x_{2}\right) \geq \alpha f\left(x_{1}\right)+(1-\alpha) f\left(x_{2}\right)\right)$, strict concavity $\left(f\left(\alpha x_{1}+(1-\alpha) x_{2}\right)>\alpha f\left(x_{1}\right)+(1-\alpha) f\left(x_{2}\right)\right)$, convexity $\left(f\left(\alpha x_{1}+(1-\alpha) x_{2}\right) \leq \alpha f\left(x_{1}\right)+(1-\alpha) f\left(x_{2}\right)\right)$ and strict convexity $\left(f\left(\alpha x_{1}+(1-\alpha) x_{2}\right)<\alpha f\left(x_{1}\right)+(1-\alpha) f\left(x_{2}\right)\right)$.
} 


\section{Density functional theory}

Lieb's choice of the density functional is [Lieb, 1983a]

$$
F_{\text {Lieb }}^{\lambda}[n]=\sup _{v \in L^{3 / 2}+L^{\infty}}\left(E_{\lambda}[v]-\int d^{3} \vec{r} v(\vec{r}) n(\vec{r})\right) .
$$

This functional is defined for all densities from the class

$$
\mathcal{R}_{N}=\left\{n \mid n(\vec{r}) \geq 0, n \in L^{3}, \int d^{3} \vec{r} n(r)=N<\infty\right\},
$$

which is convex and contains the class $\mathcal{I}_{N}$. The minimization in Eq. (4.15) is performed over all potentials from the class $L^{3 / 2}+L^{\infty}$. That is, potentials that can be written as a sum of a potential $v_{3 / 2} \in L^{3 / 2}$ and $v_{\infty} \in L^{\infty}$. This class is chosen because $n \in L^{1} \cup L^{3}$ and $^{3}$

$$
\int d^{3} \vec{r}|n(\vec{r}) v(\vec{r})| \leq \int d^{3} \vec{r}\left|n(\vec{r}) v_{3 / 2}(\vec{r})\right|+\int d^{3} \vec{r}\left|n(\vec{r}) v_{\infty}(\vec{r})\right|<\infty .
$$

Lieb's functional in Eq. (4.15) is convex

$$
F_{\text {Lieb }}^{\lambda}\left[\eta n_{1}+(1-\eta) n_{2}\right] \leq \eta F_{\text {Lieb }}^{\lambda}\left[n_{1}\right]+(1-\eta) F_{\text {Lieb }}^{\lambda}\left[n_{2}\right],
$$

because it is the result of a Legendre-Fenchel transformation [Legendre, 1787; Fenchel, 1949] of a concave quantity. Thus, the functional $F_{\text {Lieb }}^{\lambda}$ is the convex conjugate of the ground-state energy with respect to the potential. As a consequence, also the minimization

$$
E_{\lambda}\left[v_{\text {ext }}\right]=\inf _{n \in \mathcal{I}_{N}}\left(F_{\text {Lieb }}^{\lambda}+\int d^{3} \vec{r} n(\vec{r}) v_{\text {ext }}(\vec{r})\right)
$$

is convex because the domain $\mathcal{I}_{N}$ as well as the quantity to be minimized are convex. Convexity guarantees that a local minimum of Eq. (4.19) is also a global minimum ${ }^{4}$. Lieb [Lieb, 1983a] has also shown that the functional (4.15) can equivalently be written as a constrained minimization over $N$-particle density operators $\hat{\rho}^{(N)}=\sum_{i} P_{i}\left|\Psi_{i}^{N}\right\rangle\left\langle\Psi_{i}^{N}\right|$ in the form

$$
F_{\text {Lieb }}^{\lambda}[n]=\inf _{\left\{P_{i}\right\},\left\{\left|\Psi_{i}^{N}\right\rangle\right\}: 0 \leq P_{i} \leq 1,\left|\Psi_{i}^{N}\right\rangle \in \mathcal{W}_{N}, \sum_{i} P_{i}\left\langle\Psi_{i}^{N}|\hat{n}(\vec{r})| \Psi_{i}^{N}\right\rangle=n(\vec{r})} \sum_{i} P_{i}\left\langle\Psi_{i}^{N}|\hat{T}+\lambda \hat{W}| \Psi_{i}^{N}\right\rangle .
$$

This reveals that this functional is related to Levy's function in Eq. (4.10) by $F_{\text {Lieb }}^{\lambda}[n]=$ $F_{\text {Levy }}^{\lambda}[n]$ for densities of pure states and by $F_{\text {Lieb }}^{\lambda}[n] \leq F_{\text {Levy }}^{\lambda}[n] \forall n \in \mathcal{I}_{N}$.

In conclusion, the density functional $F_{\text {Lieb }}^{\lambda}[n]$ is defined for an explicitly known and convex class of densities, is convex, can be directly related to Levy's functional, and is equal to the Hohenberg-Kohn functional for all densities in $\mathcal{A}_{N}^{\lambda}$. Thus, Lieb's functional will serve as our basic definition of the density functional.

\subsection{The exchange-correlation functional}

\subsubsection{From the density functional to the exchange-correlation functional}

For practical calculations, the density functional has to be approximated because the computational effort for the exact functional is just as high as for the solution of the

\footnotetext{
${ }^{3}$ If $f \in L^{p}, g \in L^{q}$ and $\frac{1}{p}+\frac{1}{q}=1$, then $f \cdot g \in L^{1}$.

${ }^{4}$ As $F_{\text {Lieb }}^{\lambda}[n]+\int d^{3} \vec{r} n(\vec{r}) v_{\text {ext }}(\vec{r})$ is not strictly convex, there can be multiple minimizers.
} 
many-particle problem up to differences of polynomial complexity [Schuch and Verstraete, 2009; Liu et al., 2007; Whitfield et al., 2014]. The exchange-correlation functional $E_{x c}[n]$ is defined as

$$
E_{x c}[n]=F^{\lambda=1}[n]-F^{\lambda=0}[n]-\frac{1}{2} \int d^{3} \vec{r} \int d^{3} \vec{r}^{\prime} \frac{e^{2} n(\vec{r}) n\left(\vec{r}^{\prime}\right)}{4 \pi \epsilon_{0}\left|\vec{r}-\vec{r}^{\prime}\right|}
$$

where the last term is the negative of the Hartree energy $E_{H}[n] . F^{\lambda}[n]$ denotes one of the three density functionals discussed in the previous three sections. The discussion contained in this section can be performed similarly for all three variants of the density functional. However, we will give the expressions here for Lieb's functional. The minimization over densities of Eq. (4.19) can be written for $\lambda=1$ as

$$
E_{\lambda=1}\left[v_{\text {ext }}\right]=\inf _{n \in \mathcal{I}_{N}}\left(F_{\text {Lieb }}^{\lambda=0}[n]+E_{H}[n]+E_{x c}[n]+\int d^{3} \vec{r} n(\vec{r}) v_{\text {ext }}(\vec{r})\right) .
$$

Thus, the minimization over densities has been reformulated as a minimization of the noninteracting kinetic energy, the Hartree-energy, the exchange-correlation functional and the expectation value of the external potential. We define the ensemble $\left\{\bar{P}_{i}^{\lambda}\right\},\left\{\left|\bar{\Psi}_{i}^{N, \lambda}\right\rangle\right\}$ as a minimizer of Eq. (4.20) for the interaction strength $\lambda$. The non-interacting kinetic energy

$$
\begin{aligned}
T_{\lambda=0} & =F_{\text {Lieb }}^{\lambda=0}[n]=\inf _{\left\{P_{i}\right\},\left\{\left|\Psi_{i}^{N}\right\rangle\right\}: 0 \leq P_{i} \leq 1,\left|\Psi_{i}^{N}\right\rangle \in \mathcal{W}_{N}, \sum_{i} P_{i}\left\langle\Psi_{i}^{N}|\hat{n}(\vec{r})| \Psi_{i}^{N}\right\rangle=n(\vec{r})} \sum_{i} P_{i}\left\langle\Psi_{i}^{N}|\hat{T}| \Psi_{i}^{N}\right\rangle \\
& =\sum_{i} \bar{P}_{i}^{\lambda=0}\left\langle\bar{\Psi}_{i}^{N, \lambda=0}|\hat{T}| \bar{\Psi}_{i}^{N, \lambda=0}\right\rangle
\end{aligned}
$$

usually represents a large part of the interacting kinetic energy

$$
T_{\lambda=1}=\sum_{i} \bar{P}_{i}^{\lambda=1}\left\langle\bar{\Psi}_{i}^{N, \lambda=1}|\hat{T}| \bar{\Psi}_{i}^{N, \lambda=1}\right\rangle .
$$

The density functional for the full interaction strength $\lambda=1$ can be expressed assuming the existence of the derivative $d F_{\text {Lieb }}^{\lambda}[n] / d \lambda$ with an integration over the range of interacting strengths $0 \leq \lambda \leq 1$ as

$$
F_{\text {Lieb }}^{\lambda=1}[n]=F_{\text {Lieb }}^{\lambda=0}[n]+\int_{0}^{1} d \lambda \frac{d F_{\text {Lieb }}^{\lambda}[n]}{d \lambda} .
$$

The derivative $d F_{\text {Lieb }}^{\lambda}[n] / d \lambda$ can be expressed with the help of the Hellman-Feynman theorem as

$$
\frac{d F_{\text {Lieb }}^{\lambda}[n]}{d \lambda}=\sum_{i} \bar{P}_{i}^{\lambda}\left\langle\bar{\Psi}_{i}^{N, \lambda}|\hat{W}| \bar{\Psi}_{i}^{N, \lambda}\right\rangle
$$

We can connect the density functional for the non-interacting system $\lambda=0$ with the interacting system $\lambda=1$ by

$$
F_{\text {Lieb }}^{\lambda=1}[n]=F_{\text {Lieb }}^{\lambda=0}[n]+\int_{0}^{1} d \lambda \sum_{i} \bar{P}_{i}^{\lambda}\left\langle\bar{\Psi}_{i}^{N, \lambda}|\hat{W}| \bar{\Psi}_{i}^{N, \lambda}\right\rangle .
$$

The relation is has been termed the adiabatic connection [Harris and Jones, 1974; Langreth and Perdew, 1975; Gunnarsson and Lundqvist, 1976]. 


\section{Density functional theory}

The adiabatic connection allows us to rewrite the exchange-correlation functional in Eq. (4.21) as

$$
E_{x c}[n]=\int_{0}^{1} d \lambda \sum_{i} \bar{P}_{i}^{\lambda}\left\langle\bar{\Psi}_{i}^{N, \lambda}|\hat{W}| \bar{\Psi}_{i}^{N, \lambda}\right\rangle-E_{H}[n]
$$

With the two-particle density $\bar{n}^{(2), \lambda}\left(\vec{r}, \vec{r}^{\prime}\right)$ of the ensemble $\left\{\bar{P}_{i}^{\lambda}\right\},\left\{\left|\bar{\Psi}_{i}^{N, \lambda}\right\rangle\right\}$ we arrive at the final expression for the exchange-correlation functional

$$
E_{x c}[n]=\int d^{3} \vec{r} \int d^{3} \vec{r}^{\prime} \frac{1}{2} \int_{0}^{1} d \lambda \frac{\bar{n}^{(2), \lambda}\left(\vec{r}, \vec{r}^{\prime}\right)}{4 \pi \epsilon_{0}\left|\vec{r}-\vec{r}^{\prime}\right|}-E_{H}[n]
$$

Historically, the interaction-strength-dependent pair-distribution function [Perdew and Wang, 1992b]

$$
g_{\lambda}\left(\vec{r}, \vec{r}^{\prime}\right)=\frac{\bar{n}^{(2), \lambda}\left(\vec{r}, \vec{r}^{\prime}\right)}{n(\vec{r}) n\left(\vec{r}^{\prime}\right)}
$$

or the interaction-strength-dependent hole function

$$
h_{\lambda}\left(\vec{r}, \vec{r}^{\prime}\right)=\frac{\bar{n}^{(2), \lambda}\left(\vec{r}, \vec{r}^{\prime}\right)}{n\left(\vec{r}^{\prime}\right)}-n(\vec{r})
$$

have been used to express the two-particle density $\bar{n}^{(2), \lambda}\left(\vec{r}, \vec{r}^{\prime}\right)$. The fundamental sum rule for the hole function

$$
\int d^{3} \vec{r}^{\prime} h_{\lambda}\left(\vec{r}^{\prime}, \vec{r}\right)=-1 \forall \vec{r} \in \mathbb{R}^{3}
$$

directly results from Eq. (2.113). The exchange-correlation hole can be further decomposed into the exchange contribution that is explicitly known for the homogeneous noninteracting electron gas and the correlation contribution. Thus, we can split up Eq. (4.30) into

$$
E_{x c}[n]=\int d^{3} \vec{r} n(\vec{r}) \epsilon_{x}(r)+\int d^{3} \vec{r} n(\vec{r}) \epsilon_{c}(r)
$$

with the exchange(x)- and correlation(c)-energy density per particle

$$
\epsilon_{x / c}(r)=\int d^{3} \vec{r}^{\prime} \frac{1}{2} \int_{0}^{1} d \lambda \frac{h_{x / c, \lambda}\left(\vec{r}^{\prime}, \vec{r}\right)}{4 \pi \epsilon_{0}\left|\vec{r}-\vec{r}^{\prime}\right|}
$$

Local or semi-local approximations of the exchange-correlation functional are usually given in terms of the exchange- and correlation-energy densities per particle. However, expressions for the hole function or the pair-distribution function have been developed for some commonly used functionals [Perdew and Wang, 1992b; Perdew et al., 1996b; Bahmann and Ernzerhof, 2008]. 


\subsubsection{Kinetic contribution to the exchange-correlation functional}

It should be noted here, that the exchange-correlation functional contains a contribution from the kinetic energy due to the interaction-strength integration in Eq. (4.30). This kinetic contribution $T_{x c}$ is given by the difference of the interacting and the non-interacting kinetic energy

$$
T_{x c}[n]=T_{\lambda=1}[n]-T_{\lambda=0}[n] .
$$

Thus, the exchange-correlation functional can also be written as

$$
E_{x c}[n]=U_{x c}[n]+T_{x c}[n],
$$

where

$$
\begin{aligned}
U_{x c} & =\sum_{i} \bar{P}_{i}^{\lambda=1}\left\langle\bar{\Psi}_{i}^{N, \lambda=1}|\hat{W}| \bar{\Psi}_{i}^{N, \lambda=1}\right\rangle-E_{H}[n] \\
& =\frac{1}{2} \int d^{3} \vec{r} \int d^{3} \vec{r}^{\prime} \frac{\bar{n}^{(2), \lambda=1}\left(\vec{r}, \vec{r}^{\prime}\right)}{4 \pi \epsilon_{0}\left|\vec{r}-\vec{r}^{\prime}\right|}
\end{aligned}
$$

denotes the contributions from the interaction. The important difference between $E_{x c}$ in Eq. (4.30) and $U_{x c}$ in Eq. (4.38) is that $U_{x c}$ is evaluated at full interaction strength $\lambda=1$ whereas $E_{x c}$ has the average over all interaction strengths.

We define the partially integrated pair-distribution function as

$$
\bar{g}_{\lambda^{*}}\left(\vec{r}, \vec{r}^{\prime}\right)=\frac{1}{\lambda^{*}} \int_{0}^{\lambda^{*}} d \lambda g_{\lambda}(\vec{r}, \vec{r}) .
$$

It can be used to obtain the interaction-strength-dependent pair-distribution function

$$
g_{\lambda^{*}}\left(\vec{r}, \vec{r}^{\prime}\right)=\bar{g}_{\lambda^{*}}\left(\vec{r}, \vec{r}^{\prime}\right)+\lambda^{*} \partial_{\lambda^{*}} \bar{g}_{\lambda^{*}}\left(\vec{r}, \vec{r}^{\prime}\right) .
$$

In section 6.4.4 we will use this relation to obtain the interaction-strength-dependent pairdistribution function for the local-density approximation from the interaction-strength integrated pair-distribution function given by Perdew and Wang [Perdew and Wang, 1992b].

The expression for the contribution $T_{x c}[n]$ from the kinetic energy has been obtained by Levy and Perdew [Levy and Perdew, 1985] as

$$
T_{x c}[n]=-E_{x c}[n]-\int d^{3} \vec{r} n(\vec{r}) \frac{\partial E_{x c}[n]}{\partial n(\vec{r})} \geq 0 .
$$

The contribution to the kinetic energy from the exchange-part of the exchange-correlation functional is zero, i.e. $T_{x c}[n]=T_{c}[n]$. Equation (4.42) will be used later to obtain the interaction contribution $U_{x c}$ from a given exchange-correlation functional.

\subsection{Kohn-Sham system}

The minimization over densities in Eq. (4.19) can be written as the exchange-correlation functional

$$
E_{\lambda=1}\left[v_{\text {ext }}\right]=\inf _{n \in \mathcal{I}_{N}}\left(T_{\lambda=0}[n]+E_{H}[n]+E_{x c}[n]+\int d^{3} \vec{r} n(\vec{r}) v_{\text {ext }}(\vec{r})\right)
$$




\section{Density functional theory}

and can instead be performed over a Slater determinant

$$
\left.|\Phi\rangle \in \mathcal{W}_{N}^{0}=\{|\Psi\rangle|| \Psi\rangle \in \mathcal{W}_{N} \text { and }|\Psi\rangle \text { is a Slater determinant }\right\}
$$

Thus, the minimization can be written in the form

$$
E_{\lambda=1}\left[v_{\text {ext }}\right]=\inf _{|\Phi\rangle \in \mathcal{W}_{N}^{0}}\left(\langle\Phi|\hat{T}| \Phi\rangle+E_{H}\left[n_{|\Phi\rangle}\right]+E_{x c}\left[n_{|\Phi\rangle}\right]+\int d^{3} \vec{r} n_{|\Phi\rangle}(\vec{r}) v_{\text {ext }}(\vec{r})\right)
$$

This rewriting is possible because any density from the class $\mathcal{I}_{N}$ can be represented by a single Slater determinant according to Lieb [Lieb, 1983a]. Instead of using the Slater determinant directly, we can minimize over its one-particle reduced density matrix $\rho^{(1)}\left(\vec{x}, \vec{x}^{\prime}\right)$ because the density as well as the kinetic energy can be obtained from it. With the one-particle reduced density matrix in the spectral representation we obtain

$$
\begin{aligned}
E_{\lambda=1}\left[v_{\text {ext }}\right] & =\inf _{\left\{f_{i}\right\},\left\{\left|\phi_{i}\right\rangle\right\}: f_{i} \in\{0,1\}, \sum_{i} f_{i}=N,\left\langle\phi_{i} \mid \phi_{j}\right\rangle=\delta_{i j}}\left(\sum_{i} f_{i}\left\langle\phi_{i}|\hat{T}| \phi_{i}\right\rangle\right. \\
& \left.+E_{H}\left[n_{\left\{f_{i}\right\},\left\{\left|\phi_{i}\right\rangle\right\}}\right]+E_{x c}\left[n_{\left\{f_{i}\right\},\left\{\left|\phi_{i}\right\rangle\right\}}\right]+\int d^{3} \vec{r} n_{\left\{f_{i}\right\},\left\{\left|\phi_{i}\right\rangle\right\}}(\vec{r}) v_{\text {ext }}(\vec{r})\right)
\end{aligned}
$$

with the density $n_{\left\{f_{i}\right\},\left\{\left|\phi_{i}\right\rangle\right\}}(\vec{x})=\sum_{i} f_{i}\left\langle\phi_{i}|\hat{n}(\vec{x})| \phi_{i}\right\rangle$. The minimization runs over occupations $f_{i} \in\{0,1\}$ because the one-particle reduced density matrix corresponds to a single Slater determinant. The natural orbitals $\left|\phi_{i}\right\rangle$ are single-particle wave functions. The minimum condition of Eq. (4.46) results in the so-called Kohn-Sham equations [Kohn and Sham, 1965]

$$
\left(-\frac{\hbar^{2} \nabla^{2}}{2 m_{e}}+v_{\text {ext }}(\vec{x})+v_{H}(\vec{x})+v_{x c}(\vec{x})\right)\left\langle\vec{x} \mid \phi_{i}\right\rangle=\epsilon_{i}\left\langle\vec{x} \mid \phi_{i}\right\rangle
$$

with the Hartree-potential $v_{H}(\vec{x})=\partial E_{H}[n] / \partial n(\vec{x})$ and exchange-correlation potential $v_{x c}(\vec{x})=\partial E_{x c}[n] / \partial n(\vec{x})$. Thus, the natural orbitals are eigenstates of an effectively noninteracting system that has the same density as the interacting system. This effectively non-interacting system is called the Kohn-Sham system [Kohn and Sham, 1965] and, consequently, the natural orbitals of this system have been termed Kohn-Sham wave functions. The energy eigenvalues $\epsilon_{i}$ are named the Kohn-Sham energies. Although the Kohn-Sham wave functions and energy eigenvalues are frequently used in the study of chemical bonding and solid state physics, only the eigenvalue of the highest occupied state (HOMO) in a finite system has a rigorous physical meaning namely as the negative of the ionization potential $I$, i.e.

$$
\epsilon_{\mathrm{HOMO}}=-I=E(N)-E(N-1) .
$$

To simplify the notation, we write the minimization problem of DFT as

$$
E_{\lambda=1}\left[v_{\text {ext }}\right]=\inf _{\left\{f_{i}\right\},\left\{\left|\phi_{i}\right\rangle\right\}: f_{i} \in\{0,1\}, \sum_{i} f_{i}=N,\left\langle\phi_{i} \mid \phi_{j}\right\rangle=\delta_{i j}} E_{\mathrm{DFT}}\left(\left\{f_{i}\right\},\left\{\left|\phi_{i}\right\rangle\right\}\right)
$$


with $E_{\text {DFT }}$ defined as

$$
\begin{aligned}
E_{\mathrm{DFT}}\left(\left\{f_{i}\right\},\left\{\left|\phi_{i}\right\rangle\right\}\right) & =\sum_{i} f_{i}\left\langle\phi_{i}|\hat{T}| \phi_{i}\right\rangle+E_{H}\left[n_{\left\{f_{i}\right\},\left\{\left|\phi_{i}\right\rangle\right\}}\right]+E_{x c}\left[n_{\left\{f_{i}\right\},\left\{\left|\phi_{i}\right\rangle\right\}}\right] \\
& +\int d^{3} \vec{r} n_{\left\{f_{i}\right\},\left\{\left|\phi_{i}\right\rangle\right\}}(\vec{r}) v_{\mathrm{ext}}(\vec{r}) .
\end{aligned}
$$

Equivalently, the minimization problem of Eq. (4.49) can be written in the Lagrangemultiplier formalism as

$$
\begin{aligned}
E_{\lambda=1}\left[v_{\text {ext }}\right] & =\inf _{\left\{f_{i}\right\},\left\{\left|\phi_{i}\right\rangle\right\}: f_{i} \in\{0,1\}} \operatorname{stat}_{\mu, \Lambda_{i, j}}\left(E_{\mathrm{DFT}}\left(\left\{f_{i}\right\},\left\{\left|\phi_{i}\right\rangle\right\}\right)\right. \\
& \left.-\mu\left(\int d^{4} \vec{x} n_{\left\{f_{i}\right\},\left\{\left|\phi_{i}\right\rangle\right\}}(\vec{x})-N\right)-\sum_{i, j} \Lambda_{i, j}\left(\left\langle\phi_{i} \mid \phi_{j}\right\rangle-\delta_{i j}\right)\right)
\end{aligned}
$$

where $\mu$ is the Lagrange multiplier of the total particle number and $\Lambda_{i, j}$ the matrix of Lagrange multipliers of the orthonormality of the Kohn-Sham wave functions. The Lagrange multiplier $\mu$ is the chemical potential. Within the Kohn-Sham formalism Janak's theorem [Janak, 1978]

$$
\epsilon_{j}=\frac{\partial E_{\mathrm{DFT}}\left(\left\{f_{i}\right\},\left\{\left|\phi_{i}\right\rangle\right\}\right)}{\partial f_{j}}
$$

holds. The gap of the Kohn-Sham spectrum, that is, the difference of the Kohn-Sham eigenvalue $\epsilon_{\text {LUMO }}$ of the lowest unoccupied molecular orbital (LUMO) and the KohnSham eigenvalue $\epsilon_{\mathrm{HOMO}}$ of the highest occupied molecular orbital (HOMO) is defined as the Kohn-Sham gap $E_{g, K S}=\epsilon_{\mathrm{LUMO}}-\epsilon_{\mathrm{HOMO}}$. A finite derivative discontinuity of the exchange-correlation energy $E_{\text {xc }}$ also contributes to the fundamental gap $E_{g}$. It can be expressed in terms of the exchange-correlation gap

$$
E_{g, x c}(N)=\lim _{\delta \rightarrow 0^{+}}\left(\left.\frac{d E_{x c}(m)}{d m}\right|_{m=N+\delta}-\left.\frac{d E_{x c}(n)}{d n}\right|_{n=N-\delta}\right),
$$

where $E_{x c}(m)$ denotes the value of the exchange-correlation functional in the ground state of the system with $m$ electrons. Thus, the fundamental gap $E_{g}$ can be expressed in terms of the Kohn-Sham gap $E_{g, K S}$ and the exchange-correlation gap $E_{g, x c}$ as [Sham and Schlüter, 1983; Perdew and Levy, 1983; Perdew]

$$
E_{g}(N)=E_{g, K S}(N)+E_{g, x c}(N) .
$$

Local or semi-local approximations of the exchange-correlation energy $E_{x c}$ have a vanishing exchange-correlation gap $E_{g, x c}$ and usually underestimate the fundamental gap.

\subsection{Minimization of the total energy}

The minimization of the total DFT-energy in Eq. (4.46) can be performed in two different approaches. The most widespread approach is the self-consistent solution of the KohnSham equations given in Eq. (4.47). At first, a starting point for the Kohn-Sham wave functions and the occupations is chosen and then the following steps are performed: 


\section{Density functional theory}

1. The electron density $n(\vec{r})$ and the potentials $v_{\mathrm{ext}}, v_{\mathrm{H}}$ and $v_{\mathrm{xc}}$ are evaluated for the current guess of the Kohn-Sham wave functions.

2. The eigenstates and eigenvalues of the resulting effective one-particle Hamiltonian are computed. This is a conventional eigenvalue problem and scales cubically in the system size.

3. If self-consistency is reached, i.e., the new eigenstates are identical to the Kohn-Sham wave functions of the previous iteration, the algorithm terminates.

4. The eigenstates from the solution of the eigenvalue problem are used as the new Kohn-Sham wave functions, and the algorithm continues at step 1. Additional measures have to be taken to ensure convergence of the self-consistency cycle.

This approach is prevalent because the solution of large eigenvalue problems is a common task and efficient implementations exist. In practice, additional measures have to be taken to ensure the convergence of the self-consistency cycle.

The second approach to the solution of the minimization problem is to perform the minimization of Eq. (4.46) with the required constraints. An algorithm that has turned out to be particularly suitable for this task is the Car-Parrinello molecular dynamics approach [Car and Parrinello, 1985]. Here, we first discuss how the Car-Parrinello molecular dynamics approach works and show later how it can be used for the minimization of the total energy with static nuclei. The Car-Parrinello molecular dynamics approach defines the Lagrangian

$$
\begin{aligned}
L & =\frac{1}{2} \sum_{i} f_{i} m_{\phi}\left\langle\dot{\phi}_{i} \mid \dot{\phi}_{i}\right\rangle+\frac{1}{2} \sum_{i} m_{f} \dot{f}_{i}^{2}+\frac{1}{2} \sum_{i=1}^{N_{N}} M_{i} \dot{\vec{R}}_{i}^{2}-E_{\mathrm{DFT}}\left(\left\{f_{i}\right\},\left\{\left|\phi_{i}\right\rangle\right\},\left\{\vec{R}_{i}\right\}\right) \\
& -\sum_{m, n} \Lambda_{m, n}\left(\left\langle\phi_{m} \mid \phi_{n}\right\rangle-\delta_{m, n}\right)-\mu\left(\sum_{i} f_{i}-N\right) .
\end{aligned}
$$

The first term is a fictitious kinetic energy of the Kohn-Sham wave functions with the fictitious mass $m_{\phi}$ and the second term is the fictitious kinetic energy of occupations with the fictitious mass $m_{f}$. The third term denotes the kinetic energy of the nuclei. The third term is the total DFT-energy given in Eq. (4.50). The DFT-energy depends on the position of the nuclei in Eq. (4.50) via the external potential $v_{\text {ext }}$. The last two terms contain the orthonormality constraints of the Kohn-Sham wave functions with the Lagrange multipliers $\Lambda_{m, n}$ and the particle number constraint with the Lagrange multiplier $\mu$. The non-equality constraints of the occupations, i.e., $0 \leq f_{i} \leq 1$, can be enforced with the mapping to the new unconstrained variables $x_{i}$ with $f_{i}=f\left(x_{i}\right)=\left(1+\cos \left(x_{i}\right)\right) / 2$. With the Euler-Lagrange equations, we obtain the equations of motion

$$
\begin{aligned}
M_{i} \ddot{\vec{R}}_{i} & =-\nabla_{\vec{R}_{i}} E_{\mathrm{DFT}}\left(\left\{f_{i}\right\},\left\{\left|\phi_{i}\right\rangle\right\},\left\{\vec{R}_{i}\right\}\right) \\
m_{f} \ddot{f}_{i} & =\frac{1}{2} m_{\phi}\left\langle\dot{\phi}_{i} \mid \dot{\phi}_{i}\right\rangle-\frac{\partial E_{\mathrm{DFT}}\left(\left\{f_{i}\right\},\left\{\left|\phi_{i}\right\rangle\right\},\left\{\vec{R}_{i}\right\}\right)}{\partial f_{i}}-\mu \\
f_{i} m_{\phi}\left|\ddot{\phi}_{i}\right\rangle & =-\frac{\partial E_{\mathrm{DFT}}\left(\left\{f_{i}\right\},\left\{\left|\phi_{i}\right\rangle\right\},\left\{\vec{R}_{i}\right\}\right)}{\partial\left\langle\phi_{i}\right|}-\sum_{n} \Lambda_{i}\left|\phi_{n}\right\rangle .
\end{aligned}
$$


As long as the fictitious kinetic energy of the wave functions is small, i.e. they are close to their Born-Oppenheimer surface, the equations of motion in Eq. (4.56)-Eq.(4.58) describe the classical motion of the nuclei. The equations can be solved in practice by discretizing the time coordinate. The Lagrange multipliers are chosen in every propagation such that the constraints are fulfilled in the next time step. The propagation of the discretized equations of motion can be performed with the Verlet algorithm [Verlet, 1967].

We can also use the idea of the Car-Parrinello molecular dynamics approach to solve the minimization problem of Eq. (4.49) in the external potential of a static arrangement of nuclei: we only have to prevent the atoms from moving and introduce friction. The resulting equations of motion for the occupations and the Kohn-Sham wave functions,

$$
\begin{aligned}
m_{f} \ddot{f}_{i} & =\frac{1}{2} m_{\phi}\left\langle\dot{\phi}_{i} \mid \dot{\phi}_{i}\right\rangle-\frac{\partial E_{\mathrm{DFT}}\left(\left\{f_{i}\right\},\left\{\left|\phi_{i}\right\rangle\right\},\left\{\vec{R}_{i}\right\}\right)}{\partial f_{i}}-\lambda-m_{f} \alpha_{f} \dot{f}_{i} \\
f_{i} m_{\phi}\left|\ddot{\phi}_{i}\right\rangle & =-\frac{\partial E_{\mathrm{DFT}}\left(\left\{f_{i}\right\},\left\{\left|\phi_{i}\right\rangle\right\},\left\{\vec{R}_{i}\right\}\right)}{\partial\left\langle\phi_{i}\right|}-\sum_{n} \Lambda_{i}\left|\phi_{n}\right\rangle-m_{\phi} \alpha_{\phi}\left|\dot{\phi}_{i}\right\rangle,
\end{aligned}
$$

now contain velocity-dependent friction terms with the friction parameters $\alpha_{f}$ and $\alpha_{\phi}$. A simple practical minimization scheme now performs the steps starting from initial friction values $\alpha_{f}$ and $\alpha_{\phi}$ and an initial set of occupations $f_{i}(t=0)$ and Kohn-Sham wave functions $\left|\phi_{i}(t=0)\right\rangle$ :

1. The total DFT-energy $E_{\mathrm{DFT}}$ and its derivatives are evaluated.

2. The occupations and Kohn-Sham wave functions in the next time step $t+\Delta$ are constructed with a time propagation such as the Verlet algorithm.

3. The Lagrange multipliers are determined such that the constraints are fulfilled in the next time step. This yields $f_{i}(t+\Delta)$ and $\left|\phi_{i}(t+\Delta)\right\rangle$

4. If the DFT-total energy is higher than in the previous step, then large friction values are used in the next step to slow down the propagation of the system in the undesired direction.

5. If the DFT-total energy is lower than in the previous step, then friction values are reduced.

6. The algorithm terminates if the DFT-total energy is minimal. Otherwise it continues with $t \rightarrow t+\Delta$ at 1 .

The procedure is guaranteed to converge to a local minimum of the constrained minimization problem as long as the time step $\Delta$ is sufficiently small. Please note, that compared to other minimization algorithms, there is no line search necessary. Another major advantage is that the convergence speed and issues can be analyzed and understood nicely by drawing analogies from the well-known motion of classical particles in simple potentials. The Car-Parrinello-like minimization of the total DFT-energy in Eq. (4.59) and Eq. (4.59) is used in the CP-PAW code. It can easily be modified to energy functionals for which no Kohn-Sham system exists and hence can also be used in the context of reduced densitymatrix functional theory. The minimization over natural orbitals $\left|\phi_{i}\right\rangle$ and occupations $0 \leq f_{i} \leq 1$ is equivalent to a minimization over a one-particle reduced density matrix $\rho_{i j}^{(1)}$, 


\section{Density functional theory}

i.e. a hermitian matrix of dimension equal to the number of one-particle states and the semi-definiteness constraint $0 \leq \rho_{i j}^{(1)} \leq 1$. Because only natural orbitals with non-zero occupations contribute to the one-particle reduced density matrix, all natural orbitals with vanishing occupations can be excluded from the minimization in practice. For zerotemperature Kohn-Sham DFT, the occupations are integers and, hence, only a number of natural orbitals proportional to the number of electrons have to be considered explicitly in the minimization. For zero-temperature reduced density-matrix functional theory, there could in principle be as many non-zero occupations as there are one-particle basis states. Thus, for zero-temperature RDMFT calculations, one has to allow for a sufficient number of partially occupied natural orbitals in the minimization. Zero-temperature RDMFT behaves like finite-temperature DFT in this regard.

\subsection{Extensions}

\subsubsection{Spin-polarized systems}

The extension of the previous sections to a finite spin polarization and spin-dependent potentials can be obtained by replacing

$$
\begin{aligned}
& n(\vec{r}) \rightarrow n(\vec{r}, \uparrow), n(\vec{r}, \downarrow) \\
& v(\vec{r}) \rightarrow v(\vec{r}, \uparrow), v(\vec{r}, \downarrow) .
\end{aligned}
$$

However, there is no spin-dependent generalization of the Hohenber-Kohn theorems because an external magnetic field is not a unique functional of the ground-state spin-density. Levy's approach and Lieb's functional can be generalized straightforwardly [Eschrig, 2003; Ayers and Yang, 2006; Holas and Balawender, 2006].

\subsubsection{Finite temperatures}

Although Mermin [Mermin, 1965] has generalized the Hohenberg-Kohn theorems to finite temperatures, the generalization of density-functional theory to finite temperatures can be obtained much easier by starting from Lieb's functional. The only step required is the replacement of the interaction-strength dependent energy $E_{\lambda}[v]$ in Eq. (4.15) with a grand potential-like quantity

$$
\tilde{\Omega}_{\beta, \lambda}[v]=-\frac{1}{\beta} \ln \left(\operatorname{Tr} e^{-\beta(\hat{T}+\lambda \hat{W})}\right),
$$

which is concave with respect to the potential $v$. Lieb's density functional at finite temperature is

$$
F_{\text {Lieb }, \beta}^{\lambda}[n]=\sup _{v \in L^{3 / 2}+L^{\infty}}\left\{\tilde{\Omega}_{\beta, \lambda}[v]-\int d^{3} \vec{r} n(\vec{r})\right\}
$$

and convex with respect to the density $n$. It can be rewritten as a constrained minimization over many-particle density operators $\hat{\rho}^{\text {(many) }}=\sum_{n} P_{n}\left|\Psi_{n}\right\rangle\left\langle\Psi_{n}\right|$ as

$$
F_{\text {Lieb }, \beta}^{\lambda}[n]=\min _{\hat{\rho}^{(\text {many })}: \operatorname{Tr} \hat{\rho}^{(\text {many })} \hat{n}(\vec{r})=n(\vec{r})}\left\{\operatorname{Tr} \hat{\rho}^{(\text {many })} \hat{T}+\lambda \operatorname{Tr} \hat{\rho}^{(\text {many })} \hat{W}-\operatorname{TS}\left[\hat{\rho}^{(\text {many })}\right]\right\} .
$$


The entropy $S$ is defined in Eq. (2.41). Thus, the grand potential can be written as

$$
\Omega_{\beta, \mu}\left[v_{\mathrm{ext}}\right]=\min _{n \in \mathcal{I}_{N}}\left\{F_{\mathrm{Lieb}, \beta}^{\lambda=1}[n]+\int d^{3} \vec{r} n(\vec{r})\left(v_{\mathrm{ext}}(\vec{r})-\mu\right)\right\} .
$$

As done in section 4.4.1, the finite-temperature functional can be decomposed into similar contributions [Mermin, 1965; Pittalis et al., 2011; Burke et al., 2016]. Our combination of density-functional theory and reduced density matrix functional theory, that will be introduced in chapter 6 , is based on the principles of reduced density matrix functional theory. Therefore, a detailed discussion of finite temperature density functional theory is not required here.

\subsection{Approximate local functionals}

The core idea of the local density approximation (LDA) is to approximate the exchangecorrelation functional as [Vosko et al., 1980; Perdew and Zunger, 1981; Perdew and Wang, 1992a]

$$
E_{x c}[n] \approx E_{x c}^{\mathrm{LDA}}[n]=\int d^{3} \vec{r} n(\vec{r}) \epsilon_{x c}^{\mathrm{hom}}(n(\vec{r})) .
$$

The local exchange-correlation energy density per particle $\epsilon_{x c}^{\text {hom }}(n(\vec{r}))$ is derived from the homogeneous electron gas as a reference system. The three relevant quantities to characterise a homogeneous electron gas are the Fermi vector, $k_{F}=\left(3 \pi^{2} n\right)^{1 / 3}$, the density parameter $r_{s}$

$$
r_{s}=\left[\frac{3}{4 \pi n}\right]^{1 / 3}
$$

and the spin polarization

$$
\zeta=\frac{n_{\uparrow}-n_{\downarrow}}{n_{\uparrow}+n_{\downarrow}} .
$$

For example, Ceperley and Alder [Ceperley and Alder, 1980] have estimated the groundstate energy of the homogeneous electron gas at different densities with Monte Carlo-based method. By subtracting the ground-state energy of the non-interacting homogeneous electron gas

$$
E_{\lambda=0}(n)=\frac{3}{5} \frac{\hbar^{2} k_{F}^{2}}{2 m}
$$

from the numerically obtained ground-state energies of the interacting homogeneous electron gas, estimates of the exchange-correlation energy density per particle can be obtained. Together with analytical results for the limits of high-density and low-density several parametrizations for the exchange-correlation energy density per particle [Vosko et al., 1980; Perdew and Zunger, 1981; Perdew and Wang, 1992a] have been obtained. Semi-local improvements in the form of generalized gradient approximations (GGA), that additionally employ reduced gradients

$$
s=\frac{|\nabla n(\vec{r})|}{n(\vec{r})^{\alpha}}
$$

have been proposed and very successfully used. We do not discuss them here because their semi-local nature is not able to cure the problem of static correlation. 


\subsection{The symmetry-breaking dilemma}

\subsubsection{The hydrogen molecule and static correlation}

The most straightforward system for which density functional theory with local or semilocal functional fails is the hydrogen molecule in the dissociation limit. Here, we first review properties of the hydrogen molecule and then discuss the non-spin-polarized and spin-polarized solutions of local or semi-local functionals in DFT. The hydrogen molecule consists of two hydrogen atoms, one at position $\vec{R}_{1}$ and one at $\vec{R}_{2}$ with distance $d=\mid \vec{R}_{1}-$ $\vec{R}_{2} \mid$. It is well known that density functional theory with local or semi-local functionals describes the hydrogen molecule in the vicinity of the equilibrium distance accurately. Even the Hartree-Fock approximation predicts the equilibrium distance reasonably well. A comparison of the total energy of the non-magnetic ground state of the hydrogen molecule around its equilibrium distance in different theoretical descriptions is shown in figure 4.1. The results have been obtained with the quantum chemistry code Psi4 [Parrish et al., 2017] with the Gaussian basis cc-pvdz [Dunning, 1989a]. However, if the distance of the atoms is increased beyond its equilibrium distance qualitative failures in the results from local or semi-local functionals and Hartree-Fock start to emerge: if the spin-polarization $\vec{m}(\vec{r})=n_{\uparrow}(r)-n_{\downarrow}(\vec{r})$ is restricted to vanish everywhere as is the case for the exact solution, then the total energy in these approximate treatments does not approach the exact dissociation limit of

$$
\lim _{d \rightarrow \infty} E(d)=-1 \mathrm{H}
$$

but is greatly overestimated. This overestimation has been termed the static-correlation problem [Cohen et al., 2012]. In quantum chemistry usually two kinds of qualitatively different electronic correlation are distinguished: on the one side dynamical correlation and on the other side static (non-dynamical) correlation. Although there is no formal definition of static or dynamical correlation, static correlation is said to originate from degenerate or nearly degenerate states and, hence, the exact ground-state wave function is qualitatively different from the Hartree-Fock Slater determinant, i.e., the ground-state wave function in the Hartree-Fock approximation. Dynamical correlation is said to be strong if the exact ground-state wave function has a large overlap with the Hartree-Fock Slater determinant and includes a large number of excited Slater determinants with small amplitudes. Dynamical correlation can be well described by the Hartree-Fock approximation or perturbative extensions based on the Hartree-Fock Slater determinant. Local or semi-local density functionals can properly describe dynamical correlation [Langreth and Perdew, 1977; Burke and Perdew, 1995]. The dissociation limit of the hydrogen molecule is dominated by static correlation due to the fact that the ground-state wave function has significant contributions from multiple Slater determinants. In contrast, close to the equilibrium distance static correlation is small, and the system is dominated by dynamical correlation that is well described by local or semi-local functionals.

Figure 4.1 also shows results for the situation when the spin-polarization is not restricted to vanish everywhere. In this case, local or semi-local density functionals and the HartreeFock treatment give the correct dissociation energy at the cost of an unphysical broken spin-symmetry. Thus, this problem is also called the broken-symmetry dilemma. The distance $d$ beyond which a spin-polarized solution exists that has a lower total energy 
than the non-spin-polarized solution has been termed the Coulson-Fisher point [Coulson and Fischer, 1949]. The location of this point depends on the approximate treatment.

\subsubsection{The Hubbard dimer as a minimal model}

The dissociation curve of the hydrogen molecule, that is, the dependence of the total energy of the system $E$ on the distance $d$ can be most easily understood by considering a minimal one-particle basis. We choose here the minimal one-particle basis that is composed of spherical atomic 1s-orbitals $\phi_{1 s}(r)$ centered at each atom. Thus, we have the four spin-orbitals

$$
\begin{aligned}
\left\langle\vec{r}, \sigma \mid \chi_{1, \uparrow}\right\rangle & =\delta_{\sigma, \uparrow} \phi_{1 s}\left(\left|\vec{r}-\vec{R}_{1}\right|\right) \\
\left\langle\vec{r}, \sigma \mid \chi_{1, \downarrow}\right\rangle & =\delta_{\sigma, \downarrow} \phi_{1 s}\left(\left|\vec{r}-\vec{R}_{1}\right|\right) \\
\left\langle\vec{r}, \sigma \mid \chi_{2, \uparrow}\right\rangle & =\delta_{\sigma, \uparrow} \phi_{1 s}\left(\left|\vec{r}-\vec{R}_{2}\right|\right) \\
\left\langle\vec{r}, \sigma \mid \chi_{2, \downarrow}\right\rangle & =\delta_{\sigma, \downarrow} \phi_{1 s}\left(\left|\vec{r}-\vec{R}_{2}\right|\right)
\end{aligned}
$$

as a minimal basis. The Born-Oppenheimer Hamiltonian ${ }^{5}$ is given by Eq. (2.117). The required matrix elements defined in Eq. (2.118) and Eq. (2.119) can be calculated from the atomic orbital $\phi_{1 s}(r)$. We further approximate the two-particle interaction to be local on the atoms, that is,

$$
\hat{W} \approx \hat{W}_{\text {local }}=U \sum_{i \in\{1,2\}} \hat{n}_{i, \uparrow} \hat{n}_{i, \downarrow}
$$

with the matrix element of the local interaction local interaction

$$
U=\int d^{4} \vec{x} \int d^{4} \vec{x}^{\prime} \frac{e^{2}}{4 \pi \epsilon_{0}\left|\vec{r}-\vec{r}^{\prime}\right|} \phi_{1 s}(|\vec{r}|) \phi_{1 s}\left(\left|\vec{r}^{\prime}\right|\right) \phi_{1 s}(|\vec{r}|) \phi_{1 s}\left(\left|\vec{r}^{\prime}\right|\right)
$$

Thus, the hydrogen molecule is equivalent to a half-filled Hubbard dimer discussed in section 3.1 in this approximation. A change of the distance between the atoms in the hydrogen molecule amounts to a change of the matrix elements of the one-particle Hamiltonian and a change of the overlap of the one-particle orbitals. The exact ground-state energy of the half-filled Hubbard dimer with the Hamiltonian

$$
\hat{H}=-t \sum_{\sigma}\left(\hat{c}_{1 \sigma}^{\dagger} \hat{c}_{2, \sigma}+\hat{c}_{2 \sigma}^{\dagger} \hat{c}_{1, \sigma}\right)+U \sum_{i=1,2} \hat{n}_{i, \uparrow} \hat{n}_{i, \downarrow}
$$

is

$$
E=-2 t\left(\sqrt{1+\left(\frac{U}{4 t}\right)^{2}}-\frac{U}{4 t}\right) .
$$

The one-particle reduced density matrix of the exact ground state has the form

$$
\rho^{(1)}=\frac{1}{2}\left(\begin{array}{cccc}
1 & 0 & \cos (2 \theta) & 0 \\
0 & 1 & 0 & \cos (2 \theta) \\
\cos (2 \theta) & 0 & 1 & 0 \\
0 & \cos (2 \theta) & 0 & 1
\end{array}\right)
$$

\footnotetext{
${ }^{5}$ As noted in section 2.7, we will not explicitly mention the ionic interaction of the nuclei in the Hamiltonian.
} 
with

$$
\theta(U)=\arctan \left(\sqrt{1+\left(\frac{U}{4 t}\right)^{2}}+\frac{U}{4 t}\right)-\frac{\pi}{4}
$$

The occupations are

$$
\begin{aligned}
& f_{b, \sigma}=\frac{1}{2}+\frac{1}{2} \cos (2 \theta), \\
& f_{a, \sigma}=\frac{1}{2}-\frac{1}{2} \cos (2 \theta)
\end{aligned}
$$

for the natural orbitals

$$
\begin{aligned}
& |b, \sigma\rangle=\frac{1}{\sqrt{2}}\left(\left|\chi_{1, \sigma}\right\rangle+\left|\chi_{2, \sigma}\right\rangle\right), \\
& |a, \sigma\rangle=\frac{1}{\sqrt{2}}\left(\left|\chi_{1, \sigma}\right\rangle-\left|\chi_{2, \sigma}\right\rangle\right) .
\end{aligned}
$$

The dissociation limit $d \rightarrow \infty$ of the hydrogen molecule corresponds to the $U / t \rightarrow \infty$-limit of the half-filled Hubbard dimer.

\subsubsection{Broken-symmetry HF-Slater determinants}

In this section, we investigate the question if a many-particle wave function composed of broken-symmetry Hartree-Fock Slater determinants, i.e., broken-symmetry ground states of the Hartree-Fock approximation, can be constructed and distinguished from the exact ground state. We consider the half-filled Hubbard dimer as an example. In the atomic limit $U / t \rightarrow \infty$, the ground state of the half-filled Hubbard dimer is four-fold degenerate and the ground-state density operator is

$$
\begin{aligned}
\hat{\rho}_{U / t \rightarrow \infty}^{(N)} & =\sum_{i=1}^{4} \frac{1}{4}\left|\Psi_{i, U / t \rightarrow \infty}\right\rangle\left\langle\Psi_{i, U / t \rightarrow \infty}\right| \\
\left|\Psi_{1, U / t \rightarrow \infty}\right\rangle & =\frac{1}{\sqrt{2}}(|1001\rangle-|0110\rangle) \\
\left|\Psi_{2, U / t \rightarrow \infty}\right\rangle & =\frac{1}{\sqrt{2}}(|1001\rangle+|0110\rangle) \\
\left|\Psi_{3, U / t \rightarrow \infty}\right\rangle & =|1010\rangle \\
\left|\Psi_{4, U / t \rightarrow \infty}\right\rangle & =|0101\rangle .
\end{aligned}
$$

The one-particle reduced density matrix is diagonal and the occupations are equal to $1 / 2$. The symmetry-broken ground states of spin-polarized Hartree-Fock calculations in the limit $U / t \rightarrow \infty$ are

$$
\begin{aligned}
\left|\Psi_{H F, U / t \rightarrow \infty, 1}\right\rangle & =|1001\rangle \\
\left|\Psi_{H F, U / t \rightarrow \infty, 2}\right\rangle & =|0110\rangle \\
\left|\Psi_{H F, U / t \rightarrow \infty, 3}\right\rangle & =|1010\rangle \\
\left|\Psi_{H F, U / t \rightarrow \infty, 4}\right\rangle & =|0101\rangle .
\end{aligned}
$$


These Slater determinants have diagonal one-particle density matrices with integer occupations. The ensemble

$$
\hat{\rho}_{H F, U / t \rightarrow \infty}^{(N)}=\sum_{i=1}^{4} \frac{1}{4}\left|\Psi_{H F, U / t \rightarrow \infty, i}\right\rangle\left\langle\Psi_{H F, U / t \rightarrow \infty, i}\right|
$$

as a combination of all symmetry-broken Hartree-Fock ground states is equal to the ground-state density operator in Eq. (4.87) and, hence, the two cannot be distinguished by measurements. It should be noted here, that this is no longer the case if we leave away some of the Hartree-Fock states in Eq. (4.96): the density operator

$$
\hat{\tilde{\rho}}_{H F, U / t \rightarrow \infty}^{(N)}=\sum_{i=1}^{2} \frac{1}{2}\left|\Psi_{H F, U / t \rightarrow \infty, i}\right\rangle\left\langle\Psi_{H F, U / t \rightarrow \infty, i}\right|
$$

can be distinguished from the exact one in Eq. (4.87) with measurements of the spin-spin correlation function $\left\langle\hat{S}_{1, z} \hat{S}_{2, z}\right\rangle$. The spin-spin correlation functions can also be measured experimentally with neutron scattering experiments, where the spin-structure factor is proportional to the inelastic neutron scattering cross section. The spin-spin correlation functions can be obtained from the spin-structure factor by a Fourier transform. The equivalence of the exact density operator in Eq. (4.87) and the ensemble-HF density operator in Eq. (4.96) suggests to generalize this idea to other situations. We evaluate this approach for the half-filled Hubbard dimer with a large interaction strength $U / t>2$, i.e., the regime where the Hartree-Fock approximation produces antiferromagnetic symmetrybroken states to investigate the resulting reduced density matrices. A one-particle reduced density matrix that is a minimum of the Hartree-Fock energy-expression in the regime is [Kamil et al., 2016]

$$
\rho^{(1), H F}=\frac{1}{2}\left(\begin{array}{cccc}
1+\sin (2 \gamma) & 0 & \cos (2 \gamma) & 0 \\
0 & 1-\sin (2 \gamma) & 0 & \cos (2 \gamma) \\
\cos (2 \gamma) & 0 & 1-\sin (2 \gamma) & 0 \\
0 & \cos (2 \gamma) & 0 & 1+\sin (2 \gamma)
\end{array}\right)
$$

with

$$
\gamma=\frac{1}{2} \arccos (2 t / U)
$$

The corresponding spin-flipped density matrix is

$$
\tilde{\rho}^{(1), H F}=\frac{1}{2}\left(\begin{array}{cccc}
1-\sin (2 \gamma) & 0 & \cos (2 \gamma) & 0 \\
0 & 1+\sin (2 \gamma) & 0 & \cos (2 \gamma) \\
\cos (2 \gamma) & 0 & 1+\sin (2 \gamma) & 0 \\
0 & \cos (2 \gamma) & 0 & 1-\sin (2 \gamma)
\end{array}\right)
$$

The one-particle reduced density matrix of the ensemble of $\rho^{(1), H F}$ and the corresponding spin-flipped matrix similar to Eq. (4.96) is then

$$
\rho^{(1), \mathrm{HF}-\text { ensemble }}=\frac{1}{2}\left(\rho^{(1), H F}+\tilde{\rho}^{(1), H F}\right)=\frac{1}{2}\left(\begin{array}{cccc}
1 & 0 & \cos (2 \gamma) & 0 \\
0 & 1 & 0 & \cos (2 \gamma) \\
\cos (2 \gamma) & 0 & 1 & 0 \\
0 & \cos (2 \gamma) & 0 & 1
\end{array}\right) .
$$




\section{Density functional theory}

The two-fold degenerate occupations of this one-particle reduced density matrix are (1$\cos (2 \gamma)) / 2$ and $(1+\cos (2 \gamma)) / 2$. Thus, they show the same qualitative dependence on the interaction strength as the occupations of the exact one-particle reduced density matrix discussed in section 4.9.2. However, the quantitative behaviour is different because the dependence of $\gamma$ (Eq. 4.99) on the interaction strength is different from the dependence of $\theta$ on the interaction strength given in Eq. (4.82). The value of the density-matrix functional in the Hartree-Fock approximation, i.e., the Hartree-Fock interaction energy, is

$$
F_{H F}^{\hat{W}}\left[\rho^{(1), \mathrm{HF}-\mathrm{ensemble}}\right]=\frac{U}{2}
$$

for the one-particle reduced density matrix $\rho^{(1), \mathrm{HF}-\text { ensemble }}$ in Eq. (4.101). The HartreeFock density-matrix functional for the one-particle reduced density matrices of the brokensymmetry states are

$$
F_{H F}^{\hat{W}}\left[\rho_{A}^{(1)}\right]=F_{H F}^{\hat{W}}\left[\rho_{B}^{(1)}\right]=\frac{2 t^{2}}{U} .
$$

and different from the value of the ensemble-state. This is a direct consequence of concavity,

$$
F_{H F}^{\hat{W}}\left[\rho_{A}^{(1)} / 2+\rho_{B}^{(1)} / 2\right]=F_{H F}^{\hat{W}}\left[\rho^{(1), H F-\text { ensemble }}\right] \geq F_{H F}^{\hat{W}}\left[\rho_{A}^{(1)}\right] / 2+F_{H F}^{\hat{W}}\left[\rho_{B}^{(1)}\right] / 2,
$$

of the density-matrix functional in the Hartree-Fock approximation. In contrast, a convex density-matrix functional would obey

$$
F^{\hat{W}}\left[\rho^{(1), H F} / 2+\tilde{\rho}^{(1), H F} / 2\right] \leq F^{\hat{W}}\left[\rho^{(1), H F}\right] / 2+F^{\hat{W}}\left[\tilde{\rho}^{(1), H F}\right] / 2 .
$$

Thus, a convex density-matrix functional would not have produced the symmetry-broken states in the first place, but rather a spin-symmetric ground state. An alternative estimate of the interaction energy can be obtained by constructing many-particle wave functions that are Slater determinants and have the one-particle reduced density matrix in Eq. (4.98) respectively its spin-flipped modification in Eq. (4.100). We obtain the many-particle wave functions

$$
\begin{aligned}
& \left|\Psi_{H F, 1}\right\rangle=\left(\hat{c}_{1, \uparrow}^{\dagger} \cos (\gamma-\pi / 4)+\hat{c}_{2, \uparrow}^{\dagger} \cos (\gamma+\pi / 4)\right)\left(\hat{c}_{1, \downarrow}^{\dagger} \cos (\gamma+\pi / 4)+\hat{c}_{2, \downarrow}^{\dagger} \cos (\gamma-\pi / 4)\right)|\mathcal{O}\rangle \\
& \left|\Psi_{H F, 2}\right\rangle=\left(\hat{c}_{1, \downarrow}^{\dagger} \cos (\gamma-\pi / 4)+\hat{c}_{2, \downarrow}^{\dagger} \cos (\gamma+\pi / 4)\right)\left(\hat{c}_{1, \uparrow}^{\dagger} \cos (\gamma+\pi / 4)+\hat{c}_{2, \uparrow}^{\dagger} \cos (\gamma-\pi / 4)\right)|\mathcal{O}\rangle .
\end{aligned}
$$

The density operator

$$
\hat{\rho}_{H F}^{(N)}=\sum_{i=1}^{2} \frac{1}{2}\left|\Psi_{H F, i}\right\rangle\left\langle\Psi_{H F, i}\right|
$$

of these two many-particle wave functions has the one-particle reduced density matrix in Eq. (4.101) and the interaction energy $U / 2 \cos ^{2}(2 \gamma)$. This density operator $\hat{\rho}_{H F}^{(N)}$ can be distinguished from the exact ground-state density operator by measuring the spin-spin 
correlation functions. Results for the spin-spin correlation function $\left\langle\hat{\vec{S}}_{1} \cdot \hat{\vec{S}}_{2}\right\rangle$ of the density operator $\hat{\rho}_{H F}^{(N)}$ and the exact density operator are shown in figure 4.2 .

The characteristic difference in the spin-spin correlation functions also persist if an effective interactions strength $U_{\text {eff }}$ is used in the Hartree-Fock approximation that is chosen such that the reconstructed one-particle reduced density matrix in Eq. (4.101) is identical to the exact ground-state density matrix ${ }^{6}$. We conclude that the reconstruction of a spin-symmetric state from broken-symmetric states gives a qualitatively correct one-particle reduced density matrix. If the interaction energy is calculated from Slater determinants and not from the density-matrix functional in the Hartree-Fock approximation, this scheme yields the same interaction energy as for a symmetry-broken state. Other shortcomings such as the systematic overestimation of the total energy in the HartreeFock approximation or over-localization of electrons are not affected by this symmetry reconstruction scheme.

A related method that reconstructs symmetric states from broken-symmetry Slater determinants is the variational symmetry-projected Hartree-Fock method [Schmid, 2004; Schmid et al., 2005; Scuseria et al., 2011; Rodríguez-Guzmán et al., 2012, 2013]. This method has only recently made its way from nuclear physics to solid-state theory and is based on the idea to restore the symmetry of a Slater determinant with projections. Firstly, any Slater determinant with $M \in \mathbb{N}$ particles in a $d$-dimensional one-particle basis can be parametrized by a d-by-d unitary matrix $U \in U(d),|\Psi(U)\rangle$. Then required physical symmetries of the system can be restored with applications of the corresponding projection operators $\hat{P}_{i}$. The projected determinant

$$
\left|\Psi_{U, P}\right\rangle=\frac{\sum_{i} \hat{P}_{i}|\Psi(U)\rangle}{\sum_{i, j}\left\langle\Psi(U)\left|\hat{P}_{j}^{\dagger} \hat{P}_{i}\right| \Psi(U)\right\rangle}
$$

is then used as a variational wave function. The approximate ground-state energy is obtained from the minimization

$$
E_{0} \approx \min _{U \in U(d)}\left\langle\Psi_{U, P}|\hat{H}| \Psi_{U, P}\right\rangle
$$

The approximation can be improved systematically by using multiple non-orthogonal determinants instead of one [Rodríguez-Guzmán et al., 2012, 2013]. Investigation for the one-dimensional and two-dimensional Hubbard models [Schmid et al., 2005; RodríguezGuzmán et al., 2012, 2013] as well as for molecules [Scuseria et al., 2011] have shown promising results.

\subsubsection{Interpretation of the symmetry-breaking dilemma}

One possible solution of the symmetry-breaking dilemma is the reinterpretation of spindensity-functional theory given by Perdew et al. [Perdew et al., 1995]. Perdew, Savin, and Burke have constructed an alternative theory which predicts the electron density and the on-top two-electron density $n^{(2)}(\vec{r}, \vec{r})$ (on-top pair density) instead of the electron density and the magnetization density. Please note that within their theory there can be a finite magnetization density in the Kohn-Sham system, but the magnetization density of the

\footnotetext{
${ }^{6}$ This is equivalent to the choice of an effective interaction parameter $U_{\text {eff }}$ such that $\gamma\left(U_{\text {eff }}\right)=\theta(U)$.
} 


\section{Density functional theory}

Kohn-Sham system is not a prediction of the magnetization density of the real interacting system. Thus, Perdew et al. avoid the symmetry-breaking dilemma by proposing a theory that does not predict the magnetization density. The existing local spin-density approximation and the generalized gradient approximations can be reused within this theory.

However, investigations by Hollet and Gill [Hollett and Gill, 2011] show that, for example, for Be-like ions there are contributions to the correlation energy from static correlation that can not be recovered by a broken-symmetry solution. Treating the broken-symmetry state as the ground state only avoids the static-correlation error. The delocalization error, i.e., the tendency of approximate local or semi-local density functionals to unphysically favor fractional charges or delocalized charge distributions due to missing derivative discontinuities of the total energy at integer particle numbers [Cohen et al., 2012], is not cured by broken-symmetry solutions in most cases. Thus, we consider the spin-symmetrybreaking of approximate local or semi-local functionals in DFT as well as DFT+U and local hybrid functionals as a shortcoming of the functionals. We only consider the standard interpretation of the magnetization density in spin-density-functional theory as a prediction of the physical magnetization density. 

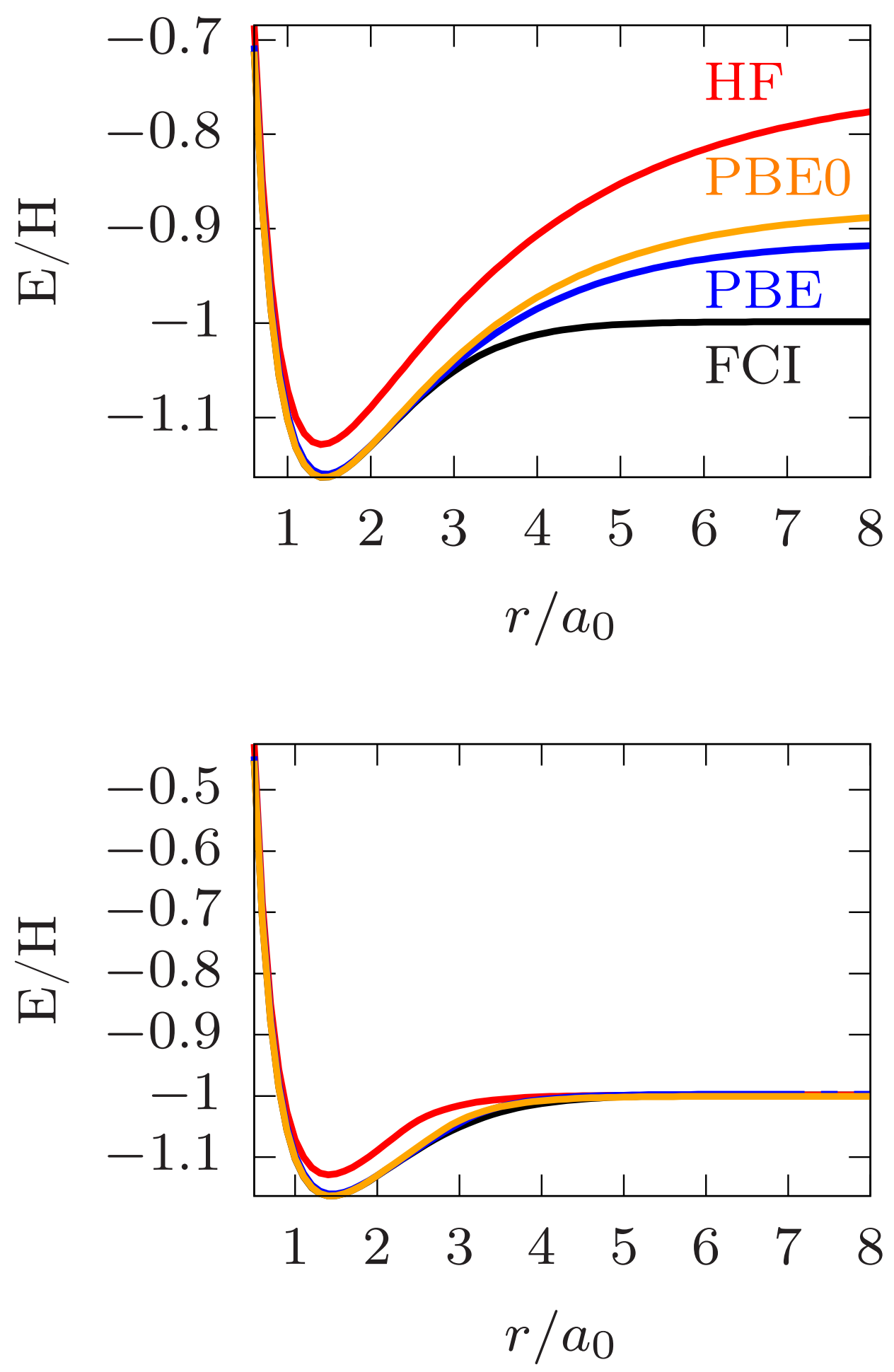

Figure 4.1.: Comparison of the dissociation curve of the hydrogen molecule from HartreeFock and density functional theory results with several functionals to exact results from the full configuration-interaction method (FCI). The upper graph shows the results when the calculation is restricted to non-spin-polarized ground states. The lower graph shows results when a finite spin polarization is allowed and the spin-polarized extension of the functionals is used. The results have been obtained with the quantum chemistry code Psi4 [Parrish et al., 2017] in the Gaussian basis cc-pvdz [Dunning, 1989a]. 


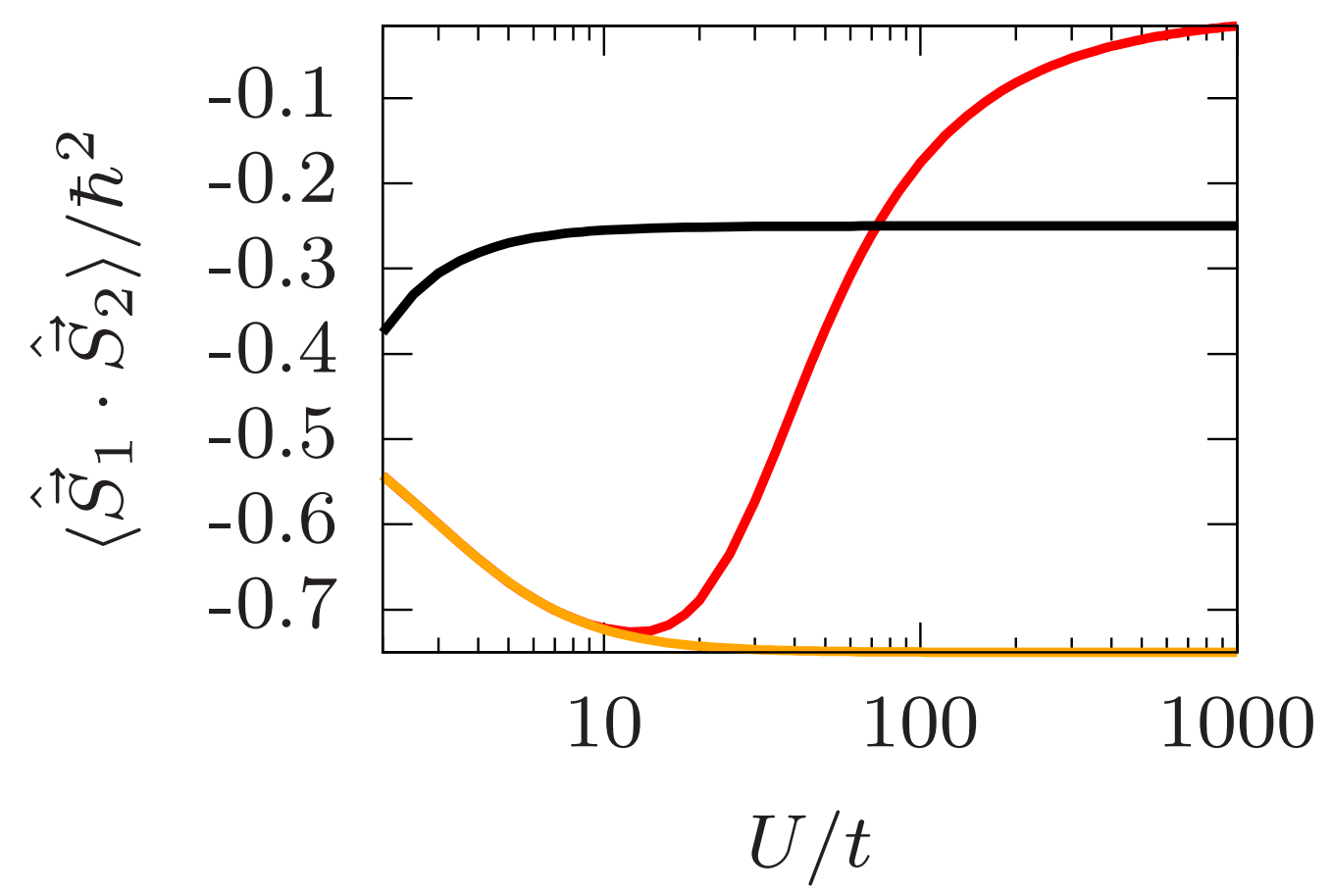

Figure 4.2.: Spin-spin correlation function $\left\langle\hat{\vec{S}}_{1} \cdot \hat{\vec{S}}_{2}\right\rangle$ of the half-filled Hubbard dimer. The orange line shows results for the zero-temperature ground state and the red line for the ground state at finite temperature $k_{B} T=t / 20$. The black line shows results for the symmetry-reconstructed Hartree-Fock ensemble density operator in Eq. 4.108. 


\section{Reduced density-matrix functional theory}

\subsection{Introduction}

We propose to use reduced density-matrix functional theory (RDMFT, [Gilbert, 1975b; Levy, 1979; Valone, 1980; Lieb, 1983b]) instead of density-functional theory for the description of strong electronic correlations. There are two main reasons for this: Firstly the density-matrix functional only contains contributions from the interaction and the entropy but no contribution from the kinetic energy like the exchange-correlation functional of DFT. Secondly, the signature of strong correlation is directly visible in the fractional character of occupations, because the one-particle reduced density matrix of the physical system is directly available. Also, effects of correlation can be much easier described in terms of the one-particle reduced density matrix instead of the electron density. This is advantageous for the construction of improved approximate functionals, for example, from many-particle perturbation theory [Blöchl et al., 2013; Baldsiefen et al., 2017, 2015]. Furthermore, there are already parametrized approximate functionals known that describe strong electronic correlation in certain situations very well [Müller, 1984].

\subsection{Basic formalism}

Within reduced density-matrix functional theory [Gilbert, 1975b; Levy, 1979; Valone, 1980; Lieb, 1983b] (RDMFT) the grand potential is written as the Legendre-Fenchel transformation [Legendre, 1787; Fenchel, 1949]

$$
\Omega_{\beta, \mu}[h]=\min _{\rho^{(1)}: 0 \leq \rho^{(1)} \leq \mathbb{1}}\left\{\operatorname{Tr}\left[\rho^{(1)}(h-\mu \mathbb{1})\right]+F_{\beta}^{\hat{W}}\left[\rho^{(1)}\right]\right\},
$$

of the density-matrix functional $F_{\beta}^{\hat{W}}\left[\rho^{(1)}\right]$ with respect to the one-particle reduced density matrix $\rho^{(1)}$. The Helmholtz potential $H_{\beta, N}(\hat{h}+\hat{W})$ for fixed particle number $N$ and inverse temperature $\beta$ defined in Eq. (2.44) can be written with the density-matrix functional as

$$
H_{\beta, N}[h]=\min _{\rho^{(1)}: 0 \leq \rho^{(1)} \leq \mathbb{1}, \operatorname{Tr}\left[\rho^{(1)}\right]=N}\left\{\operatorname{Tr}\left[\rho^{(1)} h\right]+F_{\beta}^{\hat{W}}\left[\rho^{(1)}\right]\right\} .
$$

Thus, the ground-state energy is

$$
E_{N}[h]=\min _{\rho^{(1)}: 0 \leq \rho^{(1)} \leq \mathbb{1}, \operatorname{Tr}\left[\rho^{(1)}\right]=N}\left\{\operatorname{Tr}\left[\rho^{(1)} h\right]+F^{\hat{W}}\left[\rho^{(1)}\right]\right\}
$$

with the zero-temperature density-matrix functional

$$
F^{\hat{W}}\left[\rho^{(1)}\right]=\lim _{\beta \rightarrow \infty} F_{\beta}^{\hat{W}}\left[\rho^{(1)}\right] .
$$


The density-matrix functional is the Legendre-Fenchel transform of the grand potential $\Omega_{\beta, \mu}[h]$ with respect to the matrix elements $h$ of the one-particle Hamiltonian, i.e.,

$$
F_{\beta}^{\hat{W}}\left[\rho^{(1)}\right]=\max _{h}\left[\Omega_{\beta, \mu=0}[h]-\operatorname{Tr}\left[\rho^{(1)} h\right]\right]
$$

The density-matrix functional is an universal functional of the one-particle reduced density matrix in the sense that it does not depend on the external one-particle potential of the system [Gilbert, 1975b]. As discussed in section 2.4, the grand potential is concave with respect to the matrix-elements of the one-particle Hamiltonian. Thus, Eq. (5.5) represents the Legendre-Fenchel transformation of a concave quantity and the densitymatrix functional is convex with respect to the one-particle reduced density matrix. The matrix elements of the one-particle Hamiltonian and the one-particle reduced density matrix are conjugate quantities and we have the relations

$$
\begin{gathered}
\frac{\partial \Omega_{\beta, \mu}[h]}{\partial h_{\alpha, \beta}}=\rho_{\beta, \alpha}^{(1)} \\
\frac{\partial F_{\beta}^{\hat{W}}\left[\rho^{(1)}\right]}{\partial \rho_{\alpha, \beta}^{(1)}}=-h_{\beta, \alpha} .
\end{gathered}
$$

Levy [Levy, 1979] and Valone [Valone, 1980] have shown that the density-matrix functional can be obtained from a constrained minimization over an ensemble of orthonormal fermionic many-particle wave functions $\left|\Psi_{i}\right\rangle$ and ensemble probabilities $P_{i}$ with $0 \leq P_{i} \leq 1$ and $\sum_{i} P_{i}=1$ as

$$
F_{\beta}^{\hat{W}}\left[\rho^{(1)}\right]=\min _{\left\{P_{i},\left|\Psi_{i}\right\rangle\right\} \rightarrow \rho^{(1)}}\left[\sum_{i} P_{i}\left\langle\Psi_{i}|\hat{W}| \Psi_{i}\right\rangle+\frac{1}{\beta} \sum_{i} P_{i} \ln \left(P_{i}\right)\right] .
$$

Here we denote with $\left\{P_{i},\left|\Psi_{i}\right\rangle\right\} \rightarrow \rho^{(1)}$ the set of ensembles with a given one-particle reduced density matrix $\rho^{(1)}$ according to Eq. (2.129). The density-matrix functional is usually decomposed into four contributions [Helbig, 2006; Blöchl et al., 2013; Baldsiefen et al., 2015]: the Hartree energy $F_{H}^{\hat{W}}\left[\rho^{(1)}\right]$, Fock energy $F_{x}^{\hat{W}}\left[\rho^{(1)}\right]$, an entropy contribution of a non-interacting system $F_{\beta}^{\hat{0}}\left[\rho^{(1)}\right]$ and correlation energy $F_{c, \beta}^{\hat{W}}\left[\rho^{(1)}\right]$ as

$$
\begin{aligned}
F_{\beta}^{\hat{W}}\left[\rho^{(1)}\right] & =F_{H}^{\hat{W}}\left[\rho^{(1)}\right]+F_{x}^{\hat{W}}\left[\rho^{(1)}\right]+F_{\beta}^{\hat{0}}\left[\rho^{(1)}\right]+F_{c, \beta}^{\hat{W}}\left[\rho^{(1)}\right] \\
F_{H}^{\hat{W}}\left[\rho^{(1)}\right] & =\frac{1}{2} \sum_{\alpha, \beta, \gamma, \delta} U_{\alpha, \beta, \delta, \gamma} \rho_{\delta, \alpha}^{(1)} \rho_{\gamma, \beta}^{(1)}, \\
F_{x}^{\hat{W}}\left[\rho^{(1)}\right] & =-\frac{1}{2} \sum_{\alpha, \beta, \gamma, \delta} U_{\alpha, \beta, \delta, \gamma} \rho_{\gamma, \alpha}^{(1)} \rho_{\delta, \beta}^{(1)}, \\
F_{\beta}^{\hat{0}}\left[\rho^{(1)}\right] & =\frac{1}{\beta} \operatorname{Tr}\left[\rho^{(1)} \ln \rho^{(1)}-\left(\mathbb{1}-\rho^{(1)}\right) \ln \left(\mathbb{1}-\rho^{(1)}\right)\right] .
\end{aligned}
$$

The correlation energy $F_{c, \beta}^{\hat{W}}\left[\rho^{(1)}\right]$ contains contributions from interaction as well as from the entropy and has to be approximated. Similarly to the expression for the total energy minimization within DFT given in Eq. (4.51), we can rewrite the RDMFT-minimization 
problem in Eq. (5.3) as

$$
\begin{aligned}
E_{N}[h] & =\min _{\left\{f_{i}\right\},\left\{\left|\phi_{i}\right\rangle\right\}: 0 \leq f_{i} \leq 1} \operatorname{stat}_{\mu, \Lambda_{i, j}}\left\{E_{\mathrm{RDMFT}}\left(\left\{f_{i}\right\},\left\{\left|\phi_{i}\right\rangle\right\}\right)\right. \\
& \left.-\mu\left(\sum_{i} f_{i}-N\right)-\sum_{i, j} \Lambda_{i, j}\left(\left\langle\phi_{i} \mid \phi_{j}\right\rangle-\delta_{i, j}\right)\right\}
\end{aligned}
$$

with

$$
E_{\mathrm{RDMFT}}\left(\left\{f_{i}\right\},\left\{\left|\phi_{i}\right\rangle\right\}\right)=\operatorname{Tr}\left[\rho^{(1)}\left(\left\{f_{i}\right\},\left\{\left|\phi_{i}\right\rangle\right\}\right) h\right]+F_{\beta}^{\hat{W}}\left[\rho^{(1)}\left(\left\{f_{i}\right\},\left\{\left|\phi_{i}\right\rangle\right\}\right)\right] .
$$

\subsection{Properties of the RDMF}

For the following discussion of the properties of the density-matrix functional, we assume a particle-number conserving many-particle Hamiltonian and interaction Hamiltonian. Apart from convexity, the density-matrix functional has many noteworthy properties:

1. The density-matrix functional is independent of the one-particle basis. Thus,

$$
F^{\hat{W}}\left[\rho^{(1)}\right]=F^{\hat{W}}\left[U^{\dagger} \rho^{(1)} U\right]
$$

holds for any unitary matrix $U$. This property follows directly from the independence of the grand potential of the one-particle basis. Please note, that for the density-matrix functional in the transformed basis also the matrix elements of the interaction Hamiltonian have to be transformed.

2. For an interaction Hamiltonian of the form

$$
\hat{W}=\frac{1}{2} \sum_{\alpha, \beta, \gamma, \delta \leq N_{\mathrm{W}}} U_{\alpha, \beta, \gamma, \delta} \hat{c}_{\alpha}^{\dagger} \hat{c}_{\beta}^{\dagger} \hat{c}_{\delta} \hat{c}_{\gamma}
$$

the density-matrix functional fulfills the separation property

$$
F_{\beta}^{\hat{W}}\left[\left(\begin{array}{cc}
\rho_{\text {imp }}^{(1)} & 0 \\
0 & \rho_{\text {rest }}^{(1)}
\end{array}\right)\right]=F_{\beta}^{\hat{W}}\left[\rho_{\text {imp }}^{(1)}\right]+F_{\beta}^{\hat{0}}\left[\rho_{\text {rest }}^{(1)}\right]
$$

with $\rho_{\mathrm{imp}}^{(1)} \in \mathbb{C}^{N_{\mathrm{imp}} \times N_{\mathrm{imp}}}$ and $N_{\mathrm{imp}} \geq N_{\mathrm{W}}$. The non-interacting density-matrix functional $F_{\beta}^{\hat{0}}\left[\rho_{\text {rest }}^{(1)}\right]$ only contains an entropy contribution and is defined in Eq. (5.12). The proof of this separation property has been published in [Schade and Blöchl, 2018] and can be found in appendix B.1. The separation property is related to the size-consistency [Erdahl and Smith, 1987]. Violations of size consistency can lead to unphysical delocalization of electrons especially in molecular dissociation problems [Lathiotakis et al., 2010].

3. For an idempotent one-particle reduced density matrix, the density-matrix functional is

$$
F_{\beta}^{\hat{W}}\left[\rho^{(1)}\right]=F_{\mathrm{HF}}^{\hat{W}}\left[\rho^{(1)}\right]+F^{\hat{0}}\left[\rho^{(1)}\right]
$$


The first term is the Hartree-Fock density-matrix functional

$$
F_{\mathrm{HF}}^{\hat{W}}\left[\rho^{(1)}\right]=F_{H}^{\hat{W}}\left[\rho^{(1)}\right]+F_{x}^{\hat{W}}\left[\rho^{(1)}\right]
$$

and the second term is the non-interacting functional given in Eq. (5.12).

4. At zero temperature, the Hartree-Fock density-matrix functional defined in Eq. (5.20) is an upper bound of the exact density-matrix functional, i.e.

$$
F_{\mathrm{HF}}^{\hat{W}}\left[\rho^{(1)}\right] \geq F_{\beta \rightarrow \infty}^{\hat{W}}\left[\rho^{(1)}\right]
$$

5. Frank et al. conjectured [Frank et al., 2007] and numerical results suggest that the Müller functional [Müller, 1984]

$$
F_{\mathrm{M}}^{\hat{W}}\left[\rho^{(1)}\right]=\frac{1}{2} \sum_{a, b, c, d} U_{a, b, c, d}\left[\rho_{d, a}^{(1)} \rho_{c, b}^{(1)}-\left(\left(\rho^{(1)}\right)^{\frac{1}{2}}\right)_{c a}\left(\left(\rho^{(1)}\right)^{\frac{1}{2}}\right)_{d b}\right]
$$

is a lower bound of the exact density-matrix functional, i.e.

$$
F_{\mathrm{M}}^{\hat{W}}\left[\rho^{(1)}\right] \leq F_{\beta \rightarrow \infty}^{\hat{W}}\left[\rho^{(1)}\right] .
$$

The Müller functional gives the exact ground-state energy for the half-filled Hubbard dimer [Buijse and Baerands, 2002; Di Sabatino et al., 2015]. Buijse and Baerends [Buijse and Baerands, 2002] later rederived the Müller functional starting from the exchange-correlation hole [Slater, 1951].

6. The correlation contribution is non-positive $F_{c, \beta}\left[\rho^{(1)}\right] \leq 0$ for positive two-particle interactions, i.e. $w\left(\vec{x}, \vec{x}^{\prime}\right) \geq 0$ such as the Coulomb interaction with $w\left(\vec{x}, \vec{x}^{\prime}\right)=$ $e^{2} /\left(4 \pi \epsilon_{0}|\vec{r}-\vec{r}|\right)$ [Lieb, 1981a,b; Baldsiefen, 2012].

7. The density-matrix functional scales linearly with a uniform scaling of the interaction and temperature, i.e.

$$
F_{\beta / \lambda}^{\lambda \hat{W}}\left[\rho^{(1)}\right]=\lambda F_{\beta}^{\hat{W}}\left[\rho^{(1)}\right]
$$

8. Levy's density-matrix functional with only one many-particle wave function

$$
F_{\beta, \text { Levy }}^{\hat{W}}\left[\rho^{(1)}\right]=F_{\text {Levy }}^{\hat{W}}\left[\rho^{(1)}\right]=\min _{|\Psi\rangle \rightarrow \rho^{(1)}}[\langle\Psi|\hat{W}| \Psi\rangle] \geq F_{\beta}^{\hat{W}}\left[\rho^{(1)}\right]
$$

is independent of the temperature, an upper bound of the exact functional and not always convex. If the one-particle reduced density matrix corresponds to a non-degenerate ground state, then $F_{\text {Levy }}^{\hat{W}}\left[\rho^{(1)}\right]=F^{\hat{W}}\left[\rho^{(1)}\right]$ at zero temperature.

9. The correlation contribution is particle-hole symmetric [Valdemoro, 1992; Yasuda, 2001], i.e.

$$
F_{c, \beta}^{\hat{W}}\left[\rho^{(1)}\right]=F_{c, \beta}^{\hat{W}}\left[\mathbb{1}-\rho^{(1)}\right]
$$


10. Upon homogeneous scaling of the real space coordinate $\vec{r} \mapsto \lambda \vec{r}$ the correlation contribution $F_{c, \beta \rightarrow \infty}^{\hat{W}}\left[\rho^{(1)}\right]$ at zero temperature scales as [Erdahl and Smith, 1987]

$$
F_{c, \beta \rightarrow \infty}^{\hat{W}_{\vec{r} \mapsto \lambda \vec{r}}}\left[\rho_{\vec{r} \mapsto \lambda \vec{r}}^{(1)}\right]=\lambda F_{c, \beta \rightarrow \infty}^{\hat{W}}\left[\rho^{(1)}\right] .
$$

11. Spin constancy [Yang et al., 2000; Cohen et al., 2008a; Helbig et al., 2011]: the value of the density-matrix functional is independent of the fractional spin $m_{S}$ in z-direction, $-S \leq m_{S} \leq S$, for a given total spin $S$. For a one-particle reduced density matrix

$$
\rho_{S, m_{S}}^{(1)}=\left(\frac{1}{2}+\frac{m_{S}}{2 S}\right) \rho_{S, m_{S}=S}^{(1)}+\left(\frac{1}{2}-\frac{m_{S}}{2 S}\right) \rho_{S, m_{S}=-S}^{(1)}
$$

with total spin $S$ and fractional spin $m_{s}$ the relation [Cohen et al., 2008a]

$$
F_{c, \beta}^{\hat{W}}\left[\rho_{S, m_{S}}^{(1)}\right]=F_{c, \beta}^{\hat{W}}\left[\rho_{S, m_{S}=S}^{(1)}\right]=F_{c, \beta}^{\hat{W}}\left[\rho_{S, m_{S}=-S}^{(1)}\right]
$$

holds. Violation of the spin constancy condition leads to the static correlation problem [Cohen et al., 2008b,a].

12. The ground state energy $E(N)$ is composed of a series of straight line segments [Perdew et al., 1982; Yang et al., 2000] at zero temperature. Thus, the density-matrix functional has to have derivative discontinuities at zero temperature. Continuously differentiable approximations of the density-matrix functional lead to unphysical localization or delocalization of electrons [Helbig et al., 2007; Cohen et al., 2008b; Mori-Sánchez et al., 2008; Cohen et al., 2012].

13. The exact density-matrix functional for closed-shell two-electron systems is known [Kutzelnigg, 1963; Löwdin and Shull, 1956; Kollmar and Hess, 2004].

14. Within reduced density-matrix functional theory at zero temperature, there is not always a Kohn-Sham system, i.e., a fictitious non-interacting system with the same one-particle reduced density matrix as the interacting system. For finite temperatures, there is always a Kohn-Sham system [Baldsiefen, 2012; Blöchl et al., 2013; Baldsiefen et al., 2015]. The effective one-particle Hamiltonian of the Kohn-Sham system is

$$
\hat{h}=\sum_{\alpha, \beta} h_{\alpha, \beta} \hat{c}_{\alpha}^{\dagger} \hat{c}_{\beta}
$$

with the matrix elements

$$
h=\mu \mathbb{1}+\frac{1}{\beta} \ln \left(\frac{\mathbb{1}-\rho^{(1)}}{\rho^{(1)}}\right) .
$$

15. Additional properties are known [Cioslowski and Pernal, 2002; Erdahl and Smith, 1987; Carlson and Keller, 1961; Cioslowski et al., 2002; Cioslowski, 2005; Rohr and Pernal, 2011; Benavides-Riveros and Várilly, 2012]. Known results from example systems such as the homogeneous electron gas [Cioslowski and Pernal, 1999] or closed-shell two-electron systems [Kutzelnigg, 1963; Löwdin and Shull, 1956; Kollmar and Hess, 2004] can give additional insight. 


\subsection{Parametrized approximations of the density-matrix functional}

A detailed review of approximate parametrized density-matrix functionals can be found in the review by Pernal and Giesbertz [Pernal and Giesbertz, 2016] and in [Schade et al., 2017]. Most parametrized density-matrix functionals have only been applied to small molecules. The exception is the power functional of Sharma et al. [Sharma et al., 2008]. The power functional is defined as

$$
F_{\text {power }}^{\hat{W}}\left[\rho^{(1)}\right]=\frac{1}{2} \sum_{a, b, c, d} U_{a, b, c, d}\left[\rho_{d, a}^{(1)} \rho_{c, b}^{(1)}-\left(\left(\rho^{(1)}\right)^{\alpha}\right)_{c a}\left(\left(\rho^{(1)}\right)^{\alpha}\right)_{d b}\right]
$$

It interpolates between the Müller functional $(\alpha=1 / 2)$ defined in Eq. (5.22) and the Hartree-Fock functional $(\alpha=1)$ in Eq. (5.20). It is constructed this way to cure the overcorrelating behavior [Csányi and Arias, 2000; Lathiotakis et al., 2007; Lathiotakis and Marques, 2008] of the Müller functional. The power functional has been applied to simple semiconductors and transition-metal oxides [Sharma et al., 2008; Shinohara et al., 2015b; Di Sabatino et al., 2016; Sharma et al., 2013; Shinohara et al., 2015a]. The resulting band gaps and parameters of metal-insulator transitions are in good agreement with experimental results. However, the band gaps have been estimated by an extrapolation technique [Helbig et al., 2007, 2009] due to the missing derivative discontinuity of the total energy.

We have investigated the properties of the power-functional class for the half-filled Hubbard dimer and found some severe pathologies [Kamil et al., 2016]. There is no derivative discontinuity of the total energy at integer particle numbers for $\alpha<1$. The ground state of the Müller functional, i.e., $\alpha=1 / 2$, is degenerate with a one-dimensional manifold of ferromagnetic solutions and, hence, predicts an infinite magnetic susceptibility. Furthermore, we have shown that the power functional for $1 / 2<\alpha<1$ inherits the unphysical transition to an antiferromagnetic state from the Hartree-Fock functional and shows a plethora of unphysical non-collinear magnetic structures and transitions.

These shortcomings highlight the need for systematically improvable density-matrix functionals that can be applied to solids.

\subsection{Local approximation}

The local approximation of the density-matrix functional [Blöchl et al., 2011] proposed by Blöchl, Walther and Pruschke is an important ingredient for hybrid schemes combining density-functionals and density-matrix functionals. We present here a slightly more general version of the local approximation than discussed in [Blöchl et al., 2011]. The original formulation by Blöchl et al. assumed an orbital-based separation of the interaction by introducing disjoint sets of local orbitals. Instead, we assume here any separation of the interaction Hamiltonian into local terms $\hat{W}_{R}$ and the remainder $\hat{W}_{\text {rest }}$

$$
\hat{W}=\sum_{R} \hat{W}_{R}+\hat{W}_{\text {rest }}
$$


The local approximation of the density-matrix functional approximates the density-matrix functional by using the decomposition of the interaction Hamiltonian of Eq. (5.33) as

$$
F_{\beta}^{\hat{W}}\left[\rho^{(1)}\right] \approx F_{\beta, \text { loc }}^{\hat{W}}\left[\rho^{(1)}\right]=\sum_{R} F_{\beta}^{\hat{W}_{R}}\left[\rho^{(1)}\right]+F_{\beta}^{\hat{W}_{\text {rest }}}\left[\rho^{(1)}\right] .
$$

Thus, the approximation assumes linearity of the density-matrix functional with the interaction Hamiltonian. The local approximation is a lower bound for the exact functional, i.e.

$$
F_{\beta}^{\hat{W}}\left[\rho^{(1)}\right] \geq F_{\beta, \text { loc }}^{\hat{W}}\left[\rho^{(1)}\right]
$$

This can easily be seen when considering that the minimization problem of Eq. (5.25) has been approximated by a sum of several independent minimization problems. As a consequence also the total energy obtained with the local approximation is a lower bound of the exact ground-state energy.

Results of the local approximation for small Hubbard chains at zero temperature have been reported [Blöchl et al., 2011]. Blöchl et al. have used individual sites as local clusters $R$ and the numerically exact density-matrix functionals for the local contributions $F_{\beta}^{\hat{W}_{R}}\left[\rho^{(1)}\right]$. The remaining term $F_{\beta}^{\hat{W}_{\text {rest }}}\left[\rho^{(1)}\right]$ vanishes because $\hat{W}_{\text {rest }}=0$ for the Hubbard model. The results show that the ground-state energy, double occupancies, and spin-spin correlation functions are well reproduced. However, there is no derivative discontinuity of the energy at integer particle numbers and, hence, a vanishing fundamental gap. Approximations of the gaps with Eq. (2.50) show good agreement with the exact gaps.

The unique feature of the local approximation compared to parametrized approximations of the density-matrix functional is that it can be systematically converged to the exact result by subdividing the interaction into fewer terms in Eq. (5.33).

\subsection{Gap and spectral function}

Reduced density-matrix functional theory with the exact density-matrix functional gives the exact ground-state energy and one-particle reduced density matrix of a system. Thus, for the exact ground state of a particle-number conserving Hamiltonian the energy will consist of linear segments between integer particle numbers and the fundamental gap can be evaluated with Eq. (2.48), Eq. (2.49) or Eq. (2.50). All three equations are equivalent in this case.

However, we are not aware of an approximate parametrized density-matrix functional or practical approximation scheme that leads to fractional occupation numbers at zero temperature ${ }^{1}$, is applicable to a general system and reproduces the derivative discontinuities of the exact total energy at integer particle numbers. Thus, the characterization of a material based on the definition of the fundamental gap in Eq. (2.48) is not applicable because the differences in the derivatives of the total energy approaching integer particle numbers from above and from below are equal. The approximation of the gap by Eq. (2.50) is applicable in the case of a smoothened derivative discontinuity but systematically underestimates the gap [Sharma et al., 2008].

\footnotetext{
${ }^{1}$ This excludes the HF-approximation.
} 


\subsubsection{Local spectral function}

The one-particle spectral function defined in Eq. (2.151) is not directly accessible in RDMFT. Several methods for the approximation of the spectral function in an RDMFT calculation have been proposed and analyzed [Sharma et al., 2013; Di Sabatino et al., 2015, 2016]. However, these approximations are not suitable for the application within a hybrid method combining DFT and RDMFT. For example, the approximation proposed by Sharma et al. [Sharma et al., 2013] requires many evaluations of the derivatives of the density-matrix functional and is tailored for parametrized density-matrix functionals that can be evaluated with a rather small computational effort. This approach is not suitable for density-matrix functionals that are evaluated from a numerically demanding constrained minimization problem. The evaluation of the density-matrix functional from a constrained minimization over many-particle wave functions, that will be discussed in detail in section 9, opens another route for the approximation of the spectral function. Consider the one-particle reduced density matrix $\rho^{(1)}$ and the corresponding densitymatrix functional $F^{\hat{W}}\left[\rho^{(1)}\right]$. In section 5.2 we have shown that the derivative of the density-matrix functional with respect to the elements of the one-particle reduced density matrix is

$$
\frac{\partial F_{\beta}^{\hat{W}}\left[\rho^{(1)}\right]}{\partial \rho_{\alpha, \beta}^{(1)}}=-\tilde{h}_{\beta, \alpha},
$$

where $\tilde{h}_{\beta, \alpha}$ are the Lagrange multipliers of the constraints of the one-particle reduced density matrix. The minimization problem of the ground-state energy in Eq. (5.3) has the minimum condition

$$
0=h_{\beta, \alpha}-\tilde{h}_{\beta, \alpha}-\mu \mathbb{1}
$$

with the matrix elements $h$ of the one-particle Hamiltonian of the system and the Lagrange multiplier $\mu$ of the particle-number constraint. This minimum condition only holds for a one-particle reduced density matrix $\rho^{(1)}$ that does not lie at the boundary of the subspace of ensemble N-representable one-particle reduced density matrices. So far we have not obtained anything useful, because if we could compute the spectral function of the Hamiltonian

$$
\hat{\tilde{H}}=\sum_{\alpha, \beta}\left(\tilde{h}_{\beta, \alpha}-\mu \delta_{\alpha, \beta}\right) \hat{c}_{\alpha}^{\dagger} \hat{c}_{\beta}+\hat{W}
$$

we could certainly also compute the spectral function of the original Hamiltonian

$$
\hat{H}=\sum_{\alpha, \beta} h_{\alpha, \beta} \hat{c}_{\alpha}^{\dagger} \hat{c}_{\beta}+\hat{W} .
$$

We include the local approximation introduced in section 5.5 and a simple truncation of the one-particle basis ${ }^{2}$ into our considerations. We assume that the interaction $\hat{W}$ can be split up into local contributions, i.e., $\hat{W}=\sum_{R} \hat{W}_{R}$. Thus, we approximate the density-matrix functional as

$$
F^{\hat{W}}\left[\rho^{(1)}\right] \approx \sum_{R} F^{\hat{W}_{R}}\left[\rho^{(1)}\right] \approx \sum_{R} F^{\hat{W}_{R}}\left[\rho_{R}^{(1)}\right]
$$

\footnotetext{
${ }^{2} \mathrm{~A}$ systematic truncation of the one-particle basis is introduced in section 8 in the form of the adaptive cluster approximation.
} 
where $\rho_{R}^{(1)}$ is the one-particle density matrix of the truncated one-particle basis that only contains states in the vicinity of the site $R . F^{\hat{W}_{R}}\left[\rho_{R}^{(1)}\right]$ is evaluated with a constrained minimization over many-particle wave functions. The derivatives are

$$
\frac{\partial F_{\beta}^{\hat{W}_{R}}\left[\rho_{R}^{(1)}\right]}{\partial \rho_{R, \alpha, \beta}^{(1)}}=-\tilde{h}_{R, \beta, \alpha}
$$

and the minimum condition of the total-energy minimization reads

$$
0=h_{\beta, \alpha}-\sum_{R} \tilde{h}_{R, \beta, \alpha}-\mu \mathbb{1} .
$$

Thus, the Hamiltonian

$$
\hat{\tilde{H}}_{R}=\sum_{\alpha, \beta} \tilde{h}_{R, \alpha, \beta} \hat{c}_{\alpha}^{\dagger} \hat{c}_{\beta}+\hat{W}_{R}
$$

describes the system in the vicinity of site $R$. From the constrained minimization we also know a many-particle wave function $\left|\Psi_{R}\right\rangle$ that is an eigenstate of $\hat{\tilde{H}}_{R}$ and has the correct one-particle reduced density matrix $\rho_{R}^{(1)}$. We propose to consider this state as an approximation of the ground state in the vicinity of the site $R$ and use this state together with the Hamiltonian $\hat{\tilde{H}}_{R}$ to construct the local spectral function $A_{\text {local }, \alpha, \beta}(\epsilon)$. The spectral function can be computed with the Lanczos method for spectral functions [Koch, 2011].

\subsubsection{Kohn-Sham-like spectral function}

In DFT, the derivatives of the total energy with respect to the occupations are related by Janak's theorem [Janak, 1978],

$$
\epsilon_{n}=\frac{\partial E_{\mathrm{DFT}}\left[\left\{f_{n}\right\},\left\{\left|\psi_{n}\right\rangle\right\}\right]}{\partial f_{n}}
$$

to the Kohn-Sham energy eigenvalues. In RDMFT we define Kohn-Sham-like energy eigenvalues

$$
\tilde{\epsilon}_{n}=\frac{\partial E_{\mathrm{RDMFT}}\left[\left\{f_{n}\right\},\left\{\left|\psi_{n}\right\rangle\right\}\right]}{\partial f_{n}}
$$

as derivatives of the RDMFT total energy $E_{\mathrm{RDMFT}}$ defined in Eq. (5.15). Equation (5.45) does not define the Kohn-Sham-like energy eigenvalues uniquely: the subspace of natural orbitals $\left|\psi_{n}\right\rangle$ with identical occupations can be rotated with a unitary transformation without changing the one-particle reduced density matrix. Thus, we additionally require that the matrix

$$
\tilde{H}_{i, j}=\left\langle\psi_{i}\right| \frac{\partial E_{\mathrm{RDMFT}}\left[\left\{f_{n}\right\},\left\{\left|\psi_{n}\right\rangle\right\}\right]}{\partial\left\langle\psi_{j}\right|}
$$

is diagonal for all indexes $i$ and $j$ that have identical occupations, i.e., $f_{i}=f_{j}$. With this diagonality requirement the Kohn-Sham-like energy eigenvalues $\tilde{\epsilon}_{n}$ are uniquely defined. In RDMFT, states with fractional occupations $0<f_{n}<1$ have derivatives

$$
\frac{\partial E_{\mathrm{RDMFT}}\left[\left\{f_{n}\right\},\left\{\left|\psi_{n}\right\rangle\right\}\right]}{\partial f_{n}}=\mu .
$$


In other words, if we assign Kohn-Sham-like energy eigenvalues to the natural orbitals in RDMFT, natural orbitals with fractional occupations lie at the chemical potential. Thus, a physical interpretation of the Kohn-Sham-like density of states estimated from the natural orbitals and Kohn-Sham-like energy eigenvalues $\tilde{\epsilon}_{n}$ will tend to falsely indicate a metallic state because fractionally occupied states lie by construction at the chemical potential. 


\section{DF+RDMF approaches}

In this chapter, I describe an approach to combine density functionals and reduced densitymatrix functionals in a hybrid theory geared towards the description of strong local electronic correlations in solids. Actually, I have developed the modification to the orbitalbased DF +RDMF approach of Blöchl, Walther and Pruschke [Blöchl et al., 2011], that we were using at the time, after a long and inspiring discussion with Andreas Savin about range separation, hybrid functionals, and the adiabatic connection at the International Workshop on "New challenges in Reduced Density Matrix Functional Theory: Symmetries, time-evolution and entanglement" in Lausanne $201 \%$.

The original approach [Blöchl et al., 2011] is described in detail in section 6.3. Section 6.4 presents the modification based on a decomposition of the interaction in real space. The modified approach avoids the issues of Blöchl's approach concerning the double counting of the interaction, has a parameter that smoothly controls the combination of density functionals and density-matrix functionals and, thus, might allow for an adiabatic connection approach. The important question if a screening of the bare Coulomb interaction is required in the proposed approach is discussed in section 6.5.

\subsection{Hybrid functionals, DFT+U and DFT+DMFT}

Hybrid functionals like PBE0 [Perdew et al., 1996c; Adamo and Barone, 1999] or the Heyd-Scuseria-Ernzerhof functional (HSE, [Heyd et al., 2003, 2006]) combine a local or semi-local density-functional approximation of the exchange-correlation-functional with exact exchange or, in other words, the exchange contribution in the Hartree-Fock approximation given by Eq. (5.11). Hybrid functionals have shown to improve the description of atomization energies, bond lengths and vibration frequencies [Adamo and Barone, 1999]. Hybrid functionals predict band gaps of semiconductors and many transition-metal oxides in good agreement with experimental results [Garza and Scuseria, 2016]. Please note, that this statement only considers the antiferromagnetic ground-states of transition-metal oxides. PBE0 [Perdew et al., 1996c; Adamo and Barone, 1999] uses the combination

$$
E_{x c}^{\mathrm{PBE} 0}=a E_{x}^{\mathrm{HF}}+(1-a) E_{x}^{\mathrm{PBE}}+E_{c}^{\mathrm{PBE}}
$$

of the exact exchange energy $E_{x}^{\mathrm{HF}}=F_{x}^{\hat{W}}$, the PBE-exchange energy $E_{x}^{\mathrm{PBE}}$ and the PBEcorrelation energy $E_{c}^{\mathrm{PBE}}$ with a fraction of $a=1 / 4$ as the exchange-correlation energy. PBE0 can be easily implemented in DFT codes based on local orbitals, like Gaussiantype orbitals, but the implementation of the exact exchange energy is numerically very demanding in plane-wave based codes. PBE0 is classified as a global hybrid functional because the bare Coulomb interaction is used for the evaluation of the exact exchange energy $E_{x}^{\mathrm{HF}}$. Screened hybrid functionals can drastically reduce the cost of evaluating the exact exchange energy by only considering the exact exchange energy for a short-range 
interaction. Such a screened hybrid functional is the Heyd-Scuseria-Ernzerhof functional (HSE, [Heyd et al., 2003, 2006]). It uses a range-separation of the Coulomb interaction of the form [Stoll and Savin, 1985; Savin, 1988, 1996]

$$
\frac{1}{r}=\underbrace{\frac{1-\operatorname{erf}(\omega r)}{r}}_{\text {short range }}+\underbrace{\frac{\operatorname{erf}(\omega r)}{r}}_{\text {long range }}
$$

with $\operatorname{erf}(x)=\frac{2}{\sqrt{\pi}} \int_{0}^{x} d \tau \exp \left(-\tau^{2}\right)$. The interaction Hamiltonian $\hat{W}_{\mathrm{SR}}$ of the short-range interaction is

$$
\hat{W}_{\mathrm{SR}, \omega}=\frac{1}{2} \int d^{4} \vec{x} \int d^{4} \vec{x}^{\prime} \frac{e^{2}\left(1-\operatorname{erf}\left(\omega\left|\vec{r}-\vec{r}^{\prime}\right|\right)\right) \hat{\Psi}^{\dagger}(\vec{x}) \hat{\Psi}^{\dagger}\left(\vec{x}^{\prime}\right) \hat{\Psi}\left(\vec{x}^{\prime}\right) \hat{\Psi}(\vec{x})}{4 \pi \epsilon_{0}\left|\vec{r}-\vec{r}^{\prime}\right|}
$$

and the interaction Hamiltonian $\hat{W}_{\mathrm{LR}}$ of the long-range interaction is

$$
\hat{W}_{\mathrm{LR}, \omega}=\frac{1}{2} \int d^{4} \vec{x} \int d^{4} \vec{x}^{\prime} \frac{e^{2} \operatorname{erf}\left(\omega\left|\vec{r}-\vec{r}^{\prime}\right|\right) \hat{\Psi}^{\dagger}(\vec{x}) \hat{\Psi}^{\dagger}\left(\vec{x}^{\prime}\right) \hat{\Psi}\left(\vec{x}^{\prime}\right) \hat{\Psi}(\vec{x})}{4 \pi \epsilon_{0}\left|\vec{r}-\vec{r}^{\prime}\right|} .
$$

The HSE-functional uses the combination

$$
E_{x c}^{\mathrm{HSE}}=a F_{x}^{\hat{W}_{\mathrm{SR}, \omega}}+(1-a) E_{x}^{\mathrm{PBE}, \hat{\mathrm{W}}_{\mathrm{SR}, \omega}}+E_{x}^{\mathrm{PBE}, \hat{\mathrm{W}}_{\mathrm{LR}, \omega}}+E_{c}^{\mathrm{PBE}}
$$

of the short-range exact exchange energy $F_{x}^{\hat{W}_{\mathrm{SR}, \omega}}$, the short-range PBE-exchange $E_{x}^{\mathrm{PBE}, \hat{\mathrm{W}}_{\mathrm{SR}, \omega}}$ and the long-range PBE-exchange $E_{x}^{\mathrm{PBE}, \hat{\mathrm{W}}_{\mathrm{LR}, \omega}}$. The parameters $a=1 / 4$ and $\omega=0.2$ are commonly used. Blöchl et al. [Blöchl et al., 2011; Walther, 2011] have proposed a screened variant of PBE0, named PBE0r, that only considers atom-local contributions in the exact exchange because non-local contributions are efficiently screened in many solids. Blöchl et al. define the local interaction of the correlated states as

$$
\hat{W}_{R}=\frac{1}{2} \sum_{\alpha, \beta, \gamma, \delta \in C_{R}} U_{\alpha, \beta, \gamma, \delta} \hat{c}_{\alpha}^{\dagger} \hat{c}_{\beta}^{\dagger} \hat{c}_{\delta} \hat{c}_{\gamma}
$$

where $C_{R}$ is a set of strongly correlated one-particle states localized at the atom $R$. The expression used in PBE0r for the exchange correlation energy is

$$
E_{x c}^{\mathrm{PBE} 0 \mathrm{r}}=E_{x c}^{\mathrm{PBE}}+\sum_{R} a_{R}\left(F_{x}^{\hat{W}_{\mathrm{R}}}-E_{x}^{\mathrm{PBE}, \hat{\mathrm{W}}_{\mathrm{R}}}\right) .
$$

The local hybrid functionals [Novák et al.; Tran et al., 2006; Jollet et al., 2009] like PBE0r are strongly related to the DFT $+\mathrm{U}$ method. The main idea of the $\mathrm{DFT}+\mathrm{U}$ method [Anisimov et al., 1991, 1993; Solovyev et al., 1994; Liechtenstein et al., 1995; Anisimov et al., 1997b; Dudarev et al., 1998; Cococcioni, 2012] is to correct the DFT total-energy expression $E_{\mathrm{DFT}}$ with the Hubbard Hamiltonian to describe the strongly correlated states. The total energy in $\mathrm{DFT}+\mathrm{U}$ has the form

$$
E_{\mathrm{DFT}+\mathrm{U}}=E_{\mathrm{DFT}}+E_{\text {Hubbard }}-E_{\text {double-counting. }}
$$

The energy contribution from the Hubbard-Hamiltonian $E_{\text {Hubbard }}$ is approximated as the Hartree-Fock energy of the local correlated states [Liechtenstein et al., 1995]. The doublecounting term $E_{\text {double-counting }}$ is an incarnation of the infamous double-counting problem: 
the lack of a diagrammatic expansion of the DFT total energy makes it hard to remove the local correlation effects that should be described by the Hubbard Hamiltonian in $\mathrm{DFT}+\mathrm{U}$ from the exchange-correlation functional. However, it is possible to approach this problem starting from the hole function of the DFT exchange-correlation functional and Blöchl et al. [Blöchl et al., 2011] have proposed a double counting based on the hole function in the context of the PBE0r-functional that is also applicable in the DFT+Umethod. Their definition of the interaction Hamiltonian in Eq. (6.6) of the correlated states introduces an inconsistency in practical applications of the otherwise exact definition of the double-counting contribution: it is not guaranteed that the double-counting is evaluated for the same interaction as the density-matrix functional. Details are discussed in section 6.3. We propose to use a different definition of the local interaction in section 6.4 that avoids this inconsistency and allows a numerically exact evaluation of the double-counting contribution. The introduction of a screening of the local interaction in the DFT $+\mathrm{U}$ method corresponds to a mixing parameter $a_{R}$ different from one in the PBE0r-functional in Eq. (6.7). The DFT $+\mathrm{U}$ method and local hybrid functionals predict band gaps of transition-metal oxides in the antiferromagnetic state in good agreement with experiment.

The combination of DFT with dynamical mean-field theory (DMFT, [Georges and Kotliar, 1992; Anisimov et al., 1997a; Lichtenstein and Katsnelson, 1998; Kotliar et al., 2006]), i.e. DFT+DMFT [Anisimov et al., 1997a; Lichtenstein and Katsnelson, 1998; Held, 2007; Vollhardt, 2011], improves on the DFT $+\mathrm{U}$ method by introducing a frequencydependent self-energy, while the correction in $\mathrm{DFT}+\mathrm{U}$ implicitly only considers a frequency-independent self-energy. A frequency-dependent self-energy can be related to static correlation [Kotliar, 2014].

The DFT+DMFT method is plagued by the double-counting problem. Among the many successes ${ }^{1}$ of the DFT+DMFT method is the physically correct description of the insulating behavior of the nonmagnetic state of $\mathrm{NiO}$ [Ren et al., 2006].

On the other hand, methods have been proposed to improve over hybrid functionals by using a more accurate density-matrix functional instead of exact exchange. For example, the DF+RDMF theories of Pernal [Pernal, 2010] and Rohr et al. [Rohr et al., 2010] use the same range separation of the Coulomb interaction in Eq. (6.2) as in the HSE-functional. They combine short-range PBE-exchange-correlation with a long-range variant of the Müller functional. The use of an approximate parametrized density-matrix functional potentially introduces the deficiencies of the parametrized density-matrix functional into the results. On the other hand, the need to evaluate the density-matrix functional in the full one-particle basis prevents the use of systematically improvable density-matrix functionals that might have an unfavorable scaling of the computational complexity with the size of the one-particle basis.

We propose to combine the local interaction of the correlated orbitals from $\mathrm{DFT}+\mathrm{U}$, local hybrid functionals, and DFT+DMFT with a separation of the interaction in real space and with the idea of combining density functionals and density-matrix functionals. We call the resulting method the real-space-decomposition based DF+RDMF approach. This approach improves the orbital-based DF + RDMF approach proposed by Blöchl et al. [Blöchl et al., 2011] by remedying the above-mentioned inconsistencies in the definition of the double-counting contribution. We have implemented the orbital-based approach

\footnotetext{
${ }^{1}$ For a review we refer the reader to [Held, 2007].
} 
and the real-space-decomposition based approach in the CP-PAW code and show results for the hydrogen molecule as the prototypical example for static correlation and the nonmagnetic state of $\mathrm{NiO}$.

\subsection{General DF+RDMF approach}

The starting point for an approach that combines density-functionals and density-matrix functionals is the expression for the ground-state energy within reduced density-matrix functional theory given in Eq. (5.3) as

$$
E[h]=\min _{\rho^{(1)}: 0 \leq \rho^{(1)} \leq 1, \operatorname{Tr} \rho^{(1)}=N}\left(\operatorname{Tr} \rho^{(1)} h+F^{\hat{W}}\left[\rho^{(1)}\right]\right)
$$

in a one-particle basis $\left|\chi_{\alpha}\right\rangle$. The interaction Hamiltonian

$$
\hat{W}=\frac{1}{2} \sum_{\alpha, \beta, \gamma, \delta} U_{\alpha, \beta, \gamma, \delta} \hat{c}_{\alpha}^{\dagger} \hat{c}_{\beta}^{\dagger} \hat{c}_{\delta} \hat{c}_{\gamma}
$$

is defined in the one-particle basis. For the combination of a density-functional and a density-matrix functional, the interaction is decomposed into two terms,

$$
\hat{W}=\hat{W}_{\mathrm{DF}}+\hat{W}_{\mathrm{RDMF}} .
$$

The density-matrix functional $F^{\hat{W}}\left[\rho^{(1)}\right]$ is then approximated as

$$
F^{\hat{W}}\left[\rho^{(1)}\right] \approx F^{\hat{W}_{\mathrm{DF}}}\left[\rho^{(1)}\right]+F^{\hat{W}_{\mathrm{RDMF}}}\left[\rho^{(1)}\right] .
$$

The first term is later evaluated with an approximate density-functional and the second term with a density-matrix functional, i.e.,

$$
F^{\hat{W}}\left[\rho^{(1)}\right] \approx F_{\mathrm{DF}}^{\hat{W}_{\mathrm{DF}}}\left[\rho^{(1)}\right]+F_{\mathrm{RDMF}}^{\hat{W}_{\mathrm{RDMF}}}\left[\rho^{(1)}\right]
$$

A similar starting point can be derived from the expression

$$
F^{\hat{W}}\left[\rho^{(1)}\right]=F^{\hat{W}}\left[\rho^{(1)}\right]+\left(F^{\hat{W}^{\prime}}\left[\rho^{(1)}\right]-F^{\hat{W}^{\prime}}\left[\rho^{(1)}\right]\right),
$$

by approximating the first and the last density-matrix functional with a densityfunctional. This results in

$$
F^{\hat{W}}\left[\rho^{(1)}\right] \approx F_{\mathrm{DF}}^{\hat{W}}\left[\rho^{(1)}\right]+\left(F^{\hat{W}^{\prime}}\left[\rho^{(1)}\right]-F_{\mathrm{DF}}^{\hat{W}^{\prime}}\left[\rho^{(1)}\right]\right)
$$

and the last term obtains the form of a correction contribution. Eq. (6.15) can be rearranged to

$$
F^{\hat{W}}\left[\rho^{(1)}\right] \approx F_{\mathrm{DF}}^{\hat{W}}\left[\rho^{(1)}\right]-F_{\mathrm{DF}}^{\hat{W}^{\prime}}\left[\rho^{(1)}\right]+F^{\hat{W}^{\prime}}\left[\rho^{(1)}\right] .
$$

Eq. (6.13) and Eq. (6.16) are identical for the choice $\hat{W}^{\prime}=\hat{W}_{\mathrm{RDMF}}$ if the approximate density functional $F_{\mathrm{DF}}^{\hat{W}}\left[\rho^{(1)}\right]$ is linear in the interaction. This is for example the case if it is evaluated for a fixed hole function. We will follow the starting point of Eq. (6.16), because it is more in the spirit of a correction over density-functional theory.

The decomposition of the interaction Hamiltonian in Eq. (6.11) can be performed in different ways. In the following section, we describe the orbital-based DF+RDMF approach of Blöchl, Walther and Pruschke [Blöchl et al., 2011]. In section 6.4 we introduce a new real-space-decomposition based DF+RDMF approach. 


\subsection{Orbital-based DF+RDMF approach}

\subsubsection{Foundation}

Blöchl, Walther and Pruschke [Blöchl et al., 2011] have proposed a hybrid theory combining DFT and RDMFT tailored towards the description of the local physics in materials with strong electronic correlations. We denote the complete one-particle basis as the set $\chi=\left\{\left|\chi_{\alpha}\right\rangle\right\}$. Blöchl et al. choose the interaction $\hat{W}^{\prime}$ in Eq. (6.11) as the interaction of of subset $C=\left\{\left|\chi_{\alpha}\right\rangle\right\} \subset \chi$ of one-particle states as

$$
\begin{aligned}
\hat{W}^{\prime} & =\frac{1}{2} \sum_{\alpha, \beta, \gamma, \delta \in C} U_{\alpha, \beta, \gamma, \delta} \hat{c}_{\alpha}^{\dagger} \hat{c}_{\eta}^{\dagger} \hat{c}_{\delta} \hat{c}_{\gamma} \\
U_{\alpha, \beta, \gamma, \delta} & =\int d^{4} \vec{x} \int d^{4} \vec{x}^{\prime} \frac{e^{2}}{4 \pi \epsilon_{0}\left|\vec{r}-\vec{r}^{\prime}\right|} \chi_{\alpha}^{*}(\vec{x}) \chi_{\beta}^{*}\left(\vec{x}^{\prime}\right) \chi_{\gamma}(\vec{x}) \chi_{\delta}\left(\vec{x}^{\prime}\right) .
\end{aligned}
$$

In their original publication [Blöchl et al., 2011], they have proposed to include the local one-particle states in the set $C$ that should be chosen such that the interaction $\hat{W}^{\prime}$ contains the strongly interacting states that are responsible for the strong electronic correlations. However, in principle also other choices are possible. It should be noted, that the choice of a complete one-particle basis, i.e. $C \equiv \chi$, would lead to $\hat{W}^{\prime}=\hat{W}$ and hence just reduced density-matrix functional theory because the contribution from the density-functional would vanish by construction. Thus, we assume for the following discussion, that $C \neq \chi$.

In the originally proposed approach, the functional $F_{D F}^{\hat{W}}\left[\rho^{(1)}\right]$ is approximated as [Blöchl et al., 2011]

$$
F_{D F}^{\hat{W}}\left[\rho^{(1)}\right]=E_{H}\left[n\left[\rho^{(1)}\right]\right]+E_{x c}\left[n\left[\rho^{(1)}\right]\right]
$$

without removing the kinetic correlation $T_{c}$ discussed in section 4.4.2. For the remainder of this section we define $n=n\left[\rho^{(1)}\right]$ as the density of the one-particle reduced density matrix to simplify the notation. The density-functional $F_{D F}^{\hat{W}^{\prime}}\left[\rho^{(1)}\right]$, which plays the role of a double counting of the interaction, was defined via the interaction-strength-dependent hole function $h_{\lambda}\left(\vec{r}, \vec{r}^{\prime}\right)$ of Eq. (4.32) as

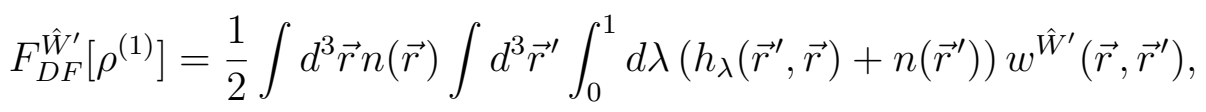

where $w^{\hat{W}^{\prime}}\left(\vec{r}, \vec{r}^{\prime}\right)$ is implicitly defined by $\hat{W}^{\prime}$ such that

$$
\begin{aligned}
& \hat{W}^{\prime}=\frac{1}{2} \int d^{4} \vec{x}_{1} \int d^{4} \vec{x}_{2} \int d^{4} \vec{x}_{3} \int d^{4} \vec{x}_{4} w^{\hat{W}^{\prime}}\left(\vec{x}_{1}, \vec{x}_{2}\right) \delta\left(\vec{x}_{1}-\vec{x}_{4}\right) \delta\left(\vec{x}_{2}-\vec{x}_{3}\right) \\
& \hat{\Psi}^{\dagger}\left(\vec{x}_{1}\right) \hat{\Psi}^{\dagger}\left(\vec{x}_{2}\right) \hat{\Psi}\left(\vec{x}_{3}\right) \hat{\Psi}\left(\vec{x}_{4}\right)
\end{aligned}
$$

holds. However, as also noted in the original publication, the interaction $\hat{W}^{\prime}$ is usually nonlocal in the coordinates $\vec{x}_{1}, \ldots, \vec{x}_{4}$ and no $w^{\hat{W}^{\prime}}\left(\vec{x}_{1}, \vec{x}_{2}\right)$ can be derived such that Eq. (6.21) holds in all situations. Therefore, the interaction needs to be approximated and the model proposed for the interaction by Blöchl et al. is ${ }^{2}$

$$
\tilde{w}^{\hat{W}^{\prime}}\left(\vec{x}_{1}, \vec{x}_{2}\right)=\frac{n_{C}(\vec{r})}{n(\vec{r})} \frac{e^{2}}{4 \pi \epsilon_{0}\left|\vec{r}-\vec{r}^{\prime}\right|} \frac{n_{C}\left(\vec{r}^{\prime}\right)}{n\left(\vec{r}^{\prime}\right)} .
$$

\footnotetext{
${ }^{2}$ We do not distinguish the density $n(\vec{r})$ from the density in the one-particle basis, which Blöchl et al. denote as $n^{\chi}(\vec{r})$. For the purpose of this discussion we assume these two quantities to be identical.
} 
The density $n_{C}(\vec{r})$ denotes the density constituted by the one-particle states in $C$ :

$$
n_{C}(\vec{r})=\sum_{\sigma} \sum_{\alpha, \beta \in C}\left\langle\vec{r}, \sigma \mid \chi_{\alpha}\right\rangle \rho_{\alpha, \beta}^{(1)}\left\langle\chi_{\beta} \mid \vec{r}, \sigma\right\rangle .
$$

The interaction $\tilde{w}^{\hat{W}^{\prime}}$ can be combined with Eq. (6.20) and, thus, the functional $F_{D F}^{\hat{W}^{\prime}}\left[\rho^{(1)}\right]$ can be evaluated approximately as

$$
F_{D F}^{\hat{W}^{\prime}}\left[\rho^{(1)}\right] \approx \frac{1}{2} \int d^{3} \vec{r} n_{C}(\vec{r}) \int d^{3} \vec{r}^{\prime} \frac{n_{C}\left(\vec{r}^{\prime}\right)}{n\left(\vec{r}^{\prime}\right)} \int_{0}^{1} d \lambda \frac{e^{2} h_{\lambda}\left(\vec{r}^{\prime}, \vec{r}\right)}{4 \pi \epsilon_{0}\left|\vec{r}-\vec{r}^{\prime}\right|}+E_{H}\left[n_{C}\right]
$$

For functionals for which the hole function is not available Blöchl et al. proposed the simple approximation

$$
F_{D F}^{\hat{W}^{\prime}}\left[\rho^{(1)}\right]=\int d^{3} \vec{r} n_{C}(\vec{r}) \epsilon_{x c}[n](\vec{r}) \frac{n_{C}(\vec{r})}{n(\vec{r})}+E_{H}\left[n_{C}\right]
$$

that only needs the exchange-correlation energy density per particle $\epsilon_{x c}[n](\vec{r})$.

The definition of the model of the interaction in Eq. (6.22) allows to approximately evaluate the functional $F_{D F}^{\hat{W}^{\prime}}\left[\rho^{(1)}\right]$ with Eq. (6.24) or Eq. (6.25) but introduces an inconsistency in the correction term in Eq. (6.15): the density-matrix functional $F^{\hat{W}^{\prime}}\left[\rho^{(1)}\right]$ is evaluated for the interaction $\hat{W}^{\prime}$ but the double counting term $F_{D F}^{\hat{W}^{\prime}}\left[\rho^{(1)}\right]$ is implicitly evaluated with the interaction $\tilde{w}^{\hat{W}^{\prime}}\left(\vec{x}_{1}, \vec{x}_{2}\right)$ that can describe a different interaction because Eq. (6.21) cannot be fulfilled in general.

The minimization problem for the ground-state energy $E$ in the orbital-based $\mathrm{DF}+\mathrm{RDMF}$ scheme is

$$
E[h]=\min _{\rho^{(1)}: 0 \leq \rho^{(1)} \leq 1, \operatorname{Tr} \rho^{(1)}=N}\left\{\operatorname{Tr}\left[\rho^{(1)} h\right]+F_{\mathrm{DF}}^{\hat{W}}\left[\rho^{(1)}\right]+F^{\hat{W}^{\prime}}\left[\rho^{(1)}\right]-F_{\mathrm{DF}}^{\hat{W}^{\prime}}\left[\rho^{(1)}\right]\right\} .
$$

\subsubsection{Local approximation}

As an additional approximation Blöchl et al. [Blöchl et al., 2011] have proposed the local approximation of the interaction and the local approximation of the density-matrix functional. We have already discussed the local approximation in section 5.5 and apply it here to the correction term of Eq. (6.26), i.e. to the term

$$
F^{\hat{W}^{\prime}}\left[\rho^{(1)}\right]-F_{\mathrm{DF}}^{\hat{W}^{\prime}}\left[\rho^{(1)}\right]
$$

For this purpose, the set of orbitals $C$ is defined as the union of disjoint sets $C_{R}$ of local orbitals, for example, d- or f-orbitals of transition metals, that are the reason for the static correlation. The interaction $\hat{W}^{\prime}$ can then be written as

$$
\begin{aligned}
\hat{W}^{\prime} & =\sum_{R} \hat{W}_{R} \\
\hat{W}_{R} & =\frac{1}{2} \sum_{\alpha, \beta, \gamma, \delta \in C_{R}} U_{\alpha, \beta, \gamma, \delta} \hat{c}_{\alpha}^{\dagger} \hat{c}_{\beta}^{\dagger} \hat{c}_{\delta} \hat{c}_{\gamma} .
\end{aligned}
$$

The second step of the local approximation, i.e., the approximation of the density-matrix functional itself, then gives

$$
F^{\hat{W}^{\prime}}\left[\rho^{(1)}\right]-F_{\mathrm{DF}}^{\hat{W}^{\prime}}\left[\rho^{(1)}\right] \approx \sum_{R}\left(F^{\hat{W}_{R}}\left[\rho^{(1)}\right]-F_{\mathrm{DF}}^{\hat{W}_{R}}\left[\rho^{(1)}\right]\right)
$$


Thus, the correction term can be approximated as a sum of local correction terms. The functional $F^{\hat{W}_{R}}\left[\rho^{(1)}\right]$ is also not known explicitly, some approximation of the densitymatrix functional has to be chosen for it that can describe static correlation sufficiently well. The minimization problem in the orbital-based DF+RDMF scheme including the local approximation is

$$
E[h]=\min _{\rho^{(1)}: 0 \leq \rho^{(1)} \leq 1, \operatorname{Tr} \rho^{(1)}=N}\left\{\operatorname{Tr}\left[\rho^{(1)} h\right]+F_{\mathrm{DF}}^{\hat{W}}\left[\rho^{(1)}\right]+\sum_{R}\left(F^{\hat{W}_{R}}\left[\rho^{(1)}\right]-F_{\mathrm{DF}}^{\hat{W}_{R}}\left[\rho^{(1)}\right]\right)\right\} .
$$

\subsubsection{Limits}

Now we explore some limits of the approach proposed in section 6.3.1:

1. In the limit where the set $C$ is empty, we get $\hat{W}^{\prime}=0$ and recover density-functional theory at zero temperature.

2. In the limit where $C \equiv \chi$, i.e., $C$ contains a complete one-particle basis, we obtain $\hat{W}=\hat{W}^{\prime}$ and, hence,reduced density-matrix functional theory, i.e.

$$
E[h]=\min _{\rho^{(1)}: 0 \leq \rho^{(1)} \leq 1, \operatorname{Tr} \rho^{(1)}=N}\left\{\operatorname{Tr}\left[\rho^{(1)} h\right]+F^{\hat{W}}\left[\rho^{(1)}\right]\right\}
$$

The limits of the orbital-based DF+RDMF scheme including the local approximation are:

1. In the limit where the set $C$ and the set sets $C_{R}$ are empty, we get $\hat{W}^{\prime}=0$ and recover density-functional theory at zero temperature.

2. If all one-particle states are contained in exactly one cluster $C$, we obtain reduced density-matrix functional theory.

3. If every one-particle state is contained in a local set $C_{R}$, i.e. $\chi=\cup C_{R}$, we obtain

$$
\begin{aligned}
E[h] & =\min _{\rho^{(1)}: 0 \leq \rho^{(1)} \leq 1, \operatorname{Tr} \rho^{(1)}=N}\left\{\operatorname{Tr}\left[\rho^{(1)} h\right]+\sum_{R} F^{\hat{W}_{R}}\left[\rho^{(1)}\right]\right. \\
& \left.+\int d^{3} \vec{r} \int d^{3} \vec{r}^{\prime} \frac{e^{2}\left(\int_{0}^{1} d \lambda h_{\lambda}\left(\vec{r}^{\prime}, \vec{r}\right)+1\right)}{4 \pi \epsilon_{0}\left|\vec{r}-\vec{r}^{\prime}\right|}\left(n(\vec{r}) n\left(\vec{r}^{\prime}\right)-\sum_{R} n_{R}(\vec{r}) n_{R}\left(\vec{r}^{\prime}\right)\right)\right\} .
\end{aligned}
$$

The hole function $h_{\lambda}\left(\vec{r}^{\prime}, \vec{r}\right)$ is the hole function of the full electron density $n(\vec{r})$. For overlapping clusters, i.e. $n_{R}(\vec{r}) n_{R^{\prime}}(\vec{r}) \neq 0$ for $R \neq R^{\prime}$ and some $\vec{r} \in \mathbb{R}^{3}$, the last term gives a non-vanishing contribution. Thus, due to the local approximation, this limit is not identical to reduced density-matrix functional theory.

4. In the limit where the approximate density functional $F_{\mathrm{DF}}^{\hat{W}_{R}}$ coincides with the chosen approximation of the local density-matrix functional $F^{\hat{W}_{R}}$, we find the expression

$$
E[h]=\min _{\rho^{(1)}: 0 \leq \rho^{(1)} \leq 1, \operatorname{Tr} \rho^{(1)}=N}\left\{\operatorname{Tr}\left[\rho^{(1)} h\right]+U_{x c}[n]+T_{c}[n]-\sum_{R} T_{c}^{\hat{W}_{R}}[n]\right\} .
$$


In this limit the approach would coincide with reduced density-matrix functional theory with a density-functional approximation of the density-matrix functional if the kinetic correlation $\sum_{R} T_{c}^{\hat{W}_{R}}[n]$ of the clusters approximately cancels the total kinetic correlation $T_{c}[n]$. However, if the sum of local kinetic correlations $\sum_{R} T_{c}^{\hat{W}_{R}}[n]$ is negligible, then the energy to be minimized is the DFT total energy.

The minimization in Eq. (6.34) is performed over all ensemble-representable oneparticle reduced density matrices and is identical to

$$
\begin{aligned}
E[h] & =\min _{n \in \mathcal{I}_{N}}\left\{U_{x c}[n]+T_{c}[n]-\sum_{R} T_{c}^{\hat{W}_{R}}[n]+\int d^{3} \vec{r} n(\vec{r}) v_{\text {ext }}(\vec{r})\right. \\
& \left.+\min _{\rho^{(1)}: 0 \leq \rho^{(1)} \leq 1, \rho^{(1)} \mapsto n} \operatorname{Tr}\left[\rho^{(1)} t\right]\right\} .
\end{aligned}
$$

The last term in the minimization can also be rewritten with wave functions as

$$
\min _{\rho^{(1)}: 0 \leq \rho^{(1)} \leq 1, \rho^{(1)} \mapsto n} \operatorname{Tr}\left[\rho^{(1)} t\right]=\min _{|\Psi\rangle \in \mathcal{W}_{N}:\langle\Psi|\hat{n}(r)| \Psi\rangle=n(r)}\langle\Psi|\hat{t}| \Psi\rangle
$$

with the operator of the kinetic energy $\hat{t}$. Thus, according to Lieb [Lieb, 1983a] a Slater determinant will be a minimizer and, consequently, a non-interacting oneparticle reduced density matrix is a minimizer of Eq. (6.34). This observation shows the tendency of density-matrix functional theory with functionals that only depend on the density to yield non-interacting one-particle reduced density matrices.

\subsubsection{Example results for $\mathbf{H}_{2}$}

We have implemented the above orbital-based scheme in the CP-PAW code and used the Car-Parrinello-like minimization of the total energy discussed in section 4.6 to obtain the ground state. The minimization problem to be solved is given by Eq. (6.26) as

$$
E[h]=\min _{\rho^{(1)}: 0 \leq \rho^{(1)} \leq 1, \operatorname{Tr} \rho^{(1)}=N}\left\{\operatorname{Tr}\left[\rho^{(1)} h\right]+F_{\mathrm{DF}}^{\hat{W}}\left[\rho^{(1)}\right]+F^{\hat{W}^{\prime}}\left[\rho^{(1)}\right]-F_{\mathrm{DF}}^{\hat{W}^{\prime}}\left[\rho^{(1)}\right]\right\} .
$$

We have employed the simple approximation of the double-counting term $F_{\mathrm{DF}}^{\hat{W}^{\prime}}\left[\rho^{(1)}\right]$ given in Eq. (6.25) and performed the three-dimensional integration with an adaptive integration [Berntsen et al., 1991] from the software package cubature ${ }^{3}$.

We have published some results of the scheme described in section 6.3.1 for the hydrogen molecule in [Schade et al., 2017]. The local basis for the for the evaluation of the densitymatrix functional $F^{\hat{W}^{\prime}}\left[\rho^{(1)}\right]$ was chosen as the atomic 1s-orbitals of hydrogen. The local one-particle reduced density matrix is obtained with the projection method discussed in section 7.3.2. We do not perform the local approximation. In other words, the local one-particle basis consists of the four states $\left.\left|\chi_{\alpha}\right\rangle \in\left\{\left|\chi_{1, \uparrow}\right\rangle,\left|\chi_{1, \downarrow},\right| \chi_{2, \uparrow}\right\rangle,\left|\chi_{2, \downarrow}\right\rangle\right\} \equiv C$ defined by Eq. (4.73)-Eq. (4.76). The interaction $\hat{W}^{\prime}$ is

$$
\begin{aligned}
\hat{W}^{\prime} & =\frac{1}{2} \sum_{\alpha, \beta, \gamma, \delta \in C} U_{\alpha, \beta, \gamma, \delta} \hat{c}_{\alpha}^{\dagger} \hat{c}_{\beta}^{\dagger} \hat{c}_{\delta} \hat{c}_{\gamma} \\
U_{\alpha, \beta, \gamma, \delta} & =\int d^{4} \vec{x} \int d^{4} \vec{x}^{\prime} \frac{e^{2}}{4 \pi \epsilon_{0}\left|\vec{r}-\vec{r}^{\prime}\right|} \chi_{\alpha}^{*}(\vec{x}) \chi_{\beta}^{*}\left(\vec{x}^{\prime}\right) \chi_{\gamma}(\vec{x}) \chi_{\delta}\left(\vec{x}^{\prime}\right) .
\end{aligned}
$$

\footnotetext{
${ }^{3}$ cubature 1.0.3 written by Steven G. Johnson https://github.com/stevengj/cubature
} 
Hence, the exact density-matrix functional $F^{\hat{W}^{\prime}}\left[\rho^{(1)}\right]$ can be approximated as the densitymatrix functional $F^{\hat{W}^{\prime}}\left[\rho^{(1), \chi}\right]$ for the one-particle-reduced density matrix $\rho^{(1), \chi}$ with respect to the four one-particle states in $C$. Thus, the density-matrix functional only has to be evaluated for a four-dimensional one-particle basis and the many-particle wave functions involved in the constrained minimization of Eq. (5.8) have at most $2^{4}=16$ Slater determinants. This minimization problem can be solved easily and no additional approximations are needed to reduce the computational complexity. The hydrogen molecule was placed in a large simulation box and an electrostatic decoupling of the periodic images was used [Blöchl, 1995].

Figure 6.1 shows the total energy of the non-spin polarized ground state of the hydrogen molecule obtained in the orbital-based $\mathrm{DF}+\mathrm{RDMF}$ approach compared to results from density-functional theory with the approximate PBE-functional [Perdew et al., 1996a] and results from full-configuration interaction (FCI, cc-pVQZ basis [Dunning, 1989a] computed with Orca [Neese, 2012]. The DFT-results show the static-correlation error as the overestimation of the total energy in the dissociation limit in non-spin-polarized calculations. The FCI-results correctly approach the total energy of -1 Hartree in the dissociation limit. The dissociation curve from orbital-based DF+RDMFT is close to the FCI-results for large atomic distances and hence cures the static-correlation error. It slightly overestimates the total energy in the dissociation limit. Close to the equilibrium distance of about $0.75 \AA$, the orbital-based DF+RDMFT drastically overestimates the total energy. This is caused by the minimal one-particle basis of only four spin-orbitals composed of atomic 1s-orbitals, which is not very suitable to describe the natural orbitals for short atomic distances. Figure 6.1 also shows the chemical potential for different particle numbers close to charge-neutrality of the molecule $(N=2)$ for a large distance of the atoms of $5 \AA$. The results from DFT with a semi-local functional show no jump in the chemical potential and, thus, a vanishing fundamental gap. The very precise results from the FCI-calculations in the finite one-particle basis show a jump in the chemical potential of about $0.5 \mathrm{H}$. A similar jump is obtained with the orbital-based DF+RDMF approach. Thus, orbital-based DF+RDMFT nicely reproduces the fundamental gap for the non-spin-polarized hydrogen molecule in the dissociation limit. This is not surprising because the chosen one-particle basis is very suitable for the dissociation limit and we are essentially in the limit of Eq. (6.32). Additional details of the ground state of the hydrogen molecule in $\mathrm{DF}+\mathrm{RDMFT}$ will be discussed in chapter 11 , because for a molecule the limit of Eq. (6.32) of orbital-based DF+RDMFT is identical to the $\mu \rightarrow 0$-limit of the real-space-decomposition DF+RDMF approach given by Eq. (6.65). 

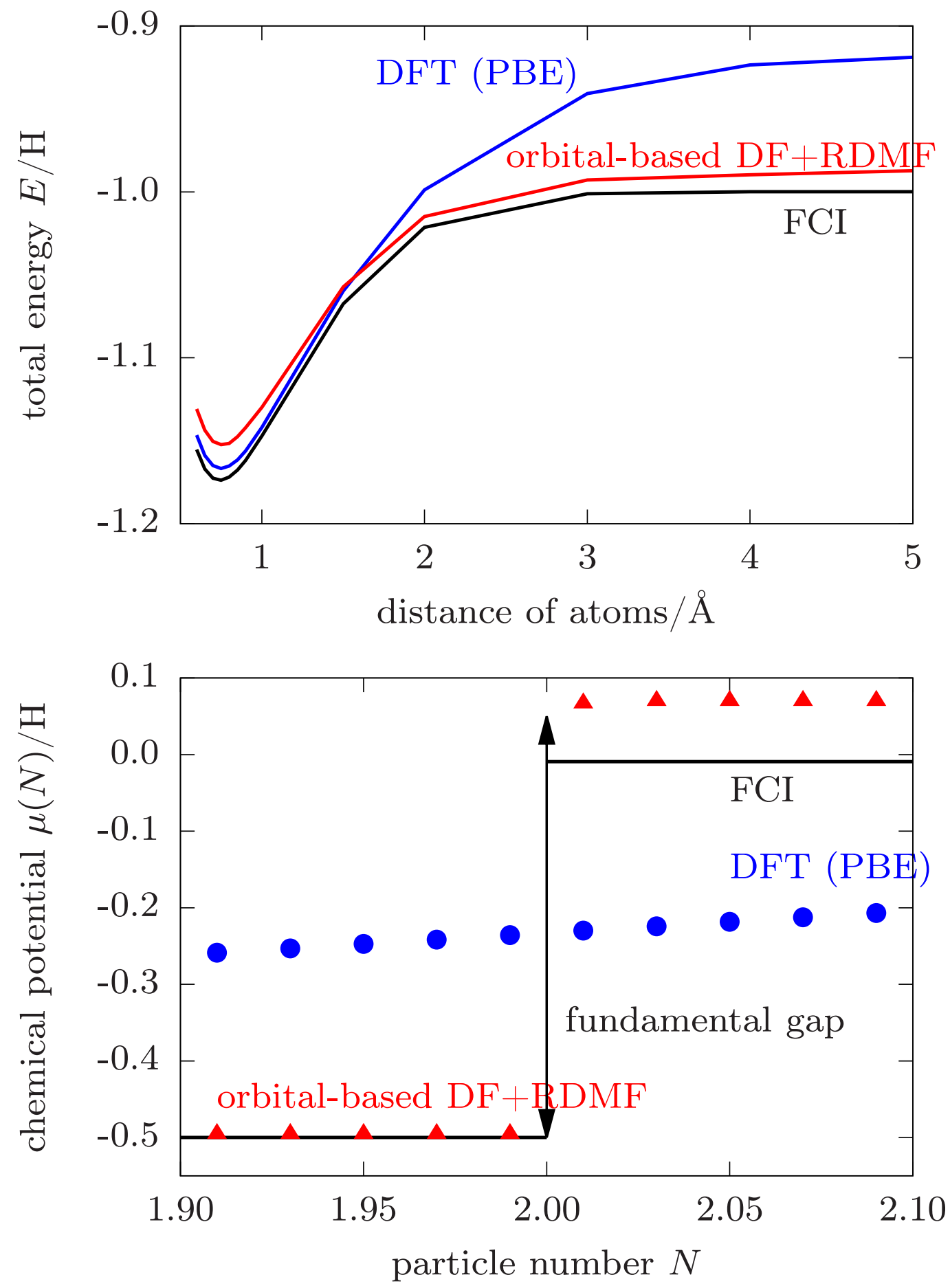

Figure 6.1.: Dissociation curve (top figure) and chemical potential for a large atomic distance (bottom figure, $5 \AA$ ) of the non-spin polarized hydrogen molecule. The figures compare results from density-functional theory with the approximate PBE functional [Perdew et al., 1996a], full-configuration interaction results (FCI, cc-pVQZ basis [Dunning, 1989a] computed with Orca [Neese, 2012]) and from the orbital-based DF+RDMF approach described in section 6.3. 


\subsection{Real-space-decomposition DF+RDMFT}

\subsubsection{Foundation}

In this section we will introduce a modification of the orbital-based DF+RDMF scheme that remedies the inconsistency of the double counting. The main change compared to the orbital-based approach is in the definition of the interaction $\hat{W}^{\prime}$ in Eq. (6.17). We start from Eq. (6.15),

$$
F^{\hat{W}}\left[\rho^{(1)}\right] \approx F_{\mathrm{DF}}^{\hat{W}}\left[\rho^{(1)}\right]+\left(F^{\hat{W}^{\prime}}\left[\rho^{(1)}\right]-F_{\mathrm{DF}}^{\hat{W}^{\prime}}\left[\rho^{(1)}\right]\right)
$$

as in the orbital-based approach and propose to define the interaction $\hat{W}^{\prime}$ by a realspace decomposition. We define the interaction as a real-space decomposition of the full Coulomb interaction

$$
w\left(\vec{r}, \vec{r}^{\prime}\right)=\frac{e^{2}}{4 \pi \epsilon_{0}\left|\vec{r}-\vec{r}^{\prime}\right|}=\left(1-\lambda\left(\vec{r}, \vec{r}^{\prime}\right)\right) w\left(\vec{r}, \vec{r}^{\prime}\right)+\lambda\left(\vec{r}, \vec{r}^{\prime}\right) w\left(\vec{r}, \vec{r}^{\prime}\right)
$$

as

$$
\begin{aligned}
w^{\prime}\left(\vec{r}, \vec{r}^{\prime}\right) & =\lambda\left(\vec{r}, \vec{r}^{\prime}\right) w\left(\vec{r}, \vec{r}^{\prime}\right) \\
\hat{W}^{\prime} & =\frac{1}{2} \int d^{4} \vec{x}_{1} \int d^{4} \vec{x}_{2} \int d^{4} \vec{x}_{3} \int d^{4} \vec{x}_{4} w^{\prime}\left(\vec{x}_{1}, \vec{x}_{2}\right) \delta\left(\vec{x}_{1}-\vec{x}_{4}\right) \delta\left(\vec{x}_{2}-\vec{x}_{3}\right) .
\end{aligned}
$$

Here, $\lambda\left(\vec{r}, \vec{r}^{\prime}\right)$ is some function with $0 \leq \lambda\left(\vec{r}, \vec{r}^{\prime}\right) \leq 1$ and determines the decomposition of the interaction. For example, with the choice

$$
\lambda_{\text {short-range-long-range }}\left(\vec{r}, \vec{r}^{\prime}\right)=\operatorname{erf}\left(\omega\left|\vec{r}-\vec{r}^{\prime}\right|\right)
$$

we would get the short-range long-range decomposition used in the HSE functional. The crucial advantage of the definition of the interaction in real space is that the density functional $F_{D F}^{\hat{W}^{\prime}}\left[\rho^{(1)}\right]$ can be evaluated without having to introduce an approximate model interaction. This change of definition allows evaluating the correction term

$$
F^{\hat{W}^{\prime}}\left[\rho^{(1)}\right]-F_{\mathrm{DF}}^{\hat{W}^{\prime}}\left[\rho^{(1)}\right]
$$

in a consistent way by using the exact same interaction for the density-matrix functional and the density functional. In contrast to the original proposal of the orbital-based DF + RDMF approach [Blöchl et al., 2011], we propose to evaluate the density-matrix functionals $F_{\mathrm{DF}}$ without the kinetic energy contribution from the exchange-correlation functional. Thus, instead of the interaction-strength averaged hole-function, we use the hole function at full interaction strength $(\lambda=1)$ and have

$$
\begin{aligned}
F^{\hat{W}}\left[\rho^{(1)}\right] & =\frac{1}{2} \int d^{3} \vec{r} n(\vec{r}) \int d^{3} \vec{r}^{\prime}\left(h_{\lambda=1}\left(\vec{r}^{\prime}, \vec{r}\right)+n\left(\vec{r}^{\prime}\right)\right) w\left(\vec{r}, \vec{r}^{\prime}\right) \\
& =U_{\mathrm{xc}}[n]+E_{H}[n] .
\end{aligned}
$$

We propose to define the local density functional $F_{\mathrm{DF}}^{\hat{W}^{\prime}}\left[\rho^{(1)}\right]$ for the interaction $\hat{W}^{\prime}$ as

$$
F_{D F}^{\hat{W}^{\prime}}\left[\rho^{(1)}\right]=\frac{1}{2} \int d^{3} \vec{r} n(\vec{r}) \int d^{3} \vec{r}^{\prime}\left(h_{\lambda\left(\vec{r}, \vec{r}^{\prime}\right)}\left(\vec{r}^{\prime}, \vec{r}\right)+n\left(\vec{r}^{\prime}\right)\right) \lambda\left(\vec{r}, \vec{r}^{\prime}\right) w\left(\vec{r}, \vec{r}^{\prime}\right) .
$$


The hole function in Eq. (6.48) depends on the interaction decomposition function $\lambda\left(\vec{r}, \vec{r}^{\prime}\right)$ and is the hole function of a system with interaction $\lambda\left(\vec{r}, \vec{r}^{\prime}\right) w\left(\vec{r}, \vec{r}^{\prime}\right)$. In principle, the hole function $h$ is a functional of the complete shape of the interaction. In Eq. (6.48), we assume a local dependency on the local strength $\lambda\left(\vec{r}, \vec{r}^{\prime}\right)$ of the interaction.

In section 6.4.4 we show that this hole function can be obtained on the level of a local density functional from the interaction-strength-dependent hole function of a local density functional. The minimization problem in the real-space-decomposition based DF + RDMF scheme,

$$
E[h]=\min _{\rho^{(1)}: 0 \leq \rho^{(1)} \leq 1, \operatorname{Tr} \rho^{(1)}=N}\left\{\operatorname{Tr}\left[\rho^{(1)} h\right]+U_{x c}[n]+E_{H}[n]+\left(F^{\hat{W}^{\prime}}\left[\rho^{(1)}\right]-F_{\mathrm{DF}}^{\hat{W}^{\prime}}\left[\rho^{(1)}\right]\right)\right\},
$$

is identical to Eq. (6.26) but the functionals are defined differently.

\subsubsection{Local approximation}

Similar to the discussion in section 6.3.2 where the local approximation for the orbitalbased DF + RDMF approach was described, we also propose a local approximation for the real-space-decomposition based DF + RDMF approach. For this purpose we define the decomposition function $\lambda\left(\vec{r}, \vec{r}^{\prime}\right)$ as a sum of localized contributions $\lambda_{R}$ in the form

$$
\lambda\left(\vec{r}, \vec{r}^{\prime}\right)=\sum_{R} \lambda_{R}\left(\vec{r}, \vec{r}^{\prime}\right) .
$$

Every local contribution $\lambda_{R}$ is supposed to be localized in the vicinity of the position $\vec{R}_{R}$. With this choice, we obtain a decomposition of the interaction $\hat{W}^{\prime}$ into local terms $\hat{W}_{R}$ that are defined as

$$
\hat{W}_{R}=\frac{1}{2} \int d^{4} \vec{x}_{1} \int d^{4} \vec{x}_{2} \int d^{4} \vec{x}_{3} \int d^{4} \vec{x}_{4} w\left(\vec{x}_{1}, \vec{x}_{2}\right) \lambda_{R}\left(\vec{r}_{1}, \vec{r}_{2}\right) \delta\left(\vec{x}_{1}-\vec{x}_{4}\right) \delta\left(\vec{x}_{2}-\vec{x}_{3}\right) .
$$

Thus, the minimization problem in the real-space-decomposition based DF+RDMF scheme including the local approximation is

$$
E[h]=\min _{\rho^{(1)}: 0 \leq \rho^{(1)} \leq 1, \operatorname{Tr} \rho^{(1)}=N}\left\{\operatorname{Tr}\left[\rho^{(1)} h\right]+U_{x c}[n]+E_{H}[n]+\sum_{R}\left(F^{\hat{W}_{R}}\left[\rho^{(1)}\right]-F_{\mathrm{DF}}^{\hat{W}_{R}}\left[\rho^{(1)}\right]\right)\right\},
$$

where the density functional $F_{\mathrm{DF}}^{\hat{W}_{R}}$ is evaluated with the interaction $w\left(\vec{r}, \vec{r}^{\prime}\right) \lambda_{R}\left(\vec{r}, \vec{r}^{\prime}\right)$ as

$$
F_{D F}^{\hat{W}_{R}}\left[\rho^{(1)}\right]=\frac{1}{2} \int d^{3} \vec{r} n(\vec{r}) \int d^{3} \vec{r}^{\prime}\left(h_{\lambda_{R}\left(\vec{r}, \vec{r}^{\prime}\right)}\left(\vec{r}^{\prime}, \vec{r}\right)+n\left(\vec{r}^{\prime}\right)\right) \lambda_{R}\left(\vec{r}, \vec{r}^{\prime}\right) w\left(\vec{r}, \vec{r}^{\prime}\right) .
$$

The local terms $\lambda_{R}$ can be chosen in a way that best suits the physical situations. We propose the symmetric choice

$$
\lambda_{\mathrm{sym}, R}\left(\vec{r}, \vec{r}^{\prime}\right)=f(|\vec{r}-\vec{R}|) f\left(\left|\vec{r}^{\prime}-\vec{R}\right|\right)
$$

and the non-symmetric choice

$$
\lambda_{\text {non-sym }, R}\left(\vec{r}, \vec{r}^{\prime}\right)=f(|\vec{r}-\vec{R}|) .
$$


The function $f(r)$ is close to unity for small arguments $r$ and falls of to zero for $r \rightarrow \infty$. The notable difference to the short-range-long-range decomposition is that the decomposition function $\lambda_{R}$ depends on the location is space relative to the atom $R$. Other choices that also include non-local terms like

$$
\begin{aligned}
\lambda_{\text {non-local }}\left(\vec{r}, \vec{r}^{\prime}\right) & =\sum_{R} \lambda_{\text {non-local }, R}\left(\vec{r}, \vec{r}^{\prime}\right) \\
\lambda_{\text {non-local }, R}\left(\vec{r}, \vec{r}^{\prime}\right) & =\sum_{R^{\prime}} \frac{1}{2}\left[f(|\vec{r}-\vec{R}|) f\left(\left|\vec{r}^{\prime}-\vec{R}^{\prime}\right|\right)+f\left(\left|\vec{r}-\vec{R}^{\prime}\right|\right) f\left(\left|\vec{r}^{\prime}-\vec{R}\right|\right)\right]
\end{aligned}
$$

or non-spherical functions instead of $f(|\vec{r}|)$ are also possible. One could also construct a decomposition function based on the model interaction proposed by Blöchl et al. and given in Eq. (6.22) with

$$
\lambda\left(\vec{r}, \vec{r}^{\prime}\right)=\frac{n_{C}(\vec{r})}{n(\vec{r})} \frac{n_{C}\left(\vec{r}^{\prime}\right)}{n\left(\vec{r}^{\prime}\right)} .
$$

In the next section we discuss limiting cases of the approach with respect to the shape of $f(r)$. In section 6.4.4 we discuss the evaluation of the terms in Eq. (6.49) that involve the hole function and explain some considerations for the practical choices of the function $f(r)$ in section 6.4.5.

\subsubsection{Limits}

The function $f(r)$ can be used to explore limiting cases of the approach: for simplicity we choose

$$
\begin{aligned}
\lambda\left(\vec{r}, \vec{r}^{\prime}\right) & =\lambda_{\mathrm{sym}}\left(\vec{r}, \vec{r}^{\prime}\right) \\
f(r) & =e^{-\mu r^{2}}
\end{aligned}
$$

here. The actual form of $f(r)$ is not relevant for the following discussion, important are only the limits

$$
\begin{gathered}
\lim _{\mu \rightarrow \infty} f(r)=0, \\
\lim _{\mu \rightarrow 0} f(r)=1 .
\end{gathered}
$$

We assume the zero-temperature limit.

1. In the limit $\mu \rightarrow \infty$, we have $\hat{W}^{\prime}=0$ and the minimization problem of Eq. (6.49) becomes

$$
E_{\mu \rightarrow \infty}[h]=\min _{\rho^{(1)}: 0 \leq \rho^{(1)} \leq 1, \operatorname{Tr} \rho^{(1)}=N}\left\{\operatorname{Tr}\left[\rho^{(1)} h\right]+E_{H}[n]+U_{\mathrm{xc}}[n]\right\}
$$

and is very much related to the limit of empty an empty set $C$ in section 6.3. However, the difference is that Eq. (6.63) lacks the term of the kinetic correlation and is therefore not identical to density-functional theory. A non-interacting one-particle reduced density matrix is a minimizer of Eq. (6.63) as discussed for Eq. (6.34) in section 6.3 . 
2. In the limit $\mu \rightarrow 0$, we have

$$
\lambda_{\mathrm{sym}, \mu \rightarrow 0}\left(\vec{r}, \vec{r}^{\prime}\right)=\lim _{\mu \rightarrow 0} f(|\vec{r}-\vec{R}|) f\left(\left|\vec{r}^{\prime}-\vec{R}\right|\right)=1 .
$$

and hence $\hat{W}^{\prime}=\hat{W}$. In this limit the minimization problem of Eq. (6.49) is equivalent to RDMFT,

$$
E_{\mu \rightarrow 0}[h]=\min _{\rho^{(1)}: 0 \leq \rho^{(1)} \leq 1, \operatorname{Tr} \rho^{(1)}=N}\left\{\operatorname{Tr}\left[\rho^{(1)} h\right]+F^{\hat{W}}\left[\rho^{(1)}\right]\right\} .
$$

3. The limit $\mu \rightarrow 0$ can also be performed with multiple local interactions

$$
\begin{aligned}
\lambda_{\text {sym }, \mu \rightarrow 0}\left(\vec{r}, \vec{r}^{\prime}\right) & =\lim _{\mu \rightarrow 0} \sum_{R} f(|\vec{r}-\vec{R}|) f\left(\left|\vec{r}^{\prime}-\vec{R}\right|\right) X_{R}(\vec{r}) X_{R}\left(\vec{r}^{\prime}\right) \\
& =\sum_{R} X_{R}(\vec{r}) X_{R}\left(\vec{r}^{\prime}\right),
\end{aligned}
$$

where

$$
X_{R}(\vec{r})= \begin{cases}1 & \vec{r} \in \Omega_{R} \\ 0 & \text { else }\end{cases}
$$

is the characteristic function of the space $\Omega_{R}$. $\Omega_{R}$ is chosen such that it is nonoverlapping and space-filling, i.e. $\cup_{R} \Omega_{R}=\mathbb{R}^{3}$ and $\Omega_{R} \cap \Omega_{R^{\prime}}=\emptyset \forall R, R^{\prime}$. We obtain the energy

$$
\begin{aligned}
E_{\mu \rightarrow 0}[h] & =\min _{\rho^{(1)}: 0 \leq \rho^{(1)} \leq 1, \operatorname{Tr} \rho^{(1)}=N}\left\{\operatorname{Tr}\left[\rho^{(1)} h\right]+\sum_{R} F^{\hat{W}_{R}}\left[\rho^{(1)}\right]\right. \\
& \left.+\sum_{R \neq R^{\prime}} \frac{1}{2} \int_{\Omega_{R}} d^{3} \vec{r} n(\vec{r}) \int_{\Omega_{R^{\prime}}} d^{3} \vec{r}^{\prime}\left(h_{\lambda=1}\left(\vec{r}^{\prime}, \vec{r}\right)+n\left(\vec{r}^{\prime}\right)\right) w\left(\vec{r}, \vec{r}^{\prime}\right)\right\}
\end{aligned}
$$

Thus, in this limit the energy is the same as for reduced density-matrix functional theory in the local approximation except for non-local terms from the interaction of one electron in $\Omega_{R}$ with another electron in $\Omega_{R^{\prime}}$ for $R \neq R^{\prime}$.

The approach can smoothly scale between RDMFT and a DFT-like limit. This smooth interpolation could possibly be used for a modified adiabatic connection construction [Yang, 1998; Toulouse et al., 2009]. The double counting of the interaction can be evaluated without additional approximations as long as the hole function of a density functional is available. We discuss in the following section how the interaction-strength dependent hole function can be obtained from the interaction-strength integrated hole function.

\subsubsection{Availability of the hole function}

The term $U_{x c}[n]$ in Eq. (6.49), that includes the hole function at full interaction strength $h_{\lambda=1}$,

$$
U_{\mathrm{xc}}[n]=\frac{1}{2} \int d^{3} \vec{r} n(\vec{r}) \int d^{3} \vec{r}^{\prime} h_{\lambda=1}\left(\vec{r}^{\prime}, \vec{r}\right) w\left(\vec{r}, \vec{r}^{\prime}\right)
$$


can be evaluated without resorting to the hole function or pair-distribution function. As this term is just the exchange-correlation functional $E_{x c}[n]$ minus the kinetic correlation $T_{c}[n]$, it can be evaluated easily if the kinetic correlation is known. In the local density approximation the kinetic correlation energy $t_{c}$ per particle can be written as [Levy and Perdew, 1985]

$$
t_{c}(n)=3 \frac{d\left[n \cdot \epsilon_{c}(n)\right]}{d n}-4 \epsilon_{c}(n)
$$

Thus, the interaction term in Eq. (6.70) can be evaluated by a simple three-dimensional integration

$$
U_{\mathrm{xc}}[n]=\int d^{3} \vec{r} n(\vec{r})\left[\epsilon_{x c}(n(\vec{r}))-t_{c}(n(\vec{r}))\right] .
$$

This term can be trivially implemented in an DFT code by replacing the exchangecorrelation energy density $\epsilon_{x c}(n)$ per particle in the evaluation of the exchange-correlation functional $E_{x c}[n]$ with the interaction energy-density $u_{x c}(n)=\epsilon_{x c}(n)-t_{c}(n)$ per particle.

Although a similar expression for the kinetic correlation can be derived for a GGA functional and models for the hole function of the PBE functional have been constructed [Perdew et al., 1996b; Bahmann and Ernzerhof, 2008], we will treat the density functional at the LDA-level. The use of a GGA functional instead of an LDA functional would make the integrations required for the density functional $F_{D F}^{\hat{W}_{R}}\left[\rho^{(1)}\right]$ more numerically involved while at the same time it gives no improvement of description of strong electronic correlations.

The interaction-strength-dependent hole function $h_{\lambda}$ can be obtained from the interaction-strength-dependent pair-distribution function $g_{\lambda}\left(\vec{r}, \vec{r}^{\prime}\right)$ in Eq. (4.41) as

$$
h_{\lambda}\left(\vec{r}, \vec{r}^{\prime}\right)=n(\vec{r})\left(g_{\lambda}\left(\vec{r}, \vec{r}^{\prime}\right)-1\right) .
$$

Within the local density approximation, the interaction-strength-dependent pairdistribution function $g_{\lambda}\left(\vec{r}, \vec{r}^{\prime}\right)$ depends on the density parameter $r_{s}(\vec{r})=\left[\frac{3}{4 \pi n(\vec{r})}\right]^{1 / 3}$ the spin polarization $\zeta(\vec{r})=\frac{n_{\uparrow}(\vec{r})-n_{\downarrow}(\vec{r})}{n_{\uparrow}(\vec{r})+n_{\downarrow}(\vec{r})}$ and is spherical. The dependence on $r=\left|\vec{r}-\vec{r}^{\prime}\right|$ is usually rewritten in terms of the product $k_{F} r$ with the Fermi vector $k_{F}(\vec{r})=\left(3 \pi^{2} n(\vec{r})\right)^{1 / 3}$ as

$$
g_{\lambda=1}\left(\vec{r}, \vec{r}^{\prime}\right)=g\left(r_{s}(\vec{r}), \zeta(\vec{r}), k_{F}(\vec{r})\left|\vec{r}-\vec{r}^{\prime}\right|\right)
$$

It depends on $\lambda$ only trough the Bohr radius

$$
a_{0, \lambda}=\frac{4 \pi \epsilon_{0} \hbar^{2}}{\lambda e^{2} m_{e}}
$$

Thus, the pair-distribution function only depends upon $\lambda$ via the density parameter $r_{s}$ [Perdew and Wang, 1992b] and we obtain for the interaction-strength-dependent pairdistribution function in the local density approximation the simple expression

$$
g_{\lambda}\left(r_{s}(\vec{r}), \zeta(\vec{r}), k_{F}(\vec{r})\left|\vec{r}-\vec{r}^{\prime}\right|\right)=g_{\lambda=1}\left(\lambda r_{s}(\vec{r}), \zeta(\vec{r}), k_{F}(\vec{r})\left|\vec{r}-\vec{r}^{\prime}\right|\right) .
$$


If only the interaction-strength-integrated pair-distribution function $\bar{g}$ is available, the pair-distribution function at full interaction strength $(\lambda=1)$ can be derived in the local density approximation as

$$
g_{\lambda=1}\left(r_{s}(\vec{r}), \zeta(\vec{r}), k_{F}(\vec{r})\left|\vec{r}-\vec{r}^{\prime}\right|\right)=\left(1+r_{s} \partial_{r_{s}}\right) \bar{g}\left(r_{s}(\vec{r}), \zeta(\vec{r}), k_{F}(\vec{r})\left|\vec{r}-\vec{r}^{\prime}\right|\right) .
$$

Thus, we can write $\sum_{R} F_{D F}^{\hat{W}_{R}}\left[\rho^{(1)}\right]$ in Eq. (6.52) in the local density approximation as

$$
F_{D F}^{\hat{W}_{R}}\left[\rho^{(1)}\right] \approx \frac{1}{2} \int d^{3} \vec{r} n(\vec{r})^{2} \int d^{3} \vec{r}^{\prime} g_{\lambda_{R}\left(\vec{r}, \vec{r}^{\prime}\right)}\left(r_{s}(\vec{r}), \zeta(\vec{r}), k_{F}(\vec{r})\left|\vec{r}-\vec{r}^{\prime}\right|\right) \lambda_{R}\left(\vec{r}, \vec{r}^{\prime}\right) w\left(\vec{r}, \vec{r}^{\prime}\right) .
$$

Even though this term could be evaluated numerically in practice, we consider here some additional simplifications. The product $\lambda \cdot g_{\lambda}\left(r_{s}, \zeta, k_{F} r\right)$ is shown in figure 6.2 for the parametrization of the pair-distribution function of the spin-polarized homogeneous electron gas by Perdew and Wang [Perdew and Wang, 1992b]. These figures show that the function $\lambda \cdot g_{\lambda}\left(r_{s}, \zeta, k_{F} r\right)$ is almost linear in $\lambda$ and, thus, can be approximated as

$$
\lambda \cdot g_{\lambda}\left(r_{s}, \zeta, k_{F} r\right) \approx \lambda \cdot g_{\lambda=1}\left(r_{s}, \zeta, k_{F} r\right)
$$

Consequently, we can approximate Eq. (6.78) further as

$$
F_{D F}^{\hat{W}_{R}}\left[\rho^{(1)}\right] \approx \frac{1}{2} \int d^{3} \vec{r} n(\vec{r})^{2} \int d^{3} \vec{r}^{\prime} g_{\lambda=1}\left(r_{s}(\vec{r}), \zeta(\vec{r}), k_{F}(\vec{r})\left|\vec{r}-\vec{r}^{\prime}\right|\right) \lambda_{R}\left(\vec{r}, \vec{r}^{\prime}\right) w\left(\vec{r}, \vec{r}^{\prime}\right)
$$

and obtain in the case of $\lambda_{R}\left(\vec{r}, \vec{r}^{\prime}\right)=\lambda_{\text {sym }, R}\left(\vec{r}, \vec{r}^{\prime}\right)=f(|\vec{r}-\vec{R}|) f\left(\left|\vec{r}^{\prime}-\vec{R}\right|\right)$ the final expression

$$
\begin{aligned}
F_{D F}^{\hat{W}_{R}}\left[\rho^{(1)}\right] & \approx \int d^{3} \vec{r} f(|\vec{r}-\vec{R}|) n(\vec{r}) \tilde{\epsilon}_{x c}(|\vec{r}-\vec{R}|, n(\vec{r}), \zeta(\vec{r})) \\
& +\frac{1}{2} \int d^{3} \vec{r} \int d^{3} \overrightarrow{r^{\prime}} \frac{e^{2} f(|\vec{r}-\vec{R}|) f\left(\left|\vec{r}^{\prime}-\vec{R}\right|\right) n(\vec{r}) n\left(\vec{r}^{\prime}\right)}{4 \pi \epsilon_{0}\left|\vec{r}-\vec{r}^{\prime}\right|} \\
\tilde{\epsilon}_{x c}(\tilde{r}, n, \zeta) & =\frac{n e^{2}}{4 \epsilon_{0}} \int_{0}^{\infty} d u \int_{0}^{\pi} d \theta u \sin (\theta) g_{\lambda=1}\left(r_{s}(n), \zeta, k_{F}(n) u\right) f\left(\left|\tilde{r} \vec{e}_{z}-\vec{r}(u, \theta, \phi=0)\right|\right) .
\end{aligned}
$$

Here, $\vec{r}(u, \theta, \phi)$ denotes the vector $\vec{r}$ parametrized in spherical coordinates. The function $\tilde{\epsilon}_{x c}(\tilde{r}, n, \zeta)$ is a function of three real variables and can be parametrized to avoid the numerical integration in practical calculations.

In the case of the non-symmetric decomposition function $\lambda_{R}\left(\vec{r}, \vec{r}^{\prime}\right)=\lambda_{\text {non-sym, } R}\left(\vec{r}, \vec{r}^{\prime}\right)=$ $f(|\vec{r}-\vec{R}|)$ we find the much simpler expression

$$
F_{D F}^{\hat{W}_{R}}\left[\rho^{(1)}\right] \approx \int d^{3} \vec{r} f(|\vec{r}-\vec{R}|) n(\vec{r}) u_{x c}(n(\vec{r}), \zeta(\vec{r}))+\frac{1}{2} \int d^{3} \vec{r} \int d^{3} \overrightarrow{r^{\prime}} \frac{e^{2} f(|\vec{r}-\vec{R}|) n(\vec{r}) n\left(\vec{r}^{\prime}\right)}{4 \pi \epsilon_{0}\left|\vec{r}-\vec{r}^{\prime}\right|}
$$



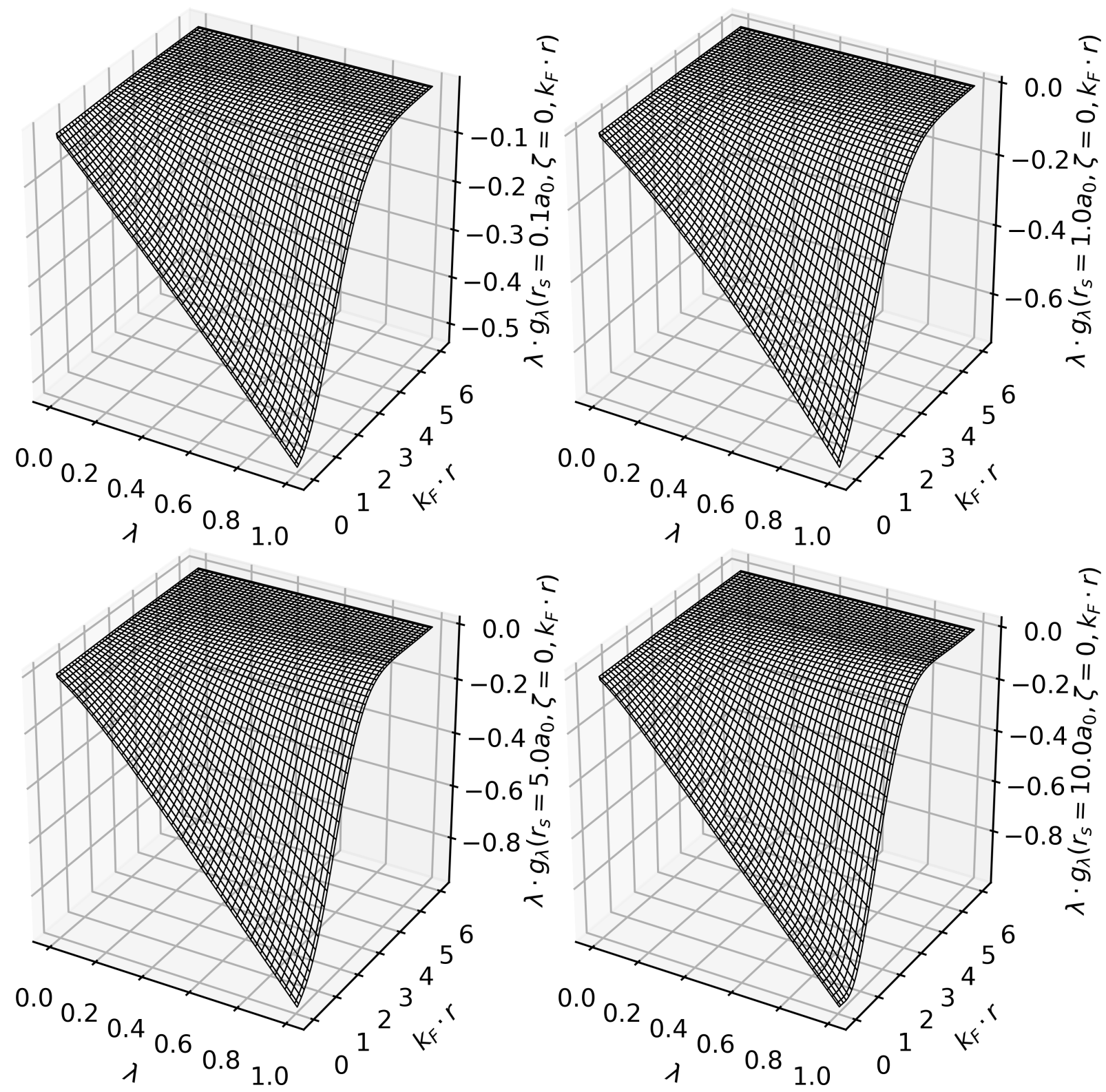

Figure 6.2.: The function $\lambda \cdot g_{\lambda}\left(r_{s}, \zeta=0, k_{F} r\right)$ with the parametrization of the pairdistribution function from Perdew and Wang [Perdew and Wang, 1992b] is shown for some important parameter ranges. The top left graph is for $r_{s}=$ $0.1 a_{0}$, the top right graph for $r_{s}=a_{0}$, to bottom left graph for $r_{s}=5 a_{0}$ and to lower right graph is for $r_{s}=10 a_{0}$. 


\subsubsection{Practical considerations for the choice of $f(r)$}

For practical calculations of correlated solids, we propose to set the function $f(r)$ such that functions from correlated atoms in different unit cells do not overlap. The main goal is to select $f(r)$ such that the local interaction $\hat{W}_{R}$ contains the interaction of the orbitals that are responsible for the static correlation. A numerically convenient choice is a superposition of Gaussian functions centered at $\vec{R}$, i.e.

$$
f(r)=\sum_{i} c_{i} e^{-a_{i} r^{2}}
$$

In the following, we assume the symmetric decomposition function $\chi_{\mathrm{sym}, R}=f(\mid \vec{r}-$ $\vec{R}) f\left(\mid \vec{r}^{\prime}-\vec{R}\right)$. The other decomposition functions can be handled analogously. With this choice the matrix elements of the local interaction are

$$
U_{R, \alpha, \beta, \gamma, \delta}=\int d^{4} \vec{x} \int d^{4} \vec{x}^{\prime} \frac{f(\mid \vec{r}-\vec{R}) f\left(\left|\vec{r}^{\prime}-\vec{R}\right|\right) \chi_{\alpha}^{*}(\vec{x}) \chi_{\beta}^{*}\left(\vec{x}^{\prime}\right) \chi_{\gamma}(\vec{x}) \chi_{\delta}\left(\vec{x}^{\prime}\right)}{4 \pi \epsilon_{0}\left|\vec{r}-\vec{r}^{\prime}\right|} .
$$

If the one-particle orbitals $\chi_{\alpha}(\vec{x})$ are known in terms spherical Gaussian-type orbitals and have the form

$$
\chi_{\alpha}(\vec{x})=S_{l_{\alpha}, m_{\alpha}}\left(\frac{\vec{x}-\vec{R}_{\alpha}}{\left|\vec{x}-\vec{R}_{\alpha}\right|}\right)\left|\vec{r}-\vec{R}_{\alpha}\right|^{l_{\alpha}} \sum_{j} c_{\alpha, j} e^{-a_{\alpha, j}\left|\vec{r}-\vec{R}_{\alpha}\right|^{2}} \delta_{\sigma_{\alpha}, \sigma}
$$

centered at $\vec{R}_{\alpha}$, we can combine the expansions of the functions $f(r)$ with the orbitals. This can be achieved by decomposing the resulting modified orbital $\tilde{\chi}_{\alpha}(\vec{r})$ as

$$
\tilde{\chi}_{\alpha}(\vec{r})=f(\mid \vec{r}-\vec{R}) \chi_{\alpha}(\vec{r})=\sum_{i, j} c_{i} c_{\alpha, j} M_{i, j}(\vec{r})
$$

with

$$
M_{i, j}(\vec{r})=S_{l_{\alpha}, m_{\alpha}}\left(\frac{\vec{x}-\vec{R}_{\alpha}}{\left|\vec{x}-\vec{R}_{\alpha}\right|}\right)\left|\vec{r}-\vec{R}_{\alpha}\right|^{l_{\alpha}} e^{-a_{i}|\vec{r}-\vec{R}|^{2}} e^{-a_{\alpha, j}\left|\vec{r}-\vec{R}_{\alpha}\right|^{2}} .
$$

With the Gaussian product rule $M_{i, j}(\vec{r})$ can be rewritten as

$$
M_{i, j}(\vec{r})=S_{l_{\alpha}, m_{\alpha}}\left(\frac{\vec{x}-\vec{R}_{\alpha}}{\left|\vec{x}-\vec{R}_{\alpha}\right|}\right)\left|\vec{r}-\vec{R}_{\alpha}\right|^{l_{\alpha}} e^{-\frac{a_{\alpha, j} a_{i}}{a_{\alpha, j}+a_{i}}\left(\vec{R}-\vec{R}_{\alpha}\right)^{2}} e^{-\left(a_{\alpha, j}+a_{i}\right)|\vec{r}-\vec{C}|^{2}} .
$$

with the new center

$$
\vec{C}=\frac{a_{\alpha, j}}{a_{\alpha, j}+a_{i}} \vec{R}_{\alpha}+\left(1-\frac{a_{\alpha, j}}{a_{\alpha, j}+a_{i}}\right) \vec{R}
$$

However, the spherical harmonic $S_{l_{\alpha}, m_{\alpha}}$ and the polynomial $\left|\vec{r}-\vec{R}_{\alpha}\right|^{l_{\alpha}}$ in Eq. (6.89) still refer to the old center $\vec{R}_{\alpha}$. They can be transformed to sums of Cartesian Gaussian-type orbitals

$$
S_{l_{\alpha}, m_{\alpha}}\left(\frac{\vec{x}-\vec{R}_{\alpha}}{\left|\vec{x}-\vec{R}_{\alpha}\right|}\right)\left|\vec{r}-\vec{R}_{\alpha}\right|^{l_{\alpha}}=\sum_{k} d_{k}\left(x-\vec{R}_{\alpha, x}\right)^{u_{k}}\left(y-\vec{R}_{\alpha, y}\right)^{v_{k}}\left(z-\vec{R}_{\alpha, z}\right)^{w_{k}} .
$$


These Cartesian Gaussian-type orbitals can then be written with $\vec{C}$ as center in the form

$$
S_{l_{\alpha}, m_{\alpha}}\left(\frac{\vec{x}-\vec{R}_{\alpha}}{\left|\vec{x}-\vec{R}_{\alpha}\right|}\right)\left|\vec{r}-\vec{R}_{\alpha}\right|^{l_{\alpha}}=\sum_{k} \tilde{d}_{k}\left(x-\vec{C}_{x}\right)^{\tilde{u}_{k}}\left(y-\vec{C}_{y}\right)^{\tilde{v}_{k}}\left(z-\vec{C}_{z}\right)^{\tilde{w}_{k}} .
$$

and transformed back to sums of spherical Gaussian-type orbitals with different angular momenta and magnetic quantum numbers but with the center $\vec{C}$. Thus, the resulting modified orbital $\tilde{\chi}_{\alpha}(\vec{r})$, can be expressed as a sum over spherical Gaussian-type orbitals with different centers in the form

$$
\begin{aligned}
\tilde{\chi}_{\alpha}(\vec{r}) & =f(\mid \vec{r}-\vec{R}) \chi_{\alpha}(\vec{r}) \\
& =\sum_{i, j} c_{i} c_{\alpha, j} e^{-\frac{a_{\alpha, j} a_{i}}{a_{\alpha, j}+a_{i}}\left(\vec{R}-\vec{R}_{\alpha}\right)^{2}} e^{-\left(a_{\alpha, j}+a_{i}\right)\left|\vec{r}-\vec{C}_{i, \alpha, j}\right|^{2}} \\
& \sum_{l, m} \beta_{i, \alpha, j, l, m} S_{l, m}\left(\frac{\vec{x}-\vec{C}_{i, \alpha, j}}{\left|\vec{x}-\vec{C}_{i, \alpha, j}\right|}\right)\left|\vec{r}-\vec{C}_{i, \alpha, j}\right|^{l} .
\end{aligned}
$$

These orbitals can then be used as an input for existing numerical routines that calculate the interaction matrix elements of spherical Gaussian-type orbitals as in section 7.3.1.

In case a given parametrization of $f(r)$ in Eq. (6.84) does not localize the interaction strongly enough, the tail of $f(r)$ needs to be reduced. In order to reduce the tail of $f(r)$ more Gaussian would be required in (6.84) which would drastically increase the numerical effort for the calculation of the interaction matrix elements. Even more importantly, a good approximation of a step function with Gaussians that are located at $\vec{R}$ requires very large positive and negative prefactors $c_{i}$ of Gaussians in $f(r)$ as well as pairs of Gaussians with similar factors $a_{i}$ in the exponent. Large floating-point numbers, however, would make the calculation of the interaction matrix elements numerically unstable. An alternative is to use a sum over Gaussians located at at different centers $\vec{g}_{i}$

$$
f_{R}(\vec{r})=\sum_{i} c_{i} e^{-a_{i}\left|\vec{r}-\vec{g}_{i}\right|^{2}}
$$

instead of the expansion of the one-dimensional function $f(r)$ in Gaussians centered at the origin. The problem of approximating a given function $f(\vec{r})$ with the expansion in Eq. (6.95) is numerically stable if the prefactors $c_{i}$ are restricted to be positive. The parameters of the Gaussians can be obtained from the solution of the minimization problem

$$
\min _{\left\{c_{i}, a_{i}, \vec{g}_{i}\right\}: c_{i} \geq 0} \int d^{3} \vec{r}\left|f(\vec{r})-\sum_{i} c_{i} e^{-a_{i}\left|\vec{r}-\vec{g}_{i}\right|^{2}}\right| .
$$

The modified orbital in Eq. (6.87) is replaced by

$$
\tilde{\chi}_{\alpha}(\vec{r})=\sum_{i} c_{i} e^{-a_{i}\left|\vec{r}-\vec{g}_{i}\right|^{2}} \chi_{\alpha}(\vec{r})=\sum_{i=1}^{N_{g}} c_{i} \sum_{j} c_{\alpha, j} M_{i, j}(\vec{r})
$$

with

$$
M_{i, j}(\vec{r})=S_{l_{\alpha}, m_{\alpha}}\left(\frac{\vec{x}-\vec{R}_{\alpha}}{\left|\vec{x}-\vec{R}_{\alpha}\right|}\right)\left|\vec{r}-\vec{R}_{\alpha}\right|^{l_{\alpha}} e^{-a_{i}\left|\vec{r}-\vec{g}_{i}\right|^{2}} e^{-a_{\alpha, j}\left|\vec{r}-\vec{R}_{\alpha}\right|^{2}} .
$$




\subsection{Screening and effective Coulomb interactions}

\subsubsection{Different aspects of screening}

In DFT $+\mathrm{U}$, local hybrid functionals or DFT+DMFT approaches usually not the bare Coulomb interaction

$$
w\left(\vec{r}, \vec{r}^{\prime}\right)=\frac{1}{4 \pi \epsilon_{0}\left|\vec{r}-\vec{r}^{\prime}\right|}
$$

but an effective Coulomb interaction $\tilde{w}\left(\vec{r}, \vec{r}^{\prime}\right)$ is used for the non-DFT part. The reason is that these methods only consider a low-energy subspace of the full Hilbert space or an otherwise truncated space. Biermann [Biermann, 2014] has nicely formulated the purpose of the estimation of screened Coulomb interactions with the constrained random-phase approximation (cRPA) as

"The cRPA provides an (approximate) answer to the following question: given the Coulomb Hamiltonian in a large Hilbert space, and a low-energy Hilbert space that is a subspace of the former, what is the effective bare interaction to be used in many-body calculations dealing only with the low-energy subspace, in order for physical predictions for the low-energy Hilbert space to be the same for the two descriptions?"

[Biermann, 2014], page 3.

It should be noted here, that one does not have to consider screening in the context of an exact-solution method of the many-particle problem: if we could solve a given manyparticle problem, for example, the electronic structure of $\mathrm{NiO}$ in a numerically exact way with a method such as exact diagonalization, no effective Coulomb interaction or screening has to be introduced. Thus, we do not consider the screening of Coulomb interactions in this context as a fundamental physical process but rather a required numerical technique to use approximate solution methods. However, we adopt the viewpoint that if the description in the low-energy subspace with the effective Coulomb interaction gives the same physical predictions as the unmodified Coulomb interaction in the full space, it is suitable to call the interaction screened. We identify three different situations where an effective Coulomb interaction can be required:

1. if the effects of non-local Coulomb interactions are to be described by a local Coulomb interaction,

2. if the effects of high-energy subspaces of the Hilbert space are to be described by only considering a low-energy subspace,

3. or if the approximation method employed requires a modification of the Coulomb interaction to describe the correct physical situation.

The first situation, i.e., the treatment of non-local Coulomb interactions with effective local Coulomb interactions has for example been discussed by Schüler et al. [Schüler et al., 2013]. They have shown that the Hamiltonian of the extended Hubbard model

$$
\hat{H}_{\text {extended }}=-\sum_{\alpha, \beta} h_{\alpha, \beta} \hat{c}_{\alpha}^{\dagger} \hat{c}_{\beta}+U \sum_{i} \hat{n}_{i, \uparrow} \hat{n}_{i, \downarrow}+\frac{1}{2} \sum_{i, j} \sum_{\sigma, \sigma^{\prime}} V_{i, j} \hat{n}_{i, \sigma} \hat{n}_{j, \sigma^{\prime}}
$$


can be mapped approximately to the effective model

$$
\hat{H}_{\text {eff. }}=-\sum_{\alpha, \beta} h_{\alpha, \beta} \hat{c}_{\alpha}^{\dagger} \hat{c}_{\beta}+U_{\text {eff. }} \sum_{i} \hat{n}_{i, \uparrow} \hat{n}_{i, \downarrow}
$$

with

$$
U_{\text {eff. }}=U+\frac{1}{2} \sum_{i, j} \sum_{\sigma, \sigma^{\prime}} V_{i, j} \frac{\partial_{U_{\text {eff. }}}\left\langle\hat{n}_{i, \sigma} \hat{n}_{j, \sigma^{\prime}}\right\rangle_{\mathrm{eff}}}{\sum_{l} \partial_{U_{\text {eff. }}\left\langle\hat{n}_{l, \uparrow} \hat{n}_{l, \downarrow}\right\rangle_{\text {eff. }}}} .
$$

The expectation values such as $\left\langle\hat{n}_{i, \sigma} \hat{n}_{j, \sigma^{\prime}}\right\rangle_{\text {eff. }}$ are evaluated with respect to the canonical density operator of the effective Hamiltonian $\hat{H}_{\text {eff. }}$. This approximate mapping is universal in the sense that it is independent of the solution method used for the system with the effective Hamiltonian $\hat{H}_{\text {eff. }}$. Thus, it allows the systematic construction of a model with local Coulomb interactions from a non-local model. The situation of the effective treatment of the effects of high-energy subspaces by only considering a low-energy subspace is of fundamental interest for approaches that combine DFT with dynamical mean-field theory. The main idea of the conventional formulation of the DFT+DMFT approach can be considered as a formulation of a local low-energy model Hamiltonian [Pavarini, 2011] for the strongly correlated degrees of freedom in terms of an impurity model. The lowenergy model is the solved with some advanced many-body technique, and the electron density of the solution is then used as the electron density in the next iteration of the Kohn-Sham self-consistency cycle till self-consistency is achieved. Due to the solution of the low-energy model instead of the model in the full Hilbert space, a modification of the Coulomb interaction is required. If a similar effective treatment of the Coulomb interactions is required in our newly proposed $\mathrm{DF}+\mathrm{RDMF}$ approach is discussed in the following section.

\subsubsection{Effective Coulomb interactions in the DF+RDMF approach}

In section 6.5.1 we have discussed three situations, where a modification of the Coulomb interaction is required. An effective description of non-local Coulomb interactions in terms of local Coulomb interactions is not required in the real-space-decomposition based $\mathrm{DF}+\mathrm{RDMF}$ approach because one could choose the space-decomposition function $\lambda\left(\vec{r}, \vec{r}^{\prime}\right)$ to include the non-local Coulomb interactions with the density-matrix functional instead of the density functional. Thus, more one-particle states in the density-matrix functional are interacting states. Such an extension is related to cluster-extensions of DFT+DMFT approaches or the inclusion of selected non-local Coulomb interactions in local hybrid functionals. Please note, that non-local interactions are naturally included at the level of the density functional. However, a different treatment of non-local interactions is possible by including them in the density-matrix functional by a suitable definition of the decomposition function and then approximating the density-matrix functional as

$$
F^{\hat{W}_{R}+\hat{W}_{\text {non-local }}}\left[\rho^{(1)}\right] \approx F^{\hat{W}_{R}}\left[\rho^{(1)}\right]+F^{\hat{W}_{\text {non-local }}}\left[\rho^{(1)}\right]
$$

The second term $F^{\hat{W}_{\text {non-local }}}\left[\rho^{(1)}\right]$ can be evaluated differently, for example with he HartreeFock approximation, from the first term. We will argue in the following that a modification of the Coulomb interaction for the description of effects of high-energy subspaces 
of the Hilbert space in the explicitly considered low-energy subspace is not required in a DF+RDMF approach that is based on RDMFT. We consider two sets of orthonormal one-particle states, i.e., $\left|\chi_{\alpha}\right\rangle$ and $\left|\tilde{\chi}_{\alpha}\right\rangle$ with $\left\langle\chi_{\alpha} \mid \chi_{\beta}\right\rangle=\delta_{\alpha, \beta}$ and $\left\langle\tilde{\chi}_{\alpha} \mid \tilde{\chi}_{\beta}\right\rangle=\delta_{\alpha, \beta}$. We assume that the space spanned by $\left|\chi_{\alpha}\right\rangle$ includes the space spanned by $\left|\tilde{\chi}_{\alpha}\right\rangle$ and $\operatorname{dim} H_{\chi}>\operatorname{dim} H_{\tilde{\chi}}$. Thus, the two statements

$$
\begin{array}{r}
\forall \alpha \exists C_{\alpha, \beta}:\left|\tilde{\chi}_{\alpha}\right\rangle=\sum_{\beta} C_{\alpha, \beta}\left|\chi_{\beta}\right\rangle \\
\exists \alpha: \nexists \tilde{C}_{\alpha, \beta} \text { with }\left|\chi_{\alpha}\right\rangle=\sum_{\beta} \tilde{C}_{\alpha, \beta}\left|\tilde{\chi}_{\beta}\right\rangle
\end{array}
$$

are assumed to be fulfilled. $\left|\chi_{\alpha}\right\rangle$ denotes a basis of the full space and $\left|\tilde{\chi}_{\alpha}\right\rangle$ can be interpreted as a basis of the smaller low-energy space. A given natural orbital $\left|\psi_{n}\right\rangle$ can be expressed in the full basis as

$$
\left|\psi_{n}\right\rangle=\sum_{\alpha} c_{n \alpha}\left|\chi_{\alpha}\right\rangle
$$

and the one-particle reduced density matrix in the one-particle basis $\left|\chi_{\alpha}\right\rangle$ as

$$
\rho_{\alpha, \beta}^{(1), \chi}=\sum_{n} f_{n}\left\langle\chi_{\alpha} \mid \psi_{n}\right\rangle\left\langle\psi_{n} \mid \chi_{\beta}\right\rangle
$$

We assume that the one-particle states $\left|\tilde{\chi}_{\alpha}\right\rangle$ have been chosen that the natural orbitals with finite occupations $f_{n}>0$ can also be represented by these states as

$$
\left|\psi_{n}\right\rangle=\sum_{\alpha} \tilde{c}_{n \alpha}\left|\tilde{\chi}_{\alpha}\right\rangle
$$

Therefore, the above assumptions the one-particle states $\left|\chi_{\alpha}\right\rangle$ and the one-particle states $\left|\tilde{\chi}_{\alpha}\right\rangle$ represent the same one-particle reduced density matrix in real space

$$
\begin{aligned}
\rho^{(1)}\left(\vec{x}, \vec{x}^{\prime}\right) & =\sum_{n} f_{n}\left\langle\vec{x} \mid \psi_{n}\right\rangle\left\langle\psi_{n} \mid \vec{x}^{\prime}\right\rangle \\
& =\sum_{\alpha, \beta, n} f_{n} c_{n \alpha} c_{n \beta}^{*}\left\langle\vec{x} \mid \chi_{\alpha}\right\rangle\left\langle\chi_{\beta} \mid \vec{x}^{\prime}\right\rangle \\
& =\sum_{\alpha, \beta, n} f_{n} \tilde{c}_{n \alpha} \tilde{c}_{n \beta}^{*}\left\langle\vec{x} \mid \tilde{\chi}_{\alpha}\right\rangle\left\langle\tilde{\chi}_{\beta} \mid \vec{x}^{\prime}\right\rangle .
\end{aligned}
$$

Now we define a unitary transformation $U$ of the one-particle states $\left|\chi_{\alpha}\right\rangle$ to $\left|\chi_{\alpha}^{\prime}\right\rangle$ that obeys

$$
\left|\tilde{\chi}_{\alpha}\right\rangle=\left|\chi_{\alpha}^{\prime}\right\rangle \forall \alpha \leq \operatorname{dim} H_{\tilde{\chi}}
$$

It follows that

$$
\begin{aligned}
& \rho_{\alpha, \beta}^{(1), \chi^{\prime}}=U^{\dagger} \rho^{(1), \chi} U=\rho^{(1), \tilde{\chi}} \forall \alpha, \beta \leq \operatorname{dim} H_{\tilde{\chi}} \\
& \rho_{\alpha, \alpha}^{(1), \chi^{\prime}}=0 \forall \alpha>\operatorname{dim} H_{\tilde{\chi}} .
\end{aligned}
$$

Thus, since the density-matrix functional is invariant with respect to unitary transformations of the one-particle basis, we have

$$
F^{\hat{W}}\left[\rho^{(1), \chi}\right]=F^{\hat{W}}\left[\rho^{(1), \chi^{\prime}}\right] .
$$


The density-matrix functional $F^{\hat{W}}\left[\rho^{(1), \chi^{\prime}}\right]$ is defined by a constrained minimization over an ensemble of many-particle wave functions $\left|\Psi_{i}\right\rangle$ by Eq. (5.8). The constraint of the diagonal elements of the one-particle reduced density matrix is

$$
\rho_{\alpha, \alpha}^{(1), \chi^{\prime}}=\sum_{i} P_{i}\left\langle\Psi_{i}\left|\hat{c}_{\alpha}^{\dagger} \hat{c}_{\alpha}\right| \Psi_{i}\right\rangle
$$

where the operators $\hat{c}_{\alpha}^{\dagger}$ and $\hat{c}_{\alpha}$ are creation and annihilation operators in the one-particle basis $\left|\chi_{\alpha}^{\prime}\right\rangle$. We consider a one-particle state $\left|\chi_{\alpha}\right\rangle$ with $\alpha>\operatorname{dim} H_{\tilde{\chi}}$ so that $\rho_{\alpha, \alpha}^{(1), \chi^{\prime}}=0$ and a general many-particle wave function in occupation number representation

$$
\left|\Psi_{i}\right\rangle=\sum_{j} a_{i, j}\left|\ldots n_{i, j, \alpha} \ldots\right\rangle
$$

with

$$
\left\langle\ldots n_{i, j, \alpha} \ldots\left|\hat{c}_{\alpha}^{\dagger} \hat{c}_{\alpha}\right| \ldots n_{i, j, \alpha} \ldots\right\rangle=n_{i, j, \alpha} .
$$

The expectation value of $\hat{c}_{\alpha}^{\dagger} \hat{c}_{\alpha}$ with respect to the ensemble of many-particle wave functions is

$$
\left\langle\hat{c}_{\alpha}^{\dagger} \hat{c}_{\alpha}\right\rangle=\sum_{n} \sum_{j} P_{i}\left|a_{i, j}\right|^{2} n_{i, j, \alpha}
$$

The condition $\rho_{\alpha, \alpha}^{(1), \chi^{\prime}}=\left\langle\hat{c}_{\alpha}^{\dagger} \hat{c}_{\alpha}\right\rangle=0$ requires that a many-particle wave function in the ensemble with a non-zero amplitude $a_{i, j}$ of a Slater determinant that has $n_{i, j, \alpha}=1$ must have $P_{i}=0$. On the other hand, it requires that if $P_{i} \neq 0$ we must have $a_{i, j}=0$ for a Slater determinant with $n_{i, j, \alpha}=1$. Thus, many-particle wave functions with finite probability $P_{i}>0$ in the constrained minimization are required to have the form

$$
\left|\Psi_{i}\right\rangle=\sum_{j} a_{i, j}|\underbrace{n_{i, j, 1} \ldots n_{i, j, \operatorname{dim} H_{\tilde{\chi}}}}_{\operatorname{dim} H_{\tilde{\chi}}} \underbrace{0 \ldots 0}_{\operatorname{dim} H_{\chi}-\operatorname{dim} H_{\tilde{\chi}}}\rangle .
$$

The expectation value of the interaction

$$
\hat{W}=\frac{1}{2} \sum_{\alpha, \beta, \gamma, \delta} U_{\alpha, \beta, \gamma, \delta} \hat{c}_{\alpha}^{\dagger} \hat{c}_{\beta}^{\dagger} \hat{c}_{\delta} \hat{c}_{\gamma}
$$

with respect to many-particle wave functions of the form in Eq. (6.120) is identical to the expectation value of the interaction operator

$$
\hat{\tilde{W}}=\frac{1}{2} \sum_{\alpha, \beta, \gamma, \delta \leq \operatorname{dim} H_{\tilde{\chi}}} U_{\alpha, \beta, \gamma, \delta} \hat{c}_{\alpha}^{\dagger} \hat{c}_{\beta}^{\dagger} \hat{c}_{\delta} \hat{c}_{\gamma} .
$$

Thus, the density-matrix functional $F^{\hat{W}}\left[\rho^{(1), \chi}\right]$ in terms of the full one-particle basis $\left|\chi_{\alpha}\right\rangle$ is identical to the density-matrix functional $F^{\hat{W}}\left[\rho^{(1), \tilde{\chi}}\right]$ in terms of the smaller one-particle basis $\left|\tilde{\chi}_{\alpha}\right\rangle$. The density functional $F_{\mathrm{DF}}^{\hat{W}}\left[\rho^{(1)}\right]$ only depends on the density $n(\vec{r})=\rho^{(1)}(\vec{r}, \vec{r})$. Because the sets of one-particle states $\left|\chi_{\alpha}\right\rangle$ and $\left|\tilde{\chi}_{\alpha}\right\rangle$ produce the same one-particle reduced density matrix in real space by construction, the density functional is not affected. In conclusion, as long as the smaller set of one-particle states $\left|\tilde{\chi}_{\alpha}\right\rangle$ can describe the oneparticle reduced density matrix of the system defined by the natural orbitals in the larger one-particle basis $\left|\chi_{\alpha}\right\rangle$ sufficiently well, the Coulomb interaction does not have to be modified. The density-matrix functional can be evaluated with respect to the smaller set of one-particle states $\left|\tilde{\chi}_{\alpha}\right\rangle$ as

$$
F^{\hat{W}}\left[\rho^{(1), \chi}\right]=F^{\hat{W}}\left[\rho^{(1), \tilde{\chi}}\right]
$$





\section{Projector augmented-wave formalism}

The efficient numerical representation of the Kohn-Sham wave functions or natural orbitals on computers is a crucial ingredient in an efficient numerical solution of the Kohn-Sham equations or the direct minimization of the DFT-energy. We employ here the projector augmented-wave formalism (PAW, [Blöchl, 1994]). Even though the combination of density-functional theory and reduced density-matrix functional theory proposed in chapter 6 is independent of the underlying representation of the one-particle wave function, we describe the PAW-method here in detail, because the representation is relevant for the construction of the local one-particle reduced density matrix. The presentation of the PAW-method presented here closely follows the discussions in [Blöchl, 1994; Blöchl et al., 2002; Schade, 2012]. This chapter only deals with single-particle states, and all states and wave functions mentioned in this chapter are single-particle states. We will also occasionally refer to the Kohn-Sham states as natural orbitals.

\subsection{Transformation theory}

The main idea of the PAW-approach [Blöchl, 1994] is the construction of a linear transformation $\mathcal{T}$ that connects the physical one-particle wave functions $\left|\psi_{n}\right\rangle$ to smooth pseudo wave function $\left|\tilde{\psi}_{n}\right\rangle$ :

$$
\left|\psi_{n}\right\rangle=\mathcal{T}\left|\tilde{\psi}_{n}\right\rangle
$$

The transformation $\mathcal{T}$ is constructed as a sum of atom-local contributions

$$
\mathcal{T}=\mathbb{1}+\sum_{R} S_{R}
$$

where the summation index $R$ runs over all atoms. The local contributions $S_{R}$ are restricted to non-overlapping augmentation spheres $\left|\vec{r}-\vec{R}_{R}\right| \leq r_{\text {aug, } R}$ centered around the atomic position $\vec{R}_{R}$. The radius $r_{\text {aug, } R}$ of the sphere, that is also called the augmentation radius, depends on the chemical element. The local augmentation at atom $R$ is constructed such that the pseudo wave functions are smooth close to the atoms and such that the transformation reproduces the strongly oscillating physical wave functions. For this purpose, the contribution $S_{R}$ to $\mathcal{T}$ are defined as the differences of physical partial waves $\left|\phi_{R, i}\right\rangle$ and smooth pseudo partial waves $\left|\tilde{\phi}_{R, i}\right\rangle$ in the form

$$
S_{R}\left|\tilde{\phi}_{R, i}\right\rangle=\left|\phi_{R, i}\right\rangle-\left|\tilde{\phi}_{R, i}\right\rangle
$$

The physical partial waves $\left|\phi_{R, i}\right\rangle$ and the pseudo partial waves $\left|\tilde{\phi}_{R, i}\right\rangle$ are chosen identical outside the augmentation sphere of atom $R$. The physical partial waves are solutions of 
a one-particle equation that describes the electrons at the chosen level of theory, e.g., Schrödinger or Dirac equation, in spherical symmetry. The partial waves are represented as products of radial functions $\phi_{i}(r)$ and spherical harmonics $Y_{l, m}$ in the form ${ }^{1}$

$$
\left\langle\vec{r}, \sigma \mid \phi_{R, i}\right\rangle=\delta_{\sigma, \sigma^{\prime}} \phi_{i}\left(\left|\vec{r}-\vec{R}_{R}\right|\right) Y_{l, m}\left(\frac{\vec{r}-\vec{R}_{R}}{\left|\vec{r}-\vec{R}_{R}\right|}\right) .
$$

Thus, the index $i$ is an abbreviation of the combined indices $l, m, \sigma^{\prime}$ and $n$ of partial wave on atom $R$. $l$ denotes the angular momentum quantum number, $m$ the magnetic quantum numbers, $\sigma^{\prime}$ the quantum number of the spin and the index $n$ counts partial waves with same angular momentum and magnetic quantum number but different radial dependencies. The primary requirement for the pseudo partial waves $\left|\tilde{\phi}_{R, i}\right\rangle$ is that any relevant pseudo wave function $|\tilde{\psi}\rangle$ must be representable by them within the augmentation region of atom $R$, i.e. $c_{R, i} \in \mathcal{C}$ should exist with

$$
\langle\vec{r}, \sigma \mid \tilde{\psi}\rangle=\sum_{i} c_{R, i}\left\langle\vec{r}, \sigma \mid \tilde{\phi}_{R, i}\right\rangle
$$

for all $\left|\vec{r}-\vec{R}_{R}\right|<r_{a u g, R}$. Inserting the definition of the local contributions defined in Eq. (7.3) into Eq. (7.2), Eq. (7.1) gives together with Eq. (7.5) the relation

$$
\left|\psi_{n}\right\rangle=\left|\tilde{\psi}_{n}\right\rangle+\sum_{R, i}\left(\left|\phi_{R, i}\right\rangle-\left|\tilde{\phi}_{R, i}\right\rangle\right) c_{R, i}
$$

The coefficients $c_{R, i}$ represent the character of the wave function $\left|\psi_{n}\right\rangle$ in terms of the partial waves $\left|\phi_{R, i}\right\rangle$. The coefficients can be written as scalar products of projector states $\left|\tilde{p}_{R, i}\right\rangle$ with the pseudo wave function $\left|\tilde{\psi}_{n}\right\rangle$, i.e.,

$$
c_{R, i}=\left\langle\tilde{p}_{R, i} \mid \tilde{\psi}_{n}\right\rangle
$$

The projector states have to obey the biorthogonality relation

$$
\left\langle\tilde{p}_{R, i} \mid \tilde{\phi}_{R, j}\right\rangle=\delta_{i, j} \forall i, j, R
$$

and can be constructed as

$$
\left\langle\tilde{p}_{i, R}\right|=\sum_{j} M_{R, i, j}^{-1}\left\langle f_{R, j}\right|
$$

where $\left|f_{R, i}\right\rangle$ are some single-particle states and $M_{R, i, j}=\left\langle f_{R, i} \mid \tilde{\phi}_{R, j}\right\rangle$. We use the construction of the partial waves and projector states that was proposed by Peter Blöchl and is documented in detail in chapter 4 of [Schade, 2012]. The PAW-transformation can be written with the projector states as

$$
\left|\psi_{n}\right\rangle=\left|\tilde{\psi}_{n}\right\rangle+\sum_{R, i}\left(\left|\phi_{R, i}\right\rangle-\left|\tilde{\phi}_{R, i}\right\rangle\right)\left\langle\tilde{p}_{R, i} \mid \tilde{\psi}_{n}\right\rangle
$$

\footnotetext{
${ }^{1}$ The CP-PAW code used in this thesis employs real spherical harmonics $S_{l, m}$ instead of the complex spherical harmonics $Y_{l, m}$.
} 
This transformation is applied to the one-particle wave functions of valence states. The one-particle states that correspond to core states are transformed by assigning exactly one partial wave to every state, i.e.

$$
\left|\psi_{n}^{c}\right\rangle=\left|\tilde{\psi}_{n}^{c}\right\rangle+\left|\phi^{c}\right\rangle-\left|\tilde{\phi}^{c}\right\rangle .
$$

Within the frozen-core approximation, the core states of the isolated atom are used as the partial waves of the core states and their occupations are assumed to be one. We use the frozen-core approximation in all calculations included in this thesis.

Expectation values of local one-particle operators like the electron density can be obtained from the natural orbitals $\left|\psi_{n}\right\rangle$ and occupations $f_{n}$ as

$$
\langle A\rangle=\sum_{n} f_{n}\left\langle\psi_{n}|\hat{A}| \psi_{n}\right\rangle+\sum_{n \in \text { core }}\left\langle\psi_{n}^{c}|\hat{A}| \psi_{n}^{c}\right\rangle .
$$

To express the expectation value in terms of the pseudo wave functions, the operator can be transformed with the PAW-transformation $\mathcal{T}$ and approximated by

$$
\begin{aligned}
\hat{\tilde{A}} & =\mathcal{T}^{\dagger} \hat{A} \mathcal{T}=\hat{A}+\sum_{R, i, j}\left|\tilde{p}_{R, i}\right\rangle\left(\left\langle\phi_{R, i}|\hat{A}| \phi_{R, j}\right\rangle-\left\langle\tilde{\phi}_{R, i}|\hat{A}| \tilde{\phi}_{R, j}\right\rangle\right)\left\langle\tilde{p}_{R, j}\right| \\
& =\hat{A}+\sum_{R}\left(\hat{A}_{R}^{1}-\hat{\tilde{A}}_{R}^{1}\right) .
\end{aligned}
$$

$\hat{A}_{R}^{1}$ and $\hat{\tilde{A}}_{R}^{1}$ are the one-center contributions to the operator. Thus, the expectation value can finally be expressed as

$$
\langle\hat{A}\rangle=\sum_{n} f_{n}\left\langle\tilde{\psi}_{n}|\hat{A}| \tilde{\psi}_{n}\right\rangle+\sum_{R} D_{R, i, j}\left(A_{R, i, j}-\tilde{A}_{R, i, j}\right)+\sum_{n \in \text { core }} A_{n}^{c},
$$

with

$$
\begin{aligned}
D_{R, i, j} & =\sum_{n} f_{n}\left\langle\tilde{\psi}_{n} \mid \tilde{p}_{R, i}\right\rangle\left\langle\tilde{p}_{R, j} \mid \tilde{\psi}_{n}\right\rangle \\
A_{R, i, j} & =\left\langle\phi_{R, i}|\hat{A}| \phi_{R, i}\right\rangle \\
\tilde{A}_{R, i, j} & =\left\langle\tilde{\phi}_{R, i}|\hat{A}| \tilde{\phi}_{R, i}\right\rangle \\
A_{n}^{c} & =\left\langle\psi_{n}^{c}|\hat{A}| \psi_{n}^{c}\right\rangle .
\end{aligned}
$$

$A_{R, i, j}, \tilde{A}_{R, i, j}$ and $A_{n}^{c}$ can be evaluated on a radial grid because the partial waves and the core states are available as products of radial function and spherical harmonics in the form of Eq. (7.4). The elements of the one-center density matrix $D_{R, i, j}$ can be evaluated in the natural representation of the pseudo wave functions $\left|\tilde{\psi}_{n}\right\rangle$. In the CP-PAW code, plane waves are used as a basis for the pseudo wave functions, i.e.

$$
\left\langle\vec{r} \mid \tilde{\psi}_{n}\right\rangle=\sum_{\vec{G}} c_{n, \vec{G}} e^{i \vec{G} \cdot \vec{r}}
$$

Thus, the complex coefficients $c_{n, \vec{G}}$ are the actual variational parameters in any minimization problem over the one-particle wave function $\left|\psi_{n}\right\rangle$. The application of Eq. (7.15) to the electron density

$$
n(\vec{r})=\sum_{n} f_{n}\left\langle\psi_{n} \mid \vec{r}\right\rangle\left\langle\vec{r} \mid \psi_{n}\right\rangle+\sum_{n \in \text { core }}\left\langle\phi_{n}^{c} \mid \vec{r}\right\rangle\left\langle\vec{r} \mid \phi_{n}^{c}\right\rangle
$$


yields the decomposition

$$
\begin{aligned}
n(\vec{r}) & =\tilde{n}(\vec{r})+\sum_{R}\left(n_{R}^{1}(\vec{r})-\tilde{n}_{R}^{1}(\vec{r})\right) \\
\tilde{n}(\vec{r}) & =\sum_{n} f_{n}\left\langle\tilde{\psi}_{n} \mid \vec{r}\right\rangle\left\langle\vec{r} \mid \tilde{\psi}_{n}\right\rangle+\sum_{n \in \text { core }}\left\langle\tilde{\psi}_{n}^{c} \mid \vec{r}\right\rangle\left\langle\vec{r} \mid \tilde{\psi}_{n}^{c}\right\rangle \\
n_{R}^{1}(\vec{r}) & =\sum_{i, j} D_{R, i, j}\left\langle\phi_{R, i} \mid \vec{r}\right\rangle\left\langle\vec{r} \mid \phi_{R, j}\right\rangle+\sum_{n \in \text { core of } R}\left\langle\phi_{n}^{c} \mid \vec{r}\right\rangle\left\langle\vec{r} \mid \phi_{n}^{c}\right\rangle \\
\tilde{n}_{R}^{1}(\vec{r}) & =\sum_{i, j} D_{R, i, j}\left\langle\tilde{\phi}_{R, i} \mid \vec{r}\right\rangle\left\langle\vec{r} \mid \tilde{\phi}_{R, j}\right\rangle+\sum_{n \in \text { core of } R}\left\langle\tilde{\phi}_{n}^{c} \mid \vec{r}\right\rangle\left\langle\vec{r} \mid \tilde{\phi}_{n}^{c}\right\rangle .
\end{aligned}
$$

For an arrangement of atoms at positions $R$ with the atomic charge densities $Z_{R}(\vec{r})$, the total DFT-energy given in Eq. (4.50) can be expressed in this fashion as

$$
\begin{aligned}
E_{\mathrm{DFT}}\left(\left\{f_{n}\right\},\left\{\left|\tilde{\psi}_{n}\right\rangle\right\}\right) & =\tilde{E}_{\mathrm{DFT}}\left(\left\{f_{n}\right\},\left\{\left|\tilde{\psi}_{n}\right\rangle\right\}\right) \\
& +\sum_{R}\left(E_{\mathrm{DFT}, R}^{1}\left(\left\{f_{n}\right\},\left\{\left|\tilde{\psi}_{n}\right\rangle\right\}\right)-\tilde{E}_{\mathrm{DFT}, R}^{1}\left(\left\{f_{n}\right\},\left\{\left|\tilde{\psi}_{n}\right\rangle\right\}\right)\right) .
\end{aligned}
$$

The contribution $\tilde{E}_{\mathrm{DFT}}\left(\left\{f_{n}\right\},\left\{\left|\tilde{\psi}_{n}\right\rangle\right\}\right)$ stems from the first term in Eq. (7.15) and has the form

$$
\begin{aligned}
\tilde{E}_{\mathrm{DFT}}\left(\left\{f_{n}\right\},\left\{\left|\tilde{\psi}_{n}\right\rangle\right\}\right) & =\sum_{n}\left\langle\tilde{\psi}_{n}\left|-\frac{\hbar^{2} \nabla^{2}}{2 m}\right| \tilde{\psi}_{n}\right\rangle+\int d^{3} \vec{r} \int d^{3} \vec{r}^{\prime} \frac{e^{2}(\tilde{n}(\vec{r})+\tilde{Z}(\vec{r}))\left(\tilde{n}\left(\vec{r}^{\prime}\right)+\tilde{Z}(\vec{r})\right)}{8 \pi \epsilon_{0}\left|\vec{r}-\vec{r}^{\prime}\right|} \\
& +\int d^{3} \vec{r} \tilde{n}(\vec{r}) \epsilon_{x c}(\vec{r}, \tilde{n})+\int d^{3} \vec{r} \tilde{v}(\vec{r}) \tilde{n}(\vec{r}) .
\end{aligned}
$$

The compensation charge density $\tilde{Z}_{R}(\vec{r})$ is a smooth localized charge density and constructed such that the electrostatic multipole moments of the density

$$
n_{R}^{1}(\vec{r})+Z_{R}(\vec{r})-\tilde{n}_{R}^{1}(\vec{r})-\tilde{Z}_{R}(\vec{r})
$$

vanish. The potential $\bar{v}(\vec{r})$ is constructed such that the Hartree and exchange-correlation potential of the pseudo charge density are smoothened. This is possible because the expectation value of an operator $\hat{C}$ of the form

$$
\hat{C}=\hat{B}-\sum_{R, i, j}\left|\tilde{p}_{R, i}\right\rangle\left\langle\tilde{\phi}_{R, i}|\hat{B}| \tilde{\phi}_{R, j}\right\rangle\left\langle\tilde{p}_{R, j}\right|
$$

with respect to the pseudo wave functions vanishes, i.e. $\langle\tilde{\psi}|\hat{C}| \tilde{\psi}\rangle=0$. Here, $\hat{B}$ is an operator that is localized within the augmentation regions. The potential $\bar{v}$ is such an 
operator. The one-center contributions to the energy can be written as

$$
\begin{aligned}
E_{\mathrm{DFT}, \mathrm{R}}^{1}\left(\left\{f_{n}\right\},\left\{\left|\tilde{\psi}_{n}\right\rangle\right\}\right) & =\sum_{i, j} D_{i, j}\left\langle\phi_{R, i}\left|-\frac{\hbar^{2} \nabla^{2}}{2 m}\right| \phi_{R, j}\right\rangle+\sum_{n \in \text { core of } R}\left\langle\phi_{n}^{c}\left|-\frac{\hbar^{2} \nabla^{2}}{2 m}\right| \phi_{n}^{c}\right\rangle \\
& +\int d^{3} \vec{r} \int d^{3} \vec{r}^{\prime} \frac{e^{2}\left(n_{R}^{1}(\vec{r})+Z_{R}(\vec{r})\right)\left(\tilde{n}_{R}^{1}\left(\vec{r}^{\prime}\right)+Z_{R}(\vec{r})\right)}{8 \pi \epsilon_{0}\left|\vec{r}-\vec{r}^{\prime}\right|} \\
& +\int d^{3} \vec{r} n^{1}(\vec{r}) \epsilon_{x c}\left(\vec{r}, n^{1}\right) \\
\tilde{E}_{\mathrm{DFT}, \mathrm{R}}^{1}\left(\left\{f_{n}\right\},\left\{\left|\tilde{\psi}_{n}\right\rangle\right\}\right) & =\sum_{i, j} D_{i, j}\left\langle\tilde{\phi}_{R, i}\left|-\frac{\hbar^{2} \nabla^{2}}{2 m}\right| \tilde{\phi}_{R, j}\right\rangle+\sum_{n \in \operatorname{core} \text { of } R}\left\langle\tilde{\phi}_{n}^{c}\left|-\frac{\hbar^{2} \nabla^{2}}{2 m}\right| \tilde{\phi}_{n}^{c}\right\rangle \\
& +\int d^{3} \vec{r} \int d^{3} \vec{r}^{\prime} \frac{e^{2}\left(\tilde{n}_{R}^{1}(\vec{r})+\tilde{Z}_{R}(\vec{r})\right)\left(\tilde{n}_{R}^{1}\left(\vec{r}^{\prime}\right)+\tilde{Z}_{R}(\vec{r})\right)}{8 \pi \epsilon_{0}\left|\vec{r}-\vec{r}^{\prime}\right|} \\
& +\int d^{3} \vec{r} \tilde{n}^{1}(\vec{r}) \epsilon_{x c}\left(\vec{r}, \tilde{n}^{1}\right)+\int d^{3} \vec{r} \tilde{v}(\vec{r}) \tilde{n}^{1}(\vec{r}) .
\end{aligned}
$$

The Kohn-Sham projected densities of states (see also Eq. (2.156)) are estimated in the CP-PAW code as

$$
D_{R, i, j}(\epsilon)=\sum_{n}\left\langle\tilde{p}_{R, i} \mid \tilde{\psi}_{n}\right\rangle \delta\left(\epsilon-\epsilon_{n}\right)\left\langle\tilde{\psi}_{n} \mid \tilde{p}_{R, j}\right\rangle
$$

from the scalar products $\left\langle\tilde{p}_{R, i} \mid \tilde{\psi}_{n}\right\rangle$. The $\epsilon_{n}$ denote the Kohn-Sham energy eigenvalues. The projectors $\left|\tilde{p}_{R, j}\right\rangle$ are scaled so that the required normalization conditions are fulfilled. The tetrahedron method of Blöchl et al. [Blöchl et al., 1994] is used to improve the Brillouin-zone integrations.

\subsection{Energy minimization}

With the expressions in Eq. (7.26), Eq. (7.27), Eq. (7.30) and Eq. (7.31), the DFT-energy can be minimized over the plane-wave coefficients of the pseudo wave functions to obtain the DFT-ground state. The minimization of the total energy is performed in CP-PAW with a Car-Parrinello-like constrained minimization [Car and Parrinello, 1985; Verlet, 1967; Ryckaert et al., 1977] because the natural orbitals have to be kept orthonormal, $\left\langle\psi_{n} \mid \psi_{m}\right\rangle=\delta_{i, j}$ and the particle-number constraint, $\sum_{i} f_{i}=N$, has to be fulfilled. The Car-Parrinello-like procedure is outlined in section 4.6.

\subsection{One-particle reduced density matrix}

\subsubsection{New one-particle basis}

In order to use the PAW-transformation together with reduced density-matrix functional theory the one-particle reduced density matrix has to be available. The natural orbitals $\left|\psi_{n}\right\rangle$ and occupations $f_{n}$ define the one-particle reduced density matrix

$$
\rho_{i, j}^{(1)}=f_{i} \delta_{i, j}
$$


in the one-particle basis of the natural orbitals. Even though the one-particle reduced density matrix is very easily expressed is the natural-orbital basis, the numerical evaluation of matrix elements of the electron-electron interaction

$$
U_{\alpha, \beta, \gamma, \delta}=\int d^{4} \vec{x} \int d^{4} \vec{x}^{\prime} \frac{e^{2}}{4 \pi \epsilon_{0}\left|\vec{r}-\vec{r}^{\prime}\right|} \psi_{\alpha}^{*}(\vec{x}) \psi_{\beta}^{*}\left(\vec{x}^{\prime}\right) \psi_{\gamma}(\vec{x}) \psi_{\delta}\left(\vec{x}^{\prime}\right)
$$

is extremely cumbersome. An additional complication arises from the fact that the natural orbitals change during the minimization of the total energy. To circumvent this problem, we derive an approximation of the natural orbitals for which we can evaluate the matrix elements of the interaction in a numerically convenient way. We choose a new one-particle basis with basis states of the general form

$$
\left\langle\vec{r} \mid \chi_{\alpha}\right\rangle=\sum_{i} c_{\alpha, i} \chi_{\alpha, i}\left(\left|\vec{r}-\vec{R}_{\alpha, i}\right|\right) S_{l_{\alpha, i}, m_{\alpha, i}}\left(\frac{\vec{r}-\vec{R}_{\alpha, i}}{\left|\vec{r}-\vec{R}_{\alpha, i}\right|}\right) .
$$

The basis state $\left|\chi_{\alpha}\right\rangle$ with index $\alpha$ is composed of individual contributions that have the radial behaviours $\chi_{\alpha, i}(r)$, the angular momenta $l_{\alpha, i}$, magnetic quantum numbers $m_{\alpha, i}$ and centers $\vec{R}_{\alpha, i}$. Please note, that we introduce one-particle basis states for the valence states as well as for the core states. We do not required that these basis states be pairwise orthonormal. The matrix elements of the interaction Hamiltonian can be evaluated conveniently by expanding the radial functions $\chi_{\alpha, i}(r)$ in terms of Gaussians, i.e.,

$$
\chi_{\alpha, i}(r)=\sum_{j} d_{\alpha, i, j} r^{l_{\alpha, i}} e^{-\eta_{j} r^{2}}
$$

Thus, the new one-particle basis states are represented as sums of spherical Gaussian-type orbitals (GTOs). We obtain the exponents $\eta_{j}$ of the expansion from spherical GTO-basis sets that are used in quantum chemistry. Although different basis sets can be easily imported into our implementation from the EMSL basis set exchange portal ${ }^{2}$, we usually use the cc-pwCV5Z basis set [Dunning, 1989b; Peterson and Dunning, 2002; Balabanov and Peterson, 2005]. For hydrogen, this basis set defines five contracted spherical GTOs for the s-states, four for the p-states, three for the d-states, two for the f-states and one for the g-state. We obtain the expansion exponents $\eta_{j}$ for the radial part $\chi_{\alpha, i}(r)$ of an s-state by collecting all exponents from the five contracted GTOs of s-states. This results in the eight expansion coefficients

$$
\begin{array}{r}
\eta_{1}=402, \eta_{2}=60.24, \eta_{3}=13.73, \eta_{4}=3.905, \\
\eta_{5}=1.283, \eta_{6}=0.4655, \eta_{7}=0.1811, \eta_{8}=0.07279 .
\end{array}
$$

We then fit the coefficients $d_{\alpha, i, j}$ such that

$$
\int_{0}^{\infty} d r\left|\sum_{j} d_{\alpha, i, j} r^{l_{\alpha, i}} e^{-\eta_{j} r^{2}}-\bar{\chi}_{\alpha, i}(r)\right|
$$

is minimized. $\bar{\chi}_{\alpha, i}(r)$ is the given radial part on a grid. In our case, $\bar{\chi}_{\alpha, i}(r)$ is an atomic orbital, i.e., the solution of the Kohn-Sham equations of the isolated atom. The quality

\footnotetext{
${ }^{2}$ https://bse.pnl.gov/bse/portal [Feller, 1996; Schuchardt et al., 2007]
} 
of the expansion exponents of the chosen GTO-basis set can be checked by inspecting the deviation of the resulting fit $\chi_{\alpha, i}(r)$ and the intended radial function $\bar{\chi}_{\alpha, i}(r)$.

With this expansion, matrix elements of the form

$$
\begin{aligned}
& \tilde{U}_{\alpha, i_{\alpha}, j_{\alpha}, \beta, i_{\beta}, j_{\beta}, \gamma, i_{\gamma}, j_{\gamma}, \delta, i_{\delta}, j_{\delta}}=\int d^{4} \vec{x} \int d^{4} \vec{x}^{\prime} \frac{e^{2}}{4 \pi \epsilon_{0}\left|\vec{r}-\vec{r}^{\prime}\right|} \\
& \cdot\left|\vec{r}-\vec{R}_{\gamma, i_{\gamma}}\right|^{l_{\gamma, i_{\gamma}}}\left|\vec{r}^{\prime}-\vec{R}_{\delta, i_{\delta}}\right|^{l_{\delta, i_{\delta}}}\left|\vec{r}-\vec{R}_{\gamma, i_{\gamma}}\right|^{l_{\gamma, i_{\gamma}}}\left|\vec{r}^{\prime}-\vec{R}_{\delta, i_{\delta}}\right|^{l_{\delta, i_{\delta}}} \\
& \cdot e^{-\eta_{j_{\alpha}}\left|\vec{r}-\vec{R}_{\alpha, i_{\alpha}}\right|^{2}} e^{-\eta_{j_{\beta}}\left|\vec{r}^{\prime}-\vec{R}_{\beta, i_{\beta}}\right|^{2}} e^{-\eta_{j_{\gamma}}\left|\vec{r}-\vec{R}_{\gamma, i_{\gamma}}\right|^{2}} e^{-\eta_{j_{\delta}}\left|\vec{r}^{\prime}-\vec{R}_{\delta, i_{\delta}}\right|^{2}} \\
& \cdot S_{l_{\alpha, i}, m_{\alpha, i}}\left(\frac{\vec{r}-\vec{R}_{\alpha, i}}{\left|\vec{r}-\vec{R}_{\alpha, i}\right|}\right) S_{l_{\beta, i}, m_{\beta, i}}\left(\frac{\vec{r}^{\prime}-\vec{R}_{\beta, i}}{\left|\vec{r}^{\prime}-\vec{R}_{\beta, i}\right|}\right) \\
& \cdot S_{l_{\gamma, i}, m_{\gamma, i}}\left(\frac{\vec{r}-\vec{R}_{\gamma, i}}{\left|\vec{r}-\vec{R}_{\gamma, i}\right|}\right) S_{l_{\delta, i}, m_{\delta, i}}\left(\frac{\vec{r}^{\prime}-\vec{R}_{\delta, i}}{\left|\vec{r}^{\prime}-\vec{R}_{\delta, i}\right|}\right)
\end{aligned}
$$

have to be computed. The evaluation of these matrix elements is a standard task in quantum chemistry and efficient implementations are available for this purpose. We use the library libcint of Qiming Sun [Sun, 2015] $]^{3}$. This library also supports matrix elements of the kinetic energy or the electron-nucleus interaction as well as derivatives of the matrix elements. The matrix elements $\tilde{U}_{\alpha, i_{\alpha}, j_{\alpha}, \beta, i_{\beta}, j_{\beta}, \gamma, i_{\gamma}, j_{\gamma}, \delta, i_{\delta}, j_{\delta}}$ can then be combined to yield the matrix elements

$$
U_{\alpha, \beta, \gamma, \delta}=\int d^{4} \vec{x} \int d^{4} \vec{x}^{\prime} \frac{e^{2}}{4 \pi \epsilon_{0}\left|\vec{r}-\vec{r}^{\prime}\right|} \chi_{\alpha}^{*}(\vec{x}) \chi_{\beta}^{*}\left(\vec{x}^{\prime}\right) \chi_{\gamma}(\vec{x}) \chi_{\delta}\left(\vec{x}^{\prime}\right) .
$$

The evaluation of matrix elements for modified Coulomb kernels is discussed in chapter 6 .

\subsubsection{Projection construction}

Of particular importance for the application of density-matrix functional theory (see section 5) is that the one-particle reduced density matrix, for which the density-matrix functional is to be evaluated, is ensemble N-representable. Ensemble N-representability of the one-particle reduced density matrix is not required for the DFT $+\mathrm{U}$ method or local hybrid functionals because a Hartree-Fock-like exchange energy functional can be evaluated for unphysical non-N-representable one-particle reduced density matrices. Let's assume that one of the occupations of the local density matrix is $f_{i}=\tilde{f}_{i}+\Delta$ with $\tilde{f}_{i}<1$ and $\Delta>0$. The deviation of the density-matrix functional in the Hartree-Fock approximation for this unphysical density matrix compared to the corresponding density matrix with $f_{i}=\tilde{f}_{i}$ is at most quadratic in $\Delta$. Thus, the deviation of the force on the occupation, that is, the derivative of the deviation with respect $\Delta$, is at most linear. In contrast the derivative of the exact density-matrix functional with respect to $\Delta$ usually diverges when approaching $\tilde{f}_{i}+\Delta=1$. Additionally, the exact density-matrix functional is not defined for $f_{i}>1$. As N-representability is not required for DFT $+\mathrm{U}$ or local hybrid functionals, very little attention had been paid to this aspect prior to the work of this thesis. The construction of a one-particle reduced density matrix that is guaranteed to be N-representable was one of the challenges of this thesis.

\footnotetext{
${ }^{3}$ The library libcint is available at https://github.com/sunqm/libcint.
} 
The goal of this section is a practical construction of the one-particle reduced density matrix

$$
\tilde{\rho}_{\beta, \alpha}^{(1), \chi}=\sum_{n} f_{n}\left\langle\chi_{\alpha} \mid \psi_{n}\right\rangle\left\langle\psi_{n} \mid \chi_{\beta}\right\rangle
$$

We construct this one-particle reduced density-matrix by projecting the natural orbitals onto a new one-particle basis composed of local one-particle states. The natural orbitals $\left|\psi_{n}\right\rangle$ are represented in the PAW-decomposition given in Eq. (7.10). For the construction of the one-particle reduced density matrix in terms of the one-particle basis defined in Eq. (7.35) that is guaranteed to be ensemble N-representable we first obtain a representation of the natural orbitals $\left|\psi_{n}\right\rangle$ in terms of the new one-particle basis. For this purpose, we explicitly consider the periodicity of the system and restrict the centers $\vec{R}_{\alpha, i}$ in Eq. (7.35) to the unit cell. The additional index $\vec{T}$ represents a translation vector of the lattice, and we define

$$
\left\langle\vec{r}+\vec{T} \mid \chi_{\alpha, \vec{T}}\right\rangle=\left\langle\vec{r} \mid \chi_{\alpha, \overrightarrow{0}}\right\rangle=\left\langle\vec{r} \mid \chi_{\alpha}\right\rangle
$$

Due to the lattice periodicity the natural orbitals are Bloch states and obey the Bloch theorem

$$
\left\langle\vec{r} \mid \psi_{n}(\vec{k})\right\rangle=u_{\vec{k}}(\vec{r}) e^{i \vec{k} \cdot \vec{r}}
$$

where $u_{\vec{k}}(\vec{r})$ is a lattice-periodic function, i.e. $u_{\vec{k}}(\vec{r}+\vec{T})=u_{\vec{k}}(\vec{r})$ with a lattice-translation vector $\vec{T}$. We adopt here the formalism and notation of Maintz et al. [Maintz et al., 2013]. We have to obtain the one-particle states

$$
\left|\mu_{n}(\vec{k})\right\rangle=\sum_{\alpha, \vec{T}} C_{\alpha, \vec{T}, n}(\vec{k})\left|\chi_{\alpha, \vec{T}}\right\rangle
$$

such that

$$
\left|\psi_{n}(\vec{k})\right\rangle \approx\left|\mu_{n}(\vec{k})\right\rangle
$$

For a small new one-particle basis $\left|\chi_{\alpha, \vec{T}}\right\rangle$ the requirement of Eq. (7.46) will only be fulfilled approximately. The new one-particle basis has to be chosen so that the difference of $\left|\psi_{n}(\vec{k})\right\rangle$ and $\left|\mu_{n}(\vec{k})\right\rangle$ for the physically relevant one-particle states is small. We will show later that one of the advantages of the projection formalism presented here is that it allows us to quantify and judge the quality of the new one-particle basis. With the PAW-transformation in Eq. (7.10), we have the representation

$$
\left|\psi_{n}(\vec{k})\right\rangle=\left|\tilde{\psi}_{n}(\vec{k})\right\rangle+\sum_{R, i, \vec{T}}\left(\left|\phi_{R, i, \vec{T}}\right\rangle-\left|\tilde{\phi}_{R, i, \vec{T}}\right\rangle\right)\left\langle\tilde{p}_{R, i, \vec{T}} \mid \tilde{\psi}_{n}(\vec{k})\right\rangle
$$

for a natural orbital. The sum over $R$ goes over all atoms in the unit cell and the sum over the index $i$ over all partial waves that belong to atom $R$. We form Bloch states of the new one-particle basis states

$$
\left|\chi_{\alpha}(\vec{k})\right\rangle=\frac{1}{\sqrt{N_{\vec{T}}}} \sum_{\vec{T}}\left|\chi_{\alpha, \vec{T}}\right\rangle e^{i \vec{k} \cdot \vec{T}} .
$$


and express $\left|\mu_{n}(\vec{k})\right\rangle$ of Eq. (7.45) as

$$
\left|\mu_{n}(\vec{k})\right\rangle=\sum_{\alpha} B_{\alpha, n}(\vec{k})\left|\chi_{\alpha}(\vec{k})\right\rangle
$$

The coefficients $B_{\alpha, n}(\vec{k})$ are related to $C_{\alpha, \vec{T}, n}$ of Eq. (7.45) by

$$
C_{\alpha, \vec{T}, n}(\vec{k})=\frac{1}{\sqrt{N_{\vec{T}}}} e^{i \vec{k} \cdot \vec{T}} B_{\alpha, n}(\vec{k})
$$

The scalar products of the Bloch states $\left|\chi_{\alpha}(\vec{k})\right\rangle$ of the one-particle basis and the states $\left|\mu_{n}(\vec{k})\right\rangle$ are simply

$$
\left\langle\chi_{\alpha}(\vec{k}) \mid \mu_{n}(\vec{k})\right\rangle=\sum_{\beta} S_{\alpha, \beta}(\vec{k}) B_{\beta, n}(\vec{k})
$$

where $S_{\alpha, \beta}=\left\langle\chi_{\alpha}(\vec{k})\right| \chi_{\beta}(\vec{k})$ denotes the overlap matrix of the Bloch states. These overlaps can be obtained from the overlaps of the new one-particle basis

$$
S_{\alpha, \overrightarrow{0}, \beta, \vec{T}}=\left\langle\chi_{\alpha, \overrightarrow{0}} \mid \chi_{\beta, \vec{T}}\right\rangle
$$

with

$$
S_{\alpha, \beta}(\vec{k})=\sum_{\vec{T}} e^{i \vec{k} \cdot \vec{T}} S_{\alpha, \overrightarrow{0}, \beta, \vec{T}}
$$

The overlaps $S_{\alpha, \overrightarrow{0}, \beta, \vec{T}}$ can be evaluated easily, because the states are defined in Eq. (7.35) as spherical GTOs. Even though Gaussians don't have a compact support, the sum can be truncated for large translations vectors $\vec{T}$, because long-range overlaps become negligible. We define the scalar products $T_{\alpha, n}(\vec{k})$ of the Bloch states $\left|\chi_{\alpha}(\vec{k})\right\rangle$ of the one-particle basis and the natural orbitals as

$$
T_{\alpha, n}(\vec{k})=\left\langle\chi_{\alpha}(\vec{k}) \mid \psi_{n}(\vec{k})\right\rangle
$$

Assuming the identity $\left|\mu_{n}(\vec{k})\right\rangle=\left|\psi_{n}(\vec{k})\right\rangle$, we have

$$
T_{\alpha, n}(\vec{k})=\sum_{\beta} S_{\alpha, \beta}(\vec{k}) B_{\beta, n}(\vec{k}) .
$$

Thus, we have the matrix identity

$$
T(\vec{k})=S(\vec{k}) B(\vec{k})
$$

The elements of $T$ are

$$
\begin{aligned}
T_{\alpha, n}(\vec{k}) & =\left\langle\chi_{\alpha}(\vec{k}) \mid \psi_{n}(\vec{k})\right\rangle \\
& =\left\langle\chi_{\alpha}(\vec{k}) \mid \tilde{\psi}_{n}(\vec{k})\right\rangle+\sum_{R, i, \vec{T}}\left(\left\langle\chi_{\alpha}(\vec{k}) \mid \phi_{R, i, \vec{T}}\right\rangle-\left\langle\chi_{\alpha}(\vec{k}) \mid \tilde{\phi}_{R, i, \vec{T}}\right\rangle\right)\left\langle\tilde{p}_{R, i, \vec{T}} \mid \tilde{\psi}_{n}(\vec{k})\right\rangle,
\end{aligned}
$$


where the scalar products $\left\langle\tilde{p}_{R, i, \vec{T}} \mid \tilde{\psi}_{n}(\vec{k})\right\rangle$ are already known. We split up $T_{\alpha, n}(\vec{k})$ into two terms:

$$
\begin{aligned}
T_{\alpha, n}(\vec{k}) & =T_{\alpha, n}^{\mathrm{PS}}(\vec{k})+T_{\alpha, n}^{\mathrm{AUG}}(\vec{k}) \\
T_{\alpha, n}^{\mathrm{PS}}(\vec{k}) & =\left\langle\chi_{\alpha}(\vec{k}) \mid \tilde{\psi}_{n}(\vec{k})\right\rangle \\
T_{\alpha, n}^{\mathrm{AUG}}(\vec{k}) & =\sum_{R, i, \vec{T}}\left(\left\langle\chi_{\alpha}(\vec{k}) \mid \phi_{R, i, \vec{T}}\right\rangle-\left\langle\chi_{\alpha}(\vec{k}) \mid \tilde{\phi}_{R, i, \vec{T}}\right\rangle\right)\left\langle\tilde{p}_{R, i, \vec{T}} \mid \tilde{\psi}_{n}(\vec{k})\right\rangle .
\end{aligned}
$$

The direct contribution $T_{\alpha, n}^{\mathrm{PS}}(\vec{k})$ from the pseudo wave function $\left|\tilde{\psi}_{n}(\vec{k})\right\rangle$ can be evaluated in the plane-wave basis with a Bessel transformation of the one-particle basis state $\left|\chi_{\alpha}(\vec{k})\right\rangle$. The contribution $T_{\alpha, n}^{\mathrm{AUG}}(\vec{k})$ from the augmentation requires the overlaps

$$
\left\langle\chi _ { \alpha } ( \vec { k } ) \left|\left(\left|\phi_{R, i, \vec{T}}\right\rangle-\left|\tilde{\phi}_{R, i, \vec{T}}\right\rangle\right)\right.\right.
$$

To evaluate these quantities we first construct the matrix $O_{\alpha, \vec{T}^{\prime}, R, i, \vec{T}}^{\mathrm{AUG}}$ of overlaps between the new one-particle basis and partial waves

$$
\begin{aligned}
O_{\alpha, \vec{T}^{\prime}, R, i, \vec{T}}^{\mathrm{AUG}} & =\left\langle\chi_{\alpha, \vec{T}^{\prime}} \mid \phi_{R, i, \vec{T}}\right\rangle-\left\langle\chi_{\alpha, \vec{T}^{\prime}} \mid \tilde{\phi}_{R, i, \vec{T}}\right\rangle \\
& =\int_{S_{R}} d^{3} \vec{r}\left\langle\chi_{\alpha, \vec{T}^{\prime}} \mid \vec{r}\right\rangle\left(\left\langle\vec{r} \mid \phi_{R, i, \vec{T}}\right\rangle-\left\langle\vec{r} \mid \tilde{\phi}_{R, i, \vec{T}}\right\rangle\right) .
\end{aligned}
$$

These real-space integrations have to be performed over the augmentation sphere of atom $R$. We use an adaptive three-dimensional integration routine [Berntsen et al., 1991] from the software package cubature ${ }^{4}$ for this purpose. The corresponding k-space overlaps are

$$
O_{\alpha, R, i}^{\mathrm{AUG}}(\vec{k})=\sum_{\vec{T}} e^{i \vec{k} \cdot \vec{T}} O_{\alpha, \overrightarrow{0}, R, i, \vec{T}}^{\mathrm{AUG}}
$$

The contribution from the augmentation can then be written as

$$
T_{\alpha, n}^{\mathrm{AUG}}(\vec{k})=\sum_{R, i}\left\langle\tilde{p}_{R, i} \mid \tilde{\psi}_{n}(\vec{k})\right\rangle O_{\alpha, R, i}^{\mathrm{AUG}}(\vec{k}) .
$$

The quality of the new one-particle basis set $\left|\chi_{\alpha}\right\rangle$ can be evaluated by computing the overlap of the states $\left|\mu_{n}(\vec{k})\right\rangle$

$$
\begin{aligned}
O_{n, n^{\prime}}(\vec{k}) & =\left\langle\mu_{n}(\vec{k}) \mid \mu_{n^{\prime}}(\vec{k})\right\rangle \\
O(\vec{k}) & =T(\vec{k})^{\dagger}\left(S^{-1}(\vec{k})\right)^{\dagger} T(\vec{k}) .
\end{aligned}
$$

The orthonormality of the natural orbitals $\left|\psi_{n}(\vec{k})\right\rangle$ would imply $O_{n, n^{\prime}}(\vec{k})=\delta_{n, n^{\prime}}$ if $\left|\psi_{n}(\vec{k})\right\rangle=\left|\mu_{n}(\vec{k})\right\rangle$. Thus, large deviations from $O_{n, n^{\prime}}(\vec{k})=\delta_{n, n^{\prime}}$ indicate that the chosen new one-particle basis is not suitable to represent the natural orbitals. If the overlap matrix $O(\vec{k})$ is sufficiently close to the unit matrix, we have obtained a faithful representation of the natural orbitals $\left|\psi_{n}(\vec{k})\right\rangle$ in terms of the new one-particle basis $\left|\chi_{\alpha, \vec{T}}\right\rangle$. This representation could also be used for other purposes, such as the evaluation of projected

\footnotetext{
${ }^{4}$ cubature 1.0.3 written by Steven G. Johnson https://github.com/stevengj/cubature
} 
densities of states or the derivation of tight-binding models. Here we use the representation of the natural orbitals in terms of the new one-particle basis in order to express the one-particle reduced density matrix of the system as

$$
\tilde{\rho}_{\alpha, \vec{T}, \beta, \vec{T}^{\prime}}^{(1), \chi}=\sum_{\vec{k}, n} f_{n}(\vec{k})\left\langle\chi_{\alpha, \vec{T}} \mid \mu_{n}(\vec{k})\right\rangle\left\langle\mu_{n}(\vec{k}) \mid \chi_{\beta, \vec{T}^{\prime}}\right\rangle
$$

The density-matrix $\tilde{\rho}^{\chi}$ has the periodicity of the lattice, i.e.,

$$
\tilde{\rho}_{\alpha, \vec{T}, \beta, \vec{T}}^{(1), \chi}=\tilde{\rho}_{\alpha, \overrightarrow{0}, \beta, \overrightarrow{0}}^{(1), \chi} \forall \vec{T} .
$$

Please note that $\left|\chi_{\alpha, \vec{T}}\right\rangle$ is not necessarily an orthonormal basis and hence the eigenvalues of $\tilde{\rho}^{(1), \chi}$ are not equal to the occupations. It can be transformed to a one-particle reduced density matrix in an orthonormal on-particle basis. The density matrix $\tilde{\rho}^{(1), \chi}$ has all information about the electronic structure of the system on the one-particle level.

\subsubsection{Local one-particle reduced density matrix}

Now we adopt a real-space view of the system. We can extract the local electronic structure in the vicinity of an atom at position $\vec{R}_{\text {center }}$ from the one-particle reduced density matrix. For this purpose, we span a sphere with radius $r_{\text {local }}$ around $\vec{R}_{\text {center }}$ and collect all one-particle basis states $\left|\chi_{\alpha, \vec{T}}\right\rangle$ with centers $\vec{R}_{\alpha}+\vec{T}$ in the sphere, i.e.

$$
\left|\vec{R}_{\alpha}+\vec{T}-\vec{R}_{\text {center }}\right|<r_{\text {local }} \text {. }
$$

This defines a set of pairs of basis state with indices $\alpha$ and translation vectors $\vec{T}$ which we reindex with $j$, such that the pair $\left(\alpha_{j}, \vec{T}_{j}\right)$ designates a one-particle state $\left|\chi_{\alpha_{j}, \vec{T}_{j}}\right\rangle$ with a center in the sphere. The sphere with radius $r_{\text {local }}$ centered at $\vec{R}_{\text {center }}$ might involve multiple unit cells. We then write the one-particle reduced density matrix of this local cluster as

$$
\tilde{\rho}_{j, j^{\prime}}^{(1), \chi}=\tilde{\rho}_{\alpha_{j}, \vec{T}_{j}, \alpha_{j^{\prime}}, \vec{T}_{j^{\prime}}}^{(1), \chi}
$$

and the corresponding overlap matrix of the one-particle states as

$$
\tilde{S}_{j, j^{\prime}}^{\chi}=\left\langle\chi_{\alpha_{j}, \vec{T}_{j}} \mid \chi_{\alpha_{j^{\prime}}, \vec{T}_{j^{\prime}}}\right\rangle .
$$

By increasing the radius of the sphere $r_{\text {local }}$ more and more long-range information of the electronic structure can be included in the local one-particle reduced density matrix. For practical purposes and to simplify the notation for the proof of N-representability in the following section, we transform the local one-particle states to an orthonormal basis. This orthonormalization gives the one-particle reduced density matrix $\rho_{j, j^{\prime}}^{(1), \chi}$ with respect to the orthonormal one-particle states.

\subsubsection{N-representability}

For the proof of N-representability of the local one-particle reduced density $\rho^{(1), \chi}$ constructed with the above projection scheme, we first introduce Cauchy's eigenvalue interlacing theorem ${ }^{5}$ : The theorem states that the eigenvalues of a principal submatrix

\footnotetext{
${ }^{5}$ Cauchy's eigenvalue interlacing theorem is sometimes called Poincaré separation theorem.
} 
$B \in \mathbb{C}^{(n-1) \times(n-1)}$ of a hermitian matrix $A \in \mathbb{C}^{n \times n}$ and the eigenvalues of $A$ interlace. This means that the ordered eigenvalues $\lambda_{1}^{B} \leq \ldots \leq \lambda_{n-1}^{B}$ of $B$ and the ordered eigenvalues $\lambda_{1}^{A} \leq \ldots \leq \lambda_{n}^{A}$ obey

$$
\lambda_{1}^{A} \leq \lambda_{1}^{B} \leq \ldots \leq \lambda_{n-1}^{B} \leq \lambda_{n}^{A}
$$

Thus, if $A$ is an N-representable one-particle reduced density, then also $B$ is a Nrepresentable one-particle reduced density matrix. The theorem also holds for the generalized eigenvalues $\lambda_{i}$ of generalized eigenvalue problems $A v_{i}=\lambda_{i} S v_{i}$ with a positive definite overlap matrix $S$. The construction of the local density matrix consists of the two steps where N-representability might be broken:

1. The first step is the construction of the density matrix $\tilde{\rho}_{\alpha, \vec{T}, \beta, \vec{T}}^{(1), \chi}$ in terms of the new one-particle basis.

2. The second step is the selection of one-particle basis states described at the beginning of this section.

We first prove that the second step preserves N-representability because this shows the application of Cauchy's eigenvalue interlacing theorem. The selection of basis states $\left|\chi_{\alpha_{j}, \vec{T}_{j}}\right\rangle$ from the full set $\left|\chi_{\alpha, \vec{T}}\right\rangle$ corresponds to a repeated application of Cauchy's eigenvalue interlacing theorem. We start from the density matrix $\tilde{\rho}_{\alpha, \vec{T}, \beta, \vec{T}}^{(1), \chi}$ in the full one-particle basis $\left|\chi_{\alpha, \vec{T}}\right\rangle$ and then remove the basis state $\left|\chi_{\gamma, \vec{t}}\right\rangle$. Thus, after a reordering of the basis states the density matrix $\tilde{\rho}_{\alpha \neq \beta, \vec{T} \neq \vec{t}, \beta \neq \gamma, \vec{T}^{\prime} \neq \vec{t}}^{(1), \chi}$ is a principal submatrix of $\tilde{\rho}_{\alpha, \vec{T}, \beta, \vec{T}^{\prime}}^{(1), \chi}$ and, hence, also N-representable. This reasoning can be repeated until only the selected basis states remain, which proves that the second step does not break N-representability.

To prove the conservation of N-representability of the first step we assume that the new one-particle basis $\left|\chi_{\alpha, \vec{T}}\right\rangle$ is not complete enough to represent a given set of natural orbitals $\left|\psi_{n}(\vec{k})\right\rangle$. Thus, we can introduce additional states $\left|\bar{\chi}_{\beta, \vec{T}}\right\rangle$ that are pairwise orthogonal to the basis states $\left|\chi_{\alpha, \vec{T}}\right\rangle$, and the combined basis can exactly represent the given natural orbitals. The eigenvalues of the one-particle reduced density matrix in the combined oneparticle basis are identical to the occupations $f_{i}$. Now we can apply Cauchy's eigenvalue interlacing theorem just as in the case of the second step. Here, we successively remove the basis states $\left|\bar{\chi}_{\beta, \vec{T}}\right\rangle$ from the combined basis till only the one-particle basis states $\left|\chi_{\alpha, \vec{T}}\right\rangle$ remain. This proves the N-representability of the one-particle reduced density matrix $\tilde{\rho}_{\alpha, \vec{T}, \beta, \vec{T}^{\prime}}^{(1), \chi}$. Please note, that the reasoning for the conservation of N-representability presented here only holds for countable one-particle basis sets. However, all basis set employed in numerical calculations have a finite dimension and are thus countable. 


\section{Adaptive cluster approximation}

\subsection{Introduction}

The density-matrix functionals $F_{\beta}^{\hat{W}_{R}}\left[\rho^{(1)}\right]$ that have to be evaluated for the local approximation of the density-matrix functional defined in Eq. (5.34) are computationally nearly as hard as the exact functional $F_{\beta}^{\hat{W}}\left[\rho^{(1)}\right]$. Thus, if the functionals $F_{\beta}^{\hat{W}_{R}}\left[\rho^{(1)}\right]$ are evaluated naively like one would evaluate the exact functional $F_{\beta}^{\hat{W}}\left[\rho^{(1)}\right]$, nothing has been gained by the local approximation of the density-matrix functional. Thus, a way to reduce the number of non-interacting one-particle states in $F_{\beta}^{\hat{W}_{R}}\left[\rho^{(1)}\right]$ is required. One way would be to neglect certain one-particle states from the one-particle reduced density matrix. However, this kind of cluster approximation leads to erratic results depending on the one-particle reduced density matrix and the criterion to select the one-particle states to neglect. Consider the pathological case of a star-like topology of the one-particle reduced density matrix

$$
\rho_{\text {star }}^{(1)}=\left(\begin{array}{cccccccc}
1 / 2 & 0 & a & 0 & a & 0 & a & 0 \\
0 & 1 / 2 & 0 & a & 0 & a & 0 & a \\
a & 0 & 1 / 2 & 0 & 0 & 0 & 0 & 0 \\
0 & a & 0 & 1 / 2 & 0 & 0 & 0 & 0 \\
a & 0 & 0 & 0 & 1 / 2 & 0 & 0 & 0 \\
0 & a & 0 & 0 & 0 & 1 / 2 & 0 & 0 \\
a & 0 & 0 & 0 & 0 & 0 & 1 / 2 & 0 \\
0 & a & 0 & 0 & 0 & 0 & 0 & 1 / 2
\end{array}\right)
$$

and an interaction on the first two one-particle states. There is no reasonable criterion to neglect one of the non-interacting one-particle states because all are related to the first and second one-particle state in the way. In this section, we propose a construction, named the adaptive cluster approximation (ACA), that performs a unitary transformation of the one-particle basis before doing a cluster approximation. The unitary transformation is constructed such that the following neglect of orbitals has a minimal impact on the density-matrix functional. The adaptive cluster approximation can be viewed as an extension of the two-level approximation of Töws and Pastor [Töws and Pastor, 2011, 2012] for the single-impurity Anderson model to an arbitrary number of interacting oneparticle states, multiorbital-interactions and an arbitrary number of effective bath levels. We have published the adaptive cluster approximation in [Schade and Blöchl, 2018]. Thus, the content of the subsequent sections is similar to the presentation in [Schade and Blöchl, 2018]. 


\subsection{Basic idea}

The main idea of the adaptive cluster approximation is to rotate the one-particle basis before performing a cluster approximation, i.e., neglecting some one-particle states. For this purpose, we assume a $N_{\chi}$-dimensional one-particle basis $\left|\chi_{\alpha}\right\rangle$ and the corresponding one-particle reduced density matrix $\rho^{(1)} \in \mathbb{C}^{N_{\chi} \times N_{\chi}}$. Additionally, we assume that the interaction is limited to the first $N_{\text {imp }}$ one-particle orbitals

$$
\hat{W}_{\text {loc }}=\frac{1}{2} \sum_{\alpha, \beta, \gamma, \delta \leq N_{\mathrm{imp}}} U_{\alpha, \beta, \gamma, \delta} \hat{c}_{\alpha}^{\dagger} \hat{c}_{\beta}^{\dagger} \hat{c}_{\delta} \hat{c}_{\gamma} .
$$

This can always be achieved by reordering the one-particle basis. We call the $N_{\text {imp }}$ interacting one-particle states the impurity and the remaining $N_{\text {bath }}=N_{\chi}-N_{\text {imp }}$ states the bath-states. We assume $N_{\text {bath }} \gg N_{\text {imp }}$.

First, we consider the case of the block-diagonal one-particle reduced density matrix

$$
\rho^{(1)}=\left(\begin{array}{cc}
\rho_{\text {imp,imp }}^{(1)} & 0 \\
0 & \rho_{\text {bath,bath }}^{(1)}
\end{array}\right)
$$

with $\rho_{\text {imp,imp }}^{(1)} \in \mathbb{C}^{N_{\text {imp }} \times N_{\text {imp }}}$ and $\rho_{\text {bath,bath }}^{(1)} \in \mathbb{C}^{N_{\text {bath }} \times N_{\text {bath }}}$. The density-matrix functional can be shown to have the separation property

$$
F_{\beta}^{\hat{W}_{\text {loc }}}\left[\rho^{(1)}\right]=F_{\beta}^{\hat{W}_{\text {loc }}}\left[\rho_{\text {imp,imp }}^{(1)}\right]+F_{\beta}^{\hat{0}}\left[\rho_{\text {bath }, \text { bath }}^{(1)}\right]
$$

in this case. The proof of this relation can be found in appendix B.1. Thus, the densitymatrix functional $F_{\beta}^{\hat{W}_{\text {loc }}}\left[\rho^{(1)}\right]$ with a complexity in $\mathcal{O}\left(2^{N_{\chi}}\right)$ can be evaluated by calculating the functional $F_{\beta}^{\hat{W}_{\text {loc }}}\left[\rho_{\text {imp,imp }}^{(1)}\right]$ with a computational complexity in $\mathcal{O}\left(2^{N_{\text {imp }}}\right)$ and a noninteracting functional $F_{\beta}^{\hat{0}}\left[\rho_{\text {bath,bath }}^{(1)}\right]$, which is given by Eq. (5.12).

For a general one-particle reduced density matrix

$$
\rho^{(1)}=\left(\begin{array}{cc}
\rho_{\text {imp,imp }}^{(1)} & \rho_{\text {imp,bath }}^{(1)} \\
\left(\rho_{\text {imp,bath }}^{(1)}\right)^{\dagger} & \rho_{\text {bath,bath }}^{(1)}
\end{array}\right)
$$

we propose a unitary transformation of the form

$$
U=\left(\begin{array}{cc}
\mathbb{1} & 0 \\
0 & U_{\text {bath,bath }}
\end{array}\right)
$$

with $U_{\text {bath,bath }} \in U\left(N_{\text {bath }}\right)$, that only acts on the bath-states and hence does not spread out the localized interaction over all one-particle states. The density-matrix functional is independent of the unitary transform, i.e.,

$$
F^{\hat{W}}\left[\rho^{(1)}\right]=F^{\hat{W}}\left[U^{\dagger} \rho^{(1)} U\right]
$$

holds. The goal of the unitary transformation is to transform the general one-particle reduced density matrix of Eq. (8.5) into a shape approximately like Eq. 8.3 so that we can make use of the separation property given in Eq. (8.4). 
We construct the unitary transform such that the transformed one-particle reduced density matrix obtains the banded form

$$
\begin{aligned}
\tilde{\rho}^{(1)} & =U^{\dagger} \rho^{(1)} U \\
& =\left(\begin{array}{cccccc}
\rho_{\text {imp,imp }}^{(1)} & \tilde{\rho}_{\text {imp,bath }}^{(1)} & 0 & \ldots & & \\
\left(\tilde{\rho}_{\text {imp }, \text { bath }_{1}}^{(1)}\right)^{\dagger} & \tilde{\rho}_{\text {bath }_{1}, \text { bath }_{1}}^{(1)} & \tilde{\rho}_{\text {bath }_{1}, \text { bath }_{2}}^{(1)} & 0 & \ldots & \\
0 & \left(\tilde{\rho}_{\text {bath }_{1}, \text { bath }_{2}}^{(1)}\right)^{\dagger} & \tilde{\rho}_{\text {bath }_{2}, \text { bath }_{2}}^{(1)} & \tilde{\rho}_{\text {bath }_{2}, \text { bath }_{3}}^{(1)} & 0 & \ldots \\
\vdots & 0 & \left(\tilde{\rho}_{\text {bath }_{2}, \text { bath }_{3}}^{(1)}\right)^{\dagger} & \tilde{\rho}_{\text {bath }_{3}, \text { bath }_{3}}^{(1)} & \tilde{\rho}_{\text {bath }_{3}, \text { bath }_{4}}^{(1)} & \ddots \\
& \vdots & 0 & \left(\tilde{\rho}_{\text {bath }_{3}, \text { bath }_{4}}^{(1)}\right)^{\dagger} & \tilde{\rho}_{\text {bath }_{4}, \text { bath }_{4}}^{(1)} & \ddots \\
& & \vdots & \ddots & \ddots & \ddots
\end{array}\right) .
\end{aligned}
$$

The unitary transformation $U$ that generates this banded shape depends on the given one-particle reduced density matrix. This is the reason why we have named it an adaptive scheme. The proof of existence of this unitary transformation and a practical construction scheme can be found in appendix B.2. For the one-particle reduced density matrix with the star-like topology of Eq. (8.1) we obtain the transformed matrix

$$
\tilde{\rho}_{\text {star }}^{(1)}=\left(\begin{array}{cccccccc}
1 / 2 & 0 & b & 0 & 0 & 0 & 0 & 0 \\
0 & 1 / 2 & 0 & b & 0 & 0 & 0 & 0 \\
b & 0 & 1 / 2 & 0 & 0 & 0 & 0 & 0 \\
0 & b & 0 & 1 / 2 & 0 & 0 & 0 & 0 \\
0 & 0 & 0 & 0 & 1 / 2 & 0 & 0 & 0 \\
0 & 0 & 0 & 0 & 0 & 1 / 2 & 0 & 0 \\
0 & 0 & 0 & 0 & 0 & 0 & 1 / 2 & 0 \\
0 & 0 & 0 & 0 & 0 & 0 & 0 & 1 / 2
\end{array}\right)
$$

with $b=\sqrt{3} a$. For the transformed matrix in Eq. (8.10) a cluster approximation, i.e. the neglect of some one-particle states is straight-forward: the last four one-particle states can be removed, because they are non-interacting states and have no finite density-matrix elements with any of the first four one-particle states. Thus, after a transformation of the one-particle basis, we can remove one-particle states without causing any deviation of the density-matrix funtional, whereas for the untransformed one-particle reduced density matrix this was not possible.

The transformation of the one-particle basis introduces no approximation. We have shown that a unitary transformation exists so that the off-diagonal matrices $\tilde{\rho}_{\text {imp,bath }}^{(1)}$ and $\tilde{\rho}_{\text {bath }_{i}, \text {,ath }_{i+1}}^{(1)}$ have a dimension of at most $N_{\text {imp }} \times N_{\text {imp }}$. Thus, the transformed one-particle reduced density matrix has a bandwidth of at most $2 N_{\mathrm{imp}}-1$. The proof of this statement can be found in appendix B.2. We call the one-particle states that belong to $\tilde{\rho}_{\mathrm{bath}_{i}, \mathrm{bath}_{i}}^{(1)}$ the $i$-th level effective bath. The off-diagonal matrix $\tilde{\rho}_{\text {bath }_{i}, \text { bath }_{i+1}}^{(1)}$ couples the $i$-th level and the $i+1$-th level effective bath.

Next, we neglect one of the off-diagonal matrices $\tilde{\rho}_{\text {bath }_{i}, \text {,ath }_{i+1}}^{(1)}$ so that we obtain a blockdiagonal one-particle reduced density matrix. If we neglect the coupling between the first 
level and second level effective bath, i.e. $\tilde{\rho}_{\text {bath }_{1}, \text { bath }_{2}}^{(1)}$, we obtain

$$
\tilde{\rho}^{(1)} \approx \tilde{\rho}_{M=1}^{(1)}=\left(\begin{array}{cc|cccc}
\rho_{\text {imp,imp }}^{(1)} & \tilde{\rho}_{\text {imp,bath }_{1}}^{(1)} & 0 & \ldots & & \\
\left(\tilde{\rho}_{\text {imp,bath }}^{(1)}\right)^{\dagger} & \tilde{\rho}_{\text {bath }_{1}, \text { bath }_{1}}^{(1)} & 0 & 0 & \ldots & \\
\hline 0 & 0 & \tilde{\rho}_{\text {bath }_{2}, \text { bath }_{2}}^{(1)} & \tilde{\rho}_{\text {bath }_{2}, \text { bath }_{3}}^{(1)} & 0 & \ldots \\
\vdots & 0 & \left(\tilde{\rho}_{\text {bath }_{2}, \text { bath }_{3}}^{(1)}\right)^{\dagger} & \tilde{\rho}_{\text {bath }_{3}, \text { bath }_{3}}^{(1)} & \tilde{\rho}_{\text {bath }_{3}, \text { bath }_{4}}^{(1)} & \ddots \\
& \vdots & 0 & \left(\tilde{\rho}_{\text {bath }_{3}, \text { bath }_{4}}^{(1)}\right)^{\dagger} & \tilde{\rho}_{\text {bath }_{4}, \text { bath }_{4}}^{(1)} & \ddots \\
& & \vdots & \ddots & \ddots & \ddots
\end{array}\right) .
$$

This defines the adaptive cluster approximation with one effective bath level $(\mathrm{ACA}(\mathrm{M}=1))$ with

$$
\begin{aligned}
& F_{\beta}^{\hat{W}}\left[\rho^{(1)}\right] \approx F_{\beta, \mathrm{ACA}(\mathrm{M}=1)}^{\hat{W}}\left[\rho^{(1)}\right]=F_{\beta}^{\hat{W}}\left[\tilde{\rho}_{M=1}^{(1)}\right]
\end{aligned}
$$

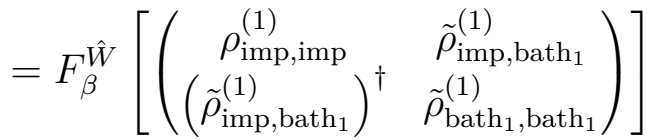

$$
\begin{aligned}
& +F_{\beta}^{\hat{0}}\left[\left(\begin{array}{cccc}
\tilde{\rho}_{\mathrm{bath}_{2}, \mathrm{bath}_{2}}^{(1)} & \tilde{\rho}_{\mathrm{bath}_{2}, \mathrm{bath}_{3}}^{(1)} & 0 & \cdots \\
\left(\tilde{\rho}_{\mathrm{bath}_{2}, \mathrm{bath}_{3}}^{(1)}\right)^{\dagger} & \tilde{\rho}_{\mathrm{bath}_{3}, \mathrm{bath}_{3}}^{(1)} & \tilde{\rho}_{\mathrm{bath}_{3}, \mathrm{bath}_{4}}^{(1)} & \ddots \\
0 & \left(\tilde{\rho}_{\mathrm{bath}_{3}, \mathrm{bath}_{4}}^{(1)}\right)^{\dagger} & \tilde{\rho}_{\mathrm{bath}_{4}, \mathrm{bath}_{4}}^{(1)} & \ddots \\
\vdots & \ddots & \ddots & \ddots
\end{array}\right)\right] .
\end{aligned}
$$

As shown in appendix B.2, the one-particle reduced density matrix $\tilde{\rho}_{M=1}^{(1)}$ has dimension $N_{\text {imp }}+N_{\text {bath }_{1}} \leq 2 N_{\text {imp }}$. We define the adaptive cluster approximation with $M>1$, $\mathrm{ACA}(\mathrm{M})$, by neglecting the coupling between the $M$-th level and $M+1$-th level effective bath, i.e. $\tilde{\rho}_{\text {bath }_{M}, \text { bath }_{M+1}}^{(1)}$. Thus, in ACA(M) the density-matrix functional is approximated as

$$
\begin{aligned}
F_{\beta, \mathrm{ACA}(\mathrm{M})}^{\hat{W}}\left[\rho^{(1)}\right] & =F_{\beta}^{\hat{W}}\left[\tilde{\rho}_{M}^{(1)}\right] \\
& =F_{\beta}^{\hat{W}}\left[T_{1, \ldots, N_{M}} \tilde{\rho}_{M}^{(1)}\right]+F_{\beta}^{\hat{0}}\left[T_{N_{M}+1, \ldots, N_{\chi}} \tilde{\rho}_{M}^{(1)}\right]
\end{aligned}
$$

with the truncation operator $\hat{T}$ that removes all columns and rows from the argument that are not listed in the subscript. Thus, $T_{1, \ldots, N_{M}} \tilde{\rho}_{M}^{(1)} \in \mathbb{C}^{N_{M} \times N_{M}}$ is the $N_{M}$-order leading principal submatrix of $\tilde{\rho}_{M}^{(1)}$. The size $N_{M}$ of the one-particle basis for which the densitymatrix functional $F_{\beta}^{\hat{W}}\left[T_{1, \ldots, N_{M}} \tilde{\rho}_{M}^{(1)}\right]$ has to be evaluated is

$$
N_{M}=N_{\mathrm{imp}}+\sum_{i=1}^{M} N_{\mathrm{bath}_{i}} \leq(M+1) N_{\mathrm{imp}} .
$$

We define

$$
\sigma_{M}\left(\tilde{\rho}^{(1)}\right)=\sum_{\alpha, \beta}\left|\left(\tilde{\rho}_{\mathrm{bath}_{M}, \operatorname{bath}_{M+1}}^{(1)}\right)_{\alpha, \beta}\right|
$$


as the discarded weight of the adaptive cluster approximation with $M$ effective bath levels. Even though the discarded weight $\sigma_{M}\left(\tilde{\rho}^{(1)}\right)$ is not necessarily a monotonically decreasing function of $M$, numerical evidence suggests that the difference of the exact density-matrix functional and the $\mathrm{ACA}(\mathrm{M})$-approximation

$$
\left|F^{\hat{W}}\left[\rho^{(1)}\right]-F_{\mathrm{ACA}(\mathrm{M})}^{\hat{W}}\left[\rho^{(1)}\right]\right|
$$

is a monotonically decreasing function of $M$.

\subsection{Related approaches}

The adaptive cluster approximation is related to the two-level approximation of Töws and Pastor for the single-impurity Anderson model [Töws and Pastor, 2011, 2012]. The unitary transformation of the one-particle basis employed in the two-level approximation is identical to the case of the adaptive cluster approximation for $M=1$ and $N_{\text {imp }}=2$. Thus, the adaptive cluster approximation represents the natural extension of the two-level approximation to an arbitrary number of interacting one-particle states $N_{\text {imp }}$, multi-orbitalinteractions and an arbitrary number of effective bath levels $M$. Unitary transformations of the one-particle Hamiltonian to transform the system to a quasi-one-dimensional structure have, for example, been used in the context of density matrix renormalization group theory (DMRG) for Anderson models [Büsser et al., 2013; Shirakawa and Yunoki, 2014]. Other related methods are [Lin and Demkov, 2013; Lu et al., 2014; Schüler et al., 2015]. However, these methods are different from the adaptive cluster approximation in the sense that they bring the one-particle Hamiltonian and not the one-particle reduced density matrix to a specific shape.

\subsection{Exact limits}

In this section, we discuss some exact limits of the adaptive cluster approximation. We assume that the exact density-matrix functional in the truncated one-particle basis is used for the density-matrix functional

$$
F_{\beta}^{\hat{W}}\left[T_{1, \ldots, N_{M}} \tilde{\rho}_{M}^{(1)}\right]
$$

in Eq. (8.15). We assume that the interaction $\hat{W}$ only acts on the first $N_{\text {imp }}$ one-particle states.

1. The adaptive cluster approximation is exact in the non-interacting limit $\hat{W} \rightarrow 0$ at zero temperature.

2. As discussed in section 8.3, the adaptive cluster approximation is identical to the two-level approximation for $M=1$ and $N_{\text {imp }}=2$. Thus, it inherits the exact limits. Töws et al. [Töws and Pastor, 2011] have proven that the two-level approximation is exact for a single-impurity Anderson model in the limit of a vanishing bath bandwidth as well as in the limit of widely separated bath levels.

3. If $\mathrm{ACA}(\mathrm{M})$ is exact, then also $\mathrm{ACA}(\mathrm{M}+1)$ is exact. 
4. The $\mathrm{ACA}(\mathrm{M})$ is exact if $\sigma_{M}\left(\tilde{\rho}^{(1)}\right)=0$, i.e., if the one-particle reduced density matrix is block-diagonal and the first diagonal block has at least $N_{\mathrm{imp}}+\sum_{i=1}^{M} N_{\mathrm{bath}_{i}}$ oneparticle states.

We also observed that if the eigenvalue spectrum of the one-particle reduced density matrix $\rho_{\text {bath,bath }}^{(1)}$ of the bath consists of $M$ distinct values with a $n_{j}$-fold degeneracy each, then the transformed one-particle reduced density matrix is block-diagonal with the first block of the size $N_{\text {imp }}+N_{B}$ and $N_{B} \leq \sum_{i=1}^{M} \min \left(n_{j}, N_{\text {imp }}\right)$. The proof of this statement can be found in appendix B.3.

\subsection{Correction using parametrized functionals}

Applications of the adaptive cluster approximations have shown that the discarded weight $\sigma_{M}\left(\tilde{\rho}^{(1)}\right)$ can grow to much larger values than the corresponding value for the one-particle reduced density matrix of the exact ground-state. This is due to the fact, that during the minimization of the total energy in Eq. (5.3) the neglected elements $\tilde{\rho}_{\text {bath }_{M}, \text { bath }_{M+1}}^{(1)}$ of the one-particle reduced density matrix only experience a force from the one-particle Hamiltonian but not from the density-matrix functional. In order to mediate this problem, we propose to add the correction term

$$
\Delta F_{\beta, \approx}^{\hat{W}}\left[\rho^{(1)}\right]=F_{\beta, \approx}^{\hat{W}}\left[\tilde{\rho}^{(1)}\right]-F_{\beta, \approx}^{\hat{W}}\left[\tilde{\rho}_{M}^{(1)}\right],
$$

where $\tilde{\rho}^{(1)}$ is given by Eq. (8.8) and $\tilde{\rho}_{M}^{(1)}$ is the corresponding one-particle reduced density matrix after the elements $\tilde{\rho}_{\text {bath }_{M}, \text { bath }_{M+1}}$ have been set to zero. $F_{\beta, \approx}^{\hat{W}}\left[\rho^{(1)}\right]$ is some approximate parametrized density-matrix functional that is numerically straight forward to evaluate. Thus, we propose the corrected adaptive cluster approximation (cACA) as

$$
F_{\beta, \mathrm{cACA}(M)}^{\hat{W}}\left[\rho^{(1)}\right]=F_{\beta, \mathrm{ACA}(M)}^{\hat{W}}\left[\rho^{(1)}\right]+\Delta F_{\beta, \approx}^{\hat{W}}\left[\rho^{(1)}\right] .
$$

\subsection{Application to Anderson models}

We study the results of the adaptive cluster approximation for the single-impurity Anderson model defined in section 3.2 by Eq. (3.2)-Eq. (3.7). We compare numerically exact results for the zero-temperature ground state obtained from exact diagonalization with results from reduced density-matrix functional theory with the (corrected) adaptive cluster approximation with $M$ effective bath levels. The results presented in this section have been published in [Schade and Blöchl, 2018]. Thus, we solve

$$
E(N)=\min _{\rho^{(1)}: 0 \leq \rho^{(1)} \leq 1, \operatorname{Tr} \rho^{(1)}=N}\left\{\operatorname{Tr}\left[\rho^{(1)} h\right]+F_{\beta,(\mathrm{c}) \operatorname{ACA}(M)}^{\hat{W}}\left[\rho^{(1)}\right]\right\},
$$

where $h$ are the matrix elements of the one-particle Hamiltonian and $N$ the particle number. We have not restricted the symmetry of the one-particle reduced density matrix and, hence, also allow ground states with collinear or non-collinear magnetization. We have used the numerically exact evaluations of the density-matrix functional of the impurity and the effective bath levels proposed in section 9.4 without additional approximations. Figure 8.2 shows the deviation of the total energy $\Delta E=E_{(\mathrm{c}) \mathrm{ACA}(\mathrm{M})}-E_{\text {exact }}$, 
the interaction energy $\Delta W=W_{(\mathrm{c}) \mathrm{ACA}(\mathrm{M})}-W_{\text {exact }}$ and the impurity occupation $\Delta n_{f}=$ $n_{f,(\mathrm{c}) \mathrm{ACA}(\mathrm{M})}-n_{f \text {,exact }}$ from the exact results shown in figure 3.2 for the interaction-strength dependence. The parameters are described in detail in section 3.2.1. The ACA(M) and the corrected $\mathrm{ACA}(\mathrm{M})$ with a correction from the Müller functional correctly predicts a vanishing impurity magnetization in this parameter range. Within the ACA with $M=1$ for this model, we have to evaluate the density-matrix functional for four one-particle states, two impurity states and two effective bath states. The results are equivalent to the results of the two-level approximation of Töws and Pastor [Töws and Pastor, 2011]. The uncorrected ACA tends to overestimate the impurity occupation $n_{f}$ because the interaction energy is underestimated. As a consequence, the total energy is underestimated. Figure 8.1 compares the discarded weight defined in Eq. (8.17) of the one-particle reduced density matrix of the exact ground state, the $\operatorname{ACA}(M=1)$ and the corrected $\operatorname{ACA}(M=1)$. The discarded weight of $\mathrm{ACA}(\mathrm{M}=1)$ is much larger than the discarded weight of the exact one-particle reduced density matrix. This is due to the missing force on these matrix elements. The corrected $\mathrm{ACA}(\mathrm{M}=1)$ proposed in section 8.5 with the Müller functional as the correction prevents the growth of the truncated matrix elements during the minimization and leads to a lower discarded weight, that agrees better with the discarded weight of the exact solution. Thus, the correction step serves its purpose and greatly improves the results of the ACA. Larger numbers of effective bath levels, i.e., $M=2$ and $M=3$, converge the results towards the exact result. The ACA with three effective bath sites is visually indistinguishable from the exact results. This is a consequence of the fact that the bath occupations, i.e., the eigenvalues of the one-particle reduced density matrix of the bath states, are contained in only three clusters with a negligible spread as shown in Figure 3.4. As discussed in detail in section 8.4 and appendix B.3 the ACA(M) is exact if the one-particle reduced density matrix of the non-interacting states contains $M$ or less distinct eigenvalues.

The deviations of the (c)ACA(M) from the exact results for single-impurity Anderson model defined in section 3.2.1 for different impurity on-site energies $\epsilon_{f}$ is shown in the left columns of figure 8.3. The $\mathrm{ACA}(\mathrm{M})$ describes the three regimes without breaking the spin-symmetry. Thus, it describes the Kondo-regime physically correctly. The deviation of the interaction energy and impurity occupation are largest during the transition from the doubly occupied impurity to the singly occupied impurity and from the singly occupied impurity to the empty impurity. The ACA with one effective bath level, $M=1$, is exact in the limit of a doubly occupied impurity and the limit of an empty impurity. The Müllercorrected ACA drastically improves the results for impurity on-site energy dependence, especially close to the mixed-valence transition points.

Finally, the right column of figure 8.3 presents the results of the ACA with $M=1$ and $M=2$ for the bandwidth dependence of the single-impurity Anderson model. The ACA with one effective bath level, i.e., $M=1$, becomes exact in the limit of widely separated bath energy levels $t \rightarrow \infty$ and in the limit of a vanishing bath bandwidth $t \rightarrow 0$ as discussed in section 8.4. In all parameter ranges studied, the ACA with $M=3$ is visually identical to the exact results and the ACA reproduces the correct non-spin-polarized ground state. In conclusion, we have shown that the adaptive cluster approximation converges rapidly to the exact results for single-impurity Anderson models, does not break the spin-symmetry, and that the correction with an approximate parametrized functional serves its purpose of preventing an uncontrolled growth of the discarded weight. 
8. Adaptive cluster approximation

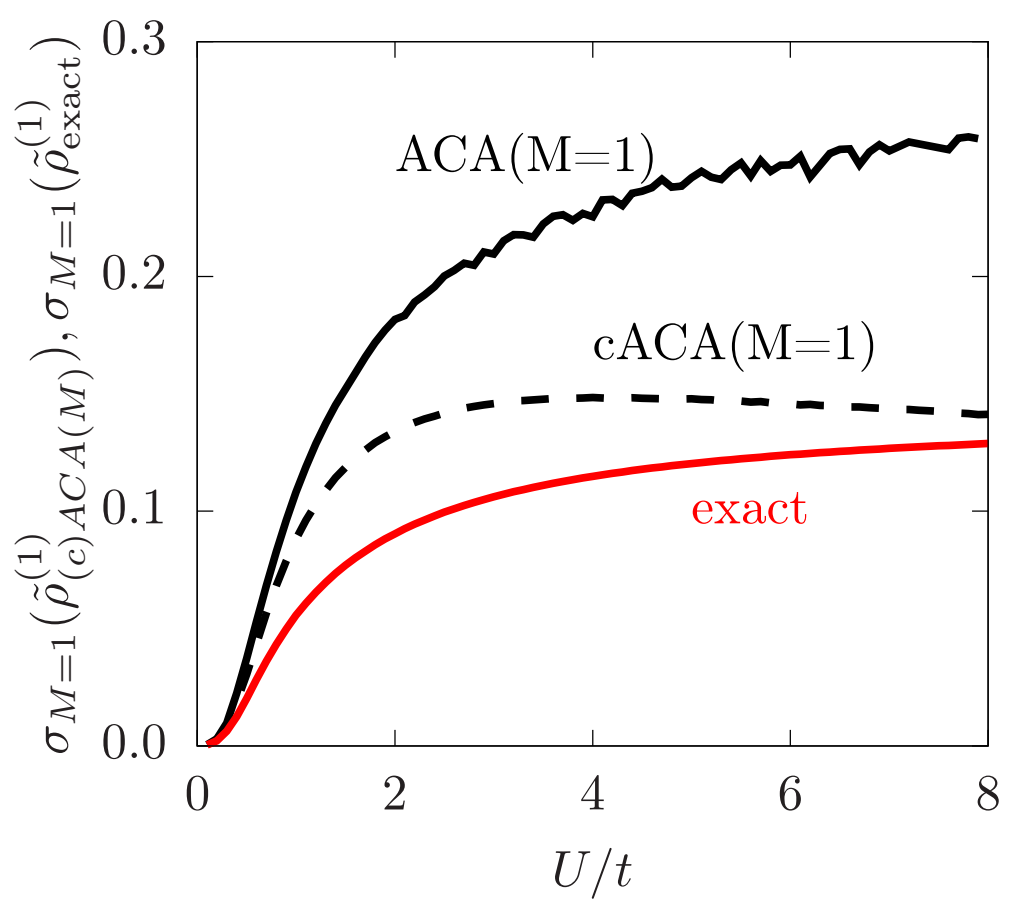

Figure 8.1.: Comparison of the discarded weight $\sigma_{M=1}$ defined Eq. (8.17) for the oneparticle reduced density matrix of the exact ground state, the $\mathrm{ACA}(\mathrm{M}=1)$ and the corrected $\mathrm{ACA}(\mathrm{M}=1)$. The model is the half-filled single-impurity Anderson model defined by Eq. (3.2)-Eq. (3.7) with $L_{\text {bath }}=11, N_{e}=L_{\text {bath }}+$ $1=12, \epsilon_{f}=0, V / t=0.4$ and $t>0$. The parameters of the model are described in detail in section 3.2.1. 


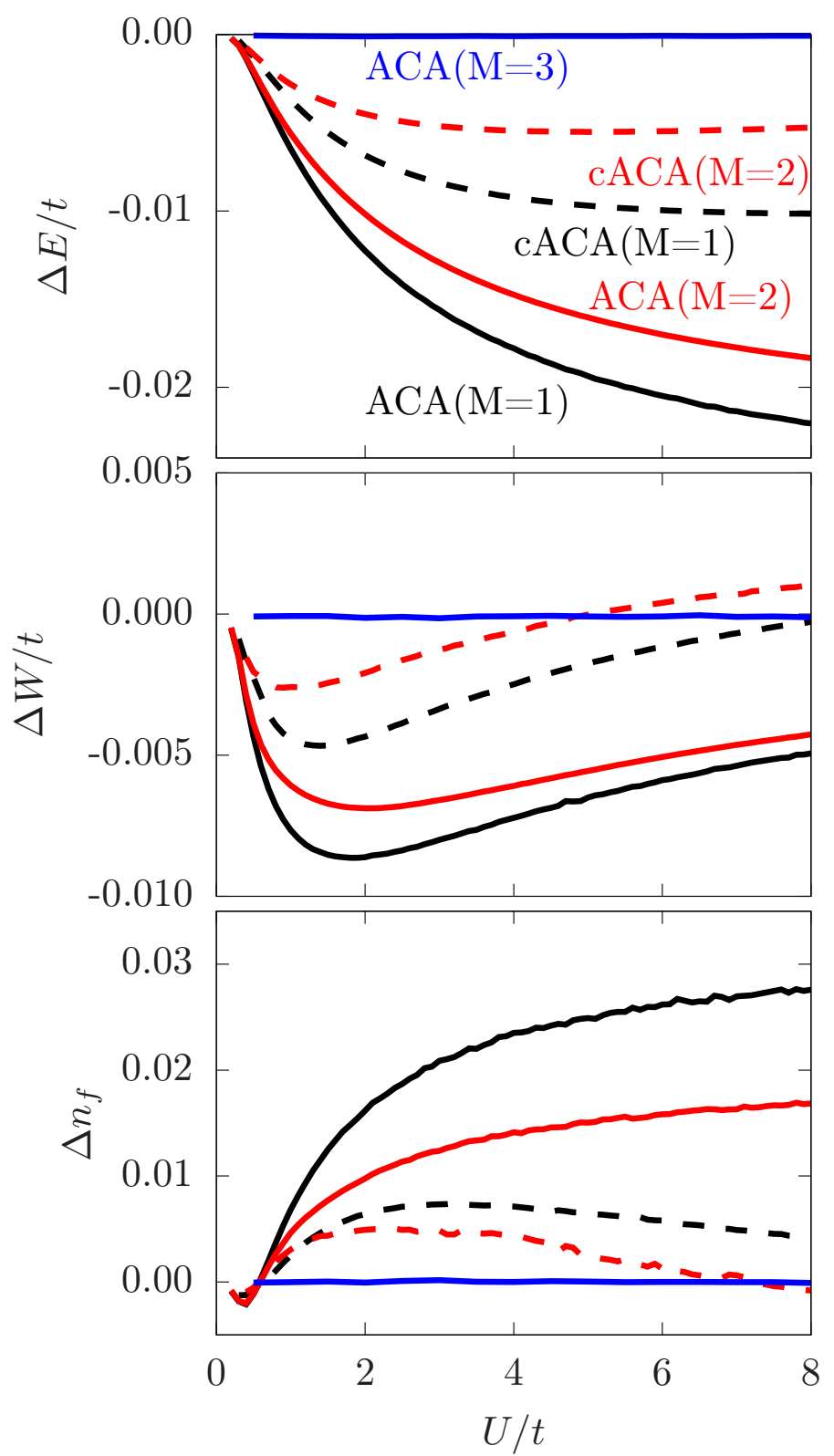

Figure 8.2.: Deviation from exact results of the (corrected) ACA(M) for the interactionstrength dependence of the ground-state of a half-filled single-impurity Anderson model defined by Eq. (3.2)-Eq. (3.7) with $L_{\text {bath }}=11, N_{e}=L_{\text {bath }}+1=12$, $\epsilon_{f}=0, V / t=0.4$ and $t>0$. The black lines show results for the ACA with $M=1$, red lines for $M=2$ and blue lines for $M=3$. The solid lines show results for the uncorrected ACA and dashed lines the corresponding results for the uncorrected ACA. The parameters of the model are described in detail in section 3.2.1 and the exact results are shown in figure 3.2. 

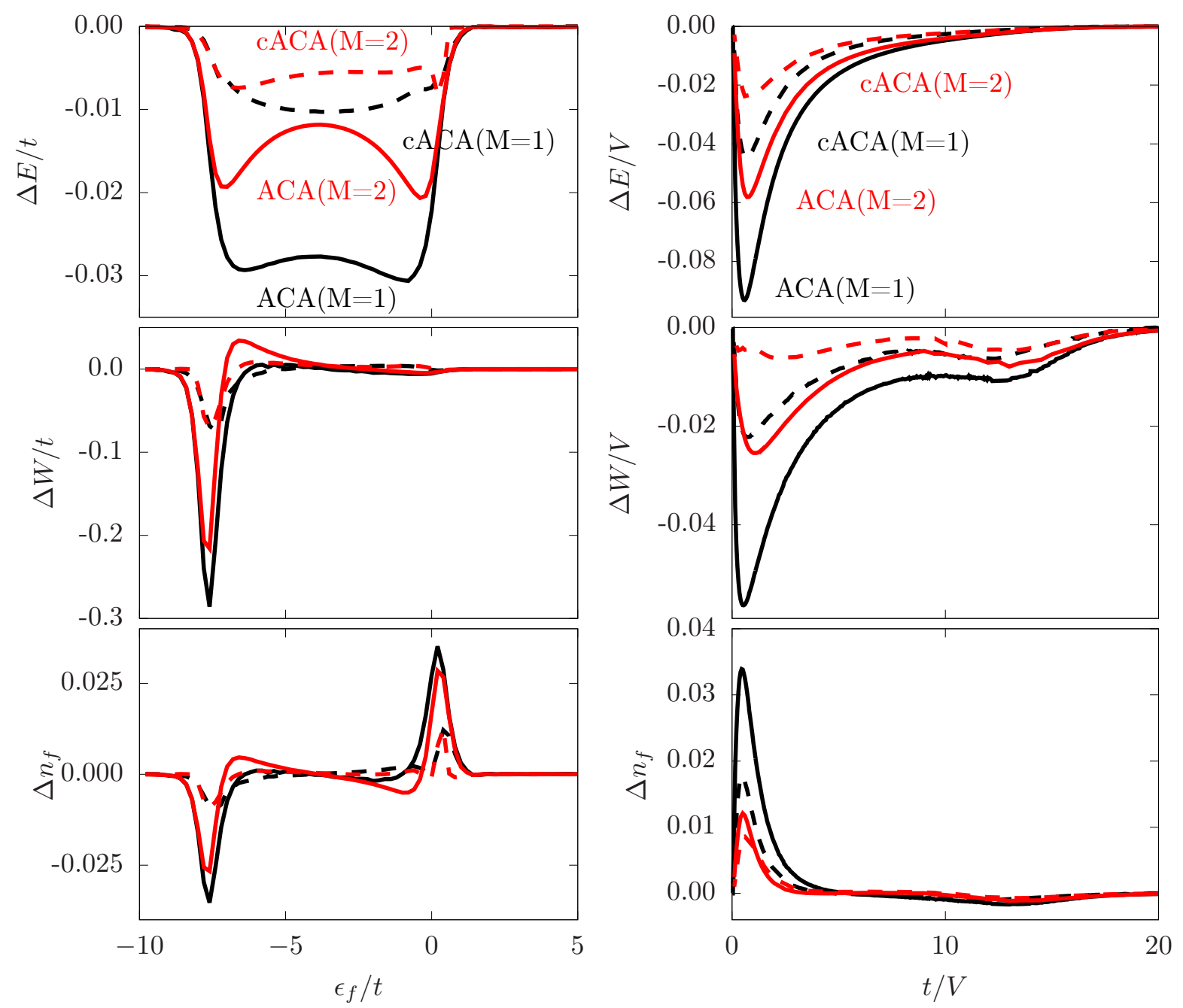

Figure 8.3.: Deviation from exact results of the (corrected) ACA(M) for the impurity onsite-energy dependence and the bandwidth dependence of the ground-state of a half-filled single-impurity Anderson model defined by Eq. (3.2)-Eq. (3.7) with $L_{\text {bath }}=11, N_{e}=L_{\text {bath }}+1=12$. The black lines show results for the ACA with $M=1$ and the red lines for $M=2$. Results for $M=3$ are not chosen, because they are visually identical with the exact results. The solid lines show results for the uncorrected ACA and dashed lines the corresponding results for the uncorrected ACA. The parameters of the model are described in detail in section 3.2.1. The corresponding exact results are shown in figure 3.3. 


\subsection{Application to Hubbard models}

To apply the adaptive cluster approximation to a Hubbard model, we first employ the local approximation of the density-matrix functional defined in section 5.5,

$$
F^{\sum_{R} \hat{W}_{R}}\left[\rho^{(1)}\right] \approx \sum_{R} F^{\hat{W}_{R}}\left[\rho^{(1)}\right]
$$

The density-matrix functionals $F^{\hat{W}_{R}}\left[\rho^{(1)}\right]$ can then be evaluated with the adaptive cluster approximation. We have published the results presented in this section in [Schade and Blöchl, 2016]. We partition the sites of the model into sets $C_{R}$ so that different variants of the local approximation can be defined. For the simplest variant, each site is in its own set $C_{R}$, and the local approximation reads

$$
F^{\sum_{i=1}^{L} U \hat{n}_{i, \uparrow} \hat{n}_{i, \downarrow}}\left[\rho^{(1)}\right] \approx \sum_{i=1}^{L} F^{U \hat{n}_{i, \uparrow} \hat{n}_{i, \downarrow}}\left[\rho^{(1)}\right]
$$

where $L$ is the number of sites in the model. The individual density-matrix functionals $F^{U \hat{n}_{i, \uparrow} \hat{n}_{i, \downarrow}}\left[\rho^{(1)}\right]$ are approximated by the ACA,

$$
F^{U \hat{n}_{i, \uparrow} \hat{n}_{i, \downarrow}}\left[\rho^{(1)}\right] \approx F_{\mathrm{ACA}(\mathrm{M})}^{U \hat{n}_{i, \uparrow} \hat{n}_{i, \downarrow}}\left[\rho^{(1)}\right]
$$

as functionals in a $2+2 M$-dimensional one-particle basis. Thus, we have approximated a density-matrix functional in a $2 L$-dimensional one-particle basis by a functional in a much smaller one-particle basis. We first investigate, if the ACA converges as rapidly with the number of effective bath sites as in the case of the single-impurity Anderson model. Figure 8.4 shows the convergence of the density-matrix functional $F_{\mathrm{ACA}(\mathrm{M})}^{U \hat{n}_{1, \uparrow} \hat{n}_{1, \downarrow}}\left[\rho^{(1)}\right]$ with the number of effective bath levels $M$ for the one-particle reduced density matrix of the exact ground state of a half-filled 10-site Hubbard ring with $U / t=5$. The numerical results show that the convergence is approximately exponential, i.e., $\left|F^{U \hat{n}_{1, \uparrow} \hat{n}_{1, \downarrow}}\left[\rho^{(1)}\right]-F_{\mathrm{ACA}(\mathrm{M})}^{U \hat{n}_{1, \uparrow} \hat{n}_{1, \downarrow}}\left[\rho^{(1)}\right]\right| \propto$ $10^{-M}$, in the number of effective bath sites $M$.

We have performed the minimization over the one-particle reduced density matrix,

$$
E_{M}=\min _{\rho^{(1)}: 0 \leq \rho^{(1)} \leq 1, \operatorname{Tr} \rho^{(1)}=N}\left\{\operatorname{Tr} \rho^{(1)} h+\sum_{i} F_{\mathrm{ACA}(\mathrm{M})}^{U \hat{n}_{i, \uparrow} \hat{n}_{i, \downarrow}}\left[\rho^{(1)}\right]\right\}
$$

in the $\mathrm{ACA}(\mathrm{M})$ over all nonmagnetic and rotational symmetric one-particle reduced density matrices. This restriction fixes the natural orbitals as momentum eigenstates and only one density-matrix functional $F_{\mathrm{ACA}(\mathrm{M})}^{U \hat{n}_{i, \uparrow} \hat{n}_{i, \downarrow}}\left[\rho^{(1)}\right]$ has to be evaluated in practice. Figure 8.5 presents results for the ground-state energy, interaction energy and next-neighbor elements $\rho_{i \sigma, i+1 \sigma}^{(1)}$ of the one-particle reduced density matrix for the local approximation with the $\mathrm{ACA}(\mathrm{M})$ for a 24 -site half-filled Hubbard ring at different interaction strengths $U / t$. The total energy $E_{M}$ converges with $M$ to a value that is lower than the exact ground-state energy. This is a consequence of the local approximation. For $M=3$ the result is visually converged so that $E_{M=3}$ is the result of the total energy in the local approximation. Thus, with the adaptive cluster approximation we can obtain results within the local approximation with a fraction of the numerical effort compared to $F^{U \hat{n}_{i, \uparrow} \hat{n}_{i, \downarrow}}\left[\rho^{(1)}\right]$. 


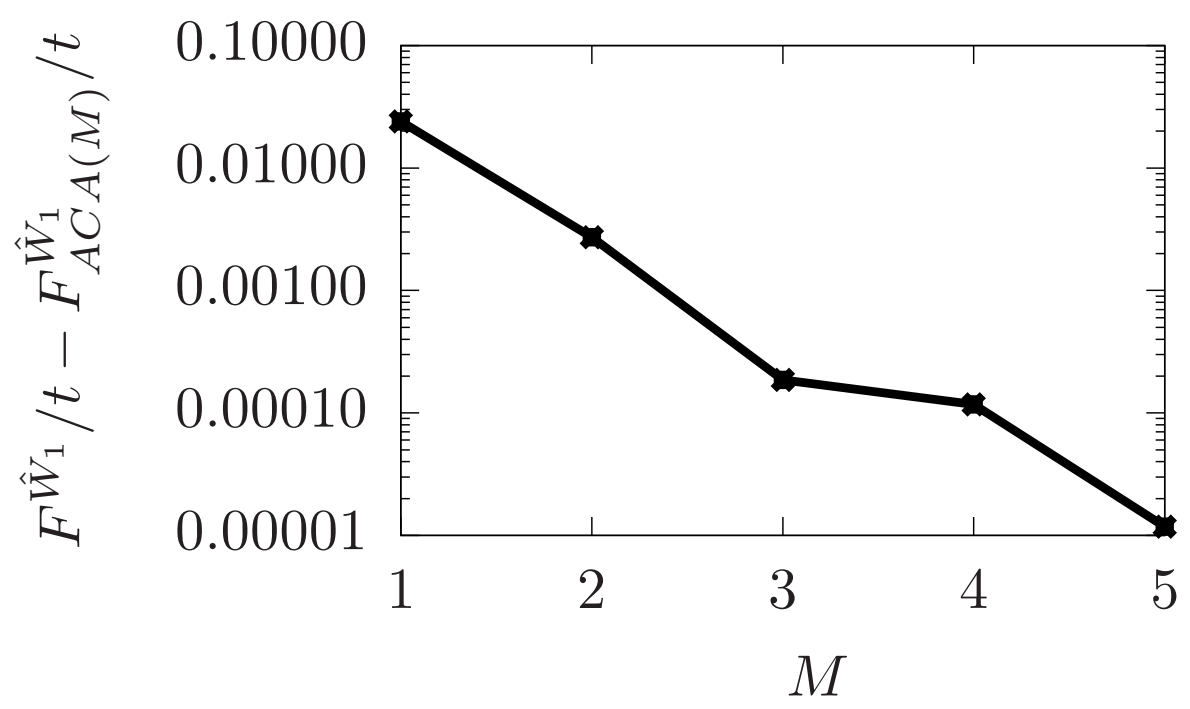

Figure 8.4.: Convergence of the adaptive cluster approximation with the level of the effective bath $M$ for the density-matrix functional $F_{\mathrm{ACA}(\mathrm{M})}^{U \hat{n}_{1, \uparrow} \hat{n}_{1, \downarrow}}\left[\rho^{(1)}\right]$ for a halffilled 10-site Hubbard ring with $U / t=5$. The density-matrix functionals have been evaluated for the one-particle reduced density matrix of the exact ground state and $\hat{W}_{1}=U \hat{n}_{1, \uparrow} \hat{n}_{1, \downarrow}$. The converged value of the density-matrix functional is $F^{U \hat{n}_{1, \uparrow} \hat{n}_{1, \downarrow}}\left[\rho^{(1)}\right] \approx 0.30936$.

We can also converge the local approximation towards the exact result by increasing the number of interacting one-particle states per density-matrix functional. If we take two sites together, we obtain the two-site local approximation

$$
F^{\sum_{i=1}^{L} U \hat{n}_{i, \uparrow} \hat{n}_{i, \downarrow}}\left[\rho^{(1)}\right] \approx F^{U\left(\hat{n}_{1, \uparrow} \hat{n}_{1, \downarrow}+\hat{n}_{2, \uparrow} \hat{n}_{2, \downarrow}\right)}\left[\rho^{(1)}\right]+\ldots+F^{U\left(\hat{n}_{L-1, \uparrow} \hat{n}_{L-1, \downarrow}+\hat{n}_{L, \uparrow} \hat{n}_{L, \downarrow}\right)}\left[\rho^{(1)}\right]
$$

Figure 8.6 shows the ground-state energy, interaction energy and next-neighbor elements $\rho_{i \sigma, i+1 \sigma}^{(1)}$ of the one-particle reduced density matrix for the two-site local approximation with the $\mathrm{ACA}(\mathrm{M})$ for a 24-site half-filled Hubbard ring at different interaction strengths $U / t$. The deviation of the total energy in the two-site local approximation from the exact results is much lower than in the one-site local approximation. In conclusion, the combination of the local approximation with the adaptive cluster approximation results in a numerical approximation scheme, that converges rapidly with the number of interacting one-particle states per density-matrix functional and also with the number of effective bath levels. 


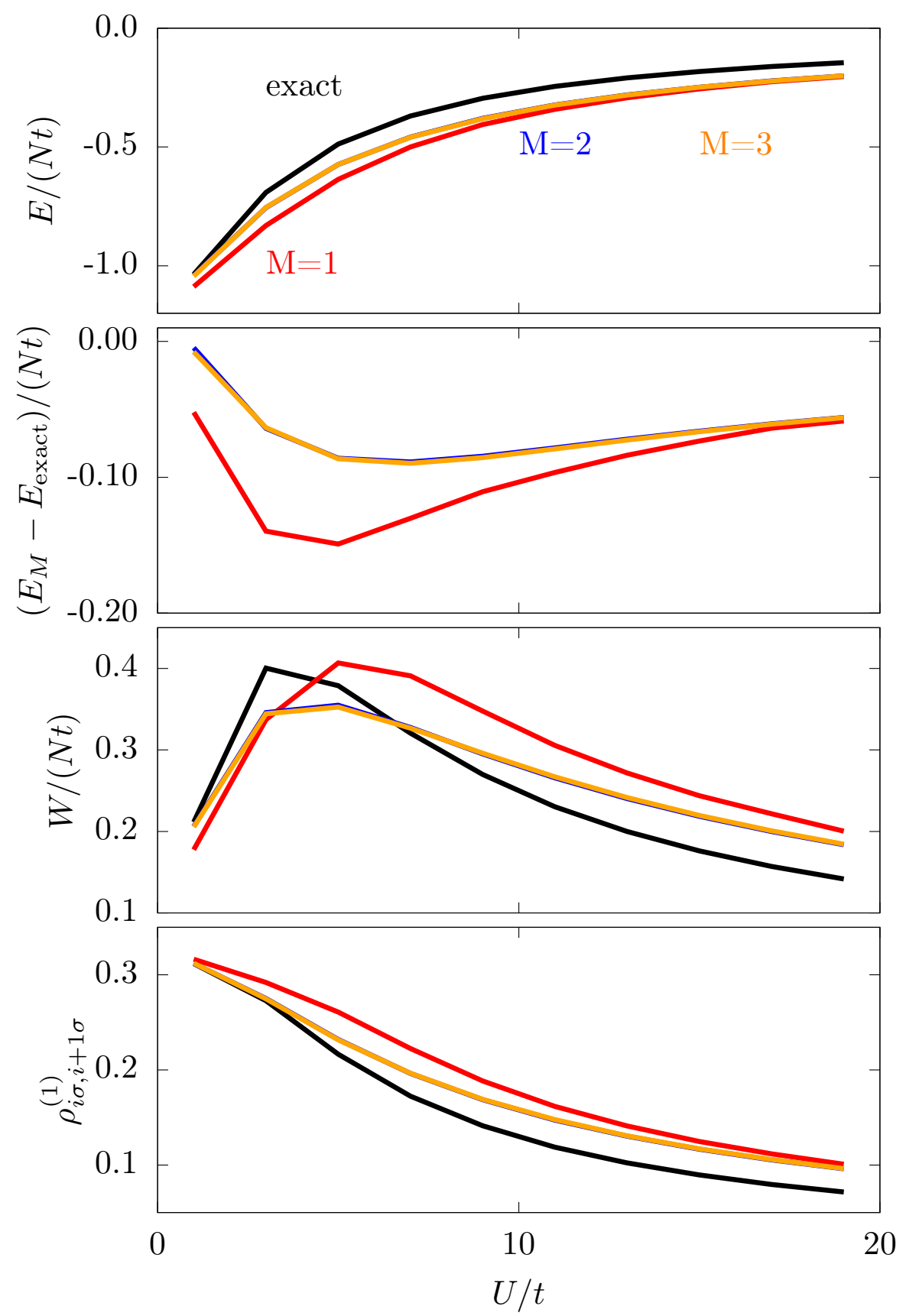

Figure 8.5.: Ground-state energy $E$, interaction energy $W$ and next-neighbor element $\rho_{i \sigma, i+1 \sigma}^{(1)}$ of the one-particle reduced density matrix in the one-site local approximation with the $\mathrm{ACA}(\mathrm{M})$ compared to exact results for the half-filled 24-site Hubbard ring. 


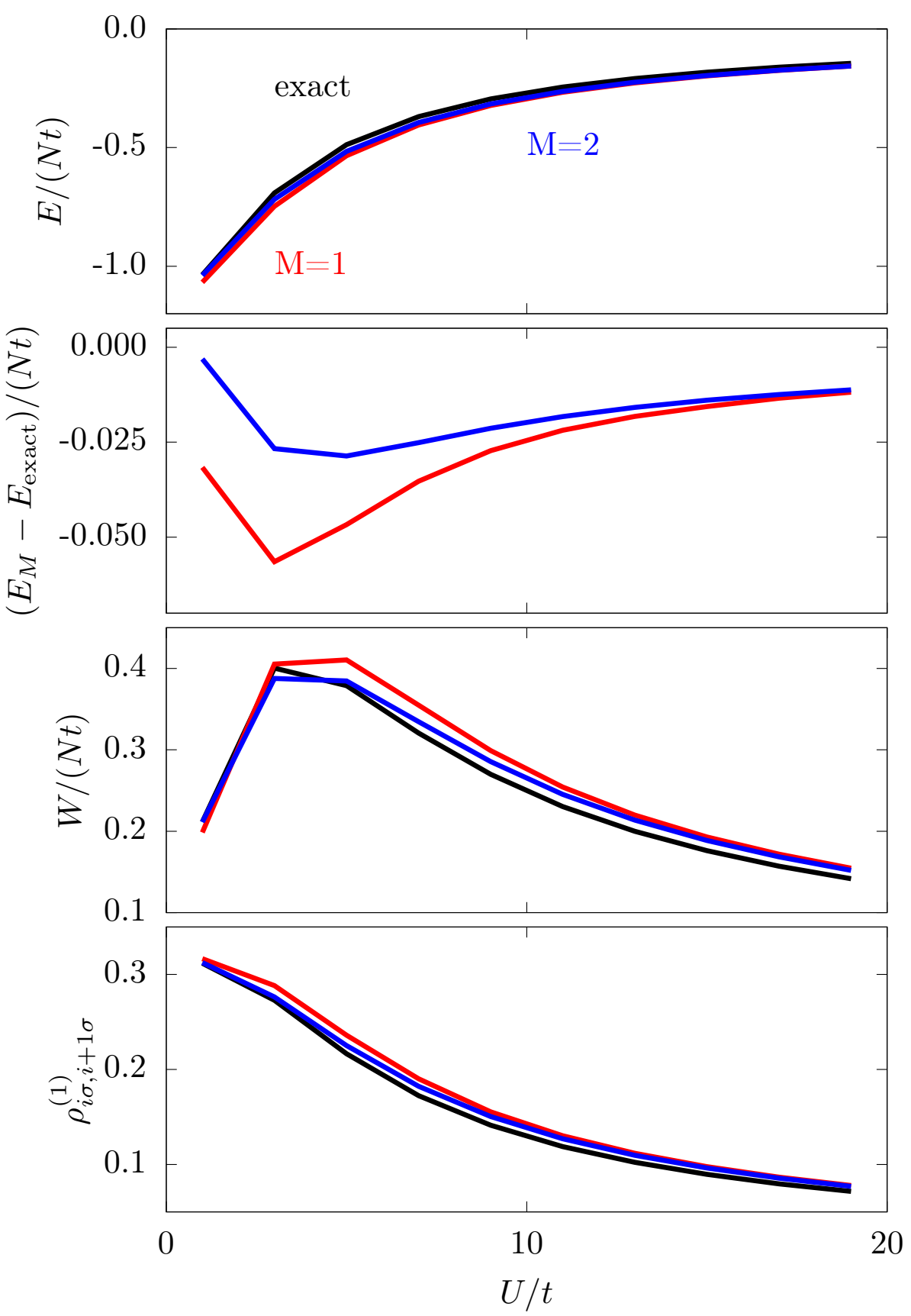

Figure 8.6.: Ground-state energy $E$, interaction energy $W$ and next-neighbor element $\rho_{i \sigma, i+1 \sigma}^{(1)}$ of the one-particle reduced density matrix in the two-site local approximation with the $\mathrm{ACA}(\mathrm{M})$ compared to exact results for the half-filled 24-site Hubbard ring. 


\subsection{Adaptive-cluster transformation for reduction of entanglement}

The idea to try matrix product states as an ansatz for the many-particle wave function for the density-matrix functional was mostly motivated by the nice seminar talks of members of the DMRG-group at Göttingen university. In the context of entanglement and matrix product states, I would especially like to acknowledge the role of Thomas Köhler who answered a lot of my beginners questions about tensor wave-functions and DMRG.

\subsubsection{Matrix product states and correlation functions}

Matrix product states (MPS) and density-matrix renormalization group theory (DMRG, [White, 1992, 1993; Östlund and Rommer, 1995; Schollwöck, 2005; Schollwöck, 2011. ${ }^{1}$ ) have been shown to be a powerful tools for quantum chemistry [Chan and Sharma, 2011; Marti and Reiher, 2011; Kurashige, 2014]. For the discussion of matrix product states, we follow the discussions in [Eisert, 2013] and [Schuch, 2013]. We focus on the case of open boundary conditions. We assume that the system consists of $L$ sites with a local basis $|0\rangle=|00\rangle,|\uparrow\rangle=|10\rangle,|\downarrow\rangle=|01\rangle$ and $|\uparrow \downarrow\rangle=|11\rangle$. Thus, the local dimension $d$ is assumed to be equal to four and the total number of one-particle basis states is $N_{\chi}=2 L$.

The central idea of matrix product states is to write the coefficients $c_{j_{1}, \ldots, j_{L}}$ of a manyparticle wave function

$$
|\Psi\rangle=\sum_{j_{1}, \ldots, j_{L} \in\{0, \uparrow, \downarrow, \uparrow \downarrow\}} c_{j_{1}, \ldots, j_{L}}\left|j_{1}, \ldots, j_{L}\right\rangle
$$

as a product [Fannes et al., 1992; Östlund and Rommer, 1995; Rommer and Östlund, 1997]

$$
\begin{aligned}
c_{j_{1}, \ldots, j_{L}} & =\sum_{i_{1}=1}^{d_{1}} \sum_{i_{2}=1}^{d_{2}} \ldots \sum_{i_{L-1}=1}^{d_{L-1}} M_{i_{1}}^{(1), j_{1}} M_{i_{1}, i_{2}}^{(2), j_{2}} \cdots M_{i_{L-2}, i_{L-1}}^{(L-1), j_{L-1}} M_{i_{L-1}}^{(L), j_{L}} \\
& =M^{(1), j_{1}} M^{(2), j_{2}} \cdots M^{(L), j_{L}}
\end{aligned}
$$

with the vectors $M^{(1), j_{1}} \in \mathbb{C}^{1 \times d_{1}}$ and $M^{(L), j_{L}} \in \mathbb{C}^{d_{L-1} \times 1}$ as well as the matrices $M^{(i), j_{i}} \in$ $\mathbb{C}^{d_{i} \times d_{i+1}}$ for $1<i<L . d_{i}$ are called bond dimensions. The maximal bond dimensions are

$$
d_{1}=d, \quad d_{2}=d^{2}, \quad d_{3}=d^{3}, \quad \ldots \quad d_{L-2}=d^{2} \quad d_{L-1}=d
$$

where $d$ is the local dimension. The maximal bond dimension $d_{l}$ of the bond between site $l$ and $l+1$ is the number of Schmidt values $q_{j}$ in the Schmidt-decomposition

$$
|\Psi\rangle=\sum_{k=1}^{d_{l}} q_{k}\left|\Psi_{A, k}\right\rangle \otimes\left|\Psi_{B, k}\right\rangle
$$

\footnotetext{
${ }^{1} \mathrm{~A}$ more complete list of references can be found in the reviews [Schollwöck, 2005], [Verstraete et al., 2008] and [Schollwöck, 2011].
} 
with

$$
\begin{aligned}
\left|\Psi_{A, k}\right\rangle & =\sum_{j_{1}, \ldots, j_{l}} c_{A, k, j_{1}, \ldots, j_{l}}\left|j_{1}, \ldots, j_{l}\right\rangle \\
\left|\Psi_{B, k}\right\rangle & =\sum_{j_{l+1}, \ldots, j_{L}} c_{B, k, j_{l+1}, \ldots, j_{L}}\left|j_{l+1}, \ldots, j_{L}\right\rangle .
\end{aligned}
$$

The von Neumann entropy of entanglement between the subsystem $A$ consisting of the sites $1, \ldots, l$ and the subsystem $B$ consisting of the sites $l+1, \ldots, L$ is defined as

$$
S_{A \mid B}=-\operatorname{Tr}\left(\rho_{A} \log _{2} \rho_{A}\right)
$$

where $\rho_{A}=\operatorname{Tr}_{B}|\Psi\rangle\langle\Psi|$ is the reduced density matrix and $\operatorname{Tr}_{B}$ is the partial trace over the basis of the system $B$. The reduced density matrix $\rho_{A}$ can be written with the Schmidt decomposition of the state $|\Psi\rangle$ as

$$
\rho_{A}=\sum_{k} q_{k}^{2}\left|\Psi_{A, k}\right\rangle\left\langle\Psi_{A, k}\right|
$$

and the entanglement entropy as

$$
S_{A \mid B}=-\sum_{k} q_{k}^{2} \log _{2} q_{k}^{2} .
$$

Thus, the entanglement entropy $S_{A \mid B}$ is directly related to the bond dimensions of a matrix product state. A matrix product state with the maximal bond dimensions can represent any many-particle wave function. Matrix product states can efficiently represent states with small numbers of non-zero Schmidt values $q_{k}$ because this situation corresponds to a small bond dimension and hence a small size of matrices $M^{(i), j_{i}}$.

A matrix product state is a representative of the much more general class of tensor wave functions. A graphical representation of tensors and operations drastically simplifies the notation. We define a scalar as a box

$$
x=\square \in \mathbb{C}
$$

and a vector as a box with a leg

$$
\vec{x}=-\square \in \mathbb{C}^{D}
$$

A vector is a tensor of rank one and has one leg. An $n$-th order tensor has $n$ legs. The leg can also have an explicitly given index $j$ to select a specific element, i.e.

$$
\vec{x}_{j}=j^{j} \square .
$$

A matrix as a tensor of rank two is represented as

$$
A=-\square \in \mathbb{C}^{D \times D}
$$

A contraction of an index is a summation over all possible values an index (a shared leg) can take. Thus, a matrix-matrix product can be written as

$$
(A \cdot B)_{a b}=\sum_{j} A_{a j} B_{j b}=a-B B^{b}=a C^{b}=C_{a b} .
$$


The coefficients $c_{j_{1}, \ldots, j_{L}}$ represent a tensor of rank $L$ and can be drawn as

$$
c_{j_{1}, \ldots, j_{L}}=\begin{array}{llll}
j_{1} j_{2} & \ldots . . & j_{L-1} & j_{L} \\
\hline & & &
\end{array} .
$$

We have oriented the legs vertically to indicate that the indexes correspond to local basis states. These indexes are called physical indexes. The matrix-product-state representation in Eq. (8.29) can be drawn as

$$
c_{j_{1}, \ldots, j_{L}}=\stackrel{j_{1}}{\square}
$$

The indexes that correspond to horizontal legs are called bond indexes.

For a variational minimization of the total energy, i.e., the expectation value of the Hamiltonian $\hat{H}$, we also need to be able to compute expectation values $\langle\Psi|\hat{O}| \Psi\rangle$ of operators $\hat{O}$. We first discuss the case where the operator $\hat{O}$ is supported on neighboring sites, $l$ and $l+1$. With this restriction, $\hat{O}$ has the general form

$$
\hat{O}=\sum_{j_{l}=1}^{d} \sum_{j_{l+1}=1}^{d} \sum_{k_{l}=1}^{d} \sum_{k_{l+1}=1}^{d} O_{k_{l}, k_{l+1}}^{j_{l}, j_{j+1}}\left|j_{j}, j_{l+1}\right\rangle\left\langle k_{l}, k_{l+1}\right| .
$$

The tensor $O$ can be represented graphically as a tensor with four physical indexes as

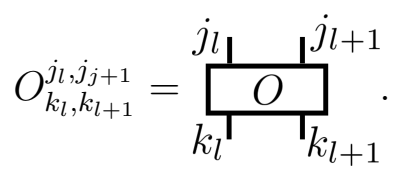

Matrix product operators will be discussed in detail in section 8.8.3. The expectation value $\langle\Psi|\hat{O}| \Psi\rangle$ can be drawn in the graphical representation as

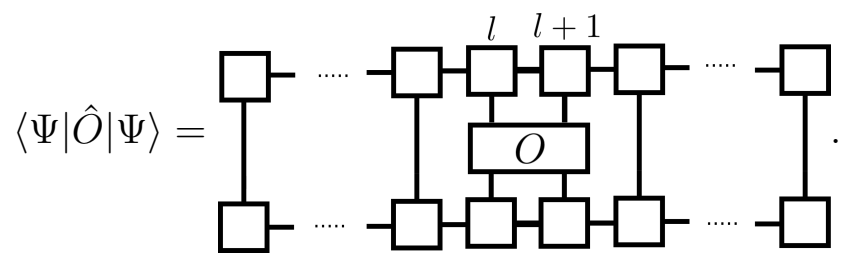

As every shared leg represents a summation, a naive evaluation of the contractions would result in an exponential complexity. Thus, a more efficient way of contracting the network is needed. For this purpose, the tensor network is contracted starting from the rightmost and the leftmost end. At first, the two ends are contracted. The tensor

$$
L_{\alpha, \beta}=L_{\beta}^{\alpha}=\sum_{j=1}^{d} M_{\alpha}^{(1), j}\left(M_{\beta}^{(1), j}\right)^{*}=\square_{\beta}^{\alpha}
$$

and the tensor

$$
R_{\alpha, \beta}=\frac{\alpha}{\beta}\left[R=\sum_{j=1}^{d} M_{\alpha}^{(L), j}\left(M_{\beta}^{(L), j}\right)^{*}=\right.
$$


are defined and represent the ends of the network. The analogous contraction of the physical index can be performed for every site. This leads to the definition of the transfer operator

$$
\left(E^{(k)}\right)_{\alpha, \beta, \gamma, \delta}=\underset{\gamma}{\stackrel{\alpha}{E^{(k)}} \frac{\beta}{E}=\sum_{j=1}^{d} M_{\alpha, \beta}^{(k), j}\left(M_{\gamma, \delta}^{(k), j}\right)^{*}=}
$$

Similarly, a two-site transfer operator for the operator $\hat{O}$ can be defined as

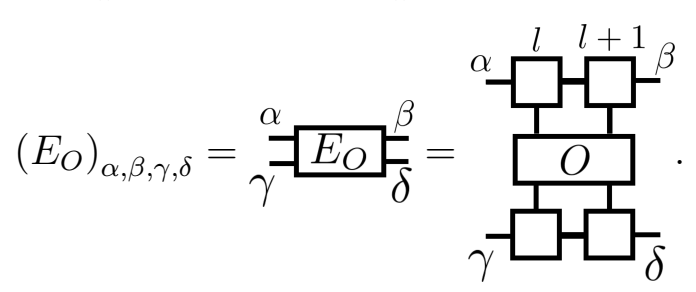

With these definitions the expectation value can be written as

$$
\langle\Psi|\hat{O}| \Psi\rangle=L E^{(2)} \cdots E^{(l-1)} E_{O} E^{(l+2)} \cdots E^{(L-1)} R .
$$

To estimate the computational complexity, we assume, that the bond dimensions $d_{i}$ are bounded by a maximal bond dimension $D$. $R$ can be viewed as an $D^{2}$-dimensional vector, because it has two bond indexes. Therefore, $E^{(L-1)} R$ is a matrix-vector multiplication with a computational complexity $\in \mathcal{O}\left(D^{4}\right)$ and hence the computation of the expectation value has a complexity $\in \mathcal{O}\left(L D^{4}\right)$. The transfer operator can be used to discuss the spatial decay of correlations functions of matrix product states. Following [Schuch, 2013; Eisert, 2013], we take an infinite translational invariant matrix product state

$$
c_{j_{1}, \ldots, j_{L}}=\operatorname{Tr} M^{j_{1}} \cdots M^{j_{L}}=
$$

and consider the thermodynamic limit $L \rightarrow \infty$. The transfer operator is

$$
E_{\alpha, \beta, \gamma, \delta}=\sum_{j=1}^{d} M_{\alpha, \beta}^{j}\left(M_{\gamma, \delta}^{j}\right)^{*}=\left(E_{\gamma, \delta, \alpha, \beta}\right)^{*}
$$

and site-independent. We estimate the correlation function $\left\langle\hat{O}_{a} \hat{O}_{b}\right\rangle$, where $\hat{O}_{a}$ is supported on site $a$ and $\hat{O}_{b}$ is supported on site $b$. We assume $a<b$. The corresponding transfer operators are

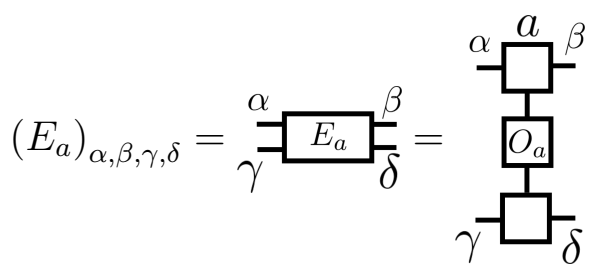

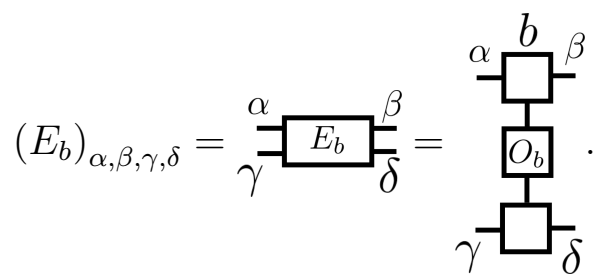


Thus, the expectation value $\left\langle\hat{O}_{a} \hat{O}_{b}\right\rangle$ can be written as

$$
\left\langle\hat{O}_{a} \hat{O}_{b}\right\rangle=\frac{\operatorname{Tr}\left(E_{a} E^{b-a-1} E_{b} E^{L-1-b+a}\right)}{\operatorname{Tr} E^{L}} .
$$

The transfer operator $E$ can be represented as a $D^{2} \times D^{2}$ hermitian matrix with the eigenvalues $\lambda_{1} \geq \lambda_{2} \geq \ldots \geq \lambda_{D^{2}}$. $E$ can be written in its eigendecomposition as

$$
E=\sum_{j=1}^{D^{2}} \lambda_{j}\left|e_{j}\right\rangle\left\langle e_{j}\right|
$$

We assume that the maximal eigenvalue $\lambda_{1}$ is unique. $E$ can be chosen such that the maximal eigenvalue $\lambda_{1}$ is equal to one and hence

$$
\lim _{m \rightarrow \infty} E^{m}=\left|e_{1}\right\rangle\left\langle e_{1}\right|
$$

Thus, in the thermodynamic limit $L \rightarrow \infty$ and for finite $b-a$, we obtain for the correlation function in Eq. (8.57) the form

$$
\begin{aligned}
\left\langle\hat{O}_{a} \hat{O}_{b}\right\rangle & =\left\langle e_{1}\left|E_{a} E^{b-a-1} E_{b}\right| e_{1}\right\rangle \\
& =\left\langle e_{1}\left|E_{a}\left(\sum_{j=1}^{D^{2}} \lambda_{j}^{b-a-1}\left|e_{j}\right\rangle\left\langle e_{j}\right|\right) E_{b}\right| e_{1}\right\rangle \\
& =\left\langle e_{1}\left|E_{a}\right| e_{1}\right\rangle\left\langle e_{1}\left|E_{b}\right| e_{1}\right\rangle+\left\langle e_{1}\left|E_{a}\left(\sum_{j=2}^{D^{2}} \lambda_{j}^{b-a-1}\left|e_{j}\right\rangle\left\langle e_{j}\right|\right) E_{b}\right| e_{1}\right\rangle \\
& =\left\langle\hat{O}_{a}\right\rangle\left\langle\hat{O}_{b}\right\rangle+\left\langle e_{1}\left|E_{a}\left(\sum_{j=2}^{D^{2}} \lambda_{j}^{b-a-1}\left|e_{j}\right\rangle\left\langle e_{j}\right|\right) E_{b}\right| e_{1}\right\rangle .
\end{aligned}
$$

The second terms governs the spatial decay of the correlation function. The correlation length $\zeta$ that quantifies the decay for $|b-a| \gg 1$ of the correlation function as

$$
\left|\left\langle\hat{O}_{a} \hat{O}_{b}\right\rangle-\left\langle\hat{O}_{a}\right\rangle\left\langle\hat{O}_{b}\right\rangle\right| \propto e^{-\zeta^{-1}|b-a|}
$$

is given by the second eigenvalue as

$$
\zeta^{-1}=-\log \left|\lambda_{2}\right|
$$

This shows that correlation functions of matrix product states decay exponentially in case the largest eigenvalue of the transfer operator is unique. This fact also indicates that matrix product states can efficiently represent ground states of gapped models because in gapped models the correlations functions decay exponentially. In case the largest eigenvalue of the transfer operator is not unique, i.e., $\lambda_{1}=\lambda_{2}$, we have

$$
\lim _{m \rightarrow \infty} E^{m}=\left|e_{1}\right\rangle\left\langle e_{1}|+| e_{2}\right\rangle\left\langle e_{2}\right|
$$


and for the correlation function

$$
\begin{aligned}
2\left\langle\hat{O}_{a} \hat{O}_{b}\right\rangle & =\operatorname{Tr}\left(E_{a} E^{b-a-1} E_{b}\left(\left|e_{1}\right\rangle\left\langle e_{1}|+| e_{2}\right\rangle\left\langle e_{2}\right|\right)\right) \\
& =\left\langle e_{1}\left|E_{a}\left(\left|e_{1}\right\rangle\left\langle e_{1}|+| e_{2}\right\rangle\left\langle e_{2}\right|+\left(\sum_{j=3}^{D^{2}} \lambda_{j}^{b-a-1}\left|e_{j}\right\rangle\left\langle e_{j}\right|\right)\right) E_{b}\right| e_{1}\right\rangle \\
& +\left\langle e_{2}\left|E_{a}\left(\left|e_{1}\right\rangle\left\langle e_{1}|+| e_{2}\right\rangle\left\langle e_{2}\right|+\left(\sum_{j=3}^{D^{2}} \lambda_{j}^{b-a-1}\left|e_{j}\right\rangle\left\langle e_{j}\right|\right)\right) E_{b}\right| e_{2}\right\rangle \\
& =\left\langle e_{1}\left|E_{a}\right| e_{1}\right\rangle\left\langle e_{1}\left|E_{b}\right| e_{1}\right\rangle+\left\langle e_{2}\left|E_{a}\right| e_{2}\right\rangle\left\langle e_{2}\left|E_{b}\right| e_{2}\right\rangle \\
& +\left\langle e_{1}\left|E_{a}\right| e_{2}\right\rangle\left\langle e_{2}\left|E_{b}\right| e_{1}\right\rangle+\left\langle e_{2}\left|E_{a}\right| e_{1}\right\rangle\left\langle e_{1}\left|E_{b}\right| e_{2}\right\rangle \\
& +\sum_{j=3}^{D^{2}} \lambda_{j}^{b-a-1}\left(\left\langle e_{1}\left|E_{a}\right| e_{j}\right\rangle\left\langle e_{j}\left|E_{b}\right| e_{1}\right\rangle+\left\langle e_{2}\left|E_{a}\right| e_{j}\right\rangle\left\langle e_{j}\left|E_{b}\right| e_{2}\right\rangle\right) .
\end{aligned}
$$

Thus, the correlation function has a constant long-range contribution $O_{\text {long-range }}$ that is independent of the distance of $a$ and $b$. The spatial decay for large $|b-a|$ is again exponential

$$
\left|\left\langle\hat{O}_{a} \hat{O}_{b}\right\rangle-\left\langle\hat{O}_{a}\right\rangle\left\langle\hat{O}_{b}\right\rangle-O_{\text {long-range }}\right| \propto e^{-\zeta^{-1}|b-a|}
$$

and the correlations length $\zeta$ is determined by the third eigenvalue $\lambda_{3}$ as

$$
\zeta^{-1}=-\log \left|\lambda_{3}\right|
$$

The generalization to $n$-times degeneracy of the largest eigenvalue, i.e. $\lambda_{1}=\ldots=\lambda_{n}$, is straight forward and also leads to constant long-range contribution. Thus, matrix product states can describe exponentially decaying and constant long-range correlation functions efficiently. However, matrix product states cannot describe algebraically decaying correlation functions, i.e.

$$
\left|\left\langle\hat{O}_{a} \hat{O}_{b}\right\rangle-\left\langle\hat{O}_{a}\right\rangle\left\langle\hat{O}_{b}\right\rangle\right| \propto|b-a|^{-\alpha}
$$

efficiently.

\subsubsection{Enhancing the spatial decay of correlation functions}

It is clear that the required maximal bond dimension $D$ for a representation of a manyparticle wave function as a matrix product state depends on the choice of the one-particle basis. A reordering of the one-particle states [Legeza and Sólyom, 2003] or the naturalorbital basis [Ma and Ma, 2013] have been used to reduce the required bond dimension of matrix product states. We propose to tailor a transformation of the one-particle basis such that two-point correlation functions of a state $|\Psi\rangle$, i.e. the one-particle reduced density matrix $\rho_{b, a}^{(1)}=\left\langle\Psi\left|\hat{c}_{a}^{\dagger} \hat{c}_{b}\right| \Psi\right\rangle$, decay much faster with distance and, hence, the state $|\Psi\rangle$ can be more efficiently represented as a matrix product state. The strongest localization is possible with a one-particle basis consisting of natural orbitals because then the oneparticle reduced density matrix is diagonal, i.e. $\rho_{b, a}^{(1)}=f_{a} \delta_{a, b}$. However, this choice has 
the drawback, that two-particle interaction that is localized to the first $N_{\text {int }}$ one-particle states in the original basis

$$
\hat{W}=\frac{1}{2} \sum_{\alpha, \beta, \gamma, \delta \leq N_{\text {int }}} U_{\alpha, \beta, \gamma, \delta} \hat{c}_{\alpha}^{\dagger} \hat{c}_{\beta}^{\dagger} \hat{c}_{\delta} \hat{c}_{\gamma}
$$

might become very long ranged in the natural-orbital basis, i.e.

$$
\hat{W}=\frac{1}{2} \sum_{\alpha, \beta, \gamma, \delta} \tilde{U}_{\alpha, \beta, \gamma, \delta} \hat{\tilde{c}}_{\alpha}^{\dagger} \hat{\tilde{c}}_{\beta}^{\dagger} \hat{\tilde{c}}_{\delta} \hat{\tilde{c}}_{\gamma}
$$

If applied to single-impurity Anderson models with large baths or quantum dots with large leads, one would obtain in the worst case $\in \mathcal{O}\left(L^{4}\right)$ non-zero elements in $\tilde{U}_{\alpha, \beta, \gamma, \delta}$. Thus, we instead propose to use the block-diagonal unitary transformation of the one-particle basis derived in section 8.2 that transforms a given one-particle reduced density matrix to the banded form given in Eq. (8.8). This transformation accomplishes the task of making the correlation functions more local while avoiding complications with the interaction Hamiltonian. The only remaining complication is that the one-particle Hamiltonian in the new one-particle basis can become non-local, i.e.,

$$
\hat{h}=\sum_{\alpha, \beta} \tilde{h}_{\alpha, \beta} \hat{\tilde{c}}_{\alpha}^{\dagger} \hat{\tilde{c}}_{\beta}
$$

We call the case of a transformation to a banded one-particle reduced density matrix the $\rho^{(1)}$-local-limit. On the other hand, one could also choose the unitary transformation so that the one-particle Hamiltonian is as local as possible. We call this the $h$-local limit. We suggest an interpolation between both limits: we could construct the unitary transformation of the one-particle basis such that both the one-particle reduced density matrix as well as the one-particle Hamiltonian have a banded form. However, then the required bandwidth will be larger than if only either one of the two is considered for the transformation. For the construction of a unitary transformation that transforms $\rho^{(1)}$ so that the matrix elements with indexes in the set $\bar{B}_{\rho}$ are zero and that transforms $h$ such that the matrix elements with indexes in the set $\bar{B}_{h}$ vanish, we can solve the minimization problem

$$
\begin{array}{r}
\min _{U \in U\left(2 L-N_{\mathrm{int}}\right)}\left\{\sum_{\{i, j\} \in B_{\rho}}\left|\left[\left(\begin{array}{cc}
\mathbb{1} & 0 \\
0 & U^{\dagger}
\end{array}\right) \rho^{(1)}\left(\begin{array}{cc}
\mathbb{1} & 0 \\
0 & U
\end{array}\right)\right]_{i, j}\right|\right. \\
\left.+\sum_{\{i, j\} \in B_{h}}\left|\left[\left(\begin{array}{cc}
\mathbb{1} & 0 \\
0 & U^{\dagger}
\end{array}\right) h\left(\begin{array}{cc}
\mathbb{1} & 0 \\
0 & U
\end{array}\right)\right]_{i, j}\right|\right\}
\end{array}
$$

over the space of $\left(2 L-N_{\text {int }}\right) \times\left(2 L-N_{\text {int }}\right)$ unitary matrices $U$. The locality of the twoparticle interaction is preserved by the block-diagonal structure of the unitary transform,

$$
\left(\begin{array}{cc}
\mathbb{1} & 0 \\
0 & U
\end{array}\right) \text {. }
$$

If the value of the minimum is zero, then such a transformation exists that transforms oneparticle density matrix and one-particle Hamiltonian to the required sparsity patterns. Because the transformed matrix elements of the one-particle Hamiltonian

$$
\tilde{h}=\left(\begin{array}{cc}
\mathbb{1} & 0 \\
0 & U^{\dagger}
\end{array}\right) h\left(\begin{array}{cc}
\mathbb{1} & 0 \\
0 & U
\end{array}\right)
$$


can have at most $\in \mathcal{O}\left(L^{2}\right)$ non-zero elements, we only consider the $\rho^{(1)}$-local limit in the following. For the application of matrix product states in the constrained minimization of the density-matrix functional we anyway have to evaluate all elements of the one-particle reduced density matrix to enforce all constraints.

The proposed idea is related to the transformation approach of Krumnow et al. [Krumnow et al., 2016]. Krumnow et al. iteratively construct local two-site unitary transformations of the one-particle basis. For the bond $i$ between sites $i$ and $i+1$ they optimize the unitary transform

$$
U_{i}=\left(\begin{array}{lll}
\mathbb{1}_{2 i-2} & & \\
& u_{i} & \\
& & \mathbb{1}_{2 L-2 i-4}
\end{array}\right)
$$

with $u_{i} \in U(2)$ such that the local bond dimension $d_{i}$ is minimal. They perform this transformation iteratively during a two-site DMRG-procedure where one sweeps trough the system from one end to the other. Thus, during one sweep from the left end to the right end they apply $L-1$ local unitary transformations of the one-particle basis of the type given in Eq. (8.79). With $\mathcal{O}\left(L^{2}\right)$ local transformations, one can in principle represent any global transformation $U \in U(2 L)$ of the one-particle basis. Thus, in Krumnow's approach $\mathcal{O}(L)$ sweeps would be required to be able to construct any global transformation of the one-particle basis. In contrast, we propose to directly construct the global transformation based on the one-particle reduced density matrix. Thus, Krumnow's approach can be seen as the bottom-up variant of a transformation approach while our proposal is a topdown variant. Our proposal can also be related to approaches that reorder the oneparticle basis [Legeza and Sólyom, 2003; Ma and Ma, 2013; Fertitta et al., 2014] to reduce the computational effort in DMRG calculations. Our approach goes beyond reorderings because we allow general unitary transformations of the one-particle basis. [Shirakawa and Yunoki, 2014] and [Büsser et al., 2013] transform the one-particle basis such that the matrix elements of the one-particle Hamiltonian form a banded matrix. This is identical to the $h$-local limit.

In the next section, we will explain additional basics of working with matrix product states. Section 8.8.4 covers how minimizations over matrix product states can be performed. In section 8.8.5 we will explain how the proposed transformation approach can be used during energy minimizations and also how it can be used for time-evolutions of many-particle states section 8.8.6.

\subsubsection{Operations with matrix product states}

In this section, we discuss how some important operations can be realized with matrix product states. For other operations and technical details, we refer the reader to [Schollwöck, 2011]. In section 8.8.1 we have described an approach to evaluate the expectation value of a local operator with a computational complexity $\in \mathcal{O}\left(D^{4}\right)$. However, this approach is only useful for introducing the transfer operators. In practice, one would contract 
the tensor network as

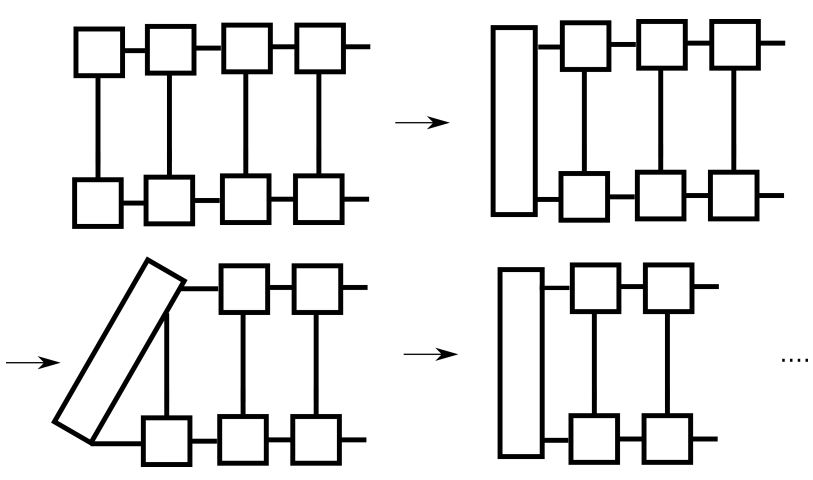

and obtain a computational complexity $\in \mathcal{O}\left(D^{3}\right)$. The matrices in a matrix product state are not uniquely defined by the many-particle wave function $|\Psi\rangle$. An invertible matrix $X$ can be introduced such that

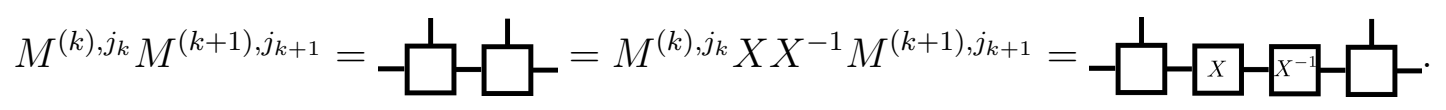

This way forms of MPS can be defined, that have particularly nice properties. These forms are called canonical forms of MPS. Left- and right canonical MPS result from the choice of $X$ such that all matrices are left- or right-normalized, i.e.,

$$
\sum_{j, i_{k-1}}\left(A^{(k), j}\right)_{\tilde{i}_{k}, i_{k-1}}^{\dagger} A_{i_{k-1}, i_{k}}^{(k), j}=\delta_{\tilde{i}_{k}, i_{k}}
$$

for left-normalized matrices $A^{(k)}$ and

$$
\sum_{j, i_{k}} B_{i_{k-1}, i_{k}}^{(k), j}\left(B^{(k), j}\right)_{i_{k}, \tilde{i}_{k-1}}^{\dagger}=\delta_{i_{k-1}, \tilde{i}_{k-1}}
$$

for left-normalized matrices $B^{(k)}$. A left- or right canonical MPS can be generated from a general MPS with repeated applications of singular value decompositions or QR decompositions. The detailed algorithm is discussed for example in [Schollwöck, 2011]. An MPS of the form

$$
|\Psi\rangle=\sum_{j_{1}, \ldots, j_{L}} A^{(1), j_{1}} \cdots A^{(l-1), j_{l-1}} M^{(l), j_{l}} B^{(l+1), j_{l+1}} \cdots B^{(L), j_{L}}\left|j_{1}, \ldots, j_{L}\right\rangle
$$

is in a mixed canonical form because it contains left-normalized and right-normalized matrices as well as a non-normalized matrix $M^{(l), j_{l}}$.

The efficient application of an operator $\hat{O}$ to a many-particle wave function in a matrix product state representation requires the concept of matrix product operators (MPO, [Verstraete et al., 2004; Zwolak and Vidal, 2004]). A matrix product representation of an operator

$$
\hat{O}=\sum_{j_{1}, \ldots, j_{L}, j_{1}^{\prime}, \ldots, j_{L}^{\prime}}\left|j_{1}, \ldots, j_{L}\right\rangle\left\langle j_{1}, \ldots, j_{L}|\hat{O}| j_{1}^{\prime}, \ldots, j_{L}^{\prime}\right\rangle\left\langle j_{1}^{\prime}, \ldots, j_{L}^{\prime}\right|
$$

with

$$
\left\langle j_{1}, \ldots, j_{L}|\hat{O}| j_{1}^{\prime}, \ldots, j_{L}^{\prime}\right\rangle=O^{(1), j_{1}, j_{1}^{\prime}} O^{(2), j_{2}, j_{2}^{\prime}} \ldots O^{(L-1), j_{L-1}, j_{L-1}^{\prime}} O^{(L), j_{L}, j_{L}^{\prime}}
$$


is very similar to a matrix product state in Eq. (8.29) in the sense that the single physical index $j_{i}$ in an MPS is replaced by a combined index $j_{i}, j_{i}^{\prime}$ in an MPO. The individual tensors in the MPO can be represented graphically as

$$
\begin{aligned}
& O_{i_{1}}^{(1), j_{1}, j_{1}^{\prime}}=\square_{j_{1}^{\prime}}^{j_{1}} i_{i_{1}} \\
& O_{i_{1}, i_{2}}^{(2), j_{2}, j_{2}^{\prime}}={ }_{i_{1}}^{j_{j_{2}}^{\prime}} i_{i_{2}} .
\end{aligned}
$$

The application of an MPO to an MPS, i.e. $\hat{O}|\Psi\rangle$, can be written as

$$
\begin{aligned}
\hat{O}|\Psi\rangle & =\sum_{j_{1}, \ldots, j_{L}, j_{1}^{\prime}, \ldots, j_{L}^{\prime}} O^{(1), j_{1}, j_{1}^{\prime}} O^{(2), j_{2}, j_{2}^{\prime}} \cdots O^{(L), j_{L}, j_{L}^{\prime}} M^{(1), j_{1}^{\prime}} M^{(2), j_{2}^{\prime}} \cdots M^{(L), j_{L}^{\prime}}\left|j_{1}, \ldots, j_{L}\right\rangle \\
& =\sum_{j_{1}, \ldots, j_{L}, j_{1}^{\prime}, \ldots, j_{L}^{\prime}} \sum_{i_{1}=1}^{d_{1}^{\prime}} \sum_{k_{1}=1}^{d_{1}} \ldots \sum_{i_{L}=1}^{d} \sum_{k_{L}=1}^{d_{L}} O_{i_{1}}^{(1), j_{1}, j_{1}^{\prime}} M_{k_{1}}^{(1), j_{1}^{\prime}} O_{i_{1}, i_{2}}^{(2), j_{2}, j_{2}^{\prime}} M_{k_{1}, k_{2}}^{(2), j_{2}^{\prime}} \cdots\left|j_{1}, \ldots, j_{L}\right\rangle \\
& =\sum_{j_{1}, \ldots, j_{L}} N^{(1), j_{1}} \cdots N^{(L), j_{l}}\left|j_{1}, \ldots, j_{L}\right\rangle
\end{aligned}
$$

or graphically represented as the contractions

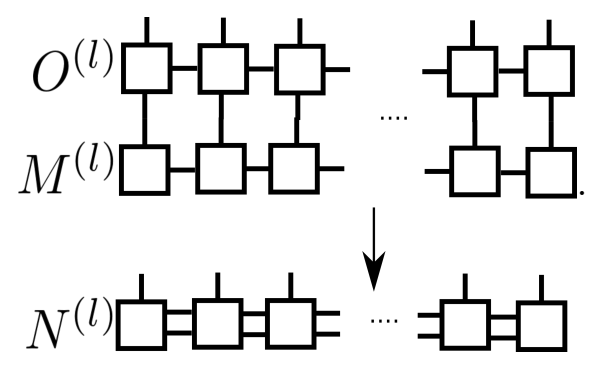

$d_{l}^{\prime}$ are the bond dimensions of the MPOs. The new site matrices $N^{(l)}$

$$
N_{\left(i_{l-1}, k_{l-1}\right),\left(i_{l}, k_{l}\right)}^{(l), j_{l}}=\sum_{j_{l}^{\prime}} O_{i_{l-1}, i_{l}}^{(l), j_{l}, j_{l}^{\prime}} M_{k_{l-1}, k_{l}}^{(l), j_{l}^{\prime}}
$$

are in $\mathbb{C}^{d_{l} d_{l}^{\prime} \times d_{l-1} d_{l-1}^{\prime}}$. Thus, the application of an MPO to an MPS increases the bond dimension drastically, and the question arises if a given MPS can be approximated by an MPS with a smaller bond dimension. This procedure has been termed the compression of an MPS. Several approaches are available to achieve this tasks, and the basic idea will be discussed in the context of two-site DMRG in section 8.8.4.

\subsubsection{Minimization over matrix product states}

Apart from the calculation of expectation values and the application of operators, especially the solution of the minimization problem

$$
E_{0}=\min _{|\Psi\rangle} \frac{\langle\Psi|\hat{H}| \Psi\rangle}{\langle\Psi \mid \Psi\rangle}
$$


is important. If the many-particle wave function is parametrized as a matrix product state, i.e.

$$
|\Psi\rangle=\sum_{j_{1}, \ldots, j_{L} \in\{0, \uparrow, \downarrow, \uparrow \downarrow\}} M^{(1), j_{1}} M^{(2), j_{2}} \cdots M^{(L), j_{L}}\left|j_{1}, \ldots, j_{L}\right\rangle,
$$

we are faced with a highly non-linear minimization problem. To avoid this non-linearity, the tensors $M^{(i)}$ are changed iteratively: in the first step only $M^{(1)}$ is varied and the others kept fixed, in the second step only $M^{(2)}$ is changed and so on. This yields the single-site DMRG procedure. Computationally it is much simpler to consider a mixed-canonical representation of an MPS than the non-canonical for in Eq. (8.94) for the discussion of the local minimization problem. Thus, we assume that the MPS is in the mixed-canonical form

$$
|\Psi\rangle=\sum_{j_{1}, \ldots, j_{L}} A^{(1), j_{1}} \cdots A^{(l-1), j_{l-1}} M^{(l), j_{l}} B^{(l+1), j_{l+1}} \cdots B^{(L), j_{L}}\left|j_{1}, \ldots, j_{L}\right\rangle
$$

and we consider the local minimization corresponding to the site $l$. The local matrixproduct operator of an operator $\hat{H}$ can be represented as

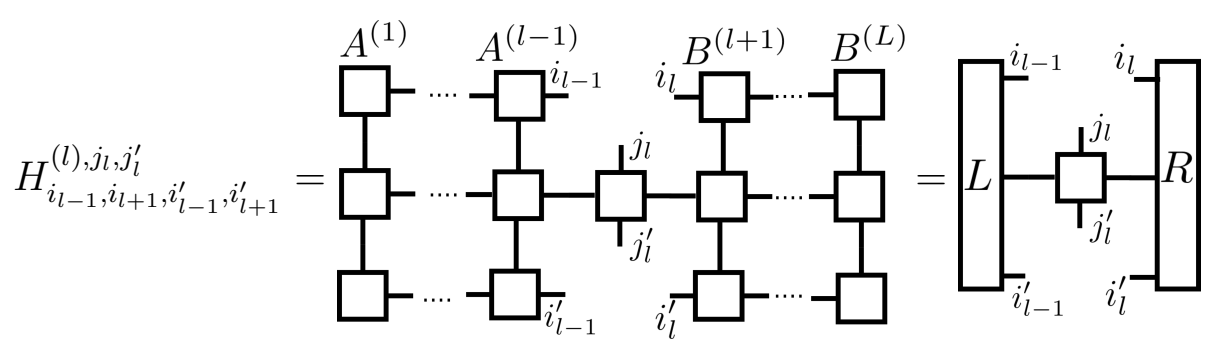

with the right environment block $R$ and the left environment block $L$. The expectation value $\langle\Psi|\hat{H}| \Psi\rangle$ can then be written as

$\langle\Psi|\hat{H}| \Psi\rangle=\sum_{j_{l}, j_{l}^{\prime}} \sum_{k_{l-1}, k_{l+1}} \sum_{i_{l-1}, i_{l-1}^{\prime}} \sum_{i_{l+1}, i_{l+1}^{\prime}} L_{i_{l-1}, i_{l-1}^{\prime}, k_{l-1}}^{(l)} W_{k_{l-1}, k_{l+1}}^{(l), j_{l}, j_{l}^{\prime}} R_{i_{l+1}, i_{l+1}^{\prime}, k_{l+1}}^{(l)}\left(M_{i_{l-1}, i_{l+1}}^{(l), j_{l}}\right)^{*} M_{i_{l-1}^{\prime}, i_{l+1}^{\prime}}^{(l), j_{l}^{\prime}}$.

To perform the minimization of Eq. (8.97) with respect to the elements of the matrix $M_{i_{l-1}^{\prime}, i_{l+1}^{\prime}}^{(l), j_{l}^{\prime}}$, the derivatives with respect to $\left(M_{i_{l-1}, i_{l+1}}^{(l), j_{l}}\right)^{*}$

$$
\begin{aligned}
& =\sum_{j_{l}^{\prime}} \sum_{k_{l-1}, k_{l+1}} \sum_{i_{l-1}^{\prime}, i_{l+1}^{\prime}} L_{i_{l-1}, i_{l-1}^{\prime}, k_{l-1}}^{(l)} W_{k_{l-1}, k_{l+1}}^{(l), j_{l}, j_{l}^{\prime}} R_{i_{l+1}, i_{l+1}^{\prime}, k_{l+1}}^{(l)} M_{i_{l-1}^{\prime}, i_{l+1}^{\prime}}^{(l), j_{l}^{\prime}} \\
= & \sum_{j_{l}^{\prime}} \sum_{i_{l-1}^{\prime}, i_{l+1}^{\prime}} H_{i_{l-1}, i_{l+1}, i_{l-1}^{\prime}, i_{l+1}^{\prime}}^{(l), j_{l}, j_{l}^{\prime}} M_{i_{l-1}^{\prime}, i_{l+1}^{\prime}}^{(l), j_{l}^{\prime}}
\end{aligned}
$$

and the derivatives of the norm $\langle\Psi \mid \Psi\rangle$

$$
\square^{(1)} \cdots \cdots-\sum_{j_{l}^{\prime}}^{A^{(l-1)}} \sum_{i_{l-1}^{\prime}, i_{l+1}^{\prime}} N_{i_{l-1}, i_{l+1}, i_{l-1}^{\prime}, i_{l+1}^{\prime}}^{(l), i_{l}, j^{\prime}} M_{i_{l-1}^{\prime}, i_{l+1}^{\prime}}^{(l), j_{l}^{\prime}}
$$


are formed. The minimization problem in Eq. (8.93) can equivalently be written as a constrained minimization problem

$$
E_{0}=\min _{|\Psi\rangle:\langle\Psi \mid \Psi\rangle=1}\langle\Psi|\hat{H}| \Psi\rangle
$$

and, hence, the normalization constraint $\langle\Psi \mid \Psi\rangle=1$ can be treated with Lagrange multipliers. The Lagrange function is

$$
L(|\Psi\rangle, \lambda)=\langle\Psi|\hat{H}| \Psi\rangle-\lambda(\langle\Psi \mid \Psi\rangle-1)
$$

or

$$
L\left(\left\{M^{(i)}\right\}, \lambda\right)=\left\langle\Psi\left(\left\{M^{(i)}\right\}\right)|\hat{H}| \Psi\left(\left\{M^{(i)}\right\}\right)\right\rangle-\lambda\left(\left\langle\Psi\left(\left\{M^{(i)}\right\}\right) \mid \Psi\left(\left\{M^{(i)}\right\}\right)\right\rangle-1\right)
$$

if $|\Psi\rangle$ is represented as a matrix product state. In an iterative minimization the stationarity conditions of the Lagrange function are solved for a single matrix $M^{(l)}$ while the other matrices are kept fixed. The stationarity conditions for a single matrix $M^{(l)}$ are the norm condition

$$
1=\left\langle\Psi\left(\left\{M^{(i)}\right\}\right) \mid \Psi\left(\left\{M^{(i)}\right\}\right)\right\rangle,
$$

which can be satisfied easily, as well as the condition

$$
0=\frac{\partial L\left(\left\{M^{(i)}\right\}, \lambda\right)}{\partial\left(M_{i_{l-1}, i_{l+1}}^{(l), j_{l}}\right)^{*}}=\sum_{j_{l}^{\prime}} \sum_{i_{l-1}^{\prime}, i_{l+1}^{\prime}}\left(H_{i_{l-1}, i_{l+1}, i_{l-1}^{\prime}, i_{l+1}^{\prime}}^{(l), j_{l}, j_{l}^{\prime}}-\lambda N_{i_{l-1}, i_{+1}, i_{l-1}^{\prime}, i_{l+1}^{\prime}}^{(l), j_{l}, j_{l}^{\prime}}\right) M_{i_{l-1}^{\prime}, i_{l+1}^{\prime}}^{(l), j_{l}^{\prime}}
$$

With the definitions

$$
\begin{aligned}
H_{\left(j_{l}, i_{l-1}, i_{l+1}\right),\left(j_{l}^{\prime}, i_{l-1}^{\prime}, i_{l+1}^{\prime}\right)} & =H_{i_{l-1}, i_{l+1}, i_{l-1}^{\prime}, i_{l+1}^{\prime}}^{(l), j_{l}, j_{l}^{\prime}} \\
N_{\left(j_{l}, i_{l-1}, i_{l+1}\right),\left(j_{l}^{\prime}, i_{l-1}^{\prime}, i_{l+1}^{\prime}\right)} & =N_{i_{l-1}, i_{l+1}, i_{l-1}^{\prime}, i_{l+1}^{\prime}}^{(l) j_{l}^{\prime},} \\
M_{\left(j_{l}^{\prime}, i_{l-1}^{\prime}, i_{l+1}^{\prime}\right)} & =M_{i_{l-1}^{\prime}, i_{l+1}^{\prime}}^{(l), j_{l}^{\prime}},
\end{aligned}
$$

the condition in Eq. (8.105) is a hermitian generalized eigenvalue problem

$$
0=H \vec{M}-\lambda N \vec{M}
$$

of dimension $d d_{l-1} d_{l+1} \in \mathcal{O}\left(D^{2}\right)$. If in Eq. (8.95) $A^{(i)}$ are left-normalized and $B^{(i)}$ rightnormalized, we have

$$
N_{\left(j_{l}, i_{l-1}, i_{l+1}\right),\left(j_{l}^{\prime}, i_{l-1}^{\prime}, i_{l+1}^{\prime}\right)}=\delta_{j_{l}, j_{l}^{\prime}} \delta_{i_{l-1}, i_{l-1}^{\prime}} \delta_{i_{l+1}, i_{l+1}^{\prime}}
$$

and hence the hermitian eigenvalue problem

$$
0=H \vec{M}-\lambda \vec{M}
$$

instead of a generalized eigenvalue problem. The eigenvalue problem, Eq. (8.109) or Eq.(8.111), can be solved by an iterative eigensolver like the Lanczos procedure or the Jacobi-Davidson algorithm. The iterative minimization repeatedly sweeps trough the system, i.e. performs local minimizations $M^{(1)} \rightarrow M^{(2)} \rightarrow \ldots \rightarrow M^{(L)} \rightarrow M^{(L-1)} \rightarrow \ldots \rightarrow$ 
$M^{(1)}$, till the ground state is reached. The single-site minimization procedure described so far is prone by local minima. Also, the bond dimensions of the matrices $M^{(l)}$ are fixed and cannot grow or shrink depending on the structure of the many-particle wave function. Thus, a two-site procedure is preferred as it naturally enlarges the ansatz space and starts from

$$
|\Psi\rangle=\sum_{j_{1}, \ldots, j_{L}} A^{(1), j_{1}} \cdots A^{(l-1), j_{l-1}} M^{(l), j_{l}} M^{(l+1), j_{l+1}} B^{(l+2), j_{l+2}} \cdots B^{(L), j_{L}}\left|j_{1}, \ldots, j_{L}\right\rangle .
$$

Instead of a minimization over a single matrix $M^{(l), j_{l}}$, the minimization is performed over the contraction of $M^{(l), j_{l}}$ and $M^{(l+1), j_{l+1}}$,

$$
P^{(l), j_{l}, j_{l+1}}=M^{(l), j_{l}} M^{(l+1), j_{l+1}},
$$

which has $d_{l-1} d_{l+1} d^{2}$ elements. The local matrix-product representation of the operator $\hat{H}$ is

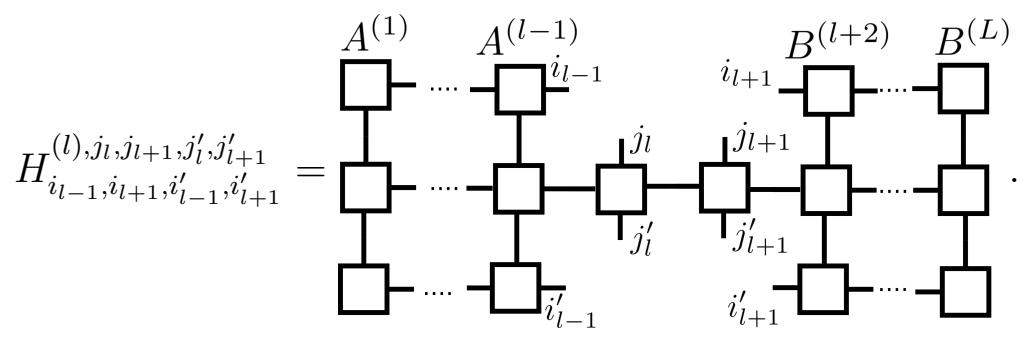

$j_{l}$ and $j_{l+1}$ can be combined into a new index $J_{l} \in\left\{1, \ldots, d^{2}\right\}$ such that $H_{i_{l-1}, i_{l+2}, i_{l-1}^{\prime}, i_{l+2}^{\prime}}^{(l), j_{l}, i_{l+1}, j_{l}^{\prime} j_{l}^{\prime}}$ obtains the form $H_{i_{l-1}, i_{l+2}, i_{l-1}^{\prime}, i_{l+2}^{\prime}}^{(l), J_{l}, J^{\prime}}$ and the minimization over $P^{(l)}$ results in an eigenvalue problem

$$
0=H \vec{P}-\lambda \vec{P}
$$

similarly to the single-site DMRG procedure. With the eigenvector $\vec{P}$ to the lowest eigenvalue, the many-particle wave function has the form

$$
|\Psi\rangle=\sum_{j_{1}, \ldots, j_{L}} A^{(1), j_{1}} \cdots A^{(l-1), j_{l-1}} P^{(l), j_{l}, j_{l+1}} B^{(l+2), j_{l+2}} \cdots B^{(L), j_{L}}\left|j_{1}, \ldots, j_{L}\right\rangle
$$

and is not in the conventional MPS-form. However, it can be brought to a suitable form by first reshaping $P^{(l), j_{l}, j_{l+1}}$ as the matrix

$$
P_{\left(j_{l}, i_{l-1}\right),\left(j_{l+1}, i_{l+1}\right)}^{(l)}
$$

and then applying the singular value decomposition (SVD) to obtain

$$
P_{\left(j_{l}, i_{l-1}\right),\left(j_{l+1}, i_{l+1}\right)}^{(l)}=\sum_{i_{l}} U_{\left(j_{l}, i_{l-1}\right), i_{l}} S_{i_{l}}\left(V^{\dagger}\right)_{i_{l},\left(j_{l+1}, i_{l+1}\right)} .
$$

$S_{i_{l}}$ are the singular values of $P_{\left(j_{l}, i_{l-1}\right),\left(j_{l+1}, i_{l+1}\right)}^{(l)} \cdot U_{\left(j_{l}, i_{l-1}\right), i_{l}} S_{i_{l}}$ can be reshaped to $M_{\left.i_{l-1}\right), i_{l}}^{(l), j_{l}}$ and $\left(V^{\dagger}\right)_{i_{l},\left(j_{l+1}, i_{l+1}\right)}$ can be reshaped to $B_{i_{l}, i_{l+1}}^{(l+1), j_{l+1}}$ so that

$$
P_{i_{l-1}, i_{l+1}}^{(l), j_{l}, j_{l+1}}=\sum_{i_{l}} M_{i_{l-1}, i_{l}}^{(l), j_{l}} B_{i_{l}, i_{l+1}}^{(l+1), j_{l+1}}
$$


Thus, the two-site tensor $P^{(l), j_{l}, j_{l+1}}$ can be decomposed into two single-site tensors and, hence, we obtain again a conventional form of the MPS

$$
|\Psi\rangle=\sum_{j_{1}, \ldots, j_{L}} A^{(1), j_{1}} \cdots v A^{(l-1), j_{l-1}} M^{(l), j_{l}} B^{(l+1), j_{l+1}} B^{(l+2), j_{l+2}} \cdots B^{(L), j_{L}}\left|j_{1}, \ldots, j_{L}\right\rangle .
$$

The local minimization of the expectation value $\langle\Psi|\hat{H}| \Psi\rangle$ on the bond $l$ over the elements $P_{\left(j_{l}, i_{l-1}\right),\left(j_{l+1}, i_{l+1}\right)}^{(l)}$ considers a larger variational space than the minimization over a single matrix $M^{(l)}$ in the single-site DMRG procedure. The subsequent decomposition of $P^{(l), j_{l}, j_{l+1}}$ into single-site tensors in Eq. (8.119) can be used to judge if the bond dimension of the bond between sites $l$ and $l+1$ is sufficiently large. The number of non-zero singular values $S_{i_{l}}$ of the decomposition of $P_{\left(j_{l}, i_{l-1}\right),\left(j_{l+1}, i_{l+1}\right)}^{(l)}$ in Eq. (8.118) determines the required bond dimension. If case one is only interested in an approximate ground state or if the required bond dimension for a numerically exact solution would exceed the available computational resources, some small non-zero singular values can be neglected. The truncation error of the bond can be defined as

$$
\epsilon=\sum_{i \in \text { neglected }} S_{i}^{2}
$$

where $S_{i}$ are the singular values in the decomposition in Eq. (8.118). The error introduced into the many-particle wave function $|\Psi\rangle$ from the truncation is in the worst case [Verstraete and Cirac, 2006; Schollwöck, 2011]

$$
\||\Psi\rangle-\left|\Psi_{\text {trunc }}\right\rangle \|_{2}^{2} \leq 2 \epsilon .
$$

An alternative way is to limit the maximal bond dimension and judge the quality of the MPS by the maximal truncation error of all bonds.

\subsubsection{ACA-transformation for ground-state calculations}

We consider the situation where the ground state of the Hamiltonian

$$
\hat{H}=\sum_{\alpha, \beta} h_{\alpha, \beta} \hat{c}_{\alpha}^{\dagger} \hat{c}_{\beta}+\frac{1}{2} \sum_{\alpha, \beta, \gamma, \delta \leq N_{\text {int }}} U_{\alpha, \beta, \gamma, \delta} \hat{c}_{\alpha}^{\dagger} \hat{c}_{\beta}^{\dagger} \hat{c}_{\delta} \hat{c}_{\gamma}
$$

is to be found by means of a DMRG ground-state search. We assume that $N_{\text {int }} \ll 2 L$ as in the case of a single-impurity Anderson model or quantum dots. For the reduction of the required bond dimension in the ground-state calculation, we propose the following approach: we first perform a ground-state calculation with a relatively small maximal bond dimension. This calculation is fast and serves the purpose of obtaining a rough estimate $\rho^{(1), \approx}$ of the one-particle reduced density matrix of the ground state. The estimated one-particle reduced density matrix can then be used to construct a new one-particle basis in which the approximate one-particle reduced density matrix $\rho^{(1), \approx}$ has a banded form as in Eq. (8.8). The construction algorithm of the unitary transformation $U$ that achieves this task is given in section 8.2 and in appendix B.2. After the transformation has been constructed, we transform the matrix elements of the one-particle Hamiltonian into the new basis, i.e.,

$$
\tilde{h}=U^{\dagger} h U
$$


The new many-particle Hamiltonian for the ground-state search has the form

$$
\hat{H}=\sum_{\alpha, \beta} \tilde{h}_{\alpha, \beta} \hat{\tilde{c}}_{\alpha}^{\dagger} \hat{\tilde{c}}_{\beta}+\frac{1}{2} \sum_{\alpha, \beta, \gamma, \delta \leq N_{\mathrm{int}}} U_{\alpha, \beta, \gamma, \delta} \hat{\tilde{c}}_{\alpha}^{\dagger} \hat{\tilde{c}}_{\beta}^{\dagger} \hat{\tilde{c}}_{\delta} \hat{\tilde{c}}_{\gamma} .
$$

Please note, that $\hat{\tilde{c}}_{\alpha}^{(\dagger)}=\hat{c}_{\alpha}^{(\dagger)}$ for $\alpha \leq N_{\text {int }}$ by construction. The final DMRG ground-state calculation is performed in the transformed one-particle basis. An MPS-DMRG-code only needs to support the calculation of all two-point correlation functions, $\rho_{\beta, \alpha}^{(1)}=\left\langle\hat{c}_{\alpha}^{\dagger} \hat{c}_{\beta}\right\rangle$, for a given state in an MPS representation and the solution of the minimization of the total energy for an arbitrary one-particle Hamiltonian in order to be able to apply this scheme. We evaluate the proposed approach with the single-impurity Anderson model defined in section 3.2 by Eq. (3.2)-Eq. (3.7). We choose a total system size of $L_{\text {bath }}+1=40$ sites, a filling with $N_{e}=40$ electrons, $t>0, U / t=2, \epsilon_{f}=0$ and $V / t=0.4$. The results of the calculations performed with ITensor $^{2}$ are summarized in the following table:

\begin{tabular}{l|l|l|l|l} 
one-particle basis & original & original & $\rho^{(1), \approx}$ banded & $\rho^{(1), \approx^{\prime} \text { banded }}$ \\
\hline maximal bond dim. of MPS & 186 & 30 & 98 & 57 \\
\hline maximal bond dim. of MPO & 6 & 6 & 80 & 82 \\
\hline truncation error & $10^{-10}$ & $5 \cdot 10^{-6}$ & $10^{-10}$ & $10^{-10}$ \\
\hline deviation of energy & 0 (reference) & $0.00023 t$ & $<10^{-8} t$ & $<10^{-8} t$ \\
\hline runtime per sweep/s & 7.8 & 3.3 & 3.9 & 3.7 \\
\hline $\begin{array}{l}\text { resulting one-particle } \\
\text { reduced density matrix }\end{array}$ & & $\rho^{(1), \approx}$ & $\rho^{(1), \approx^{\prime}}$ &
\end{tabular}

The second column lists details of a reference calculation in the original one-particle basis, where the bond dimensions of the MPS have been allowed to grow till a required truncation error of $10^{-10}$ was achieved. This resulted in a maximal bond dimension of 186 . We use the ground-state energy obtained with this calculation as the reference value. The initial DMRG calculation with a small maximal bond dimension of 30 listed in the third column produced a truncation error of $5 \cdot 10^{-6}$. The one-particle reduced density matrix $\rho^{(1)} \approx$ that resulted from this fast calculation was used to generate a new one-particle basis. The calculation in the new one-particle basis only required a maximal bond dimension of 98 to reach a truncation error of $10^{-10}$. We call the resulting one-particle reduced density matrix $\rho^{(1), \approx^{\prime}}$. The bond dimension required in the transformed one-particle basis is only about half of the bond dimension of the MPS in the original basis with the same truncation error. The maximal bond dimension of the MPO depends on the one-particle basis because the matrix elements of the one-particle Hamiltonian depend on the oneparticle basis. The transformation of the one-particle basis increases the bond dimensions of the MPO of the Hamiltonian, because there are only $\in \mathcal{O}(L)$ non-zero matrix elements of one-particle Hamiltonian, Eq. (3.3) and Eq. (3.7), in the original one-particle basis of the single-impurity Anderson model investigated here, but there are $\in \mathcal{O}\left(L^{2}\right)$ non-zero matrix elements of the one-particle Hamiltonian in the transformed basis. However, the speedup of the computational runtime per sweep listed in the above table shows that the reduction of the bond dimensions of the MPS outweighs the increase of the bond dimensions of the MPO. We can also iterate this scheme and use the one-particle reduced density matrix $\rho^{(1), \approx^{\prime}}$ from the calculation in the already transformed one-particle basis

\footnotetext{
${ }^{2}$ ITensor C++ library (version 2.1.1), http://itensor.org/
} 
to create a one-particle basis in which $\rho^{(1), \approx^{\prime}}$ is banded. A ground state calculation in this one-particle basis requires an even smaller bond dimension of 57 to reach a truncation error of $10^{-10}$. Even though the second iteration is of less practical importance here, because the numerically exact ground state was already obtained in the first transformed oneparticle basis, one could set up a scheme with multiple transformation steps interleaved with DMRG-ground-state calculations with decreasing truncation errors.

The transformation approach, either with a single transformation or multiple transformations, presented in this section drastically reduces the required bond dimension to reach a given truncation error in DMRG ground state calculations for the class of manyparticle Hamiltonians in Eq. (8.123). The growth of the bond dimension of the MPOs only moderately increases the computation time per sweep.

\subsubsection{ACA-transformation for time-evolutions}

The approach proposed in the previous section does not allow to reuse the MPS from the original one-particle basis after the transformation of the one-particle basis. If we assume that the MPS-code can apply arbitrary single-site or two-site unitary operators to an MPS, we can also transform the MPS to a different one-particle basis. This would extend the proposed approach to time evolutions and other situations where the MPS needs to be preserved by the transformation of the one-particle basis.

As an example we consider the case of a real-time evolution of a many-particle state in an MPS-representation, i.e.,

$$
|\Psi(t)\rangle=e^{i \hat{H} t / \hbar}|\Psi(t=0)\rangle
$$

We propose to following computational protocol:

1. The time evolution is performed for the time interval $\Delta$

$$
|\Psi(t+\Delta)\rangle=e^{i \hat{H} \Delta / \hbar}|\Psi(t)\rangle
$$

2. Then the one-particle reduced density matrix

$$
\rho_{\alpha, \beta}^{(1)}(t+\Delta)=\left\langle\Psi(t+\Delta t)\left|\hat{c}_{\beta}^{\dagger} \hat{c}_{\alpha}\right| \Psi(t+\Delta)\right\rangle
$$

of the state $|\Psi(t+\Delta)\rangle$ is computed. A unitary transformation $U$ of the one-particle basis is constructed such that the one-particle reduced density matrix of the state $|\Psi(t+\Delta)\rangle$ in the transformed basis is in a banded form.

3. The MPS-representation of $|\Psi(t+\Delta)\rangle$ is only known with respect to the old oneparticle basis. The MPS-representation with respect to the transformed one-particle basis can be obtained by performing the steps:

a) At first the unitary transform $U$ is decomposed into a product

$$
U=U_{1} U_{2} \cdots U_{M}
$$


of at most $M \leq 2 L \cdot(2 L-1) / 2$ two-level unitary matrices $U_{i} \in S U(2 L)$ of the form

$$
U_{i}=\left(\begin{array}{ccccccc}
1 & & & & & & \\
& \ddots & & & & & \\
& & 1 & & & & \\
& & & u_{i} & & & \\
& & & & 1 & & \\
& & & & & \ddots & \\
& & & & & & 1
\end{array}\right) .
$$

with $u_{i} \in S U(2)$. A numerical procedure for this decomposition was given by $\mathrm{Li}$ et al. [Li et al., 2013] in the context of the decomposition of quantum gates. Figure 8.7 schematically shows the resulting pattern of two-level unitary matrices. In the general case where the unitary transform $U$ can mix all sites and all spin channels, the local unitary transformations $u_{i}$ either act on a single site or on the spin-up state of one site and the spin-down state of the right-neighbor site. In the case, where the one-particle reduced density matrix $\rho_{\alpha, \beta}^{(1)}(t+\Delta)$ is collinearly spin-polarized or non-spin-polarized, i.e.

$$
\rho_{i, \uparrow, j, \downarrow}^{(1)}(t+\Delta)=0 \forall i \text { and } j,
$$

the unitary transformation of the one-particle basis can be constructed for every spin-channel independently and fulfills

$$
U_{i, \uparrow, j, \downarrow}=0 \forall i \text { and } j \text {. }
$$

Thus, in total only $2 L(L-1) / 2$ two-level unitary matrices are required and the local unitary transformations $u_{i}$ act on the spin-up (-down) state of one site and the spin-up (-down) state of a neighboring site. The computational effort for the construction of the decomposition is $\in \mathcal{O}\left(L^{3}\right)$.

b) The local two-level unitary transformations $U_{i}$ of the one-particle basis are now translated into local unitary operators $\hat{\tilde{U}}_{i}$ of the many-particle basis. We consider here the case where the unitary transformation $U$ acts on each spinchannel independently. The operator that performs the local transformation of the $\sigma$-spin-channel of the one-particle basis in the many-particle wave functions can be chosen as

$$
\begin{aligned}
\hat{\tilde{U}}_{i, \sigma} & =u_{i, \sigma, 11} \hat{n}_{a(i), \sigma}+\hat{c}_{a(i), \sigma} \hat{c}_{a(i), \sigma}^{\dagger}+\left(u_{i, \sigma, 22}-1\right) \hat{n}_{a(i)+1, \sigma} \\
& +u_{i, \sigma, 12} \hat{c}_{a(i), \sigma}^{\dagger} \hat{c}_{a(i)+1, \sigma}+u_{i, \sigma, 21} \hat{c}_{a(i)+1, \sigma}^{\dagger} \hat{c}_{a(i), \sigma} \\
& +\left(2-u_{i, \sigma, 11}-u_{i, \sigma, 22}\right) \hat{n}_{a(i), \sigma} \hat{n}_{a(i)+1, \sigma} .
\end{aligned}
$$

$a(i)$ denotes the first site that the local two-level unitary transformations $U_{i}$ non-trivially acts on.

c) The representation of the many-particle wave function is then transformed to the new one-particle basis with successive applications of the MPOs of the 
unitary operators $\hat{\tilde{U}}_{i}$ to the MPS. After this step, the state $|\Psi(t+\Delta)\rangle$ is represented as an MPS with respect to the transformed one-particle basis. The bond dimensions and the entanglement entropies should be significantly smaller than for the untransformed MPS. The computational effort for this step is quadratic in system size because there are $\mathcal{O}\left(L^{2}\right)$ operators to be applied.

4. The matrix elements of the one-particle Hamiltonian in the transformed one-particle basis have to be calculated with $\tilde{h}=U h U^{\dagger}$. This step is cubic in system size because of the matrix multiplication.

5. The time evolution can now proceed with the transformed MPS-representation of the state $|\Psi(t+\Delta)\rangle$ and with the representation of the Hamiltonian $\hat{H}$ in the transformed one-particle basis.

This scheme can also be applied to imaginary time evolutions, during sweeps of DMRGlike ground-state calculation or other operations involving MPS. This approach requires that the interaction Hamiltonian acts only on $N_{\text {int }} \ll L$ one-particle states. These states are not transformed, and, hence, the bond dimension of the MPO-representation of the Hamiltonian $H$ originates only from the one-particle Hamiltonian. This scheme is, for example, applicable to quantum dots with an arbitrary number of leads as well as impurity models in arbitrary dimensions and with arbitrary bath geometries. Thus, it could be a valuable tool to study the dynamics of quantum quenches and transport through quantum dots.

As an example, we demonstrate the proposed algorithm for the time-evolution of the Néel-state on a 16-site system with the Hamiltonian

$$
\hat{H}=-T \sum_{<i, j>} \sum_{\sigma} \hat{c}_{i, \sigma}^{\dagger} \hat{c}_{j, \sigma}+U \hat{n}_{1, \uparrow} \hat{n}_{1, \downarrow}
$$

with $U / T=1^{3}$. We have implemented the approach proposed in this section into the MPS-library ITensor ${ }^{4}$. ITensor performs the time evolution by constructing the unitary operator $e^{i \hat{H} \tau / \hbar}$ up to terms of order $\tau^{2}$ [Zaletel et al., 2015] for arbitrary long-ranged Hamiltonians $\hat{H}$. We have used a time evolution step of $\tau=0.01 \hbar / T$ and transformed the one-particle basis every $\Delta=0.25 \hbar / T$. We did not limit the maximal bond dimension of the MPS during the time evolution but enforced a maximal truncation error of $10^{-14}$ to ensure a numerically exact representation of the many-particle state as an MPS. Figure 8.8 shows the entanglement entropies of all bonds of the MPS for the time evolution without transformations of the one-particle basis and with transformations. The entanglement entropy in the untransformed case grows linearly with time while for the case with transformation it only grows linearly with time in the beginning and then saturates at a constant entanglement entropy. The saturated values of the entanglement entropy of $S_{A \mid B}=2$ are equal to the maximal entanglement entropy of a system consisting of a single site as subsystem $A$ and the subsystem $B$ that contains the remaining $L-1$ sites. The entanglement entropy is drastically reduced by the transformation of the one-particle basis. Figure 8.9 shows the Schmidt coefficients at time $t=\hbar / T$. The Schmidt coefficients of the MPS with a transformation of the one-particle basis decay faster than without

\footnotetext{
${ }^{3}$ Please note, that we have renamed the hopping parameter to $T$ to distinguish it from the time $t$.

${ }^{4}$ ITensor $\mathrm{C}++$ library (version 2.1.1), http://itensor.org/
} 
8.8. Adaptive-cluster transformation for reduction of entanglement

the transformation. The faster decay is even more obvious at time $t=2 \hbar / T$ shown in Fig. 8.10. 


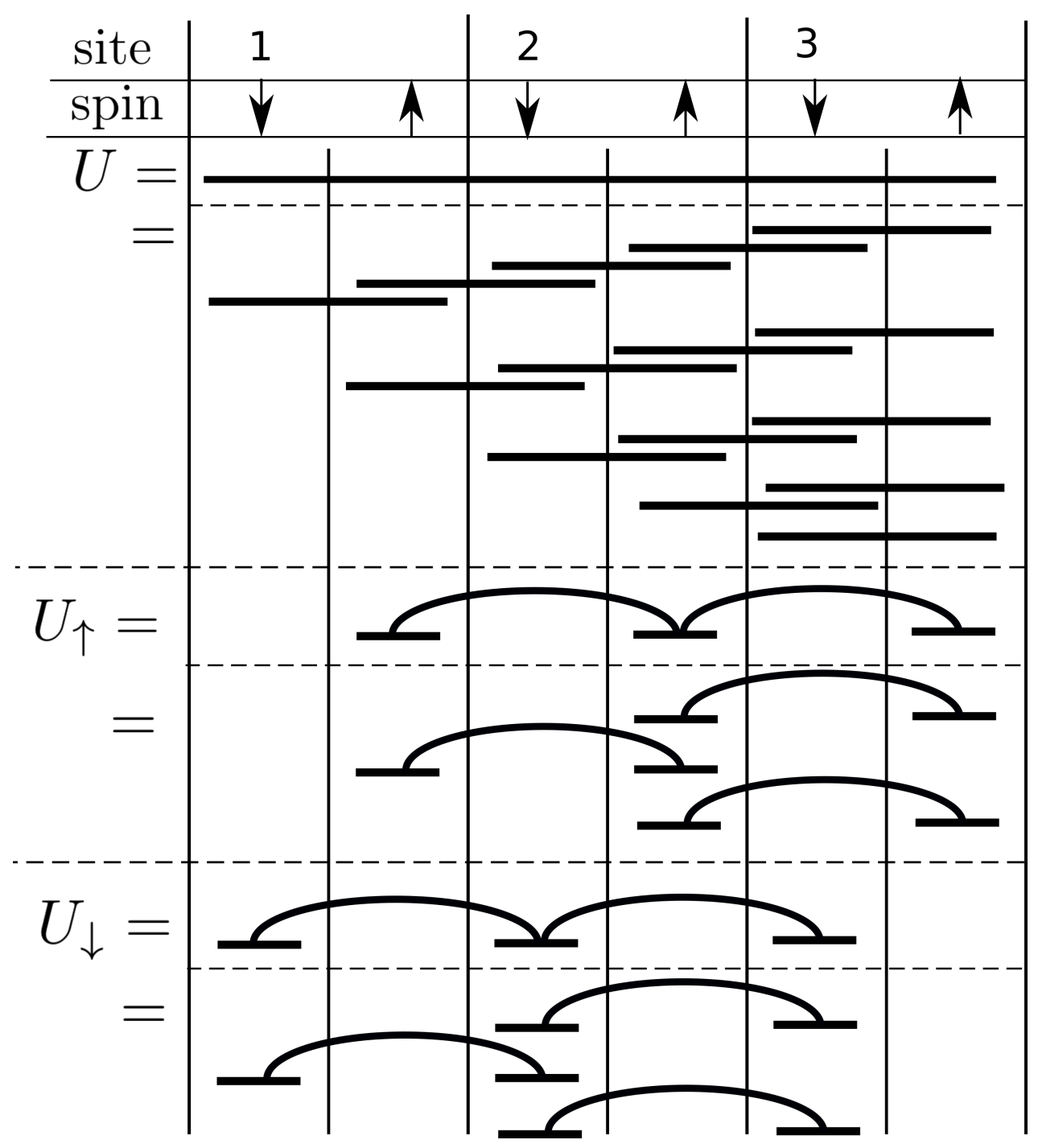

Figure 8.7.: Three-site $(L=3)$ example for the decomposition of a unitary transformation of the one-particle basis into local transformations. A general unitary transformation $U$ of the one-particle basis is decomposed into $15=2 L(2 L-1) / 2$ local transformations. If the unitary transformation individually acts on the spin-up and spin-down channel, then the transformation of the individual spin-channels can be decomposed into $2 \cdot 3=2 L(L-1) / 2$ local unitary transformations. 


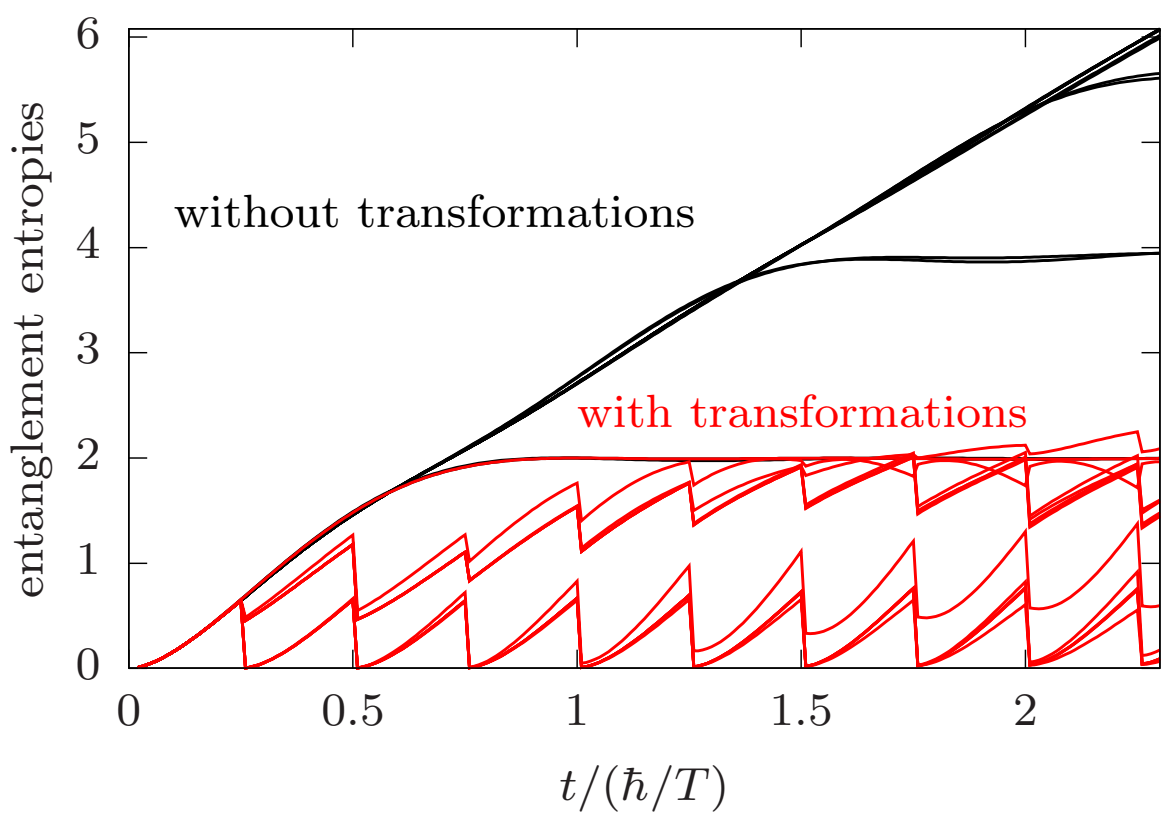

Figure 8.8.: Entropies of entanglement of all bonds during the time evolution of a 16-site Néel state with the Hamiltonian in Eq. (8.134) and $U / T=1$. The black lines show results when the one-particle basis is not transformed. Red lines denote results with a transformation of the one-particle basis every $\Delta=0.25 \hbar / T$.

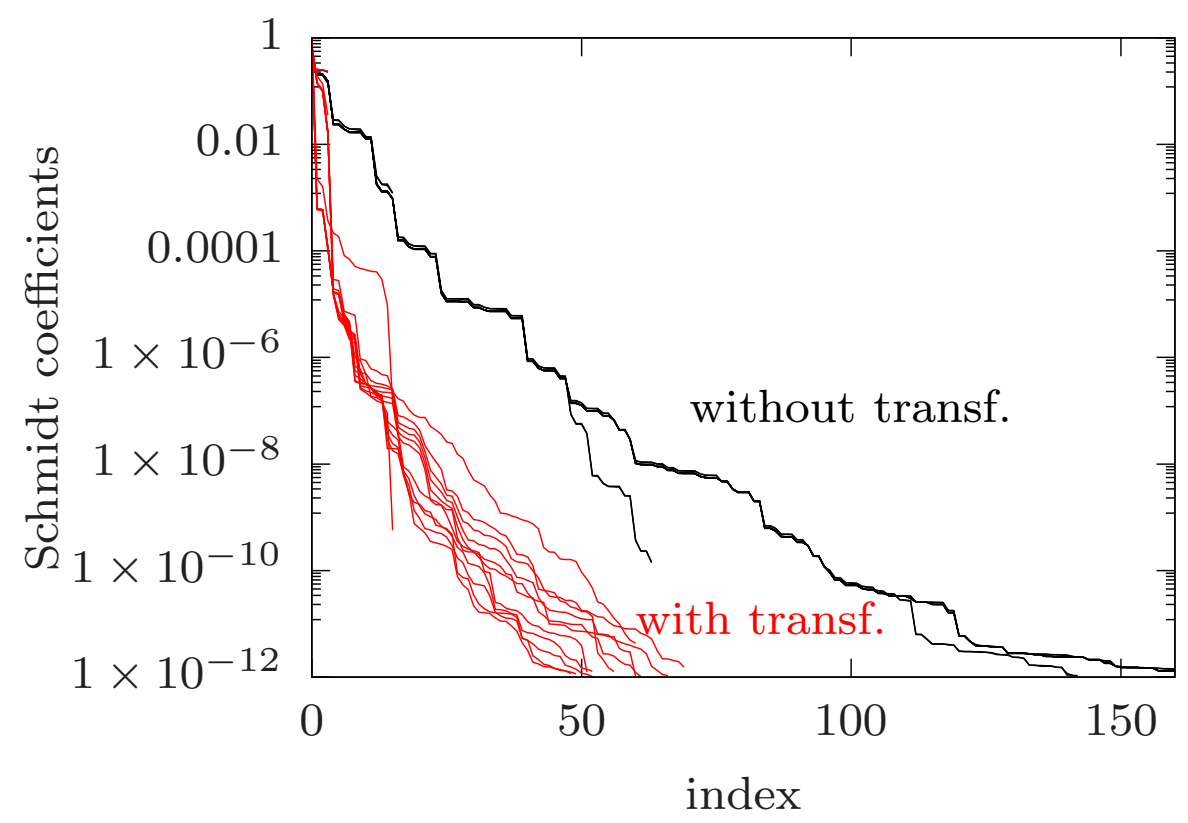

Figure 8.9.: Schmidt coefficients of all bonds of the MPS at time $t=\hbar / T$ without transformations of the one-particle basis (black lines) and with a transformation of the one-particle basis (red lines). Same system and initial state as in Figure 8.8 . 
8. Adaptive cluster approximation

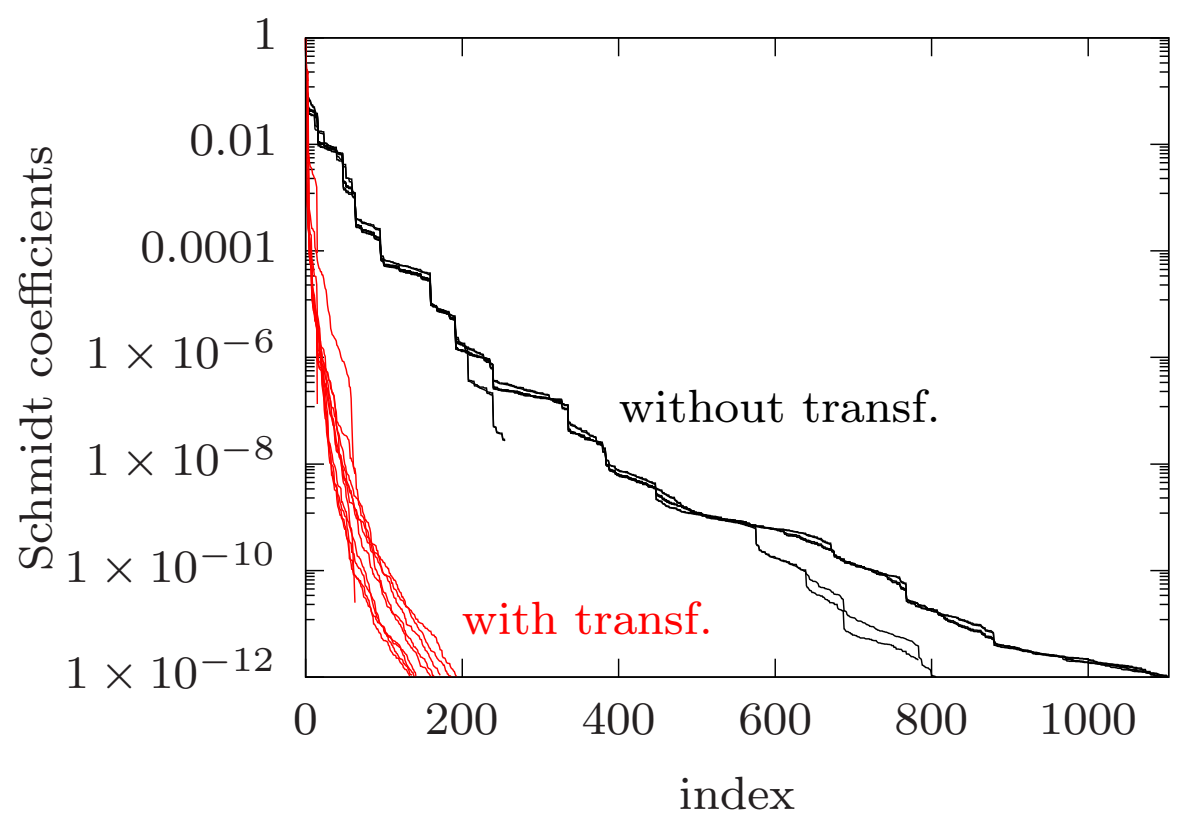

Figure 8.10.: Schmidt coefficients of all bonds of the MPS at time $t=2 \hbar / T$ without transformations of the one-particle basis (black lines) and with a transformation of the one-particle basis (red lines). Same system and initial state as in Figure 8.8. 


\section{Wave-function based approach for the RDMF}

\subsection{Introduction}

As shown in chapter 5, the density-matrix functional can be expressed either with a Legendre-Fenchel transformation

$$
F^{\hat{W}}\left[\rho^{(1)}\right]=\max _{h}\left[\Omega_{\beta, \mu=0}^{\hat{W}}[h]-\operatorname{Tr}\left(\rho^{(1)} h\right)\right]
$$

of the grand potential $\Omega_{\beta, \mu=0}^{\hat{W}}[h]$ or a constrained minimization as in Eq. (5.8), i.e.,

$$
F_{\beta}^{\hat{W}}\left[\rho^{(1)}\right]=\min _{\left\{P_{i},\left|\Psi_{i}\right\rangle\right\} \rightarrow \rho^{(1)}}\left[\sum_{i} P_{i}\left\langle\Psi_{i}|\hat{W}| \Psi_{i}\right\rangle+\frac{1}{\beta} \sum_{i} P_{i} \ln \left(P_{i}\right)\right]
$$

over an ensemble of normalized many-particle wave functions $\left|\Psi_{i}\right\rangle$ and probabilities $P_{i}$ with $\sum_{i} P_{i}=1$. Details can be found in section 5.2. Most methods in use for the investigation of properties of molecules or solids can estimate either the ground-state energy or the grand potential. Thus it would be easy to use them in the Legendre-Fenchel transformation. This approach was used together with an open-systems treatment in the Ph.D. project of Ebad Kamil [Kamil et al., 2013] to calculate density-matrix functionals of Hubbard models but later abandoned due to numerical complexity.

The derivatives,

$$
\frac{\partial}{\partial h_{\alpha, \beta}}\left[\Omega_{\beta, \mu=0}^{\hat{W}}[h]-\operatorname{Tr}\left(\rho^{(1)} h\right)\right]=\frac{\operatorname{Tr} e^{-\beta(\hat{h}+\hat{W})} \hat{c}_{\alpha}^{\dagger} \hat{c}_{\beta}}{\operatorname{Tr} e^{-\beta(\hat{h}+\hat{W})}}-\rho_{\beta, \alpha}^{(1)},
$$

can be used the guide the minimization of the matrix elements of the one-particle Hamiltonian. The derivative information in Eq. (9.3) is determined by the estimate of the one-particle reduced density matrix of the method for the grand potential. Here an additional problem can arise: If we, for example, use a DMRG method for the grand potential. For very low temperatures we can neglect the entropy contribution and the grand potential can be calculated with only one many-particle wave function. Thus, a conventional zero-temperature DMRG calculation would give the correct grand potential. However, the one-particle reduced density matrix from this one many-particle wave function is completely unphysical if the ground state that corresponds to the maximum is degenerate. As a consequence, the derivative information that is used from Eq. (9.3) for the construction of a new search direction in the iterated maximization procedure would be incorrect.

An additional problem arises from the fact that nearly all numerical non-perturbative techniques for the grand potential involve a minimization procedure for the determination 
of the grand potential. In consequence, there are two conflicting extremalisation procedures: A maximization over the one-particle Hamiltonian and a nested minimization in every iteration for the grand potential. The conflicting maximization and minimization lead to numerical instabilities if the solution of the grand potential is not well converged to the minimum. A similar conflict exists between the maximization within the LegendreFenchel transform and the minimization for the grand potential in

$$
\Omega_{\beta, \mu}[h]=\min _{\rho^{(1)}: 0 \leq \rho^{(1)} \leq \mathbb{1}}\left\{\operatorname{Tr}\left[\rho^{(1)}(h-\mu \mathbb{1})\right]+F_{\beta}^{\hat{W}}\left[\rho^{(1)}\right]\right\}
$$

or any other minimization procedure such as DFT+RDMFT in section 6 in which the density matrix functional enters. A highly precise calculation of the grand potential in every energy maximization step of the grand potential would be computationally very expensive. Additional problems can arise if the path of the one-particle Hamiltonians during the maximization crosses trough regions where the approximation or numerical method used for the grand potential breaks down or gives unphysical results. Experiments with an exact diagonalization solver for the grand potential have shown that discontinuities in the grand potential that exists in the zero-temperature limit can cause significant problems for traditional derivative based maximization algorithms. This problem also persists at low temperatures and becomes weaker when increasing the temperature.

These issues together make the Legendre-Fenchel transformation undesirable as a practical method for the practical evaluation of the density-matrix functional. Even though it could be done in practice, the expected numerical effort will be very high, because the convergence criteria must be very tight. The numerical algorithm is pretty straightforward, because the grand potential from any method can be used as a black box. Even if the method used for the grand potential cannot estimate the one-particle reduced density matrix, one can always use a derivative-free minimization algorithm like the downhill simplex method to perform the maximization procedure. Thus, we will not consider the practical evaluation of the density-matrix functional from the Legendre-Fenchel transformation in the remainder of this thesis but focus on more promising avenues.

In contrast, the evaluation of the density-matrix functional via the constrained minimization as defined by Eq. (5.8) has the advantage that it is a minimization procedure. It integrates nicely with any minimization of the total energy or grand potential. It is, however, a constrained minimization, whereas the Legendre-Fenchel transform is an unconstrained problem, and cannot be formulated as a problem with a Hamiltonian. This uncommon form makes the development of new solvers very challenging. In this work, we explore different routes, that can be distinguished by the form of the many-particle wave function in the constrained minimization: We explore a solver based on a configurationinteraction-like expansion in terms of Slater determinants in some one-particle basis in section 9.4. An impurity-bath separation ansatz where the representation of the manyparticle wave function distinguishes between interacting and non-interacting one-particle states is constructed in section 9.5. This idea is related to matrix-product states which are used in section (9.6). We construct a DMRG-like iterative algorithm of the solution of the constrained minimization over matrix product states. Finally, the application of stochastic optimization techniques allows us to use parametrizations of many-particle wave functions for which the expectation values cannot be calculated in a closed form in practice. In section 9.7 we demonstrate the use of Gutzwiller-correlated and Jastrowcorrelated many-particle wave functions for the constrained optimization. Finally, in 
section 9.8 we propose an algorithm that make use of an existing gate-based quantum computer to estimate the density-matrix functional.

\subsection{Simplifications}

The density-matrix functional $F^{\hat{W}}\left[\rho^{(1)}\right]$ defined by Eq. (5.8) requires a constrained minimization over an ensemble of many-particle wave functions. At zero temperature and for one-particle reduced density matrices that correspond to non-degenerate ground states, the density-matrix functional with an ensemble of many-particle wave functions is identical to Levy's density-matrix functional $F_{\text {Levy }}^{\hat{W}}\left[\rho^{(1)}\right]$ with only one many-particle wave function defined in Eq. (5.25). In this chapter, we only consider Levy's density-matrix functional. The extension of the ansatzes presented in the following sections to an ensemble of many-particle wave functions is straightforward.

A different simplification arises if we analyze the implications of certain occupations of the one-particle reduced density matrix on the possible many-particle wave functions. Consider a given one-particle reduced density matrix $\rho^{(1)}$ with ordered occupations

$$
\begin{array}{r}
f_{1}, \ldots, f_{n_{0}}=0 \\
f_{n_{0}+1}, \ldots, f_{N_{\chi}-n_{1}} \neq 0 \\
f_{N_{\chi}-n_{1}+1}, \ldots, f_{N_{\chi}}=1 .
\end{array}
$$

Thus, $n_{0}$ is the number of zero occupations and $n_{1}$ the number of occupations equal to one. We introduce the natural orbitals as a new one-particle basis in which the one-particle reduced density matrix is diagonal

$$
\tilde{\rho}^{(1)}=\operatorname{diag}\left(f_{1}, \ldots, f_{N_{\chi}}\right) .
$$

An ensemble on many-particle wave functions in the constrained minimization of the density-matrix functional has to generate this one-particle reduced density matrix by

$$
\tilde{\rho}_{\beta, \alpha}^{(1)}=\sum_{i} P_{i}\left\langle\Psi_{i}\left|\hat{c}_{\alpha}^{\dagger} \hat{c}_{\beta}\right| \Psi_{i}\right\rangle
$$

We can expand the many-particle wave functions in the occupation-number representation as

$$
\begin{aligned}
\left|\Psi_{i}\right\rangle & =\sum_{j} c_{i, j} \prod_{\alpha}\left(\hat{c}_{\alpha}^{\dagger}\right)^{\vec{s}_{j, \alpha}}|\mathcal{O}\rangle \\
& =\sum_{j} c_{i, j}\left|s_{j, 1} \ldots s_{j, N_{\chi}}\right\rangle .
\end{aligned}
$$

The requirement $\tilde{\rho}_{\alpha, \alpha}^{(1)}=f_{\alpha}$ can be shown to have a strong impact on the type of Slater determinants $\left|s_{j, 1} \ldots s_{j, N_{\chi}}\right\rangle$ that can have non-zero prefactors $c_{i, j}$. Consider $\alpha$ with $f_{\alpha} \in$ $\{0,1\}$, then we have

$$
\begin{aligned}
f_{\alpha} & =\tilde{\rho}_{\alpha, \alpha}^{(1)}=\sum_{i} P_{i} \sum_{j, j^{\prime}} c_{i, j} c_{i, j^{\prime}}^{*}\left\langle s_{j^{\prime}, 1} \ldots s_{j^{\prime}, N_{\chi}}\left|\hat{c}_{\alpha}^{\dagger} \hat{c}_{\alpha}\right| s_{j, 1} \ldots s_{j, N_{\chi}}\right\rangle \\
& =\sum_{i} P_{i} \sum_{j}\left|c_{i, j}\right|^{2} s_{j, \alpha} .
\end{aligned}
$$


For $f_{\alpha}=0$ if follows from $P_{i} \geq 0, \sum_{i} P_{i}=1,\left|c_{i, j}\right|^{2} \geq 0$ and $\sum_{j}\left|c_{i, j}\right|^{2}=1$ that

$$
c_{i, j}=0 \forall j \text { with } s_{j, \alpha}=1
$$

and similarly for $f_{\alpha}=1$ that

$$
c_{i, j}=0 \forall j \text { with } s_{j, \alpha}=0 .
$$

Thus, the many-particle wave functions can only contain Slater determinants with nonzero amplitudes that have the form

$$
|\underbrace{0 \ldots 0}_{n_{0}} \underbrace{s_{j, n_{0}+1} \ldots s_{j, n_{1}-1}}_{N_{\chi}-n_{0}-n_{1}} \underbrace{1 \ldots 1}_{n_{1}}\rangle \text { times }
$$

and the wave functions can be written as

$$
\left|\Psi_{i}\right\rangle=|\underbrace{0 \ldots 0}_{n_{0} \text { times }}\rangle \otimes\left|\tilde{\Psi}_{i}\right\rangle \otimes|\underbrace{1 \ldots 1}_{n_{1} \text { times }}\rangle .
$$

This decomposition is identical to the complete-active-space wave function used in quantum chemistry. However, here the occupations of the orbitals are known because the one-particle reduced density matrix is given and, hence, there it is no approximation to use the complete-active-space wave function within the constrained minimization of the density-matrix functional. Following the terminology of quantum chemistry we call the first $n_{0}$ one-particle states the virtual states (v), the $N_{\chi}-n_{0}-n_{1}$ states with fractional occupations the active states (a) and the $n_{1}$ states with unit occupations the core states (c). The two-particle reduced density matrix $\left\langle\Psi_{i}\left|\hat{c}_{\alpha}^{\dagger} \hat{c}_{\beta}^{\dagger} \hat{c}_{\gamma} \hat{c}_{\delta}\right| \Psi_{i}\right\rangle$ of the wave function in Eq. (9.17) can be non-zero only for the six cases

$$
\begin{aligned}
& \alpha \in \text { core, } \beta \in \text { core, } \gamma \in \text { core, } \delta \in \text { core } \\
& \alpha \in \text { core, } \beta \in \text { active, } \gamma \in \text { core, } \delta \in \text { active } \\
& \alpha \in \text { core, } \beta \in \text { active, } \gamma \in \text { active, } \delta \in \text { core } \\
& \alpha \in \text { active, } \beta \in \text { core, } \gamma \in \text { core, } \delta \in \text { active } \\
& \alpha \in \text { active, } \beta \in \text { core, } \gamma \in \text { active, } \delta \in \text { core } \\
& \alpha \in \text { active, } \beta \in \text { active, } \gamma \in \text { active, } \delta \in \text { active. }
\end{aligned}
$$

The expectation value of the interaction $\hat{W}$ is

$$
\begin{aligned}
\langle\hat{W}\rangle & =\frac{1}{2} \sum_{\alpha, \beta, \gamma, \delta \leq N_{\chi}-n_{0}-n_{1}} U_{\alpha+n_{0}, \beta+n_{0}, \delta+n_{0}, \gamma+n_{0}} \sum_{i} P_{i}\left\langle\tilde{\Psi}_{i}\left|\hat{c}_{\alpha}^{\dagger} \hat{c}_{\beta}^{\dagger} \hat{c}_{\gamma} \hat{c}_{\delta}\right| \tilde{\Psi}_{i}\right\rangle \\
& +\frac{1}{2} \sum_{\alpha, \beta \in \text { core }}\left(U_{\alpha, \beta, \alpha, \beta}-U_{\alpha, \beta, \beta, \alpha}\right)+\frac{1}{2} \sum_{\alpha \in \text { core } ; \beta, \gamma \in \operatorname{active}} U_{\alpha, \beta, \alpha, \gamma} \tilde{\rho}_{\gamma, \beta}^{(1)} .
\end{aligned}
$$

The first term is the interaction energy of the active states, the second term the interaction of the core states and the last term is often called the core-valance exchange.

For one-particle basis sets with large dimensions, it is a numerically cumbersome task to transform the matrix elements of the interaction to a different one-particle basis, in this case to the natural-orbital basis. However, we can also employ the idea of the completeactive-space wave function in Eq. (9.17) in the original basis instead of the natural-orbital 
basis. We first consider that the occupations obey $0 \leq f_{i} \leq 1$ and inspect the diagonal elements on the one-particle reduced density matrix $\rho_{\alpha, \alpha}^{(1)}$. If a diagonal element is integer for some $\alpha$, i.e. $\rho_{\alpha, \alpha}^{(1)} \in\{0,1\}$, it follows that $\rho_{\alpha, \beta}^{(1)}=\delta_{\alpha, \beta} \rho_{\alpha, \alpha}^{(1)}$ for this $\alpha$. This can easily be seen from Gershgorin's circle theorem [Gershgorin, 1931]

$$
\begin{gathered}
\max _{\beta} f_{\beta} \geq \max _{\beta}\left(\rho_{\beta, \beta}^{(1)}+\sum_{\gamma \neq \beta}\left|\rho_{\gamma, \beta}^{(1)}\right|\right) \\
\min _{\beta} f_{\beta} \leq \min _{\beta}\left(\rho_{\beta, \beta}^{(1)}-\sum_{\gamma \neq \beta}\left|\rho_{\gamma, \beta}^{(1)}\right|\right) .
\end{gathered}
$$

Thus, an integer diagonal element of the one-particle reduced density matrix directly implies the existence of an integer occupation. The converse is not true. With the decomposition of the one-particle basis into a core-space, active-space and virtual-space wave function in Eq. (9.17), we only need to consider the active-space wave function $\left|\tilde{\Psi}_{i}\right\rangle$ for the constrained minimization of the density-matrix functional. We include the expectation values of the interaction between core-states and valence states and, thus, account for core-valence exchange.

\subsection{Solution of the minimization problem}

\subsubsection{Lagrange function}

The constrained minimization problem for the density-matrix functional defined in Eq. (5.8) can be written as

$$
F_{\beta}^{\hat{W}}\left[\rho^{(1)}\right]=\min _{\left\{P_{i},\left|\Psi_{i}\right\rangle\right\}}\left[\sum_{i} P_{i}\left\langle\Psi_{i}|\hat{W}| \Psi_{i}\right\rangle+\frac{1}{\beta} \sum_{i} P_{i} \ln \left(P_{i}\right)\right]
$$

with the constraints

$$
\begin{aligned}
P_{i} & \geq 0, \quad \sum_{i} P_{i}=1 \quad\left\langle\Psi_{i} \mid \Psi_{i}\right\rangle=1 \\
\rho_{\beta, \alpha}^{(1)} & =\sum_{i} P_{i}\left\langle\Psi_{i}\left|\hat{c}_{\alpha}^{\dagger} \hat{c}_{\beta}\right| \Psi_{i}\right\rangle .
\end{aligned}
$$

This is a mixed equality-inequality constrained non-linear minimization problem. We can write it as an equality-constrained problem by introducing the auxiliary variables $x_{i}$ with

$$
P\left(x_{i}\right)=\left(1+\cos x_{i}\right) / 2 .
$$

Thus, the constraint $P_{i} \geq 0$ can be replaced by the unconstrained variable $x_{i}$ and the minimization problem written in the Lagrange formalism as

$$
\begin{aligned}
F_{\beta}^{\hat{W}}\left[\rho^{(1)}\right] & =\min _{\left\{P\left(x_{i}\right),\left|\Psi_{i}\right\rangle\right\}} \operatorname{stat}_{\lambda_{i}, h_{\alpha, \beta}, \Lambda}\left[\sum_{i} P\left(x_{i}\right)\left\langle\Psi_{i}|\hat{W}| \Psi_{i}\right\rangle+\frac{1}{\beta} \sum_{i} P\left(x_{i}\right) \ln \left(P_{i}\right)\right. \\
& -\Lambda\left(\sum_{i} P\left(x_{i}\right)-1\right)-\sum_{i} \lambda_{i}\left(\left\langle\Psi_{i} \mid \Psi_{i}\right\rangle-1\right) \\
& \left.-\sum_{\alpha, \beta} h_{\alpha, \beta}\left(\sum_{i} P\left(x_{i}\right)\left\langle\Psi_{i}\left|\hat{c}_{\alpha}^{\dagger} \hat{c}_{\beta}\right| \Psi_{i}\right\rangle-\rho_{\beta, \alpha}^{(1)}\right)\right] .
\end{aligned}
$$


$\Lambda, \lambda_{i}$ and $h$ are the Lagrange multipliers for enforcing the equality constraints. For ease of notation, we define the Lagrange function

$$
\begin{aligned}
L\left(\left\{x_{i}\right\},\left\{\left|\Psi_{i}\right\rangle\right\}, \Lambda,\left\{\lambda_{i}\right\},\left\{h_{\alpha, \beta}\right\}\right) & =\left[\sum_{i} P\left(x_{i}\right)\left\langle\Psi_{i}|\hat{W}| \Psi_{i}\right\rangle+\frac{1}{\beta} \sum_{i} P\left(x_{i}\right) \ln \left(P_{i}\right)\right. \\
& -\Lambda\left(\sum_{i} P\left(x_{i}\right)-1\right)-\sum_{i} \lambda_{i}\left(\left\langle\Psi_{i} \mid \Psi_{i}\right\rangle-1\right) \\
& \left.-\sum_{\alpha, \beta} h_{\alpha, \beta}\left(\sum_{i} P\left(x_{i}\right)\left\langle\Psi_{i}\left|\hat{c}_{\alpha}^{\dagger} \hat{c}_{\beta}\right| \Psi_{i}\right\rangle-\rho_{\beta, \alpha}^{(1)}\right)\right] .
\end{aligned}
$$

\subsubsection{Conceptual challenges}

The equality-constrained non-linear minimization problem of Eq. (9.27) or Eq. (9.31) includes some important conceptual and practical challenges. One can imagine two different classes of iterative minimization algorithms: one that enforces the constraints in every minimization step and one that does not require the fulfillment of the constraints in every step. The first variant can suffer from the so-called Maratos effect [Maratos, 1978]. The Maratos effect means that the algorithm can fail to rapidly converge to the solution because steps that would make good progress would violate the constraints.

The derivatives of the density-matrix functional with respect to the one-particle reduced density matrix

$$
\frac{\partial F^{\hat{W}}\left[\rho^{(1)}\right]}{\partial \rho_{\alpha, \beta}^{(1)}}
$$

are required for the efficient minimization of the total energy. Ignoring mathematical peculiarities for a moment, the derivatives can be obtained from the Lagrange multipliers $h$ of the density-matrix constraint as

$$
\frac{\partial F^{\hat{W}}\left[\rho^{(1)}\right]}{\partial \rho_{\alpha, \beta}^{(1)}}=h_{\beta, \alpha}
$$

However, this is only true if the Lagrange multipliers are unique. To simplify the notation for the investigation of the uniqueness of the Lagrange multipliers, we consider the densitymatrix functional with only one many-particle wave function $|\Psi\rangle$, i.e. $P_{1}=1$. The derivative of the Lagrange function in Eq. (9.32) with respect to the many-particle wave function $|\Psi\rangle$ is

$$
\frac{\partial L\left(\{|\Psi\rangle\}, \Lambda, \lambda,\left\{h_{\alpha, \beta}\right\}\right)}{\partial\langle\Psi|}=\left(\hat{W}|\Psi\rangle-\lambda|\Psi\rangle-\sum_{\alpha, \beta} h_{\alpha, \beta} \hat{c}_{\alpha}^{\dagger} \hat{c}_{\beta}|\Psi\rangle\right)
$$

and has to vanish for the solution. Thus, $|\Psi\rangle$ is an eigenstate of the effective Hamiltonian

$$
-\sum_{\alpha, \beta} h_{\alpha, \beta} \hat{c}_{\alpha}^{\dagger} \hat{c}_{\beta}+\hat{W}
$$

with the eigenvalue $\lambda$. It is not necessarily the ground-state. 
The derivatives of the density-matrix constraint

$$
\frac{\partial\left\langle\Psi\left|\hat{c}_{\alpha}^{\dagger} \hat{c}_{\beta}\right| \Psi\right\rangle}{\partial\langle\Psi|}=\hat{c}_{\alpha}^{\dagger} \hat{c}_{\beta}|\Psi\rangle
$$

determine the uniqueness of the Lagrange multipliers. To illustrate the connection, we consider two matrices of Lagrange multipliers, $h$ and $\tilde{h}$, for the density-matrix constraint while all other variables are identical. We assume that the minimum condition is fulfilled for both matrices, i.e. Eq. (9.35) vanishes. Thus, we have the condition

$$
\sum_{\alpha, \beta}\left(h_{\alpha, \beta}-\tilde{h}_{\alpha, \beta}\right) \hat{c}_{\alpha}^{\dagger} \hat{c}_{\beta}|\Psi\rangle=0 .
$$

We show now that the uniqueness of the Lagrange multipliers, i.e. $h=\tilde{h}$, is not required to fulfill the minimum condition in Eq. (9.38). For this purpose, we consider the situation where the two index-pairs $\left(\alpha^{\prime}, \beta^{\prime}\right) \neq\left(\alpha^{\prime \prime}, \beta^{\prime \prime}\right)$ and $a \neq 0$ exist such that

$$
\hat{c}_{\alpha^{\prime \prime}}^{\dagger} \hat{\beta}_{\beta^{\prime \prime}}|\Psi\rangle=a \hat{c}_{\alpha^{\prime}}^{\dagger} \hat{c}_{\beta^{\prime}}|\Psi\rangle
$$

with $\hat{c}_{\alpha^{\prime}}^{\dagger} \hat{c}_{\beta^{\prime}}|\Psi\rangle \neq 0$. All other $\hat{c}_{\alpha}^{\dagger} \hat{c}_{\beta}|\Psi\rangle$ are assumed to be linearly independent of $\hat{c}_{\alpha^{\prime}}^{\dagger} \hat{c}_{\beta^{\prime}}|\Psi\rangle$. We show that Eq. (9.38) does not imply $h=\tilde{h}$ in this case. The condition in Eq. (9.38) reads

$$
\begin{aligned}
0 & =\sum_{\alpha, \beta:(\alpha, \beta) \neq\left(\alpha^{\prime}, \beta^{\prime}\right) \wedge(\alpha, \beta) \neq\left(\alpha^{\prime \prime}, \beta^{\prime \prime}\right)}\left(h_{\alpha, \beta}-\tilde{h}_{\alpha, \beta}\right) \hat{c}_{\alpha}^{\dagger} \hat{c}_{\beta}|\Psi\rangle \\
& +\left(h_{\alpha^{\prime}, \beta^{\prime}}-\tilde{h}_{\alpha^{\prime}, \beta^{\prime}}\right) \hat{c}_{\alpha^{\prime}}^{\dagger} \hat{c}_{\beta^{\prime}}|\Psi\rangle+\left(h_{\alpha^{\prime \prime}, \beta^{\prime \prime}}-\tilde{h}_{\alpha^{\prime \prime}, \beta^{\prime \prime}}\right) \hat{c}_{\alpha^{\prime \prime}}^{\dagger} \hat{c}_{\beta^{\prime \prime}}|\Psi\rangle .
\end{aligned}
$$

With Eq. (9.39) we obtain

$$
\begin{aligned}
0 & =\sum_{\alpha, \beta:(\alpha, \beta) \neq\left(\alpha^{\prime}, \beta^{\prime}\right) \wedge(\alpha, \beta) \neq\left(\alpha^{\prime \prime}, \beta^{\prime \prime}\right)}\left(h_{\alpha, \beta}-\tilde{h}_{\alpha, \beta}\right) \hat{c}_{\alpha}^{\dagger} \hat{c}_{\beta}|\Psi\rangle \\
& +\left(h_{\alpha^{\prime}, \beta^{\prime}}-\tilde{h}_{\alpha^{\prime}, \beta^{\prime}}+a h_{\alpha^{\prime \prime}, \beta^{\prime \prime}}-a \tilde{h}_{\alpha^{\prime \prime}, \beta^{\prime \prime}}\right) \hat{c}_{\alpha^{\prime}}^{\dagger} \hat{c}_{\beta^{\prime}}|\Psi\rangle .
\end{aligned}
$$

The linear independence of $\hat{c}_{\alpha^{\prime}}^{\dagger} \hat{c}_{\beta^{\prime}}|\Psi\rangle$ from other $\hat{c}_{\alpha}^{\dagger} \hat{c}_{\beta}|\Psi\rangle$ for $(\alpha, \beta) \neq\left(\alpha^{\prime}, \beta^{\prime}\right) \wedge(\alpha, \beta) \neq$ $\left(\alpha^{\prime \prime}, \beta^{\prime \prime}\right)$ requires

$$
h_{\alpha^{\prime}, \beta^{\prime}}-\tilde{h}_{\alpha^{\prime}, \beta^{\prime}}=-a \cdot\left(h_{\alpha^{\prime \prime}, \beta^{\prime \prime}}-\tilde{h}_{\alpha^{\prime \prime}, \beta^{\prime \prime}}\right) \text {. }
$$

Thus, the minimum condition in Eq. (9.38) can be fulfilled with $h \neq \tilde{h}$ if $a \neq 0$. More generally, the Lagrange multipliers are not unique if the gradients of the constraints

$$
\frac{\partial\left\langle\Psi\left|\hat{c}_{\alpha}^{\dagger} \hat{c}_{\beta}\right| \Psi\right\rangle}{\partial\langle\Psi|}=\hat{c}_{\alpha}^{\dagger} \hat{c}_{\beta}|\Psi\rangle
$$

are linearly dependent. This is also known as the linear-independence-constraintqualification (LICQ). In other words, if the gradients of the constraints are linearly dependent, then the Lagrange multipliers do not have to correspond to the derivatives of the density-matrix functional. We have only observed this issue in evaluations of the density-matrix functional for highly symmetric one-particle reduced density matrices and highly symmetric interaction Hamiltonians. In those cases, the linear dependence can be removed by adding a very small perturbation to the one-particle reduced density matrix. 


\subsubsection{Powell-Hestenes augmented Lagrangian}

We propose to use the Powell-Hestenes augmented Lagrangian [Powell, 1969; Hestenes, 1969] for the numerical solution of the constrained minimization problem of the densitymatrix functional. Consider the generic equality-constrained minimization problem

$$
\min _{\vec{x}} f(\vec{x})
$$

with the constraints

$$
c_{i}(\vec{x})=0 .
$$

The Lagrange function $L\left(\vec{x}, \lambda_{i}\right)$ for this problem is

$$
L\left(\vec{x},\left\{\lambda_{i}\right\}\right)=f(\vec{x})-\sum_{i} \lambda_{i} c_{i}(\vec{x}) .
$$

The augmented Lagrangian $L\left(\vec{x},\left\{\lambda_{i}\right\},\left\{\mu_{i}\right\}\right)$ adds a penalty function $p$ (augmentation) to the Lagrange function and is defined

$$
L\left(\vec{x},\left\{\lambda_{i}\right\},\left\{\mu_{i}\right\}\right)=f(\vec{x})-\sum_{i} \lambda_{i} c_{i}(\vec{x})+\frac{1}{2} \sum_{i} \mu_{i} p\left(c_{i}(\vec{x})\right) .
$$

$\mu_{i}$ are the penalty parameters. The penalty function $p(c)$ can be chosen rather freely and properties of penalty functions have been studied in the literature. For a review we refer the reader to [Nocedal and Wright, 2006]. We choose the quadratic penalty function $p(c)=c^{2}$, because it has smooth derivatives and does not involve the derivatives of the constraints. The augmented Lagrangian method maps the constrained minimization to a series of unconstrained minimization problems whose solution converges to the solution of the constrained problem. A generic augmented Lagrangian algorithm first chooses initial values for the penalty parameters $\mu_{i}^{(0)}$, tolerance $\tau^{(0)}$, Lagrange multipliers $\lambda_{i}^{(0)}$ and a starting point $\vec{x}^{(0)}$. Then the following step are executed for $k=0,1,2, \ldots$ :

1. The unconstrained problem

$$
\min _{\vec{x}} L\left(\vec{x},\left\{\lambda_{i}\right\},\left\{\mu_{i}\right\}\right)
$$

is solved up to a tolerance of $\left\|\nabla_{x} L\left(\vec{x},\left\{\lambda_{i}\right\},\left\{\mu_{i}\right\}\right)\right\| \leq \tau^{(k)}$. The minimizer is used as $\vec{x}^{(k)}$.

2. The convergence is checked. If the current $\vec{x}^{(k)}$ and estimates for the Lagrange multipliers $\lambda_{i}^{(k)}$ satisfy the minimum conditions, then the algorithm terminates.

3. The Lagrange multipliers are updated with the first-order multiplier update

$$
\lambda_{i}^{(k+1)}=\lambda_{i}^{(k)}-\mu_{i}^{(k)} c_{i}\left(\vec{x}^{(k)}\right) .
$$

4. New penalty parameters $\mu_{i}^{(k+1)} \geq \mu_{i}^{(k)}$ are chosen.

5. A new tolerance $\tau^{(k+1)}$ is chosen. 
The convergence of the augmented Lagrangian-approach is governed by the theorem [Nocedal and Wright, 2006]: let $\vec{x}^{*}$ be a local minimizer of the constrained minimization problem defined in Eq. (9.44) and Eq. (9.45) and let the linear-independenceconstraint-qualification be fulfilled at $\vec{x}^{*}$. Let $\lambda_{i}^{*}$ be the exact Lagrange multipliers. Then there exists a threshold value $\bar{\mu}$ such that for all penalty parameters $\mu_{i} \geq \bar{\mu}, \vec{x}^{*}$ is a local minimizer of $L\left(\vec{x},\left\{\lambda_{i}^{*}\right\},\left\{\mu_{i}\right\}\right)$.

This theorem states that in contrast to the penalty method the penalty parameters in the augmented Lagrangian do not have to be increased to infinity to find the exact solution. As a consequence, the unconstrained subproblems of the augmented Lagrangian are much less prone to ill-conditioning. Additional theorems by Bertsekas [Bertsekas, 1999; Nocedal and Wright, 2006] apply to the situation of approximate solutions of the Lagrange multipliers. With the assumption of the previous theorem, there exists $\delta>0$, $\epsilon>0$ and $M>0$ such that:

1. For all $\lambda_{i}$ and $\mu_{i}$ that satisfy

$$
\| \vec{\lambda}-\vec{\lambda}^{*} \mid \leq \delta \max _{i} \mu_{i} \text { and } \mu_{i} \geq \bar{\mu}
$$

the problem

$$
\min _{\vec{x}} L\left(\vec{x},\left\{\lambda_{i}\right\},\left\{\mu_{i}\right\}\right)
$$

has the solution $\vec{x}$ with

$$
\left\|\vec{x}-\vec{x}^{*}\right\| \leq M\left\|\vec{\lambda}-\vec{\lambda}^{*}\right\| / \bar{\mu}
$$

2. For all $\lambda_{i}$ and $\mu_{i}$ that satisfy

$$
\| \vec{\lambda}-\vec{\lambda}^{*} \mid \leq \delta \max _{i} \mu_{i} \text { and } \mu_{i} \geq \bar{\mu}
$$

we have

$$
\left\|\vec{\lambda}^{(k+1)}-\vec{\lambda}^{*}\right\| \leq M\left\|\vec{\lambda}^{(k)}-\vec{\lambda}^{*}\right\| / \min _{i} \mu_{i}
$$

This first theorem shows that the solution $\vec{x}$ of the unconstrained subproblem will be close to the exact solution $\vec{x}^{*}$ of the constrained problem, if the estimates of the Lagrange multiplies $\vec{\lambda}$ are close to the exact Lagrange multipliers $\vec{\lambda}^{*}$ or if the penalty parameters are large. The second theorem relates the change of the multipliers by the multiplier update in Eq. (9.49) to the size of the penalty parameters. Improvement is guaranteed as long as the penalty parameters are sufficiently large.

We have implemented the augmented Lagrangian scheme in a general way with welldefined interfaces so that it can work with arbitrary parametrizations of the many-particle wave function and arbitrary constraints. The unconstrained subproblems can be solved with any suitable unconstrained minimization algorithm. In situations, where the derivatives of the augmented Lagrangian with respect to the variational parameters are available, we solve the unconstrained subproblems either with the non-linear conjugate gradient method or the limited-memory Broyden-Fletcher-Goldfarb-Shanno quasi-Newton 
method [Broyden, 1970; Fletcher, 1970; Goldfarb, 1970; Shanno, 1970]. For parametrizations of many-particle wave functions that contain complex parameters, we employ the complex generalizations of the minimization algorithms [Sorber et al., 2012] to preserve the compact structure. The line searches are solved either numerically exact, if $L\left(\vec{x}+\alpha \vec{y},\left\{\lambda_{i}\right\},\left\{\mu_{i}\right\}\right)$ can be easily written as a polynomial function in $\alpha$, or otherwise with a secant line search. In cases where the derivatives are not available or if there is noise in the augmented Lagrangian, we use the simultaneous perturbation stochastic approximation approach (SPSA, [Spall, 1987, 1992]). The SPSA will be discussed in detail in section 9.7.5.

The augmented Lagrangian approach presented here is a general algorithm for the solution of constrained minimization problems. The main benefits are a rather simple formulation, the availability of derivatives and, most importantly, the numerical stability ${ }^{1}$.

\subsection{Configuration-interaction ansatz}

\subsubsection{Ansatz for the many-particle wave function}

A simple ansatz for the many-particle wave function is the configuration-interaction-like (CI) ansatz $^{2}$

$$
|\Psi(\vec{d})\rangle=\sum_{i=1}^{N_{\operatorname{det}}} d_{i}\left|n_{i, 1} \ldots . n_{i, N_{\chi}}\right\rangle
$$

as a sum of $N_{\text {det }}$ Slater determinants with complex coefficients $d_{i}$. We don't consider the CI-ansatz as the traditional sum of excitations over a reference Slater determinant, because in our application to the density-matrix functional the reference Slater determinant is not well-defined. The CI-ansatz in Eq. (9.55) is completely defined by a set of Slater determinants $\left\{\left|n_{i, 1} \ldots . n_{i, N_{\chi}}\right\rangle\right\}$ and the one-particle basis $\left|\chi_{\alpha}\right\rangle\left(\alpha \in\left\{1, . ., N_{\chi}\right\}\right)$. We consider strategies for the selection of the Slater determinants and the one-particle basis in section 9.4.3.

The constrained minimization of the density-matrix functional of (5.25) with a CIansatz takes the form ${ }^{3}$

$$
F_{\text {Levy }}^{\hat{W}}\left[\rho^{(1)}\right]=\min _{\vec{d}}\langle\Psi(\vec{d})|\hat{W}| \Psi(\vec{d})\rangle
$$

with the constraints

$$
\begin{aligned}
1 & =\langle\Psi(\vec{d}) \mid \Psi(\vec{d})\rangle \\
\rho_{\beta, \alpha}^{(1)} & =\left\langle\Psi(\vec{d})\left|\hat{c}_{\alpha}^{\dagger} \hat{c}_{\beta}\right| \Psi(\vec{d})\right\rangle .
\end{aligned}
$$

\footnotetext{
${ }^{1}$ For example, after suggestion from us, Bünemann et al. adopted [Bünemann et al., 2016; Bünemann et al., 2017] the augmented Lagrangian approach for their Gutzwiller-DFT approach and found a significantly improved stability over the previous constrained minimization approach.

${ }^{2}$ For a review we refer the reader for example to [SHAVITT, 1998].

${ }^{3}$ For simplicity of notation, we consider the density-matrix functional with only one many-particle wave function here.
} 
It can be solved with the augmented Lagrangian approach. The augmented Lagrangian reads in this case

$$
\begin{aligned}
L\left(\vec{d},\left\{h_{\alpha, \beta}\right\}, \lambda,\left\{\mu_{\alpha, \beta}\right\}, \mu\right) & =\langle\Psi(\vec{d})|\hat{W}| \Psi(\vec{d})\rangle \\
& -\sum_{\alpha, \beta} h_{\alpha, \beta}\left(\left\langle\Psi(\vec{d})\left|\hat{c}_{\alpha}^{\dagger} \hat{c}_{\beta}\right| \Psi(\vec{d})\right\rangle-\rho_{\beta, \alpha}^{(1)}\right)-\lambda(\langle\Psi(\vec{d}) \mid \Psi(\vec{d})\rangle-1) \\
& +\sum_{\alpha, \beta} \frac{\mu_{\alpha, \beta}}{2}\left(\left\langle\Psi(\vec{d})\left|\hat{c}_{\alpha}^{\dagger} \hat{c}_{\beta}\right| \Psi(\vec{d})\right\rangle-\rho_{\beta, \alpha}^{(1)}\right)^{2}+\frac{\mu}{2}(\langle\Psi(\vec{d}) \mid \Psi(\vec{d})\rangle-1)^{2} .
\end{aligned}
$$

For convenience in numerical calculations, we use the matrix representations

$$
\begin{aligned}
\left\langle\Psi\left(\vec{e}_{i}\right)|\hat{W}| \Psi\left(\vec{e}_{j}\right)\right\rangle & =W_{i, j} \\
\left\langle\Psi\left(\vec{e}_{i}\right)\left|\hat{c}_{\alpha}^{\dagger} \hat{c}_{\beta}\right| \Psi\left(\vec{e}_{j}\right)\right\rangle & =C_{\alpha, \beta, i, j} \in\{-1,0,1\},
\end{aligned}
$$

where $\vec{e}_{i}$ is a unit basis vector in the $i$-th direction. We have found that this representation is advantageous to an on-the-fly calculation of the matrix elements for up to $\approx 10^{6}$ Slater determinants. We calculate the matrix elements on the fly for larger CI-spaces and the considerations in section (9.4.2) also hold for on-the-fly evaluations. The augmented Lagrangian takes the form

$$
\begin{aligned}
L\left(\vec{d},\left\{h_{\alpha, \beta}\right\}, \lambda,\left\{\mu_{\alpha, \beta}\right\}, \mu\right) & =\vec{d} \cdot W \vec{d}-\sum_{\alpha, \beta} h_{\alpha, \beta}\left(\vec{d} \cdot C_{\alpha, \beta} \vec{d}-\rho_{\beta, \alpha}^{(1)}\right)-\lambda(\vec{d} \cdot \vec{d}-1) \\
& +\sum_{\alpha, \beta} \frac{\mu_{\alpha, \beta}}{2}\left(\vec{d} \cdot C_{\alpha, \beta} \vec{d}-\rho_{\beta, \alpha}^{(1)}\right)^{2}+\frac{\mu}{2}(\vec{d} \cdot \vec{d}-1)^{2} .
\end{aligned}
$$

We solve the unconstrained subproblems

$$
\min _{\vec{d}} L\left(\vec{d},\left\{h_{\alpha, \beta}\right\}, \lambda,\left\{\mu_{\alpha, \beta}\right\}, \mu\right)
$$

of the augmented Lagrangian approach with the complex-generalized variants [Sorber et al., 2012] of the non-linear conjugate gradient method or the limited-memory BFGSmethod [Broyden, 1970; Fletcher, 1970; Goldfarb, 1970; Shanno, 1970]. The space of Slater determinants is not changed during the solution of an unconstrained subproblem.

\subsubsection{Numerical challenges}

The efficient practical solution of the unconstrained subproblems given in Eq. (9.63) presents several numerical challenges. We first consider the shape of the matrices $W$ and $C_{\alpha, \beta}$ : The matrix $W$ contains complex coefficients and the number of non-zero elements strongly depends on the matrix elements of the interaction Hamiltonian $\hat{W}$. However, $W$ is rather sparse in practice. The matrix $C_{\alpha, \beta}$ contains on average only $N_{\text {det }} / 4$ nonzero elements. The elements are integers and $C_{\alpha, \beta}$ is similar to a permutation matrix. We have implemented custom routines that handle $W$ and $C_{\alpha, \beta}$ as sparse matrices and explicitly consider their sparsity patterns. The central numerical challenge is the evaluation of sparse matrix-vector (SpMV) products, like $W \vec{d}$ or $\hat{W}|\Psi(\vec{d})\rangle$. The performance of sparse matrix-vector products is memory-bandwidth bound for large vectors, and hence 
measures must be taken to reduce the amount of data required from the main memory per arithmetic operation.

The computationally intensive quantities for the solution of the unconstrained subproblem are expectation values,

$$
w(\vec{d})=\vec{d} \cdot W \vec{d}, \quad c_{\alpha, \beta}(\vec{d})=\vec{d} \cdot C_{\alpha, \beta} \vec{d}-\rho_{\beta, \alpha}^{(1)} \quad \text { and } \quad n(\vec{d})=\vec{d} \cdot \vec{d}-1,
$$

and the derivative

$$
\frac{\partial L\left(\vec{d},\left\{h_{\alpha, \beta}\right\}, \lambda,\left\{\mu_{\alpha, \beta}\right\}, \mu\right)}{\partial \vec{d}^{*}}=W \vec{d}-\sum_{\alpha, \beta}\left(h_{\alpha, \beta}-\mu_{\alpha, \beta} c_{\alpha, \beta}(\vec{d})\right) C_{\alpha, \beta} \vec{d}-(\lambda-\mu n(\vec{d})) \vec{d} .
$$

The solution of the line search

$$
\min _{\alpha} L\left(\vec{d}+\alpha \vec{p},\left\{h_{\alpha, \beta}\right\}, \lambda,\left\{\mu_{\alpha, \beta}\right\}, \mu\right)
$$

with the step length $\alpha$ and the proposed step $\vec{p}$ is cumbersome. For the augmented Lagrangian in Eq. (9.59) and also its generalization to more than one many-particle wave functions at zero temperature, we can solve the line search problem by writing $L(\vec{d}+$ $\left.\alpha \vec{p},\left\{h_{\alpha, \beta}\right\}, \lambda,\left\{\mu_{\alpha, \beta}\right\}, \mu\right)$ as a polynomial function of the form

$$
L\left(\vec{d}+\alpha \vec{p},\left\{h_{\alpha, \beta}\right\}, \lambda,\left\{\mu_{\alpha, \beta}\right\}, \mu\right)=L_{0}+L_{1} \alpha+L_{2} \alpha^{2}+L_{3} \alpha^{3}+L_{4} \alpha^{4} .
$$

For multiple many-particle wave functions we have to consider orders up to eight in $\alpha$, because we enforce the positivity condition of the ensemble probabilities with the unconstrained auxiliary variable $x_{i}$ and set $P_{i}=x_{i}^{2}$. We determine the solution $\alpha_{\min }$ of the minimization problem of the one-dimensional polynomial function with Newton's method. In cases where Newton's method finds a stationary point that is not a minimum, we employ the Jenkins-Traub method [Traub, 1966; Jenkins and Traub, 1970; Jenkins, 1975] to determine all stationary points and select the global minimum. The coefficients $L_{i}$ can be calculated from the separate terms in the augmented Lagrangian in Eq. (9.62) by first computing the coefficients of the polynomial functions

$$
\begin{aligned}
w(\vec{d}+\alpha \vec{p}) & =w_{0}+w_{1} \alpha+w_{2} \alpha^{2} \\
c_{\alpha, \beta}(\vec{d}+\alpha \vec{p}) & =c_{\alpha, \beta, 0}+c_{\alpha, \beta, 1} \alpha+c_{\alpha, \beta, 2} \alpha^{2} \\
n(\vec{d}+\alpha \vec{p}) & =n_{0}+n_{1} \alpha+n_{2} \alpha^{2} .
\end{aligned}
$$

This has the nice side-effect that the expectation values don't have to be calculated explicitly in the next iteration of the unconstrained minimization but can be easily evaluated as $w\left(\vec{d}+\alpha_{\min } \vec{p}\right)$.

The evaluations of the coefficients require sparse matrix-vector products of the form $\vec{y} \cdot A \vec{x}$. We make extensive use of cache-blocking optimizations to reduce the amount of data that has to be transferred from main memory to the caches of the central processor. The main idea of cache-blocking optimizations is to group arithmetic operations in a different way than one would intuitively do to reuse data, that has been loaded from main memory to the cache, as often as possible before loading other data to the cache. The sizes of blocks depend on the size of the cache and the layout of the cache hierarchy. 
Modern CPUs usually have three cache levels of different sizes ${ }^{4}$ and depending on the microarchitecture several CPU-cores can either share the cache at a certain cache level or have their core-private cache. The different possibilities make an a-priori determination of the block sizes used in the cache blocking optimization difficult. We have implemented an automatic benchmarking scheme, that determines the optimal block sizes at runtime. With this implementation, we have been able to treat CI-spaces of sizes up to $5 \cdot 10^{7}$ on a regular recent desktop $\mathrm{CPU}$ in the constrained minimization. However, one evaluation of the density-matrix functional took about one day on the desktop CPU for such large CI-spaces ${ }^{5}$. Practical evaluations of the density-matrix functional in moderately sized CIspaces of $10^{3}-10^{5}$ Slater determinants can be performed in a matter of seconds or minutes because then the memory interface is not yet a bottleneck thanks to our cache-blocking optimizations. Detailed runtimes for $\mathrm{NiO}$ are presented in section 11.3.4.

\subsubsection{Selection of one-particle basis and Slater determinants}

It has been argued rather early [Löwdin, 1955; Davidson, 1972] in the development of the configuration-interaction method, that the expansion should be most efficient in a one-particle basis of natural orbitals, i.e., for a diagonal one-particle reduced density matrix. Although the strict optimality of the natural orbitals for a CI-ansatz also been disputed [Giesbertz, 2014], we use it here, because it can be constructed in a straightforward way. In contrast to methods from quantum chemistry that aim at the determination or approximation of the ground state, for the evaluation of the density-matrix functional we already know the one-particle reduced density matrix that the many-particle wave function should correspond to. Therefore, we can easily perform a basis transformation before the constrained minimization. However, a one-particle basis of natural orbitals has the main drawback, that any one-particle states of the original basis might be mixed with any other one-particle state. Thus, if we started out with a one-particle basis in which the interaction Hamiltonian only acted on a limited set $C$ of $N_{C}$ one-particle states

$$
\hat{W}=\frac{1}{2} \sum_{\alpha, \beta, \gamma, \delta \in C} U_{\alpha, \beta, \gamma, \delta} \hat{c}_{\alpha}^{\dagger} \hat{c}_{\beta}^{\dagger} \hat{c}_{\delta} \hat{c}_{\gamma}
$$

it would, in the worst case, act on all orbitals of the transformed basis, i.e., we have the form

$$
\hat{W}=\frac{1}{2} \sum_{\alpha, \beta, \gamma, \delta} \tilde{U}_{\alpha, \beta, \gamma, \delta} \hat{\tilde{c}}_{\alpha}^{\dagger} \hat{\tilde{c}}_{\beta}^{\dagger} \hat{\tilde{c}}_{\delta} \hat{\tilde{c}}_{\gamma} .
$$

As a consequence the number of non-zero elements of the matrix elements of the interaction Hamiltonian would grow from $\mathcal{O}\left(N_{C}^{4}\right)$ to $\mathcal{O}\left(N_{\chi}^{4}\right)$, where $N_{\chi}$ denotes the total number of one-particle states.

\footnotetext{
${ }^{4}$ There is usually $32 \mathrm{kB}$ of first level cache per CPU-core with a bandwidth of 64-128 Byte/cycle (200-400 $\mathrm{GB} / \mathrm{s}$ per core). The second-level cache has sizes from $256 \mathrm{kB}-1 \mathrm{MB}$ per core and a bandwidth of about $100 \mathrm{~GB} / \mathrm{s}$ per core. The third-level cache is usually shared among all cores with sizes of $5 \mathrm{MB}-$ $50 \mathrm{MB}$ and a bandwidth of $20-60 \mathrm{~GB} / \mathrm{s}$ per core. Thus, a few CPU cores can saturate a modern six-channel memory interface, that has a maximal bandwidth of $80 \mathrm{~GB} / \mathrm{s}$.

${ }^{5}$ The desktop CPU was an Intel Core $17-5820 \mathrm{~K}$ with a quad-channel memory interface with a maximal memory bandwidth of $60 \mathrm{~GB} / \mathrm{s}$. An expensive dual-socket compute node usually used in computing centers with two six-channel memory interfaces has a maximal memory bandwidth of only about 160 $\mathrm{GB} / \mathrm{s}$. Thus, at most a speedup of 2.5 can be expected from a modern computing node.
} 
To avoid this growth, we propose a one-particle basis composed of what we named partial natural orbitals: we define partial natural orbitals for the one-particle reduced density matrix

$$
\rho^{(1)}=\left(\begin{array}{cc}
\rho_{C, C}^{(1)}, & \rho_{C, B}^{(1)} \\
\left(\rho_{C, B}^{(1)}\right)^{\dagger} & \rho_{B, B}^{(1)}
\end{array}\right)
$$

by the unitary transformation $U$ of the one-particle basis with the form

$$
U=\left(\begin{array}{cc}
\mathbb{1} & 0 \\
0 & U_{B}
\end{array}\right)
$$

such that $U_{B} \in U\left(N_{\chi}-N_{C}\right)$ and

$$
\tilde{\rho}^{(1)}=U^{\dagger} \rho^{(1)} U=\left(\begin{array}{cc}
\rho_{C, C}^{(1)}, & \tilde{\rho}_{C, B}^{(1)} \\
\left(\tilde{\rho}_{C, B}^{(1)}\right)^{\dagger} & \operatorname{diag}\left(\lambda_{1}, \ldots, \lambda_{N_{\chi}-N_{C}}\right)
\end{array}\right) .
$$

$\lambda_{1}, \ldots, \lambda_{N_{\chi}-N_{C}}$ denote the eigenvalues of the one-particle reduced density matrix $\rho_{B, B}^{(1)}$ of the non-interacting states. This form guarantees that no interacting and non-interacting states are mixed and the interaction stays localized. The unitary matrix $U_{B}$ can be easily obtained from a diagonalization of the one-particle reduced density matrix of the non-interacting states.

The remaining question is how we actually select Slater determinants from the exponentially large Fock space. We perform this selection before a minimization of an unconstrained subproblem of the augmented Lagrangian approach. That means that the unconstrained minimization is performed in a fixed space of Slater determinants. We start from a small space of Slater determinants that allows the exact or approximate fulfillment of the constraints. For a diagonal one-particle reduced density matrix, i.e., in the naturalorbital basis, Walther and Blöchl [Walther, 2011] have proposed a simple construction of a many-particle wave function with at most $N_{\chi}+1$ Slater determinants that fulfills all constraints. We describe this scheme in appendix $\mathrm{C}$ and also employ this scheme for nondiagonal one-particle reduced density matrices to generate a many-particle wave function that approximately fulfills the constraints.

The space of Slater determinants is iteratively increased by adding the $N_{\text {expand }}$ determinants $\left\langle n_{1} \ldots n_{N_{\chi}}\right|$ for which

$$
\mid\left\langle n_{1} \ldots n_{N_{\chi}}\left|\frac{\partial L\left(\vec{d},\left\{h_{\alpha, \beta}\right\}, \lambda,\left\{\mu_{\alpha, \beta}\right\}, \mu\right)}{\partial\langle\Psi|}\right|\right.
$$

is largest. This criterion on the one hand respects the reduction of the objective $\langle\Psi|\hat{W}| \Psi\rangle$ but on the other hand also the reduction of constraint violations. The selection criterion can only add Slater determinants that are connected to existing Slater determinants by single-excitations $\hat{c}_{i}^{\dagger} \hat{c}_{j}$ or double excitations $\hat{c}_{i}^{\dagger} \hat{c}_{j}^{\dagger} \hat{c}_{k} \hat{c}_{l}$. Because we allow the expansion of the space of Slater determinants by some $N_{\text {expand }} \ll N_{\text {CI }}$ before every unconstrained subproblem, in principle any Slater determinant can be generated. Figure 9.1 shows the convergence of the CI-expansion with Eq. (9.76) for the density-matrix functional of oneparticle reduced density matrix of the exact ground state of a single-impurity Anderson 
model. The Anderson model is the model discussed in chapter 3 with 11 bath sites, 12 electrons, $t>0, U=4 t, V / t=0.4$ and $\epsilon_{f}=0$. The convergence in the one-particle basis constructed from natural orbitals is extremely rapid. The convergence with partial natural orbitals is slower than in the natural-orbital basis but still quite rapid. The reason is that most occupations of the exact ground state are close to zero or one. The rapid convergence of a CI-expansion for single-impurity Anderson models has, for example, been used to solve the impurity problem that occurs within dynamical mean-field theory with a CI-ansatz [Lu et al., 2014]. Figure 9.2 presents results for the convergence of the CI-expansion for a half-filled 12-site Hubbard chain with different interaction strengths. The convergence is rapid for small interaction strengths but becomes slower when increasing the interaction strength. Here, one can observe a situation where the selection criterion does not work well: in the limit $U / t \rightarrow \infty$, the exact solution contains only a few Slater determinants and should in principle be easy to describe with a finite CI-expansion. However, the selection criterion in Eq. (9.76) is not able to find these Slater determinants. This problem could be solved by adding some Slater determinants of the lowest eigenstates of the interaction Hamiltonian to the initial space of Slater determinants. We will investigate the convergence of the CI-ansatz for a real material in chapter 11. 
9. Wave-function based approach for the RDMF

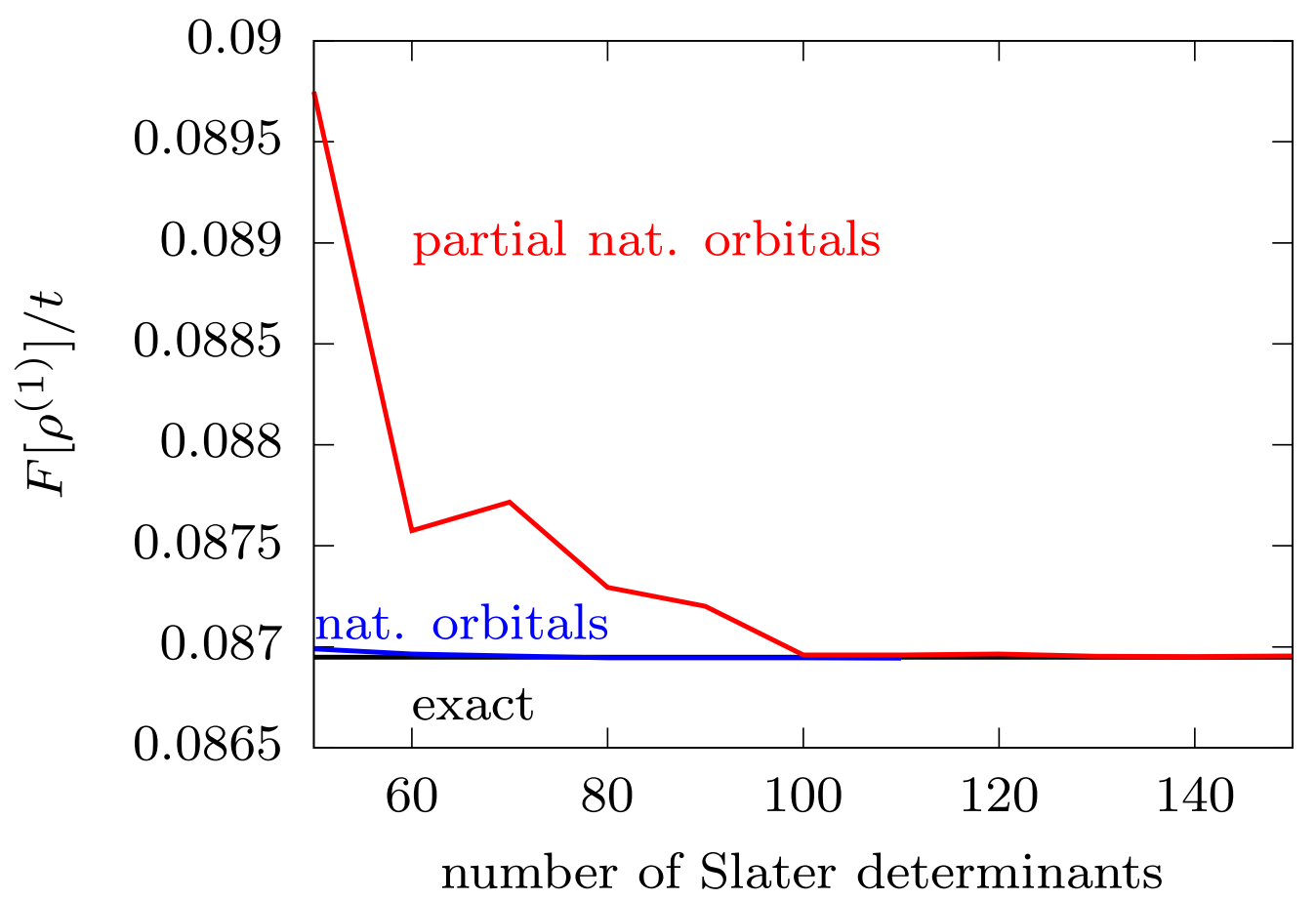

Figure 9.1.: Convergence of the density-matrix functional with the CI-ansatz with the number of Slater determinants for a single-impurity Anderson model introduced in chapter 3 with 11 bath sites, 12 electrons, $t>0, U=4 t, V / t=0.4$ and $\epsilon_{f}=0$. The blue line indicates the results for a one-particle basis, where the one-particle reduced density matrix is diagonal (natural orbitals), and the red line shows results for a one-particle basis, where only the one-particle reduced density matrix of the non-interacting states is diagonal (partial natural orbitals). The black line shows the exact result. The Slater determinants have been selected according to the criterion in Eq. (9.76). 


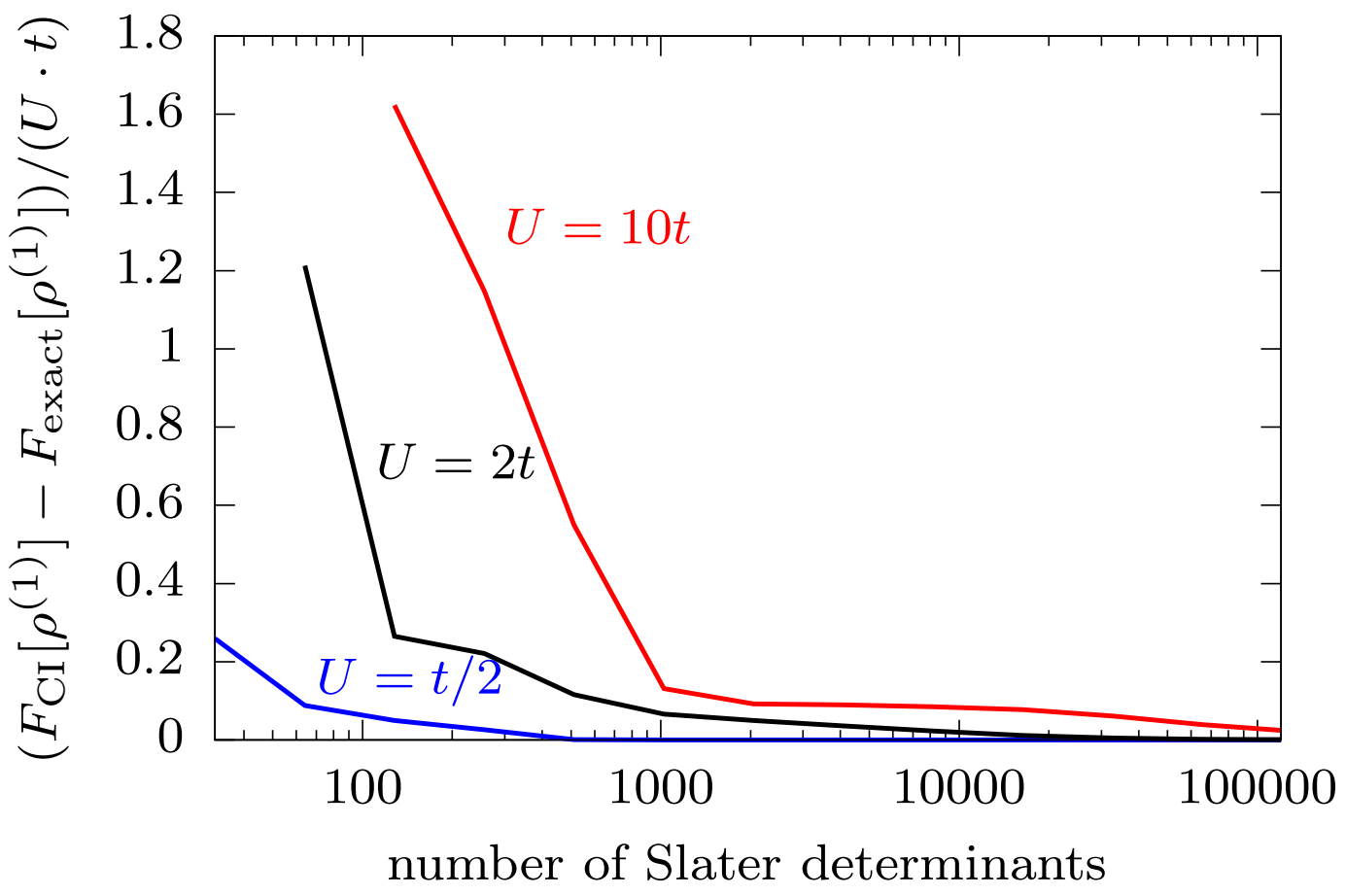

Figure 9.2.: Convergence of the density-matrix functional with the CI-ansatz with the number of Slater determinants for a half-filled 12-site Hubbard chain introduced in chapter 3 . The one-particle reduced density matrix $\rho^{(1)}$ is the oneparticle reduced density matrix of the exact ground state for the given interaction strength $U / t$. The Slater determinants have been selected according to the criterion in Eq. (9.76). 


\subsection{Impurity-bath separation ansatz}

\subsubsection{Wave-function ansatz}

The impurity-bath separation ansatz [Blöchl, 2013] is tailored for applications where the two-particle interaction only includes a few one-particle basis states. This is the case for the density-matrix functionals that have to be evaluated within the local approximation, Anderson models or quantum dots. The overall idea [Blöchl, 2013] is to describe degrees of freedom of the interacting one-particle states (impurity) with general many-particle wave functions and the degrees of freedom of the non-interacting states (bath) with manyparticle wave functions that only contain a few Slater determinants.

We assume that we have $N_{A}$ interacting one-particle states with the interaction Hamiltonian $\hat{W}$ and $N_{B}$ non-interacting one-particle states. We assume $N_{A} \ll N_{B}$. A general many-particle wave function can be written as a sum over Slater determinants as

$$
|\Psi\rangle=\sum_{m, n} \hat{Q}_{A, n}^{\dagger} \hat{Q}_{B, m}^{\dagger}|\mathcal{O}\rangle c_{n, m}
$$

with

$$
\begin{aligned}
& \hat{Q}_{A, n}^{\dagger}=\prod_{\alpha \leq N_{A}}\left(\hat{c}_{\alpha}^{\dagger}\right)^{s_{n, \alpha}^{(A)}} \\
& \hat{Q}_{B, n}^{\dagger}=\prod_{\alpha>N_{A}}\left(\hat{c}_{\alpha}^{\dagger}\right)^{s_{n, \alpha}^{(B)}} .
\end{aligned}
$$

$\vec{s}_{n}^{(A)}$ and $\vec{s}_{n}^{(B)}$ are vectors containing zeros and ones with $1 \leq n \leq 2^{N_{A}}$ and $1 \leq m \leq 2^{N_{B}}$. All matrix elements, like

$$
\left\langle\Psi\left|\hat{c}_{\alpha}^{\dagger} \hat{c}_{\beta}\right| \Psi\right\rangle=\sum_{m, n, m^{\prime}, n^{\prime}}\left\langle\mathcal{O}\left|\hat{Q}_{B, m} \hat{Q}_{A, n} \hat{c}_{\alpha}^{\dagger} \hat{c}_{\beta} \hat{Q}_{A, n^{\prime}}^{\dagger} \hat{Q}_{B, m^{\prime}}^{\dagger}\right| \mathcal{O}\right\rangle c_{n, m}^{*} c_{n^{\prime}, m^{\prime}}
$$

can be evaluated in this representation. The coefficients $c_{n, m}$ can be written as a Schmidtdecomposition

$$
c_{n, m}=\sum_{j=1}^{2^{N_{A}}} q_{j} a_{j, n} \otimes b_{j, m}
$$

with $q_{j} \geq 0$. With $\langle\Psi \mid \Psi\rangle=1$, the orthonormality of $\vec{a}_{j}$ as well as $\vec{b}_{j}$, we have $\sum_{j} q_{j}^{2}=1$. We now define the impurity wave function as

$$
\left|\Psi_{A, j}\right\rangle=\sum_{n} \hat{Q}_{A, n}^{\dagger}|\mathcal{O}\rangle a_{j, n}
$$

and the bath wave functions as

$$
\left|\Psi_{B, j}\right\rangle=\sum_{m} \hat{Q}_{B, m}^{\dagger}|\mathcal{O}\rangle b_{j, m}
$$

The many-particle wave function in Eq. (9.77) can be written as

$$
|\Psi\rangle=\sum_{j=1}^{2^{N_{A}}} q_{j}\left|\Psi_{A, j}\right\rangle \otimes\left|\Psi_{B, j}\right\rangle .
$$


Thus, the density-matrix functional with one many-particle wave function defined in Eq. (5.8) can be written as [Blöchl, 2013]

$$
F^{\hat{W}}\left[\rho^{(1)}\right]=\min _{\left\{q_{j},\left|\Psi_{A, j}\right\rangle,\left|\Psi_{B, j}\right\rangle\right\}: \text { constraints fulfilled }} \sum_{j} q_{j}^{2}\left\langle\Psi_{A, j}|\hat{W}| \Psi_{A, j}\right\rangle
$$

and the constraints to be fulfilled during the minimization as

$$
\begin{aligned}
q_{j} & \geq 0 \forall j \\
\sum_{j} q_{j}^{2} & =1 \\
\left\langle\Psi_{A, i} \mid \Psi_{A, j}\right\rangle & =\delta_{i, j} \forall i, j \\
\left\langle\Psi_{B, i} \mid \Psi_{B, j}\right\rangle & =\delta_{i, j} \forall i, j \\
\rho_{\alpha, \beta}^{(1)} & =\sum_{j} q_{j}^{2}\left\langle\Psi_{A, j}\left|\hat{c}_{\beta}^{\dagger} \hat{c}_{\alpha}\right| \Psi_{A, j}\right\rangle \forall \alpha \leq N_{A}, \beta \leq N_{A} \\
\rho_{\alpha, \beta}^{(1)} & =\sum_{j} q_{j}^{2}\left\langle\Psi_{B, j}\left|\hat{c}_{\beta}^{\dagger} \hat{c}_{\alpha}\right| \Psi_{B, j}\right\rangle \forall \alpha>N_{A}, \beta>N_{A} \\
\rho_{\alpha, \beta}^{(1)} & =\sum_{j, j^{\prime}} q_{j} q_{j^{\prime}}\left\langle\Psi_{A, j}\left|\hat{c}_{\beta}^{\dagger}(-1)^{\hat{N}}\right| \Psi_{A, j^{\prime}}\right\rangle\left\langle\Psi_{B, j}\left|\hat{c}_{\alpha}\right| \Psi_{B, j^{\prime}}\right\rangle \forall \alpha>N_{A}, \beta \leq N_{A} \\
\rho_{\alpha, \beta}^{(1)} & =\sum_{j, j^{\prime}} q_{j} q_{j^{\prime}}\left\langle\Psi_{A, j}\left|\hat{c}_{\alpha}(-1)^{\hat{N}+1}\right| \Psi_{A, j^{\prime}}\right\rangle\left\langle\Psi_{B, j}\left|\hat{c}_{\beta}^{\dagger}\right| \Psi_{B, j^{\prime}}\right\rangle \forall \alpha \leq N_{A}, \beta>N_{A} .
\end{aligned}
$$

The extension to an ensemble of many-particle wave functions is straight forward. So far we have only rewritten the many-particle wave function. However, we can use the above form to treat the impurity wave functions $\left|\Psi_{A, j}\right\rangle$ and the bath wave functions $\left|\Psi_{B, j}\right\rangle$ differently. We propose to use general many-particle wave functions for the impurity wave functions and simplified bath wave functions. Hence, a impurity wave functions $\left|\Psi_{A, j}\right\rangle$ is represented as a sum of Slater determinants and has $2^{N_{A}}$ complex variational parameters. We have at most $2^{N_{A}}$ such wave functions, because $1 \leq j \leq 2^{N_{A}}$. The bath wave functions $\left|\Psi_{B, j}\right\rangle$ are parametrized as sums of Slater determinants with different particle numbers

$$
\left|\Psi_{B, j}\right\rangle=\sum_{N=0}^{N_{B}} p_{j, N}\left|\Psi^{N}\left(U_{j, N}\right)\right\rangle .
$$

Here, $\left|\Psi^{N}\left(U_{j, N}\right)\right\rangle$ denotes a Slater determinant with $N$ particles in the one-particle basis defined by the unitary matrix $U_{j, N} \in U\left(N_{B}\right)$. We have also evaluated to use only a single Slater determinant for $\left|\Psi_{B, j}\right\rangle$. However, this turned out to give not enough variational freedom for the bath wave functions to represent the given one-particle reduced density matrix. It should be noted that also the ansatz in Eq. (9.94) does not guarantee that a given one-particle reduced density matrix can be represented. However, it drastically reduces this problem while only introducing a small additional computational complexity. The bath wave functions in Eq. (9.94) have $\left(N_{B}+1\right) \cdot 2 \cdot N_{B}^{2}+\left(N_{B}+1\right)$ real variational parameters each. The impurity-bath separation ansatz can be converged to the exact density-matrix functional by increasing the number of Slater determinants $N_{\text {bath-dets }}$ in the bath wave functions. Thus, the ansatz in Eq. (9.94) is an ansatz with $N_{\text {bath-dets }}=N_{B}+1$. We have not investigated constructions with higher values of $N_{\text {bath-dets }}$. 
The overlaps of the bath wave functions can be written as [Utsuno et al., 2013]

$$
\left\langle\Psi_{B, j} \mid \Psi_{B, j^{\prime}}\right\rangle=\sum_{N=0}^{N_{B}} p_{j, N}^{*} p_{j^{\prime}, N} \operatorname{det}\left(U_{j, N}^{\dagger} U_{j^{\prime}, N}\right)
$$

and the elements of the one-particle reduced density matrix as

$$
\begin{aligned}
\left\langle\Psi_{B, j}\left|\hat{c}_{\beta}^{\dagger} \hat{c}_{\alpha}\right| \Psi_{B, j}\right\rangle & =\sum_{N=0}^{N_{B}}\left|p_{j, N}\right|^{2} \rho_{B, j, N, \alpha, \beta}^{(1)} \\
\rho_{B, j, N}^{(1)} & =U_{j, N}^{\dagger} \operatorname{diag}(\underbrace{1, \ldots, 1}_{N \text { times }}, 0, \ldots 0) U_{j, N} .
\end{aligned}
$$

The elements $\left\langle\Psi_{B, j}\left|\hat{c}_{\alpha}\right| \Psi_{B, j^{\prime}}\right\rangle$ and $\left\langle\Psi_{B, j}\left|\hat{c}_{\beta}^{\dagger}\right| \Psi_{B, j^{\prime}}\right\rangle$ can be written as

$$
\begin{aligned}
\left\langle\Psi_{B, j}\left|\hat{c}_{\alpha}\right| \Psi_{B, j^{\prime}}\right\rangle & =\sum_{N=0}^{N_{B}-1} p_{j, N}^{*} p_{j^{\prime}, N+1}\left\langle\Psi^{N}\left(U_{j, N}\right)\left|\hat{c}_{\alpha}\right| \Psi^{N+1}\left(U_{j^{\prime}, N+1}\right)\right\rangle \\
\left\langle\Psi_{B, j}\left|\hat{c}_{\beta}^{\dagger}\right| \Psi_{B, j^{\prime}}\right\rangle & =\sum_{N=1}^{N_{B}} p_{j, N}^{*} p_{j^{\prime}, N-1}\left\langle\Psi^{N}\left(U_{j, N}\right)\left|\hat{c}_{\beta}^{\dagger}\right| \Psi^{N-1}\left(U_{j^{\prime}, N-1}\right)\right\rangle .
\end{aligned}
$$

The individual terms $\left\langle\Psi^{N}\left(U_{j, N}\right)\left|\hat{c}_{\alpha}\right| \Psi^{N+1}\left(U_{j^{\prime}, N+1}\right)\right\rangle$ or $\left\langle\Psi^{N}\left(U_{j, N}\right)\left|\hat{c}_{\alpha}\right| \Psi^{N-1}\left(U_{j^{\prime}, N-1}\right)\right\rangle$ can be evaluated with basis transformations. The requirement of unitarity of $U_{j, N} \in U\left(N_{B}\right)$ is problematic for numerical purposes because parametrizations of unitary matrices or the addition of the unitarity requirement as an additional constraint are computationally cumbersome. The unitarity of $U_{j, N} \in U\left(N_{B}\right)$ can be relaxed to allow general complex matrices $U_{j, N} \in \mathbb{C}^{N_{B} \times N_{B}}$ if Eq. (9.97) is replaced with [Utsuno et al., 2013]

$$
\rho_{B, j, N}^{(1)}=U_{j, N}\left(U_{j, N}^{\dagger} U_{j, N}\right)^{-1} U_{j, N}^{\dagger}
$$

Furthermore, the individual terms $\left\langle\Psi^{N}\left(U_{j, N}\right)\left|\hat{c}_{\alpha}\right| \Psi^{N+1}\left(U_{j^{\prime}, N+1}\right)\right\rangle \quad$ or $\left\langle\Psi^{N}\left(U_{j, N}\right)\left|\hat{c}_{\alpha}\right| \Psi^{N-1}\left(U_{j^{\prime}, N-1}\right)\right\rangle$ now have to be evaluated by a modified variant of Eq. (9.95).

The overall computational complexity of the evaluation of the all quantities that include the bath wave functions is in $\mathcal{O}\left(2^{2 N_{A}} \cdot N_{B}^{5}\right)$ for the ansatz of Eq. (9.94). With the quantities defined in the current section, we can apply the augmented Lagrangian to solve the constrained optimization problem. As in the case of the configuration-interaction ansatz, also here no constrained minimization scheme that requires the numerically exact fulfillment of the constraints can be used because no practical scheme is known to create an initial guess of the parameters in the ansatz that fulfills all constraints.

\subsubsection{Example results}

\section{Single-impurity Anderson model}

We evaluate the performance of the impurity-bath separation ansatz for the singleimpurity Anderson model defined in section 3.2. The one-particle states treated as the bath in the impurity-bath separation ansatz are non-interacting one-particle states in the 
physical model. This situation is in contrast to an application of the impurity-bath separation ansatz within the local approximation of the density-matrix functional, where the one-particle states of the bath correspond to interacting one-particle states of the physical system. Thus, we expect the single-impurity Anderson model to show the best-case performance of the impurity-bath separation ansatz.

We investigate the performance of the ansatz for the single-impurity Anderson model defined in section 3.2 with an impurity consisting of one site, a bath consisting of 5 sites and a filling of 6 electrons. We consider the density-matrix functional for the exact ground state of this system at zero temperature. The other parameters were chosen as $t>0$, $\epsilon_{f}=0$ and $V / t=0.4$. Figure 9.3 compares the density-matrix functional obtained with the ansatz (9.94) and a constrained minimization based on the augmented Lagrangian to the exact density-matrix functional of the ground state. The absolute difference is below $10^{-5} t$ which shows that the impurity-bath separation ansatz proposed here describes the ground state very well. Figure 9.4 shows the maximal absolute constraint violation of the numerical solution for the constraints listed in Eq. (9.86)-Eq. (9.93). We have not been able to obtain a numerical solution with a maximal absolute constraint violation below $10^{-6}$ with this ansatz. The fact that in most cases $F_{\text {imp-bath }}<F_{\text {exact }}$ shown in figure 9.3 is due to the fact, that a sufficiently small maximal absolute constraint violation could not be reached in the numerical minimization. Thus, the expectation that the density-matrix functional from the ansatz is an upper limit to the exact density-matrix functional can be violated. We can understand this behavior by inspecting the wave function $\left|\Psi_{\text {exact }}\right\rangle$ of the exact ground state. For the following we consider the smaller system with one impurity site, three bath sites and the parameters $t>0, U / t=4, \epsilon_{f}$ and $V / t=0.4$. With the Schmidt decomposition in Eq. (9.84), we obtain the Schmidt coefficients

$$
q_{1}=0.6641, \quad q_{2}=0.516, \quad q_{3}=0.516, \quad q_{4}=0.16259 .
$$

Thus, this state cannot be approximated further by reducing the number of impurity-and bath wave functions below $2^{N_{A}}$ without introducing large deviations. The one-particle reduced density matrices of the impurity- and bath wave functions, i.e.

$$
\begin{aligned}
& \rho_{A, j, \alpha, \beta}^{(1)}=\left\langle\Psi_{A, j}\left|\hat{c}_{\beta}^{\dagger} \hat{c}_{\alpha}\right| \Psi_{A, j}\right\rangle \\
& \rho_{B, j, \alpha, \beta}^{(1)}=\left\langle\Psi_{B, j}\left|\hat{c}_{\beta}^{\dagger} \hat{c}_{\alpha}\right| \Psi_{B, j}\right\rangle,
\end{aligned}
$$

have the occupations

$$
\begin{aligned}
f_{A, 1, i} & \in\{0\} \\
f_{A, 2, i} & \in\{0,1\} \\
f_{A, 3, i} & \in\{0,1\} \\
f_{A, 4, i} & \in\{0,1\} \\
f_{B, 1, i} & \in\left\{0,5.0 \cdot 10^{-5}, 0.99995,1\right\} \\
f_{B, 2, i} & \in\left\{0,5.4 \cdot 10^{-5}, 0.999946,1\right\} \\
f_{B, 3, i} & \in\left\{0,5.4 \cdot 10^{-5}, 0.999946,1\right\} \\
f_{B, 4, i} & \in\left\{0,2.1 \cdot 10^{-4}, 0.99979,1\right\} .
\end{aligned}
$$

The occupations of the one-particle reduced density matrices of the bath $\rho_{B, j}^{(1)}$ have nearly integer occupations and hence can be represented well with single Slater determinants. 
The occupations also show the reason for the finite constraint violation shown in Figure 9.4 .

\section{Hubbard chain within the local approximation}

To apply the impurity-bath separation ansatz to the Hubbard chain, we have to employ the local approximation of the density-matrix functional discussed in section 5.5. Consider the four-site Hubbard chain with the interaction

$$
\hat{W}=U \sum_{i=1}^{4} \hat{n}_{i, \uparrow} \hat{n}_{i, \downarrow}
$$

In the local approximation, the density-matrix functional is approximated as

$$
F^{\hat{W}}\left[\rho^{(1)}\right] \approx \sum_{i=1}^{4} F^{U \hat{n}_{i, \uparrow} \hat{n}_{i, \downarrow}}\left[\rho^{(1)}\right] .
$$

We now consider the first density-matrix functional $F^{U \hat{n}_{1, \uparrow} \hat{n}_{1, \downarrow}}\left[\rho^{(1)}\right]$ for the one-particle reduced density matrix of the exact ground state at zero temperature. We tried to apply the impurity-bath separation ansatz for this density-matrix functional but have not been able to find a parametrization that fulfills all constraints in Eq. (9.86)-Eq. (9.93) sufficiently well. To understand the reason for this, we consider the exact ground-state wave function of the half-filled four-site Hubbard chain with $U / t=4$ and perform a Schmidt decomposition. We obtain the Schmidt values

$$
q_{1}=0.6536, \quad q_{2}=0.6536, \quad q_{3}=0.2698, \quad q_{4}=0.26298 .
$$

The one-particle reduced density matrices of the impurity- and bath wave functions, i.e.

$$
\begin{aligned}
\rho_{A, j, \alpha, \beta}^{(1)} & =\left\langle\Psi_{A, j}\left|\hat{c}_{\beta}^{\dagger} \hat{c}_{\alpha}\right| \Psi_{A, j}\right\rangle \\
\rho_{B, j, \alpha, \beta}^{(1)} & =\left\langle\Psi_{B, j}\left|\hat{c}_{\beta}^{\dagger} \hat{c}_{\alpha}\right| \Psi_{B, j}\right\rangle,
\end{aligned}
$$

have the occupations

$$
\begin{aligned}
& f_{A, 1, i} \in\{0,1\} \\
& f_{A, 2, i} \in\{0,1\} \\
& f_{A, 3, i} \in\{0\} \\
& f_{A, 4, i} \in\{0,1\} \\
& f_{B, 1, i} \in\{0,0.0158,0.1203,0.1361,0.8638,0.8797,0.9842\} \\
& f_{B, 2, i} \in\{0,0.0158,0.1203,0.1361,0.8638,0.8797,0.9842\} \\
& f_{B, 3, i} \in\{0,0.1355,0.8649,0.9996\} \\
& f_{B, 4, i} \in\left\{0,3.921 \cdot 10^{-4}, 0.1351,0.8645\right\} .
\end{aligned}
$$

Thus, the occupation of the bath wave functions are very fractional and cannot be represented by a single or a few Slater determinants. Please note, that these results are for the exact ground-state wave function. We can also consider the many-particle wave 
function $\left|\Psi_{\text {Levy,1 }}\right\rangle$ that corresponds to minimum of the Levy's constrained search of the local functional

$$
F^{U \hat{n}_{1, \uparrow} \hat{n}_{1, \downarrow}}\left[\rho^{(1)}\right]=\min _{\left|\Psi_{\text {Levy }, 1}\right\rangle \rightarrow \rho^{(1)}}\left\langle\Psi_{\text {Levy }, 1}\left|U \hat{n}_{1, \uparrow} \hat{n}_{1, \downarrow}\right| \Psi_{\text {Levy }, 1}\right\rangle
$$

For this many-particle wave function, we obtain the Schmidt coefficients

$$
q_{1}=0.6559, \quad q_{2}=0.6559, \quad q_{3}=0.2640, \quad q_{4}=0.2640 .
$$

and the occupations of the impurity- and bath wave functions

$$
\begin{aligned}
& f_{A, 1, i} \in\{0,1\} \\
& f_{A, 2, i} \in\{0,1\} \\
& f_{A, 3, i} \in\{0,1\} \\
& f_{A, 4, i} \in\{0\} \\
& f_{B, 1, i} \in\left\{0,1.670 \cdot 10^{-4}, 0.1379,0.1380,0.8619,0.8621\right\} \\
& f_{B, 2, i} \in\left\{0,1.670 \cdot 10^{-4}, 0.1379,0.1380,0.8619,0.8621\right\} \\
& f_{B, 3, i} \in\left\{0,1.332 \cdot 10^{-5}, 0.1413,0.8587\right\} \\
& f_{B, 4, i} \in\{0,0.1413,0.8587,0.999987\} .
\end{aligned}
$$

The Schmidt coefficients and the occupation are very similar to the corresponding values of the ground-state wave function and the same conclusions apply.

\subsubsection{Conclusion and outlook}

In conclusion, we have shown that the impurity-bath separation ansatz with a few Slater determinants for the bath wave functions works well for the single-impurity Anderson model introduced in section 3.2. However, the constraints that arise from the densitymatrix functional could not be fulfilled to machine precision. We found the reason for this issue in the occupations of the one-particle reduced density matrices of the bath wave functions. These occupations are only approximately integer with deviations from integer numbers of the order of $10^{-4}$. In contrast, for a Hubbard chain within the local approximation, the occupations of the one-particle reduced density matrices of the bath wave functions are very fractional. We conclude that the impurity-bath separation ansatz with a few Slater determinants for the bath wave functions is not able to represent the oneparticle reduced density matrix of the exact ground state or an approximate one-particle reduced density matrix with a similar structure at all.

If many Slater determinants would be allowed for the bath wave functions, i.e., if we approach a complete representation of $\left|\Psi_{B, j}\right\rangle$, then the impurity-bath separation ansatz can represent any state. However, then the whole underlying idea of representing the bath wave functions in a simpler form than the impurity wave functions would be abandoned, and the computational complexity would be exponential in the size of the bath in the worst case. 

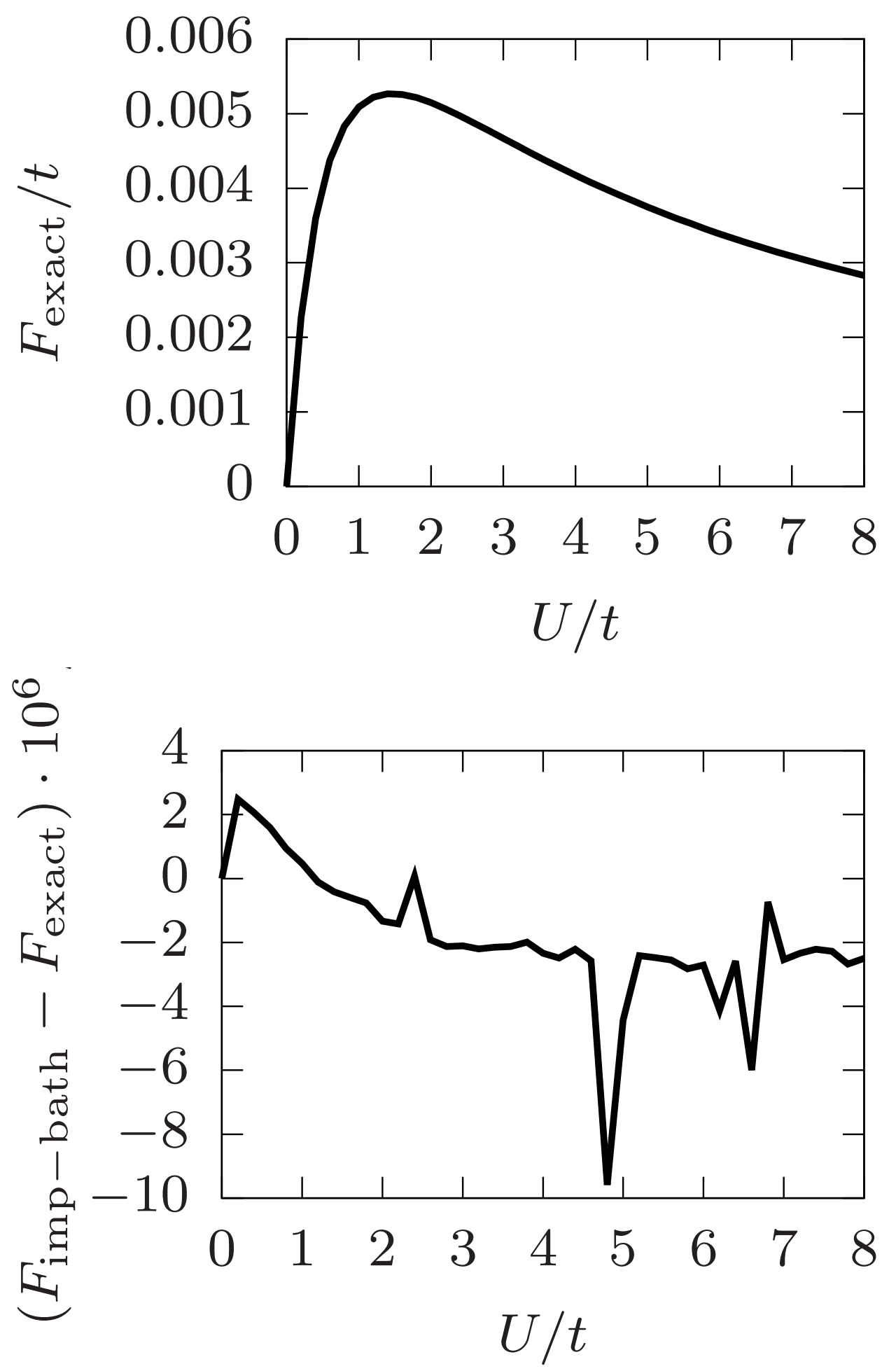

Figure 9.3.: The top figure shows the value of the exact density-matrix functional of the one-particle reduced density matrix of the exact zero-temperature groundstate of a half-filled single-impurity Anderson model defined in section 3.2 with a one-site impurity and a 5 -site bath. The interaction $U$ on the impurity and the hopping parameter $t>0$ in the bath give the effective interaction strength $U / t$. The impurity on-site energy is $\epsilon_{f}=0$ and the parameter of the impurity-bath hybridization is $V / t=0.4$. The bottom figure shows the difference of the exact density matrix functional to the density-matrix functional obtained with the impurity-bath ansatz of Eq. (9.94). 


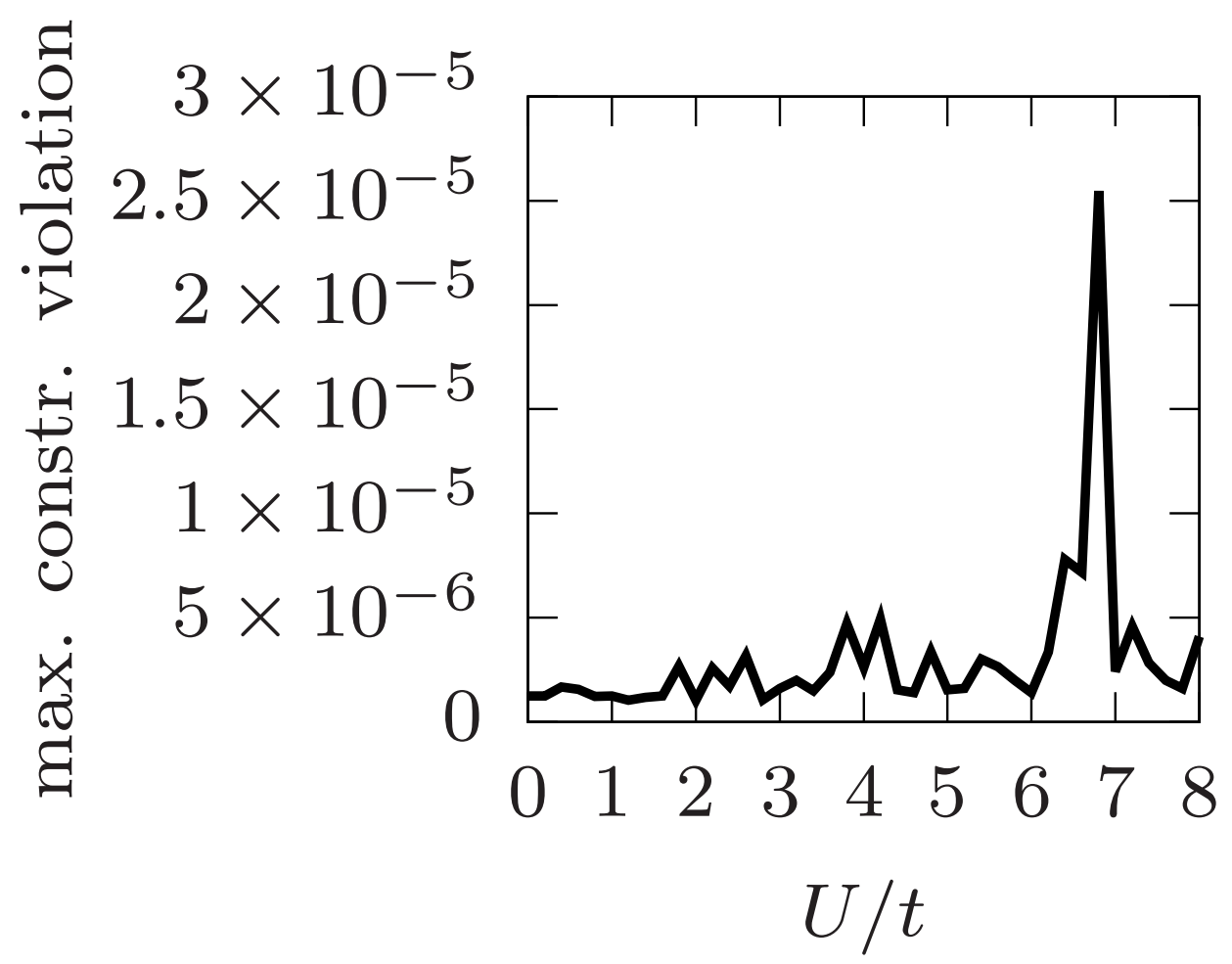

Figure 9.4.: Maximal absolute violation of the constraints given in Eq. (9.86)-Eq. (9.93) by the numerical solution of the impurity-bath separation ansatz. The model and parameters are identical to figure 9.3. 


\subsection{Matrix product states}

The use of matrix product states (MPS, [White, 1992, 1993; Östlund and Rommer, 1995; Schollwöck, 2005; Schollwöck, 2011] $]^{6}$ ) as the ansatz for the many-particle wave functions in solid-state physics and quantum chemistry [Chan and Sharma, 2011; Marti and Reiher, 2011; Kurashige, 2014] has emerged as a new powerful method. We have discussed matrix product states (MPS) and matrix product operators (MPO) in detail in section 8.8. As a systematically improvable ansatz, MPS are suitable for the evaluation of the densitymatrix functional.

\subsubsection{Constrained optimization over MPS}

We propose to solve the minimization problem of the density-matrix functional given by Eq. (9.27) and the constraints in Eq. (9.28) and Eq. (9.29) with MPS-parametrizations of the many-particle wave functions. For simplicity we only consider a single many-particle wave function at zero temperature, i.e. Levy's functional $F_{\text {Levy }}^{\hat{W}}\left[\rho^{(1)}\right]$ of Eq. (5.25). The problem to be solved is

$$
F_{\text {Levy }}^{\hat{W}}\left[\rho^{(1)}\right]=\min _{\{|\Psi\rangle\}}\langle\Psi|\hat{W}| \Psi\rangle
$$

with the constraints

$$
\begin{aligned}
1 & =\langle\Psi \mid \Psi\rangle, \\
\rho_{\beta, \alpha}^{(1)} & =\sum_{i}\left\langle\Psi\left|\hat{c}_{\alpha}^{\dagger} \hat{c}_{\beta}\right| \Psi\right\rangle .
\end{aligned}
$$

The corresponding Lagrange function is

$$
L\left(|\Psi\rangle, \lambda, h_{\alpha, \beta}\right)=\langle\Psi|\hat{W}| \Psi\rangle-\lambda(\langle\Psi \mid \Psi\rangle-1)-\sum_{\alpha, \beta} h_{\alpha, \beta}\left(\left\langle\Psi\left|\hat{c}_{\alpha}^{\dagger} \hat{c}_{\beta}\right| \Psi\right\rangle-\rho_{\beta, \alpha}^{(1)}\right)
$$

with the Lagrange multipliers $\lambda$ for the norm constraint and $h_{\alpha, \beta}$ for the density-matrix constraint. For an MPS-representation of the many-particle wave function

$$
\begin{aligned}
|\Psi\rangle & =\sum_{j_{1}, \ldots, j_{L} \in\{0, \uparrow, \downarrow, \uparrow \downarrow\}} M^{(1), j_{1}} M^{(2), j_{2}} \cdots M^{(L), j_{L}}\left|j_{1}, \ldots, j_{L}\right\rangle \\
M^{(1), j_{1}} M^{(2), j_{2}} \cdots M^{(L), j_{L}} & =j_{1}^{j_{1}}
\end{aligned}
$$

the entries of the matrices $M^{(i), j_{i}}$ are the variational parameters and one would obtain a highly nonlinear constrained minimization problem. To avoid this non-linearity, we adopt the DMRG-like iterative minimization described in section 8.8.4. We propose to use the augmented-Lagrangian formalism described in section 9.3.3 to map the constrained minimization problem to a series of unconstrained minimization problems. The augmented Lagrangian that corresponds to the given constrained minimization problem is

$$
\begin{aligned}
L\left(|\Psi\rangle, \lambda, \mu_{\lambda}, h_{\alpha, \beta}, \mu_{\alpha, \beta}\right) & =L\left(|\Psi\rangle, \lambda, h_{\alpha, \beta}\right)+\frac{\mu_{\lambda}}{2}(\langle\Psi \mid \Psi\rangle-1)^{2} \\
& +\sum_{\alpha, \beta} \frac{\mu_{\alpha, \beta}}{2}\left(\left\langle\Psi\left|\hat{c}_{\alpha}^{\dagger} \hat{c}_{\beta}\right| \Psi\right\rangle-\rho_{\beta, \alpha}^{(1)}\right)^{2} .
\end{aligned}
$$

\footnotetext{
${ }^{6} \mathrm{~A}$ more complete list of references can be found in the reviews [Schollwöck, 2005], [Verstraete et al., 2008] and [Schollwöck, 2011].
} 
The unconstrained subproblems defined by Eq. (9.48) have here the form

$$
\min _{|\Psi\rangle} L\left(|\Psi\rangle, \lambda, \mu_{\lambda}, h_{\alpha, \beta}, \mu_{\alpha, \beta}\right)
$$

with fixed Lagrange multipliers and penalty parameters. We propose to solve an unconstrained problem with a DMRG-like iterative minimization. That means, that the minimization is performed stepwise by only minimizing over a subset of the matrices, i.e. a single $M^{(l), j_{l}}$ in the single-site approach or $M^{(l), j_{l}}$ and $M^{(l+1), j_{l+1}}$ in the two-site approach, while the other matrices are not changed. We consider the two-site approach and assume that the in the current step the matrices $M^{(l), j_{l}}$ and $M^{(l+1), j_{l+1}}$ at site $l$ and $l+1$ are to be optimized. We also assume that the MPS representation is in a mixed-canonical form

$$
|\Psi\rangle=\sum_{j_{1}, \ldots, j_{L}} A^{(1), j_{1}} \cdots A^{(l-1), j_{l-1}} M^{(l), j_{l}} M^{(l+1), j_{l+1}} B^{(l+2), j_{l+2}} \cdots B^{(L), j_{L}}\left|j_{1}, \ldots, j_{L}\right\rangle
$$

with left-normalized matrices $A^{(i), j_{i}}$ and right-normalized matrices $B^{(i), j_{i}}$. The MPOs for the interaction operator $\hat{W}$,

$$
\begin{gathered}
\hat{W}=\sum_{j_{1}, \ldots, j_{L}, j_{1}^{\prime}, \ldots, j_{L}^{\prime}} W^{(1), j_{1}, j_{1}^{\prime}} W^{(2), j_{2}, j_{2}^{\prime}} \ldots W^{(L-1), j_{L-1}, j_{L-1}^{\prime}} W^{(L), j_{L}, j_{L}^{\prime}}\left|j_{1}, \ldots, j_{L}\right\rangle\left\langle j_{1}^{\prime}, \ldots, j_{L}^{\prime}\right| \\
W^{(1), j_{1}, j_{1}^{\prime}} W^{(2), j_{2}, j_{2}^{\prime}} \cdots W^{(L-1), j_{L-1}, j_{L-1}^{\prime}} W^{(L), j_{L}, j_{L}^{\prime}}=\frac{1}{W^{(1)}} \cdots-\frac{1}{\mathbf{l}} \cdots \frac{W^{(L)}}{\mathbf{l}}
\end{gathered}
$$

and the operators $\hat{c}_{\alpha}^{\dagger} \hat{c}_{\beta}$

$$
\begin{gathered}
\hat{c}_{\alpha}^{\dagger} \hat{c}_{\beta}=\sum_{j_{1}, \ldots, j_{L}, j_{1}^{\prime}, \ldots, j_{L}^{\prime}} C_{\alpha, \beta}^{(1), j_{1}, j_{1}^{\prime}} \cdots C_{\alpha, \beta}^{(L), j_{L}, j_{L}^{\prime}}\left|j_{1}, \ldots, j_{L}\right\rangle\left\langle j_{1}^{\prime}, \ldots, j_{L}^{\prime}\right| \\
C_{\alpha, \beta}^{(1), j_{1}, j_{1}^{\prime}} \cdots C_{\alpha, \beta}^{(L), j_{L}, j_{L}^{\prime}}=\frac{1}{C_{\alpha, \beta}^{(1)}} \cdots-\frac{-\frac{1}{C_{\alpha, \beta}^{(l)}}}{\mathbf{I}} \cdots-\frac{\frac{1}{C_{\alpha, \beta}^{(L)}}}{\mathbf{I}}
\end{gathered}
$$

are constructed. The minimization will be performed over elements of $P^{(l), j_{l}, j_{l+1}}$, which is the contraction

$$
P^{(l), j_{l}, j_{l+1}}=M^{(l), j_{l}} M^{(l+1), j_{l+1}},
$$

to make use of the larger ansatz space. Then we implicitly form the local matrix-product representations of the interaction operator $\hat{W}$,

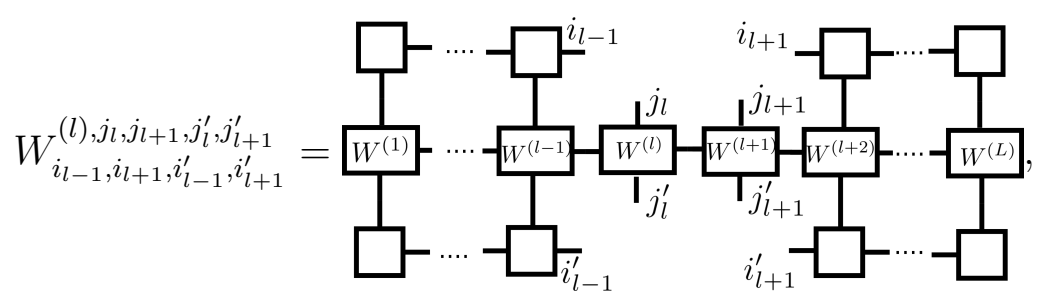

and the corresponding local matrix-product representations of the operators $\hat{c}_{\alpha}^{\dagger} \hat{c}_{\beta}$

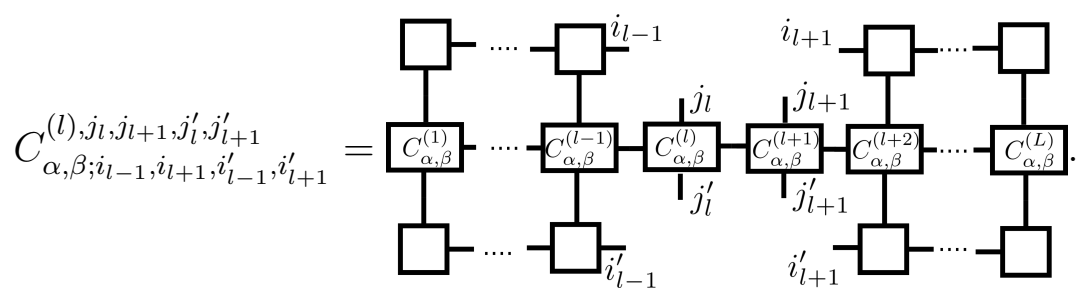


Please note, that at this point we don't restricted the form of the interaction operator. The derivative of the augmented Lagrangian in Eq. (9.141) with respect to $P^{(l), j_{l}, j_{l+1}}$ takes the form

$$
\begin{aligned}
\frac{\partial L\left(P^{(l), j_{l}, j_{l+1}}, \lambda, \mu_{\lambda}, h_{\alpha, \beta}, \mu_{\alpha, \beta}\right)}{\partial\left(P^{\left.(l), j_{l}, j_{l+1}\right)^{*}}\right.} & =\sum_{j_{l}^{\prime}, j_{l+1}^{\prime}} W^{(l), j_{l}, j_{l+1}, j_{l}^{\prime}, j_{l+1}^{\prime}} P^{(l), j_{l}^{\prime}, j_{l+1}^{\prime}} \\
& -\left[\lambda-\mu_{\lambda} c_{\mathrm{norm}}\left(P^{(l), j_{l}, j_{l+1}}\right)\right] P^{(l), j_{l}, j_{l+1}} \\
& -\sum_{\alpha, \beta}\left[h_{\alpha, \beta}-\mu_{\alpha, \beta} c_{\alpha, \beta}\left(P^{(l), j_{l}, j_{l+1}}\right)\right] \sum_{j_{l}^{\prime}, j_{l+1}^{\prime}} C^{(l), j_{l}, j_{l+1}, j_{l}^{\prime}, j_{l+1}^{\prime}} P^{(l), j_{l}^{\prime}, j_{l+1}^{\prime}}
\end{aligned}
$$

with

$$
\begin{aligned}
c_{\mathrm{norm}}\left(P^{(l), j_{l}, j_{l+1}}\right) & =\sum_{j_{l}^{\prime}, j_{l+1}^{\prime}} P^{(l), j_{l}^{\prime}, j_{l+1}^{\prime}}\left(P^{(l), j_{l}^{\prime}, j_{l+1}^{\prime}}\right)^{*}-1 \\
c_{\alpha, \beta}\left(P^{(l), j_{l}, j_{l+1}}\right) & =\sum_{j_{l}, j_{l+1}, j_{l}^{\prime}, j_{l+1}^{\prime}} C^{(l), j_{l}, j_{l+1}, j_{l}^{\prime}, j_{l+1}^{\prime}} P^{(l), j_{l}^{\prime}, j_{l+1}^{\prime}}\left(P^{(l), j_{l}, j_{l+1}}\right)^{*}-\rho_{\beta, \alpha}^{(1)} .
\end{aligned}
$$

In contrast to the minimization of the total energy over MPS discussed in section 8.8.4, the derivative in Eq. (9.149) is not linear in $P^{(l), j_{l}, j_{l+1}}$ and the minimization condition cannot be written as an eigenvalue problem. However, we can solve the local minimization problem over $P^{(l), j_{l}^{\prime}, j_{l+1}^{\prime}}$ with a conjugate-gradient approach or the limited memory BFGSmethod [Broyden, 1970; Fletcher, 1970; Goldfarb, 1970; Shanno, 1970]. We have implemented this scheme with the MPS-library ITensor ${ }^{7}$ by extending the existing DMRG-like ground state search to the minimization of the augmented Lagrangian with a conjugategradient method. This scheme could also be used to enforce other constraints on the MPS as long as the constraints can be written as an expectation value of some operator.

\subsubsection{Example results}

We investigate the convergence of the iterative solution of the constrained minimization problem proposed in the previous section. We consider the case of a half-filled 8-site Hubbard chain with an interaction strength of $U / t=2$. We have obtained the ground state of this system with the total-energy minimization implemented in ITensor. The ground state can be represented as an MPS with a maximal bond dimension of 120 and a maximal truncation error of $10^{-10}$. We have also obtained the one-particle reduced density matrix $\rho^{(1)}$ of this ground state and consider the evaluation of the density-matrix functional $F^{\hat{W}}\left[\rho^{(1)}\right]$ for this one-particle reduced density matrix with the approach presented in section 9.6.1. Figure 9.5 shows the interaction energy $\langle\Psi|\hat{W}| \Psi\rangle$ after each solution of an unconstrained subproblem, i.e. a two-site minimization problem. The convergence with the number of sweeps is moderately slower than the convergence of the total energy in a total-energy minimization for the same system, which is also shown in Figure 9.5 for comparison. A complete sweep through the 8-site system consists of 14 two-site problems. However, every minimization of an unconstrained subproblem is much more computationally involved than in a DMRG-ground-state search, because it is a non-linear problem and instead of

\footnotetext{
${ }^{7}$ ITensor C++ library (version 2.1.1), http://itensor.org/
} 


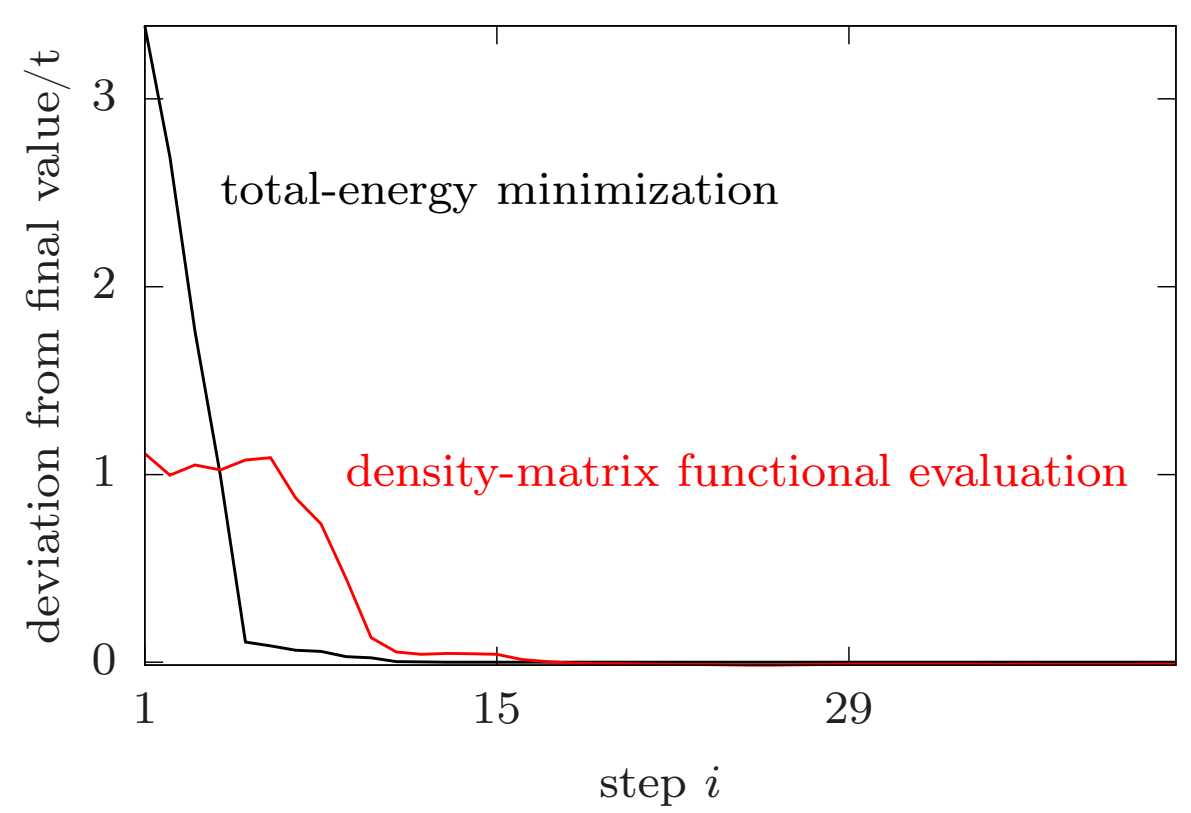

Figure 9.5.: Convergence of the interaction energy (red line) during the DMRG-like constrained minimization algorithm proposed in section 9.6.1 for the one-particle reduced density matrix of the numerically exact ground state of a half-filled 8 -site Hubbard chain with interaction strength $U / t=2$. A step denotes the solution of the two-site unconstrained minimization problem at a bond. The black line shows the convergence of the total energy during a conventional DMRG-like total-energy minimization. One sweep through the 8-site system consists of 14 steps. Thus, the figure shows three sweeps.

one MPO for the Hamiltonian there are $\in \mathcal{O}\left(L^{2}\right)$ MPOs for the interaction Hamiltonian and the operators $\hat{c}_{\alpha}^{\dagger} \hat{c}_{\beta}$. Figure 9.6 shows the maximal absolute constraint violation after each solution of an unconstrained subproblem. The presented results show that the modification of the DMRG-like total-energy minimization to a DMRG-like augmentedLagrangian based iterative solution of the constrained minimization problem over matrix product states preserves the fast convergence speed in terms of sweeps of the DMRG-like total energy minimization. Together with a reduction of the entanglement entropy by a transformation of the one-particle basis discussed in section 8.8, the parametrization of a many-particle wave function as a matrix product state could be a valuable approach for the evaluation of the density-matrix functional within hybrid theories that combine DFT and RDMFT. The transformation of the one-particle basis does not lead to an increase of the bond dimension of the MPOs here because the MPO of the interaction Hamiltonian is not affected by the unitary transformation and the elements of the one-particle reduced density matrix are evaluated in the transformed basis. 
9. Wave-function based approach for the RDMF

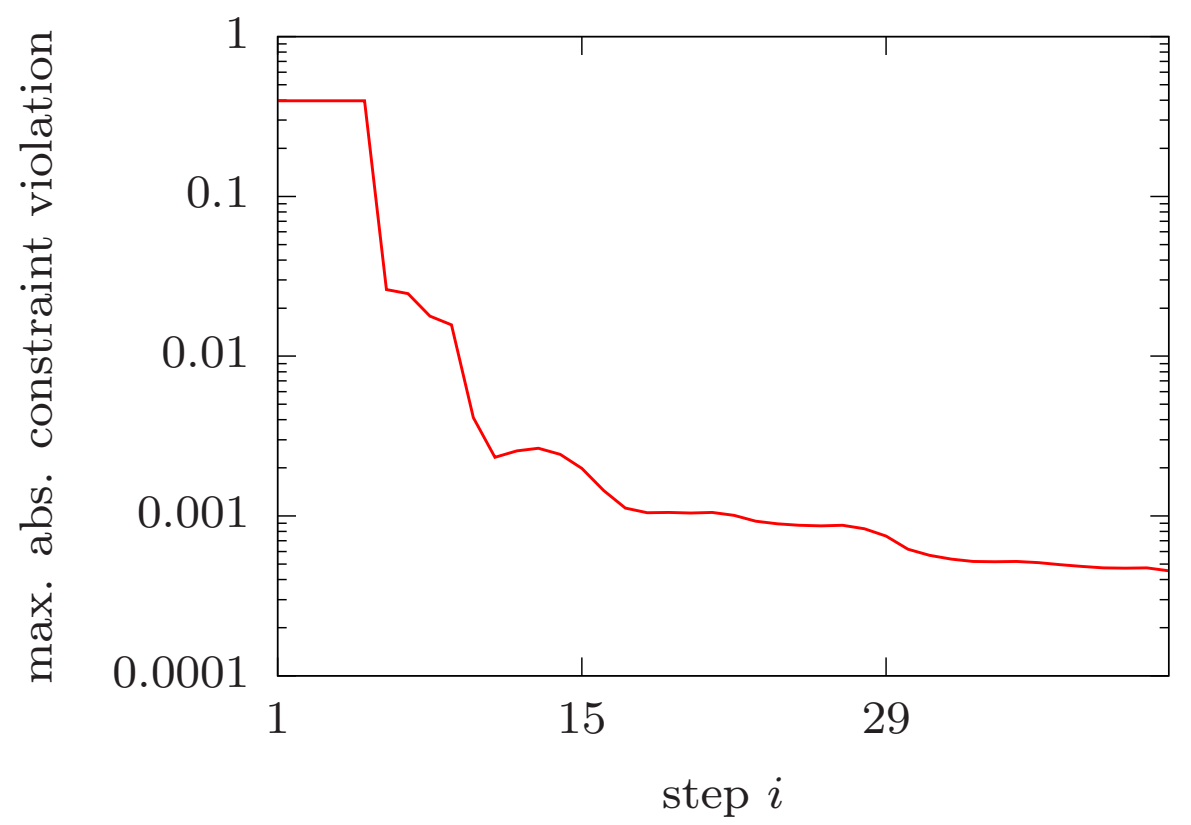

Figure 9.6.: Convergence of the maximal absolute constraint violation during the DMRGlike constrained minimization algorithm proposed in section 9.6.1 for the oneparticle reduced density matrix of the numerically exact ground state of a half-filled 8-site Hubbard chain with interaction strength $U / t=2$. A step denotes the solution of the two-site unconstrained minimization problem at a bond. One sweep through the 8-site system consists of 14 steps. Thus, the figure shows three sweeps. 


\subsection{Gutzwiller-and Jastrow-correlated wave functions}

\subsubsection{Context}

I had considered the use of Gutzwiller-correlated wave functions as variational wave functions for the density-matrix functional for the first time when Peter Blöchl, Ebad Kamil and I visited Florian Gebhard and his group in Marburg. The two main challenges of this idea were the lack of access to an implementation that could compute arbitrary elements of the one-particle and two-particle reduced density matrix and the lack of a derivative-free minimization algorithm that could deal with the noise inherent in a Monte-Carlo procedure. The former problem was solved when I talked to Benedikt Fauseweh who visited Göttingen in early 2018 during a coffee break, and he told me about the openly available mVMC-code from Misawa and coworkers. The latter problem of a suitable minimization algorithm was solved when I came across the simultaneous perturbation stochastic approximation (SPSA) in the context of the variational quantum eigensolver.

\subsubsection{General considerations}

In principle any quantum Monte Carlo method (QMC) can be used to calculate the density-matrix functional via the Legendre-Fenchel-transformation. As discussed in section 9.1 we will not follow this route here but Levy's approach via the constrained minimization problem. Thus, we can only consider quantum Monte Carlo methods where the many-particle wave function is parametrized somehow. Another challenge arises from the fact that most quantum Monte Carlo methods are based on the imaginary-time-dependent Schrodinger equation

$$
\hbar \partial_{\tau}|\Psi(\tau)\rangle=-\hat{H}|\Psi(\tau)\rangle
$$

Examples are diffusion Monte Carlo (DMC, [Grimm and Storer, 1971]), variational Monte Carlo (VMC, [McMillan, 1965; Ceperley et al., 1977]) and full configuration-interaction quantum Monte Carlo (FCIQMC, [Booth et al., 2009]). For a review on quantum Monte Carlo methods we refer the reader to [Foulkes et al., 2001]. Because the constrained minimization problem can not be formulated as a Hamiltonian, we cannot use approaches involving the imaginary-time dynamics. Just like in the previous cases we can leverage the formulation of the constrained minimization problem via the augmented Lagrangian in section 9.3.3 to produce a series of non-linear unconstrained minimization problems.

We construct an algorithm exactly along the lines of the previous wave-function-based evaluations of the density matrix functional presented in this work. We first choose a parametrization of a many-particle wave function and then apply the augmentedLagrangian scheme to solve the constrained minimization problem in the space spanned by the parametrized many-particle states. We use the mVMC-code ${ }^{8}$ by Misawa et al. [Misawa et al., 2017] for the Monte Carlo evaluation of expectation values of variational wave functions. The types of variational wave functions are discussed in section 9.7.3. The Monte Carlo procedure is outlined in section 9.7.4. We propose to use the simultaneous perturbation stochastic approximation (SPSA, [Spall, 1987, 1992]) as a derivative-free and noise-tolerant minimization algorithm in section 9.7.5. We have added the possibility in

\footnotetext{
${ }^{8}$ https://github.com/issp-center-dev/mVMC version 1.0.2
} 
the mVMC code to keep a fixed Markov chain for different sets of variational parameters and have implemented a reweighting approach to remove statistical noise almost completely from minimization procedure. The reweighting procedure is discussed in detail in section 9.7.6. Finally, some example results are presented in section 9.7.7.

\subsubsection{Variational wave function}

\section{Gutzwiller-correlated wave functions}

Here we choose the Gutzwiller-correlated wave function [Gutzwiller, 1963a, 1965; Bünemann et al., 1998]

$$
\left|\Psi_{G}\right\rangle=\frac{\mathcal{P}_{G}\left|\Psi_{0}\right\rangle}{\left\langle\Psi_{0}\left|\mathcal{P}_{G}^{\dagger} \mathcal{P}_{G}\right| \Psi_{0}\right\rangle},
$$

for the parametrized many-particle state. The term $\mathcal{P}_{G}=e^{\sum_{i} g_{i} \hat{n}_{i \uparrow} \hat{n}_{i, \downarrow}}$ is the Gutzwillercorrelator. The sum runs over all sites and the variational parameters $g_{i}$ are named Gutzwiller-correlation factors. $\left|\Psi_{0}\right\rangle$ is a Slater determinant and also has some variational parameters. The physical meaning of the Gutzwiller wave function becomes immediately clear when we consider the half-filled Hubbard dimer

$$
\hat{H}=-t \sum_{\sigma \in\{\uparrow, \downarrow\}}\left(\hat{c}_{1, \sigma}^{\dagger} \hat{c}_{2, \sigma}+\hat{c}_{2, \sigma}^{\dagger} \hat{c}_{1, \sigma}\right)+U\left(\hat{n}_{1, \uparrow} \hat{n}_{1, \downarrow}+\hat{n}_{2, \uparrow} \hat{n}_{2, \downarrow}\right)
$$

$\hat{c}_{i, \sigma}^{(\dagger)}$ is the annihilation (creation) operator for an electron with spin $\sigma$ on site $i$ and $\hat{n}_{i, \downarrow}=$ $\hat{c}_{i, \sigma}^{\dagger} \hat{c}_{i, \sigma}$. Thus, $\hat{n}_{i, \uparrow} \hat{n}_{i, \downarrow}$ is the operator of the double occupancy on site $i$. We take $\left|\Psi_{0}\right\rangle$ as the non-interacting solution

$$
\left|\Psi_{0}\right\rangle=\frac{1}{2}(|0011\rangle+|1100\rangle+|1001\rangle-|0110\rangle)
$$

with a total double occupancy of $\sum_{i} \hat{n}_{i, \uparrow} \hat{n}_{i, \downarrow}=\frac{1}{2}$. Because of the symmetry of the Hubbard dimer we now choose $g_{i}=g$ and obtain the dependency of the total double occupancy on the Gutzwiller correlation factor $g$ shown in Fig. 9.7. In the limit $g \rightarrow-\infty$ we get the state

$$
\lim _{g \rightarrow-\infty}\left|\Psi_{G}\right\rangle=\frac{1}{\sqrt{2}}(|1001\rangle-|0110\rangle)
$$

with a vanishing double occupancy. Thus, the double occupancies are suppressed for large $g$. In the opposite limit of $g \rightarrow \infty$ we obtain the state

$$
\lim _{g \rightarrow \infty}\left|\Psi_{G}\right\rangle=\frac{1}{\sqrt{2}}(|1100\rangle+|0011\rangle) .
$$

For a filling with two electrons, the maximal possible total double occupancy is 1 . In this way, the Gutzwiller correlator $\mathcal{P}_{G}$ mimics what happens at repulsive $(U>0, g<0)$ or attractive $(U<0, g>0)$ interaction strengths, namely a suppression or amplification of the double occupancy. The Gutzwiller-ground state can be obtained as for any other variational wave function by a minimization of the expectation value $\left\langle\Psi_{G}|\hat{H}| \Psi_{G}\right\rangle$ with 


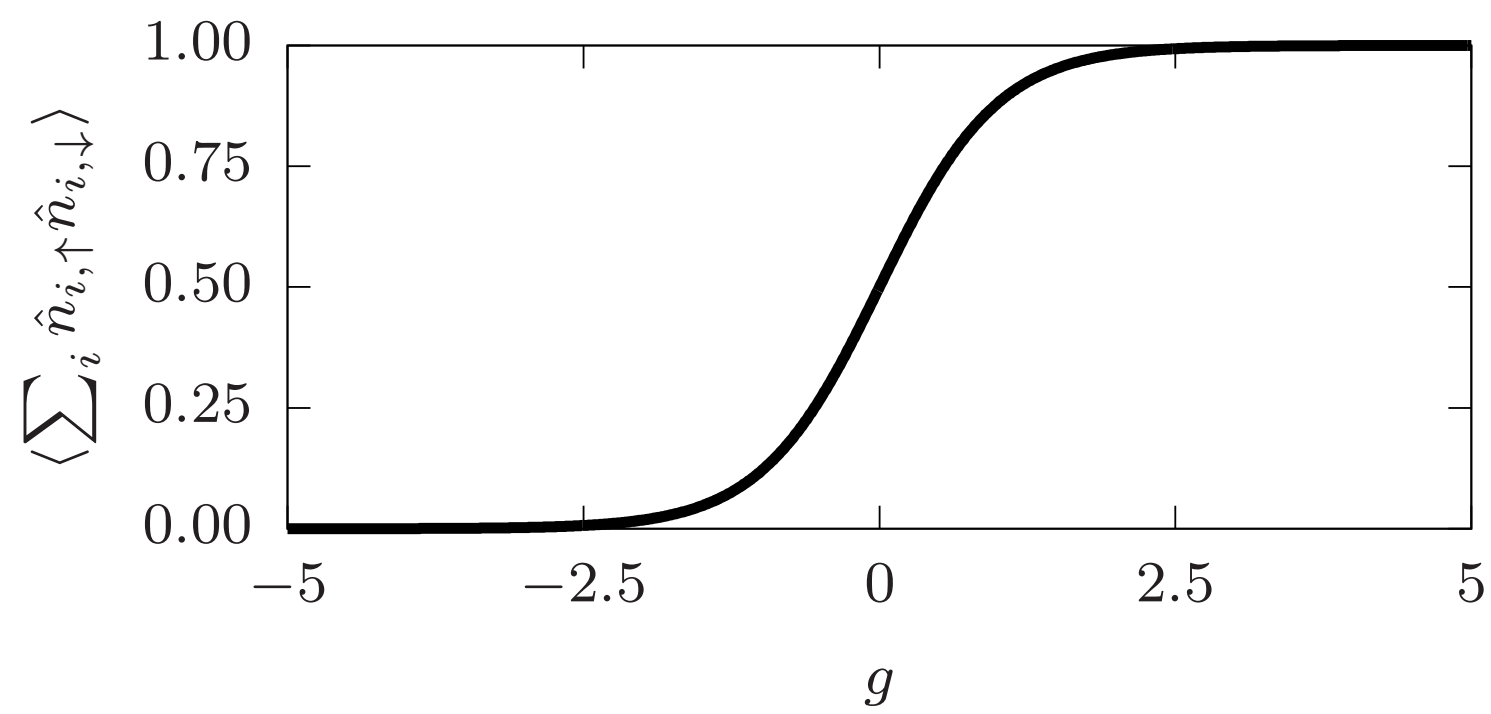

Figure 9.7.: Total double occupancy $\left\langle\sum_{i} \hat{n}_{i, \uparrow} \hat{n}_{i, \downarrow}\right\rangle$ of the Gutzwiller-correlated wave function $\left|\Psi_{G}\right\rangle=\mathcal{P}_{G}\left|\Psi_{0}\right\rangle /\left\langle\Psi_{0}\left|\mathcal{P}_{G}^{\dagger} \mathcal{P}_{G}\right| \Psi_{0}\right\rangle$ with $\mathcal{P}_{G}=\exp \left(g \sum_{i} \hat{n}_{i \uparrow} \hat{n}_{i, \downarrow}\right)$ for a halffilled Hubbard dimer. $\left|\Psi_{0}\right\rangle$ is the non-interacting ground state in Eq. 9.155.

respect to the Gutzwiller-correlation factors. The minimal energy obtained this way is shown in figure 9.8 in comparison to the exact result. In fact, the Gutzwiller correlator gives the exact ground state for the half-filled Hubbard dimer. The optimal parameter $g_{\text {opt }}$ is given as

$$
g_{\mathrm{opt}}=\ln \left[\frac{1}{4 t}\left(-U+\sqrt{U^{2}+(4 t)^{2}}\right)\right]
$$

and shown in figure 9.9 .

\section{Jastrow correlator and relation to the coupled-cluster ansatz}

The Gutzwiller correlator $\mathcal{P}_{G}$ is one representative of the larger class of Jastrow-Feenberg correlators [Jastrow, 1955; Clark and Feenberg, 1959]. Jastrow-Feenberg correlators can be written in the form

$$
\mathcal{P}_{\mathrm{JF}}=\exp \left(\hat{O}_{2}\right)
$$

where $\hat{O}_{2}$ is some two-particle operator. It has been conjectured by some authors [Nooijen, 2000; Piecuch et al., 2003] that the exact ground state of any Hamiltonian with at most two-particle interactions can be written in the form $|\Psi\rangle=\mathcal{P}_{\mathrm{JF}}\left|\Psi_{0}\right\rangle$ with a Slater determinant $\left|\Psi_{0}\right\rangle$. Numerical results exists that oppose this conjecture [Mazziotti, 2004a]. It should be noted that the general form of the correlator in Eq. (9.159) is more general than the exponential wave function ansatz of coupled-cluster theory [Coester, 1958; Coester and Kümmel, 1960; Čížek, 1966; Bartlett and Musiał, 2007]

$$
\begin{aligned}
\left|\Psi_{C C}\right\rangle & =\exp \left(\hat{T}_{1}+\hat{T}_{2}+\ldots\right)\left|\Psi_{0}\right\rangle \\
\hat{T}_{n} & =\frac{1}{(n !)^{2}} \sum_{i, j, \ldots ; a, b, \ldots} t_{i j \ldots}^{a b \ldots} \hat{C}_{a, b \ldots}^{i, j \ldots} .
\end{aligned}
$$




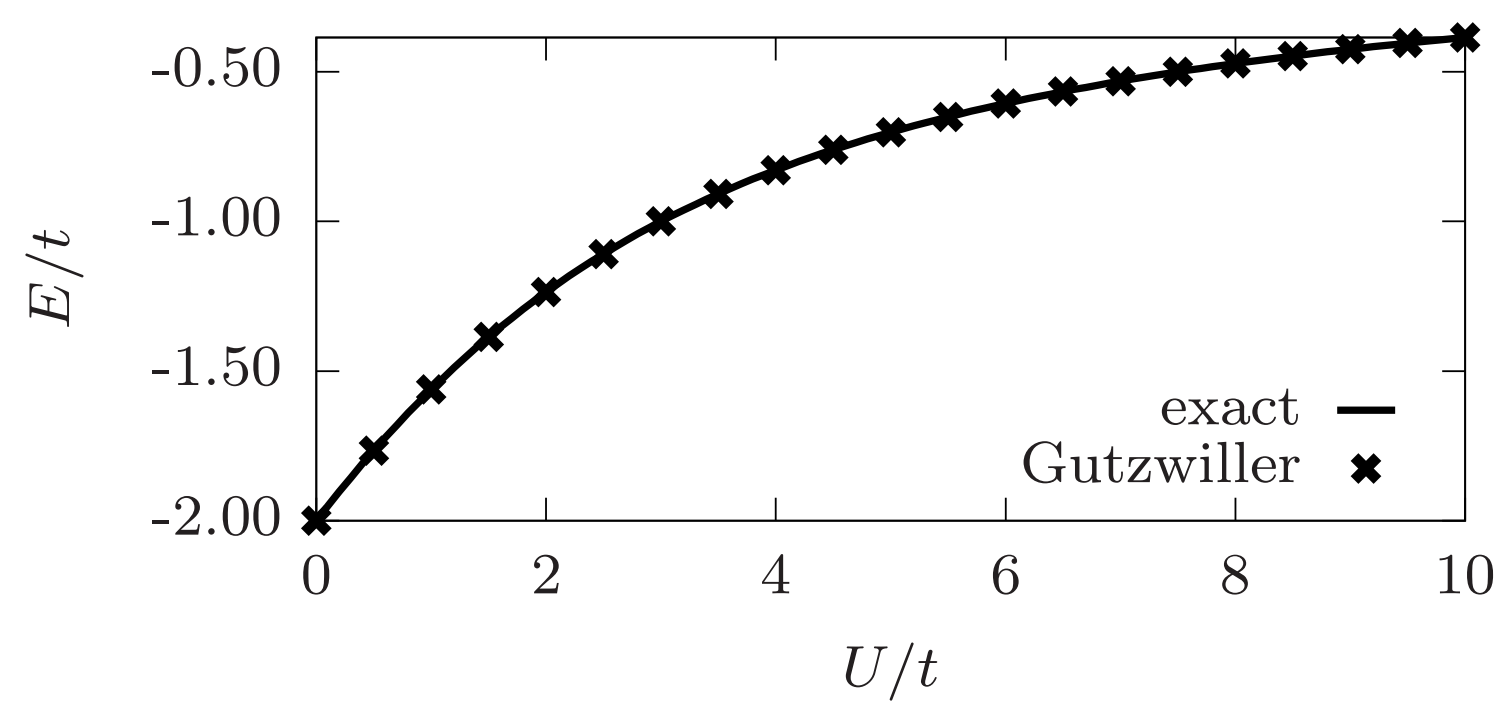

Figure 9.8.: Ground-state energy from the Gutzwiller-correlator, $\min _{g}\left\langle\Psi_{G}|\hat{H}| \Psi_{G}\right\rangle$, (crosses) compared to the exact result (line) for a half-filled Hubbard dimer for different interaction strengths $U / t$.

The coefficients $t_{i j \ldots}^{a b \ldots}$ are the variational parameters. The $n$-fold excitation operator $\hat{C}_{a, b \ldots}^{i, j \ldots}=\hat{c}_{a}^{\dagger} \hat{c}_{b}^{\dagger} \ldots \hat{c}_{j} \hat{c}_{i}$ excites electrons of the Slater determinant $\left|\Psi_{0}\right\rangle$ from the occupied orbital $i$ to the unoccupied orbital $a$, from occupied orbital $j$ to unoccupied orbital $b$ and so on. Thus, if the orbitals are ordered such that the first $N_{e}$ orbitals in $\left|\Psi_{0}\right\rangle$ are occupied, then we have $i, j \ldots \leq N_{e}$ and $a, b \ldots>N_{e}$. The Gutzwiller- or Jastrow correlator in contrast is not based on excitations but on the concept of probing the double occupancy and then penalizing it. The coupled-cluster ansatz with $\hat{T}_{1}+\hat{T}_{2}$ (CCSD), i.e. single and double excitations, and $n_{o}$ occupied orbitals and $n_{v}$ unoccupied orbitals in $\left|\Psi_{0}\right\rangle$ has $n_{o} n_{v}+\frac{1}{4} n_{o} n_{v}\left(n_{o}-1\right)\left(n_{v}-1\right)$ variational parameters. For a large half-filled system $\left(L=n_{o}=n_{v}\right)$ we therefore have about $\frac{1}{4} L^{4}$ variational parameters in the coupled-cluster ansatz.

It should also be noted that practical coupled-cluster calculations in quantum chemistry such as the famous $\operatorname{CCSD}(\mathrm{T})$, which adds perturbative triple excitations to CCSD, are usually not performed by variational minimization of the energy

$$
E=\frac{\left\langle\Psi_{0}\left|\exp \left(\hat{T}^{\dagger}\right) \hat{H} \exp (\hat{T})\right| \Psi_{0}\right\rangle}{\left\langle\Psi_{0}\left|\exp \left(\hat{T}^{\dagger}\right) \exp (\hat{T})\right| \Psi_{0}\right\rangle}
$$

but via the solution of the coupled-cluster equations, here given for CCSD

$$
\begin{aligned}
\left\langle\Psi_{0}\left|\exp \left(-\hat{T}_{1}-\hat{T}_{2}\right) \hat{H} \exp \left(\hat{T}_{1}+\hat{T}_{2}\right)\right| \psi_{0}\right\rangle & =E \\
\left\langle\Psi_{0}\left|\left(\hat{C}_{a}^{i}\right)^{\dagger} \exp \left(-\hat{T}_{1}-\hat{T}_{2}\right) \hat{H} \exp \left(\hat{T}_{1}+\hat{T}_{2}\right)\right| \psi_{0}\right\rangle & =0 \\
\left\langle\Psi_{0}\left|\left(\hat{C}_{a, b}^{i, j}\right)^{\dagger} \exp \left(-\hat{T}_{1}-\hat{T}_{2}\right) \hat{H} \exp \left(\hat{T}_{1}+\hat{T}_{2}\right)\right| \psi_{0}\right\rangle & =0 .
\end{aligned}
$$

This form is solved, because the relation [Bartlett and Musiał, 2007]

$\exp (-\hat{T}) \hat{H} \exp (\hat{T})=\hat{H}+[\hat{H}, \hat{T}]+\frac{1}{2 !}[[\hat{H}, \hat{T}], \hat{T}]+\frac{1}{3 !}[[[\hat{H}, \hat{T}], \hat{T}], \hat{T}]+\frac{1}{4 !}[[[[\hat{H}, \hat{T}], \hat{T}], \hat{T}], \hat{T}]$ 


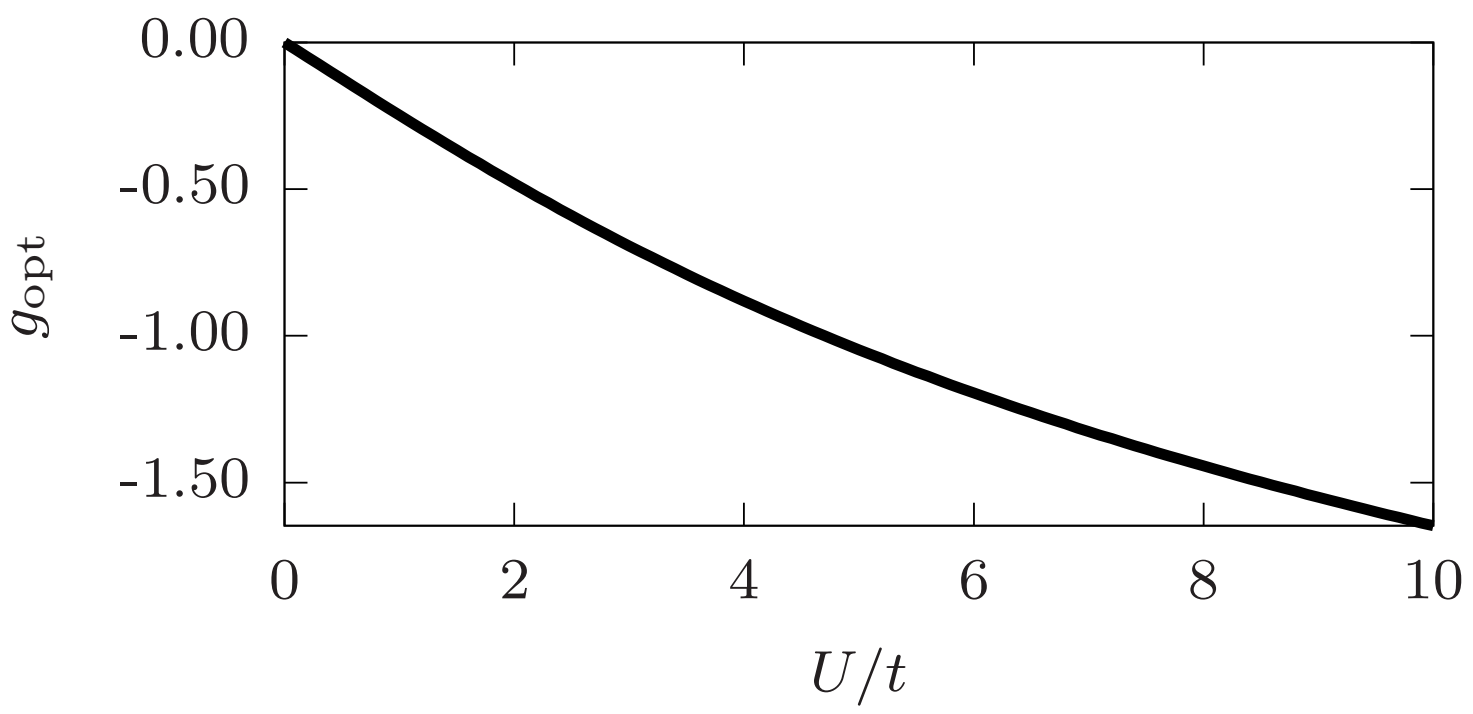

Figure 9.9.: Gutzwiller correlation factor $g$ for which the Gutzwiller energy is minimal for the half-filled Hubbard dimer.

holds for a Hamiltonian with only has one- and two-particle operators. Thus, the coupledcluster equations like Eq. (9.163) only have a finite number of terms and only a polynomial computational complexity. Even though this trick makes the coupled-cluster wave function usable in practice, it breaks down for strongly correlated systems. One example is the $N_{2}$ molecule: The solution of the CCSD-equations in Eq. (9.163) gives a much to low energy for intermolecular distances larger than $1.7 \AA$ whereas the variational solution with the CCSD-wave function gives the proper physical result [Cooper and Knowles, 2010]. Thus, the traditional non-variational coupled-cluster method is not suitable for the density-matrix functional of strongly correlated systems. On the other hand, the coupledcluster wave function is promising, and it might be worth the implementation effort to extend mVMC to this class of wave functions.

\section{Specification of the variational wave function}

To sum up, of the correlation factors supported by mVMC we use the Gutzwiller-correlator and the Jastrow-correlator

$$
\mathcal{P}_{J}=\exp \left[\frac{1}{2} \sum_{i \neq j} v_{i j}\left(\sum_{\sigma} \hat{n}_{i, \sigma}-1\right)\left(\sum_{\sigma} \hat{n}_{j, \sigma}-1\right)\right] .
$$

This factor has been shown by Capello et al. [Capello et al., 2005] to be essential for the proper description of Mott insulators. Thus, the full correlator that we use is

$$
\mathcal{P}=\mathcal{P}_{J} \mathcal{P}_{G}
$$

The Slater determinant $\left|\Psi_{0}\right\rangle$ used in the original Gutzwiller-method described in section 9.7.3 is replaced in the $\mathrm{mVMC}$-code with a more general pair-product wave func- 
tion [Bouchaud, J.P. et al., 1988]

$$
\left|\Psi_{P P}\right\rangle=\left[\sum_{i, j=0}^{L-1} \sum_{\sigma_{i}, \sigma_{j}} F_{i \sigma_{i} j \sigma j} \hat{c}_{i \sigma_{i}}^{\dagger} \hat{c}_{j \sigma_{j}}^{\dagger}\right]^{\frac{N_{e}}{2}}|0\rangle,
$$

where $L$ is the number of sites and $N_{e}$ the number of electrons. This state can describe any Slater determinant but additionally also, for example, certain superconducting phases.

For a system with a vanishing magnetic moment, we can use the simpler form

$$
\left|\Psi_{P P, S_{z}=0}\right\rangle=\left[\sum_{i, j=0}^{L-1} f_{i j} \hat{c}_{i \uparrow}^{\dagger} \hat{c}_{j \downarrow}^{\dagger}\right]^{\frac{N_{e}}{2}}|0\rangle .
$$

Therefore the full variational wave function used in this work is

$$
|\Psi(\vec{u})\rangle=\frac{\left|\Psi_{\mathcal{P}}\right\rangle}{\left\langle\Psi_{\mathcal{P}} \mid \Psi_{\mathcal{P}}\right\rangle}=\frac{\mathcal{P}_{J} \mathcal{P}_{G}\left|\Psi_{P P, S_{z}=0}\right\rangle}{\left\langle\Psi_{P P, S_{z}=0}\left|\mathcal{P}_{G}^{\dagger} \mathcal{P}_{J}^{\dagger} \mathcal{P}_{J} \mathcal{P}_{G}\right| \Psi_{P P, S_{z}=0}\right\rangle},
$$

where the variational parameters are expressed as the vector $\vec{u}$. If no additional spatial symmetries are used, this parametrization has

$$
\underbrace{L^{2}}_{\left|\Psi_{P P, S_{z}=0}\right\rangle}+\underbrace{L}_{\mathcal{P}_{G}}+\underbrace{\frac{1}{2} L \cdot(L-1)}_{\mathcal{P}_{J}}=\frac{3}{2} L^{2}+\frac{1}{2} L
$$

real variational parameters.

\subsubsection{Monte-Carlo sampling of expectation values Metropolis-Hastings algorithm}

For the minimization of the augmented Lagrangian $L(\vec{u}, \vec{\lambda}, \vec{\mu})$ in section 9.3.3 over the variational parameters $\vec{u}$, we need all expectation values of the one-particle reduced density matrix $\rho_{\alpha, \beta}^{(1)}(\vec{u})=\left\langle\Psi(\vec{u})\left|\hat{c}_{\beta}^{\dagger} \hat{c}_{\alpha}\right| \Psi(\vec{u})\right\rangle$ as well some of the expectation values of the two-particle reduced density matrix $\rho_{\alpha \beta \gamma \delta}^{(2)}(\vec{u})=\left\langle\Psi(\vec{u})\left|\hat{c}_{\delta}^{\dagger} \hat{c}_{\gamma}^{\dagger} \hat{c}_{\alpha} \hat{c}_{\beta}\right| \Psi(\vec{u})\right\rangle$. If possible, also their derivatives with respect to the variational parameters $\vec{u}$ should be evaluated because they are helpful to speed up convergence. For practical calculations the expectation values of the Gutzwiller-correlated wave function can be estimated in two ways, either with the Gutzwiller approximation or a Monte Carlo procedure. The Gutzwiller approximation [Ogawa et al., 1975; Vollhardt, 1984] originally estimated the expectation value by counting of classical spin configurations and, thus, effectively neglects spatial correlations between spins. Later a diagrammatic expansion in terms of the spatial dimension was developed [Gebhard, 1990].

Here we go the more straightforward route of estimating the expectation values with a Monte Carlo procedure [Binder et al., 2012]. For this purpose, we use the mVMC-code by Misawa et al. [Misawa et al., 2017]. The mVMC-code implements their many-variable variational Monte Carlo method. The main functionality is the minimization over the chosen variational parameters with the stochastic reconfiguration method, that is based on the imaginary-time Schrödinger equation. Thus, we can not use this part for the 
purpose of the evaluation of the density-matrix functional. However, the code also allows the estimation of the one-particle and two-particle reduced density matrix for a given set of parameters via a Monte Carlo sampling of the Fock space. This is the functionality that we will use within the augmented Lagrangian. The basis representation used in mVMC are Slater determinants in occupation number representation

$$
|\vec{n}\rangle=\left|n_{1, \uparrow} n_{1, \downarrow} n_{2, \uparrow \ldots .}\right\rangle=\prod_{i, \sigma}\left(\hat{c}_{i, \sigma}^{\dagger}\right)^{n_{i, \sigma}}|0\rangle .
$$

Certain parts of the Fock space can be omitted from the start such as parts with different particle numbers $N=\sum_{i} \sum_{\sigma} \vec{n}_{i, \sigma}$ from the requested $N_{e}$ electrons or states with a total electron spin $\frac{\hbar}{2} \sum_{i}\left(\vec{n}_{i, \uparrow}-n_{i, \downarrow}\right)$ different from the requested total spin.

The goal is the estimation of the expectation value of an operator $\hat{A}$

$$
\langle\hat{A}\rangle=\frac{\left\langle\Psi_{\mathcal{P}}|\hat{A}| \Psi_{\mathcal{P}}\right\rangle}{\left\langle\Psi_{\mathcal{P}} \mid \Psi_{\mathcal{P}}\right\rangle}=\sum_{\vec{n}} \frac{\left\langle\Psi_{\mathcal{P}}|\hat{A}| \vec{n}\right\rangle\left\langle\vec{n} \mid \Psi_{\mathcal{P}}\right\rangle}{\left\langle\Psi_{\mathcal{P}} \mid \Psi_{\mathcal{P}}\right\rangle}=\sum_{\vec{n}} p(\vec{n}) \frac{\left\langle\Psi_{\mathcal{P}}|\hat{A}| \vec{n}\right\rangle}{\left\langle\Psi_{\mathcal{P}} \mid \vec{n}\right\rangle}
$$

with the probability density

$$
p(\vec{n})=\frac{\left|\left\langle\vec{n} \mid \Psi_{\mathcal{P}}\right\rangle\right|^{2}}{\left\langle\Psi_{\mathcal{P}} \mid \Psi_{\mathcal{P}}\right\rangle} .
$$

To sample only the important contributions instead of the sum over all Slater determinants in Eq. (9.174), a Markov-chain $\left|\vec{n}^{(1)}\right\rangle \rightarrow\left|\vec{n}^{(2)}\right\rangle \rightarrow \ldots$ is constructed. The probability to be at state $|\vec{n}\rangle$ in step $i$ is denoted as $P(\vec{n}, i)$. For a Markov-chain the conditional probability $T\left(\left|\vec{n}^{(1)}\right\rangle \rightarrow\left|\vec{n}^{(2)}\right\rangle\right)$ for the transition from state $\left|\vec{n}^{(1)}\right\rangle$ to state $\left|\vec{n}^{(2)}\right\rangle$ only depends on the state $\left|\vec{n}^{(1)}\right\rangle$. The probability for the path $\left|\vec{n}^{(1)}\right\rangle \rightarrow\left|\vec{n}^{(2)}\right\rangle \rightarrow\left|\vec{n}^{(3)}\right\rangle$ is, thus, the product $P\left(\vec{n}^{(1)}, 1\right) T\left(\left|\vec{n}^{(1)}\right\rangle \rightarrow\left|\vec{n}^{(2)}\right\rangle\right) T\left(\left|\vec{n}^{(2)}\right\rangle \rightarrow\left|\vec{n}^{(3)}\right\rangle\right)$ of the transition probabilities and the probability of the initial state $P\left(\left|\vec{n}^{(1)}\right\rangle, 1\right)$. The transition probabilities are non-negative $T\left(\left|\vec{n}^{(i)}\right\rangle \rightarrow\left|\vec{n}^{(j)}\right\rangle\right) \geq 0$ and sum up to one, i.e. $\sum_{j} T\left(\left|\vec{n}^{(i)}\right\rangle \rightarrow\left|\vec{n}^{(j)}\right\rangle\right)=1$.

The key requirement for the transition probabilities is that the probability $P(\vec{n}, i)$ to be at state $|\vec{n}\rangle$ in step $i$ becomes stationary and equal to the intended distribution $p(\vec{n})$ of Eq. (9.175) after a sufficiently large number of steps in the Markov process. Stationarity means here that probability $P(\vec{n}, i)$ is independent of the step $i$, i.e. $P(\vec{n}, i)=P(\vec{n}, i+1)$. The detailed balance criterion

$$
p\left(\vec{n}^{(i)}\right) T\left(\left|\vec{n}^{(i)}\right\rangle \rightarrow\left|\vec{n}^{(j)}\right\rangle\right)=p\left(\vec{n}^{(j)}\right) T\left(\left|\vec{n}^{(j)}\right\rangle \rightarrow\left|\vec{n}^{(i)}\right\rangle\right)
$$

of a Markov chain is a necessary condition for the stationarity of the desired distribution $p(\vec{n})$. An additional necessary condition is ergodicity, that is, for every $\left|\vec{n}^{(i)}\right\rangle$ and $\left|\vec{n}^{(e)}\right\rangle$ there exists a Markov process $\left|\vec{n}^{(i)}\right\rangle \rightarrow \ldots \rightarrow\left|\vec{n}^{(e)}\right\rangle$ with a finite total transition probability $T_{\text {tot }}\left(\left|\vec{n}^{(i)}\right\rangle \rightarrow\left|\vec{n}^{(e)}\right\rangle\right)$. This means in other words, that in principle every state can be reached from any other state. As we exclude some parts of the Hilbert space a priori, it should be understood that ergodicity is meant here with respect to the parts of the Fock space that have not been omitted intentionally.

Metropolis [Metropolis et al., 1953] used a Markov process to create states according to a Boltzmann distribution. Hastings [Hastings, 1970] later generalized the Metropolis approach to arbitrary probability densities. The resulting Metropolis-Hastings algorithm 
factorizes the transition probability into a proposal distribution $\pi\left(\vec{n}^{(i)}, \vec{n}^{(j)}\right)$ and an acceptance distribution $W\left(\left|\vec{n}^{(i)}\right\rangle \rightarrow\left|\vec{n}^{(j)}\right\rangle\right)$, i.e.,

$$
T\left(\left|\vec{n}^{(i)}\right\rangle \rightarrow\left|\vec{n}^{(j)}\right\rangle\right)=\pi\left(\vec{n}^{(i)}, \vec{n}^{(j)}\right) W\left(\left|\vec{n}^{(i)}\right\rangle \rightarrow\left|\vec{n}^{(j)}\right\rangle\right) .
$$

The proposal distribution is symmetric $\pi\left(\vec{n}^{(i)}, \vec{n}^{(j)}\right)=\pi\left(\vec{n}^{(j)}, \vec{n}^{(i)}\right)$ and independent of the intended probability density $p(\vec{n})$. The acceptance probability is chosen as

$$
W\left(\left|\vec{n}^{(i)}\right\rangle \rightarrow\left|\vec{n}^{(j)}\right\rangle\right)=\min \left(1, \frac{p\left(\vec{n}^{(j)}\right)}{p\left(\vec{n}^{(i)}\right)}\right)
$$

and fulfills the detailed balance condition in Eq. (9.175).

The Metropolis-Hastings algorithm starts from an initial state $\left|\vec{n}_{0}\right\rangle$ and iteratively preforms the following steps:

1. An small update $\left|\vec{n}^{(i-1)}\right\rangle \rightarrow\left|\vec{n}^{\left(i^{\prime}\right)}\right\rangle$ is proposed.

2. The ratio $w=p\left(\vec{n}^{\left(i^{\prime}\right)}\right) / p\left(\vec{n}^{(i-1)}\right)$ is calculated.

3. A uniformly distributed random number $r \in[0,1]$ is chosen.

4. If $r \leq w$ then the update is accepted and $\left|\vec{n}^{(i)}\right\rangle=\left|\vec{n}^{\left(i^{\prime}\right)}\right\rangle$ set.

5. If $r>w$ then the update is discarded.

For the first step of the algorithm the mVMC code supports various types of updates. We use the hopping update

$$
n_{i, \sigma}=1, n_{j, \sigma}=0 \rightarrow n_{i, \sigma}=0, n_{j, \sigma}=1
$$

where a randomly chosen electron jumps from site $i$ to a randomly chosen site $j$. The states $\left|\vec{n}^{(i)}\right\rangle$ generated by this algorithm sample the probability density $p(\vec{n})$. The expectation value of an operator can be estimated as

$$
\langle\hat{A}\rangle=\frac{1}{N_{\mathrm{MC}}} \sum_{i} \frac{\left\langle\Psi_{\mathcal{P}}|\hat{A}| \vec{n}^{(i)}\right\rangle}{\left\langle\Psi_{\mathcal{P}} \mid \vec{n}^{(i)}\right\rangle} .
$$

The mVMC code calculates the expectation values not after every update, but after sweeps. For a system composed of $L$ sites a sweep contains $L$ updates. Thus, $k$ will count the number of sweeps in the following.

Because the expectation value is estimated from a stochastic process, it has some statistical uncertainty. The measurements of the observable

$$
A(k)=\frac{\left\langle\Psi_{\mathcal{P}}|\hat{A}| \vec{n}^{(k)}\right\rangle}{\left\langle\Psi_{\mathcal{P}} \mid \vec{n}^{(k)}\right\rangle}
$$

after the $k$-sweep of the algorithm are correlated. The unnormalized autocorrelation function for the measurements is defined as

$$
\rho_{A}(k)=\left\langle A\left(k_{0}+k\right) A\left(k_{0}\right)\right\rangle-\langle A\rangle^{2} .
$$


For large numbers of sweeps $k \gg \tau_{A}$ the autocorrelation function usually shows an exponential decaying behaviour

$$
\rho_{A}(k) \sim e^{-k / \tau_{A}}
$$

with the exponential autocorrelation time $\tau_{A}$. The autocorrelation time $\tau_{A}$ describes over how many steps measurements are correlated. Thus, one, on the one hand, has to discard the first steps corresponding to a few multiples of the autocorrelation time in the Metropolis-Hastings algorithm (warmup, thermalization) because these states are still correlated to the initial state. On the other hand, only measurements that are a few autocorrelation times apart can be considered as uncorrelated for the estimate of the statistical error. The integrated autocorrelation time

$$
\tau_{A, i n t}=\frac{1}{2}+\sum_{k=1}^{\infty} \frac{\rho_{A}(k)}{\rho_{A}(0)} \stackrel{\tau \gg 1}{\approx} \tau
$$

can be calculated easily and is directly related to the statistical error of the correlated measurements

$$
\sigma_{A}=\sqrt{2 \tau_{A, i n t} \rho_{A}(0)}
$$

where $\rho_{A}(0)=\left\langle A^{2}\right\rangle-\langle A\rangle^{2}$ is the variance of the measurements if they would be considered as uncorrelated measurements. Therefore the correlation between the measurements increases the statistical error of the expectation values.

\section{Negative sign problem}

The negative sign problem arises in quantum Monte Carlo calculations and generally describes the situation where the statistical errors increase exponentially with increasing system size or decreasing temperature [Loh et al., 1990; Troyer and Wiese, 2005; Gubernatis et al., 2016]. It should be noted that a sign problem can not only arise for fermions but also for example for frustrated quantum-spin systems. The exact reason for the existence of a sign problem depends on the Monte Carlo algorithm used and physical problem under investigation. The general reason for the fermionic sign problem is that the weights $p(x)$ of some configurations $x$ in

$$
\langle\hat{A}\rangle=\frac{\sum_{x} p(x) A(x)}{\sum_{x} p(x)}
$$

are negative for fermions due to the Pauli principle. This can easily be understood for world-line quantum Monte Carlo [Sandvik and Kurkijärvi, 1991; Troyer and Wiese, 2005]: In this technique the partition function

$$
Z=\operatorname{Tr} \exp [-\beta(\hat{H}-\mu \hat{N})]
$$

is written as a series expansion

$$
Z=\sum_{n=0}^{\infty} \frac{(-\beta)^{n}}{n !} \operatorname{Tr}(\hat{H}-\mu \hat{N})^{n}=\sum_{n=0}^{\infty} \frac{(-\beta)^{n}}{n !} \sum_{i_{1}}\left\langle i_{1}\left|(\hat{H}-\mu \hat{N})^{n}\right| i_{1}\right\rangle
$$


Then $n-1$ complete ones $\sum_{i}|i\rangle\langle i|$ are inserted in the sum

$$
Z=\sum_{n=0}^{\infty} \frac{(-\beta)^{n}}{n !}\left\langle i_{1}|\hat{H}-\mu \hat{N}| i_{2}\right\rangle\left\langle i_{2}|\hat{H}-\mu \hat{N}| i_{3}\right\rangle \ldots\left\langle i_{n}|\hat{H}-\mu \hat{N}| i_{1}\right\rangle
$$

A configuration $x$ in the sense of Eq. (9.186) is a path $\left|i_{1}\right\rangle \rightarrow\left|i_{2}\right\rangle \ldots \rightarrow\left|i_{n}\right\rangle \rightarrow\left|i_{1}\right\rangle$. Thus, when sampling over all possible paths of all lengths the partition function can be calculated as $Z=\sum_{x} p(x)$ with the weight function

$$
p(x)=\frac{(-\beta)^{n(x)}}{n(x) !} \sum_{i_{1}, \ldots, i_{n(x)}}\left\langle i_{1}|\hat{H}-\mu \hat{N}| i_{2}\right\rangle\left\langle i_{2}|\hat{H}-\mu \hat{N}| i_{3}\right\rangle \ldots\left\langle i_{n(x)}|\hat{H}-\mu \hat{N}| i_{1}\right\rangle,
$$

where $n(x)$ means the length of the path described by the configuration $x$. If we now take the simple example of $\mu=0$ and $\hat{H}$ as the hopping Hamiltonian of spin-less fermions on a four-site ring

$$
\hat{H}=-t \sum_{\langle i, j\rangle}\left(\hat{c}_{i}^{\dagger} \hat{c}_{j}+\hat{c}_{j}^{\dagger} \hat{c}_{i}\right)
$$

we get for the $n=4$-path

$$
x=(|1100\rangle \rightarrow|1010\rangle \rightarrow|0110\rangle \rightarrow|0101\rangle \rightarrow|1100\rangle)
$$

the negative weight $p(x)=-t^{4} \beta^{4} / 24<0$. The reason for the negative sign is the exchange of the electrons along the path. Rewriting the weight function as the product of a non-negative part and the sign, $p(x)=|p(x)| S(x)$, and introducing the normalized non-negative weight function

$$
\bar{p}(x)=\frac{|p(x)|}{\sum_{x^{\prime}}\left|p\left(x^{\prime}\right)\right|}
$$

the expectation value in Eq. (9.186) can be written as

$$
\langle\hat{A}\rangle=\frac{\langle\hat{A} S\rangle_{\bar{p}}}{\langle S\rangle_{\bar{p}}}
$$

The averages are taken with respect to the weight function $\bar{p}$. Basically, the sampling over the fermionic system has been replaced by sampling over a corresponding bosonic system. The sign problem now means that the average sign $\langle S\rangle_{\bar{p}}$ becomes small and statistical fluctuations will make reliable estimates of the expectations value $\langle\hat{A}\rangle$ very hard.

Fortunately, the variational Monte Carlo algorithm described above does not suffer from the fermionic sign problem unlike most other QMC methods. This is due to the fact that the weights $p(\vec{n})$ given in Eq. 9.175 are non-negative by construction. Phrased differently, the average sign $\langle S\rangle_{\bar{p}}$ for the VMC algorithm is equal to one and independent of the physical system under investigation.

\subsubsection{Simultaneous perturbation stochastic approximation}

The minimization in the unconstrained subproblems of the augmented Lagrangian in Eq. (9.48) must be handled here differently than in the previously presented schemes 
because of the stochastic nature of the Monte Carlo method used for the estimate of the expectation values. In order to simplify the notation used in this section we define

$$
L(\vec{u})=L(\vec{u}, \vec{\lambda}, \vec{\mu})
$$

Thus, we have to devise a custom minimization scheme that tolerates the statistical noise in the expectation values and, hence, also in the objective function $L(\vec{u})$. The conventional VMC for ground state energies as used in $\mathrm{mVMC}$ employs the stochastic reconfiguration method [Sorella, 2001; Sorella et al., 2007]. It uses the imaginary-time Schrödinger equation given in Eq. (9.152) and is based on the time-dependent variational principle [McLachlan, 1964] to obtain the optimal imaginary-time evolution. Therefore, derivatives of expectation values with respect to the variational parameters are not necessary for the conventional minimization procedure and therefore not available in the mVMC-code. As a consequence, we propose to use a minimization algorithm that does not require us to provide derivatives and tolerates some noise.

A good candidate for the solution of the minimization problem of Eq. (9.48) in the context of a quantum Monte Carlo method is the simultaneous perturbation stochastic approximation (SPSA, [Spall, 1987, 1992]). The core idea is based on a gradient decent iteration

$$
\vec{u}^{(k+1)}=\vec{u}^{(k)}-\alpha \vec{g}\left(\vec{u}^{(k)}\right),
$$

where $\vec{u}^{(i)}$ is the vector of variational parameters in the $i$-th iteration, $\vec{g}\left(\vec{u}^{(k)}\right)$ is some estimate of the gradient of the objective at $\vec{u}^{(k)}$ and $\alpha$ quantifies the length of the step taken in the direction of the negative gradient. The first modification introduced by the SPSA is the approximation of the gradient with the stochastic perturbation gradient

$$
\vec{g}_{i}\left(\vec{u}^{(k)}\right) \approx \frac{L\left(\vec{u}^{(k)}+c^{(k)} \vec{\Delta}^{(k)}\right)-L\left(\vec{u}^{(k)}-c^{(k)} \vec{\Delta}^{(k)}\right)}{2 c^{(k)} \vec{\Delta}_{i}^{(k)}} .
$$

$\vec{\Delta}^{(k)}$ is a random perturbation vector. A simple choice is $\vec{\Delta}_{i}^{(k)} \in\{ \pm 1\}$ randomly and independently distributed. The parameter $c^{(k)}$ should be small but not so small that the noise in $L$ dominates the numerator of Eq. (9.197). The series of parameters $c^{(k)}$ is constructed such that it converges to zero for large $k$. The estimation of the derivative only requires two evaluations of the objective function and is therefore much more efficient than a conventional finite-difference estimation especially for computationally expensive objective functions.

The line search along $\alpha^{(k)}>0$ in the $k$-th minimization iteration

$$
\min _{\alpha^{(k)}>0} L\left(\vec{u}^{(k)}-\alpha^{(k)} \vec{g}\left(\vec{u}^{(k)}\right)\right)
$$

is replaced in the SPSA with a fixed choice of the step parameter $\alpha^{(k)}$. The series of step parameters $\alpha^{(k)}$ is constructed such that it converges monotonically to zero for large $k$. In this way, the SPSA avoids the line search altogether and is unaffected by noise in the objective function. 
The following assumptions are introduced for the parameters $\alpha^{(k)}, c^{(k)}$ and $\Delta^{(k)}$

$$
\begin{gathered}
\alpha^{(k)>0, \quad} \quad c^{(k)}>0, \quad \alpha^{(k) \stackrel{k \rightarrow \infty}{\rightarrow} 0, \quad c^{(k)} \stackrel{k \rightarrow \infty}{\rightarrow} 0} \\
\sum_{k=0}^{\infty} \alpha^{(k)}=\infty \\
\sum_{k=0}^{\infty}\left(\frac{\alpha^{(k)}}{c^{(k)}}\right)^{2}<\infty \\
\left\langle\vec{\Delta}^{(k)}\right\rangle_{k}=\overrightarrow{0} .
\end{gathered}
$$

They can be fulfilled with the choices

$$
\alpha^{(k)}=\frac{\alpha^{(0)}}{(k+\beta)^{\gamma}}, \quad c^{(k)}=\frac{c^{(0)}}{k^{\delta}},
$$

$\alpha^{(0)}>0, \beta>0,0 \leq \gamma \leq 1$ and $0<\delta<\gamma-1 / 2$. These assumptions and some additional mild preconditions [Spall, 1992] guarantee the convergence

$$
\vec{u}^{(k) \stackrel{k \rightarrow \infty}{\rightarrow}} \vec{u}^{*}
$$

of the sequence of variational parameters to a local minimizer $\vec{u}^{*}$ of $L(\vec{u})$. The values of $\alpha^{(0)}, c^{(0)}, \beta, \gamma$ and $\delta$ are parameters of the algorithm and must be chosen so that the noise does not dominate the estimate of the derivative in Eq. (9.197). The step lengths $\alpha^{(k)}$ should not be too small for sufficient progress towards the minimum but also not too long as to go beyond the region of validity of the derivative. With some knowledge about the statistical errors of the Monte Carlo sampling of the expectation values and a simple error propagation to the augmented Lagrangian a lower bound for the parameter $c^{(k)}$ can be estimated. However, the sequence $c^{(k)}$ by construction has to go to zero for large $k$ and thus the problem that the estimate of the derivative in Eq. (9.197) is dominated by noise can easily happen for slowly converging minimizations. An ad-hoc way around this problem is to reset $c^{(k)}$ to its initial value $c^{(0)}$ after a large number of steps. For a MonteCarlo based evaluation of the expectation values that runs on a classical deterministic computer, we propose an alternative mediation of this problem based on a reweighting technique in the subsequent section.

\subsubsection{Reweighting}

The reason for the noise in the derivative estimate, Eq. (9.197), is the statistical independence of the evaluation of the two sets of expectations values used for the augmented Lagrangian. In a single run of the mVMC-code, we can measure an arbitrary number of matrix elements of the one- or two-particle reduced density matrix. We propose to evaluate these two sets of expectation values for the same Markov chain $\left|\vec{n}^{(1)}\right\rangle \rightarrow\left|\vec{n}^{(2)}\right\rangle \rightarrow \ldots$ This removes the need to choose the parameter $c^{(k)}$ larger than the noise. On the other hand, since we can choose the parameter $c^{(k)}$ now arbitrarily small ${ }^{9}$, the perturbation $c^{(k)} \vec{\Delta}^{(k)}$ of the variational parameters is small which makes the use of the same Markov chain a legitimate approach. To get the proper derivatives we have to reweight [Binder

\footnotetext{
${ }^{9}$ One should still has to pay attention to the finite floating-point precision.
} 
et al., 2012] the configurations for the evaluation of the expectations values. The modified probability function $p_{\vec{v}}(\vec{n})$ for a perturbed vector of variational parameters $\vec{v}$ in relation to another probability function $p_{\vec{u}}(\vec{n})$ is

$$
\begin{aligned}
p_{\vec{v}}(\vec{n}) & =\frac{|\langle\vec{n} \mid \Psi(\vec{v})\rangle|^{2}}{\langle\Psi(\vec{v}) \mid \Psi(\vec{v})\rangle} \\
& =p_{\vec{u}}(\vec{n}) \frac{|\langle\vec{n} \mid \Psi(\vec{v})\rangle|^{2}}{|\langle\vec{n} \mid \Psi(\vec{u})\rangle|^{2}} \frac{\langle\Psi(\vec{u}) \mid \Psi(\vec{u})\rangle}{\langle\Psi(\vec{v}) \mid \Psi(\vec{v})\rangle} \\
& \approx p_{\vec{u}}(\vec{n}) \frac{|\langle\vec{n} \mid \Psi(\vec{v})\rangle|^{2}}{|\langle\vec{n} \mid \Psi(\vec{u})\rangle|^{2}} .
\end{aligned}
$$

If a Markov chain $\left|\vec{n}^{(1)}\right\rangle \rightarrow\left|\vec{n}^{(2)}\right\rangle \rightarrow \ldots$ was constructed for the vector of variational parameters $\vec{u}$, we can use the modified weight function $p_{\vec{v}}(\vec{n})$ given by Eq. (9.207) to reweight the estimate of the expectation values as

$$
\begin{aligned}
\langle\hat{A}\rangle_{\vec{v}} & =\frac{\langle\Psi(\vec{v})|\hat{A}| \Psi(\vec{v})\rangle}{\langle\Psi(\vec{v}) \mid \Psi(\vec{v})\rangle} \\
& =\frac{1}{N_{\mathrm{MC}}} \sum_{i=1}^{N_{\mathrm{MC}}} \frac{\left\langle\Psi(\vec{v})|\hat{A}| \vec{n}^{(i)}\right\rangle}{\left\langle\Psi(\vec{v}) \mid \vec{n}^{(i)}\right\rangle} \\
& \approx \frac{1}{\sum_{i} w_{i}} \sum_{i=1}^{N_{\mathrm{MC}}} \frac{w_{i}\left\langle\Psi(\vec{v})|\hat{A}| \vec{n}^{(i)}\right\rangle}{\left\langle\Psi(\vec{v}) \mid \vec{n}^{(i)}\right\rangle}
\end{aligned}
$$

with the reweighting function

$$
w_{i}=\frac{p_{\vec{v}}\left(\vec{n}^{(i)}\right)}{p_{\vec{u}}\left(\vec{n}^{(i)}\right)}=\frac{\left|\left\langle\Psi(\vec{v}) \mid \vec{n}^{(i)}\right\rangle\right|^{2}}{\left|\left\langle\Psi(\vec{u}) \mid \vec{n}^{(i)}\right\rangle\right|^{2}}
$$

The quantity $\sum_{i} w_{i} \leq N_{\mathrm{MC}}$ represents the effective number of sampling points. It is a measure for the effective distance of the wave functions $|\Psi(\vec{u})\rangle$ and $|\Psi(\vec{v})\rangle$ and directly measures how suitable the Markov chain is for the perturbed vector of variational parameters $\vec{v}$. Numerical experiments have shown that for a small parameter $c^{(k)}$ in Eq. (9.197) the Markov chains for the variational parameters $\vec{u}^{(k)}+c^{(k)} \vec{\Delta}^{(k)}$ and the variational parameters $\vec{u}^{(k)}-c^{(k)} \vec{\Delta}^{(k)}$ are essentially identical and hence $\sum_{i} w_{i} \approx N_{\mathrm{MC}}$. We can use the reweighting procedure beyond the noise-free estimation of the derivatives to remove noise from the numerical minimization: it turned out to work rather well if we only construct a new Markov chain when the number of effective sampling points $\sum_{i} w_{i}$ drops below $0.9 \cdot N_{\mathrm{MC}}$. As a consequence we now have a noise-free objective function for all gradient decent iterations between two updates of the Markov chain.

The overall algorithm for the unconstrained minimization of $L(\vec{u})=L_{\rho^{(1)}}^{\hat{W}}(\vec{u}, \vec{\lambda}, \vec{\mu})$ over the variational parameters $\vec{u}$ with a Monte-Carlo evaluation of the expectation values in one subproblem of the augmented Lagrangian can be stated as:

1. Setting the parameters for the SPSA according to requirements of Eq. (9.199)(9.201), an initial guess or random initial values of the variational parameters $\vec{u}^{(0)}$ and $k=0$. 
2. A Markov chain for the initial variation parameters $\vec{u}^{(0)}$ is constructed.

3. The derivative vector $\vec{g}\left(\vec{u}^{(k)}\right)$ is estimated via Eq. (9.197). If the number of effective sampling points is lower than $0.9 \cdot N_{\mathrm{MC}}$ the Markov chain is discarded and a new Markov chain is constructed for the current vector of variational parameters $\vec{u}^{(k)}$.

4. The estimate of the variational parameters is updated according to $\vec{u}^{(k+1)}=\vec{u}^{(k)}-$ $\alpha^{(k)} \vec{g}\left(\vec{u}^{(k)}\right)$.

5. If the value of the augmented Lagrangian is converged, then the algorithm stops. Otherwise, the next step $k \rightarrow k+1$ is performed by continuing at step 3 .

The convergence of the augmented Lagrangian in this algorithm is hard to judge because of the jumps in value when changing the Markov chain. If the Markov chain is sufficiently long and, thus, the statistical errors of the expectations values sufficiently small, the convergence for one Markov chain is a good indicator for convergence of any other possible Markov chain.

For the practical implementation of this algorithm, we have modified the mVMC-code to be able to output and read in the constructed Markov chain $\left|\vec{n}^{(1)}\right\rangle \rightarrow\left|\vec{n}^{(2)}\right\rangle \rightarrow \ldots$ and the scalar products $\left\langle\Psi(\vec{u}) \mid \vec{n}^{(1)}\right\rangle$ so that they can be reused for multiple evaluations of the expectation values and for the reweighting procedure. Instead of writing the Markov chain in a full occupation number representation to disk we write the logical value of the decisions in the fourth step of the Metropolis-Hastings algorithm as single bits to disk. This conserves orders of magnitude of disk storage and input-output time. For better performance, it is advisable to integrate the SPSA directly into mVMC-code so that initialization times and input-output overhead is avoided.

\subsubsection{Example results}

As a simple example system, we have chosen a $L=20$-site half-filled Hubbard chain as defined in chapter 3 with an interaction strength of $U / t=1$. We show here proof-of-concept results from the evaluation of the density-matrix functional via a Monte Carlo procedure for the one-particle reduced density matrix of the numerically exact zero-temperature ground state. The numerically exact ground state has been calculated with the MPSDMRG-code ITensor as discussed in chapter 3 with a maximal truncation error of $10^{-10}$. The exact density-matrix functional for the ground state of this system, that is, the difference of the total energy of the ground state and the one-particle energy, is

$$
F_{\text {exact }}^{\sum_{i=1}^{L} U \hat{n}_{i \uparrow} \hat{n}_{i \downarrow}}\left[\rho_{\text {exact }}^{(1)}\right]=4.2194 t .
$$

We have employed the variational wave function in Eq. (9.171): a Gutzwiller- and Jastrow-correlated wave function with a $S_{z}=0$-pair-product wave function as a noninteracting wave function. We have not assumed any spatial symmetries and, hence, have 610 real variational parameters. For the estimation of the expectation values we used $N_{\mathrm{MC}}=10^{5}$ Monte Carlo sweeps in mVMC. Thus, the $L \cdot N_{\mathrm{MC}}=2 \cdot 10^{6}$ states in the Markov chain only span a tiny fraction of the full Fock space of dimension $2^{40} \approx 10^{12}$ or of the Hilbert space of half-filled- $S_{z}=0$-Slater determinants, that has a dimension of

$$
\left(\begin{array}{l}
L \\
\frac{L}{2}
\end{array}\right)^{2} \approx 10^{10.5}
$$


The density-matrix functional converged to a value of

$$
F_{\text {Monte Carlo }}^{\sum_{i=1}^{L} U \hat{n}_{i \uparrow} \hat{n}_{i \downarrow}}\left[\rho_{\text {exact }}^{(1)}\right]=(4.236 \pm 0.007) \cdot t
$$

and a maximal constraint violation of

$$
\max _{\alpha, \beta} \frac{\left\langle\Psi \mathcal{P}\left|\hat{c}_{\alpha}^{\dagger} \hat{c}_{\beta}\right| \Psi_{\mathcal{P}}\right\rangle}{\left\langle\Psi \mathcal{P} \mid \Psi_{\mathcal{P}}\right\rangle}=0.006 \pm 0.004
$$

This example shows that the minimization scheme works in practice and that even a tiny number of Monte Carlo sweeps can yield satisfactory results. The situation that the obtained value of the density-matrix functional is larger than the exact value, even if we consider the error interval, is probably due to the parametrization of the manyparticle wave function. The size of the error agrees with results from ground-state energy calculation: if we perform a ground-state energy minimization in $\mathrm{mVMC}$ for the same system and the same parametrization of the wave function, we obtain a ground state energy of $(-20.1329 \pm 0.0002) \cdot t$ which is about $0.02 t$ higher than the numerically exact result from an MPS-DMRG calculation $(-20.15042 t)$.

The statistical errors stated in Eq. (9.215) and Eq. (9.216) were estimated from the integrated autocorrelation times according to equation (9.185) and we have verified them with a binning analysis.

The normalized autocorrelation function

$$
\rho_{W, \text { norm }}(k)=\frac{\left\langle W\left(k_{0}+k\right) W\left(k_{0}\right)\right\rangle-\langle W\rangle^{2}}{\left\langle W^{2}\right\rangle-\langle W\rangle^{2}} .
$$

for the expectation value $W=\langle\hat{W}\rangle$ of the interaction is shown in Figure 9.10. It should be noted that $k$ is counted in sweeps where one sweep is comprised of $L$ Metropolis-Hastings iterations. The small autocorrelation time of about 1.5 sweeps shows the efficiency of the hopping update. The integrated autocorrelation for the expectation values of the one-particle reduced density matrix in Figure 9.11 show the autocorrelation times for the off-diagonal elements are very small as well. However, the autocorrelation times for density-matrix elements close to the diagonal are much larger and lie in the range between 2 and 4 sweeps. The strong diagonal dominance of the integrated autocorrelation times

The corresponding statistical errors of the density-matrix elements are shown in figure 9.12. The statistical errors show that the same qualitative behaviour as the autocorrelation times. The reason is that the uncorrelated statistical errors $\sqrt{\rho_{A}(0)}$ are roughly identical for all matrix elements of the one-particle reduced density matrix as shown in figure 9.13 .

\subsubsection{Outlook}

The results presented in the previous section show that the evaluation of the densitymatrix functional with a QMC-based procedure works in practice. However, there a few optimizations that are necessary to make it an efficient scheme within DFT+RDMFT. Especially the evaluation of expectation values for only slightly perturbed sets of parameters must be made more efficient, that is, without input and output via the file system but internally in mVMC. It might also be fruitful to implement derivatives of the expectation values with respect to the variational parameters directly in $\mathrm{mVMC}$ and to 


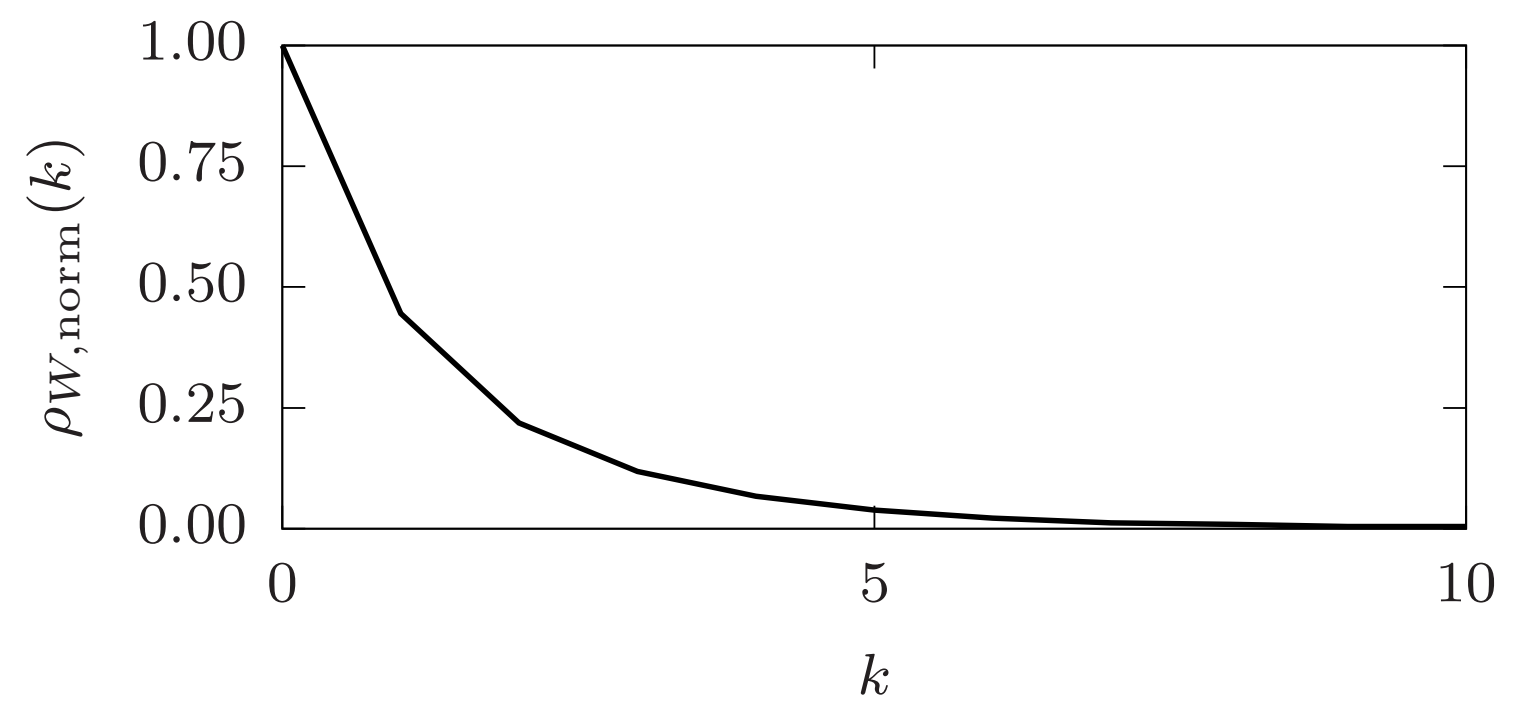

Figure 9.10.: Normalized autocorrelation function $\rho_{W, \text { norm }}(k)$ for the expectation value of the interaction Hamiltonian. The variational wave function resulted from the constrained minimization procedure of the density-matrix functional using a Monte Carlo method. The density-matrix functional was evaluated for the exact ground-state density matrix of a $L=20$-site half-filled Hubbard chain with $U / t=1$. The iteration index $k$ is counted in sweeps where one sweep contains $L$ Metropolis-Hastings updates.

use a conjugate-gradient-based- or LBFGS-minimization scheme instead of the SPSA. It should be noted that the algorithm for the evaluation of the density-matrix functional is not advisable for ground-state energy calculations where the full one-particle basis is treated with the same density-matrix functional. For this purpose, the existing stochastic reconfiguration method [Sorella, 2001; Sorella et al., 2007] is more efficient than the SPSA. However, the evaluation of the density-matrix functional using a variational Monte Carlo procedure over Gutzwiller-correlated and higher-order-correlated wave functions is suitable for a local one-particle basis within DFT+RDMFT. Especially the possibility of warm-starting with the variational parameters from the previous outer iteration and the scalability of the computational effort make it a promising candidate. An open question is the representability of one-particle reduced density matrices with Gutzwiller- or higher-order correlated wave functions. This question can only be answered when applying this scheme to real situations which are not possible at the moment due to the lacking computational efficiency of the current proof-of-concept implementation. 


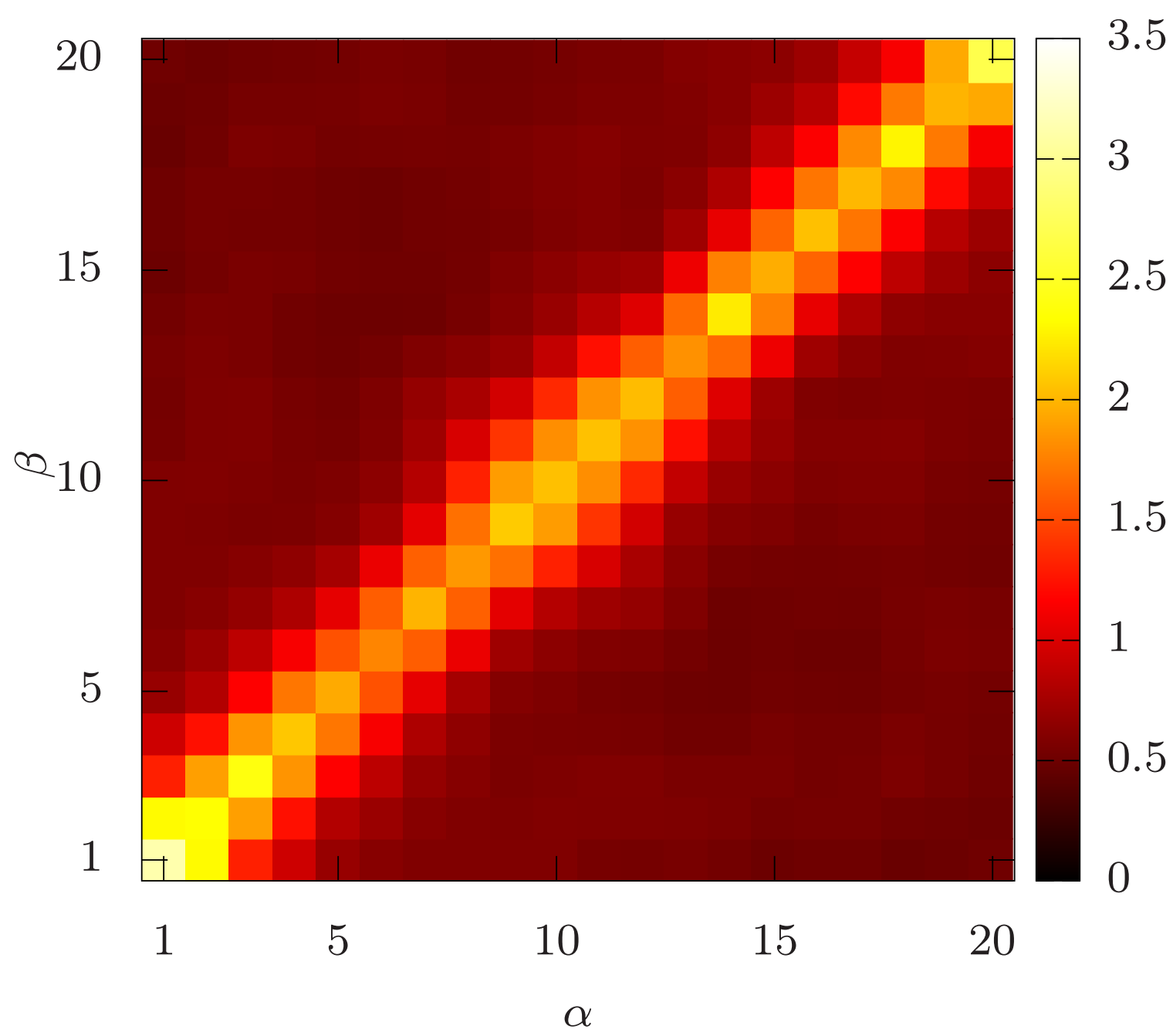

Figure 9.11.: Integrated autocorrelation times $\tau_{\left\langle\hat{c}_{\alpha \uparrow}^{\dagger} \hat{c}_{\beta \uparrow}\right\rangle, \text { int }}$ for the one-particle reduced density matrix. The variational wave function resulted from the constrained minimization procedure of the density-matrix functional using a Monte Carlo method. The density-matrix functional was evaluated for the exact groundstate one-particle reduced density matrix of a $L=20$-site half-filled Hubbard chain with $U / t=1$. The iteration index $k$ is counted in sweeps where one sweep contains $L$ Metropolis-Hastings updates. The autocorrelation times for the opposite spin-direction are quantitatively very similar to the ones shown. 
9. Wave-function based approach for the RDMF

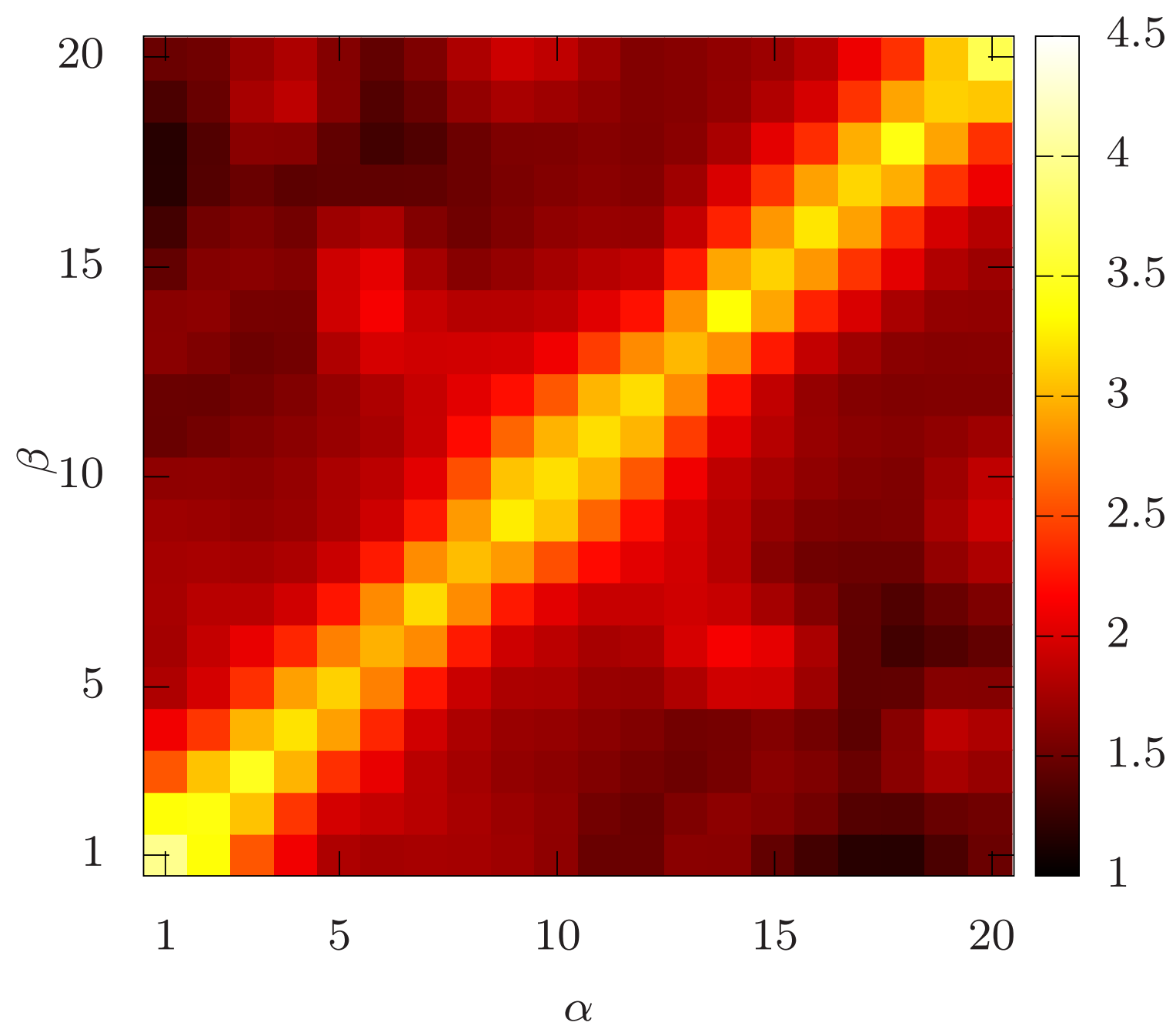

Figure 9.12.: Statistical errors $10^{3} \cdot \sigma_{\left\langle\hat{c}_{\alpha \uparrow}^{\dagger} \hat{c}_{\beta \uparrow}\right\rangle}$ for the one-particle reduced density matrix. The variational wave function resulted from the constrained minimization procedure of the density-matrix functional using a Monte Carlo method. The density-matrix functional was evaluated for the exact ground-state oneparticle reduced density matrix of a $L=20$-site half-filled Hubbard chain with $U / t=1$. The iteration index $k$ is counted in sweeps where one sweep contains $L$ Metropolis-Hastings updates. The statistical errors of the opposite spin-direction are quantitatively very similar to the ones shown. 


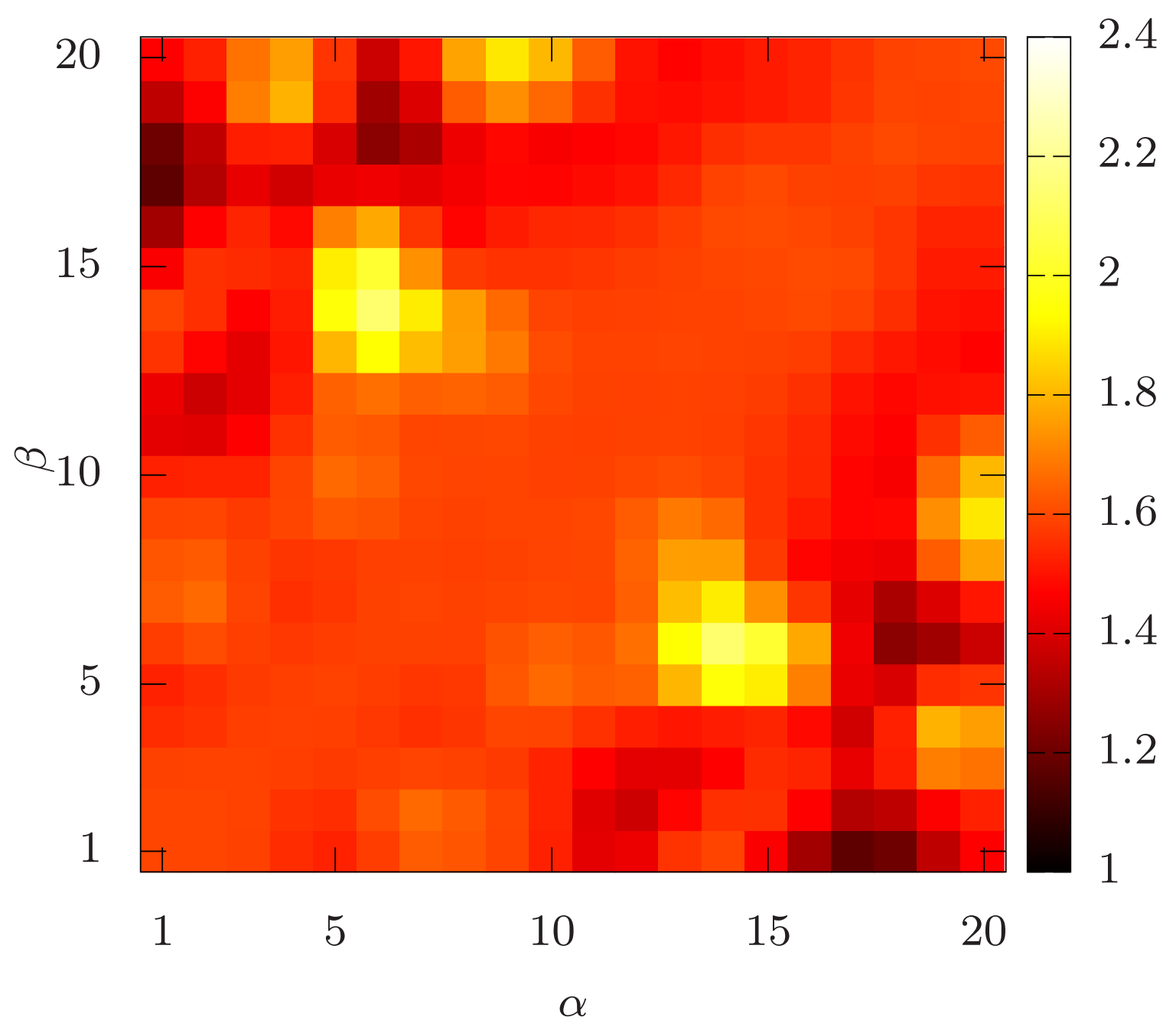

Figure 9.13.: Uncorrelated statistical errors $10^{3} \cdot \sqrt{\rho_{\left\langle\hat{c}_{\alpha \uparrow}^{\dagger} \hat{c}_{\beta \uparrow}\right\rangle}(0)}$ of the one-particle reduced density matrix. The variational wave function resulted from the constrained minimization procedure of the density-matrix functional using a Monte Carlo method. The density-matrix functional was evaluated for the exact groundstate one-particle reduced density matrix of a $L=20$-site half-filled Hubbard chain with $U / t=1$. The iteration index $k$ is counted in sweeps where one sweep contains $L$ Metropolis-Hastings updates. The uncorrelated statistical errors of the opposite spin-direction are quantitatively very similar to the ones shown. 


\subsection{Density-matrix functional on quantum computers}

\subsubsection{Context}

The idea of using quantum computers was inspired by a personal project of mine together with Thomas Köhler and Sebastian Paeckel. Thomas Köhler and Sebastian Paeckel have recently developed a quantum-computer-emulator, that is based on matrix-product states and can emulate quantum circuit models. As they were looking for non-trivial test cases, I have implemented the factorization of numbers with Shor's algorithm [Shor, 1997] for their description language by using the existing implementation of the algorithm in libquantum $^{10}$. As a proof-of-concept, I successfully factorized the number 15 . In this process, I became familiar with circuit-based quantum computers and the variational quantum eigensolver. In this section, I describe how the main idea of the variational quantum eigensolver can be used to evaluate the density-matrix functional on near-term quantum computers.

\subsubsection{Quantum Turing machines and quantum circuit models}

A quantum computer is a computing device which is not based on digital-information theory with binary numbers but quantum-mechanical effects of many-particle wave functions such as entanglement and superposition of states. There are two main classes of quantum computers: adiabatic and gate-based quantum computers. Adiabatic quantum computing [Apolloni et al., 1989; Albash and Lidar, 2018] starts from an initial Hamiltonian for which the ground state is experimentally easy to prepare. The Hamiltonian is adiabatically changed to the final Hamiltonian. The final Hamiltonian is chosen such that measurable observables of its ground state contain the solution of the computational problem. Adiabatic quantum computing is sometimes also called quantum stochastic optimization or quantum annealing.

Gate-based quantum computing [Fredkin and Toffoli, 1982; Deutsch, 1985; Barenco et al., 1995; Nielsen and Chuang, 2010] is more universal than adiabatic quantum computing in the sense that the many-particle states involved form a complete set. In this way, it can be seen as a generalization of a classical computing machine to quantum mechanics. Gate-based quantum computers are often called circuit-based quantum computers to highlight their structure. In the following, we will discuss the computability of functions with classical computers and then generalize these notions to circuit-based quantum computing.

The concept of Turing machines [Turing, 1936] can be used to formally understand the computability ${ }^{11}$ of classical functions. At first, we define an alphabet $\Gamma$, that is a non-empty set of symbols and contains the blank character $\square$. Additionally we need a finite set of states $Q$, an initial state $q_{0} \in Q$, a set of final states $F \subseteq Q$ and a transition function $\delta:(Q \backslash F) \times \Gamma \rightarrow Q \times \Gamma \times\{L, N, R\}$. A Turing machine consists of four parts:

1. a one-dimensional tape that is infinite in both directions and is divided into cells. Each cell can hold exactly one symbol from the alphabet. Cells that have not been

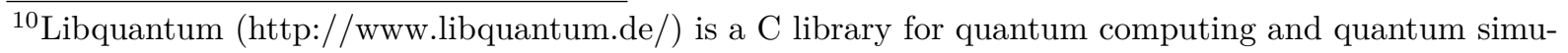
lation written by written by Björn Butscher and Hendrik Weimer

${ }^{11}$ The Church-Turing thesis states that the set of functions that would be naturally regarded as computable is identical to the class of functions that are computable by a Turing machine [Nielsen and Chuang, 2010].
} 
written yet contain the blank symbol $\square$. The input for the Turing machine is written to the tape prior to execution of the machine.

2. a read-write head with the following capabilities:

- shifting to the left $(L)$ or right cell $(R)$ from the current position or not moving at all $(N)$

- reading the symbol from the current cell or writing a symbol to the current cell

3. a state register for storing the current state $q \in Q$ of the Turing machine

4. a program that in every execution step of the Turing machine executes the steps

- stops execution if $q \in F$,

- instructs the head to read the symbol $s \in \Gamma$ from the current cell,

- evaluates the transition function $\delta(q, s)=\left(s_{\text {new }}, q_{\text {new }}, t \in\{L, N, R\}\right)$

- instructs the head to write the new symbol $s_{\text {new }}$ in the current cell

- sets the state to $q_{\text {new }}$ and

- instructs the head to move according to $t$.

With this straightforward machine one can emulate any computation that can be done on a classical computer. A computing device or programming language is called Turingcomplete if it can compute any function that can be computed by a Turing machine. This definition of Turing-completeness ignores the limitation of the finite memory of a physical computer. The quantum-mechanical generalization of the Turing machine is the quantum Turing machine [Deutsch, 1985]. A quantum Turing machine can simulate every finite quantum-mechanical physical system, every other quantum computer, and quantum simulator as well as every classical Turing machine. In this way its computational capability is analogous to the power of a classical Turing machine. Thus, we call a quantum computer or a quantum programming language as quantum Turing-complete if it can in principle compute any function that a quantum Turing machine could compute. This definition of quantum Turing-completeness ignores the limited available sizes of practical realizations of quantum computers. An adiabatic quantum computer is not quantum Turing-complete.

The critical difference of a quantum Turing machine to a classical Turing machine is that instead of symbols of an alphabet $\Gamma$ it works with quantum-mechanical observables. For the quantum Turing machine of Deutsch [Deutsch, 1985], these observables have the spectrum $\{0,1\}$. This is called the computational basis, whereas, for example, the eigenstates of the $S_{z}$-operator of a spin- $\frac{1}{2}$-particle with the spectrum $\left\{-\frac{\hbar}{2}, \frac{\hbar}{2}\right\}$ is named the physical basis. The computational basis shows that a single observable is the quantum analog of a classical one-bit memory cell. Therefore, these observables are called qubits. We will use the computational basis for the remaining discussion concerning quantum computers. The analog of the tape position is the observable $\hat{x}$ that has the whole number range $\mathbb{Z}$ as its spectrum. The internal state of the quantum Turing machine, that is analog to the state $q \in Q$ of the classical Turing machine, is stored in the $M$-dimensional vector $\overrightarrow{\hat{n}}$ of observables $\hat{n}_{i}$. The cells on the tape consist of observables $\hat{m}_{i}$ with $i \in \mathbb{Z}$ and hence, the tape can be described by the vector $\hat{\vec{m}}$. Thus, the overall state of a quantum Turing machine $|\Psi\rangle$ can be written as a superposition of the simultaneous eigenstates of $\hat{x}$ as well 
as the elements of $\hat{\vec{n}}$ and $\hat{\vec{m}}$ as

$$
|\Psi\rangle=\sum_{x, \vec{n}, \vec{m}} c_{x, \vec{n}, \vec{m}}|x, \vec{n}, \vec{m}\rangle .
$$

The initial state $|\Psi(t=0)\rangle$ of the quantum Turing machine is prepared in the state

$$
|\Psi(t=0)\rangle=\sum_{i} \lambda_{i}\left|0, \overrightarrow{0}, \vec{m}_{i}\right\rangle
$$

with $\sum_{i}\left|\lambda_{i}\right|^{2}=1$. An iteration of the quantum Turing machine is performed by the application an unitary operator $U$

$$
|\Psi(t+\Delta t)\rangle=\hat{U}|\Psi(t)\rangle
$$

that is restricted to the form [Deutsch, 1985]

$$
\left\langle x^{\prime}, \vec{n}^{\prime}, \vec{m}^{\prime}|\hat{U}| x, \vec{n}, \vec{m}\right\rangle=\left[\delta_{x^{\prime}, x+1} U^{+}\left(\vec{n}^{\prime}, m_{x}^{\prime}, \vec{n}, m_{x}^{\prime}\right)+\delta_{x^{\prime}, x-1} U^{-}\left(\vec{n}^{\prime}, m_{x}^{\prime}, \vec{n}, m_{x}\right)\right] \prod_{x \neq y} \delta_{m_{y}^{\prime}, m_{y}} .
$$

The functions $U^{+}\left(\vec{n}^{\prime}, m_{x}^{\prime}, \vec{n}, m_{x}^{\prime}\right)$ and $U^{-}\left(\vec{n}^{\prime}, m_{x}^{\prime}, \vec{n}, m_{x}^{\prime}\right)$ describe the changes of the internal state and only depend of the local state of the tape in cell $x$. The delta-functions $\delta_{x^{\prime}, x+1}$ and $\delta_{x^{\prime}, x-1}$ implement the requirement that the position of the read-write head can only change by one cell per iteration. One of the internal observables $\vec{n}$ can be used to signal halting of the machine. The unitary operator $\hat{U}$ can be constructed such that the expectation value of this observable goes to one if the machine has halted.

Yao [Yao, 1993] has shown that any function that is computable in polynomial time with a quantum Turing machine can also be computed by a quantum circuit model of polynomial size. Thus, we do not need to experimentally construct a quantum Turing machine to harness its computational power but can use a quantum circuit model instead. A quantum circuit model consists of a defined initial state, a number of qubits, gates, and measurements. The gates are unitary transformations that are iteratively applied to the quantum state of these qubits. The input of the circuit is either encoded in the initial state or in the circuit model itself. The output is encoded in measurements $S_{z}$ of the $z$-component $\hat{S}_{z}$ of the effective spin- $\frac{1}{2}$-particle represented by the qubits if the physical basis is considered or, equivalently, in measurements $q$ of some qubit-local operator $\hat{q}$ with the spectrum $\{0,1\}$ if the computational basis is considered. Thus, to obtain expectation values $\langle\hat{q}\rangle=1 / N \sum_{i=1}^{N} q_{i}$ or $\left\langle\hat{S}_{z}\right\rangle=1 / N \sum_{i=1}^{N} S_{z, i}$, the quantum program has to be executed $N \gg 1$ times. A quantum circuit model can be seen as the quantum analog of an acyclic Boolean circuit model that all our current classical computers as based on. A Boolean circuit model consists of inputs, outputs and logic gates like AND- $(\wedge)$, OR- $(\vee)$ or NOT- $(\neg)$ gates that are connected by wires. It should be noted here that there are universal gates that can be used to express any circuit model. One such universal gate is the NAND-gate $(\neg(A \wedge B))$, another possible one is the NOR-gate $(\neg(A \vee B))$. Thus, any logical function can be realized with wires and only NAND-gates or with wires and only NOR-gates. They are called functionally complete or universal sets of gates.

The commonly used quantum gates are shown in table 9.1. Not all gates listed are necessary to express any given unitary transformation. A popular choice of a functionally 
complete set consists of the phase shift gate, the general rotation gate, and the CNOTgate [Barenco et al., 1995]. An equivalent set is composed of the phase shift gate, the $R_{y}$ gate, the $R_{z}$ gate and the CNOT-gate because any unitary $2 \times 2$ matrix can be written as a product of the phase shift gate, the $R_{y}$ gate and the $R_{z}$ gate. For practical applications, The phase shift gate can be dropped from these sets because it only adds an overall phase to the wave function that cannot be measured. Thus, we define a universal quantum computer as a quantum computer that can perform all operation of some set of universal quantum gates natively, that can be set to a well defined initial state $(|000 \ldots\rangle$.$) , and$ can measure expectation values of all qubits. In practice, not the whole solution of the problem at hand is computed on a quantum computer but only the part that profits from quantum effects. All other steps of the solution are executed on a classical computer. As the initial state of a universal quantum computer as defined here is fixed and does not depend on the parameters of the problem to be solved, these parameters have to be encoded into the circuit model. So, for example, in Shor's algorithm for the factorization of the number $N$ only the determination of the period $r$, that is, the smallest positive integer such that $f(x+r)=f(x)$, of the function

$$
f(x)=a^{x} \bmod N
$$

is executed on a quantum computer. The determination of the parameter $a$ and the reconstruction of the prime factors from the period $r$ are performed on a classical computer. Also, the construction of the circuit model for the determination of the period that depends on $a$ and $N$ must be done on a classical computer. This kind of hybrid classical-quantum computing reduces the computational demands on the quantum computer greatly. A program for a universal quantum computer can be written in different equivalent ways. We prefer here the form of circuit diagrams using the basic gates shown in table 9.1 or the textual representation of the same information in the open quantum assembly language (OpenQASM, [Cross et al., 2017]). A circuit diagram written down in OpenQASM is analogous to program code for classical computers written in assembly language. An example of a program that implements the modular multiplication $7 x$ mod 15 [Markov and Saeedi, 2012] of a number $x$ and is used during the factorization of the number $N=15$ in Shor's algorithm (with $a=7$ ) is shown in figure 9.14. The corresponding OpenQASM code is

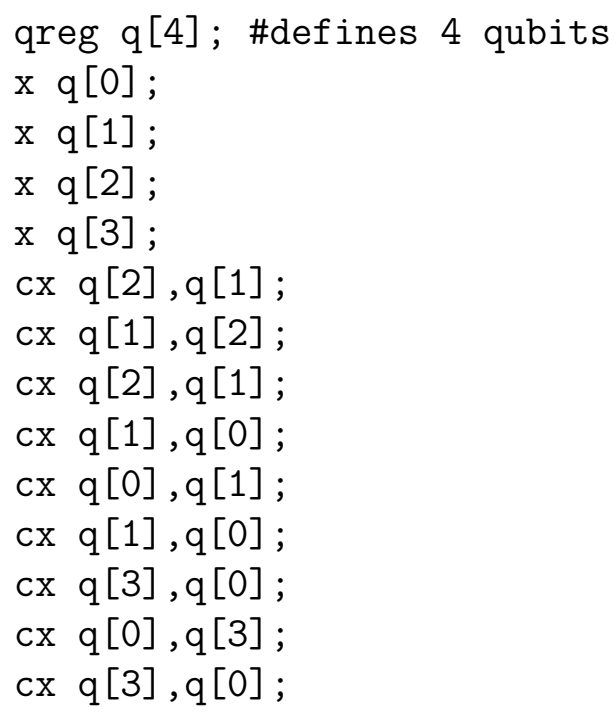




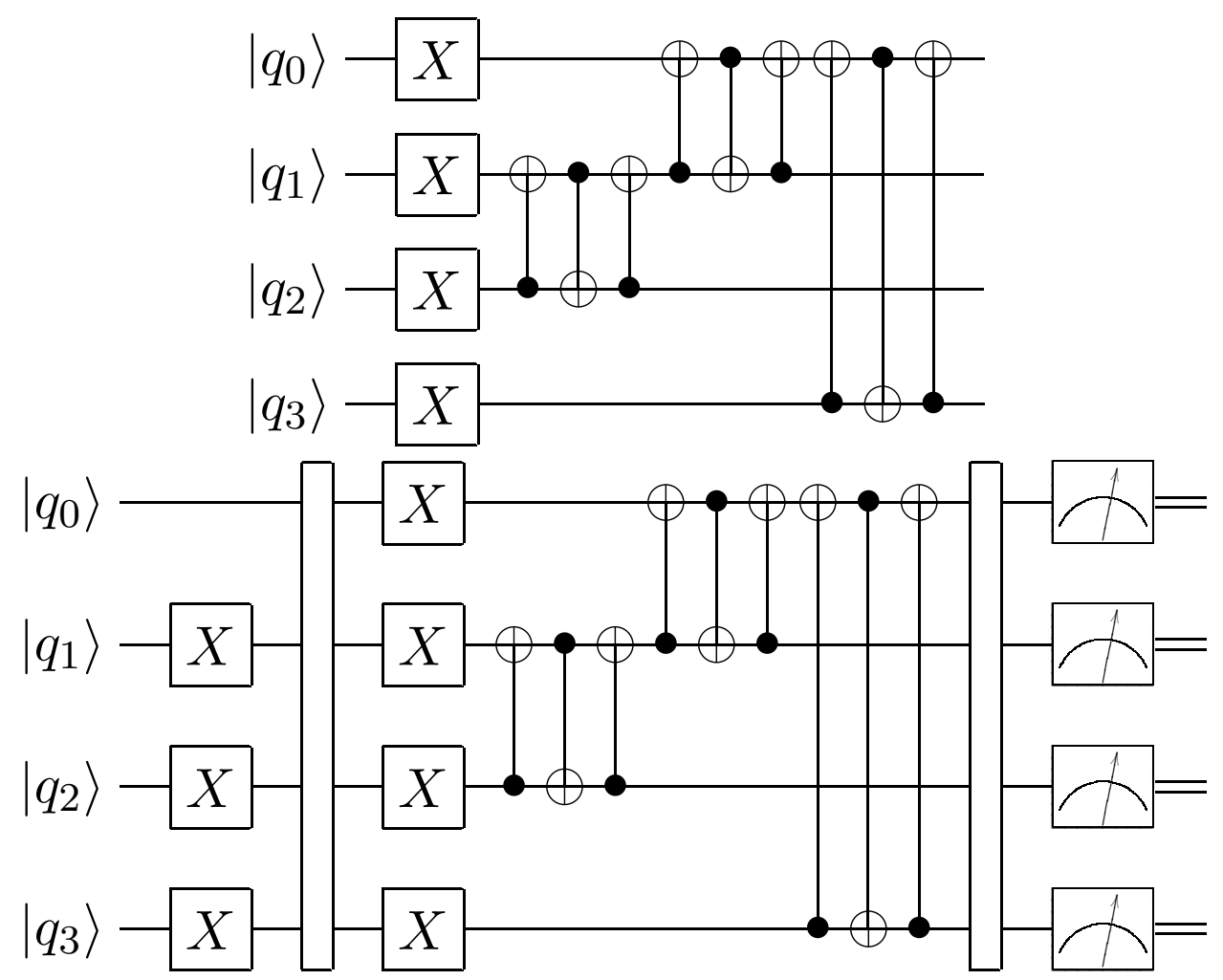

Figure 9.14.: Visual representation of the program for the modular multiplication of a state with $7 x \bmod 15$ is shown in the upper figure. The lower figure shows a full quantum program that applies the unitary transformation to a state that encodes the number $x=7$ and measures the result. The gates are defined in table 9.1. The vertical empty boxes are barriers to visually separate different stages of the quantum program.

This program is applied to a state that encodes numbers $N$ according to

$$
|\Psi\rangle=\left(\sigma_{x}^{b(N, 4)} \otimes \sigma_{x}^{b(N, 3)} \otimes \sigma_{x}^{b(N, 2)} \otimes \sigma_{x}^{b(N, 1)}\right)|0000\rangle,
$$

where $b(N, i)$ gives the $i$-th bit in the binary representation of the number $N$ starting from least significant bit. The lower circuit diagram in figure 9.14 shows a full quantum program including input and measurement. It first encodes the number 7 in a state, i.e. $|\Psi\rangle=\left(\mathbb{1} \otimes \sigma_{x} \otimes \sigma_{x} \otimes \sigma_{x}\right)|0000\rangle$, then applies the unitary transformation and measures the results. The outcome are the measurements $\left\langle q_{0}\right\rangle=0,\left\langle q_{1}\right\rangle=1,\left\langle q_{2}\right\rangle=0$ and $\left\langle q_{3}\right\rangle=0$. This corresponds to the number 4 which is indeed the result of $7 \cdot 7 \bmod 15=49 \bmod 15$. In Shor's algorithm many modular multiplications are applied to a state and the period is measured with a quantum Fourier transform.

\section{Computational power and quantum volume}

The concept of a universal quantum computer defined in the previous section gives five requirements for experimental realizations [DiVincenzo, 2000; Nielsen and Chuang, 2010]:

1. a scalable physical system with well-characterized quantum-mechanical observables to represent the qubits, 


\begin{tabular}{|c|c|c|}
\hline name & unitary matrix & visual representation \\
\hline Hadamard gate (h) & $\frac{1}{\sqrt{2}}\left(\begin{array}{cc}1 & 1 \\
1 & -1\end{array}\right)$ & $\left|q_{0}\right\rangle-H$ \\
\hline$\sigma_{x}$ gate $(\mathrm{x})$ & $\left(\begin{array}{ll}0 & 1 \\
1 & 0\end{array}\right)$ & $\left|q_{0}\right\rangle-X$ \\
\hline$\sigma_{y}$ gate $(\mathrm{y})$ & $\left(\begin{array}{cc}0 & -i \\
i & 0\end{array}\right)$ & $\left|q_{0}\right\rangle-Y$ \\
\hline$\sigma_{z}$ gate $(\mathrm{z})$ & $\left(\begin{array}{cc}1 & 0 \\
0 & -1\end{array}\right)$ & $\left|q_{0}\right\rangle-Z$ \\
\hline$R_{x}$ gate $(\mathrm{rx})$ & $e^{-i \theta \sigma_{x} / 2}=\left(\begin{array}{cc}\cos (\theta / 2) & -i \sin (\theta / 2) \\
-i \sin (\theta / 2) & \cos (\theta / 2)\end{array}\right)$ & $\left|q_{0}\right\rangle-R_{x}(\theta)$ \\
\hline$R_{y}$ gate (ry) & $e^{-i \theta \sigma_{y} / 2}=\left(\begin{array}{cc}\cos (\theta / 2) & -\sin (\theta / 2) \\
\sin (\theta / 2) & \cos (\theta / 2)\end{array}\right)$ & $\left|q_{0}\right\rangle-R_{y}(\theta)$ \\
\hline$R_{z}$ gate $(\mathrm{rz})$ & $e^{-i \theta \sigma_{z} / 2}=\left(\begin{array}{cc}e^{-i \theta / 2} & 0 \\
0 & e^{i \theta / 2}\end{array}\right)$ & $\left|q_{0}\right\rangle-R_{z}(\theta)$ \\
\hline phase shift & $e^{i \theta \mathbb{1}}=\left(\begin{array}{cc}e^{i \theta} & 0 \\
0 & e^{i \theta}\end{array}\right)$ & $\left|q_{0}\right\rangle-\operatorname{Ph}(\theta)-$ \\
\hline rotation gate (u3) & $\left(\begin{array}{cc}e^{-i(\phi+\lambda) / 2} \cos (\theta / 2) & -e^{-i(\phi-\lambda) / 2} \sin (\theta / 2) \\
e^{i(\phi-\lambda) / 2} \sin (\theta / 2) & e^{i(\phi+\lambda) / 2} \cos (\theta / 2)\end{array}\right)$ & $\left|q_{0}\right\rangle-U 3(\theta, \phi, \lambda)-$ \\
\hline phase gate (s) & $\left(\begin{array}{ll}1 & 0 \\
0 & i\end{array}\right)$ & $\left|q_{0}\right\rangle-S-$ \\
\hline phase gate $^{\dagger}$ (sdag) & $\left(\begin{array}{cc}1 & 0 \\
0 & -i\end{array}\right)$ & $\left|q_{0}\right\rangle-S^{\dagger}$ \\
\hline swap gate & $\left(\begin{array}{llll}1 & 0 & 0 & 0 \\
0 & 0 & 1 & 0 \\
0 & 1 & 0 & 0 \\
0 & 0 & 0 & 1\end{array}\right)$ & 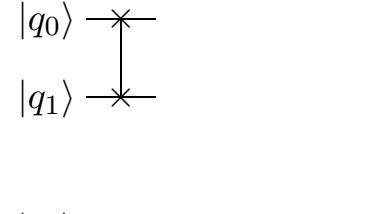 \\
\hline CNOT gate (cx) & $\left(\begin{array}{llll}1 & 0 & 0 & 0 \\
0 & 1 & 0 & 0 \\
0 & 0 & 0 & 1 \\
0 & 0 & 1 & 0\end{array}\right)$ & $\begin{array}{l}\left|q_{0}\right\rangle \multimap \\
\left|q_{1}\right\rangle-\emptyset\end{array}$ \\
\hline Toffoli gate $(\mathrm{cxx})$ & $\left(\begin{array}{llllllll}1 & 0 & 0 & 0 & 0 & 0 & 0 & 0 \\
0 & 1 & 0 & 0 & 0 & 0 & 0 & 0 \\
0 & 0 & 1 & 0 & 0 & 0 & 0 & 0 \\
0 & 0 & 0 & 1 & 0 & 0 & 0 & 0 \\
0 & 0 & 0 & 0 & 1 & 0 & 0 & 0 \\
0 & 0 & 0 & 0 & 0 & 1 & 0 & 0 \\
0 & 0 & 0 & 0 & 0 & 0 & 0 & 1 \\
0 & 0 & 0 & 0 & 0 & 0 & 1 & 0\end{array}\right)$ & 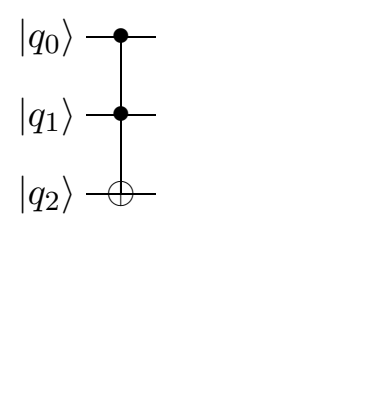 \\
\hline
\end{tabular}

Table 9.1.: List of commonly used quantum gates. The abbreviation in the brackets after the name denotes the operation code in the open quantum assembly language (OpenQASM, [Cross et al., 2017]). 
2. a preparation of an initial qubit state like $|000 \ldots\rangle$,

3. a controllable unitary evolution with single qubit-gates and at least one type of universal two-qubit gate,

4. decoherence times that are much longer than gate-operation times and

5. the possibility to measure final qubit states.

The computational power of a physical realization of a gate-based quantum computer can be quantified with the measure called quantum volume [Moll et al., 2017]. We denote $n$ as the minimal number of qubits to run a specific algorithm and $d$ as the number of steps, i.e., the circuit depth. To be able to compare the circuit depth between different quantum computers that might use different sets of natively supported universal gates, a step is defined as a unitary operator that can be written as a product of arbitrary twoqubit gates that act on disjoint pairs of qubits. As some quantum computer architecture might not support the required two-qubit gates directly or might not provide the required connectivity paths, the two-qubit gates might have to be emulated by a composition of natively supported two-qubit gates. For example, if the two-qubit gate required for a computation step of the algorithm is a swap gate, this would count as a single step but on a quantum computer that does not natively support swap gates this has to be emulated by three CNOT-gates

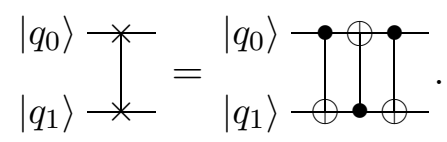

This gate overhead is included effectively in the effective error rate $\epsilon_{\text {eff }}$ that describes the averaged error rate of the application of a general two-qubit gate. For a formal evaluation of this quantity, first all possible two-qubit gates, that is, any unitary transformation from $\mathrm{SU}(4)$, are expressed with natively supported gates. Then the error rates for the resulting combinations of natively supported gates are evaluated and averaged. Thus, a quantum computer that supports more types of one- and two-qubit gates natively with small error rates and has a more complete connectivity graph is assigned a smaller effective error rate. Due to crosstalk between the qubits, the effective error rate of a given architecture will depend on the number of qubits used. The quantity

$$
D(N)=\max _{n \leq N} \frac{1}{n \epsilon_{\mathrm{eff}}(n)}
$$

represents the maximal executable circuit depth in terms of general two-qubit gates depending on the number $n$ of qubits used. Thus, the product $D \cdot N$ of the maximal depth $D$ and the number of qubits $N$ gives an estimate of the volume in space (qubits) and time (depth of the circuit) of programs executable on the quantum computer. The definition of the quantum volume given by Moll et al. [Moll et al., 2017] is

$$
V_{Q}(n)=\max _{n \leq N}\left[\min \left(n, \frac{1}{n \epsilon_{\mathrm{eff}}(n)}\right)^{2}\right] \leq D(N) \cdot N,
$$

where $n$ is the number of qubits required for the algorithm and $N$ the number of qubits of the quantum computer. The maximization is performed over subsets of qubits if not all available qubits are required for the algorithm, that is $n<N$. In the limit of perfect 
gate-application fidelity and native support of any two-qubit gate as a native gate, that is, $\epsilon_{\text {eff }} \rightarrow 0$, we get $V_{Q}(n)=n^{2}$. Thus, the quantum volume of a quantum computer indicates if an algorithm with a certain complexity in terms of qubits and circuit depth can be run on physical realization of this quantum computer. The publicly accessible 5-qubit quantum computers $^{12}$, IBM Q5 Teneriffe and IBM Q5 Yorktown, have an average single-qubit gate errors of $2 \cdot 10^{-3}$ and averaged CNOT-gate errors of $4 \cdot 10^{-2}$ to $6 \cdot 10^{-2}$ measured using simultaneous randomized benchmarking [Gambetta et al., 2012]. Therefore, the quantum volume of these quantum computers can be roughly estimated to between 11 and 25 . Similarly, the estimated maximal circuit depth lies between 3 and 5 . These estimates do not consider that the connectivity graphs are not all-to-all graphs and that the errors of natively supported CNOT-gates along different paths can vary greatly, namely between $2.5 \cdot 10^{-2}$ and about $10 \cdot 10^{-2}$. The estimate of the quantum volume can also be used when some form of quantum error correction is employed: instead of the number of hardware-qubits of the quantum computer then the number of logical qubits is used for the quantum volume. Codes for quantum error correction will not be discussed here because they require much more qubits due to the fact that every logical qubit is encoded in two or more hardware-qubits.

\subsubsection{Physical realization of quantum computers: transmon qubit}

\section{Basic physical principle of the transmon qubit}

There are several possibilities to realize a qubit experimentally. For example [Nielsen and Chuang, 2010]:

- ions in electromagnetic traps manipulated with laser pulses,

- nuclear spins of molecules manipulated with nuclear magnetic resonance,

- single photons in non-linear optical media or

- cooper pairs in quantum dots manipulated with Josephson junctions.

The latter type is used by IBM's publicly accessible quantum computers, and we will only describe the physical principles underlying this type of qubit. We restrict ourselves to this type because we have evaluated the practical viability of our algorithm on a 5-qubit quantum computer from IBM. The publicly accessible 5-qubit quantum computers, IBM Q5 Teneriffe and IBM Q5 Yorktown, as well as their 16-qubit and 20-qubit quantum computers $^{13}$ belong to the class of superconducting quantum computers with transmission-line shunted plasma oscillation (transmon) qubits [Koch et al., 2007]. The working principle of a superconducting transmon qubit is the same as the principle of a Cooper-pair box qubit [Bouchiat et al., 1998]. The main element of a Cooper-pair box qubit is a superconducting tunnel junction in the form of two BCS-type [Bardeen et al., 1957] superconductors coupled by a thin insulator (superconductor-insulator-superconductor Josephson junction). The observable used to physically encode the states of the quantum computer are charge states of a superconducting island. We follow here the description of the

\footnotetext{
${ }^{12}$ IBM Q initiative (https://www.research.ibm.com/ibm-q/), access through IBM Q Experience (https://quantumexperience.ng.bluemix.net/qx)

${ }^{13}$ https://quantumexperience.ng.bluemix.net/qx/devices accessed: 2018-06-07. The larger quantum computers are not publicly accessible. For some results obtained on these devices see [Wang et al., 2018].
} 


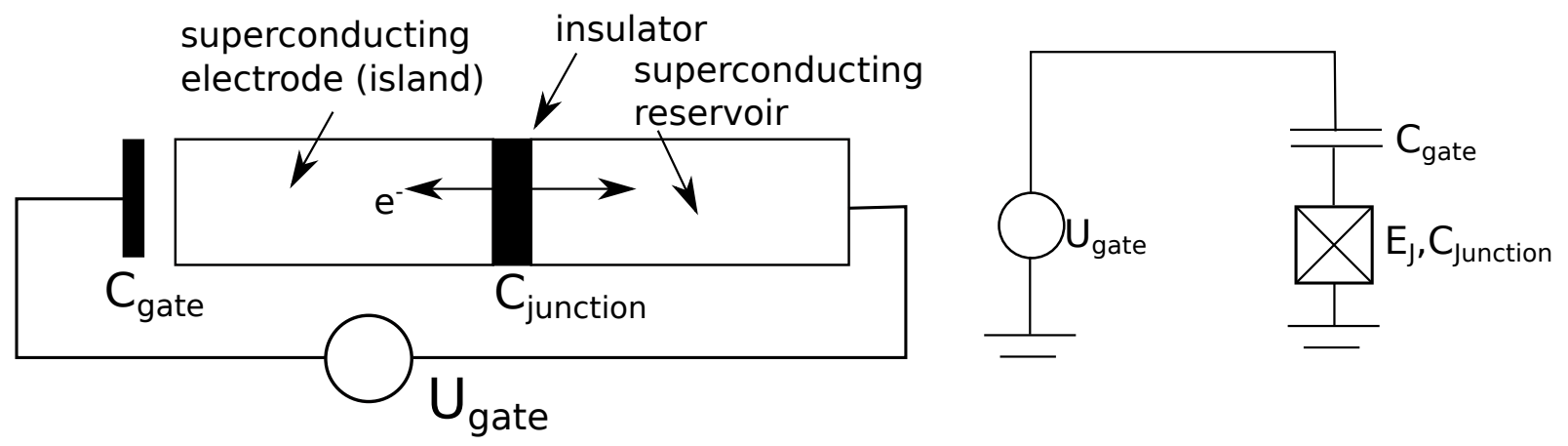

Figure 9.15.: Schematical representation of a Cooper-pair box qubit (left) and corresponding circuit schematic (right). The superconducting electrode (island) is connected to the superconducting reservoir by a thin isolating barrier. This forms an effective capacitance $C_{\text {junction }}$. The gate voltage $U_{\text {gate }}$ is applied between the reservoir and the gate electrode with a capacitance $C_{\text {gate }}$.

Cooper-pair box qubit given by Bouchiat et al. in [Bouchiat et al., 1998]. The layout of a Cooper-pair box qubit is schematically shown in figure 9.15. The two superconductors are not equivalent, but one is connected directly to the gate voltage $U_{\text {gate }}$ and is called the superconducting reservoir. The other superconductor is named the superconducting island and indirectly coupled to the gate voltage by a capacitance $C_{\text {gate }}$. The insulator in between the two superconductors also represents a capacitance $C_{\text {junction. }}$. The total island capacitance $C_{\text {tot }}$ is $C_{\text {tot }}=C_{\text {gate }}+C_{\text {junction }}$. The gate voltage can be used to drive electrons from the reservoir to the superconducting island.

In the following, $\Delta$ denotes the superconducting gap of the island and $N_{\text {eff }}$ the effective number of states available for excitations. The difference of the free energies $\tilde{\Delta}(T)$ of the island with an even number of electrons and an odd number of electrons at low temperatures $N_{\text {eff }} e^{\frac{-\Delta}{k_{B} T}} \ll 1$ is approximately [Tuominen et al., 1992]

$$
\tilde{\Delta}(T)=\Delta-k_{B} T \ln \left(N_{\text {eff }}\right) .
$$

Now we observe the conditions under which all electrons on the island are paired [Bouchiat et al., 1998]: on the one hand, the even-odd free energy difference $\tilde{\Delta}(T)$ has to be much larger than the energy of thermal fluctuations $k_{B} T$ and the Coulomb energy $E_{c}=\frac{4 e^{2}}{2 C_{\text {tot }}}$. The Coulomb energy is the classical electrostatic charging energy of a Cooper pair on the island at zero gate voltage. On the other hand, also the Coulomb energy has to be much larger than the energy of thermal fluctuations so that the fluctuations of the number of electrons on the island become negligible (Coulomb blockage, [Lafarge et al., 1993]). Thus, in total we have the condition $\tilde{\Delta}(T) \gg E_{c} \gg k_{B} T$. The number $n \in \mathbb{Z}$ of excess or missing Cooper pairs on the island determines the charge of the island as $q=-2 \mathrm{en}$. The number $n$ is discrete, and the corresponding operator $\hat{n}$ is the operator of the number of Cooper pairs on the island. In the basis of eigenstates $|n\rangle$ the effective Hamiltonian ${ }^{14}$ can be written [Bouchiat et al., 1998] as a sum of two contributions. The first contribution is the Coulomb energy

$$
\hat{H}_{c}=E_{c} \sum_{n}\left(n-n_{\text {gate }}\right)^{2}|n\rangle\langle n|,
$$

\footnotetext{
${ }^{14}$ Detailed derivations of the effective Hamiltonian can be found in [Wendin and Shumeiko, 2005] or [Ågren, 2002].
} 
where $n_{\text {gate }}=\frac{C_{\text {gate }} U_{\text {gate }}}{2 e}$ is the charge that is induced by the finite gate voltage. The ground-state energy and expectation value of the number of excess Cooper pairs on the superconducting island is shown in figure 9.16. The energies $E(n)=\left\langle n\left|\hat{H}_{c}\right| n\right\rangle$ for neighboring $n$, i.e. $E(n)$ and $E(n+1)$, cross at $n+1 / 2$ at an energy value of $E_{c} / 4$. The second contribution to the effective Hamiltonian is the Josephson coupling Hamiltonian

$$
\hat{H}_{j}=-E_{J} \cos (\hat{\phi})
$$

$\hat{\phi}$ denotes the operator of the difference of the phases of the superconducting order parameters of the two superconductors. $E_{J}$ denotes the Josephson energy and is related to the total electrical tunnel-junction conductance $G_{T}$ in the normal state by $E_{J}=\frac{h G_{T}}{8 e^{2}} \Delta$. With

$$
\begin{aligned}
\hat{n}|n\rangle & =n|n\rangle \\
{\left[\hat{n}, e^{ \pm i \hat{\phi}}\right] } & = \pm e^{ \pm i \hat{\phi}} \\
e^{ \pm i \hat{\phi}}|n\rangle & =|n \pm 1\rangle,
\end{aligned}
$$

the Josephson coupling Hamiltonian can also be written as

$$
\hat{H}_{J}=-\frac{E_{J}}{2} \sum_{n}(|n\rangle\langle n+1|+| n+1\rangle\langle n|) .
$$

Now we consider the case of $E_{c} \gg E_{J}$. Then the Josephson Hamiltonian Eq. (9.233) can be considered as a perturbation and it lifts the degeneracy at the crossings of the Coulomb Hamiltonian of Eq. (9.228). This opens a gap around $E_{c} / 4$ of width $E_{J}$. At low temperatures $E_{c} \gg k_{B} T$ and for $0 \leq n_{\text {gate }}=\frac{C_{\text {gate }} U_{\text {gate }}}{2 e} \leq 1$ we only have to consider the two states $|0\rangle$ and $|1\rangle$ with the matrix-representation of the Hamiltonian

$$
H_{2}=\left(\begin{array}{cc}
E_{C} n_{\text {gate }}^{2} & -\frac{1}{2} E_{J} \\
-\frac{1}{2} E_{J} & E_{C}\left(1-2 n_{\text {gate }}+n_{\text {gate }}^{2}\right)
\end{array}\right)=\frac{1}{2}\left(\begin{array}{cc}
-E_{2} & -E_{J} \\
-E_{J} & E_{2}
\end{array}\right)+E_{c}\left(\frac{1}{2}-n_{\text {gate }}+n_{\text {gate }}^{2}\right) \mathbb{1}_{2}
$$

with $E_{2}=E_{C}\left(1-2 n_{\text {gate }}\right)$. Using the Pauli matrices, this Hamiltonian can be rewritten as

$$
\hat{H}_{2}-E_{c}\left(\frac{1}{2}-n_{\text {gate }}+n_{\text {gate }}^{2}\right) \mathbb{1}_{2}=-\frac{1}{2} E_{J} \hat{\sigma}_{x}-\frac{1}{2} E_{2} \hat{\sigma}_{z}=-\vec{h} \cdot \hat{\vec{S}}
$$

with $\hat{\vec{S}}=\frac{\hbar}{2} \hat{\vec{\sigma}}$ the magnetic field $\vec{h}=\left(E_{J} / \hbar, 0, E_{2} / \hbar\right)$. Thus, under the previously mentioned conditions, this system corresponds to a spin- $\frac{1}{2}$-particle in a magnetic field. The operator $\hat{n}$ of the number of excess Cooper pairs corresponds to the z-component of the spin-operator via

$$
\hat{n}=\frac{1}{2}-\frac{\hat{S}_{z}}{\hbar}
$$

On the other hand, the state $|0\rangle$ corresponds to the spin-up state $|\uparrow\rangle$ and the state $|1\rangle$ corresponds to the spin-down state $|\downarrow\rangle$. For example, the state $(|\downarrow\rangle+|\uparrow\rangle) / \sqrt{2}$ can be obtained as the ground state of the qubit at $n_{\text {gate }}=1 / 2$ and the state $(|\downarrow\rangle-|\uparrow\rangle) / \sqrt{2}$ 


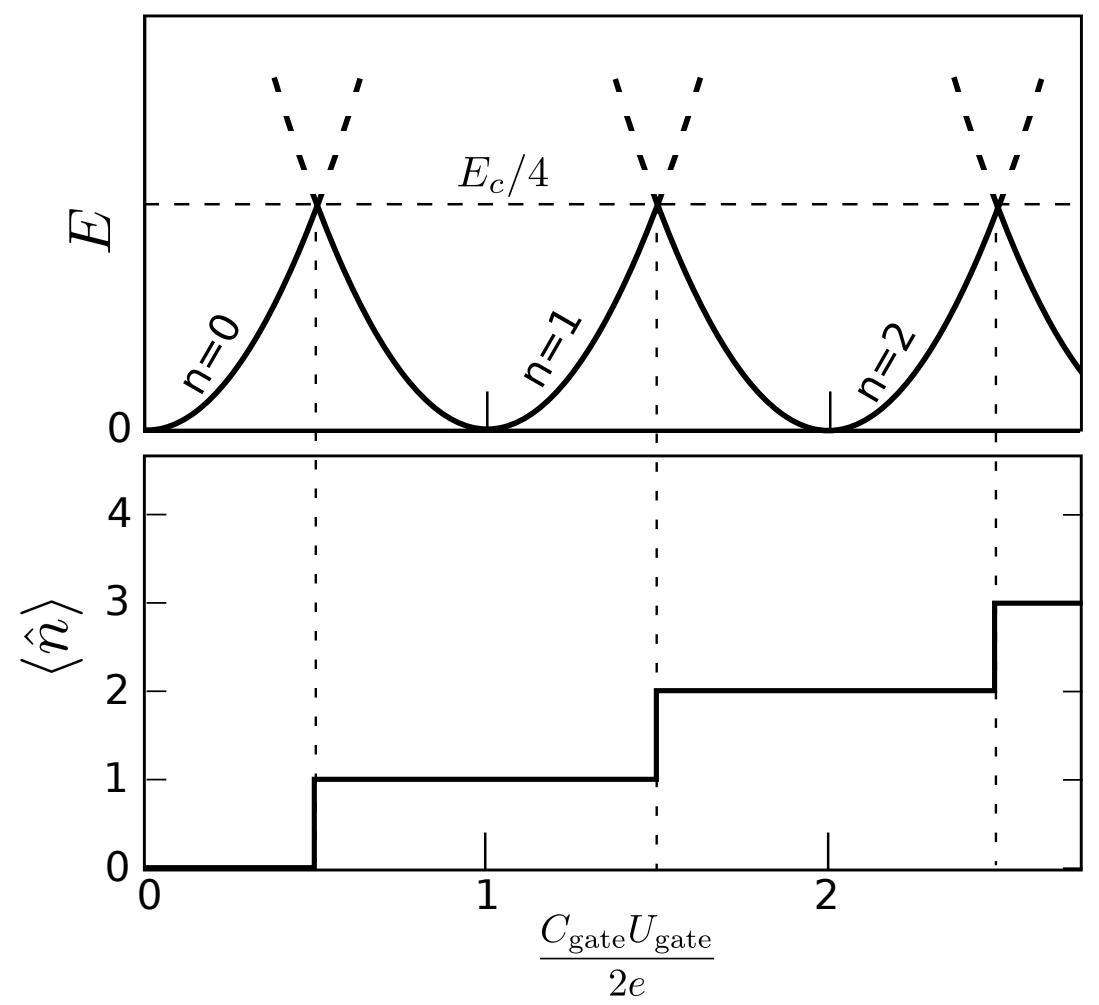

Figure 9.16.: Energy $E$ and expectation value $\langle\hat{n}\rangle$ of the number of excess Cooper pairs on the island in the ground state of the Hamiltonian $\hat{H}_{c}$ in Eq. 9.228, that only contains the Coulomb energy of the Cooper-pair box qubit as a function of $\frac{C_{\text {gate }} U_{\text {gate }}}{2 e} . U_{\text {gate }}$ is the gate voltage and $C_{\text {gate }}$ the gate capacitance. $E_{c}=\frac{4 e^{2}}{2 C_{\text {tot }}}$ denotes the classical electrostatic charging energy of a Cooper pair on the island.

as the first excited state at $n_{\text {gate }}=1 / 2$. Therefore, a Cooper-pair box can be used as a physical realization of a single qubit. Thus, the first requirement for a realization of a universal quantum computer given in section 9.8.2, a scalable physical system with wellcharacterized quantum-mechanical observables to represent the qubits, is fulfilled. Also, the fourth criterion, decoherence times that are much longer than gate-operation times, is fulfilled because the decoherence times of this simple qubit are estimated to about 10 $\mu \mathrm{s}$ [Bouchiat et al., 1998]. The decoherence is mainly caused by dissipation due to the electromagnetic environment [Bouchiat et al., 1998]. Therefore, the main challenges of experimental realizations of this type of qubit are the cooling to temperatures of a few milli-Kelvin as well as the electromagnetic isolation with respect to charge noise.

A transmon qubit [Koch et al., 2007] is an advanced version of the Cooper-pair box qubit. The two main changes are the doubling of the Josephson junction to a split junction and the operation in the regime $E_{c} \ll E_{J}$ by shunting with a large capacitance. The doubling of the Josephson junction is schematically shown in figure 9.18. For simplicity the two junctions are assumed to have the same characteristics, that is, we only consider a symmetric split junction here. The split junction has a shape similar to a DC-SQUID (direct current superconducting quantum interference device, [Jaklevic et al., 1964]). Let $\hat{\phi}_{1}$ and $\hat{\phi}_{2}$ be the operators of the respective differences of the phases of the superconduct- 


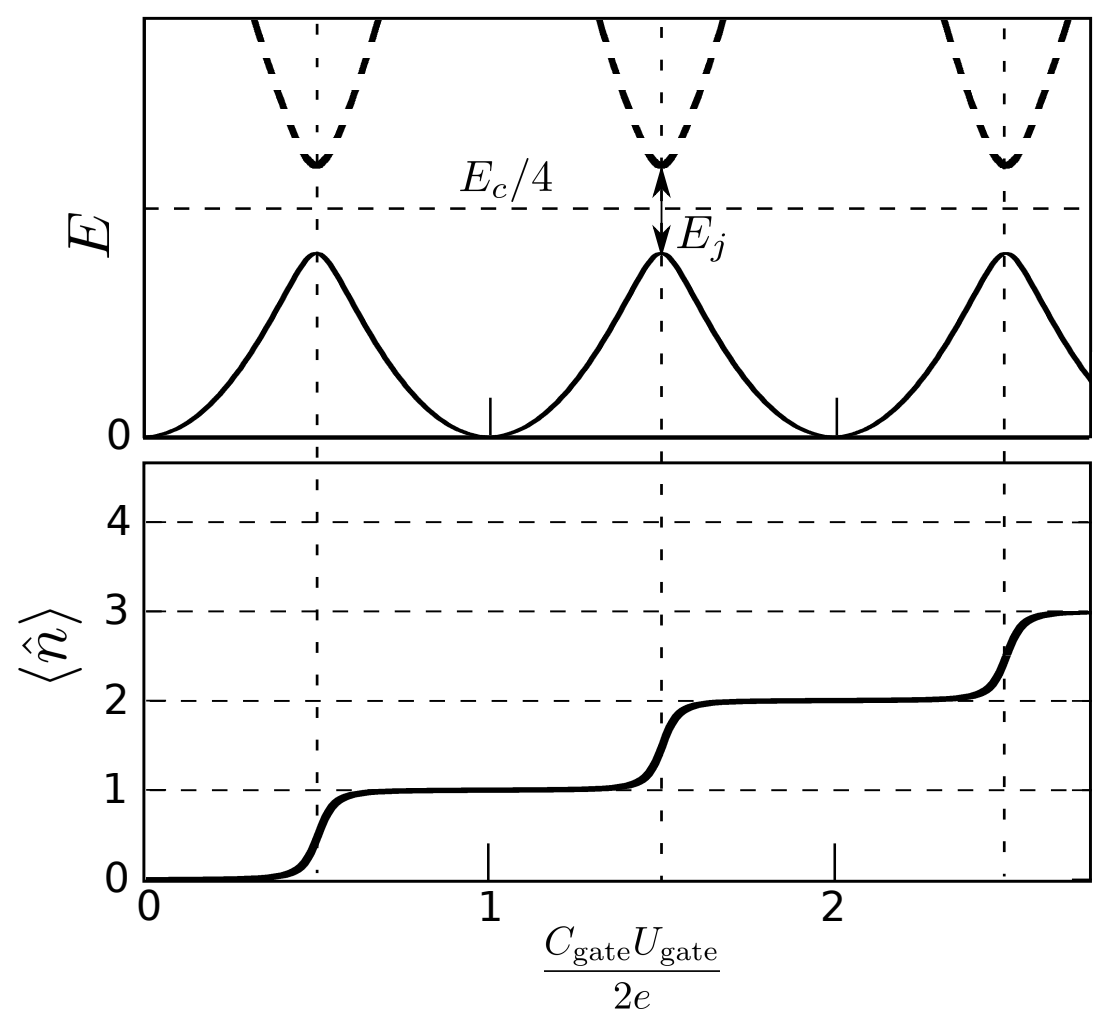

Figure 9.17.: Energy $E$ and expectation value $\langle\hat{n}\rangle$ of the number of excess Cooper pairs on the island in the ground state of the Hamiltonian $\hat{H}_{c}+\hat{H}_{J}$ in Eq. 9.228 and Eq. 9.233 as a function of $\frac{C_{\text {gate }} U_{\text {gate }}}{2 e}$, where $U_{\text {gate }}$ is the gate voltage and $C_{\text {gate }}$ the gate capacitance. $E_{c}=\frac{4 e^{2}}{2 C_{\text {tot }}}$ denotes the classical electrostatic charging energy of a Cooper pair on the island. $E_{J}$ denotes the Josephson energy. The results shown are for $E_{c}=10 E_{J} \gg E_{J}$.

ing order parameters of the first and second junction. Then the magnetic flux $\Phi$ through the junction is quantized and follows the relation

$$
\phi_{1}-\phi_{2}=2 \pi n+2 \pi \frac{\Phi}{\Phi_{0}}
$$

with $n \in \mathbb{Z}$ and $\Phi_{0}=\frac{h}{2 e}$ as the magnetic flux quantum. This leads to the effective Josephson coupling Hamiltonian [Koch et al., 2007]

$$
\hat{H}_{J \text {,split }}=-2 E_{J} \cos \left(\frac{\pi \Phi}{\Phi_{0}}\right) \cos \left(\frac{\hat{\phi}_{1}+\hat{\phi}_{2}}{2}\right),
$$

which is equivalent to the Josephson coupling Hamiltonian of a single junction Eq. (9.229) with the choices

$$
\begin{aligned}
E_{J} & \mapsto E_{J, \text { split }}=2 E_{J} \cos \left(\frac{\pi \Phi}{\Phi_{0}}\right) \\
\hat{\phi} & \mapsto \frac{\hat{\phi}_{1}+\hat{\phi}_{2}}{2} .
\end{aligned}
$$



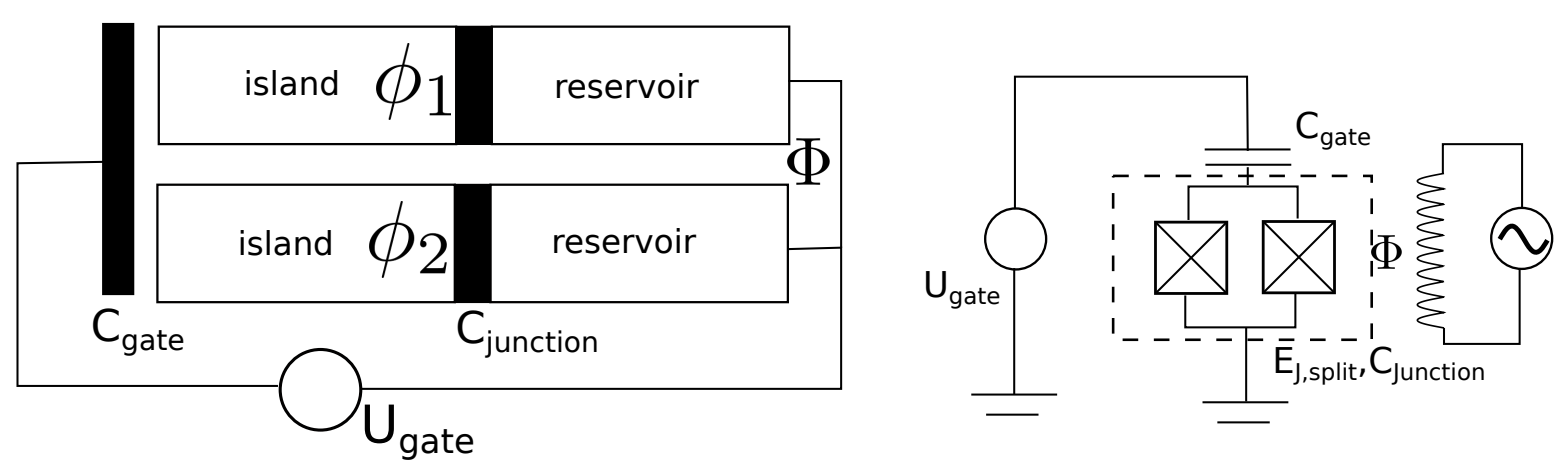

Figure 9.18.: Schematical layout of a split junction Cooper-pair box (left) and the corresponding circuit schematic (right). The two Josephson junctions are assumed to be identical. $\phi_{1}$ and $\phi_{2}$ denote the corresponding superconducting phase differences. $\Phi$ denotes the magnetic flux through the split junction Cooper-pair box and is used to tune its properties. The gate capacitance $C_{\text {gate }}$, the gate voltage $U_{\text {gate }}$ and the junction capacitance $C_{\text {junction }}$ are identical to figure 9.15 . The effective Josephson energy $E_{J, \text { split }}$ depends on the magnetic flux $\Phi$ through the junctions and is given by Eq. (9.239).

Thus, the magnetic flux $\Phi$ can be used to tune the effective Josephson energy $E_{J \text {,split }}$ of the split junction. For the previously discussed Cooper-pair-box qubit, the regime of large Coulomb energy $E_{c} \gg E_{J}$ was used. As shown in figure 9.17 , the energy spectrum in this region strongly depends on $n_{\text {gate }}$. Thus, small changes in $n_{\text {gate }}$ due to charge noise from the environment can cause large changes in the energy levels. However, $E_{c} \gg E_{J}$ has the positive feature that the energy differences for $n_{\text {gate }}=1 / 2$ between the lowest energy state and the second state are much smaller than between the second state and higher states. Motivated by the energy levels of an anharmonic oscillator these nonuniform differences of the energy levels are called anharmonicity. Larger anharmonicity means a larger non-uniformity of the differences. This large anharmonicity for $E_{c} \gg E_{J}$ results in very different frequencies for the excitations between these states, which is an experimentally favourable situation for the control of the qubit. The opposite situations, $E_{c} \ll E_{J}$, results in the energy level diagram shown on the right side of figure 9.19. In this case, the differences in the lowest energy levels are similar in size and, hence, the anharmonicity low. Consequently, this qubit will be harder to control experimentally. Thus, a compromise between a low influence of charge noise from $E_{c} \ll E_{J}$ and high anharmonicity at $E_{c} \gg E_{J}$ has to be found. A transmon uses the fact that anharmonicity decreases only with a slow power law in $E_{J} / E_{c}$ whereas the sensitivity to charge noise reduces exponentially in $E_{J} / E_{c}$ [Koch et al., 2007]. To achieve a decrease of $E_{c}$ a large capacitance $C_{\text {shunt }}$ is added in parallel to the Josephson junctions. In the sense of where it is placed, the added capacitance is similar to a shunt resistor for measuring large electric currents. The Coulomb energy now reads

$$
E_{c}=\frac{4 e^{2}}{2\left(C_{\text {shunt }}+C_{\text {gate }}+C_{\text {junction }}\right)} .
$$

In practical realizations usually values of $10<E_{J} / E_{c}<1000$ are used [Koch et al., 2007]. 

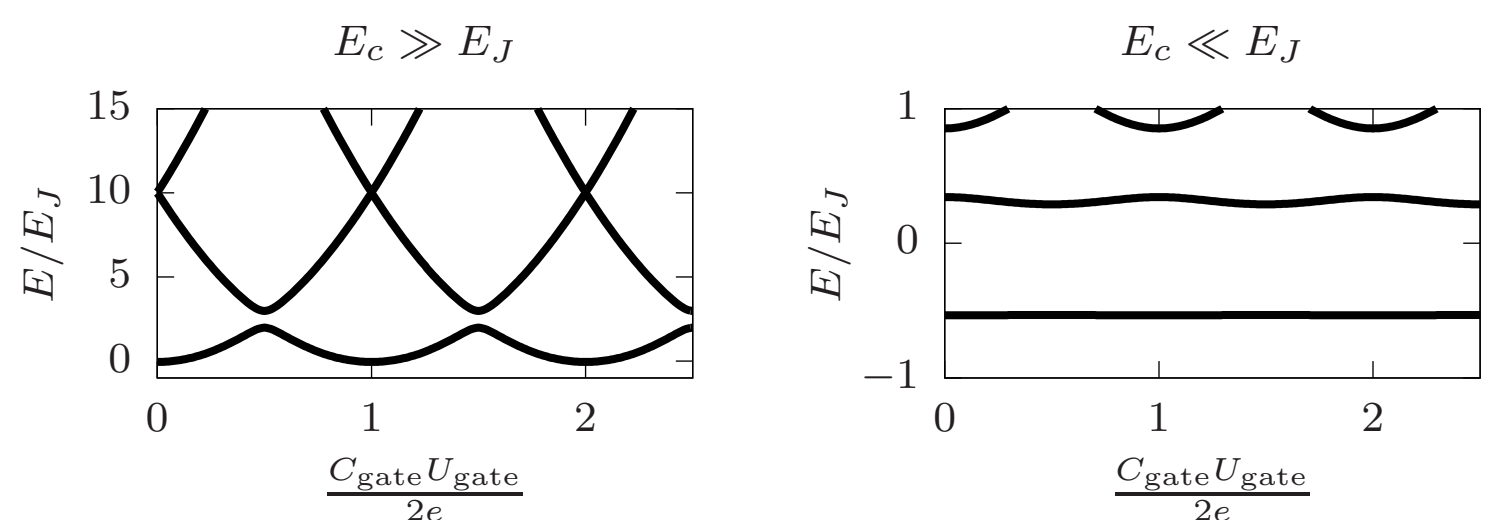

Figure 9.19.: Energy levels of the Hamiltonian $\hat{H}_{c}+\hat{H}_{J}$ in Eq. 9.228 and Eq. 9.233 as a function of $\frac{C_{\text {gate }} U_{\text {gate }}}{2 e}$, where $U_{\text {gate }}$ is the gate voltage and $C_{\text {gate }}$ the gate capacitance. $E_{c}=\frac{4 e^{2}}{2 C_{\text {tot }}}$ denotes the classical electrostatic charging energy of a Cooper pair on the island. $E_{J}$ denotes the Josephson energy. The left figure is for $E_{c}=5 E_{J} \gg E_{J}$ (Cooper-pair box qubit) and the right figure for $E_{c}=E_{J} / 2 \ll E_{J}$ (transmon qubit).

In this regime the energy difference between the $|0\rangle$ state and the $|1\rangle$ state is

$$
E_{|0\rangle \leftrightarrow|1\rangle}=\hbar \omega_{|0\rangle \leftrightarrow|1\rangle}=\sqrt{2 E_{c} E_{J}}-\frac{E_{c}}{4}=\hbar \omega_{p}-\frac{E_{c}}{4} .
$$

$\omega_{p}=\sqrt{2 E_{c} E_{J}} / \hbar$ denotes the Josephson plasma oscillation frequency [Koch et al., 2007]. Thus, because $E_{c}$ is small, the time scale of the dynamics of a transmon qubit is given by the Josephson plasma oscillation frequency. Typical transmon qubit frequencies are in the range of $5 \mathrm{GHz}<\omega_{|0\rangle \leftrightarrow|1\rangle} / 2 \pi<10 \mathrm{GHz}$.

By optimizing the ratio $E_{J} / E_{c}$ to find a sweet spot between anharmonicity and sensitivity to charge noise decoherence times of the order of 40-100 $\mu$ s [Barends et al., 2013; Rigetti et al., 2012] have been achieved. The transmon qubits in the 5-qubit quantum computers from IBM use aluminum as the BCS-superconductor, aluminum-oxide as the insulator in the Josephson junction, a temperature of $15 \mathrm{mK}$ and qubit frequencies $\omega_{|0\rangle \leftrightarrow|1\rangle} / 2 \pi$ between $5.0 \mathrm{GHz}$ and $5.4 \mathrm{GHz}^{15}$. With this setup, decoherence times of 15-80 $\mu$ s are reached depending on the location of the qubit on the device and gate application times of 100-400 $\mathrm{ns}^{16}$.

In conclusion, we have shown how the transmon qubit is related to the Cooper-pair box qubit and how the states of a spin- $\frac{1}{2}$-particle can be encoded in it such that high decoherence times can be achieved. This fulfills the first criterion that requires a scalable physical system with well characterized quantum-mechanical observables and the fourth criterion that requires long decoherence times of section 9.8.2.

\section{Control and measurements with a transmon qubit}

The remaining requirements to fulfill from the list in section 9.8.2 are the preparation of an initial state, the application of single qubit gates and at least one type of universal

\footnotetext{
${ }^{15}$ https://quantumexperience.ng.bluemix.net/qx/devices accessed: 2018-06-07

${ }^{16}$ https://quantumexperience.ng.bluemix.net/qx/devices accessed: 2018-06-07
} 

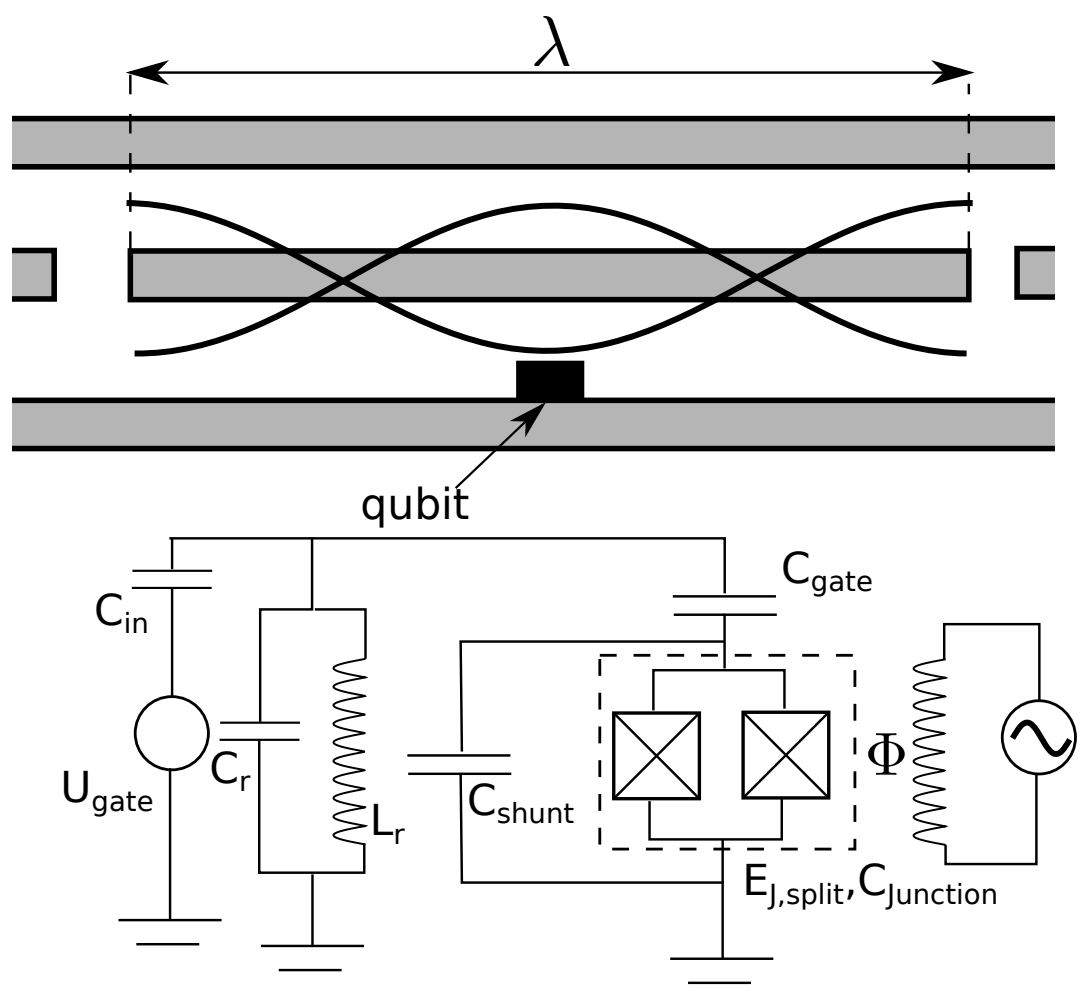

Figure 9.20.: Simplified schematic layout of a coplanar waveguide transmission line resonator for the wavelength $\lambda$ that is coupled to a qubit (upper figure) and the corresponding circuit schematic (lower figure). The sinusoid waves illustrate the standing wave in the resonator and the qubit is placed at the maximum of the amplitude. In the circuit schematic the resonator is modelled as an LC-oscillator.

two-qubit gate as well as a possibility to measure observables in the final state. In case of a transmon qubit, these aspects are realized with a transmission line resonators. A schematic layout of a transmon qubit coupled to a coplanar waveguide transmission line resonator is shown in Figure 9.20. The qubit is placed at the maximum of the standing wave generated by the resonator.

In the framework of circuit quantum electrodynamics Koch et al. [Koch et al., 2007] have obtained the effective Hamiltonian for a transmon qubit coupled to a transmission line resonator with a large effective resonator capacitance $C_{r} \gg C_{\text {shunt }}, C_{\text {in }}, C_{\text {gate }}, C_{\text {junction }}$ (see figure 9.20) as

$$
\hat{H}=E_{c}\left(\hat{n}-n_{\text {gate }}\right)^{2}-E_{J} \cos (\hat{\phi})+\hbar \omega_{r} \hat{a}^{\dagger} a+\frac{2 C_{\text {gate }} e V_{\text {rms }}^{0}}{C_{\text {shunt }}+C_{\text {gate }}+C_{\text {junction }}} \hat{n}\left(\hat{a}^{\dagger}+\hat{a}\right) .
$$

The oscillation frequency of the resonator $\omega_{r}=1 / \sqrt{L_{r} C_{r}}$ and the root mean voltage $V_{\mathrm{rms}}^{0}=\sqrt{\hbar \omega_{r} / 2 C_{r}}$ of the resonator depend on the effective inductance $L_{r}$ and capacitance $C_{r}$ of the resonator. $\hat{a}^{\dagger}$ is the creation operator and $\hat{a}$ the annihilation operator of a photon in the resonator with the mode shown in figure 9.20. The coupling between the qubit and the resonator is given by the last term of Eq. (9.243). This coupling can be used to construct procedures for measurements [Blais et al., 2007], setting initial states [Geerlings et al., 2013], application of single-qubit gates [Chow et al., 2010] and applications of 
two-qubit gates [Chow et al., 2011]. The IBM quantum computers natively support the single-qubit rotation gate $u_{3}(\mathrm{u} 3$ in table 9.1$)$ and the CNOT gate as a universal twoqubit gate ${ }^{17}$. These gates are implemented with sequences of electromagnetic pulses to the resonators with durations of about 100 ns. Single-qubit gates for the rotation around the z-axis

$$
R_{z}(\lambda)=u_{3}(0,0, \lambda)=\left(\begin{array}{cc}
e^{-i \lambda / 2} & 0 \\
0 & e^{i \lambda / 2}
\end{array}\right)
$$

can be applied with a simple frame change in software instead of an electromagnetic pulse and thus requires no interaction with the qubits. Single-qubit gates of the form

$$
u_{2}(\phi, \lambda)=u_{3}(\pi / 2, \phi, \lambda)=\frac{1}{\sqrt{2}}\left(\begin{array}{cc}
e^{-i(\phi+\lambda) / 2} & -e^{-i(\phi-\lambda) / 2} \\
e^{i(\phi-\lambda) / 2} & e^{i(\phi+\lambda) / 2}
\end{array}\right)
$$

require one resonator pulse whereas general rotations $u_{3}(\theta, \phi, \lambda)$ require two resonator pulses. CNOT gates on the IBM quantum computers require

- two resonator pulses to the control qubit,

- one resonator pulse to the target qubit and

- two pulses to the bus connecting the target and control qubit.

It should be noted here, that CNOT gates on the IBM quantum computers cannot be executed between all pairs of qubits but some qubits are not coupled by resonators. Figure 9.21 shows the simplified layout of the IBM Q5 Teneriffe quantum computer. Only qubits $Q_{2}, Q_{3}$ and $Q_{4}$ are connected to the transmission line bus $B_{1}$ and, thus, CNOT gates can only be applied among these qubits. On the other hand, qubits $Q_{0}, Q_{1}$ and $Q_{2}$ are connected to the transmission line bus $B_{2}$ so that CNOT gates can also be applied among these qubits. Qubit $Q_{2}$ has the special role of being the coupling qubit in this system. The natively supported CNOT-gates are not only restricted by the availability of a transmission line bus but also by the direction, that is, which qubit serves as the control qubit and which qubit serves as the target qubit in the CNOT-gate. The natively supported directions are also shown in figure 9.21. In case a CNOT-gate with the opposite direction is needed than available, we can use the transformation

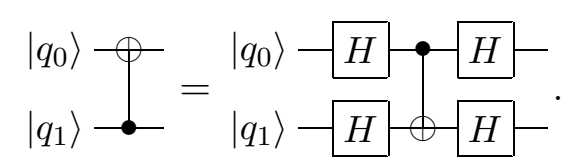

\subsubsection{Representing fermionic wave functions on quantum computers}

As discussed above, universal quantum computers are composed of spin- $\frac{1}{2}$ qubits. Thus, the many-particle wave functions are bosonic wave functions. However, in the context of electronic structure theory, we have to deal with fermionic many-particle problems. The goal is to map one qubit to one spin-orbital. By explicitly considering some spatial

\footnotetext{
${ }^{17}$ https://github.com/QISKit/qiskit-backend-information/tree/master/backends/tenerife/V1 accessed: 2018-06-07
} 


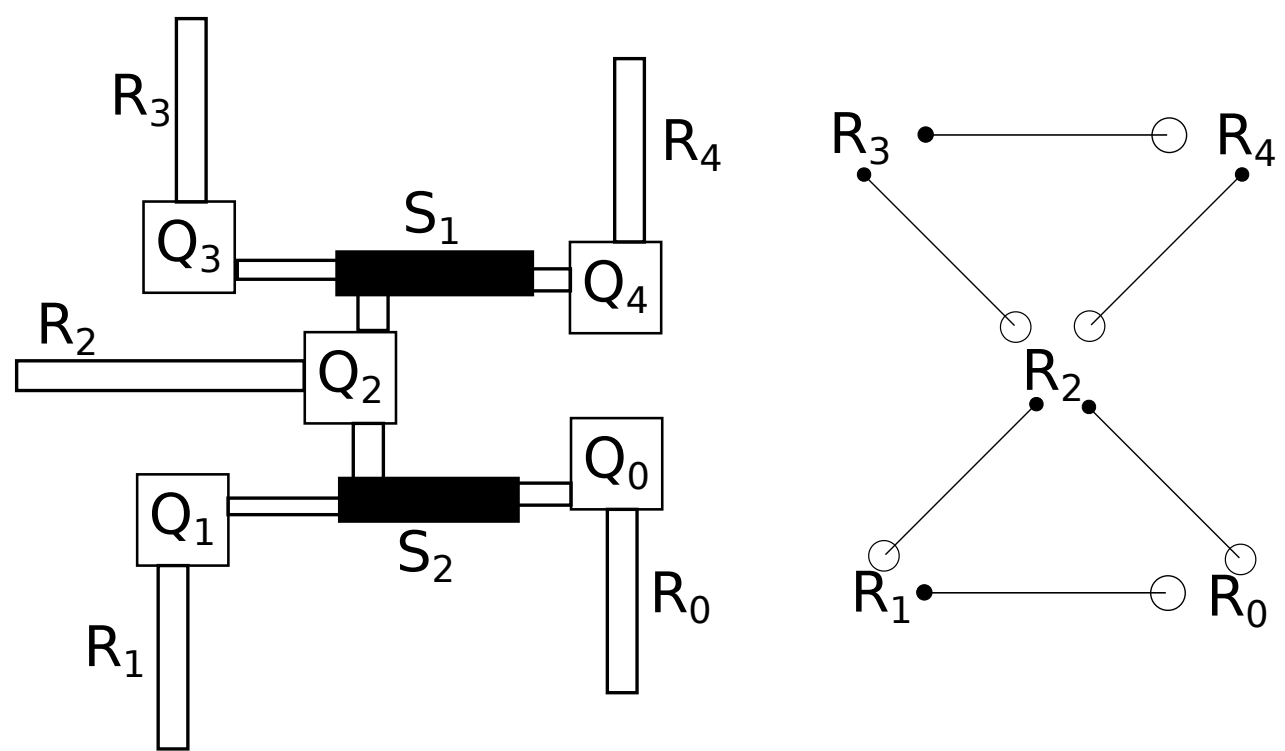

Figure 9.21.: Schematic layout of the IBM Q5 Teneriffe quantum computer (left figure) and natively supported CNOT-gates (right figure). $Q_{i}$ are the qubits, $R_{i}$ the resonators for single-qubit gates and measurements. $S_{1}$ as well as $S_{2}$ are the transmission line buses for the application of two-qubit gates. Filled circles denote control qubits, and open circles denote the target qubits of the CNOT-gate.

symmetries of the fermionic problem or restricting the Hilbert space the number of qubits to represent the fermionic system can be reduced [Lanyon et al., 2010]. In the proof-ofconcept calculation of the density-matrix functional presented here, we do not employ this reduction of the number of qubits. However, it should be noted here that the implementation of symmetries or restrictions of the Hilbert space is equivalent to the inclusion of symmetries or Hilbert-space restriction in the variational quantum eigensolver.

One possible mapping between bosonic annihilation and creation operators $\hat{\sigma}^{ \pm}$and fermionic annihilation and creation operators $\hat{c}^{(\dagger)}$ is the Jordan-Wigner transformation [Jordan and Wigner, 1993]

$$
\begin{aligned}
& \hat{c}_{\alpha}^{\dagger}=\left(\otimes_{i=1}^{\alpha-1} \mathbb{1}\right) \times \hat{\sigma}^{-} \otimes\left(\otimes_{i=1}^{n-\alpha-1} \hat{\sigma}_{z}\right) \\
& \hat{c}_{\alpha}=\left(\otimes_{i=1}^{\alpha-1} \mathbb{1}\right) \times \hat{\sigma}^{+} \otimes\left(\otimes_{i=1}^{n-\alpha-1} \hat{\sigma}_{z}\right)
\end{aligned}
$$

in the Hilbert space $\mathcal{H}=\otimes_{i=1}^{n} \mathbb{C}^{2}$. Other mappings, like the Bravyi-Kitaev transformation [Bravyi and Kitaev, 2002], are possible and the choice of the mapping can influence the efficiency of the method for larger quantum computers. In our implementation, we made sure that the Jordan-Wigner transformation can easily be exchanged for a different mapping.

\subsubsection{Measurement of observables}

We have shown how to store fermionic many-particle wave functions on universal quantum computers. The remaining question is how to measure certain observables like one-particle 
and two-particle reduced density matrices in practice. We have discussed in section 2.9.3 that these are the only observables necessary to obtain the expectation value of a fermionic many-particle Hamiltonian with one-particle and two-particle interactions. As discussed in section 9.8.2 the actual output of a universal quantum computer consists of the selection of a value from the spectrum of a qubit-local operator with the spectrum $\{0,1\}$ (computational basis) or $\{-\hbar / 2, \hbar / 2\}\left(\hat{S}_{z}\right.$, physical basis). The postprocessing on the classical computer has to derive the solution of the problem from these measurements. Thus, to measure reduced density matrices, we have to perform the averaging

$$
\begin{gathered}
\left\langle\hat{c}_{\alpha}^{\dagger} \hat{c}_{\alpha}\right\rangle=\left(\otimes_{i=1}^{\alpha-1} \mathbb{1}\right) \times \hat{\sigma}^{-} \hat{\sigma}^{+} \otimes\left(\otimes_{i=1}^{n-\alpha-1} \mathbb{1}\right) \\
=\frac{1}{N} \sum_{i=1}^{N} q_{\alpha}^{(i)}
\end{gathered}
$$

over $N$ measurements $q_{\alpha}^{(i)} \in\{0,1\}$ of $\hat{\sigma}_{z}$ of the $\alpha$-th qubit in the computational basis from the quantum computer. Some other expectation values like

$$
\begin{aligned}
\left\langle\hat{c}_{1}^{\dagger} \hat{c}_{3}\right\rangle & =\hat{\sigma}^{-} \otimes \hat{\sigma}_{z} \otimes \hat{\sigma}_{z} \hat{\sigma}^{+} \otimes\left(\otimes_{i=1}^{n-3} \mathbb{1}\right) \\
& =\hat{\sigma}_{x} \otimes \hat{\sigma}_{z} \otimes \hat{\sigma}_{z} \hat{\sigma}_{x} \otimes\left(\otimes_{i=1}^{n-3} \mathbb{1}\right)+i \hat{\sigma}_{x} \otimes \hat{\sigma}_{z} \otimes \hat{\sigma}_{z} \hat{\sigma}_{y} \otimes\left(\otimes_{i=1}^{n-3} \mathbb{1}\right) \\
& -i \hat{\sigma}_{y} \otimes \hat{\sigma}_{z} \otimes \hat{\sigma}_{z} \hat{\sigma}_{x} \otimes\left(\otimes_{i=1}^{n-3} \mathbb{1}\right)-\hat{\sigma}_{y} \otimes \hat{\sigma}_{z} \otimes \hat{\sigma}_{z} \hat{\sigma}_{y} \otimes\left(\otimes_{i=1}^{n-3} \mathbb{1}\right) \\
& =i \hat{\sigma}_{x} \otimes \hat{\sigma}_{z} \otimes \hat{\sigma}_{y} \otimes\left(\otimes_{i=1}^{n-3} \mathbb{1}\right)+\hat{\sigma}_{x} \otimes \hat{\sigma}_{z} \otimes \hat{\sigma}_{x} \otimes\left(\otimes_{i=1}^{n-3} \mathbb{1}\right) \\
& +\hat{\sigma}_{y} \otimes \hat{\sigma}_{z} \otimes \hat{\sigma}_{y} \otimes\left(\otimes_{i=1}^{n-3} \mathbb{1}\right)+i \hat{\sigma}_{y} \otimes \hat{\sigma}_{z} \otimes \hat{\sigma}_{x} \otimes\left(\otimes_{i=1}^{n-3} \mathbb{1}\right)
\end{aligned}
$$

can not be derived from measurements in the computational basis but additional gates have to be added to the quantum circuit such that measurements in the computational basis allow a reconstruction of the desired expectation value. The example in Eq. (9.252)Eq. (9.254) shows that for every element of the one-particle reduced density matrix up to four multi-qubit measurements are necessary. For an element of the two-particle reduced density matrix up to eight multi-qubit measurements are necessary in the worst case. Thus, any element of the one-particle reduced density matrix or of the two-particle reduced density matrix can be decomposed as

$$
\sum_{j} c_{j}\left(\otimes_{i=1}^{n} \hat{a}_{j, i}\right)
$$

with $\hat{a}_{i, j} \in\left\{\mathbb{1}, \hat{\sigma}_{x}, \hat{\sigma}_{y}, \hat{\sigma}_{z}\right\}$ and $c_{j} \in \mathbb{C}$. Measurements of multi-qubit observables of the form

$$
\otimes_{i=1}^{n} \hat{a}_{i}
$$

with $\hat{a}_{i} \in\left\{\mathbb{1}, \hat{\sigma}_{x}, \hat{\sigma}_{y}, \hat{\sigma}_{z}\right\}$ can be evaluated as single-qubit measurements in the computational basis if we transform the state, that is, apply some gates before the measurement. For this purpose the following fundamental identities are necessary

$$
\begin{aligned}
\sigma_{x} & =\frac{1}{2}\left(\begin{array}{cc}
1 & 1 \\
1 & -1
\end{array}\right) \sigma_{z}\left(\begin{array}{cc}
1 & 1 \\
1 & -1
\end{array}\right)=H \sigma_{z} H \\
\sigma_{y} & =\frac{1}{2}\left(\begin{array}{ll}
1 & 0 \\
0 & i
\end{array}\right)\left(\begin{array}{cc}
1 & 1 \\
1 & -1
\end{array}\right) \sigma_{z}\left(\begin{array}{cc}
1 & 1 \\
1 & -1
\end{array}\right)\left(\begin{array}{cc}
1 & 0 \\
0 & -i
\end{array}\right)=S H \sigma_{z} H S^{\dagger} .
\end{aligned}
$$


$H$ is the Hadamard gate and $S^{\dagger}$ and $S$ are the phase gates listed in table 9.1. Consequently, we can measure for example the two-qubit measurement $\hat{\sigma}_{x} \otimes \hat{\sigma}_{y}$ by first applying $H \otimes H S^{\dagger}$ to the state and then measuring $\hat{\sigma}_{z} \otimes \hat{\sigma}_{z}$. Thus, we can transform observables $\otimes_{i=1}^{n} \hat{a}_{i}$ with $\hat{a}_{i} \in\left\{\mathbb{1}, \hat{\sigma}_{x}, \hat{\sigma}_{y}, \hat{\sigma}_{z}\right\}$ to the form $\otimes_{i=1}^{n} \hat{\sigma}_{z}^{s_{i}}$ with $s_{i} \in\{0,1\}$. However, even $\hat{\sigma}_{z} \otimes \hat{\sigma}_{z}$ cannot be measured directly with an universal quantum computer and needs to be reduced further. Here, we have the two-qubit identity

$$
\begin{aligned}
\hat{\sigma}_{z} \otimes \hat{\sigma}_{z} & =\left(\begin{array}{cccc}
1 & 0 & 0 & 0 \\
0 & -1 & 0 & 0 \\
0 & 0 & -1 & 0 \\
0 & 0 & 0 & 1
\end{array}\right)=\left(\begin{array}{cccc}
1 & 0 & 0 & 0 \\
0 & 0 & 0 & 1 \\
0 & 0 & 1 & 0 \\
0 & 1 & 0 & 0
\end{array}\right)\left(\hat{\sigma}_{z} \otimes \mathbb{1}\right)\left(\begin{array}{cccc}
1 & 0 & 0 & 0 \\
0 & 0 & 0 & 1 \\
0 & 0 & 1 & 0 \\
0 & 1 & 0 & 0
\end{array}\right) \\
& =C N O T_{c=2, t=1}\left(\hat{\sigma}_{z} \otimes \mathbb{1}\right) C N O T_{c=2, t=1},
\end{aligned}
$$

where $C N O T_{c=2, t=1}$ is the CNOT gate with the second qubit as control qubit and first qubit as target qubit. For a two-qubit observable Eq. (9.259) would be sufficient to transform it to a measurable observable. However, for more-than-two-qubit observables we have to apply Eq. (9.259) repeatedly. For $\hat{\sigma}_{x} \otimes \hat{\sigma}_{z} \otimes \hat{\sigma}_{y} \otimes\left(\otimes_{i=1}^{n-3} \mathbb{1}\right)$, which is the first term of Eq. (9.254), we get the transformation

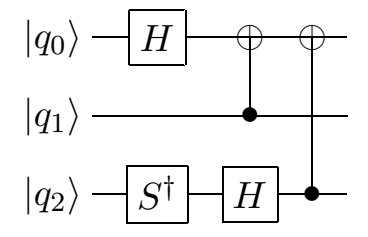

of the state in terms of quantum gates. After these gates have been applied to the state, $\hat{\sigma}_{x} \otimes \hat{\sigma}_{z} \otimes \hat{\sigma}_{y} \otimes\left(\otimes_{i=1}^{n-3} \mathbb{1}\right)$ can be measured by measuring the single-qubit observable $\hat{\sigma}_{z} \otimes\left(\otimes_{i=1}^{n-1} \mathbb{1}\right)$. In this way we can reduce $n$-qubit observables to single-qubit observables with the use of at most two single-qubit gates per qubit $\left(H\right.$ and $\left.S^{\dagger}\right)$ and $n-1$ CNOTgates. These additional gates can be arranged as a quantum circuit with a depth of at most $2+\left\lceil\log _{2}(n)\right\rceil$. In conclusion, we have defined a way to store fermionic wave function on a universal quantum computer. We can evaluate one-particle and two-particle reduced density matrices from single-qubit measurements after the state has been transformed with a small number of quantum gates.

\subsubsection{Ab-initio quantum chemistry on quantum computers}

There are two main approaches to ab-initio quantum chemistry on universal gate-based quantum computers: the quantum phase-estimation algorithm [Lloyd, 1996; AspuruGuzik et al., 2005] and the variational quantum eigensolver [Peruzzo et al., 2014]. In order to be able to evaluate which approaches of both can be adapted to estimate the density-matrix functional on a quantum computer, we discuss the core ideas of the two approaches here.

\section{Quantum phase-estimation algorithm}

The quantum phase-estimation algorithm [Lloyd, 1996; Aspuru-Guzik et al., 2005] estimates the eigenvalue $e^{2 \pi i \theta}$ of an eigenstate $|\Psi\rangle$ of a unitary operator $\hat{U}$, i.e.

$$
\hat{U}|\Psi\rangle=e^{2 \pi i \theta}|\Psi\rangle
$$




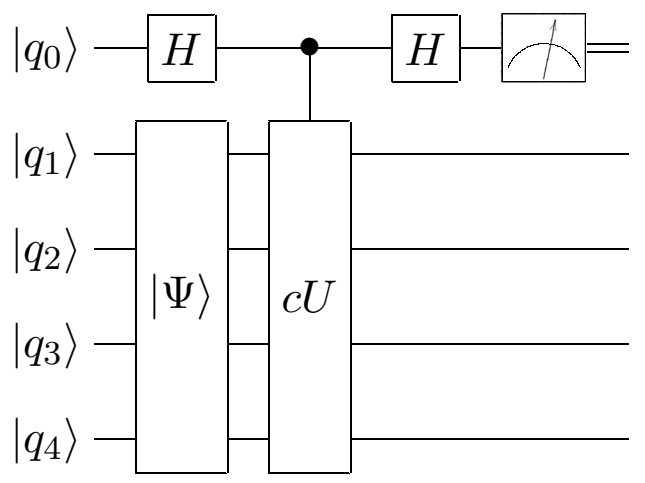

Figure 9.22.: Quantum phase-estimation circuit for an eigenstate $|\Psi\rangle$ of a unitary operator $\hat{U}$. The $|\Psi\rangle$-gate generates the state $|\Psi\rangle$ in the lower four qubits from the initial state $|0\rangle \otimes \ldots \otimes|0\rangle$ of the qubits. The controlled-U gate $c U$ is controlled by the first qubit and applies the unitary operator $\hat{U}$ on the state $|\Psi\rangle$.

The required number of qubits depends on the requested precision of the eigenvalue estimate. The functionality of the phase-estimation circuit can be understood with the help of the simple phase-estimation circuit shown in figure 9.22 . The qubits $q_{1}, \ldots, q_{4}$ encode an eigenstate of the unitary operator $\hat{U}, \hat{U}|\Psi\rangle=e^{2 \pi i \phi}|\Psi\rangle$ for which the phase $\phi$ is to be measured. The state of the first qubit $q_{0}$ is $(|0\rangle+|1\rangle) / \sqrt{2}$ after the application of the Hadamard gate. Thus, the state of the system before the application of the controlled$\hat{U}$-gate is

$$
\frac{1}{\sqrt{2}}(|0\rangle+|1\rangle) \otimes|\Psi\rangle
$$

The controlled-U gate transforms this state to

$$
c U \frac{1}{\sqrt{2}}(|0\rangle+|1\rangle) \otimes|\Psi\rangle=\frac{1}{\sqrt{2}}\left(|0\rangle \otimes|\Psi\rangle+e^{2 \pi i \phi}|1\rangle \otimes|\Psi\rangle\right) .
$$

The subsequent Hadamard-gate on the control qubit gives the final state of the quantum circuit as

$$
\frac{1}{2}\left(\left(1+e^{2 \pi i \phi}\right)|0\rangle+\left(1-e^{2 \pi i \phi}\right)|1\rangle\right) \otimes|\Psi\rangle .
$$

Hence the probability for the measurement of 0 at the first qubit is $(1+\cos (2 \pi \phi)) / 2$ and the phase can be estimated from repeated executions of this quantum circuit. However, a large number of executions and measurements are required to estimate the phase to high precision. Thus, instead of applying the unitary operator $\hat{U}$ to the state $|\Psi\rangle$ only once as in figure 9.22 , the quantum phase-estimation applies the repeated squares $\hat{U}, \hat{U}^{2}, \hat{U}^{4}, \ldots, \hat{U}^{2^{n-1}}$ to the eigenstate to estimate the phase with $n$-bit precision. Instead of one control qubit now $n$ control qubits are used so that the state of the system after the Hadamard transforms on the control qubits and the generation of the eigenstate $|\Psi\rangle$ is

$$
\left(\frac{1}{\sqrt{2}}\right)^{n}\left[\otimes_{i=1}^{n}(|0\rangle+|1\rangle)\right] \otimes|\Psi\rangle .
$$


The applications of the unitary operator $\hat{U}^{2^{j}}$ with $j=0, \ldots, n-1$ controlled by the $n-j$-th qubit results in the state

$$
\left(\frac{1}{\sqrt{2}}\right)^{n}\left[\left(|0\rangle+e^{2^{n-1} 2 \pi i \phi}|1\rangle\right) \otimes\left(|0\rangle+e^{2^{n-2} 2 \pi i \phi}|1\rangle\right) \otimes \ldots \otimes\left(|0\rangle+e^{2^{0} 2 \pi i \phi}|1\rangle\right)\right] \otimes|\Psi\rangle .
$$

Thus, the $n$-bit quantum phase-estimation algorithm can be seen as simultaneous estimation of the phases $\phi, 2 \phi, 4 \phi, \ldots, 2^{n-1} \phi$ with the simple quantum phase estimation shown in figure 9.22. An $n$-bit integer number $k$ can be represented with $n$-qubits as a quantum state $|k\rangle$ of the form

$$
|k\rangle=\otimes_{i=1}^{n-1} 2^{i}\left(\left(1-b_{i}(k)\right)|0\rangle+b_{i}(k)|1\rangle\right),
$$

where $b_{i}(k)$ are the bits of the binary representation of the number $k$. Therefore, the state in Eq. (9.266) can be written in the short form

$$
\left(\frac{1}{\sqrt{2}}\right)^{n}\left(\sum_{k=0}^{2^{n}-1} 2^{2 \pi i \phi k}|k\rangle\right) \otimes|\Psi\rangle
$$

The quantum Fourier transformation transforms the $n$-qubit state $|j\rangle$ to [Nielsen and Chuang, 2010]

$$
\operatorname{QFT}_{1, \ldots, n}|j\rangle=\left(\frac{1}{\sqrt{2}}\right)^{n} \sum_{l=0}^{2^{n}-1} e^{2 \pi i j l 2^{-n}}|l\rangle .
$$

The inverse of the quantum Fourier transform applied to the first $n$ qubits transforms the state in Eq. (9.268) as

$$
Q F T_{1, \ldots, n}^{-1}\left(\frac{1}{\sqrt{2}}\right)^{n}\left(\sum_{k=0}^{2^{n}-1} 2^{2 \pi i \phi k}|k\rangle\right) \otimes|\Psi\rangle=\frac{1}{2^{n}}\left(\sum_{l=0}^{2^{n}-1} \sum_{k=0}^{2^{n}-1} e^{-\frac{2 \pi i k}{2^{n}}\left(l-2^{n} \phi\right)}|l\rangle\right) \otimes|\Psi\rangle .
$$

For simplicity, we assume here that $2^{n} \phi$ is integer. Then the measurement of the first $n$ qubits yields the number $x$ with probability

$$
\begin{aligned}
p(x) & =\left|\langle x| \frac{1}{2^{n}}\left(\sum_{l=0}^{2^{n}-1} \sum_{k=0}^{2^{n}-1} e^{-\frac{2 \pi i k}{2^{n}}\left(l-2^{n} \phi\right)}|l\rangle\right)\right|^{2} \\
& =\left|\frac{1}{2^{n}}\left(\sum_{k=0}^{2^{n}-1} e^{-\frac{2 \pi i k}{2^{n}}\left(x-2^{n} \phi\right)}\right)\right|^{2}=\delta_{x, 2^{n} \phi .}
\end{aligned}
$$

That means that the number $x=2^{n} \phi$ is measured with probability 1 . If $2^{n} \phi$ is not integer the best $n$-bit estimate of $2^{n} \phi$ is measured with probability $4 / \pi^{2}$ [Cleve et al., 1998]. An example of a 4-bit quantum phase-estimation algorithm is shown in figure 9.23

Shor's algorithm for the factorization of a large number $N$ can be seen as the application of the quantum phase-estimation algorithm to determine the eigenvalue of the modular multiplication $\hat{U}_{a}|x\rangle=\left|a^{x} \bmod N\right\rangle$ in the form

$$
\hat{U}_{a}\left|\Psi_{j}\right\rangle=e^{\frac{2 \pi i j}{r}}\left|\Psi_{j}\right\rangle
$$




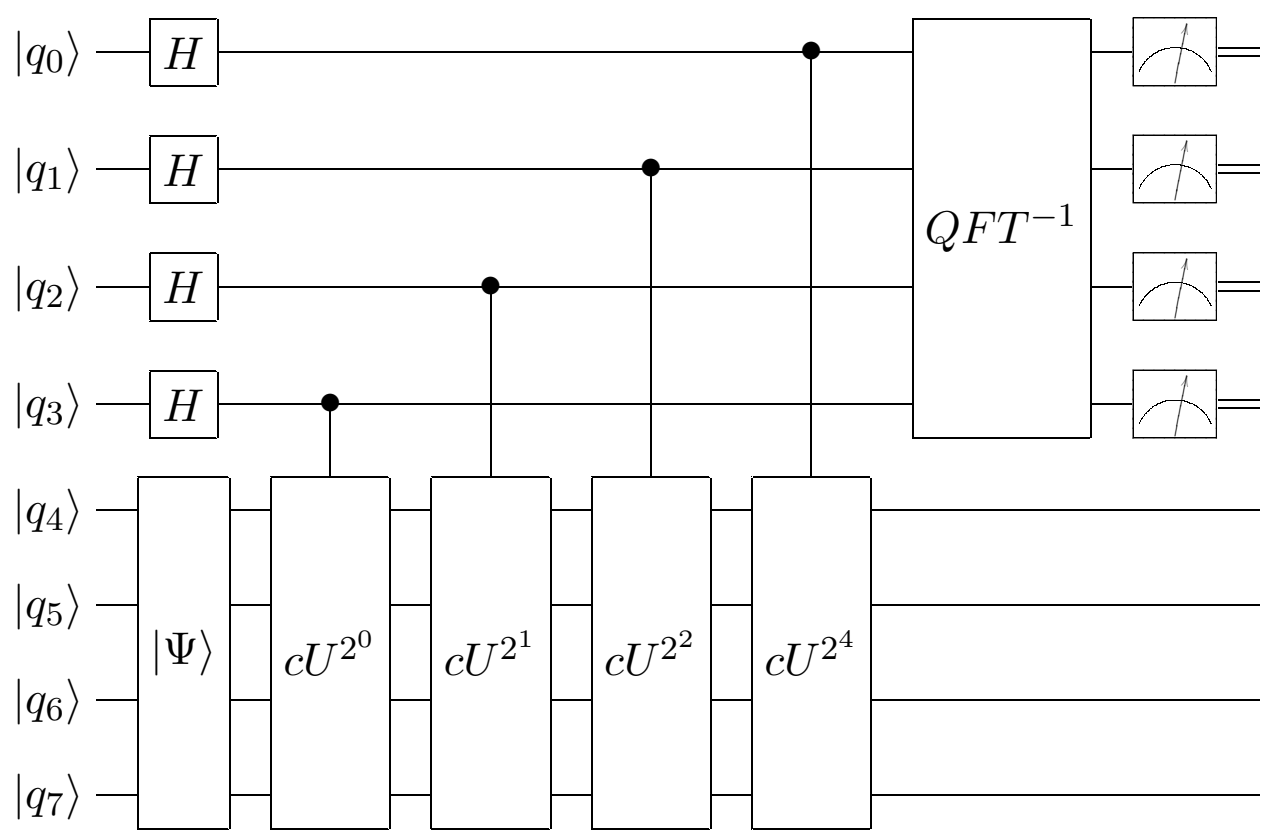

Figure 9.23.: 4-bit quantum phase estimation circuit for an eigenstate $|\Psi\rangle$ of a unitary operator $\hat{U}$. The first 4 qubits make up the control register. The last four qubits store the state $|\Psi\rangle$. The qubits in the control register control the application of repeated squares of the unitary operator $\hat{U}$ on the state $|\Psi\rangle$ for which the phase $\phi$ in $\hat{U}|\Psi\rangle=e^{2 \pi i \phi}|\Psi\rangle$ is to be estimated. The result of the inverse quantum Fourier transform $Q F T^{-1}$ is measured.

where $\left|\Psi_{j}\right\rangle$ is an eigenstate of $\hat{U}_{a}$ and $r$ is the desired period of $N$ with respect to $a$. It should be noted here, that Shor's algorithm also generates the eigenstate $\left|\Psi_{j}\right\rangle$ starting from the simple state $|100 \ldots\rangle$. In this way it goes beyond a simple application of the quantum phase-estimation algorithm.

For the application of the quantum phase-estimation algorithm in quantum chemistry, the unitary operator is chosen as $\hat{U}=e^{i \hat{H} \tau / \hbar}$ such that the energy eigenvalue $E$ of an eigenstate $|\Psi\rangle$ of the Hamiltonian is related to the phase by $E=2 \pi \hbar \phi / \tau$. On the other hand, the eigenstate $|\Psi\rangle$ or a state that has a high overlap with the desired eigenstate must be prepared somehow. It is not necessary that the prepared $|\Phi\rangle$ is an exact eigenstate because

$$
\hat{U}|\Phi\rangle=\sum_{n} e^{-i E_{n} \tau / \hbar}\left|\Psi_{n}\right\rangle\left\langle\Psi_{n} \mid \Phi\right\rangle
$$

where $\left|\Psi_{n}\right\rangle$ are eigenstates of the Hamiltonian $\hat{H}$. Consequently, the measurement process at the end of the quantum phase-estimation algorithm selects the state $\left|\Psi_{n}\right\rangle$ with probability

$$
p(n)=\left|\left\langle\Psi_{n} \mid \Phi\right\rangle\right|^{2}
$$

and returns the binary representation of the phase $\phi$ that corresponds to the energy eigenvalue $E_{n}$. Several investigations have shown that the Hartree-Fock ground state as the prepared state $|\Phi\rangle$ has sufficient overlap with the ground state so that the quantum phaseestimation algorithm can be used to determine the eigenvalue of the exact ground state 
with high probability [Lanyon et al., 2010; O'Malley et al., 2016]. The major downside of the quantum phase estimation is the execution of repeated squares $\hat{U}, \hat{U}^{2}, \hat{U}^{4}, \ldots$ which require the execution of many quantum gates and consequently long coherence times. Thus, this algorithm is not practically usable on current or near-term quantum computers.

\section{Variational quantum eigensolver}

The variational quantum eigensolver (VQE, [Peruzzo et al., 2014; McClean et al., 2016]) proceeds along the line of variational approaches on classical computers: the normalized many-particle wave function is parametrized with a small set of variational parameters $\vec{u}$ and the expectation value of the Hamiltonian $\hat{H}$ is minimized with respect to these variational parameters, i.e.,

$$
E_{0, \approx}=\min _{\vec{u}}\langle\Psi(\vec{u})|\hat{H}| \Psi(\vec{u})\rangle \geq E_{0, \text { exact }} .
$$

For some parametrization of the variational wave function then the expectation value of the Hamiltonian is measured by decomposing it into separate terms. As shown in section 9.8.5, all expectation values of one- and two-particle reduced density matrices can be transformed to single-qubit measurements in the computational basis. Thus, once the many-particle wave function $|\Psi(\vec{u})\rangle$ has been prepared on the quantum computer the corresponding energy can be measured after the application of a small number of gates. The number of necessary measurements depends on the required precision and the number of terms in the many-particle Hamiltonian.

Peruzzo et al. [Peruzzo et al., 2014] proposed to use the unitary coupled-cluster ansatz [Taube and Bartlett, 2006]

$$
|\Psi(\vec{u})\rangle=\left|\Psi_{\text {unitary CC }}\right\rangle=e^{\hat{T}_{n}-\hat{T}_{n}^{\dagger}}\left|\Psi_{0}\right\rangle
$$

with the $n$-fold cluster operator $\hat{T}_{n}$ from Eq. (9.161) and the reference Slater determinant $\left|\Psi_{0}\right\rangle$. However, even in low excitation levels $n$ this leads to a large number of terms and, thus, a large number of quantum gates for the preparation of the state. Kandala et al. [Kandala et al., 2017] have recently proposed to use a parametrisation of the form

$$
|\Psi(\vec{u})\rangle=\prod_{i=1}^{d}\left[\left(\prod_{q=1}^{N} U^{q, d-i+1}(\vec{u})\right) U_{\text {entangler }}\right] \prod_{q=1}^{N} U^{q, 0}(\vec{u})|00 \ldots\rangle
$$

for the wave function of the qubits for a $N$-qubit system. They named these states hardware-efficient trial states because they are directly written in terms of quantum gates that are natively supported on a quantum computer. Thus, they avoid the overhead introduced by Jordan-Wigner transformation or similar transformation from the fermionic wave function to a bosonic wave function by directly specifying the bosonic wave function. The single-qubit rotation for the $q$-th qubit $U^{q, i}(\vec{u})$ are composed of rotations around the $\mathrm{x}$ - and z-axis

$$
U^{q, i}(\vec{u})=R_{z}\left(u_{1}^{q, i}\right) R_{x}\left(u_{2}^{q, i}\right) R_{z}\left(u_{3}^{q, i}\right) .
$$

The rotation angles are the variational parameters. The entanglers $U_{\text {entangler }}$ are composed of a sequence of two-qubit gates that create entanglement between the qubits. Thus, the 


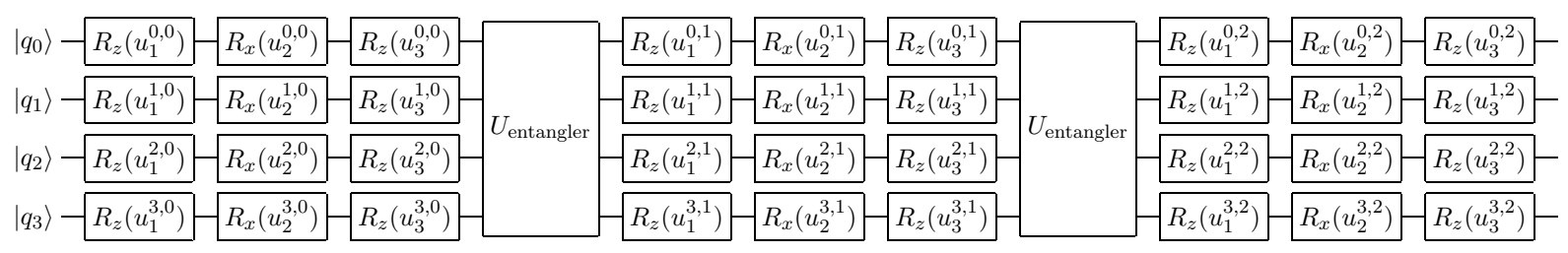

Figure 9.24.: Quantum circuit for the preparation of the hardware-efficient trail state given in Eq. 9.278 with two blocks $(d=2)$ on four qubits.

hardware-efficient trial state in Eq. (9.278) consists of $d$ blocks of entanglers and singlequbit rotations and the circuit to prepare this state is shown in Figure 9.24 for four qubits and two blocks $(d=2)$. For $N$ qubits, the parametrization of the wave function with $d$ blocks in Eq. 9.278 has $N \cdot(3 d+2)$ independent variational parameters. The initial rotations around the z-axis $R_{z}\left(u_{1}^{q, 0}\right)$ can be left away because they act on the initial state $|00 \ldots\rangle$ and only produce an overall phase for the wave function. The total circuit depth $D(d, N)$ for the preparation of the trial state in terms of general single- and two-qubit gates (see section 9.8.2) is

$$
D(d, N)=\underbrace{1+d}_{\text {single-qubit gates }}+\underbrace{d \cdot n_{\text {entangler }}}_{\text {two-qubit gates }},
$$

where $n_{\text {entangler }}$ is the number of general two-qubit gates that correspond to the $U_{\text {entangler }}{ }^{-}$ circuit. The two-qubit gates in the entangler $U_{\text {entangler }}$ must connect all qubits such that the system does not trivially decompose into two independent subsystems. Therefore, the entangler must consist of at least $N-1$ two-qubit gates. Consequently, the total depth of the circuit is linear in the number $N$ of qubits and linear in the number of blocks $d$, i.e., $D(d, N) \in \mathcal{O}(d \cdot N)$. Recent investigations [Barkoutsos et al., 2018] have shown that with the structure of the hardware-efficient trial states in Eq. 9.278 ground state energies of simple molecules can be obtained with circuit depths that are much lower than in the unitary coupled cluster ansatz given in Eq. (9.277) with single and double excitations (UCCSD). This suggests the use of the hardware-efficient trial states on current or near-term quantum computers instead of the conventional coupled-cluster-ansatz wave function.

The minimization of the expectation value of the Hamiltonian with respect to the variational parameters requires a derivative-free algorithm that can tolerate noise. Thus, the requirements are identical to the case when the expectation value is determined from a Monte Carlo procedure on a classical computer discussed in section 9.7.5. Consequently, the simultaneous perturbation stochastic approximation introduced in section 9.7.5 can also be used for the variational quantum eigensolver.

\subsubsection{RDMF from a VQE-like algorithm}

We propose the evaluation of the density-matrix functional from a procedure based on the idea of the variational quantum eigensolver. As discussed in section 9.1, two approaches for the density-matrix functional are possible: the Legendre-Fenchel-transformation of the grand potential or Levy's constrained minimization over many-particle wave functions. For the reasons explained in section 9.1 we expect Levy's constrained minimization ansatz to result in a much more efficient approach than the Legendre-Fenchel-transformation. 
Thus, we use the augmented Lagrangian to form a sequence of unconstrained problems from the given constrained optimization problem. The unconstrained minimization problems are solved with the SPSA. We have implemented the construction of hardwareefficient trial states and measurement circuits for all elements of the one-particle and two-particle reduced density matrix in terms of quantum circuits.

In the following, we estimate the overall computational complexity of the proposed algorithm. For this purpose, we assume that the Jordan-Wigner transformation ${ }^{18}$ is employed for the mapping of the fermionic wave function. The construction of the quantum program to prepare a hardware efficient trial state of Eq. 9.278 has a computational complexity that is polynomial in the number $N$ of qubits. As discussed in section 9.8.6 the number of gates and circuit depth of the state preparation circuit is linear in the number of blocks. It is currently unknown how the number of required blocks scales with the number of qubits for typical physical many-particle wave functions.

For the measurement of the full one-particle reduced density matrix we need to evaluate $2 N^{2}-N$ expectation values of single- and multi-qubit observables of the form $\otimes_{i=1}^{n} \hat{a}_{i}$ with $\hat{a}_{i} \in\left\{\mathbb{1}, \hat{\sigma}_{x}, \hat{\sigma}_{y}, \hat{\sigma}_{z}\right\}$. The diagonal elements of the one-particle reduced density matrix require $N$ single-qubit measurements of the form $\left\langle\ldots \otimes \mathbb{1} \otimes \sigma_{z} \otimes \mathbb{1} \otimes \ldots\right\rangle$ and can be performed with a single quantum program. Elements of the one-particle reduced density matrix $\rho_{i, j}^{(1)}$ with $|i-j|=1$ require $4(N-1)$ two-qubit measurements that can be evaluated with $\min (8,4(N-1))$ quantum programs. In general, elements of the one-particle reduced density matrix $\rho_{i, j}^{(1)}$ with $|i-j|=n-1$ require $4(N-n+1) n$-qubit measurements that can be evaluated with $\min (4 n, 4(N-n+1)$ quantum programs. Thus, for an $N$-qubit system we have to evaluate

$$
1+2(\lceil N / 2\rceil-1)(\lceil N / 2\rceil+2)+2\lfloor N / 2\rfloor(\lfloor N / 2\rfloor+1) \approx N^{2}+2 N-3
$$

quantum programs to get all elements of the one-particle reduced density matrix. The difference of the number of quantum programs required given in Eq. 9.281 to the theoretical number $N^{2}$ of real parameters of the one-particle reduced density matrix represents the overhead introduced by the Jordan-Wigner transformation. Because the overhead is only linear in system size and because the Jordan-Wigner transformation is not suitable for large systems sizes, we do not analyse this difference any further here. A circuit for the measurement of an $n$-qubit observable requires at most $2 n$ single-qubit gates and $n-1$ CNOT-gates to reduce the $n$-qubit operator to a single-qubit operator. It has a depth of at most $2+\left\lceil\log _{2}(n)\right\rceil$.

The number of quantum programs for the expectation value of the interaction Hamiltonian depends strongly on sparsity of the matrix elements $U_{\alpha, \beta, \gamma, \delta}$. For a Hubbard interaction of the form

$$
\hat{W}_{\text {Hubbard }}=\sum_{i} U_{i} \hat{n}_{i, \uparrow} \hat{n}_{i, \downarrow}
$$

the two-qubit expectation values $\left\langle\sigma_{z} \otimes \sigma_{z} \otimes \mathbb{1} \otimes \ldots\right\rangle,\left\langle\mathbb{1} \otimes \mathbb{1} \otimes \sigma_{z} \otimes \sigma_{z} \otimes \mathbb{1} \otimes \ldots\right\rangle, \ldots$ are required additionally to the single-qubit measurements $\left\langle\ldots \otimes \mathbb{1} \otimes \sigma_{z} \otimes \mathbb{1} \otimes \ldots\right\rangle$, that are already known from the evaluation of the diagonal elements of the one-particle reduced density matrix. All of these two-qubit expectation values can be measured with a single quantum program. Thus, for a system with a Hubbard interaction it is required to execute

\footnotetext{
${ }^{18}$ For large systems the Bravyi-Kitaev transformation [Bravyi and Kitaev, 2002] is preferable.
} 
approximately $N^{2}+2 N-2$ quantum programs to obtain all elements of the one-particle reduced density matrix and the expectation value of the interaction.

The number of iterations for the augmented Lagrangian is typically below 100, and we have not observed a dependence on the system size. The dependence of the number of SPSA-iterations for the unconstrained subproblems on the number of variational parameters is hard to estimate. However, a polynomial dependence is a reasonable assumption. Thus, the overall computational complexity of the approach to estimate the density-matrix functional with hardware-efficient trial states on a quantum computer is polynomial in system size if the many-particle state can be described with hardware-efficient trail state with a polynomial number of blocks.

As a proof of concept we try to evaluate the zero-temperature density-matrix functional for the ground state of a half-filled Hubbard dimer with interaction strength $U=2 t$. Thus, the one-particle reduced density matrix in the one-particle basis $\left\{\left|\chi_{1, \downarrow}\right\rangle,\left|\chi_{1, \uparrow}\right\rangle,\left|\chi_{2, \downarrow}\right\rangle,\left|\chi_{2, \uparrow}\right\rangle\right\}$ is

$$
\rho_{\text {exact }}^{(0)}=\left(\begin{array}{cccc}
\frac{1}{2} & 0 & \sqrt{\frac{2}{10}} & 0 \\
0 & \frac{1}{2} & 0 & \sqrt{\frac{2}{10}} \\
\sqrt{\frac{2}{10}} & 0 & \frac{1}{2} & 0 \\
0 & \sqrt{\frac{2}{10}} & 0 & \frac{1}{2}
\end{array}\right)
$$

and the two-particle interaction is

$$
\hat{W}=U \sum_{i=1}^{2} \hat{n}_{i, \uparrow} \hat{n}_{i, \downarrow} .
$$

The density-matrix functional, that we try to evaluate on the quantum computer, is therefore

$$
F_{\mathrm{qc}}^{\hat{W}}\left[\rho_{\text {exact }}^{(0)}\right]=\min _{\vec{u}:\left\langle\Psi(\vec{u})\left|\hat{c}_{\alpha}^{\dagger} \hat{c}_{\beta}\right| \Psi(\vec{u})\right\rangle=\rho_{\text {exact }, \beta, \alpha}^{(0)}}\langle\Psi(\vec{u})|\hat{W}| \Psi(\vec{u})\rangle .
$$

The exact result is

$$
\begin{aligned}
F^{\hat{W}}\left[\rho_{\text {exact }}^{(0)}\right] & =\min _{|\Psi\rangle:\langle\Psi \mid \Psi\rangle=1,\left\langle\Psi\left|\hat{c}_{\alpha}^{\dagger} \hat{c}_{\beta}\right| \Psi\right\rangle=\rho_{\text {exact }, \beta, \alpha}^{(0)}}\langle\Psi|\hat{W}| \Psi\rangle \\
& =2 t \cos ^{2}\left(\arctan \frac{\sqrt{5}+1}{2}\right) \approx 0.5528 t .
\end{aligned}
$$

We adjust the parametrization of the hardware-efficient trial states introduced in the previous section to the physical characteristics of the IBM Q5 Tenerife 5-qubit quantum computer. We use the first three qubits $Q_{0}, Q_{1}, Q_{2}$ and the fifth qubit $Q_{4}$ as qubits $q_{0}, q_{1}, q_{2}$ and $q_{3}$ for our calculation. We skipped the fourth qubit because it showed two-qubit error rates of above $10 \%$ whereas the other qubits had two-qubit error rates of below 5\%. The composition of the entanglers is limited by the physical layout of the quantum computer shown in figure 9.20. We chose to use the entangler shown in figure 9.25. This entangler consists of three CNOT gates and, thus, has the minimal possible number. To reduce the number of necessary resonator pulses required to execute the quantum program and with that the execution time, we replace the general rotations 


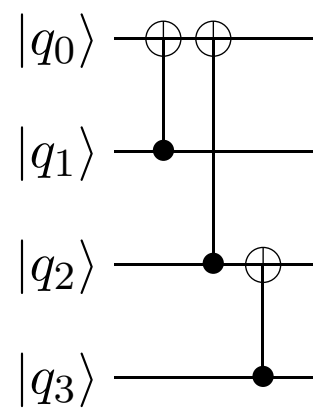

Figure 9.25.: Entangler in terms of CNOT gates used for the evaluation of the densitymatrix functional on the quantum computer IBM Q5 Tenerife. Qubit $q_{3}$ corresponds to qubit $Q_{4}$ in Figure 9.21.

$U^{q, i}\left(u_{1}^{q, i}, u_{2}^{q, i}, u_{3}^{q, i}\right)=R_{z}\left(u_{1}^{q, i}\right) R_{x}\left(u_{2}^{q, i}\right) R_{z}\left(u_{3}^{q, i}\right)$ in the originally proposed hardware-efficient trial states by the rotation

$$
\tilde{U}^{q, i}\left(u_{1}^{q, i}\right)=\frac{1}{\sqrt{2}}\left(\begin{array}{cc}
e^{-i u_{1}^{q, i} / 2} & -e^{-i u_{1}^{q, i} / 2} \\
e^{i u_{1}^{q, i} / 2} & e^{i u_{1}^{q, i} / 2}
\end{array}\right) \stackrel{E q .}{=}{ }^{9.245} u_{2}\left(u_{1}^{q, i}, 0\right)=u_{3}\left(\pi / 2, u_{1}^{q, i}, 0\right) .
$$

The general rotation $U^{q, i}\left(u_{1}^{q, i}, u_{2}^{q, i}, u_{3}^{q, i}\right)$ needs two pulses to execute whereas the rotation $\tilde{U}^{q, i}\left(u_{1}^{q, i}\right)$ only needs one pulse to execute. On the IBM quantum computers, there is no access to lower level two-qubit gates so that in contrast to Kandala et al. [Kandala et al., 2017] we have to use CNOT gates in the entangler and cannot reduce the number of pulses. Figure 9.26 shows the full quantum circuit with a depth of $d=3$ for the preparation of the trial state. The trial state prepared by this circuit has 16 variational parameters and can be executed with

- 10 resonator pulses to the first qubit,

- 10 resonator pulses to the second qubit,

- 13 resonator pulses to the third qubit,

- 10 resonator pulses to the fourth qubit,

- 12 pulses to the bus connecting the first three qubits and

- 6 pulses to the bus connecting the third and fourth qubit.

The preparation circuit has a depth of 40 pulses, which corresponds to an execution time of about $4 \mu \mathrm{s}$. Thus, the required execution time is well below the decoherence time of the device. In total, we need to run 22 quantum programs to estimate the expectation value of the interaction Hamiltonian and all matrix elements of the one-particle reduced density matrix. These quantum programs do not only have to be executed once but many times to obtain the expectation value from many measurements.

The available execution time on the IBM quantum computer is shared among many users worldwide. Thus, we cannot run the whole minimization loop of the augmented Lagrangian, which requires many evaluations of the expectation values. Therefore, we have obtained the solution of the constrained optimization problem by simulating the 


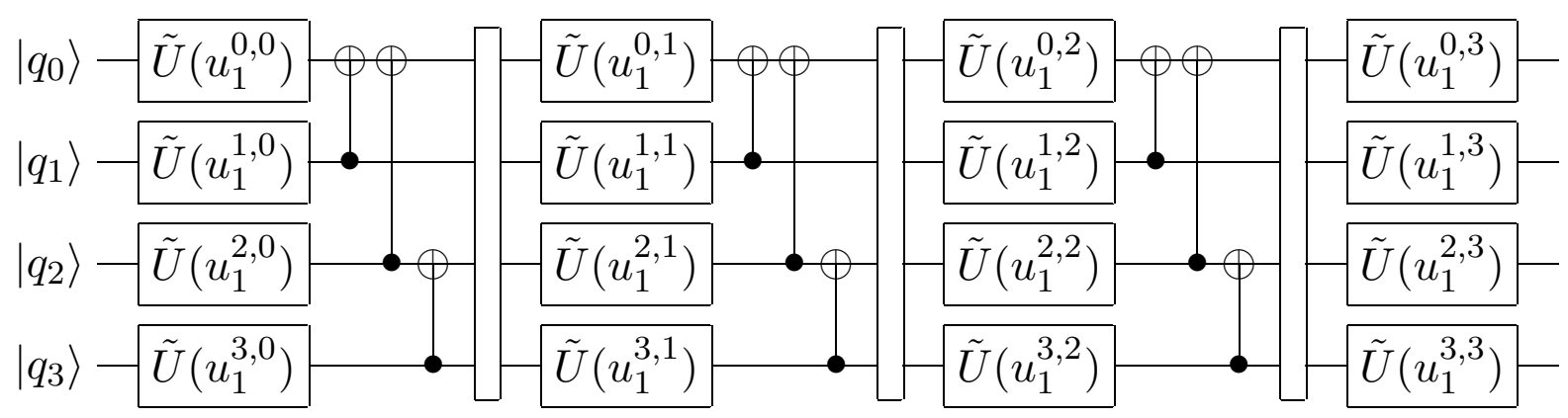

Figure 9.26.: Quantum circuit for the preparation of a 4-qubit trial state on the IBM Q5 Tenerife quantum computer. The empty vertical blocks indicate the centers of the three blocks. Qubit $q_{3}$ corresponds to qubit $Q_{4}$ in Figure 9.21.

execution of the quantum program on a classical computer. The resulting values of the optimal variational parameters are provided together with the QASM-code of the quantum circuit for the preparation of the trail state in Appendix A.1. We then verify the solution by estimating the expectation values for the optimal variational parameters once with the quantum computer. We assume that if one estimation of the expectation values gives results in agreement with the simulation on a classical computer, this is also true for all steps during the minimization. Thus, with this assumption, the minimization that uses the quantum computer for all evaluations of the expectation values should converge to the same result as the minimization that uses a classical simulation of the circuits.

The results for the individual multi-qubit measurements and the corresponding measurement circuits are listed in appendix A together with the exact results from the numerically exact simulation of the circuits on a classical computer and results from a Monte Carlo simulation of the circuits performed in IBM Q experience ${ }^{19}$. The average deviation between the expectation values from the Monte Carlo simulation of the circuits with $N_{\mathrm{MC}}$ shots and the exact expectation values is $\sigma_{\mathrm{avg}, \mathrm{MC}}=0.009$. Thus, the average deviation is similar to the expected deviation of

$$
\sigma_{\mathrm{avg}, \mathrm{MC}, \text { expect }}=\frac{1}{\sqrt{N_{\mathrm{MC}}}} \stackrel{N_{\mathrm{MC}}=8192}{\approx} 0.011
$$

However, this is not the case for the expectation values from the quantum computer, which have been calculated from $N_{\mathrm{qc}}=8192$ executions of the quantum programs. The average deviation of the expectation values from the exact results is

$$
\sigma_{\mathrm{avg}, \mathrm{qc}}=0.16 \gg \frac{1}{\sqrt{N_{\mathrm{qc}}}} \approx 0.011 .
$$

The one-particle reduced density matrix and the expectation value of the interaction can be reconstructed from the measurements with the help of decompositions like Eq. 9.252. From the Monte Carlo simulation of the quantum programs in appendix A.2 with IBM

${ }^{19}$ IBM Q experience (https://quantumexperience.ng.bluemix.net) is IBM's online platform for the access to the publicly available quantum computers. It also contains a backend for a Monte Carlo simulation of circuits that takes the same input and gives results in the same format as the quantum computer. This backend can be used to verify the input and interpretation of results. 
QISKit we get the one-particle reduced density matrix

$$
\rho_{\mathrm{MC}}^{(1)}=\left(\begin{array}{cccc}
0.498 & -0.004-0.003 i & 0.449+0.001 i & 0+0.007 i \\
-0.004+0.003 i & 0.493 & 0.001-0.005 i & 0.447-0.010 i \\
0.449-0.001 i & 0.001+0.005 i & 0.504 & -0.001+0.007 i \\
0-0.007 i & 0.447+0.010 i & -0.001-0.007 i & 0.501
\end{array}\right)
$$

and the value of the density matrix functional $\langle\hat{W}\rangle_{\mathrm{MC}}=0.549$. As expected, these values agree with the exact expectation values given in Eq. (9.283) and Eq. (9.287) within the average deviation of $1 / \sqrt{N_{\mathrm{qc}}} \approx 0.011$.

The execution of the quantum programs in appendix A on the IBM Q5 Teneriffe quantum computer yields the value of the density-matrix functional $\langle\hat{W}\rangle_{\mathrm{qc}}=0.696 t$, which is much higher than the exact result. We obtained the one-particle reduced density matrix

$$
\rho_{\mathrm{qc}}^{(1)}=\left(\begin{array}{cccc}
0.369 & 0.051+0.008 i & 0.232+0.001 i & 0.099-0.036 i \\
0.051-0.008 i & 0.520 & 0.015-0.008 i & 0.221-0.012 i \\
0.232-0.001 i & 0.015+0.008 i & 0.524 & 0.021-0.008 i \\
0.099+0.036 i & 0.221+0.012 i & 0.021+0.008 i & 0.452
\end{array}\right) .
$$

This density matrix qualitatively corresponds to the exact result but large deviations occur especially for the diagonal density matrix element $\rho_{1,1}^{(1)}$ and the off-diagonal elements $\rho_{1,3}^{(1)}$ and $\rho_{2,4}^{(1)}$. Small elements of the exact density matrix are also small in the density matrix evaluated on the quantum computer. This shows, that the preparation of the state on the quantum computer creates the qualitatively correct state.

Michielsen et al. [Michielsen et al., 2017] have benchmarked the correctness of results of the IBM quantum computer for small quantum programs such as the generator of a singlet state of two qubits. They have found systematic deviations that can not be described by probabilistic error models. Willsch et al. [Willsch et al., 2017] have investigated the results of sequences of CNOT-gates on the IBM quantum computers compared to exact results. They found that the deviation from the exact result in terms of the statistical distance [Willsch et al., 2017], i.e., the probability of measuring a state compared to the exact result is linear in the number of CNOT-gates in the sequence. They have also observed systematic deviations when trying to create a singlet state. Thus, we expect these systematic deviations to be the reason for the large deviations of the off-diagonal elements of the one-particle reduced density matrix. These deviations only occur because we obtained the variational parameters from an exact simulation of the quantum circuits on a classical computer. If all quantum programs during the constrained minimization are executed on the quantum computer, then systematic deviations in the implementation of quantum gates on the quantum computer will be compensated by the variational parameters. Thus, the deviations shown here do not imply that the algorithm would not work correctly on a current quantum computer, because for the actual use of the algorithm all quantum programs are supposed to be executed on a quantum computer. The algorithm presented here does not rely on the precise implementation of gates on a quantum computer but only on a small level of random noise.

In conclusion, we have presented an algorithm for the evaluation of the density-matrix functional on current and near-term quantum computers that scales polynomially in system size. Finally, we would like to note here, that the intended applicability of this 
algorithm is not the evaluation of the density-matrix functional within total energy minimizations where all one-particle states of the system are considered for the density-matrix functional. In these cases, a straightforward application of the variational quantum eigensolver would be more efficient. We see the main applicability of our algorithm for the evaluation of the density-matrix function on a quantum computer in the context of hybrid methods such as DFT+RDMFT, where the density-matrix functional only needs to be evaluated for a rather small one-particle basis. A hybrid approach that treats only a few one-particle states on a quantum computer would also alleviate the need for quantum computers with huge numbers of qubits that is the main drawback of the variational quantum solver. In late 2017 IBM has presented a 50-qubit quantum computer ${ }^{20}$. This number of qubits would already be sufficient for a hybrid algorithm because most interesting correlated orbitals in solids, like 3d-orbitals, including some environment states would easily fit into this quantum computer. Additionally, the augmented Lagrangian or the penalty method used here to enforce the one-particle reduced density matrix could be used within the variational quantum eigensolver to enforce certain constraints on the wave function like particle number or spin states.

\subsection{Comparison of approaches}

In this final section, we compare the number of parameters and the computational complexity of the approaches to evaluate the density-matrix functional presented in this chapter. All ansatzes for the many-particle wave function presented in this chapter are systematically improvable:

- The configuration-interaction ansatz presented in section 9.4 converges to the exact density-matrix functional with the number of Slater determinants $N_{\mathrm{CI}}$.

- The impurity-bath separation ansatz presented in section 9.5 converges to the exact density-matrix functional with the number of Slater determinants $N_{\text {bath-dets }}$ of the bath wave functions.

- The ansatz with matrix product states presented in section 9.6 states converges to the exact density-matrix functional with the maximal bond dimension $D$ of the MPS. For the estimate of the computational complexity, we assume matrix product states with open-boundary conditions and a system-size-independent number of sweeps.

- The Gutzwiller- and Jastrow wave-function ansatz presented in section 9.7 itself does not converge to the exact density-matrix functional [Mazziotti, 2004a]. However, the ansatz can be extended similarly to the excitation hierarchy of the coupled-cluster ansatz to converge to the exact result. This generalized ansatz converges with the number of terms in the correlator $N_{\text {corr }}$.

- The ansatz with hardware-efficient trial states on quantum computers presented in section 9.8 converges to the exact density-matrix functional with the number of entangler-blocks $d$.

\footnotetext{
$\overline{{ }^{20} \text { https://www.ibm.com/blogs/research/2017/11/the-future-is-quantum/ accessed: } 2018-06-07}$
} 
We assume an orthonormal one-particle basis with $N_{\chi}$ one-particle states and that the two-particle interaction is restricted to only $N_{A} \ll N_{\chi}$ one-particle states. We additionally assume $N_{A}^{4} \ll N_{\chi}$ so that the evaluations of expectation values of the interaction Hamiltonian is negligible even in the worst case. The number of parameters for the parametrization of a one many-particle wave function and runtime complexities of one minimization step without derivatives are listed in the following table. The last two rows of the table show the corresponding results for the N-representability ansatz proposed in chapter 10 .

\begin{tabular}{l|c|c} 
ansatz & $\begin{array}{c}\text { number of } \\
\text { parameters }\end{array}$ & $\begin{array}{c}\text { runtime } \\
\text { complexity }\end{array}$ \\
\hline configuration interaction & $N_{\mathrm{CI}}$ & $\mathcal{O}\left(N_{\mathrm{CI}} N_{\chi}^{2}\right)$ \\
\hline $\begin{array}{l}\text { impurity-bath separation } \\
\left(\left|\Psi_{B, j}\right\rangle \text { from Eq. }(9.94)\right)\end{array}$ & $\mathcal{O}\left(2^{2 N_{A}} N_{\chi}^{3}\right)$ & $\mathcal{O}\left(2^{2 N_{A}} N_{\chi}^{5}\right)$ \\
\hline $\begin{array}{l}\text { impurity-bath separation } \\
\left(\left|\Psi_{B, j}\right\rangle \text { as CI-expansion) }\right.\end{array}$ & $\mathcal{O}\left(2^{2 N_{A}} N_{\text {bath-dets }}\right)$ & $\mathcal{O}\left(2^{2 N_{A}} N_{\text {bath-dets }} N_{\chi}^{2}\right)$ \\
\hline matrix product states & $\mathcal{O}\left(D^{2} N_{\chi}\right)$ & $\mathcal{O}\left(D^{3} N_{\chi}^{3}\right)$ \\
\hline $\begin{array}{l}\text { Gutzwiller- and Jastrow } \\
\text { (Eq. }(9.171))\end{array}$ & $\mathcal{O}\left(N_{\chi}^{2}\right)$ & $\mathcal{O}\left(N_{\mathrm{MC}} N_{\chi}^{4}\right)$ \\
\hline $\begin{array}{l}\text { hardware-efficient trial-states } \\
\text { on quantum computers }\end{array}$ & $\mathcal{O}\left(3 d N_{\chi}\right)$ & $\mathcal{O}\left(d N_{\mathrm{qc}} N_{\chi}^{3}\right)$ \\
\hline $\begin{array}{l}\text { N-representability of } \rho^{(2)} \\
(\mathcal{D} \mathcal{Q G})\end{array}$ & $\mathcal{O}\left(N_{\chi}^{4}\right)$ & $\mathcal{O}\left(N_{\chi}^{6}\right)$ \\
\hline $\begin{array}{l}\text { N-representability of } \rho^{(2)} \\
\left(\mathcal{D} \mathcal{Q \mathcal { G }} \mathcal{T}_{1} \mathcal{T}_{2}\right)\end{array}$ & $\mathcal{O}\left(N_{\chi}^{4}\right)$ & $\mathcal{O}\left(N_{\chi}^{9}\right)$
\end{tabular}

The high computational scaling of all methods with the size of the one-particle basis highlights the importance of methods for the reduction of the system size such as the adaptive cluster approximation. For the evaluation of the suitability of an ansatz for the density-matrix functional in a specific physical situation, one can consider the plethora of existing insights about the different wave functions. We have not found a one-size-fitsall ansatz, and the choice depends on the physical situation. However, we have shown that for a broad spectrum of wave-function ansatzes, one can construct algorithms that solve the constrained minimization problem of the density-matrix functional even if Monte Carlo-evaluations, quantum computers or semi-definiteness constraints are involved. 


\section{N-representability based approach to the RDMF}

\subsection{Introduction}

A non-perturbative approach to the electronic structure problem, that is complementary to the wave-function based approaches in chapter 9, is variational two-particle reduced density-matrix functional theory (2RDMFT, [Erdahl, 1979; Mazziotti and Erdahl, 2001; Nakata et al., 2001; Mazziotti, 2002; Zhao et al., 2004; Hammond and Mazziotti, 2005; Verstichel et al., 2011; Mazziotti, 2011; Verstichel, 2012]). The main idea of this approach is to use the minimization in Eq. (2.131),

$$
\tilde{E}=\min _{\rho_{\alpha, \beta, \gamma, \delta}^{(2)}: \exists\left\{\left|\Psi_{i}^{N}\right\rangle, P_{i}\right\}} \sum_{\text {that fulfills Eq. } 10.2} \sum_{\alpha, \beta, \gamma, \delta} \rho_{\alpha, \beta, \gamma, \delta}^{(2)}\left(\frac{1}{N-1} h_{\gamma, \alpha} \delta_{\beta, \delta}+\frac{1}{2} U_{\delta, \gamma, \beta, \alpha}\right),
$$

and to approximate the N-representability constraints

$$
\rho_{\alpha, \beta, \gamma, \delta}^{(2)}=\sum_{i} P_{i}\left\langle\Psi_{i}^{N}\left|\hat{c}_{\delta}^{\dagger} \hat{c}_{\gamma}^{\dagger} \hat{c}_{\alpha} \hat{c}_{\beta}\right| \Psi_{i}^{N}\right\rangle
$$

of the two-particle reduced density matrix. Following Levy's constrained search approach, the density-matrix functional can be written as

$$
F^{\hat{W}}\left[\rho^{(1)}\right]=\min _{\rho_{\alpha, \beta, \gamma, \delta}^{(2)}: \exists\left\{\left|\Psi_{i}^{N}\right\rangle, P_{i}\right\}} \sum_{\text {that fulfills Eq. }} \sum_{10.2, \rho^{(1)}\left(\rho^{(2)}\right)=\rho^{(1)}} \frac{1}{\alpha, \beta, \gamma, \delta} U_{\delta, \gamma, \beta, \alpha},
$$

where the two-particle reduced density matrix has to reduce to the given one-particle reduced density matrix via Eq. (2.116) with $p=2$, i.e.,

$$
\rho_{\alpha, \beta}^{(1)}\left(\rho^{(2)}\right) \stackrel{!}{=} \frac{1}{N-1} \sum_{\gamma} \rho_{\alpha, \gamma, \beta, \gamma}^{(2)} .
$$

Obviously, if the exact N-representability constraints of the two-particle reduced density matrix are used, the scheme would have an exponential complexity just as the manyparticle problem itself. However, approximate schemes with polynomial computational complexity can be set up by considering a subset of the necessary N-representability conditions. A scheme based on the two-particle reduced density matrix is complementary to the wave-function-based approaches discussed in chapter 9 in the sense that instead of implicitly enforcing the N-representability of the two-particle reduced density matrix by some form of a wave-function ansatz, the N-representability of two-particle reduced density matrix is explicitly enforced.

In sections 10.2-10.4 we describe some of the conventional N-representability conditions for the two-particle reduced density matrix [Harriman, 1978; Coleman and Yukalov, 2000; Coleman, 1963]. In section 10.5 we outline a numerical minimization scheme and propose two different ways of including the constraints of the one-particle reduced density matrix. Some example results of our proof-of-concept implementation are presented in section 10.8. 


\subsection{One-index $\mathrm{N}$-representability conditions}

In this and the two following sections, we closely follow the discussion of the Nrepresentability conditions by [Verstichel, 2012]. We assume a system with $N$ electrons in $M$ spin-orbitals. N-representability conditions of the two-particle reduced density matrix can be systematically derived by considering classes of Hamiltonians for which the ground-state energy is known to be non-negative. One such Hamiltonian is

$$
\hat{H}=\sum_{i} \hat{B}_{i}^{\dagger} \hat{B}_{i}
$$

with p-particle operators $\hat{B}_{i}$. The well known ensemble-N-representability conditions of the one-particle reduced density matrix can be derived with the two choices

$$
\hat{B}^{\dagger}=\sum_{\alpha} p_{\alpha} \hat{c}_{\alpha}^{\dagger}
$$

and

$$
\hat{B}^{\dagger}=\sum_{\alpha} q_{\alpha} \hat{c}_{\alpha}
$$

with $p_{\alpha}, q_{\alpha} \in \mathbb{C}$. The first choice results in the condition

$$
\sum_{\alpha, \beta} p_{\alpha} \hat{c}_{\alpha}^{\dagger} \hat{c}_{\beta} p_{\beta}^{*} \geq 0
$$

which is equivalent to the condition that the one-particle reduced density matrix is positive semidefinite, $\rho^{(1)} \geq 0$. The second choice results in the condition

$$
\sum_{\alpha, \beta} q_{\alpha} \hat{c}_{\alpha} \hat{c}_{\beta}^{\dagger} q_{\beta}^{*} \geq 0
$$

This condition requires that the one-hole reduced density matrix $q_{\beta \alpha}^{(1)}=\left\langle\hat{c}_{\alpha} \hat{c}_{\beta}^{\dagger}\right\rangle=\delta_{\alpha \beta}-\rho_{\beta \alpha}^{(1)}$ is positive semidefinite, $q^{(1)}=\mathbb{1}-\rho^{(1)} \geq 0$. Because of the single summation index in Eq. (10.6) and Eq. (10.7), these conditions are known as the one-index conditions.

\subsection{Two-index $\mathrm{N}$-representability conditions}

A generalization of the operator $\hat{B}$ to two summation indexes leads to the operators of the four two-index conditions

$$
\begin{aligned}
\hat{B}_{\mathcal{D}}^{\dagger} & =\sum_{\alpha, \beta} p_{\alpha, \beta} \hat{c}_{\alpha}^{\dagger} \hat{c}_{\beta}^{\dagger}, \\
\hat{B}_{\mathcal{Q}}^{\dagger} & =\sum_{\alpha, \beta} q_{\alpha, \beta} \hat{c}_{\alpha} \hat{c}_{\beta}, \\
\hat{B}_{\mathcal{G}}^{\dagger} & =\sum_{\alpha, \beta} g_{\alpha, \beta} \hat{c}_{\alpha}^{\dagger} \hat{c}_{\beta}, \\
\hat{B}_{\mathcal{G}^{\prime}}^{\dagger} & =\sum_{\alpha, \beta} g_{\alpha, \beta} \hat{c}_{\alpha} \hat{c}_{\beta}^{\dagger} .
\end{aligned}
$$


From the first operator $\hat{B}_{\mathcal{D}}^{\dagger}$, we obtain the condition

$$
\sum_{\alpha, \beta, \gamma, \delta} p_{\alpha, \beta} \hat{c}_{\alpha}^{\dagger} \hat{c}_{\beta}^{\dagger} \hat{c}_{\delta} \hat{c}_{\gamma} p_{\gamma, \delta}^{*} \geq 0
$$

which is just a different expression for the positive semidefiniteness of the two-particle reduced density matrix, $\rho^{(2)} \geq 0$. Positive semidefiniteness of two-particle reduced density matrix $\rho^{(2)}$ implies positive semidefiniteness of one-particle reduced density matrix $\rho^{(1)}$. The condition of the semidefiniteness of the two-particle reduced density matrix is also known as the $\mathcal{D}$-condition or the $\mathcal{I}$-condition [Coleman, 1963].

The $\mathcal{Q}$-condition expresses the positive semidefiniteness of the two-hole reduced density matrix [Garrod and Percus, 1964]

$$
Q_{\alpha, \beta, \gamma, \delta}=\left\langle\hat{c}_{\alpha} \hat{c}_{\beta} \hat{c}_{\delta}^{\dagger} \hat{c}_{\gamma}^{\dagger}\right\rangle
$$

and can be derived from the operator $\hat{B}_{\mathcal{Q}}^{\dagger}$. The matrix $Q$ reduces to the one-hole reduced density matrix $q$ with

$$
q_{\alpha, \beta}^{(1)}=\frac{1}{M-N-1} \sum_{\gamma} Q_{\alpha, \gamma, \beta, \gamma}
$$

and can be expressed in terms of the two-particle reduced density matrix and one-particle reduced density matrix as

$$
Q_{\alpha, \beta, \gamma, \delta}=\delta_{\alpha, \gamma} \delta_{\beta, \delta}-\delta_{\beta, \gamma} \delta_{\alpha, \delta}+\rho_{\alpha, \beta, \gamma, \delta}^{(2)}-\delta_{\alpha, \gamma} \rho_{\beta, \delta}^{(1)}+\delta_{\alpha, \delta} \rho_{\beta, \gamma}^{(1)}+\delta_{\beta, \gamma} \rho_{\alpha, \delta}^{(1)}-\delta_{\beta, \delta} \rho_{\alpha, \gamma}^{(1)}
$$

Positive semidefiniteness of the two-hole reduced density matrix $Q$ implies positive semidefiniteness of one-hole reduced density matrix $q^{(1)}$.

The $\mathcal{G}$-condition [Garrod and Percus, 1964] is derived from the operator $\hat{B}_{\mathcal{G}}^{\dagger}$. The operator $\hat{B}_{\mathcal{G}}^{\dagger}$ has the form of a particle-hole operator and leads to the condition

$$
\sum_{\alpha, \beta, \gamma, \delta} g_{\alpha, \beta} \hat{c}_{\alpha}^{\dagger} \hat{c}_{\beta} \hat{c}_{\delta}^{\dagger} \hat{c}_{\gamma} g_{\gamma, \delta}^{*} \geq 0
$$

The matrix $G$ is defined as

$$
G_{\alpha, \beta, \gamma, \delta}=\left\langle\hat{c}_{\alpha}^{\dagger} \hat{c}_{\beta} \hat{c}_{\delta}^{\dagger} \hat{c}_{\gamma}\right\rangle
$$

and can be expressed in terms of the one-particle and two-particle reduced density matrices as

$$
G_{\alpha, \beta, \gamma, \delta}=\delta_{\beta, \delta} \rho_{\alpha, \gamma}^{(1)}-\rho_{\alpha, \delta, \gamma, \beta}^{(2)}
$$

The $\mathcal{D}$ - $\mathcal{Q}$ - and $\mathcal{G}$-condition are known as the standard two-index conditions. The last operator of two-index form, $\hat{B}_{\mathcal{G}^{\prime}}^{\dagger}$, produces a condition that is redundant if the $\mathcal{G}$-condition is included. 


\subsection{Three-index $\mathrm{N}$-representability conditions}

The extension of $\hat{B}$ to a three-index form, that is,

$$
\hat{B}^{\dagger}=\sum_{\alpha, \beta, \gamma} p_{\alpha, \beta, \gamma} \hat{c}_{\alpha}^{\dagger} \hat{c}_{\beta}^{\dagger} \hat{c}_{\gamma}^{\dagger}
$$

with the Hamiltonian $\hat{H}=\hat{B}^{\dagger} \hat{B}$ leads to the condition

$$
\sum_{\alpha, \beta, \gamma, \alpha^{\prime}, \beta^{\prime}, \gamma^{\prime}} p_{\alpha, \beta, \gamma} \hat{c}_{\alpha}^{\dagger} \hat{c}_{\beta}^{\dagger} \hat{c}_{\gamma}^{\dagger} \hat{c}_{\gamma^{\prime}} \hat{c}_{\beta^{\prime}} \hat{c}_{\alpha^{\prime}} p_{\alpha^{\prime}, \beta^{\prime}, \gamma^{\prime}}^{*} \geq 0
$$

However, the matrix $T_{\alpha, \beta, \gamma, \alpha^{\prime}, \beta^{\prime}, \gamma^{\prime}}^{\prime}=\left\langle\hat{c}_{\alpha}^{\dagger} \hat{c}_{\beta}^{\dagger} \hat{c}_{\gamma}^{\dagger} \hat{c}_{\gamma^{\prime}} \hat{c}_{\beta^{\prime}} \hat{c}_{\alpha^{\prime}}\right\rangle$ cannot be expressed with the twoparticle reduced density matrix. In contrast, the expectation value of the anticommutator

$$
\begin{aligned}
\hat{H} & =\hat{B}^{\dagger} \hat{B}+\hat{B} \hat{B}^{\dagger} \\
& =\sum_{\alpha, \beta, \gamma, \alpha^{\prime}, \beta^{\prime}, \gamma^{\prime}} p_{\alpha, \beta, \gamma} p_{\alpha^{\prime}, \beta^{\prime}, \gamma^{\prime}}^{*}\left(\hat{c}_{\alpha}^{\dagger} \hat{c}_{\beta}^{\dagger} \hat{c}_{\gamma}^{\dagger} \hat{c}_{\gamma^{\prime}} \hat{c}_{\beta^{\prime}} \hat{c}_{\alpha^{\prime}}+\hat{c}_{\gamma^{\prime}} \hat{c}_{\beta^{\prime}} \hat{c}_{\alpha^{\prime}} \hat{c}_{\alpha}^{\dagger} \hat{c}_{\beta}^{\dagger} \hat{c}_{\gamma}^{\dagger}\right)
\end{aligned}
$$

can be expressed solely by the two-particle reduced density matrix [Erdahl, 1978; Zhao et al., 2004; Hammond and Mazziotti, 2005]. The resulting matrix is usually named the $T_{1}$-matrix and is defined as

$$
T_{1, \alpha, \beta, \gamma, \alpha^{\prime}, \beta^{\prime}, \gamma^{\prime}}=\left\langle\hat{c}_{\alpha}^{\dagger} \hat{c}_{\beta}^{\dagger} \hat{c}_{\gamma}^{\dagger} \hat{c}_{\gamma^{\prime}} \hat{c}_{\beta^{\prime}} \hat{c}_{\alpha^{\prime}}+\hat{c}_{\gamma^{\prime}} \hat{c}_{\beta^{\prime}} \hat{c}_{\alpha^{\prime}} \hat{c}_{\alpha}^{\dagger} \hat{c}_{\beta}^{\dagger} \hat{c}_{\gamma}^{\dagger}\right\rangle .
$$

The expression of this matrix in terms of the two-particle reduced density matrix is rather lengthy and has, for example, been given by Verstichel [Verstichel, 2012] as

$$
\begin{aligned}
T_{1, \alpha, \beta, \gamma, \alpha^{\prime}, \beta^{\prime}, \gamma^{\prime}} & =\delta_{\gamma, \gamma^{\prime}} \delta_{\beta, \beta^{\prime}} \delta_{\alpha, \alpha^{\prime}}-\delta_{\gamma, \beta^{\prime}} \delta_{\alpha, \alpha^{\prime}} \delta_{\beta, \gamma^{\prime}}+\delta_{\alpha, \gamma^{\prime}} \delta_{\gamma, \beta^{\prime}} \delta_{\beta, \alpha^{\prime}}-\delta_{\gamma, \gamma^{\prime}} \delta_{\alpha, \beta^{\prime}} \delta_{\beta, \alpha^{\prime}}+\delta_{\beta, \gamma^{\prime}} \delta_{\alpha, \beta^{\prime}} \delta_{\gamma, \alpha^{\prime}} \\
& -\delta_{\alpha \gamma^{\prime}} \delta_{\beta \beta^{\prime}} \delta_{\gamma \alpha^{\prime}}+\left(\delta_{\beta \gamma^{\prime}} \delta_{\gamma \beta^{\prime}}-\delta_{\gamma \gamma^{\prime}} \delta_{\beta \beta^{\prime}}\right) \rho_{\alpha \alpha^{\prime}}^{(1)}+\left(\delta_{\gamma \gamma^{\prime}} \delta_{\alpha \beta^{\prime}}-\delta_{\alpha \gamma^{\prime}} \delta_{\gamma \beta^{\prime}}\right) \rho_{\beta \alpha^{\prime}}^{(1)} \\
& +\left(\delta_{\alpha, \gamma^{\prime}} \delta_{\beta, \beta^{\prime}}-\delta_{\beta, \gamma^{\prime}} \delta_{\alpha, \beta^{\prime}}\right) \rho_{\gamma, \alpha^{\prime}}^{(1)}+\left(\delta_{\gamma, \gamma^{\prime}} \delta_{\beta, \alpha^{\prime}}-\delta_{\beta \gamma^{\prime}} \delta_{\gamma \alpha^{\prime}}\right) \rho_{\alpha \beta^{\prime}}^{(1)} \\
& +\left(\delta_{\alpha, \gamma^{\prime}} \delta_{\gamma, \alpha^{\prime}}-\delta_{\gamma, \gamma^{\prime}} \delta_{\alpha, \alpha^{\prime}}\right) \rho_{\beta^{\prime}, \beta}^{(1)}+\left(\delta_{\beta, \gamma^{\prime}} \delta_{\alpha, \alpha^{\prime}}-\delta_{\alpha \gamma^{\prime}} \delta_{\beta \alpha^{\prime}}\right) \rho_{\gamma \beta^{\prime}}^{(1)} \\
& +\left(\delta_{\beta, \beta^{\prime}} \delta_{\gamma, \alpha^{\prime}}-\delta_{\beta, \alpha^{\prime}} \delta_{\gamma, \beta^{\prime}}\right) \rho_{\alpha, \gamma^{\prime}}^{(1)}+\left(\delta_{\gamma, \beta^{\prime}} \delta_{\alpha, \alpha^{\prime}}-\delta_{\alpha \beta^{\prime}} \delta_{\gamma \alpha^{\prime}}\right) \rho_{\beta \gamma^{\prime}}^{(1)} \\
& +\left(\delta_{\alpha, \beta^{\prime}} \delta_{\beta, \alpha^{\prime}}-\delta_{\beta, \beta^{\prime}} \delta_{\alpha, \alpha^{\prime}}\right) \rho_{\gamma, \gamma^{\prime}}^{(1)}+\delta_{\gamma, \gamma^{\prime}} \rho_{\alpha, \beta, \alpha^{\prime}, \beta^{\prime}}^{(2)}-\delta_{\beta, \gamma^{\prime}} \rho_{\alpha, \gamma, \alpha^{\prime}, \beta^{\prime}}^{(2)} \\
& +\delta_{\alpha, \gamma^{\prime}} \rho_{\beta, \gamma, \alpha^{\prime}, \beta^{\prime}}^{(2)}-\delta_{\gamma, \beta^{\prime}} \rho_{\alpha, \beta, \alpha^{\prime}, \gamma^{\prime}}^{(2)}+\delta_{\beta, \beta^{\prime}} \rho_{\alpha, \gamma, \alpha^{\prime}, \gamma^{\prime}}^{(2)}-\delta_{\alpha, \beta^{\prime}} \rho_{\beta, \gamma, \alpha^{\prime}, \gamma^{\prime}}^{(2)} \\
& +\delta_{\gamma, \alpha^{\prime}} \rho_{\alpha, \beta, \beta^{\prime}, \gamma^{\prime}}^{(2)}-\delta_{\beta, \alpha^{\prime}} \rho_{\alpha, \gamma, \beta^{\prime}, \gamma^{\prime}}^{(2)}+\delta_{\alpha, \alpha^{\prime}} \rho_{\beta, \gamma, \beta^{\prime}, \gamma^{\prime}}^{(2)}
\end{aligned}
$$

The positive semidefiniteness of the matrix $T_{1}, T_{1} \geq 0$, defines the $\mathcal{T}_{1}$-condition.

Similarly, the $\mathcal{T}_{2}$-condition can be derived with

$$
\hat{B}^{\dagger}=\sum_{\alpha, \beta, \gamma} p_{\alpha, \beta, \gamma} \hat{c}_{\alpha}^{\dagger} \hat{c}_{\beta}^{\dagger} \hat{c}_{\gamma} .
$$

The $\mathcal{T}_{2}$-condition is the positive semidefiniteness of the matrix [Verstichel, 2012]

$$
\begin{aligned}
T_{2, \alpha, \beta, \gamma, \alpha^{\prime}, \beta^{\prime}, \gamma^{\prime}} & =\left(\delta_{\alpha, \alpha^{\prime}} \delta_{\beta, \beta^{\prime}}-\delta_{\alpha, \beta^{\prime}} \delta_{\beta, \alpha^{\prime}}\right) \rho_{\gamma, \gamma^{\prime}}^{(1)}+\delta_{\gamma, \gamma^{\prime}} \rho_{\alpha, \beta, \alpha^{\prime}, \beta^{\prime}}^{(2)}-\delta_{\alpha, \alpha^{\prime}} \rho_{\gamma, \beta^{\prime}, \gamma^{\prime}, \beta}^{(2)} \\
& +\delta_{\beta, \alpha^{\prime}} \rho_{\gamma, \beta^{\prime}, \gamma^{\prime}, \alpha}^{(2)}+\delta_{\alpha, \beta^{\prime}} \rho_{\gamma, \alpha^{\prime}, \gamma^{\prime}, \beta}^{(2)}-\delta_{\beta, \beta^{\prime}} \rho_{\gamma, \alpha^{\prime}, \gamma^{\prime}, \alpha}^{(2)} .
\end{aligned}
$$

A systematic construction scheme for all necessary conditions of the two-particle reduced density matrix has been given by Mazziotti [Mazziotti, 2012]. However, for the proof-of-concept work in this thesis we only consider the $\mathcal{D}$-, $\mathcal{Q}_{-}^{-}, \mathcal{G}_{-}, \mathcal{T}_{1^{-}}$and $\mathcal{T}_{2}$-conditions. It has been shown that for strongly correlated systems the $\mathcal{T}_{1}$ - and $\mathcal{T}_{2}$-conditions have to be enforced to obtain physically correct ground states [Verstichel et al., 2013]. 


\subsection{Numerical scheme}

The necessary conditions for N-representability of the two-particle reduced density matrix considered have the form of positive semidefiniteness relations. Thus, the Nrepresentability condition given by Eq. (10.2) is approximated by the $\mathcal{D}_{-}, \mathcal{Q}_{-}^{-}, \mathcal{G}_{-}, \mathcal{T}_{1^{-}}$ and $\mathcal{T}_{2}$-conditions discussed in the previous section. The minimization problem to be solved is

$$
F_{2 \operatorname{RDMFT}}^{\hat{W}}\left[\rho^{(1)}\right]=\min _{\rho_{\alpha, \beta, \gamma, \delta}^{(2)}: \rho^{(2)} \geq 0, Q \geq 0, G \geq 0, T_{1} \geq 0, T_{2} \geq 0, \rho^{(1)}\left(\rho^{(2)}\right)=\rho^{(1)}} \frac{1}{2} \sum_{\alpha, \beta, \gamma, \delta} U_{\delta, \gamma, \beta, \alpha} .
$$

Because the employed N-representability condition are necessary but not sufficient for the N-representability of the two-particle reduced density matrix, the approximate densitymatrix functional in Eq. (10.29) systematically underestimates the exact density-matrix functional, $F_{2 \operatorname{RDMFT}}^{\hat{W}}\left[\rho^{(1)}\right] \leq F_{\text {exact }}^{\hat{W}}\left[\rho^{(1)}\right]$.

The related minimization problem of the two-particle reduced density-matrix functional theory is

$$
E_{2 \operatorname{RDMFT}}(N)=\min _{\rho_{\alpha, \beta, \gamma, \delta}^{(2)}: \rho^{(2)} \geq 0, Q \geq 0, G \geq 0, T_{1} \geq 0, T_{2} \geq 0, \operatorname{Tr} \rho^{(1)}=N} \sum_{\alpha, \beta, \gamma, \delta} \rho_{\alpha, \beta, \gamma, \delta}^{(2)}\left(\frac{1}{N-1} h_{\gamma, \alpha} \delta_{\beta, \delta}+\frac{1}{2} U_{\delta, \gamma, \beta, \alpha}\right) .
$$

Several algorithms have been proposed [Vandenberghe and Boyd, 1996; Burer and Monteiro, 2003; Zhao et al., 2004; Mazziotti, 2004b; Fukuda et al., 2007; Malick et al., 2009; Mazziotti, 2011; Verstichel, 2012] for the numerical solution of this problem. The most promising is the boundary-point semidefinite programming algorithm [Malick et al., 2009; Mazziotti, 2011; Verstichel, 2012]. In the following subsection we first discuss this algorithm for the problem of the energy minimization of Eq. (10.30). Afterward, we propose two different routes to include the constraints of the one-particle reduced density matrix in the minimization scheme to estimate the density-matrix functional. The constraint of particle number in Eq. (10.30) is handled implicitly and, hence, not considered in the following discussion.

\subsection{Boundary-point semidefinite programming algorithm for energy minimization}

For the minimization of the energy within two-particle reduced density-matrix functional theory given by Eq. (10.30), we first define

$$
E\left(\rho^{(2)}\right)=\sum_{\alpha, \beta, \gamma, \delta} \rho_{\alpha, \beta, \gamma, \delta}^{(2)}\left(\frac{1}{N-1} h_{\gamma, \alpha} \delta_{\beta, \delta}+\frac{1}{2} U_{\delta, \gamma, \beta, \alpha}\right)
$$

and the matrix $Z$ for the positive semidefiniteness constraints as

$$
Z\left(\rho^{(2)}\right)=\left(\begin{array}{lllll}
\rho^{(2)} & & & & \\
& Q\left(\rho^{(2)}\right) & & & \\
& & G\left(\rho^{(2)}\right) & & \\
& & & T_{1}\left(\rho^{(2)}\right) & \\
& & & & T_{2}\left(\rho^{(2)}\right)
\end{array}\right) .
$$


Thus, the N-representability constraints for the two-particle reduced density matrix can be written simply as $Z\left(\rho^{(2)}\right) \geq 0$. The matrix $Z\left(\rho^{(2)}\right)$ can be decomposed into a sum of a positive semidefinite part $Z_{+} \geq 0$ and a negative semidefinite part $Z_{-}$. The positive (negative) semidefinite part can be obtained from the eigendecomposition $Z=U D U^{\dagger}$ by only considering the positive (negative) eigenvalues $D_{+/-}$in the decomposition, i.e.

$$
Z_{+/-}=U D_{+/-} U^{\dagger}
$$

The boundary-point semidefinite programming algorithm is essentially the application of the alternating direction method of multipliers [Gabay and Mercier, 1976]. We follow here the description of the algorithm given by Verstichel [Verstichel, 2012]. The main idea of the alternating direction method of multipliers is to rewrite the minimization problem

$$
\min _{x}[f(x)+g(x)]
$$

in the form

$$
\min _{x, y: x=y}[f(x)+g(y)] .
$$

At first glance, this complicates the problem by introducing additional constraints but it opens the door for the application of constrained optimization methods. For the situation at hand, we use this approach to treat $Z$ as an additional variable and add the constraint $Z=Z\left(\rho^{(2)}\right)$ such that the minimization problem obtains the form

$$
\min _{\rho^{(2)}: Z\left(\rho^{(2)}\right) \geq 0} E\left(\rho^{(2)}\right)=\min _{\rho^{(2)}, Z: Z \geq 0, Z=Z\left(\rho^{(2)}\right)} E\left(\rho^{(2)}\right) .
$$

Now we can apply the augmented Lagrangian method [Hestenes, 1969; Powell, 1969] to the constraint $Z=Z\left(\rho^{(2)}\right)$ which results in the augmented Lagrangian

$$
L\left(\rho^{(2)}, Z, X, \mu\right)=E\left(\rho^{(2)}\right)+\operatorname{Tr}\left[X\left(Z-Z\left(\rho^{(2)}\right)\right)\right]+\frac{\mu}{2}\left\|Z-Z\left(\rho^{(2)}\right)\right\|^{2}
$$

with the matrix of Lagrange multipliers $X$ and the penalty parameter $\mu$. The positive semidefiniteness constraint is carried over to the subproblem of the augmented Lagrangian. By introducing the matrix

$$
V\left(\rho^{(2)}\right)=Z\left(\rho^{(2)}\right)-X / \mu
$$

we can rewrite the augmented Lagrangian as

$$
L\left(\rho^{(2)}, Z, X, \mu\right)=E\left(\rho^{(2)}\right)+\frac{\mu}{2}\left\|Z-V\left(\rho^{(2)}\right)\right\|^{2}-\frac{1}{2 \mu}\|X\|^{2} .
$$

Thus, in the framework of the augmented Lagrangian we have to solve the subproblems

$$
\min _{\rho^{(2)}, Z: Z \geq 0}\left(E\left(\rho^{(2)}\right)+\frac{\mu}{2}\left\|Z-V\left(\rho^{(2)}\right)\right\|^{2}\right)
$$

for fixed $\mu$ and $X$. The solution $\rho^{(2), *}$ of this subproblem determines the update for the Lagrange multipliers $X$ by

$$
X \rightarrow X+\mu\left(Z-Z\left(\rho^{(2), *}\right)\right)
$$


These two steps have to be iterated until the constraint violation $\left\|Z-Z\left(\rho^{(2)}\right)\right\|$ and the derivative of the augmented Lagrangian $L\left(\rho^{(2)}, Z, X, \mu\right)$ with respect to the two-particle reduced density matrix is sufficiently small.

The Lagrangian for the subproblems reads

$$
\min _{\rho^{(2)}, Z: Z \geq 0}\left(E\left(\rho^{(2)}\right)+\frac{\mu}{2}\left\|Z-V\left(\rho^{(2)}\right)\right\|^{2}\right) .
$$

The solution of the subproblem in Eq. (10.42) can be obtained by alternating minimization. If we fix $Z$ we have the unconstrained minimization problem

$$
\min _{\rho^{(2)}}\left(E\left(\rho^{(2)}\right)+\frac{\mu}{2}\left\|Z-V\left(\rho^{(2)}\right)\right\|^{2}\right),
$$

which can be solved efficiently with a conjugate gradient approach for the solution of the minimum condition. When the two-particle reduced density matrix $\rho^{(2)}$ is fixed, we have to solve the minimization problem

$$
\min _{Z: Z \geq 0} \frac{\mu}{2}\left\|Z-V\left(\rho^{(2)}\right)\right\|^{2}
$$

This minimization problem can be solved with the decomposition of the $V\left(\rho^{(2)}\right)$ into its positive definite $V\left(\rho^{(2)}\right)_{+}$and negative semidefinite contribution $V\left(\rho^{(2)}\right)_{-}$,

$$
V\left(\rho^{(2)}\right)=V\left(\rho^{(2)}\right)_{+}+V\left(\rho^{(2)}\right)_{-} .
$$

Equation (10.44) is minimized by $Z^{*}=V\left(\rho^{(2)}\right)_{+}$because $Z^{*}-V\left(\rho^{(2)}\right)=V\left(\rho^{(2)}\right)_{-}$contains no positive definite contribution. The alternating minimization over $Z$ and $\rho^{(2)}$ is performed until the minimum conditions of Eq. (10.43) are fulfilled up a finite accuracy after the solution of Eq. (10.44). This concludes the solution of the subproblem given by Eq. (10.42). In the next step the Lagrange multiplier $X$ is updated according to Eq. (10.41), which is identical to

$$
X \rightarrow X+\mu V\left(\rho^{(2)}\right)_{-} .
$$

For a one-particle basis with $M$ basis states, the matrices $\rho^{(2)}, Q$ and $G$ of the twoindex conditions $\mathcal{D}, \mathcal{Q}$ and $\mathcal{G}$ have dimension $\in \mathcal{O}\left(M^{2}\right)$. The matrices $T_{1}$ and $T_{2}$ of the three-index condition have dimension $\in \mathcal{O}\left(M^{3}\right)$. The computational effort for the diagonalization of a matrix of dimension $d$ scales as $\mathcal{O}\left(d^{3}\right)$. Thus, the resulting scheme scales as $M^{6}$ if only the two-index conditions are considered or $M^{9}$ if also the three-index conditions are considered. The computational effort for the setup of the matrices or the solution of the subproblem in Eq. (10.43) with a conjugate gradient approach is negligible compared to the matrix diagonalization.

\subsection{Boundary-point semidefinite programming algorithm for the density-matrix functional}

\subsubsection{Explicit restriction of the two-particle reduced density matrix}

We propose two different ways to include the constraints of the one-particle reduced density matrix in two-particle reduced density-matrix functional theory: on the one hand 
by explicitly restricting the possible two-particle reduced density matrices or, on the other hand, by enforcing the constraints with a method from constrained optimization like the augmented Lagrangian. The restriction of the possible two-particle reduced density matrices is based on the reduction

$$
\rho_{\alpha, \beta}^{(1)}\left(\rho^{(2)}\right) \stackrel{!}{=} \frac{1}{N-1} \sum_{\gamma} \rho_{\alpha, \gamma, \beta, \gamma}^{(2)}
$$

of the two-particle reduced density matrix to the one-particle reduced density matrix. The explicit inclusion of this reduction property into the numerical scheme can be accomplished by decomposing the two-particle reduced density matrix into one matrix $\tilde{a}$ with a non-zero trace and a complete set of traceless matrices $a_{i}$. The matrix $\tilde{a}$ is chosen such that the trace is

$$
\operatorname{Tr} \tilde{a}=N \cdot(N-1) .
$$

This matrix fixes the total particle number to $N$. All matrices $a_{i}$, as well as the matrix $\tilde{a}$, have the same symmetries as the two-particle reduced density matrix,

$$
\begin{aligned}
a_{i, \alpha, \beta, \gamma, \delta} & =-a_{i, \alpha, \beta, \delta, \gamma}=-a_{i, \beta, \alpha, \gamma, \delta}=a_{i, \beta, \alpha, \delta, \gamma} \\
& =a_{i, \gamma, \delta, \alpha, \beta}^{*}=-a_{i, \delta, \gamma, \alpha, \beta}^{*}=-a_{i, \gamma, \delta, \beta, \alpha}^{*}=a_{i, \delta, \gamma, \beta, \alpha}^{*} \forall i=0,1,2 \ldots
\end{aligned}
$$

There are $M^{4} / 8-M^{3} / 4+3 / 8 M^{2}-M / 4$ real traceless matrices $a_{i}$ and $M^{4} / 8-M^{3} / 4-$ $M^{2} / 8+M / 4$ imaginary traceless matrices $a_{i}$ that are compatible with the symmetry of the two-particle reduced density matrix required by Eq. (10.49). These matrices $a_{i}$ can then be orthogonalized to from a new set of orthogonal matrices $b_{i}$ that fulfills

$$
\operatorname{Tr} b_{i} b_{j}=\delta_{i, j} .
$$

The set of matrices $b_{i}$ contains $M^{4} / 4-M^{3} / 2+M^{2} / 4$ matrices. Together with the matrix $\tilde{a}$, any two-particle reduced density matrix can be written as

$$
\rho^{(2)}=\tilde{a}+\sum_{i} x_{i} b_{i}
$$

where $x_{i}$ are real coefficients. The constraint of the one-particle reduced density matrix can be explicitly enforced for a two-particle reduced density matrix by first constructing the matrices $C_{\rho^{(1)}, \alpha, \beta}$ with $\alpha \geq \beta$, that implement the reduction condition in Eq. (10.47) as

$$
\rho_{\alpha, \beta}^{(1)}=\operatorname{Tr} \rho^{(2)} C_{\rho^{(1)}, \alpha, \beta}=\frac{1}{N-1} \sum_{\gamma} \rho_{\alpha, \gamma, \beta, \gamma}^{(2)} .
$$

The matrices $C_{\rho^{(1)}, \alpha, \beta}$ of the constraints are then orthogonalized among each other to yield the matrices $\tilde{C}_{\rho^{(1)}, \alpha, \beta}$ that fulfill

$$
\operatorname{Tr} \tilde{C}_{\rho^{(1)}, \alpha, \beta} \tilde{C}_{\rho^{(1)}, \alpha^{\prime}, \beta^{\prime}}=\delta_{\alpha, \alpha^{\prime}} \delta_{\beta, \beta^{\prime}}
$$

The constraint for the two-particle reduced density matrix now reads

$$
\operatorname{Tr} \rho^{(2)} \tilde{C}_{\rho^{(1)}, \alpha, \beta}=\tilde{\rho}_{\alpha, \beta}^{(1)},
$$


where $\tilde{\rho}_{\alpha, \beta}^{(1)}$ results from the orthogonalization of the constraint matrices. The matrices $\tilde{a}$ and $b_{i}$ can then be orthogonalized to the matrices $\tilde{C}_{\rho^{(1)}, \alpha, \beta}$. The constraint of the total particle number, that is represented by the matrix $\tilde{a}$, is automatically enforced by the constraints for the diagonal elements of the one-particle reduced density matrix. We obtain the new set of matrices $\tilde{b}_{i}$ with $M^{4} / 4-M^{3} / 2-3 / 4 M^{2}$ matrices. Then, any two-particle reduced density matrix $\rho^{(2)}$ that reduces to the one-particle reduced density matrix $\rho^{(1)}$ can be written as

$$
\rho^{(2)}=\sum_{\alpha \geq \beta} \tilde{\rho}_{\alpha, \beta}^{(1)} \tilde{C}_{\rho^{(1)}, \alpha, \beta}+\sum_{i} \tilde{x}_{i} \tilde{b}_{i} .
$$

Thus, we have rewritten the minimization problem of Eq. (10.29) in terms of the variables $\tilde{x}_{i}$. The matrix $Z\left(\rho^{(2)}\right)$, that has to be positive semidefinite, can be written in terms of the variable $\tilde{x}_{i}$ as

$$
Z\left(\tilde{x}_{i}\right)=Z\left(\sum_{\alpha \geq \beta} \tilde{\rho}_{\alpha, \beta}^{(1)} \tilde{C}_{\rho^{(1)}, \alpha, \beta}\right)+\sum_{i} \tilde{x}_{i} Z\left(\tilde{b}_{i}\right) .
$$

This scheme only allows two-particle reduced density matrices during the minimization that reduce to the given one-particle reduced density matrix. In this way, this scheme is different from all other schemes presented in this thesis. However, the number of $\mathcal{O}\left(M^{4}\right)$ variational parameters of the unconstrained two-particle reduced density matrix is only reduced by $\mathcal{O}\left(M^{2}\right)$ with the explicit inclusion of the one-particle reduced density matrix. We have implemented the scheme outlined in this section for small systems. Even though the scheme is attractive due to the exact enforcing of the constraints of the one-particle reduced density matrix, the numerical effort for the orthogonalizations turned out to be large. With $\mathcal{O}\left(M^{4}\right)$ matrices $b_{i}$ of dimension $M^{2}$ and $\mathcal{O}\left(M^{2}\right)$ matrices $\tilde{C}_{\rho^{(1)}, \alpha, \beta}$ of dimension $M^{2}$, we obtain a computational complexity of the orthogonalization of at least $\mathcal{O}\left(M^{10}\right)$. This contains $\mathcal{O}\left(M^{4}\right)$ for the trace of a product of two $M^{2} \times M^{2}$ matrices and a factor of $\mathcal{O}\left(M^{4+2}\right)$ for all pairs of $b_{i}$ 's and $\tilde{C}_{\rho^{(1)}, \alpha, \beta}$ 's. Regardless of this unfavorable scaling, this scheme might be useful as a benchmark for schemes that only allow an approximate enforcing of the constraints.

\subsubsection{Enforcing of constraints with the augmented Lagrangian}

The boundary-point semidefinite programming algorithm introduced in section 10.7 can be easily modified to enforce additional equality constraints $c_{i}\left(\rho^{(2)}\right)=0$ on the twoparticle reduced density matrix. This can be achieved by replacing the objective function $E\left(\rho^{(2)}\right)$ with the augmented Lagrangian [Hestenes, 1969; Powell, 1969]

$$
L\left(\rho^{(2)}, \lambda_{i}, \mu_{i}\right)=E\left(\rho^{(2)}\right)-\sum_{i} \lambda_{i} c_{i}\left(\rho^{(2)}\right)+\frac{\mu}{2} \sum_{i} c_{i}^{2}\left(\rho^{(2)}\right)
$$

In this way, we have an augmented Lagrangian, Eq. (10.36), nested inside another augmented Lagrangian, Eq. (10.57). The derivatives of the density-matrix functional with respect to the one-particle reduced density matrix can be obtained from the Lagrange multipliers $\lambda_{i}$ in Eq. (10.57). The main advantage of this scheme is that existing codes for two-particle reduced density-matrix functional theory can be used. The only modification 
necessary is that in Eq. (10.43) instead of the energy $E\left(\rho^{(2)}\right)$ the augmented Lagrangian of Eq. (10.57) with fixed Lagrange multipliers $\lambda_{i}$ and penalty parameters $\mu_{i}$ has to be minimized. We have adapted the implementation of the boundary-point semidefinite programming algorithm of Brecht Verstichel and Ward Poelmans [Verstichel, 2012; Verstichel et al., 2012] to include equality constraints based on the augmented Lagrangian.

\subsection{Example results}

As a benchmark for the method, we have investigated the $L$-site Hubbard chain at half filling. We investigate the performance of the $\mathcal{D}_{-}, \mathcal{Q}_{-}, \mathcal{G}_{-}, \mathcal{T}_{1^{-}}$and $\mathcal{T}_{2}$-conditions for the estimation of the density-matrix functional with the boundary-point semidefinite programming algorithm while enforcing the constraints of the one-particle reduced density matrix with an augmented Lagrangian. We have adapted the publicly available implementation of the boundary-point semidefinite programming algorithm of Brecht Verstichel and Ward Poelmans [Verstichel, 2012; Verstichel et al., 2012] to include equality constraints based on the augmented Lagrangian. No spin symmetry or spatial symmetries have been assumed. We have also improved their implementation by using a divide-and-conquer based diagonalization of symmetric matrices, that is more suitable for multi-core CPUs. In contrast to the original implementation, we perform the matrix diagonalization with floating-point numbers in single precision and automatically decompose the $\rho^{(2)}{ }_{-}, Q_{-}, G_{-}$, $T_{1^{-}}$and $T_{2}$-matrices into block-diagonal matrices. These two changes greatly reduce the computation time for the matrix diagonalization and allow us to treat larger system sizes. In order to be able to compare with numerically exact results, we chose to calculate the density-matrix functional for the one-particle reduced density matrix of the numerically exact ground state obtained from MPS-DMRG calculations with ITensor.

As a first step, we investigate the dependence of the interaction strength for a $L=10$ site Hubbard chain at half filling. Figure 10.1 shows the values of the reduced densitymatrix functional obtained with the numerical scheme outlined in section 10.7.2 when only the $\mathcal{D}$-, $\mathcal{Q}$ - and $\mathcal{G}$-conditions for the N-representability of the two-particle reduced density matrix are enforced. For small interaction strengths $U / t \ll 1$, even only enforcing the $\mathcal{D}$ and $\mathcal{Q}$-conditions yields results in good agreement with exact results. Figure 10.2 shows results when also the three-index conditions $\mathcal{T}_{1}$ and $\mathcal{T}_{2}$ are enforced. The density-matrix functional estimated with the $\mathcal{D Q G} \mathcal{T}_{1} \mathcal{T}_{2}$-conditions gives results that are in good quantitative agreement with the exact results. The largest deviation occurs for intermediate interactions strengths around $U / t \approx 5$.

To evaluate the suitability of this scheme for larger models, we applied this scheme to the 20-site Hubbard chain at half filling and $U / t=1$, that was already used as an example case in section 9.7.7. The exact density-matrix functional for the one-particle reduced density matrix $\rho_{\text {exact }}^{(1)}$ of the exact ground state of this system is

$$
F_{\text {exact }}^{\hat{W}}\left[\rho_{\text {exact }}^{(1)}\right]=4.2194 t
$$

Enforcing the $\mathcal{D}_{-}, \mathcal{Q}_{-}, \mathcal{G}_{-}, \mathcal{T}_{1^{-}}$and $\mathcal{T}_{2}$-conditions we obtained the value of the densitymatrix functional of

$$
F_{\text {2RDMFT }}^{\hat{W}}\left[\rho_{\text {exact }}^{(1)}\right]=4.2019 t .
$$




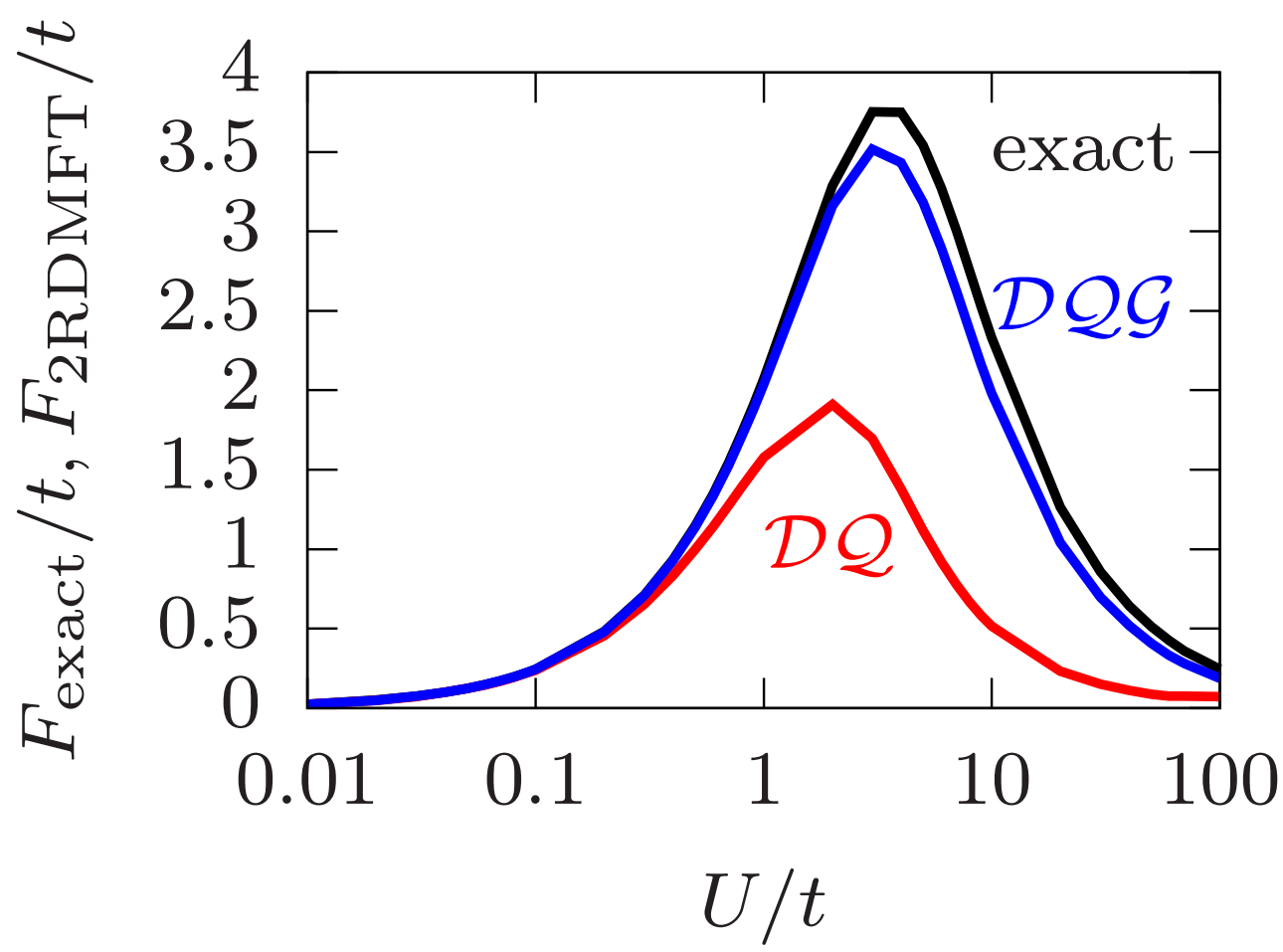

Figure 10.1.: Comparison of the exact density-matrix functional to values obtained from the scheme based on the N-representability conditions of the two-particle reduced density matrix proposed in section 10.7 .2 for a 10-site half-filled Hubbard chain at different interaction strengths $U / t$. The solid red line shows results when the $\mathcal{D}$ - and $\mathcal{Q}$-conditions are enforced and the solid blue line when the additional $\mathcal{G}$-condition is also enforced.

The result from the scheme based on the Monte Carlo-evaluation of the Gutzwiller- and Jastrow-correlator proposed in section 9.7 was

$$
F_{\text {Monte Carlo }}^{\hat{W}}\left[\rho_{\text {exact }}^{(1)}\right]=(4.236 \pm 0.007) \cdot t .
$$

Thus, the scheme based on the N-representability conditions $\left(\mathcal{D Q} \mathcal{Q} \mathcal{T}_{1} \mathcal{T}_{2}\right)$ underestimates the density-matrix functional by approximately the same amount by which the scheme based on a Monte Carlo-treatment of Gutzwiller- and Jastrow-correlator overestimates the density-matrix functional. The dimension of the matrix $T_{1}$ after removing all zero elements is $M \cdot(M-1) \cdot(M-2) / 6=9880$ and the dimension of the $T_{2}$ matrix is $M^{2} \cdot(M-1) / 2=31200$. Thanks to our automatic detection of block-diagonal matrices the largest matrix to be diagonalized in the case of the 20-site Hubbard chain for the $\mathcal{T}_{1}$-condition only has a dimension of 3800 and for the $\mathcal{T}_{2}$-condition the largest submatrix has a dimension of 11800 .

We compare the runtime needed to diagonalize real symmetric matrices of the given dimensions on a modern high-performance computing (HPC) node and the general-purpose graphics processing unit (GPGPU). The diagonalization includes the computation of all eigenvalues and all eigenvectors. As the modern high-performance computing node, we choose a dual-socket system with two Intel Xeon Gold 6150 with a total peak floating 
point performance in single precision of 5.75 TFlops/s and a memory bandwidth of 180 GB/s in the STREAM ${ }^{1}$ benchmark. As the GPGPU we used a Nvidia GTX 970 with a peak floating point performance in single precision of 3.9 TFlops/s and a memory bandwidth of $224 \mathrm{~GB} / \mathrm{s}^{2}$. To compute the matrix diagonalization on the two different devices, we use the divide-and-conquer-implementation of the LAPACK routine ssyevd from the Intel Math-kernel-Library ${ }^{3}$ on the HPC-node and from Magma ${ }^{4}$ on the GPGPU. The runtime comparison of the two devices is shown in table 10.1 for the matrix sizes of interest. The results show that a low-cost GPGPU is very competitive especially in terms of initial cost and power usage compared to a HPC-computing node for this type of workload. More powerful GPGPUs like the Nvidia GTX 1080 ti (11.3 TFlops/s, 484 GB/s, approx. 700 Euro, 250 Watt) are available and would lead to a much wider gap between the performance of the HPC-node and GPGPUs.

\begin{tabular}{l|l|l} 
& HPC-node & GPGPU \\
\hline cost & approx. 9000 Euro & approx. 300 Euro \\
power usage-idle power usage & $370 \mathrm{~W}$ & $190 \mathrm{~W}$ \\
implementation & Intel MKL & Magma \\
dimension 3800 & $0.9 \mathrm{~s}$ & $0.94 \mathrm{~s}$ \\
dimension 11800 & $12.4 \mathrm{~s}$ & $10.2 \mathrm{~s}$ \\
dimension 20000 & $38.6 \mathrm{~s}$ & $36.7 \mathrm{~s}$ \\
dimension 24000 & $63.2 \mathrm{~s}$ & $50.4 \mathrm{~s}$
\end{tabular}

Table 10.1.: Comparison of the runtime needed for the diagonalization of a real symmetric matrix with a modern HPC-node (dual-socket Intel Xeon Gold 6150) and a GPGPU (Nvidia GTX 970).

In conclusion, we have proposed a new non-perturbative scheme to estimate the densitymatrix functional without using many-particle wave functions. The scheme can be systematically improved by enforcing more N-representability conditions of the two-particle reduced density matrix. We have outlined, implemented and tested two numerical schemes to enforce the constraints of the one-particle reduced density matrix and found that a scheme using the augmented Lagrangian works much more efficiently than explicitly enforcing these constraints by restricting the space of possible two-particle reduced density matrices. The requirement of a one-particle reduced density matrix with an integer total particle number can be circumvented by adding an additional site to the system with a fractional particle number so that the total particle number is an integer number. The extension to of this approach to finite temperatures has not been accomplished yet.

The main drawback of this method is the scaling of the computational complexity, $M^{6}$ for two-index conditions $(\mathcal{D Q \mathcal { G }})$ and $M^{9}$ for three-index conditions $\left(\mathcal{T}_{1} \mathcal{T}_{2}\right)$. This scaling stems from the required splitting up of the matrices of the N-representability constraints into positive and negative semidefinite contributions. Currently, a full matrix diagonalization is used for this purpose, and it scales as $\mathcal{O}\left(d^{3}\right)$, where $d$ is the dimension of the matrix. If the full matrix diagonalization could be replaced by a method with a lower

\footnotetext{
${ }^{1}$ https://www.cs.virginia.edu/stream/

${ }^{2}$ The host system was running an Intel Core i7-5820k (approx. cost 800 Euro).

${ }^{3}$ Intel MKL 18.0.3.222 https://software.intel.com/en-us/mkl

${ }^{4}$ Magma 2.4.0 http://icl.cs.utk.edu/magma/software/
} 
computational scaling maybe inspired by ideas from linear-scaling density functional theory, variational two-particle reduced density-matrix functional theory and the evaluation of the density-matrix functional based on it would become very competitive methods. In the context of the density-matrix functional, the main advantages of this method are that it is non-perturbative, systematically improvable, a guaranteed lower bound, and defined for any ensemble-representable one-particle reduced density matrix ${ }^{5}$.

\footnotetext{
${ }^{5}$ For example, the density-matrix functional based on a CI-expansion of the many-particle wave function is not guaranteed to be defined, because it is possible that there is no many-particle wave function in the given CI-space that corresponds to the required one-particle reduced density matrix.
} 

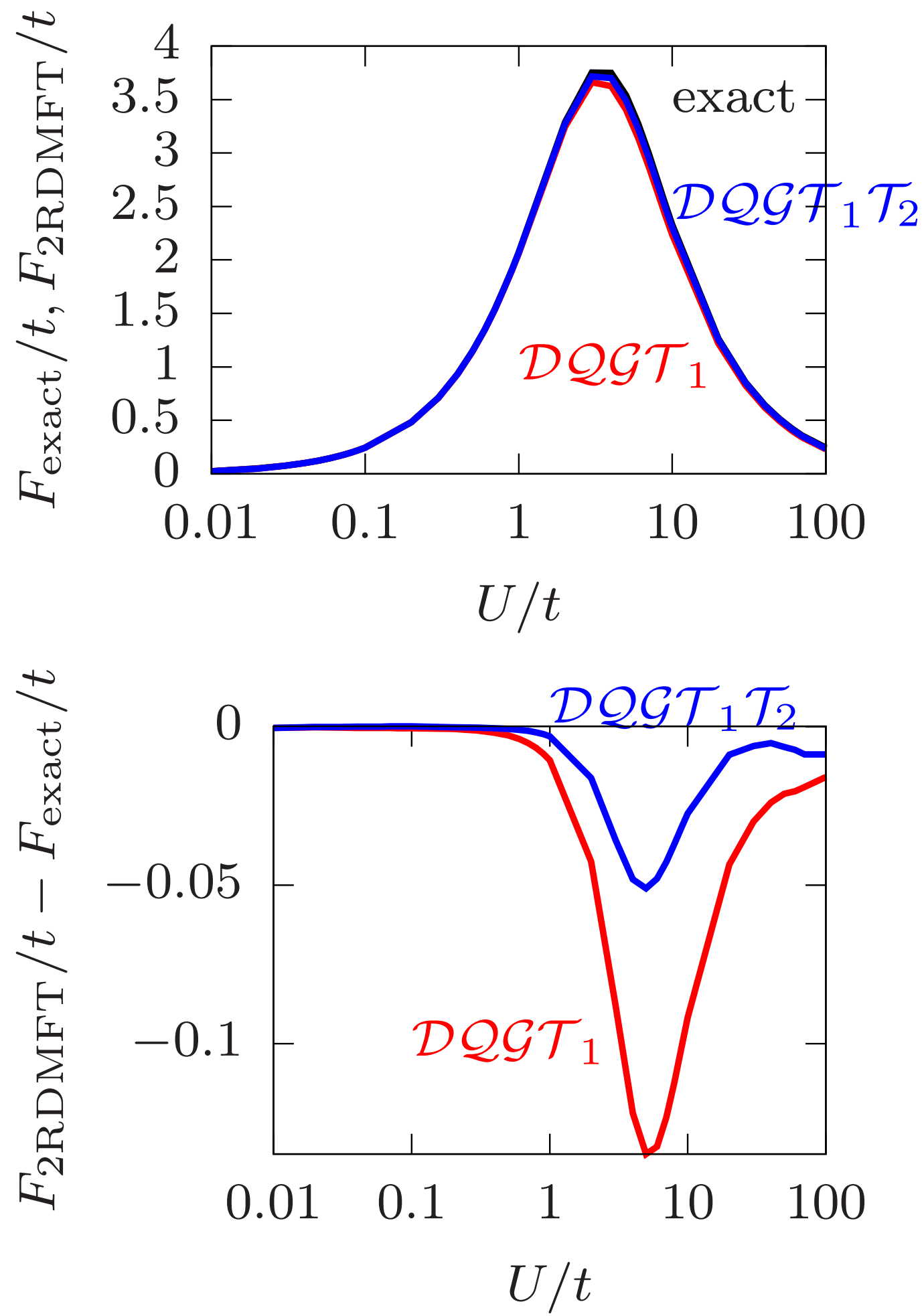

Figure 10.2.: Comparison of the exact density-matrix functional to values obtained from the scheme based on the N-representability conditions of the two-particle reduced density matrix proposed in section 10.7 .2 for a 10 -site half-filled Hubbard chain at different interaction strengths $U / t$. The solid red line shows results when the $\mathcal{D}$-, $\mathcal{Q}$-, $\mathcal{G}$ - and $\mathcal{T}_{1}$-conditions are enforced and the solid blue line when the additional $\mathcal{T}_{2}$-condition is also enforced. The lower graph shows the numerical difference between the exact results and the approximate results. 


\section{Application of the DF+RDMF scheme to real systems}

\subsection{General implementation details}

We have implemented the real-space-decomposition based DF+RDMF approach outlined in section 6.4 into the CP-PAW code. The minimization problem of the total energy in Eq. (6.52) is

$$
E[h]=\min _{\rho^{(1)}: 0 \leq \rho^{(1)} \leq 1, \operatorname{Tr} \rho^{(1)}=N}\left\{\operatorname{Tr}\left[\rho^{(1)} h\right]+U_{x c}[n]+E_{H}[n]+\sum_{R}\left(F^{\hat{W}_{R}}\left[\rho^{(1)}\right]-F_{\mathrm{DF}}^{\hat{W}_{R}}\left[\rho^{(1)}\right]\right)\right\} .
$$

We describe in this section the required additional approximations to solve this minimization problem in practice. We use the parametrization of LDA from Perdew and Wang [Perdew and Wang, 1992a], which contains a parametrization of the QMC-results of Ceperley and Alder [Ceperley and Alder, 1980]. We also include DFT-results obtained with the PBE functional [Perdew et al., 1996a] for comparison. The contribution $U_{x c}$ from the interaction to the exchange-correlation functional $E_{x c}$ is obtained by removing the contribution $T_{x c}$ of the kinetic energy from the exchange-correlation functional, i.e.,

$$
U_{x c}=E_{x c}-T_{x c} .
$$

$U_{x c}$ is given in Eq. (6.72). Results presented in this chapter are for the symmetric variant of the space decomposition function

$$
\begin{aligned}
\lambda_{\mathrm{sym}}\left(\vec{r}, \vec{r}^{\prime}\right) & =\sum_{R} \lambda_{\mathrm{sym}, \mathrm{R}}\left(\vec{r}, \vec{r}^{\prime}\right) \\
& =\sum_{R} f(|\vec{r}-\vec{R}|) f\left(\left|\vec{r}^{\prime}-\vec{R}\right|\right),
\end{aligned}
$$

because it allows a stronger spatial localization of the interaction $\hat{W}_{R}$,

$$
\hat{W}_{R}=\frac{1}{2} \int d^{4} \vec{x} \int d^{4} \vec{x}^{\prime} \frac{e^{2} \hat{\Psi}^{\dagger}(\vec{x}) \hat{\Psi}^{\dagger}\left(\vec{x}^{\prime}\right) \hat{\Psi}\left(\vec{x}^{\prime}\right) \hat{\Psi}(\vec{x}) \lambda_{\mathrm{sym}, \mathrm{R}}\left(\vec{r}, \vec{r}^{\prime}\right)}{4 \pi \epsilon_{0}\left|\vec{r}-\vec{r}^{\prime}\right|}
$$

The one-particle states $\left|\chi_{\alpha}\right\rangle$, Eq. (7.35), that were introduced in section 7.3.1 for the representation of the natural orbitals, are non-orthonormal. Please note, that the one-particle states include valence-like states as well as core-like states. Consequently, the local oneparticle reduced density matrix $\tilde{\rho}_{j, j^{\prime}}^{(1), \chi}$ defined in Eq. (7.72) is expressed in non-orthogonal one-particle states $\left|\chi_{j}\right\rangle=\left|\chi_{\alpha_{j}, \vec{T}_{j}}\right\rangle$ with the overlap matrix $\tilde{S}_{j, j^{\prime}}$ given by Eq. (7.73). We orthonormalize the states and obtain the orthonormal one-particle states $\left|\bar{\chi}_{j}\right\rangle$ as well as 
the transformed one-particle reduced density matrix $\bar{\rho}_{j, j^{\prime}}^{(1), \chi}$. We perform the orthonormalization with Löwdin's symmetric orthonormalization [Löwdin, 1948] that guarantees that the orthonormalized states are as similar to the original states as possible.

The density-matrix functional $F^{\hat{W}_{R}}\left[\rho^{(1)}\right]$ can only be evaluated approximately because $\rho^{(1)}$ denotes one-particle reduced density matrix in the complete one-particle basis. Instead, we approximate the density-matrix functional by the density-matrix functional of the local one-particle reduced density matrix $\tilde{\rho}_{j, j^{\prime}}^{(1), \chi}$ defined in Eq. (7.72) as

$$
F^{\hat{W}_{R}}\left[\rho^{(1)}\right] \approx F^{\hat{W}_{R}}\left[\bar{\rho}^{(1), \chi}\right] .
$$

The last step is to transform the matrix elements $U_{R, \alpha, \beta, \gamma, \delta}$ of the interaction $\hat{W}_{R}$ discussed in section 6.4.5 from the non-orthonormal states $\left|\chi_{j}\right\rangle$ to the orthonormal states $\left|\bar{\chi}_{j}\right\rangle$. Optionally, we neglect small elements of the $\bar{U}_{R, \alpha, \beta, \gamma, \delta}$, that result from the orthonormalization, to make the adaptive cluster approximation applicable. With these ingredients, i.e., the matrix elements of the local interaction $\bar{U}_{R, \alpha, \beta, \gamma, \delta}$ and the one-particle reduced density matrix $\bar{\rho}_{j, j^{\prime}}^{(1), \chi}$, we can evaluate the density-matrix functional with one of the methods proposed in the previous chapters. We employ the complete-active-space wave function defined in Eq. (9.17) We evaluate the density-matrix functional with the configurationinteraction-like ansatz proposed in section 9.4. If numerically viable, like in case of the hydrogen molecule, we include all Slater determinants in the ansatz. Otherwise, we apply the adaptive cluster approximation. Details for $\mathrm{NiO}$ will be discussed in section 11.3.4.

The local double-counting contribution $F_{D F}^{\hat{W}_{R}}$ from the density-functional defined in Eq. (6.53) is evaluated in the local density approximation as given in Eq. (6.81) and Eq. (6.82). It is evaluated for the electron density of the local one-particle reduced density matrix $\bar{\rho}^{(1), \chi}$. The difference of the results of a full six-dimensional adaptive integration of Eq. (6.78) and the full six-dimensional adaptive integration of Eq. (6.80) are negligible for the cases investigated in this thesis. The three-dimensional integration in Eq. (6.81) is performed with the help of a Lebedev-Laikov grid [Lebedev and Laikov, 1999] of order 35 for the angular dependency and Chebyshev-Gauss grids [Treutler and Ahlrichs, 1995; Becke, 1988] for the radial dependency. The center of the grid is the position $\vec{R}$. We have generated the grids with the software package pyscf [Sun et al.] ${ }^{1}$. The two-dimensional integration in Eq. (6.82) can be written as a one-dimensional integration if we perform the angular integration analytically. The resulting one-dimensional integration is solved with an adaptive integration [Berntsen et al., 1991] from the software package cubature ${ }^{2}$. The proposed combination of a three-dimensional fixed grid with a one-dimensional adaptive integration allows the efficient numerical evaluation of the local double-counting term, and the evaluation can easily be optimized further by constructing an approximate parametrization of $\tilde{\epsilon}_{x c}(\tilde{r}, n, \eta)$ defined in Eq. (6.82).

In practice the minimization is not performed over all elements of the one-particle reduced density matrix $\rho^{(1)}$ but over natural orbitals $\left|\psi_{n}\right\rangle$ and occupations $f_{n}$ so that only natural orbitals with non-zero occupations have to be considered explicitly. The natural orbitals are represented in the PAW-representation, i.e., $\left|\psi_{n}\right\rangle=\mathcal{T}\left|\tilde{\psi}_{n}\right\rangle$. The minimization is performed over the parameters of the pseudo partial waves $\left|\tilde{\psi}_{n}\right\rangle$ defined in Eq. (7.20). The minimization over the pseudo partial waves is solved with the CarParrinello-like minimization scheme outlined in section 4.6. The resulting form of the

\footnotetext{
${ }^{1}$ pyscf 1.5 .3 https://github.com/sunqm/pyscf

${ }^{2}$ cubature 1.0.3 written by Steven G. Johnson https://github.com/stevengj/cubature
} 
minimization problem of the real-space-decomposition based $\mathrm{DF}+\mathrm{RDMF}$ approach in the PAW-formalism is

$$
\begin{aligned}
E_{N}=\min _{\left\{f_{n}\right\},\left\{\left|\tilde{\psi}_{n}\right\rangle\right\}: \sum_{n} f_{n}=N,\left\langle\tilde{\psi}_{i}\left|\mathcal{T}^{\dagger} \mathcal{T}\right| \tilde{\psi}_{j}\right\rangle=\delta_{i, j}} & \left\{E_{\mathrm{DFT}, u_{x c}}\left[\left\{f_{n}\right\},\left\{\left|\tilde{\psi}_{n}\right\rangle\right\}\right]\right. \\
& +\sum_{R}\left(F^{\hat{W}_{R}}\left[\rho^{(1), \chi}\right]-F_{\mathrm{DF}}^{\hat{W}_{R}}\left[n\left[\rho^{(1), \chi}\right]\right)\right\} .
\end{aligned}
$$

The first term $E_{\mathrm{DFT}, \mathrm{u}_{\mathrm{xc}}}\left[\left\{f_{n}\right\},\left\{\left|\tilde{\psi}_{n}\right\rangle\right\}\right]$ denotes the DFT-total energy given in Eq. (7.26) with $\epsilon_{x c}$ replaced by $u_{x c}=\epsilon_{x c}-t_{x c}$. The local one-particle reduced density matrix $\rho^{(1), \chi}$ depends on the occupations $f_{n}$ and pseudo wave functions $\left|\tilde{\psi}_{n}\right\rangle$.

The DFT and DF+RDMF calculations presented in the subsequent sections have been performed with the CP-PAW code. For this purpose, the hydrogen molecule was placed in a large cell so that atoms of periodic images of hydrogen molecules had a least a distance of 10 Åand we have used an electrostatic decoupling of periodic images [Blöchl, 1995]. The augmentation in the PAW method was generated with the scheme proposed by Blöchl and is documented in detail in [Schade, 2012]. The full-configuration-interaction calculations for the hydrogen molecule have been performed in the cc-pVQZ basis [Dunning, 1989a] in the quantum-chemistry code Orca [Neese, 2012].

The $\mathrm{k}$-point grid for $\mathrm{NiO}$ was chosen as a grid of $7 \times 7 \times 7$ divisions. No spatial symmetries have been employed in the calculations. For nickel, the $3 \mathrm{~d}-$ and $4 \mathrm{~s}-$ states have been treated as the valence states in the PAW construction, Eq. (7.10). For oxygen, the $2 \mathrm{~s}-$ and $2 \mathrm{p}-$ states have been treated as valence states. We have used one projector for every valence state. The augmentation in the PAW method was generated with the scheme proposed by Blöchl and is documented in detail in [Schade, 2012]. We chose the low-temperature lattice constant of $4.17 \AA$ for all calculations of $\mathrm{NiO}$ presented in this thesis.

\subsection{Hydrogen molecule}

We have discussed the properties of the hydrogen molecule as the simplest case of the static-correlation problem in section 4.9. Here, we explore if the proposed DF+RDMF scheme can describe static correlation in the non-spin polarized hydrogen molecule correctly. A proper treatment of the hydrogen molecule requires the $\mu \rightarrow 0$-limit, i.e., the space-decomposition function $\lambda\left(\vec{r}, \vec{r}^{\prime}\right)=1$ discussed in section 6.4.3. This is the RDMFT-limit given by Eq. (6.65) and identical to the RDMFT-limit of the orbital-based $\mathrm{DF}+\mathrm{RDMF}$ approach given by Eq. (6.32). However, we expect the results to differ slightly because the local one-particle states do not form a complete basis. We chose the atomic 1s-orbitals of both atoms as the local one-particle basis. Thus, the local one-particle states consists of the four states $\left.\left|\chi_{\alpha}\right\rangle \in\left\{\left|\chi_{1, \uparrow}\right\rangle,\left|\chi_{1, \downarrow},\right| \chi_{2, \uparrow}\right\rangle,\left|\chi_{2, \downarrow}\right\rangle\right\}=C$ defined by Eq. (4.73)Eq. (4.76). We approximate the density-matrix functional $F^{\hat{W}}\left[\rho^{(1)}\right]$ as the density-matrix 
functional in the four-dimensional one-particle basis, i.e.,

$$
\begin{aligned}
F^{\hat{W}}\left[\rho^{(1)}\right] & \approx F^{\hat{W}^{\prime}}\left[\rho^{\chi,(1)}\right] \\
\hat{W}^{\prime} & =\frac{1}{2} \sum_{\alpha, \beta, \gamma, \delta \in C} U_{\alpha, \beta, \gamma, \delta} \hat{c}_{\alpha}^{\dagger} \hat{c}_{\beta}^{\dagger} \hat{c}_{\delta} \hat{c}_{\gamma} \\
U_{\alpha, \beta, \gamma, \delta} & =\int d^{4} \vec{x} \int d^{4} \vec{x}^{\prime} \frac{e^{2}}{4 \pi \epsilon_{0}\left|\vec{r}-\vec{r}^{\prime}\right|} \chi_{\alpha}^{*}(\vec{x}) \chi_{\beta}^{*}\left(\vec{x}^{\prime}\right) \chi_{\gamma}(\vec{x}) \chi_{\delta}\left(\vec{x}^{\prime}\right) .
\end{aligned}
$$

The resulting dissociation curve of the non-spin-polarized hydrogen molecule within the real-space-decomposition based DF+RDMF approach is shown in Figure 11.1 in comparison to results from DFT with a semi-local density-functional and exact results from full configuration interaction in the cc-pVQZ basis [Dunning, 1989a]. The correct dissociation limit is reproduced in the real-space-decomposition based DF+RDMF approach as well as in the orbital-based DF+RDMF scheme. However, the total energy close to the equilibrium distance is overestimated even more than in the orbital-based DF+RDMF scheme. The main reason is a different treatment of the double counting term $F_{D F}^{\hat{W}}\left[\rho^{(1)}\right]$ : in our implementation of the orbital-based scheme it is evaluated for the electron density of the natural orbitals whereas in our implementation of the real-space scheme it is evaluated for the electron density of the local one-particle reduced density matrix. Figure 11.2 compares the particle number of the local one-particle states to the total particle number $N=2$. In the vicinity of the equilibrium distance the local one-particle states consisting of atomic 1s-states of the hydrogen atom fails to describe the natural orbitals and thus leads to a deficiency of the total particle number. Figure 11.3 shows the norm $O_{n, n}=\left\langle\mu_{n} \mid \mu_{n}\right\rangle$ of the projected natural orbitals $\left|\mu_{n}\right\rangle$ for all natural orbitals used in the calculation. A norm below one, i.e., $O_{n, n}<1$, indicates that the chosen one-particle states are not suitable to describe the natural orbitals. Thus, all natural orbitals except two cannot be described by the 1s-states at all. The two natural orbitals that can be described show a deficiency around the equilibrium distance. The deficiency of the local one-particle states can be cured by either adding some suitable one-particle states to the set of local one-particle states or by choosing a minimal basis that depends on the distance of the atoms. Finally, figure 11.4 shows the occupations of the ground state of the hydrogen molecule in the real-space-decomposition $\mathrm{DF}+\mathrm{RDMF}$ approach compared to exact full-configuration interaction results. The dependence of the occupations on the distance of the hydrogen atoms is nicely reproduced by the occupations $f_{i}$ and by the occupation of the local oneparticle reduced density matrix. The results of the chemical potential in the dissociation limit from the real-space-decomposition DF+RDMF approach are visually identical to the results shown for the orbital-based approach in Figure 6.1 and, thus, not shown here. In conclusion, for the hydrogen molecule, the orbital-based DF+RDMF approach and the real-space-decomposition based approach give very similar results. The underlying reason is that the RDMFT-limit is identical in both approaches. Numerical differences result only from implementation choices. 


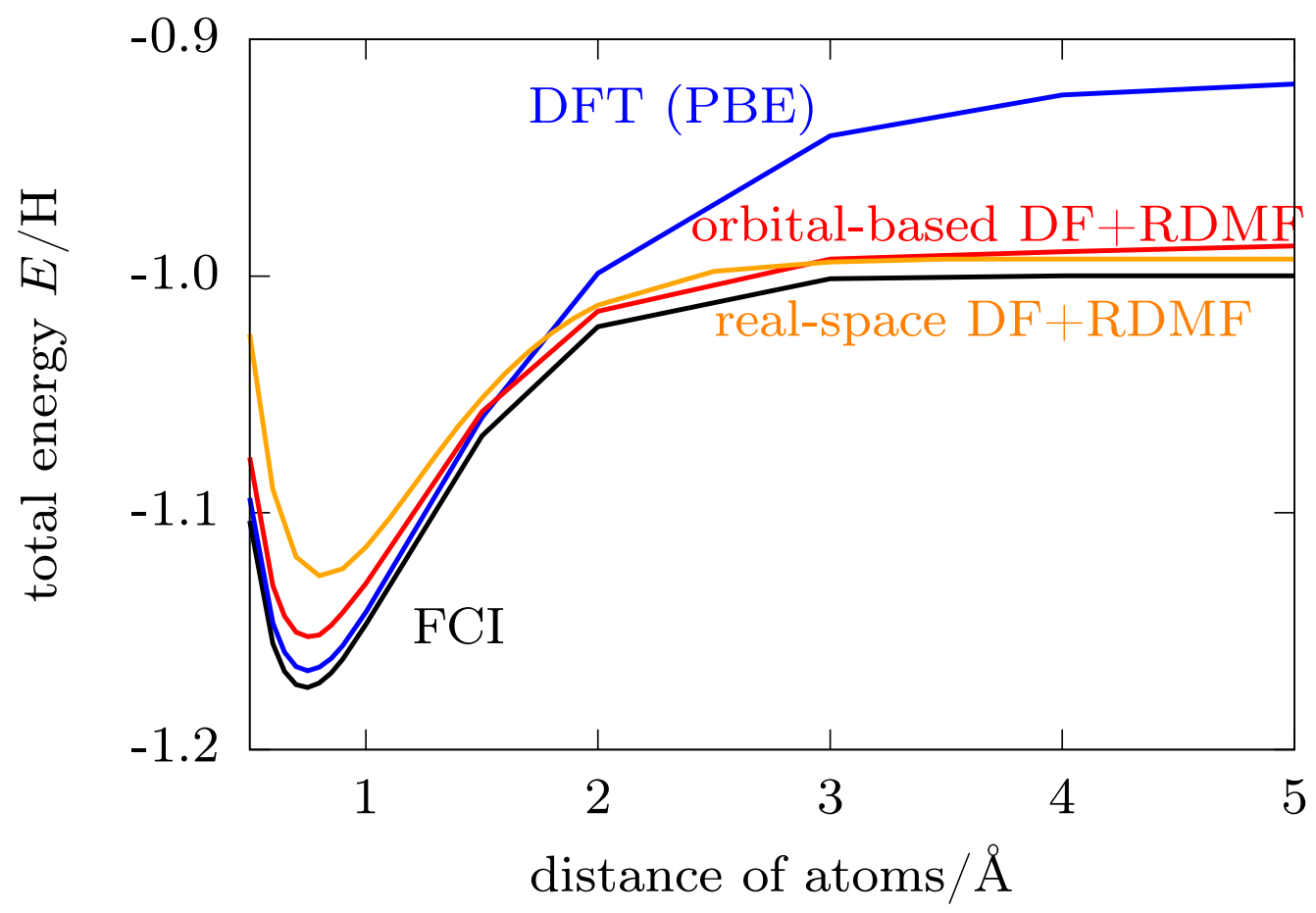

Figure 11.1.: Dissociation curve of the non-spin-polarized hydrogen molecule. The figures compare results from density-functional theory with the approximate PBE functional [Perdew et al., 1996a], full-configuration interaction results (FCI, cc-pVQZ basis [Dunning, 1989a] computed with Orca [Neese, 2012]), from the orbital-based DF+RDMF approach described in section 6.3 and results from the real-space-decomposition based approach. 


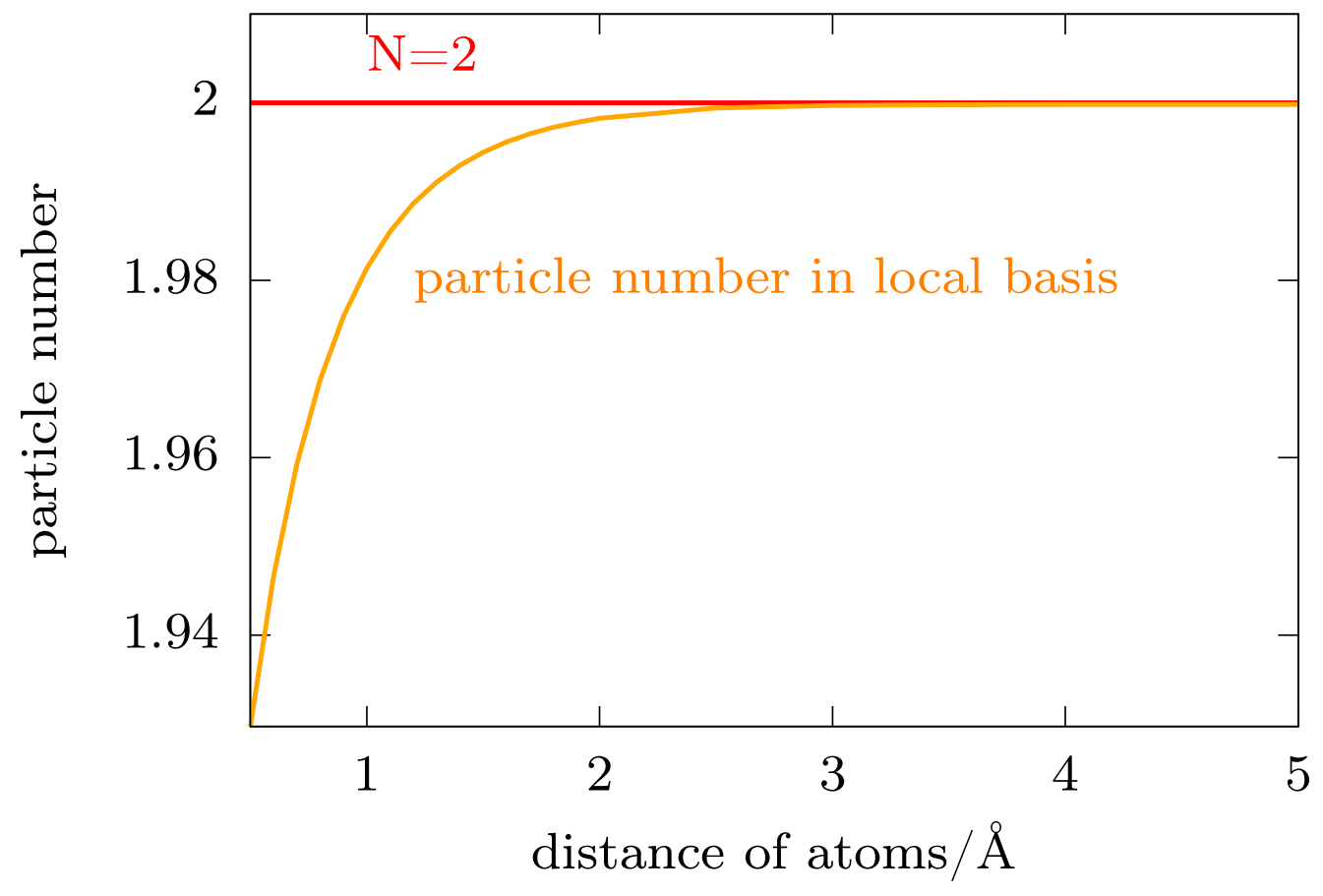

Figure 11.2.: Total particle number in the local one-particle basis within the real-spacedecomposition DF+RDMF approach for the non-spin-polarized hydrogen molecule.

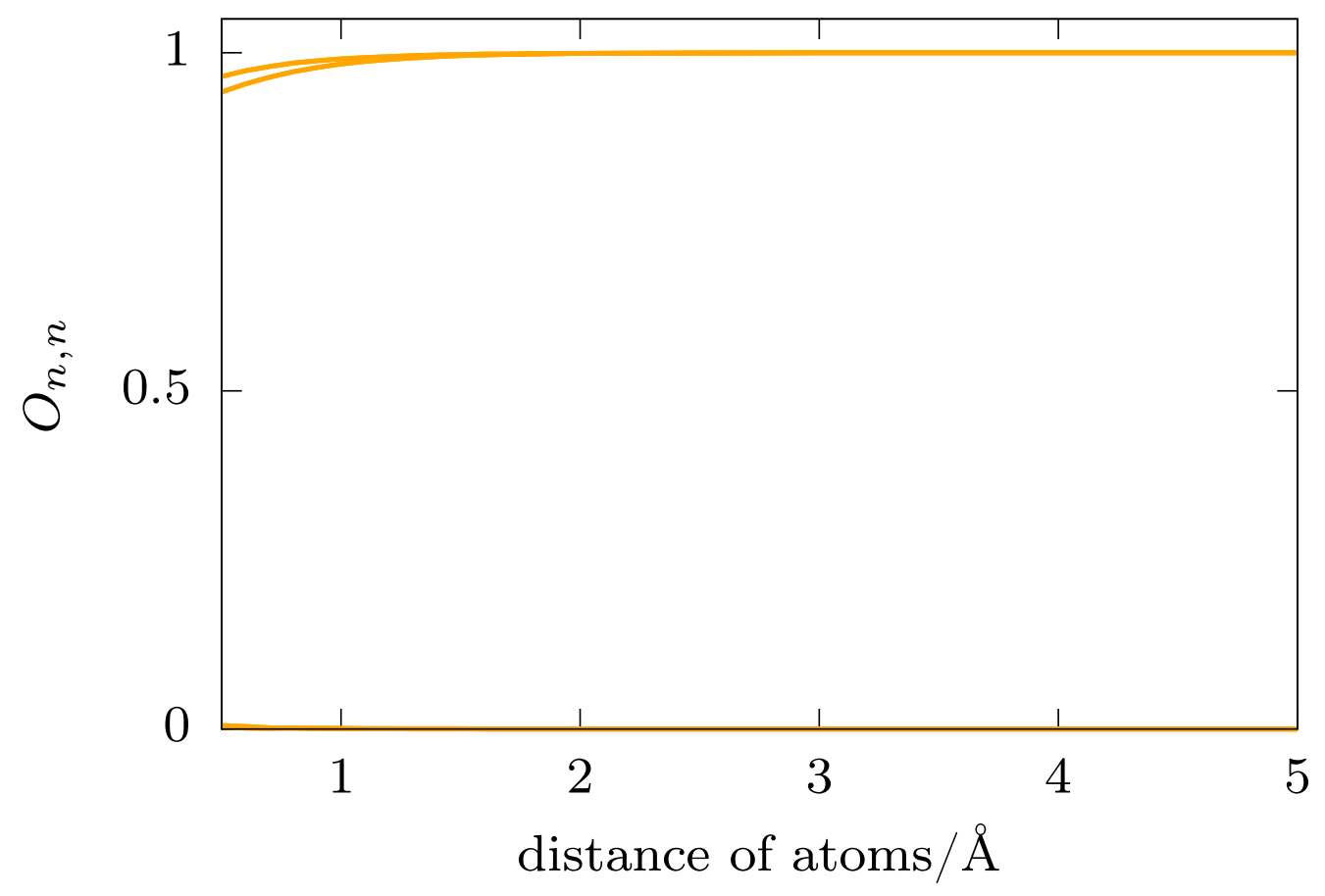

Figure 11.3.: Norm $O_{n, n}=\left\langle\mu_{n} \mid \mu_{n}\right\rangle$ of the projected natural orbitals $\left|\mu_{n}\right\rangle$ for the ground state of the non-spin-polarized hydrogen molecule within the real-spacedecomposition DF+RDMF approach. 


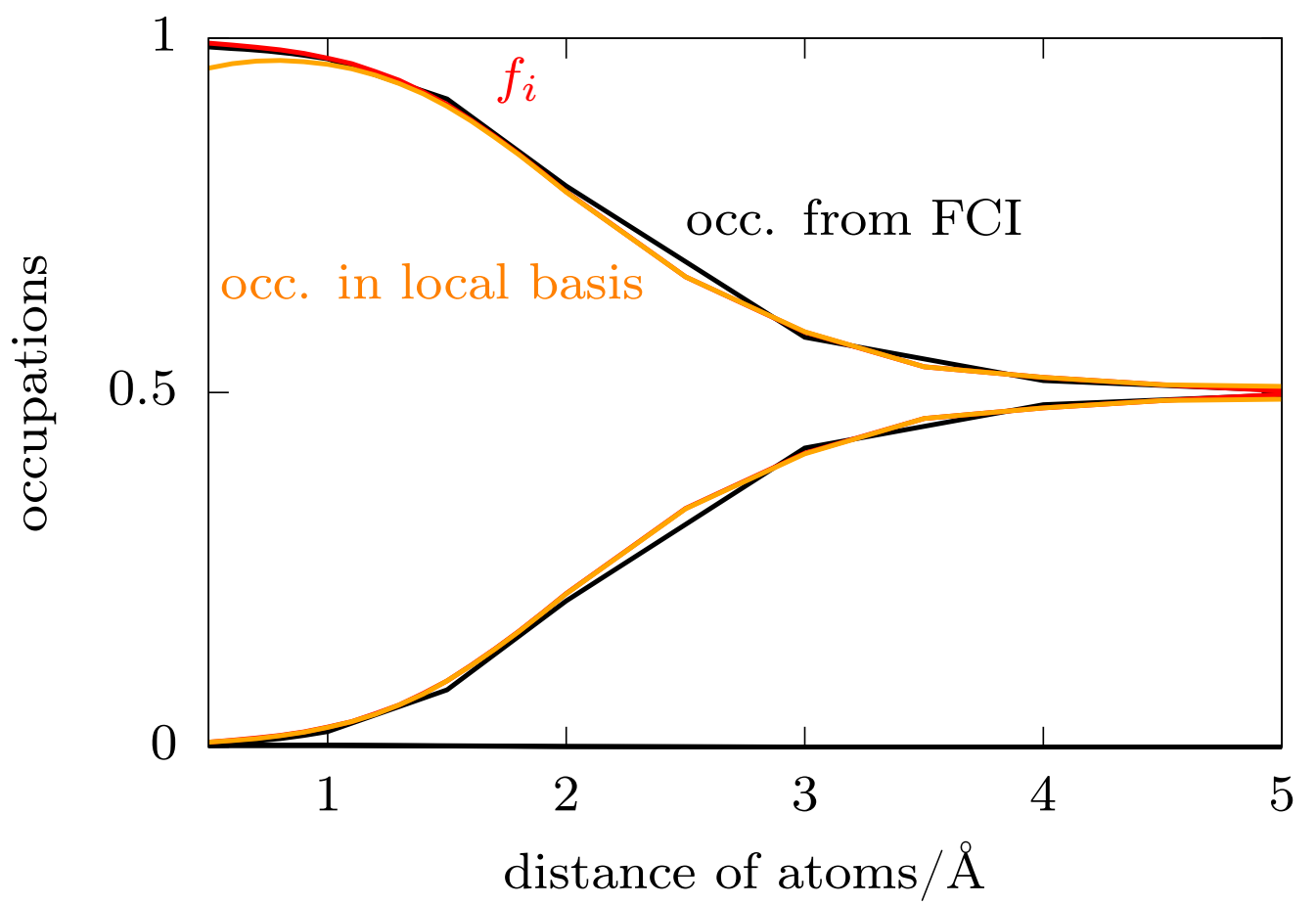

Figure 11.4.: Occupations of the non-spin-polarized ground state of the non-spinpolarized hydrogen molecule. The occupations $f_{i}$ of the real-space-based $\mathrm{DF}+\mathrm{RDMF}$ approach are compared to the occupations of the local oneparticle reduced density matrix and full-configuration interaction results (FCI, cc-pVQZ basis [Dunning, 1989a] computed with Orca [Neese, 2012]). 


\section{3. $\mathrm{NiO}$}

\subsubsection{Properties of $\mathrm{NiO}$}

The results for the dissociation of the non-spin-polarized hydrogen molecule presented in the previous section have shown that the proposed DF+RDMF schemes can describe static correlation. As an example of a solid with strong local electronic correlations, we study nickel oxide in this section. $\mathrm{NiO}$ has been studied intensely with theoretical and experimental methods. For a review, we refer the reader to [Kuo et al., 2017]. The crystal structure of $\mathrm{NiO}$ is the rocksalt structure $(\mathrm{Fm} \overline{3} \mathrm{~m})$ shown in figure 11.5 [Roth, 1958a,b]. Below its Néel-temperature of $523 \mathrm{~K}$ [Roth, 1958a] it is an antiferromagnetic insulator with antiferromagnetic (111)-planes. The lattice constant is $4.17 \AA$ and NiO has a small trigonal distortion [Bartel and Morosin, 1971] at low temperatures. Upon increase of the temperature the, distortion is reduced and the lattice constant increases. The distortion vanishes at the Néel-temperature and the lattice constant is $4.19 \AA$ at this temperature. At $700 \mathrm{~K}$, the lattice constant reaches about $4.20 \AA$ [Bartel and Morosin, 1971]. The nickel ion in $\mathrm{NiO}$ is in a $3 \mathrm{~d}^{8}$ configuration. A nickel atom is surrounded by six oxygen atoms in an octahedral arrangement. The $\mathrm{t}_{2 g}$ states are lower in energy than the $\mathrm{e}_{g}$ states $\left(\mathrm{d}_{z^{2}}\right.$ and $\mathrm{d}_{x^{2}-y^{2}}$ orbitals). The crystal-field splitting $10 D q$ has been estimated to about $0.5 \mathrm{eV}$ [Haverkort et al., 2012].

Already De Boer and Verwey [de Boer and Verwey, 1937] have pointed out that the insulating nature of $\mathrm{NiO}$ cannot be understood in a simple band theory, because it is insulating even though it has a partially filled valence band, which is the $3 \mathrm{~d}$-band of nickel. The reason for the insulating behavior is the strong Coulomb interaction between the $3 \mathrm{~d}-$ states, that are partially filled and localized [Mott, 1949; Hubbard, 1963; Sawatzky and Allen, 1984; Brandow, 1977]. The band gap in antiferromagnetic NiO has been measured to about $4 \mathrm{eV}$ by a combination of x-ray-photoemission and bremsstrahlungisochromatspectroscopy [Sawatzky and Allen, 1984; Hüfner et al., 1984]. DFT calculations with a local or semi-local density-functional predict a metal in non-spin-polarized calculations and an antiferromagnetic ground state with a tiny gap of $0.1-0.4 \mathrm{eV}$ in spin-polarized calculations [Oguchi et al., 1983]. This failure of local and semi-local density-functionals explains the interest in the treatment of $\mathrm{NiO}$ with more advanced methods.

The Hubbard $U$ and the charge-transfer-gap $\Delta$ discussed in section 2.10 .2 are comparable in size for $\mathrm{NiO}$ [Kuo et al., 2017]. Therefore, it is not obvious if $\mathrm{NiO}$ should be defined as a Mott insulator or a charge-transfer insulator and the widely accepted classification as a charge-transfer insulator [Hüfner and Riesterer, 1986; Kuo et al., 2017] has been challenged by some theoretical [Hugel and Kamal, 1997] and experimental studies [Schuler et al., 2005]. The first ionization state for a Mott insulator is a $\mathrm{d}^{n-1}$ state of the atomic multiplet. In contrast, for a charge-transfer insulator, the first ionization state is a $\mathrm{d}^{n} \mathrm{~L}^{-1}$ state, where $\mathrm{L}^{-1}$ denotes a hole on the oxygen 2 p ligand. Figure 11.6 shows the valenceband photoemission spectra of $\mathrm{NiO}$ obtained by Tjernberg et al. [Tjernberg et al., 1996]. The figure, on the one hand, shows the photoemission spectrum for the antiferromagnetic state at $500 \mathrm{~K}$ and, on the other hand, the spectrum for the paramagnetic state at $615 \mathrm{~K}$. Both spectra are fairly similar. Only the peak-widths and heights of peaks show small differences between the antiferromagnetic and the paramagnetic state. Thus, the long-range antiferromagnetic order has no significant influence on the electronic structure. The resistivity measurements in Figure 11.7 obtained by Yamaka et al. [Yamaka and Sawamoto, 


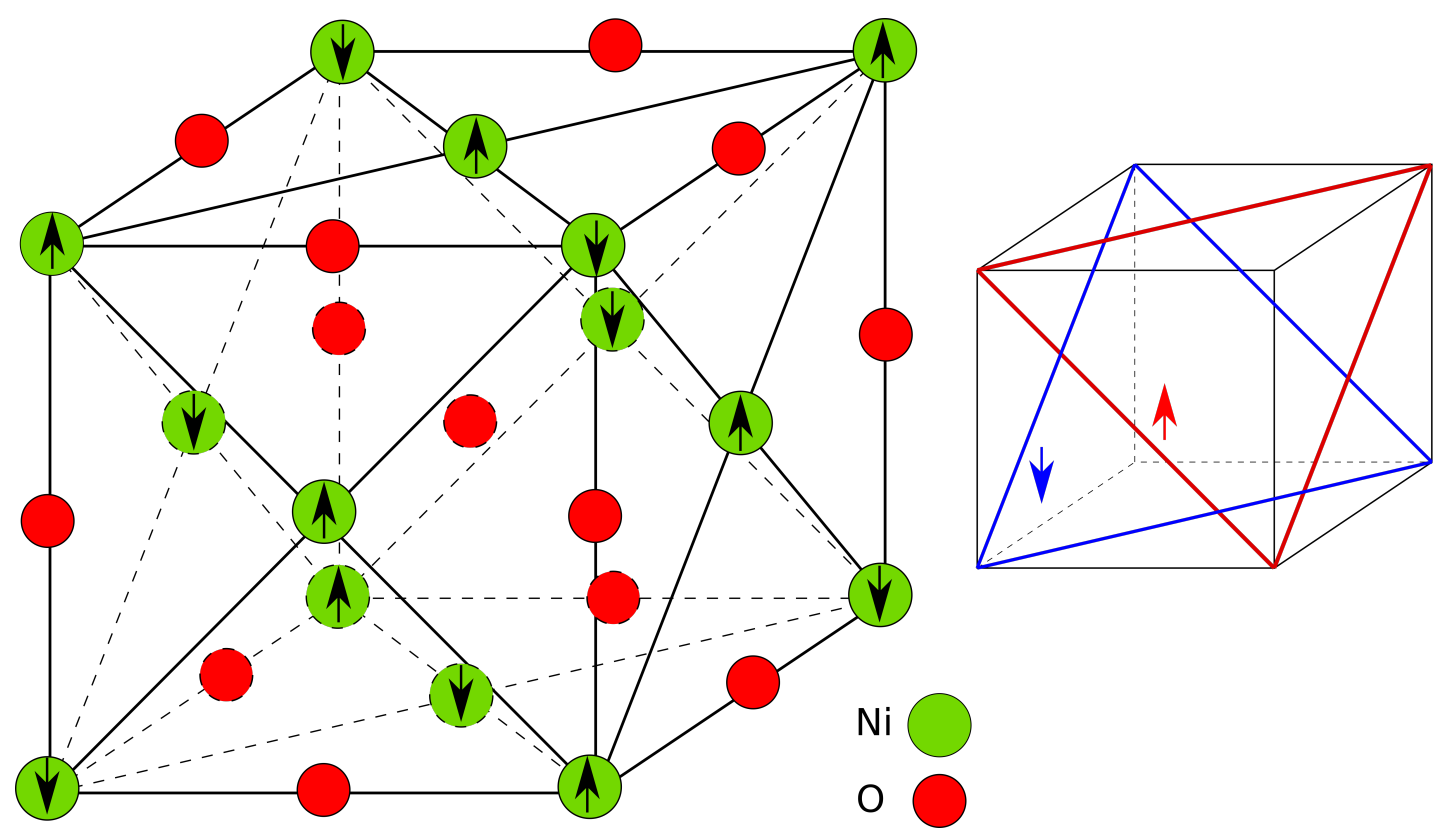

Figure 11.5.: Schematic representation of the crystal structure of $\mathrm{NiO}$ (left) and the arrangement of the antiferromagnetic planes (right). The arrows indicate the low-temperature antiferromagnetic structure.

1958] show no insulator-to-metal transition in the vicinity of the Néel-temperature. We conclude that the insulating behavior does not depend on the antiferromagnetic order and that $\mathrm{NiO}$ is an insulating paramagnet above the Néel-temperature.

In order to be able to obtain a paramagnetic state of a material that is an antiferromagnet at low temperatures, a finite-temperature theory is required. Even though it is in principle straightforward to extend the proposed DF+RDMF approaches to finite temperatures, the evaluation of the density-matrix functional at finite temperatures with a wave-function based approach is challenging. The challenge originates from the need to use a sufficiently large ensemble of many-particle wave functions instead of a single manyparticle wave function. The number of required many-particle wave functions depends on the temperature so that low temperatures only require a small number of wave functions. For the evaluation of the density-matrix functional at finite temperatures other methods like [Blöchl et al., 2013] are more promising than the wave fucntion based approach. As a workaround we consider the nonmagnetic state of $\mathrm{NiO}$ at zero temperature as an approximation of the high-temperature paramagnetic phase. Thus, the primary goal of this chapter is to study the predictions of the real-space-decomposition based DF + RDMF approach for the nonmagnetic phase of $\mathrm{NiO}$.

\subsubsection{DFT ground state}

We have calculated the nonmagnetic and the antiferromagnetic ground state of $\mathrm{NiO}$ in LDA/LSDA-DFT and GGA-DFT with the CP-PAW code. Figure 11.8 shows the projected density of states of the non-spin-polarized ground state of $\mathrm{NiO}$ with the LDAfunctional [Perdew and Wang, 1992a] and the PBE-functional [Perdew et al., 1996a]. Both functionals predict a metal in the nonmagnetic ground state and there are no qual- 


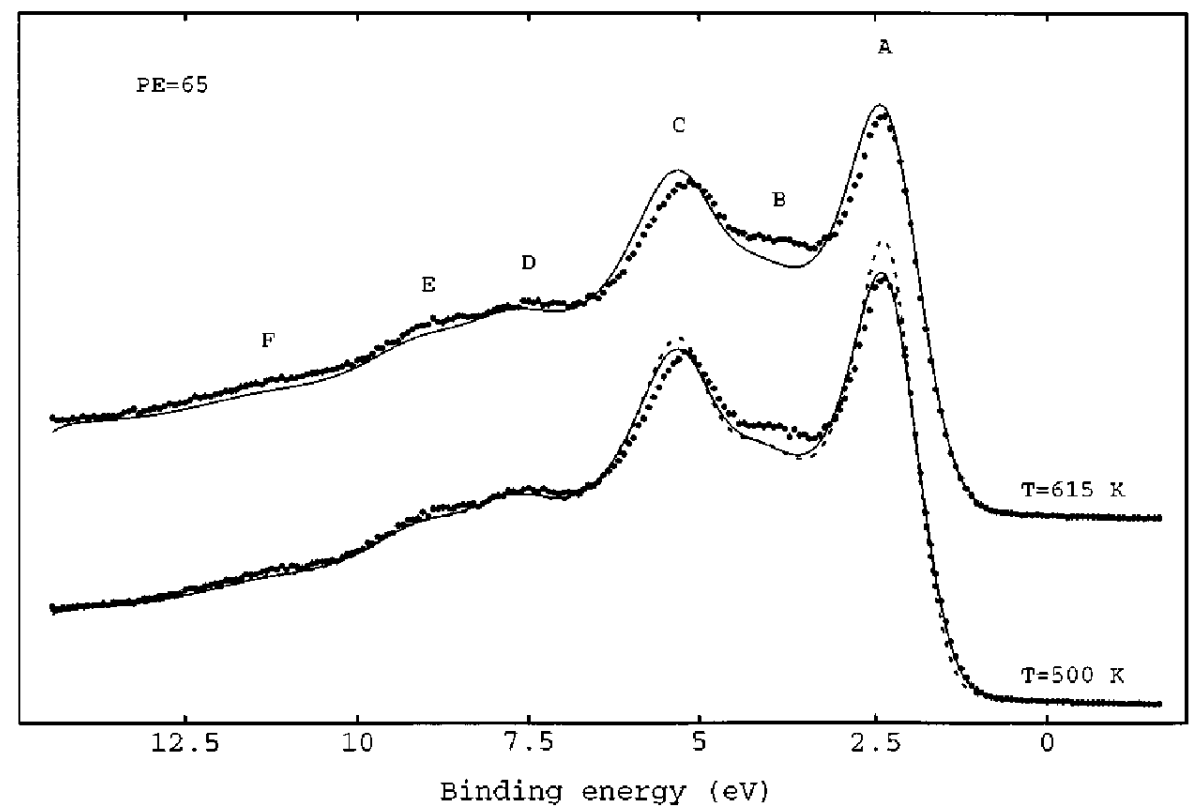

Figure 11.6.: Valence-band photoemission spectra of $\mathrm{NiO}$ in the antiferromagnetic state at $500 \mathrm{~K}$ and in the paramagnetic state at $615 \mathrm{~K}$ from [Tjernberg et al., 1996]. The measurements have been performed for photon energies of 65 $\mathrm{eV}$. The spectra have been shifted horizontally to improve visibility. The dots represent measurement results, and the lines include a broadening with Gaussians (solid line: 0.55 eV FWHM, dashed line: 0.35 eV FWHM). Graph from [Tjernberg et al., 1996]. 


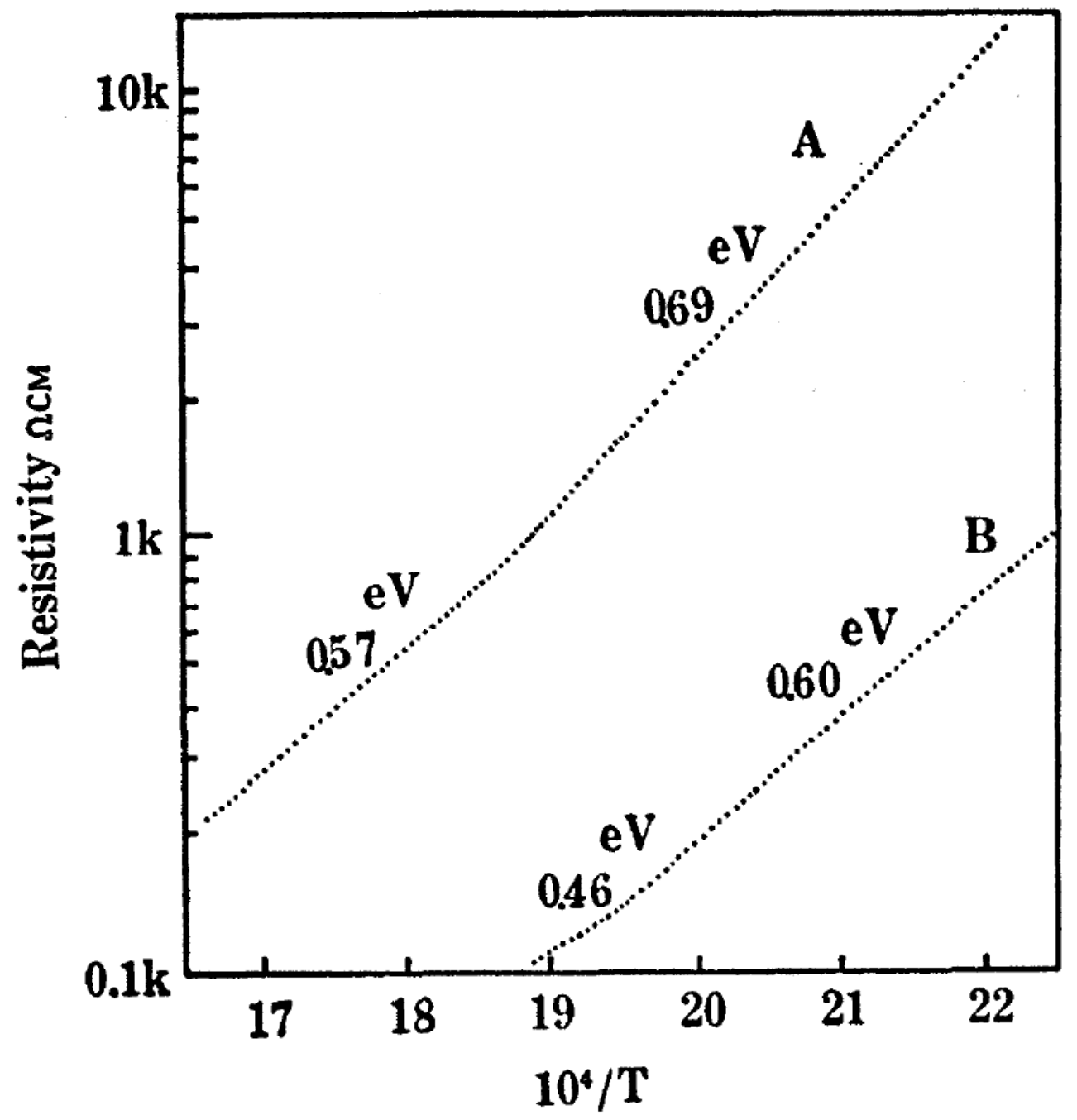

Figure 11.7.: Resistivity of $\mathrm{NiO}$ single crystals (upper line) and with $\mathrm{Li}$ ions added as impurities (lower line) from [Yamaka and Sawamoto, 1958]. Graph from [Yamaka and Sawamoto, 1958]. 
itative differences of the projected density of states of the two functionals. The states at the Fermi level are mostly of Ni-e $g$ character $\left(\mathrm{d}_{z^{2}}\right.$ and $\mathrm{d}_{x^{2}-y^{2}}$ orbitals $)$. The $\mathrm{t}_{2 g}$ states $\left(\mathrm{d}_{x y}, \mathrm{~d}_{y z}\right.$ and $\mathrm{d}_{x z}$ orbitals) of nickel lie just below the Fermi level. Thus, we only consider the LDA-functional for the remaining calculations because the GGA exchange-correlation functional does not improve the description of static correlation. The removal of the kinetic correlation $T_{c}$ of the exchange-correlation functional does not influence the projected densities of states shown in Figure 11.9 in a qualitative way but only shifts the states slightly. The generalization of the LDA-functional to systems with non-zero magnetization, i.e., the local spin-density functional theory (LSDA) predicts an antiferromagnetic ground state of $\mathrm{NiO}$ with a band gap of about $0.5 \mathrm{eV}$. We have performed the antiferromagnetic calculation in a unit cell with two nickel atoms, i.e., $\mathrm{Ni} 1$ and $\mathrm{Ni} 2$, and two oxygen atoms. The projected densities of states of the antiferromagnetic LDA-ground state are shown in Figure 11.10. The states just below the band gap have predominately $\mathrm{Ni}-\mathrm{t}_{2 g}$-character and the states just above the band gap have predominately $\mathrm{Ni}-\mathrm{e}_{g}$ character. However, the states just above and just below the band gap also have some oxygen-p character which indicates a small hybridization between the states of the oxygen ligands and the localized 3d-states of nickel. The bottom plot in Figure 11.10 shows the spin-resolved projected densities of states of the $t_{2 g^{-}}$and $\mathrm{e}_{g^{-}}$-states of the individual nickel atoms. The two $t_{2 g^{-}}$and $\mathrm{e}_{g^{-}}$-states of the two nickel atoms have opposite spin-directions.

\subsubsection{Local one-particle states and decomposition function $f(r)$}

The new one-particle basis $\left|\chi_{\alpha}\right\rangle$ in Eq. (7.35) for the definition of the local one-particle reduced density matrix $\rho^{(1), \chi}$ is constructed by first performing a DFT-calculation of isolated atoms for oxygen and nickel. We expanded the radial dependencies of the resulting Kohn-Sham wave functions in terms of Gaussians with exponents from the cc-pwCV5Z basis set [Dunning, 1989b; Peterson and Dunning, 2002; Balabanov and Peterson, 2005]. Details of this approach are described in section 7.3.1. Thus, the new one-particle basis consists of 15 one-particle states located at every nickel atom and five one-particle states at every oxygen atom. These counts include core-like one-particle states. This is the basis used for the GTO-approximation of the natural orbitals $\left|\psi_{n}\right\rangle$, that are represented with augmented plane waves in the PAW-formalism.

The choice of the space-decomposition function $\lambda_{R}\left(\vec{r}, \vec{r}^{\prime}\right)$ depends on the physical situation at hand. To describe the local atomic physics of the transition-metal ions of $\mathrm{NiO}$ with the density-matrix functional, we choose the symmetric variant of the space-decomposition function in Eq. (6.54)

$$
\lambda_{\text {sym }, \vec{R}}\left(\vec{r}, \vec{r}^{\prime}\right)=f(|\vec{r}-\vec{R}|) f\left(\left|\vec{r}^{\prime}-\vec{R}\right|\right)
$$

and select the positions of the nickel atoms as the centers $\vec{R}$. We choose the function $f(r)$ as a sum of five Gaussians

$$
f(r)=e^{-3.025 r^{2}}+4.2 \cdot\left(e^{-0.6 r^{2}}-e^{-1.11 r^{2}}\right)+85 \cdot\left(e^{-3.625 r^{2}}-e^{-3.6365 r^{2}}\right),
$$

so that it approximates a smoothened step function. The function $f(r)$ is shown in Figure 11.11. 

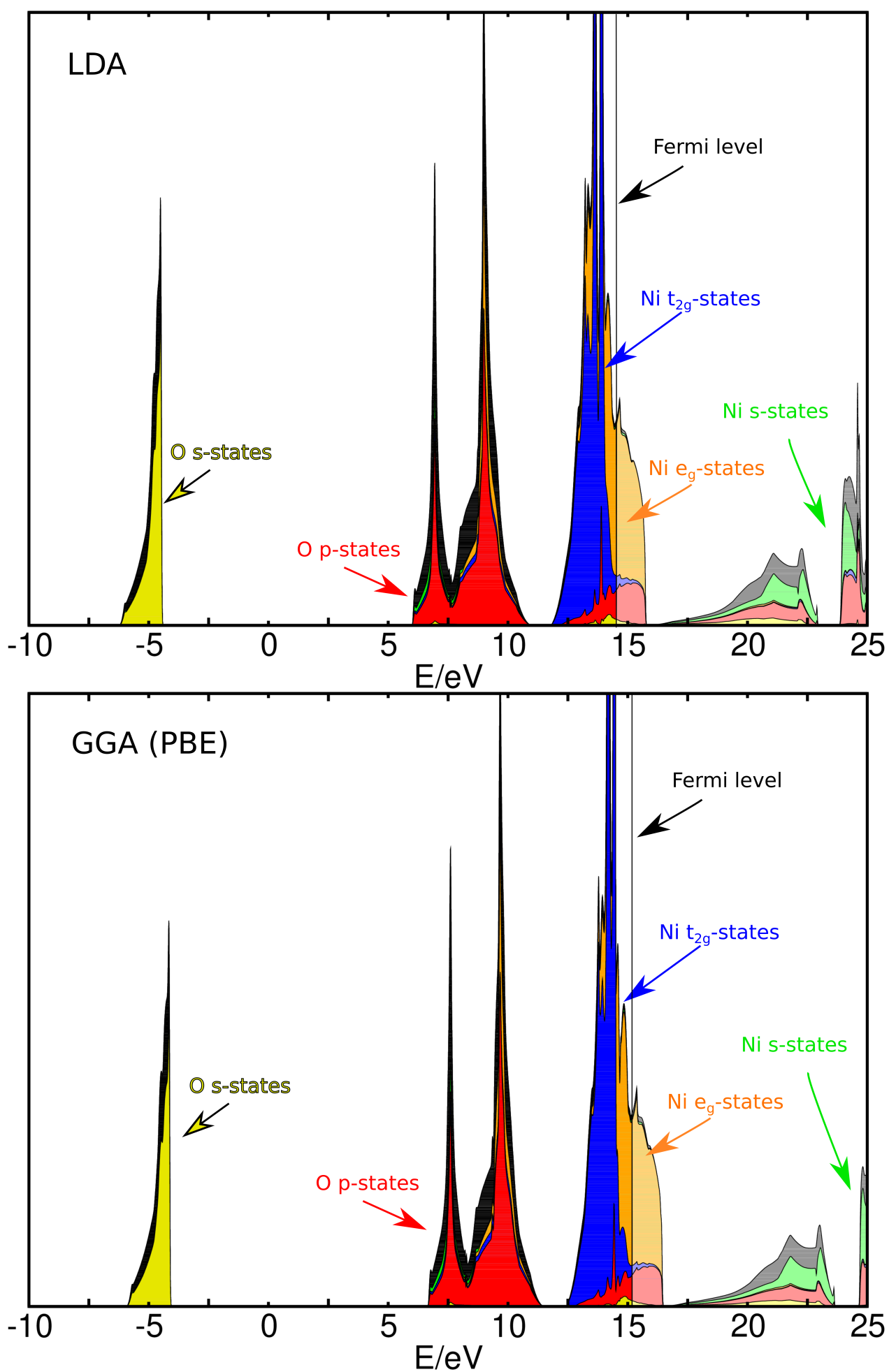

Figure 11.8.: Projected density of states of the nonmagnetic ground state of $\mathrm{NiO}$ in DFT with the LDA-functional (top figure) and the PBE functional (bottom figure). Details of the calculation as discussed in section 11.1. The projected densities of states are stacked in front of the total density of states (black). The Fermi level is indicated by a vertical line and unoccupied states are indicated by pale colors. 


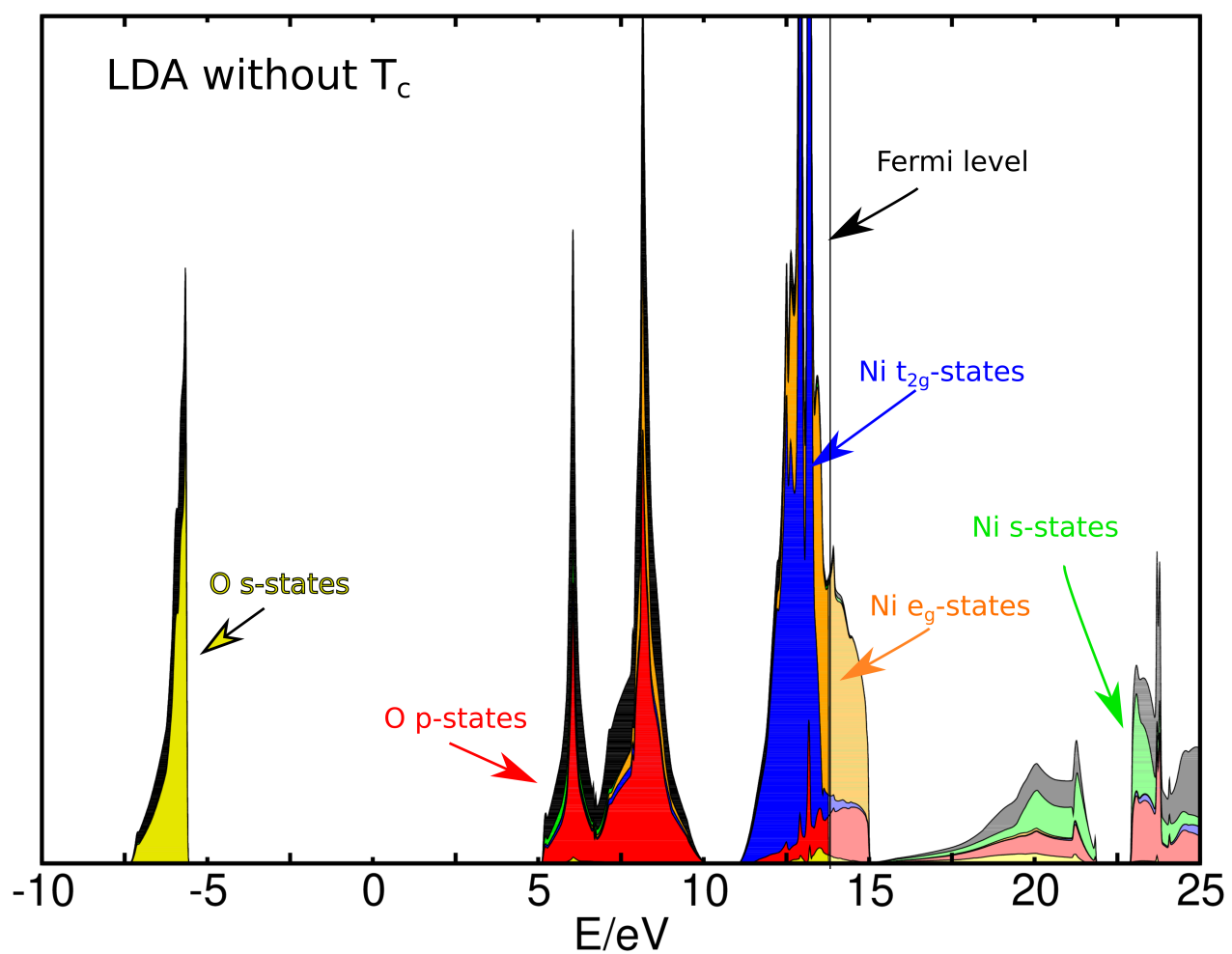

Figure 11.9.: Projected density of states of the antiferromagnetic ground state of $\mathrm{NiO}$ in DFT with the LDA-functional where the kinetic contribution to the exchange-correlation functional was removed. Details of the calculation as discussed in section 11.1. The projected densities of states are stacked in front of the total density of states (black). The Fermi level is indicated by a vertical line and unoccupied states are indicated by pale colors. 

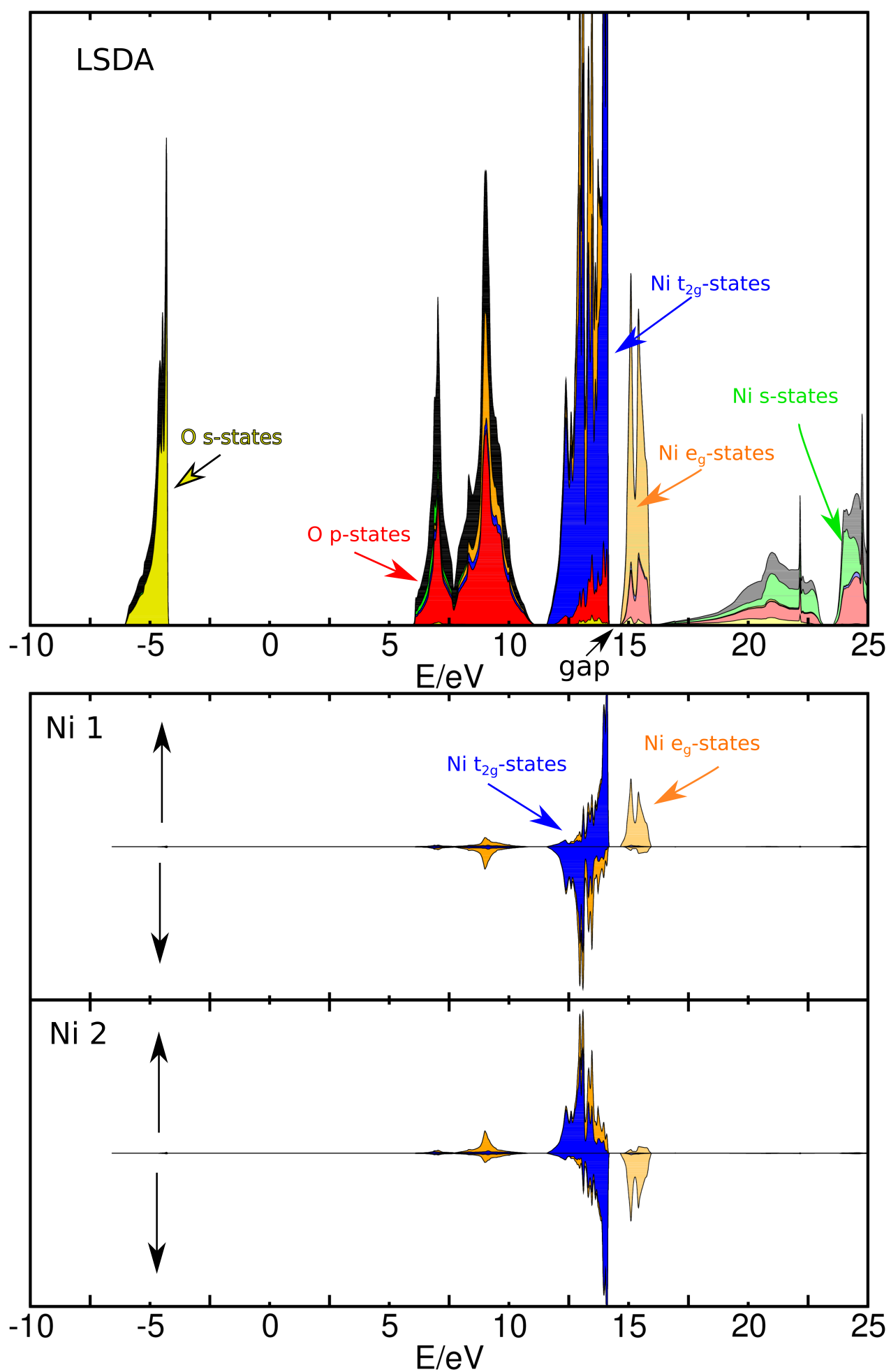

Figure 11.10.: Projected density of states (top figure) and spin-projected densities of states of the nickel 3d-states (bottom figure) of the antiferromagnetic ground state of $\mathrm{NiO}$ and in DFT with the LSDA-functional. Details of the calculation as discussed in section 11.1. The projected densities of states are stacked in front of the total density of states (black). Unoccupied states are indicated by pale colors. 


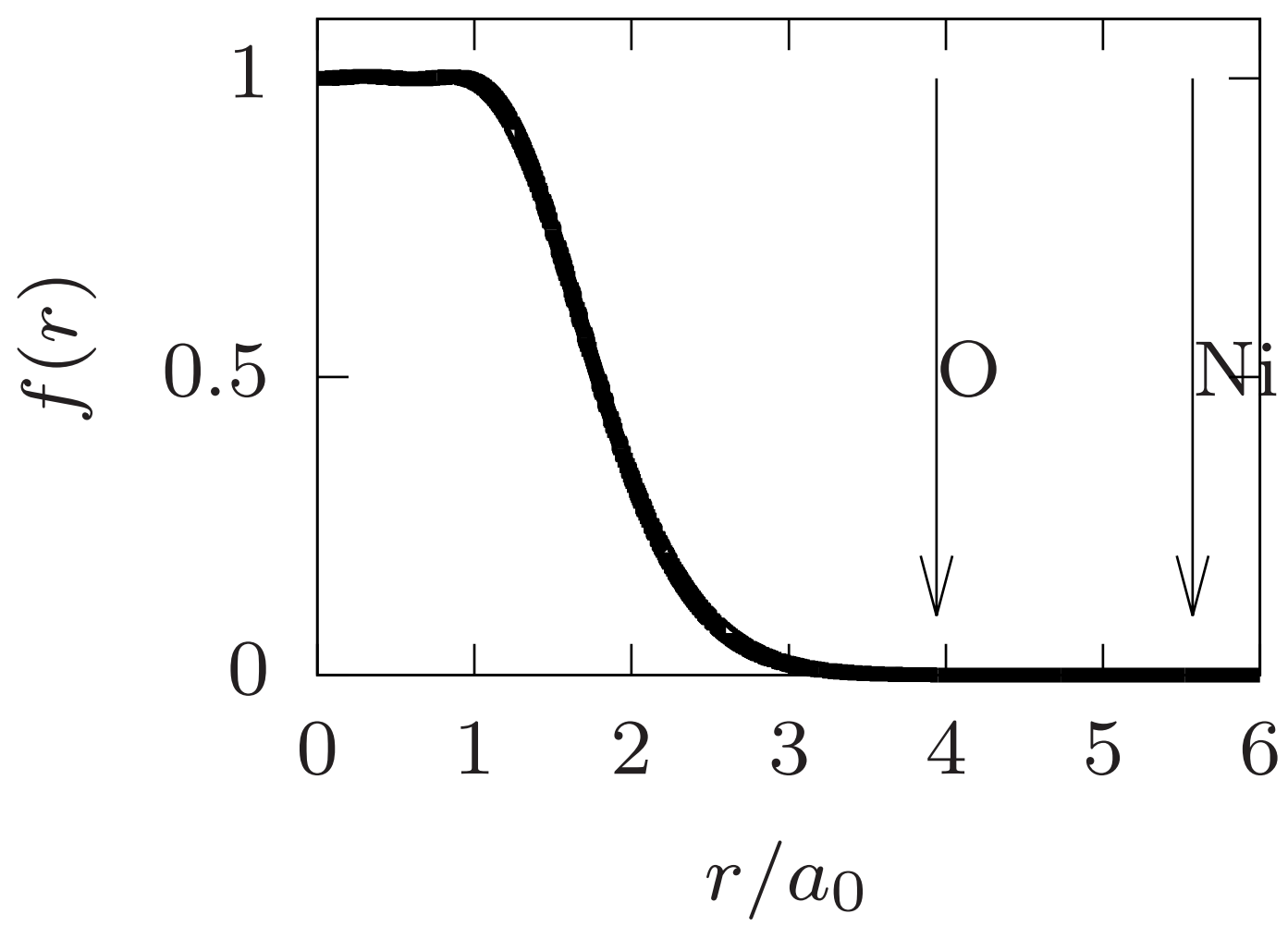

Figure 11.11.: The function $f(r)$ given by Eq. (11.12). The nickel atoms is located at the origin. The vertical arrow at $r=3.94 a_{0}$ indicates the distance to the neighboring oxygen atoms and the vertical arrow at $r=5.57 a_{0}$ denotes the distance to the neighboring nickel atoms. 
For the choice of the space-decomposition function defined by Eq. 11.11 and Eq. (11.12) as well as the new local basis $\left|\chi_{\alpha}\right\rangle$, we have to evaluate the matrix elements

$$
U_{R, \mathrm{sym}, \alpha, \beta, \gamma, \delta}=\int d^{4} \vec{x} \int d^{4} \vec{x}^{\prime} \frac{\chi_{\alpha}^{*}(\vec{x}) \chi_{\beta}^{*}\left(\vec{x}^{\prime}\right) \chi_{\gamma}(\vec{x}) \chi_{\delta}\left(\vec{x}^{\prime}\right) f(|\vec{r}-\vec{R}|) f\left(\left|\vec{r}^{\prime}-\vec{R}\right|\right)}{4 \pi \epsilon_{0}\left|\vec{r}-\vec{r}^{\prime}\right|}
$$

of the local interaction

$$
\hat{W}_{R}=\frac{1}{2} \sum_{\alpha, \beta, \gamma, \delta} U_{\alpha, \beta, \gamma, \delta} \hat{c}_{\alpha}^{\dagger} \hat{c}_{\beta}^{\dagger} \hat{c}_{\delta} \hat{c}_{\gamma}
$$

Figure 11.12 and Figure 11.13 compare the matrix elements of the interaction $\hat{W}_{R}$ in Eq. (6.85) to the matrix elements of the unmodified interaction

$$
U_{\alpha, \beta, \gamma, \delta}=\int d^{4} \vec{x} \int d^{4} \vec{x}^{\prime} \frac{\chi_{\alpha}^{*}(\vec{x}) \chi_{\beta}^{*}\left(\vec{x}^{\prime}\right) \chi_{\gamma}(\vec{x}) \chi_{\delta}\left(\vec{x}^{\prime}\right)}{4 \pi \epsilon_{0}\left|\vec{r}-\vec{r}^{\prime}\right|}
$$

and the matrix elements

$$
U_{R, \text { non-sym } \alpha, \beta, \gamma, \delta}=\int d^{4} \vec{x} \int d^{4} \vec{x}^{\prime} \frac{\chi_{\alpha}^{*}(\vec{x}) \chi_{\beta}^{*}\left(\vec{x}^{\prime}\right) \chi_{\gamma}(\vec{x}) \chi_{\delta}\left(\vec{x}^{\prime}\right) f(|\vec{r}-\vec{R}|)}{4 \pi \epsilon_{0}\left|\vec{r}-\vec{r}^{\prime}\right|}
$$

of the non-symmetrically localized interaction with $\lambda_{\text {non-sym, } \vec{R}}\left(\vec{r}, \vec{r}^{\prime}\right)$ to the unmodified interaction. The case where all orbitals involved in the interaction matrix elements are centered on the nickel atom is shown in the top row of figure 11.12. These interaction matrix elements are practically identical to the corresponding unmodified matrix elements $U_{\alpha, \beta, \gamma, \delta}$. If all orbitals are on one neighboring oxygen atom, then the matrix elements $U_{R \text {,sym } / \text { non-sym }, \alpha, \beta, \gamma, \delta}$ of the local interaction are negligibly small as shown in the bottom row of Figure 11.12. Thus, the proposed interaction decomposition serves its purpose of generating a local interaction. Figure 11.13 shows the corresponding results for exactly one, exactly two or exactly three orbitals on the nickel atom and the remaining orbitals on a neighboring oxygen atom. When we compare these results between the symmetric decomposition function $\lambda_{\mathrm{R} \text {,sym }}$ and the non-symmetric decomposition function $\lambda_{\mathrm{R}, \mathrm{non} \text {-sym }}$, we observe that the symmetric variant yields a stronger localization of the interaction as expected. For exactly one or exactly two orbitals on the nickel atom, the interaction matrix elements of the local interaction are small. However, in the case where there are three orbitals on the nickel atom an one on the neighboring oxygen atom, we observe a weaker localization with interaction matrix elements of up to $0.04 \mathrm{H}$. The four orbitals involved in these matrix elements are mainly the s-orbitals of core states of nickel. For example, the largest interaction matrix element in this class is from two 2s- and one 3sorbital on nickel and one p-orbital of oxygen. We use $f(r)$ as defined in Eq. (11.12) for calculations of $\mathrm{NiO}$ and neglect the non-local interaction matrix elements of the resulting local interaction $\hat{W}_{R}$ later. Please note, that this neglect is not a limitation of the proposed approach but merely for numerical convenience. For an exact implementation of the realspace-decomposition $\mathrm{DF}+\mathrm{RDMF}$ approach, all finite interaction matrix elements of the local interaction

$$
w_{R}\left(\vec{r}, \vec{r}^{\prime}\right)=\frac{\lambda_{R}\left(\vec{r}, \vec{r}^{\prime}\right)}{4 \pi \epsilon_{0}\left|\vec{r}-\vec{r}^{\prime}\right|}
$$


in the new one-particle basis $\left|\chi_{\alpha}\right\rangle$ should be considered.

The choice and number of the one-particle states for the local one-particle reduced density matrix $\rho^{(1), \chi}$ depends on the choice of the decomposition function $\lambda_{R}\left(\vec{r}, \vec{r}^{\prime}\right)$. The counts of one-particle states include the factor of two for the spin. The set of local oneparticle states at least has to consist of the one-particle states that have finite matrix elements for the local interaction $w_{R}\left(\vec{r}, \vec{r}^{\prime}\right)$. Due to the neglect of non-local matrix elements we consider the one-particle states located at the nickel atom as the minimal choice here. This minimal choice consists of 18 core-like states (Ni 1s, 2s, 2p, 3s, 3d) and 12 valence-like states ( $\mathrm{Ni} 3 \mathrm{~d}$ and $4 \mathrm{~s}$ ). We call the minimal choice the $N N=0$-choice because no neighbor of a nickel atom is considered. The next larger choice would be the one-particle states located at the nickel atom and the neighboring six oxygen atoms. This larger choice results in 30 one-particle states for nickel plus a total of 60 one-particle states for the surrounding oxygen atoms. We call this choice the $N N=1$-choice because the first shell of neighbors of a nickel atom is considered. There are 30 core-like one-particle states and 60 valence-like one-particle states in the $N N=1$-choice. Adding the next-neighbor shell of nickel atoms, i.e., $N N=2$, there are 246 core-like one-particle states and 204 valence-like one-particle states. The three choices $N N=0, N N=1$ and $N N=2$ are schematically shown in figure 11.14. In both DF+RDMF approaches, the orbital-based and the real-space-decomposition based, the size of the local one-particle reduced density matrix $\rho^{(1), \chi}$ for the calculation of the density-matrix functional and the double counting term have to be increased until convergence. An examination of the convergence of the term

$$
F_{R}=F^{\hat{W}_{R}}\left[\rho^{(1), \chi}\right]+F_{\mathrm{DF}}^{\hat{W}_{R}}\left[n\left(\rho^{(1), \chi}\right)\right]
$$

with a numerically exact evaluation of the density-matrix functional $F^{\hat{W}_{R}}\left[\rho^{(1), \chi}\right]$ is not possible for local one-particle reduced density matrices $\rho^{(1), \chi}$ that result from choices $N N \geq 1$. To make sure that the numerical solution of constrained search of the density-matrix functional does not influence the convergence, we evaluate the two terms in Eq. (11.18) and choose the Müller functional as the density-matrix functional. We evaluate $F_{R}$ for the nonmagnetic LDA-ground state of $\mathrm{NiO}$. The resulting values are shown in Figure 11.15. As expected, there is a large difference between $N N=0$ and $N N=1$ but only little change beyond $N N=1$. Thus, we consider $N N=1$ to be converged.

\subsubsection{Evaluation of the density-matrix functional}

The evaluation of the density-matrix functional $F^{\hat{W}_{R}}\left[\bar{\rho}^{(1), \chi}\right]$ in the local orthonormal oneparticle basis $\left|\bar{\chi}_{\alpha}\right\rangle$ for the local interaction

$$
\hat{W}_{R}=\frac{1}{2} \sum_{\alpha, \beta, \gamma, \delta \leq N_{\text {int }}} \bar{U}_{R, \alpha, \beta, \gamma, \delta} \hat{\bar{c}}_{\alpha}^{\dagger} \hat{\bar{c}}_{\beta}^{\dagger} \hat{\bar{c}}_{\delta} \hat{\bar{c}}_{\gamma}
$$

is the most numerically demanding step of the DF+RDMF approach if a constrained minimization over many-particle wave functions is used. We evaluate with only a single many-particle wave function, i.e., we calculate Levy's density-matrix functional defined in Eq. (5.25) as

$$
F_{\text {Levy }}^{\hat{W}_{R}}\left[\bar{\rho}^{(1)}\right]=\min _{|\Psi\rangle \rightarrow \bar{\rho}^{(1)}}\left\langle\Psi\left|\hat{W}_{R}\right| \Psi\right\rangle
$$



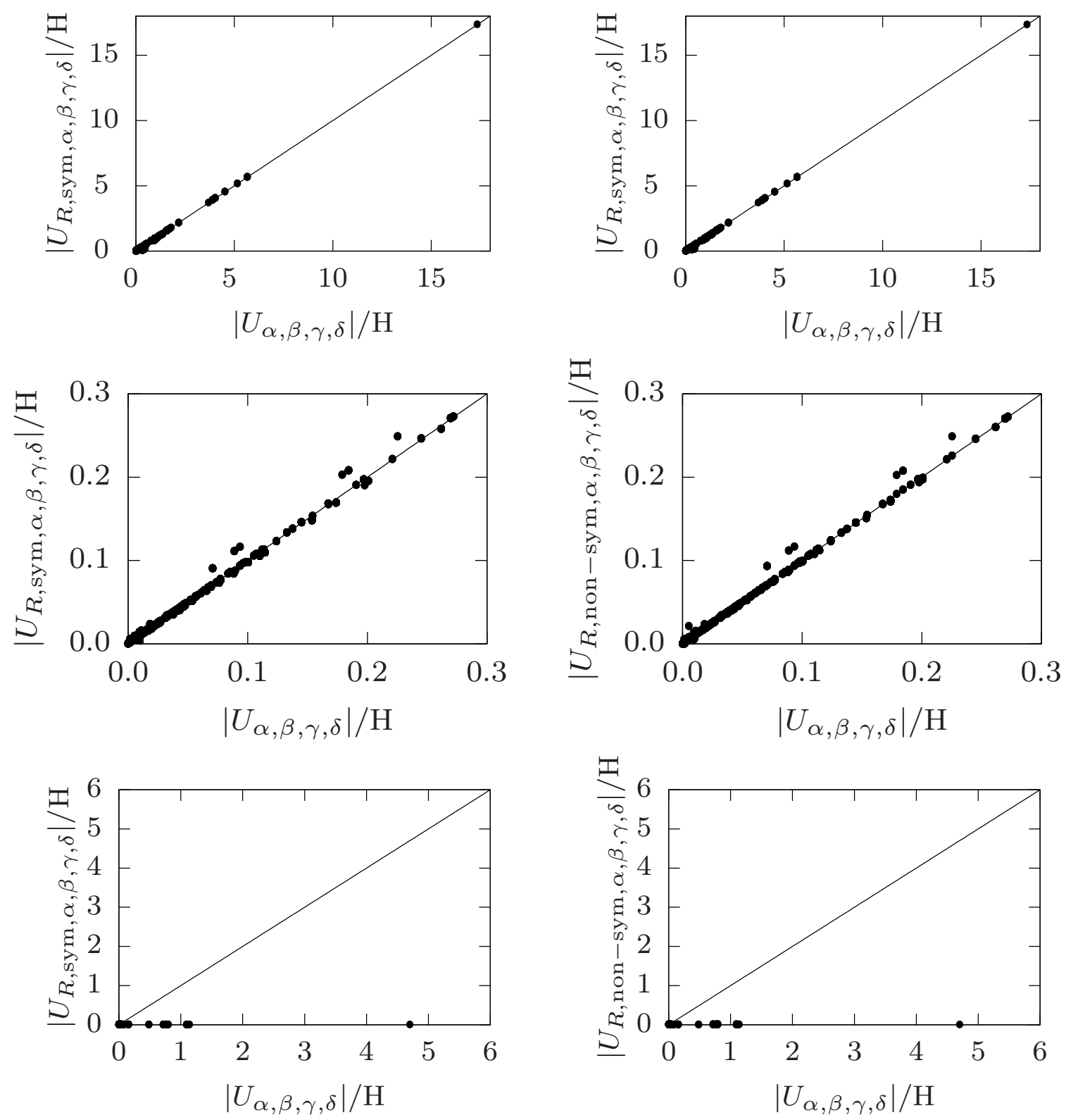

Figure 11.12.: Comparison between the matrix elements $U_{R, \mathrm{sym}, \alpha, \beta, \gamma, \delta}$ (Eq. (11.13)) and $U_{R, \text { non-sym }, \alpha, \beta, \gamma, \delta}$ (Eq. (11.16)) of the local interaction to the matrix elements $U_{\alpha, \beta, \gamma, \delta}$ of the unmodified interaction (Eq. (11.15)). The decomposition function is centered at the nickel atom, the left column shows results for the decomposition function $\lambda_{\text {sym }}$ in Eq. 11.11 and the right column results for $\lambda_{\text {non-sym }}$ in Eq. 6.55. The function $f(r)$ is defined by Eq. 11.12 in both cases. The graphs in the top row show the interactions matrix elements in the case where all orbitals are centered on the nickel atom. The middle row shows a zoom of the data of the first row. The graphs in the bottom row show the case where all four orbitals are on the neighboring oxygen atom. The oneparticle basis is the minimal basis of nickel and oxygen (see section 11.3.3). The solid diagonal line denotes $U_{\alpha, \beta, \gamma, \delta}=U_{R \text {,sym } / \text { non-sym }, \alpha, \beta, \gamma, \delta}$. 

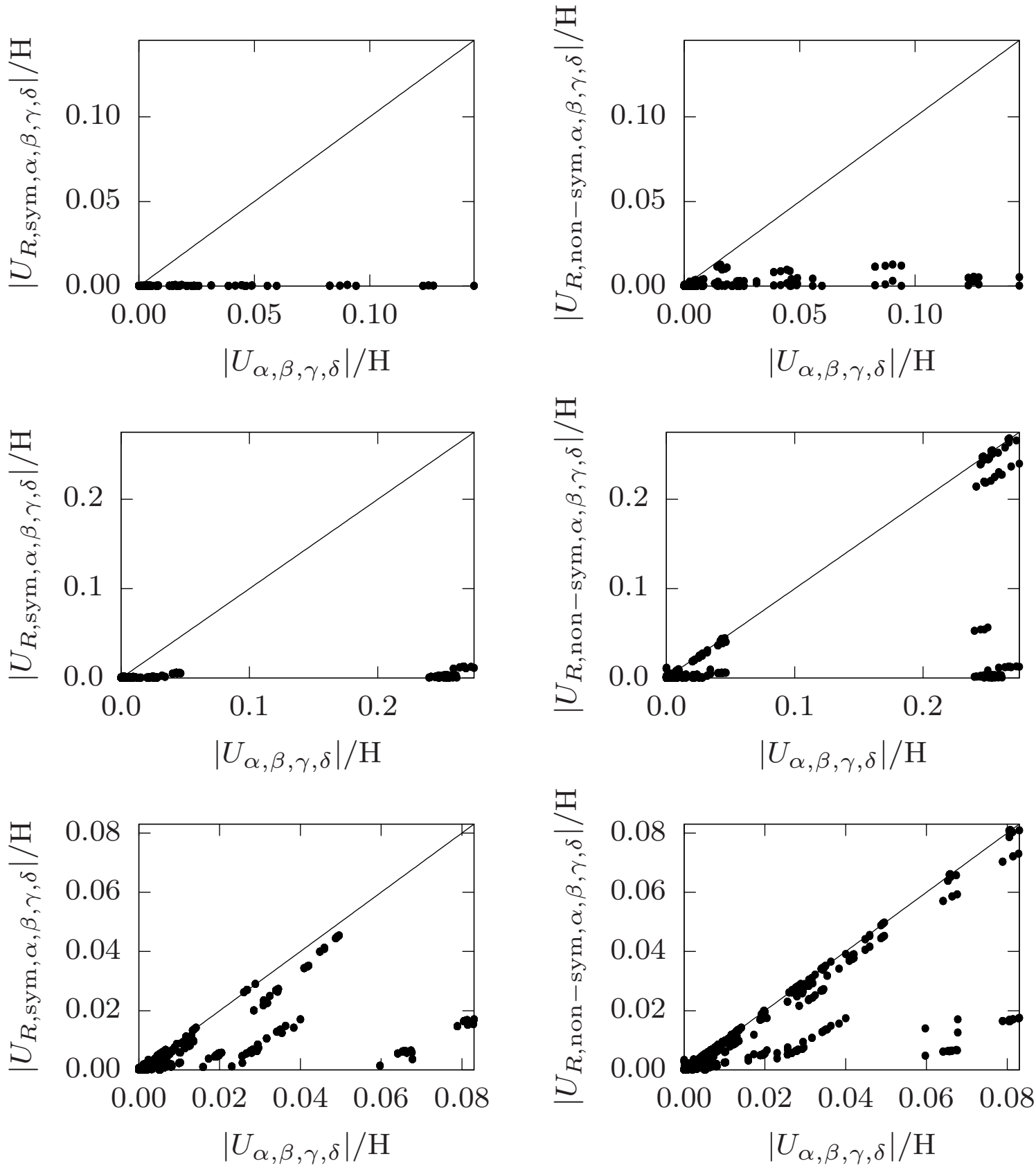

Figure 11.13.: Comparison between the matrix elements $U_{R, s y m, \alpha, \beta, \gamma, \delta}$ (Eq. (11.13)) and $U_{R, \text { non-sym }, \alpha, \beta, \gamma, \delta}$ (Eq. (11.16)) of the local interaction to the matrix elements $U_{\alpha, \beta, \gamma, \delta}$ of the unmodified interaction (Eq. (11.15)). Same representation as in figure 11.12 except that the graphs in the top row shows the case where exactly one of the four orbitals is on the nickel atom and the remaining orbitals are on the neighboring oxygen atom, the middle row the case of two orbitals on nickel and two on oxygen and finally the bottom row shows the case of exactly three orbitals on nickel and one on an oxygen atom. 

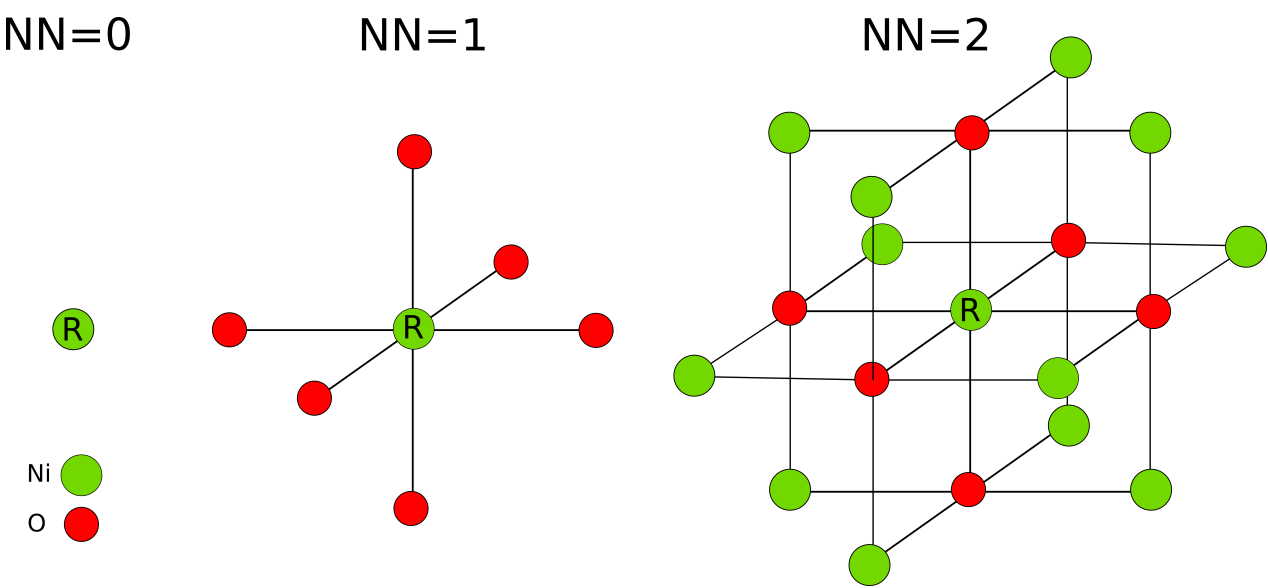

Figure 11.14.: Different choices of the atoms for which the one-particle basis states are considered for the local one-particle reduced density matrix $\rho^{(1), \chi}$. The nickel atom with the label $\mathrm{R}$ denotes the center of the local interaction $w_{R}$. $N N=0$ means no next neighbor, $N N=1$ denotes one shell of neighbors and $N N=2$ two shells of neighbors.

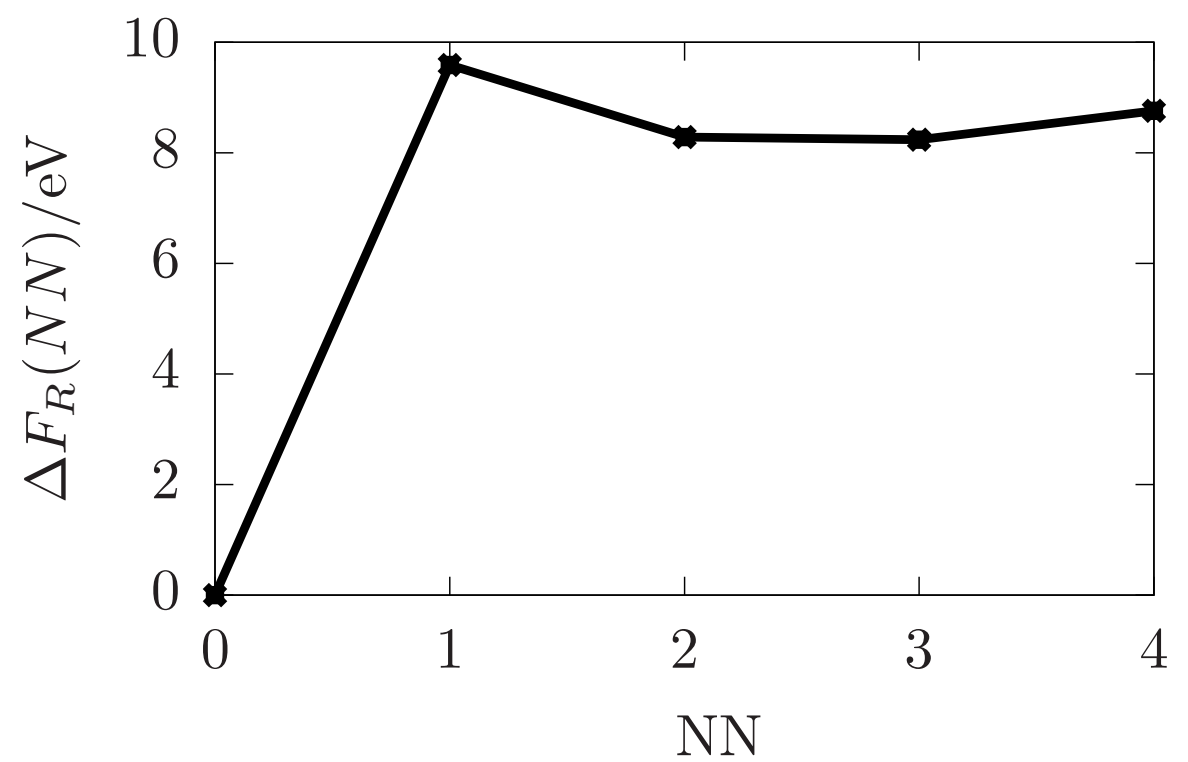

Figure 11.15.: Convergence of $\Delta F_{R}(N N)=F_{R}(N N)-F_{R}(N N=0)$ defined in Eq. (11.18) with the number of neighbor-shells $N N$ for the nonmagnetic LDA-ground state of $\mathrm{NiO}$. 
The frozen-core approximation that we employ within the PAW-formalism leads to a distinction of fully occupied core-like and valence-like states with fractional occupations. With this distinction, we can use the complete-active-space wave function in Eq. (9.17), which reads in this case

$$
|\Psi\rangle=|\underbrace{1 \ldots 1}_{\text {core-like states }}\rangle \otimes|\tilde{\Psi}\rangle
$$

We found no one-particle states with vanishing occupations during our calculations for $\mathrm{NiO}$ and, hence, there are no virtual states in the complete-active-space wave function. The density-matrix functional can then be written as

$$
\begin{aligned}
F_{\text {Levy }}^{\hat{W}_{R}}\left[\bar{\rho}^{(1)}\right] & =F_{\text {core-valence }}^{\hat{W}_{R}}\left[\bar{\rho}^{(1)}\right]+F_{\text {Levy }}^{\hat{W}_{R}}\left[\bar{\rho}_{v}^{(1)}\right] \\
F_{\text {Levy }}^{\hat{W}_{R}}\left[\bar{\rho}_{v}^{(1)}\right] & =\min _{|\tilde{\Psi}\rangle \rightarrow \bar{\rho}_{v}^{(1)}}\left\langle\tilde{\Psi}\left|\hat{W}_{R, v}\right| \tilde{\Psi}\right\rangle,
\end{aligned}
$$

where $F_{\text {core-valence }}^{\hat{W}_{R}}\left[\bar{\rho}^{(1)}\right]$ denotes the core-valence exchange, $\bar{\rho}_{v}^{(1)}$ is the one-particle reduced density matrix of the valence-like states and $\hat{W}_{R, v}$ is the interaction Hamiltonian of the valence-like states. $\hat{W}_{R, v}$ only contains the interactions between the 4 s- and 3d-states of nickel.

For $\mathrm{NiO}$ with only the nickel states as the local one-particle states, i.e., $N N=0$, we have to evaluate the density-matrix functional $F_{\text {Levy }}^{\hat{W}_{R}}\left[\bar{\rho}_{v}^{(1)}\right]$ in a 12-dimensional oneparticle basis. This can be done numerically exact in a few seconds, and no additional approximations are required. One evaluation of the density-matrix functional takes about 30 seconds with a single CPU core of a current desktop computer. This evaluation also gives the derivatives of the density matrix functional with respect the one-particle reduced density matrix.

For $\mathrm{NiO}$ with the nickel states and the first shell of neighboring oxygen atoms, i.e., $N N=1$, we have to evaluate the density-matrix functional $F_{\text {Levy }}^{\hat{W}_{R}}\left[\bar{\rho}_{v}^{(1)}\right]$ in a 60 -dimensional one-particle basis. As this is not possible in practice, we employ the adaptive cluster approximation to reduce the size of the one-particle basis. The adaptive cluster approximation with the $M$-th level effective bath $(\mathrm{ACA}(\mathrm{M}))$ results in a density-matrix functional in a $12 \cdot(M+1)$-dimensional one-particle basis. $\mathrm{ACA}(\mathrm{M}=0)$ is equivalent to the case of $N N=0$. We apply the ACA with $M=1$ and approximate the manyparticle wave function in the $2^{24}=16777216$-dimensional space of Slater determinants with the configuration-interaction-like ansatz with $M_{\mathrm{CI}}$ Slater determinants described in section 9.4. We do not employ a correction with an approximate parametrized functional in the ACA here in order to avoid possible unphysical effects from an approximate parametrized density-matrix functional and to be able to evaluate the local spectral function based on the minimum condition in Eq. 5.42 as discussed in section 5.6.1.

Thus, we are required to investigate the convergence with respect to the three parameters $N N, M$ and $M_{\mathrm{CI}}$. We have evaluated the convergence with respect to $N N$ for the example of the Müller functional for $\mathrm{NiO}$ in section 11.3.3 because an evaluation with a more advanced density-matrix functional is not possible due to the large dimension of the resulting one-particle basis. We study the convergence with the effective bath level $M$ in the adaptive cluster approximation for $N N=1$ with the Müller functional for the ground state of $\mathrm{NiO}$ with $N N=1, M=1, M_{\mathrm{CI}}=16384$. Figure 11.16 shows the convergence of 
the Müller functional with the effective bath level $M$ and the discarded weight $\sigma_{M}$ of the one-particle reduced density matrix defined in Eq. (8.17). $M=4$ is the full one-particle reduced density matrix of $N N=1$ with 60 valence-like one-particle states. The difference of the Müller functional between the $\operatorname{ACA}(M=1)$ and the $\operatorname{ACA}(M=4)$ is negligible and, hence, we consider $M=1$ as converged. Figure 11.17 shows the convergence of the density-matrix functional for many-particle wave functions with different numbers $M_{\mathrm{CI}}$ of Slater determinants. The rate convergence of the deviation $F_{\mathrm{CI}}\left(M_{\mathrm{CI}}\right)-F_{\mathrm{CI}}\left(M_{\mathrm{CI}}=65536\right)$ with $M_{\mathrm{CI}}$ is quite rapid and in $\mathcal{O}\left(M_{\mathrm{CI}}^{-4.9}\right)$ in this case. Thus, we assume $M_{\mathrm{CI}}=16384$ to be converged and use this value in all calculations with $N N=1$ and $M=1$. Figure 11.18 shows the dependence of the runtime of the constrained minimization for the density-matrix functional on the number of Slater determinants in the many-particle wave function. The runtimes have been obtained on a desktop CPU (Intel Core i7-5820k). We distinguish three different situations in the figure:

1. The solution of the minimization problem starting from the construction of a manyparticle wave function with a minimal number of Slater determinants outlined in appendix $\mathrm{C}$ and zero values for the Lagrange multipliers. This is the case if a minimization procedure of the total energy is started newly. The runtime obtained in this case can be seen as an upper bound.

2. The solution of the minimization problem starting for the many-particle wave function, space of Slater determinants and Lagrange multipliers of a previous evaluation of the density-matrix functional (warm-start). If the one-particle reduced density matrix is not changed, we obtain an estimate of the minimal runtime.

3. An identical situation as in the previous case except that the one-particle reduced density matrix is changed slightly. This is the situation close to convergence of the minimization of the total energy or during an ab-initio molecular dynamics simulation. Care has to be taken, because the relevant selection of Slater determinants depends on the given one-particle reduced density matrix. Therefore, the space of Slater determinants should occasionally be either constructed newly starting from a minimal many-particle wave function or some Slater determinants with small amplitudes in the many-particle wave function should be removed from the space and then the space grown to the intended size again.

The dependence is linear for large $M_{\mathrm{CI}}$ and the evaluation for $M_{\mathrm{CI}}=16384$ takes about five minutes from scratch, 7 seconds when restarted for the same one-particle reduced density matrix, and 20 seconds when restarted for a slightly changed one-particle reduced density matrix on a desktop CPU.

\subsubsection{Results for nickel-only cluster $(N N=0)$}

We have performed calculations for nonmagnetic $\mathrm{NiO}$ in the proposed real-spacedecomposition based $\mathrm{DF}+\mathrm{RDMF}$ approach with the density-matrix functional evaluated with a many-particle wave function in a representation with Slater determinants. We begin our discussion with the results of the case $N N=0$, where only states at the nickel atom are considered for the local one-particle reduced density matrix. Figure 11.19 shows the overlap $O_{i i}(\vec{k})=\left\langle\mu_{i}(\vec{k}) \mid \mu_{i}(\vec{k})\right\rangle$ of the projected wave functions $\left|\mu_{i}(\vec{k})\right\rangle$ in the ground 

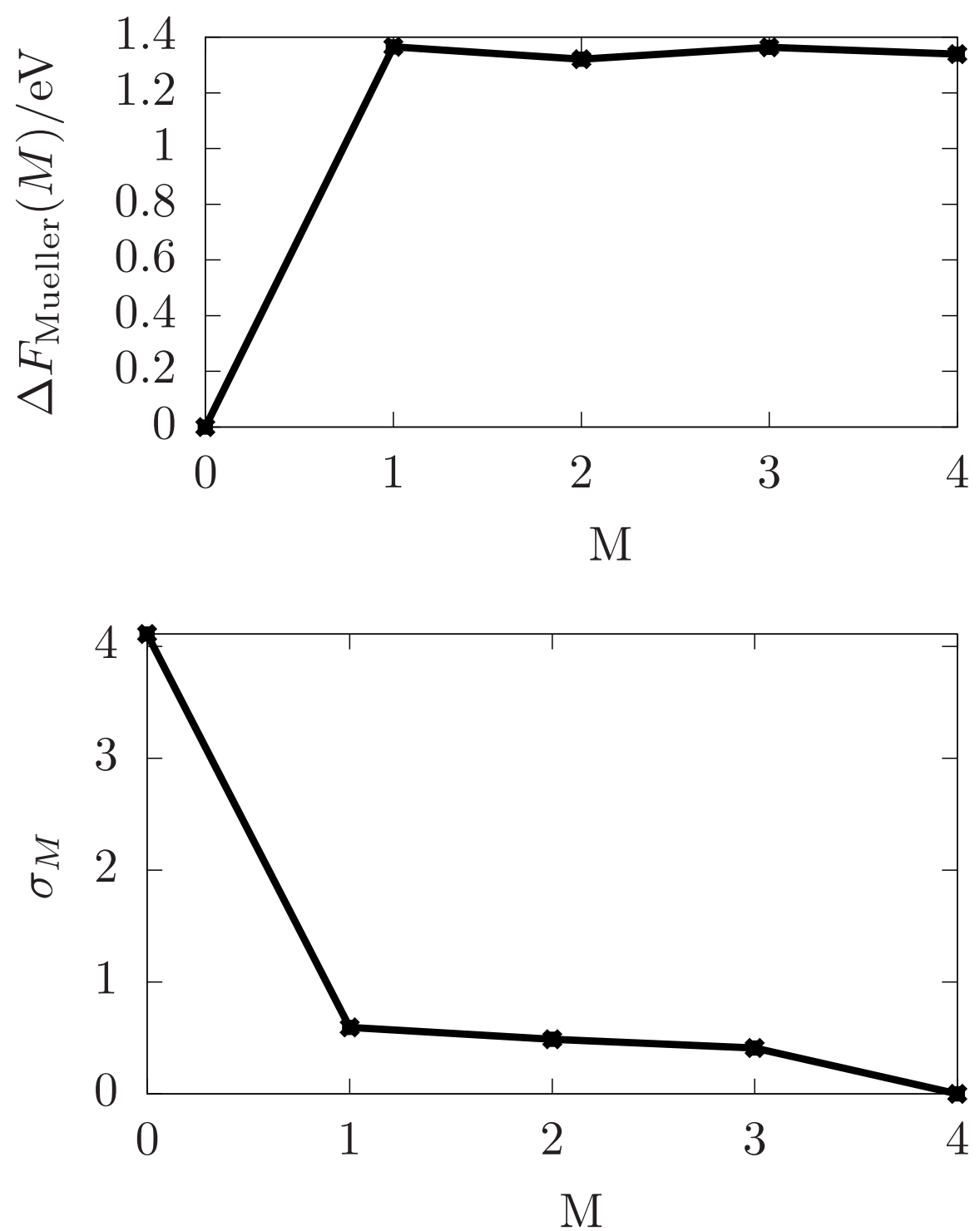

Figure 11.16.: Convergence of $\Delta F_{\text {Müller }}(M)=F_{\text {Müller }}(M)-F_{\text {Müller }}(M=0)$ the Müller functional (top graph) and the discarded weight $\sigma_{M}$ of Eq. (8.17) (bottom graph) with the level of the effective bath $M$ of the adaptive cluster approximation for the local one-particle reduced density matrix of the nonmagnetic ground state of $\mathrm{NiO}$ with $N N=1, M=1, M_{\mathrm{CI}}=16384$. 


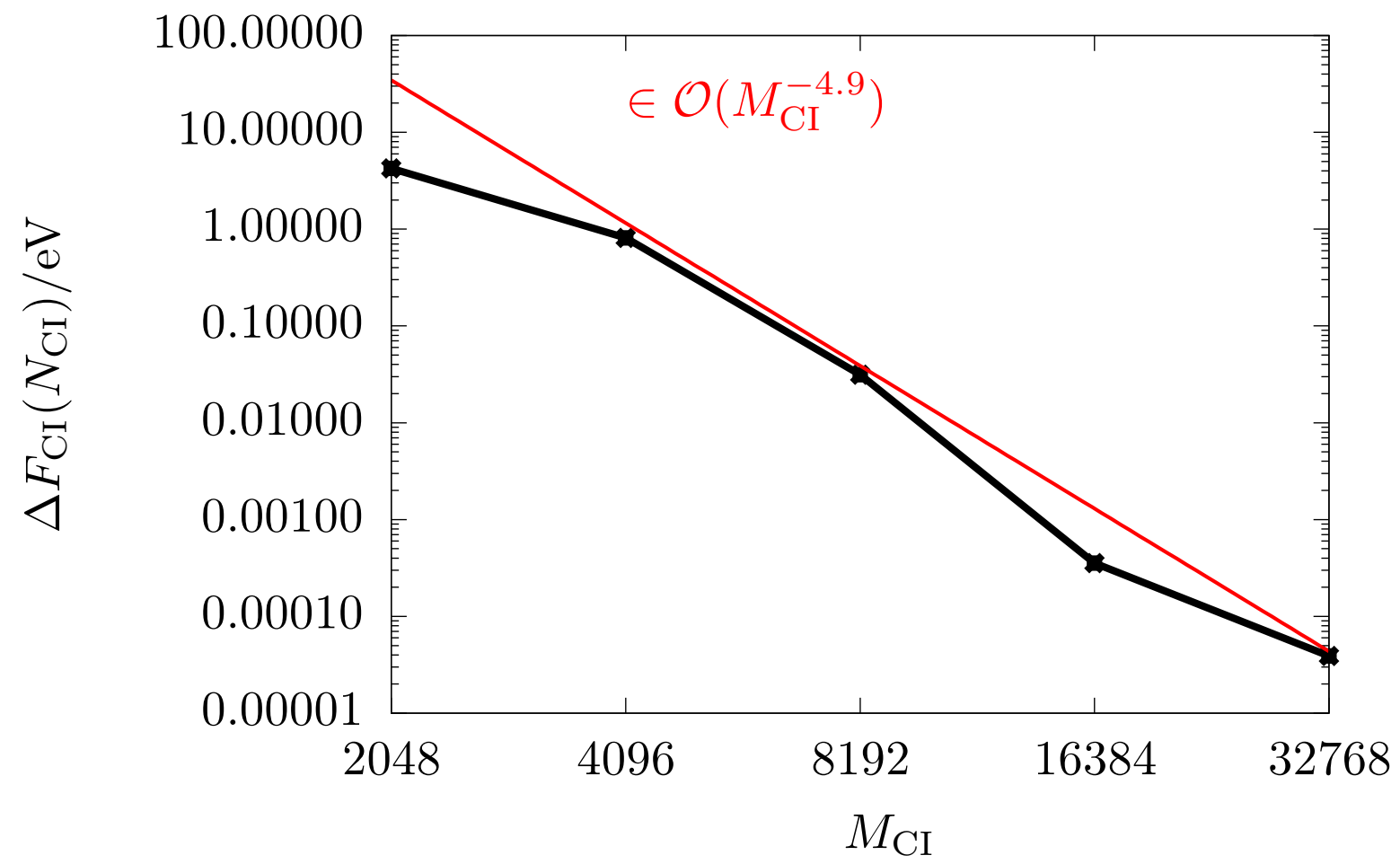

Figure 11.17.: Convergence of $\Delta F_{\mathrm{CI}}\left(M_{\mathrm{CI}}\right)=F_{\mathrm{CI}}\left(M_{\mathrm{CI}}\right)-F_{\mathrm{CI}}\left(M_{\mathrm{CI}}=65536\right)$ with the number of Slater determinants $M_{\mathrm{CI}}$ in the many-particle wave function in the constrained minimization for the local one-particle reduced density matrix of the nonmagnetic ground state of $\mathrm{NiO}$ with $N N=1, M=1$, $M_{\mathrm{CI}}=16384$. The red line shows the function $M_{\mathrm{CI}}^{-4.9} \cdot 10^{17.764} \mathrm{eV}$ that resulted from a fit of the data points. 


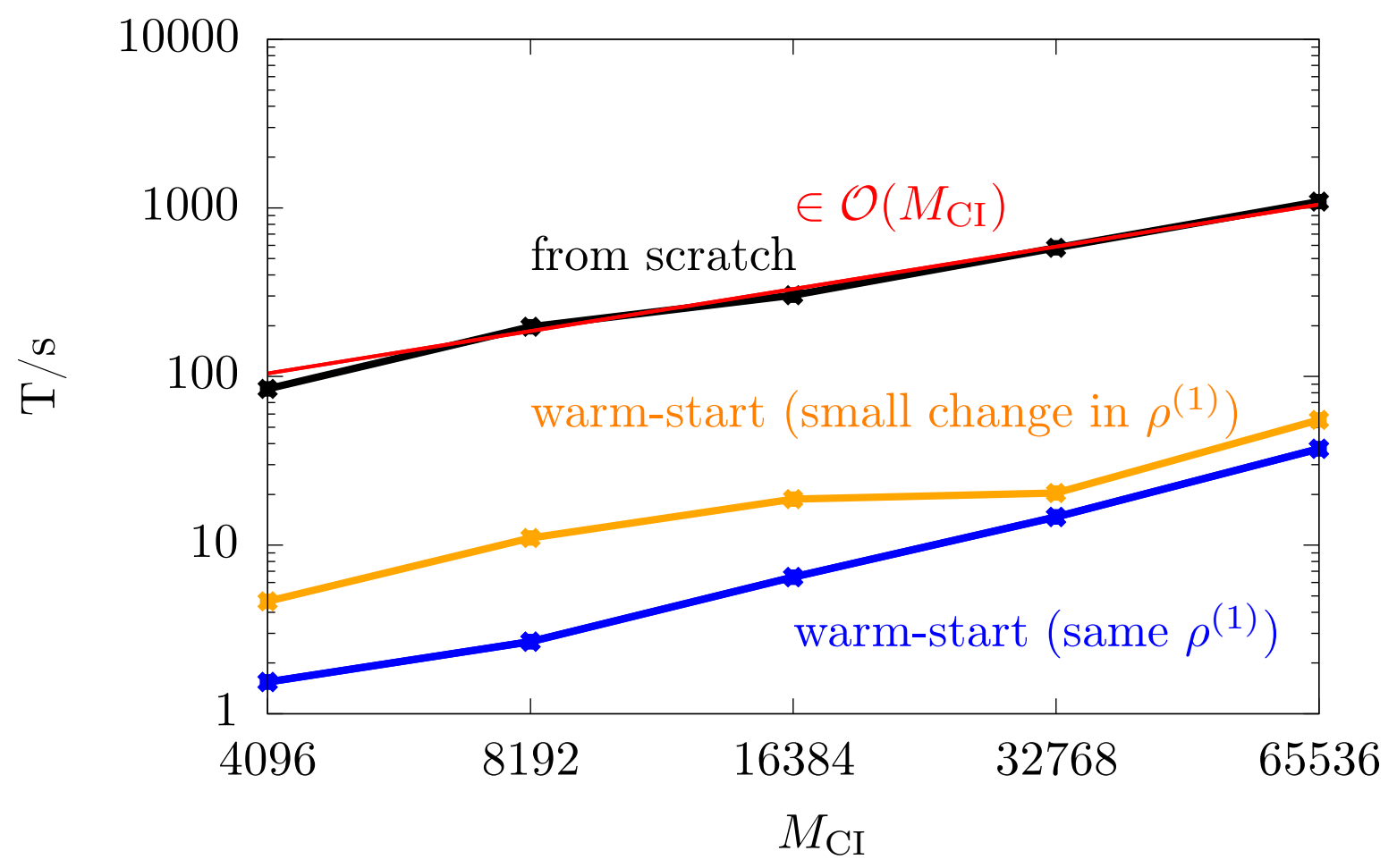

Figure 11.18.: Dependence of the runtime for the evaluation of the density-matrix functional $F_{\mathrm{CI}}\left(M_{\mathrm{CI}}\right)$ on the number of Slater determinants $M_{\mathrm{CI}}$ in the manyparticle wave function in the constrained minimization for the local oneparticle reduced density matrix of the nonmagnetic ground state of $\mathrm{NiO}$ with $N N=1, M=1, M_{\mathrm{CI}}=16384$. The black line shows the runtime if the evaluation is started from a minimial many-particle wave function constructed in appendix $\mathrm{C}$ and with zero as the values of the Lagrange multipliers. In contrast, the blue line shows the runtime if the many-particle wave function and Lagrange multipliers are reused (warm-start) from a previous iteration and the one-particle reduced density matrix is not changed, whereas the orange line depicts the situation if the one-particle reduced density matrix is changed slightly. Calculations have been performed on an Intel Core i7-5820K desktop CPU. 
state with $N N=0$. As discussed in section 7.3.2 the local one-particle basis $\left|\chi_{\alpha}\right\rangle$ has to be sufficiently complete to be able to represent the natural orbitals $\left|\psi_{i}(\vec{k})\right\rangle$, that are represented in a basis of augmented plane waves. Thus, the deviation of $O_{i i}$ from the value one quantifies how well the approximation

$$
\left|\psi_{i}(\vec{k})\right\rangle \approx\left|\mu_{i}(\vec{k})\right\rangle=\sum_{\alpha, \vec{T}} C_{\alpha, \vec{T}, i}\left|\chi_{\alpha, \vec{T}}\right\rangle
$$

is fulfilled. The one-particle reduced density matrix, for which the density-matrix functional is evaluated, is constructed from the states $\left|\mu_{i}(\vec{k})\right\rangle$. A faithful representation of the one-particle reduced density matrix requires a $O_{i i}(\vec{k}) \approx 1$ for all states $i$ that have finite occupations $f_{i}(\vec{k})>0$. The top graph in Figure 11.19 shows the overlaps $O_{i i}(\vec{k})$ versus the occupations $f_{i}(\vec{k})$. All states with $f_{i}(\vec{k})>0$ have $O_{i i}(\vec{k})>0.8$. The bottom graph of Figure 11.19 depicts the overlaps $O_{i i}(\vec{k})$ for all states with $f_{i}>0.1$ and shows that only a very small number of overlaps drop below a value of 0.95 . Thus, the chosen one-particle basis $\left|\chi_{\alpha}\right\rangle$ is sufficient to allow for a proper representation of the natural orbitals $\left|\psi_{i}(\vec{k})\right\rangle$. The occupations $f_{i}(\vec{k})$ of the one-particle reduced density matrix of the ground state are almost exclusively integer as shown in Figure 11.19.

The prevalent integer occupations in the ground state suggest that a Kohn-Sham-like spectral function introduced in section 5.6.2 might give some physical insight into the system. Figure 11.20 shows the Kohn-Sham-like projected densities of states of the nonmagnetic ground state of $\mathrm{NiO}$ in the real-space-decomposition based DF+RDMF approach with $N N=0$. In comparison to the nonmagnetic LDA ground state shown in Fig. 11.8, qualitative changes in the oxygen p-states and 3d-states of nickel are visible: the distance between the oxygen p-band between $5-10 \mathrm{eV}$ in the LDA ground state and the nickel $3 \mathrm{~d}-$ states is drastically increased in the $\mathrm{DF}+\mathrm{RDMF}$ ground state. In the $\mathrm{DF}+\mathrm{RDMF}$ ground state, the 3d-states of nickel form a peak at the Fermi level. There is no visible gap in the Kohn-Sham-like density of states at the Fermi energy. The peak at the Fermi level suggests that the $3 \mathrm{~d}$-states have the character of fractionally occupied states even though they have integer occupations in the $\mathrm{DF}+\mathrm{RDMF}$ approach. A different point of view is the local spectral function defined in section 5.6.1. The local spectral function shown in figure 11.21 was calculated with the recipe discussed in section 5.6.1 and predicts a gap of about $15 \mathrm{eV}$. The local spectral function is the spectral function of a finite system and, hence, consists of delta-peaks at zero temperature. We have broadened the peaks with a Gaussian broadening of $0.2 \mathrm{eV}$ for better visibility. We ascribe this unphysical prediction to the fact that if one only considers the nickel-states for the local one-particle reduced density matrix, the core assumption of section 6.5.2 is violated. This core assumption is that the one-particle states $\left|\chi_{\alpha}\right\rangle$ used for the representation of the local one-particle reduced density matrix $\rho^{(1), \chi}$ are chosen such that they can describe the real-space oneparticle reduced density matrix

$$
\rho^{(1)}\left(\vec{x}, \vec{x}^{\prime}\right)=\sum_{n} f_{n}\left\langle\vec{x} \mid \psi_{n}\right\rangle\left\langle\psi_{n} \mid \vec{x}^{\prime}\right\rangle,
$$

that is represented by the natural orbitals $\left|\psi_{n}\right\rangle$, in the vicinity of the center $R$ of the interaction sufficiently well. Thus, we conclude that not only for the Müller functional discussed in section 11.3.3 but also for the numerically exact density-matrix functional a local basis of at least $N N=1$, i.e., the nickel-states and the states of the neighboring 
oxygen atoms, are required for a faithful representation of the real-space one-particle reduced density matrix. The contradiction between the Kohn-Sham-like density of states and the local spectral function is resolved if we consider that the proposed real-spacedecomposition based DF+RDMF approach is based on reduced density-matrix functional theory and not density-functional theory. Thus, the Kohn-Sham-like density of states only carry a physical interpretation for the states that are not directly affected by the density-matrix functional.

\subsubsection{Results for nickel-and-oxygen cluster $(N N=1)$}

We extend the set of one-particle states used for the local one-particle reduced density matrix to include the one-particle states of the six oxygen atoms that are close to one nickel atom, i.e., $N N=1$. The density-matrix functional is evaluated within the adaptive cluster approximation with one effective bath level $(M=1)$ and with $M_{\mathrm{CI}}=16384$ Slater determinants. Similarly to the discussion of the results of $N N=0$, we first analyze the representability of the natural orbitals $\left|\psi_{n}\right\rangle$ in the local one-particle basis $\left|\chi_{\alpha}\right\rangle$. The overlaps $O_{i i}(\vec{k})=\left\langle\mu_{i}(\vec{k}) \mid \mu_{i}(\vec{k})\right\rangle$ of the projected states $\left|\mu_{i}(\vec{k})\right\rangle \approx\left|\psi_{i}(\vec{k})\right\rangle$ are shown in figure 11.23. Most values of $O_{i i}(\vec{k})$ for natural orbitals with finite occupations $f_{i}$ lie above $O_{i i}(\vec{k})=0.95$. Thus, most natural orbitals are well represented in the one-particle basis $\left|\chi_{\alpha}\right\rangle$. In comparison to the case of $N N=0$ shown in figure 11.19, there are more states with smaller values $0.7<O_{i i}(\vec{k})<0.95$. The Kohn-Sham-like projected density of states of the ground state for $N N=1, M=1$ and $M_{\mathrm{CI}}=16384$ are shown in figure 11.22. The large gap between the oxygen-p band and the nickel 3d-states visible in the $N N=0$-ground state is not visible in the $N N=1$-ground state. Compared to the density of states of the LDA-ground state, the shape of the density of states in broadened and nickel $t_{2 g}$-states hybridize more strongly with the oxygen p-states. The Kohn-Sham-like projected density of states of the ground state for $N N=1, M=1$ and $M_{\mathrm{CI}}=16384$ show no gap at the Fermi level. The density of states from the local spectral function are shown in figure 11.24. A gap of about $6 \mathrm{eV}$ is visible. This prediction is much smaller than the value of $15 \mathrm{eV}$ predicted with $N N=0$ and shows the importance of a faithful representation of the real-space one-particle reduced density matrix in the vicinity of the local interaction with the one-particle basis used for the evaluation of the density-matrix functional. The reduction of the gap compared to $N N=0$ is also a practical confirmation of the considerations presented in section 6.5.2, namely that no screening of the interaction has to be considered in the DF+RDMF approach as long as the real-space one-particle reduced density matrix is represented faithfully. The slight overestimation of the gap of $6 \mathrm{eV}$ compared to DFT+DMFT results of $4.3 \mathrm{eV}$ [Ren et al., 2006] probably results from the adaptive cluster approximation because the adaptive cluster approximation effectively neglects some of the oxygen-p states that otherwise would lead a reduction of the effective Coulomb interaction. A full minimization of the $\mathrm{DF}+\mathrm{RDMF}$-total energy with the density-matrix functional evaluated with $M>1$, i.e., multiple evaluations of a density-matrix functional with at least 36 one-particle states that is converged with respect to the number of Slater determinants, are not possible in a reasonable runtime with the current implementation of the configuration-interaction ansatz. We expect this to be possible with a properly tuned implementation of the MPSbased ansatz or the Gutzwiller-Jastrow-ansatz for the many-particle wave function for the 

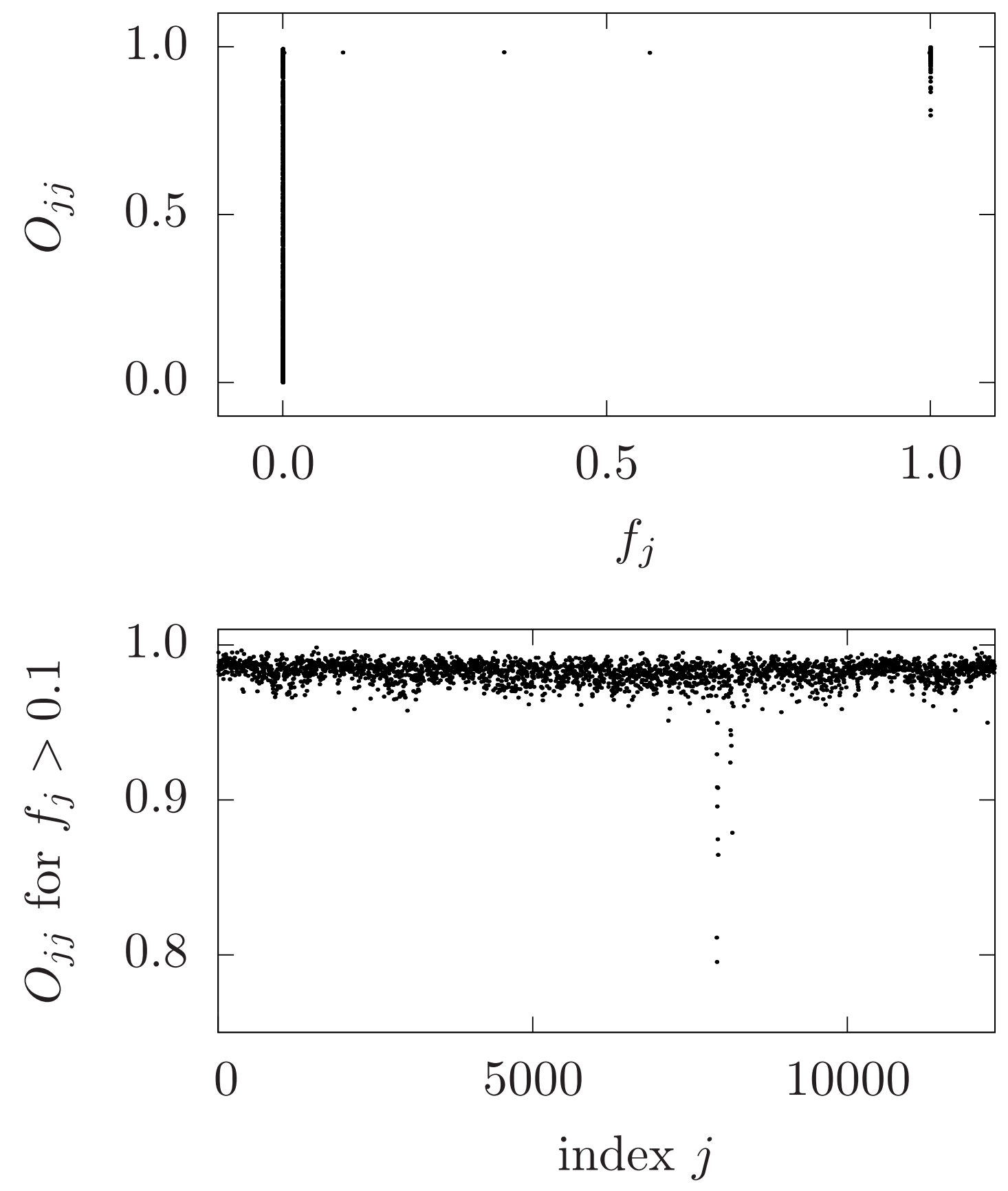

Figure 11.19.: Overlap $O_{j j}=O_{i i}(\vec{k})=\left\langle\mu_{i}(\vec{k}) \mid \mu_{i}(\vec{k})\right\rangle$ of the projected states $\left|\mu_{i}(\vec{k})\right\rangle \approx$ $\left|\psi_{i}(\vec{k})\right\rangle$ in the nonmagnetic ground state of $\mathrm{NiO}$ with $N N=0$. The index $j$ is a combined index of the band index $i$ and the index of a k-point $\vec{k}$ in a discrete k-point grid. The top graph shows the overlaps $O_{j j}$ versus the occupations $f_{j}$ of the states. The bottom graph shows only the overlaps of states with $f_{i}>0.1$ for closer inspection. 


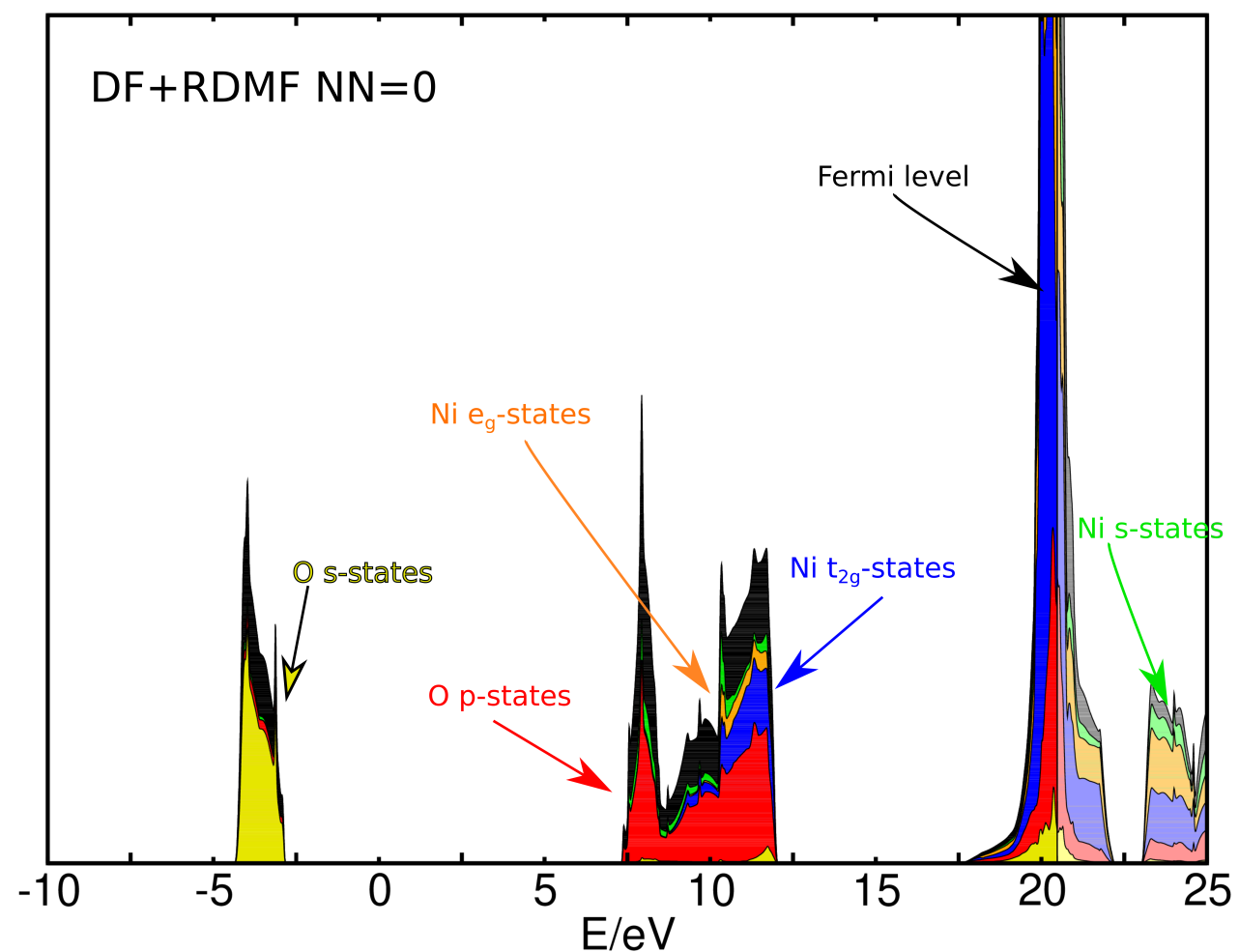

Figure 11.20.: Kohn-Sham-like projected density of states of the nonmagnetic ground state of $\mathrm{NiO}$ in the real-space-decomposition based $\mathrm{DF}+\mathrm{RDMF}$ approach with only the nickel states used in the local one-particle reduced density matrix for the evaluation of the numerically exact density-matrix functional. Details of the calculation as discussed in section 11.1. The projected densities of states are stacked in front of the total density of states (black). Unoccupied states are indicated by pale colors. 


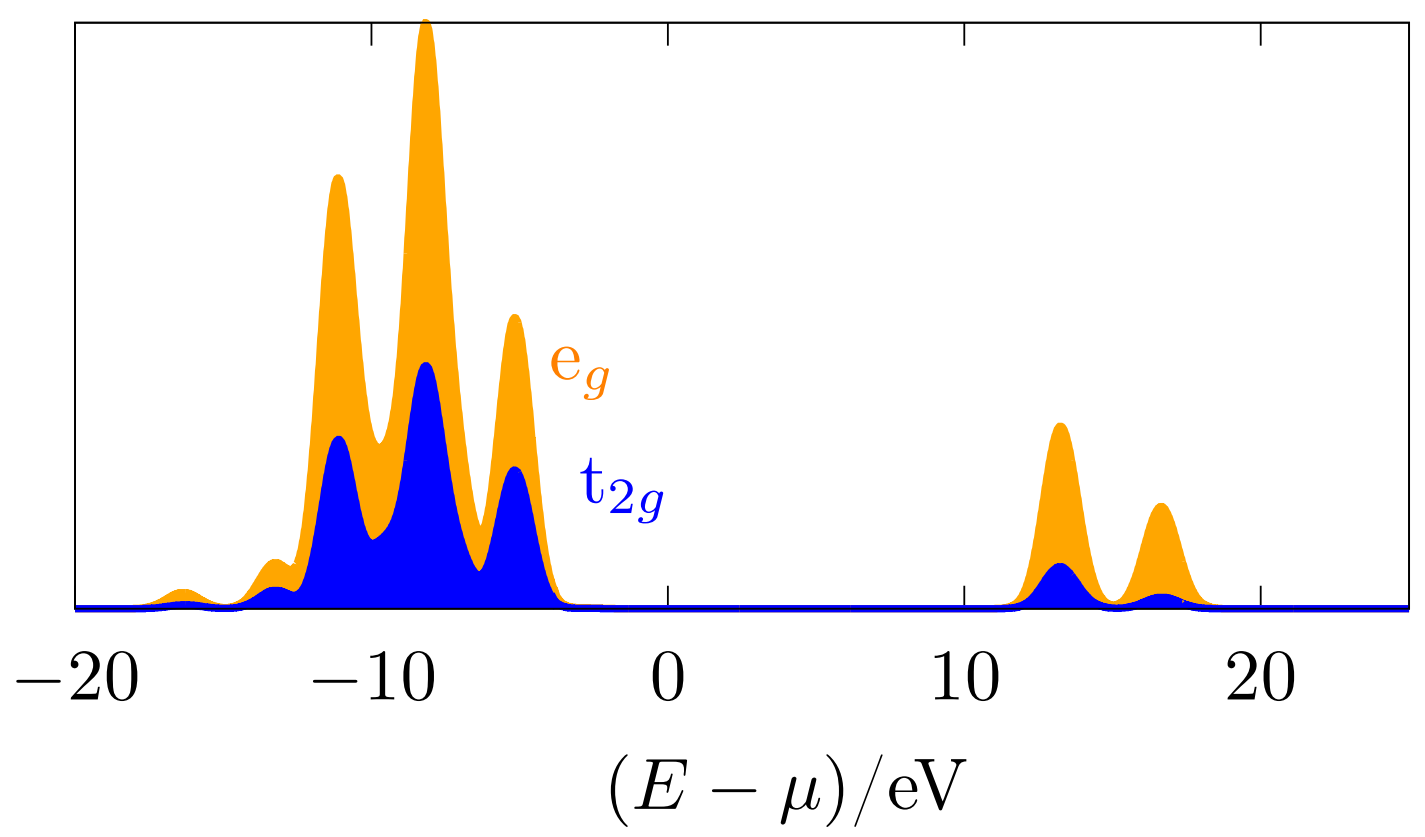

Figure 11.21.: Local density of states of the nickel 3d-states for the nonmagnetic ground state of $\mathrm{NiO}$ in the real-space-decomposition based $\mathrm{DF}+\mathrm{RDMF}$ approach with only the nickel states used in the local one-particle reduced density matrix for the evaluation of the numerically exact density-matrix functional $(N N=0)$. The densities of states are stacked on top of each other. Details of the calculation as discussed in section 11.1. The peaks have been broadened with a Gaussian broadening of $0.2 \mathrm{eV}$ for better visibility. 
density-matrix functional.

About $10 \mathrm{eV}$ below the main peak there is a satellite peak in the local spectral function that is not visible in LDA+U-results [Kuo et al., 2017]. Figure 11.25 shows a direct comparison of the local spectral function to the experimental valence-band XPS spectrum [Kuo et al., 2017] and cluster calculations of $\mathrm{NiO}$ of van Elp at al. [van Elp et al., 1992] and Fujimori et al. [Fujimori and Minami, 1984]. These cluster calculations are based on a model Hamiltonian with empirical parameters. The local spectral function in our real-space-decomposition based $\mathrm{DF}+\mathrm{RDMF}$ approach agrees qualitatively with the experimental XPS results. The distance of the main two peaks is larger and the satellite structure lies at higher binding energies in the $\mathrm{DF}+\mathrm{RDMF}$ ground state than in the experimental results. In the local spectral function, the satellite structure is composed of just two peaks because the local spectral function is the spectral function of a finite system, whereas the satellite structure is very broad in the experimental results. The satellite peak in the cluster calculations is composed of many small peaks. DFT + DMFT results for the density of states of Kunes et al. [Kuneš et al., 2007a,b], Yin et al. [Yin et al., 2008], Miura et al. [Miura and Fujiwara, 2008], Nekrasov et al. [Nekrasov et al., 2012], Thunström et al. [Thunström et al., 2012] and results from the variational cluster approximation (VCA) of Eder [Eder, 2007, 2008, 2015] are shown in figure 11.26 in direct comparison to results from our real-space-decomposition based DF+RDMF approach. All $\mathrm{DFT}+\mathrm{DMFT}$ result qualitatively agree, but the position of the satellite structure or the structure of the main peaks are predicted differently. Compared to experimental results and DFT + DMFT results, the peaks of the local spectral function in our DF + RDMF approach lie further apart.

Our results for the local spectral function discussed in this section have been calculated with the ACA with the first level effective bath $(M=1)$. As calculations for for higher effective bath levels $(M>1)$ are not possible at the moment, we can include the screening due to the truncated states effectively by an empirical screening of the Coulomb interaction. We can estimate the effect of a homogeneous screening of the interaction with a factor $a$ on the local spectral functional. We assume that the one-particle reduced density matrix of the ground state is not significantly affected by the change from $\hat{W}_{R}$ to $a \hat{W}_{R}$. We have then

$$
F^{a \hat{W}_{R}}\left[\rho_{R}^{(1), \chi}\right]=a F^{\hat{W}_{R}}\left[\rho_{R}^{(1), \chi}\right]
$$

and for the derivatives

$$
\frac{\partial F_{\beta}^{a \hat{W}_{R}}\left[\rho_{R}^{(1)}\right]}{\partial \rho_{R, \alpha, \beta}^{(1), \chi}}=a \frac{\partial F_{\beta}^{a \hat{W}_{R}}\left[\rho_{R}^{(1), \chi}\right]}{\partial \rho_{R, \alpha, \beta}^{(1)}} .
$$

As a consequence, the many-particle Hamiltonian for which the spectral function is calculated is linear in $a$ and, hence, the spectral function $A_{a, \text { local }, \alpha \beta}(\epsilon)$ for the effective interaction $a \hat{W}_{R}$ is related to the spectral function $A_{\text {local, } \alpha \beta}(\epsilon)$ for the full interaction $\hat{W}_{R}$ by

$$
A_{a, \mathrm{local}, \alpha \beta}(\epsilon)=A_{\text {local }, \alpha \beta}(\epsilon / a) \text {. }
$$

Thus, a linear scaling of the interaction leads to a linear scaling of the energy axis of the local spectral function. If we estimate the required screening to obtain the experimental value of the gap in a calculation with $N N=1$ and $M=1$ to $a=4 \mathrm{eV} / 6 \mathrm{eV}=2 / 3$. If we 


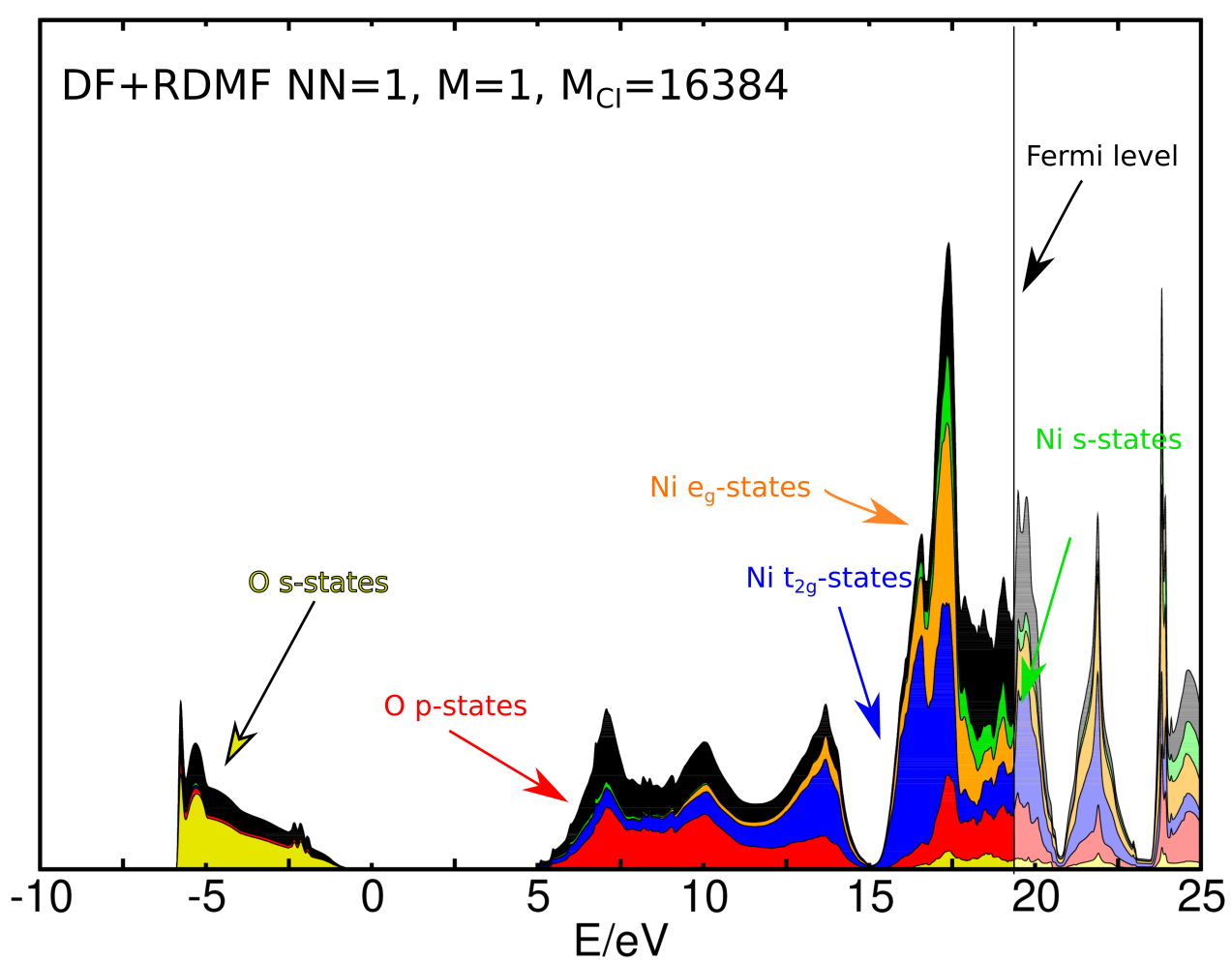

Figure 11.22.: Kohn-Sham-like projected density of states of the nonmagnetic ground state of $\mathrm{NiO}$ in the real-space-decomposition based $\mathrm{DF}+\mathrm{RDMF}$ approach with $N N=1, M=1$ and $M_{\mathrm{CI}}=16384$. Details of the calculation as discussed in section 11.1. The projected densities of states are stacked in front of the total density of states (black). Unoccupied states are indicated by pale colors.

scale the energy axis of the local spectral function by $2 / 3$, the distances in the peaks of the local spectral function agree much better with the experimental XPS results. A scaling factor of $2 / 3$ is much larger than the scaling factors of $0.125-0.25$ commonly used in local hybrid functionals. Thus, our method covers a large portion of the screening already for $M=1$. We expect this portion to converge to one with increasing $M>1$. Please note, that all results shown in figures in this section do not include the factor $a \neq 1$ but the unscaled results from the calculation with $M=1$.

We conclude that the real-space-decomposition based approach with $N N=1, M=1$ and $M_{\mathrm{CI}}=16384$ correctly predicts the nonmagnetic state of $\mathrm{NiO}$ as an insulator. The screening of the interaction is implicitly accounted for by the method. One effective bath level in the adaptive cluster approximation $(M=1)$ is not sufficient for $\mathrm{NiO}$ and, hence, the value of the gap of $6 \mathrm{eV}$ is overestimated compared to the experimental result of $4 \mathrm{eV}$. The spectral function qualitatively agrees with the experimental results and results from DFT+DMFT. 

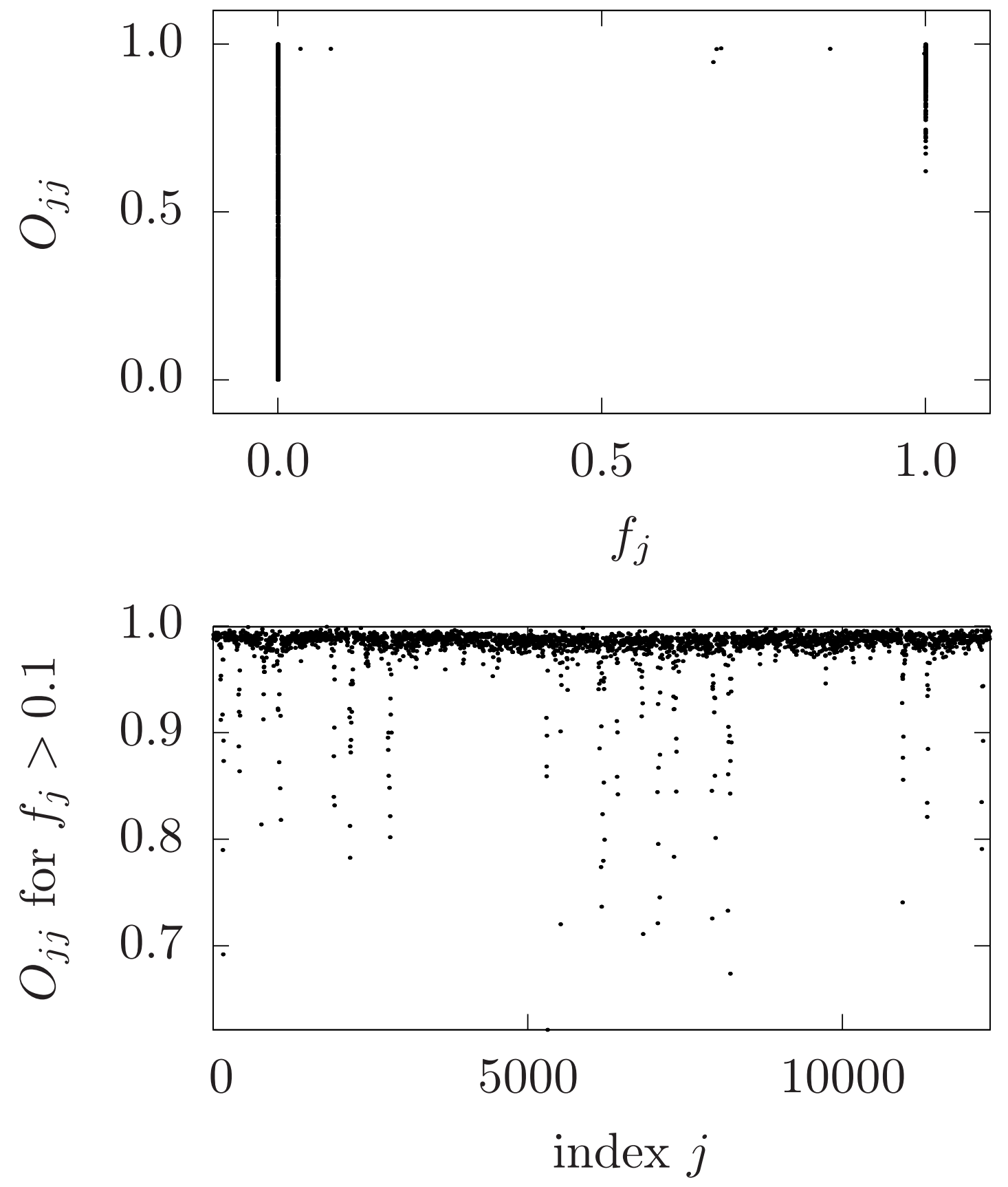

Figure 11.23.: Overlap $O_{j j}=O_{i i}(\vec{k})=\left\langle\mu_{i}(\vec{k}) \mid \mu_{i}(\vec{k})\right\rangle$ of the projected states $\left|\mu_{i}(\vec{k})\right\rangle \approx$ $\left|\psi_{i}(\vec{k})\right\rangle$ in the nonmagnetic ground state of $\mathrm{NiO}$ with $N N=1, M=1$ and $M_{\mathrm{CI}}=16384$. The index $j$ is a combined index of the band index $i$ and the index of a k-point $\vec{k}$ in a discrete k-point grid. The top graph shows the overlaps $O_{j j}$ versus the occupations $f_{j}$ of the states. The bottom graph shows only the overlaps of states with $f_{i}>0.1$ for closer inspection. 


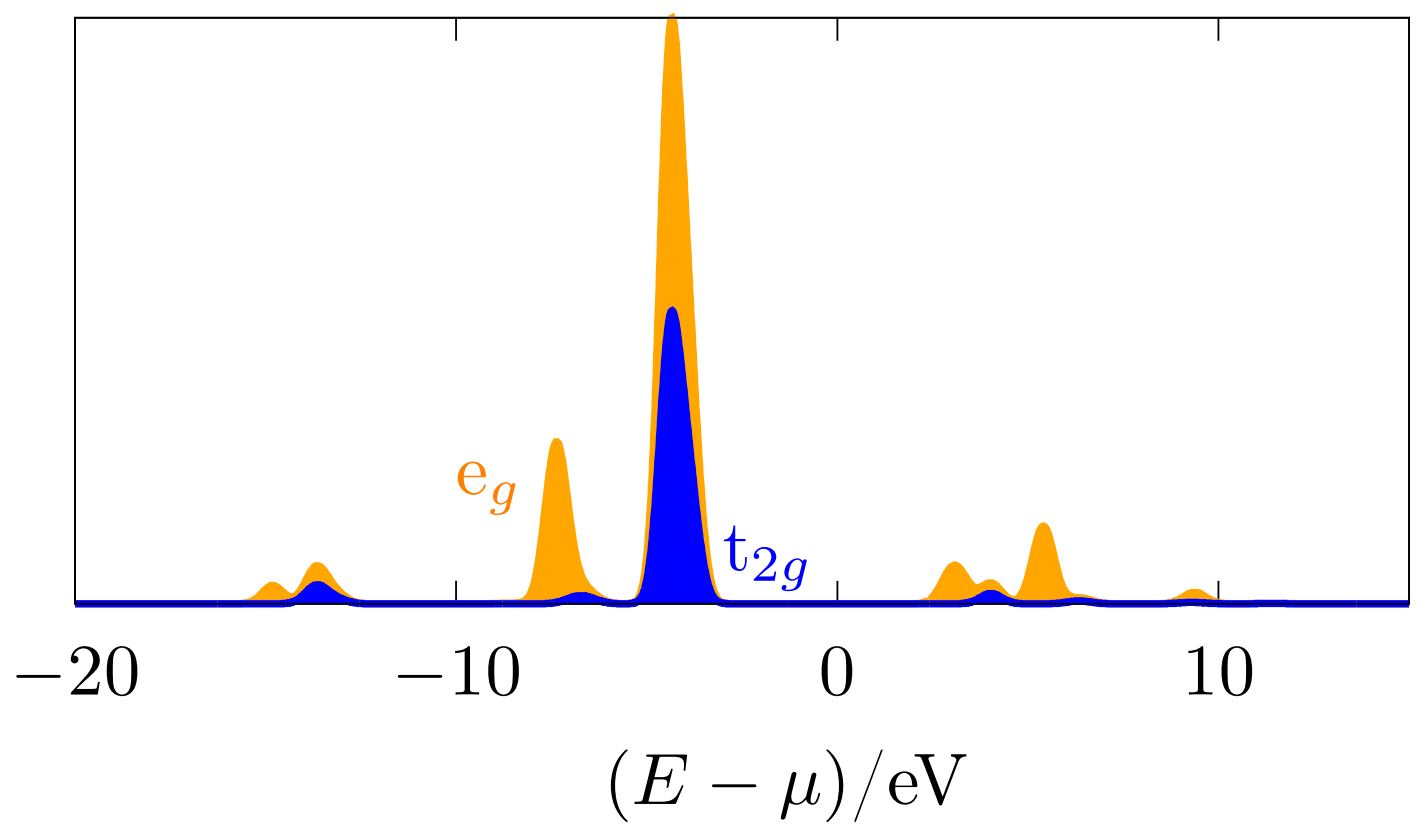

Figure 11.24.: Local density of states of the nickel 3d-states for the nonmagnetic ground state of $\mathrm{NiO}$ in the real-space-decomposition based DF+RDMF approach with $N N=1, M=1$ and $M_{\mathrm{CI}}=16384$. The densities of states are stacked on top of each other. Details of the calculation as discussed in section 11.1. The peaks have been broadened with a Gaussian broadening of $0.2 \mathrm{eV}$ for better visibility. 

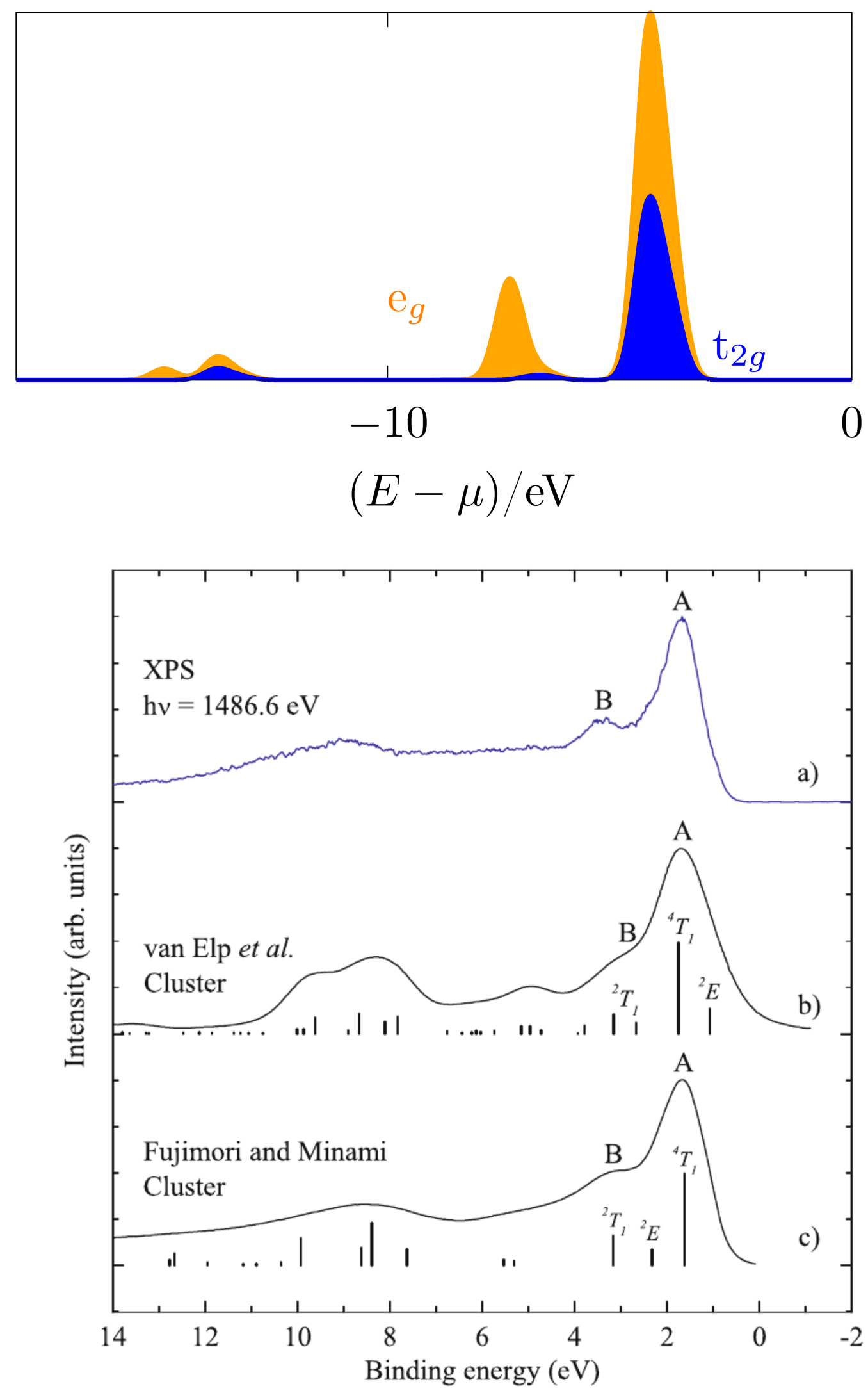

Figure 11.25.: Local density of states of the nickel 3d-states for the nonmagnetic ground state of $\mathrm{NiO}$ in the real-space-decomposition based DF+RDMF approach with $N N=1, M=1$ and $M_{\mathrm{CI}}=16384$ compared to cluster results of van Elp at al. [van Elp et al., 1992] and Fujimori et al. [Fujimori and Minami, 1984]. The bottom figure from [Kuo et al., 2017]. 

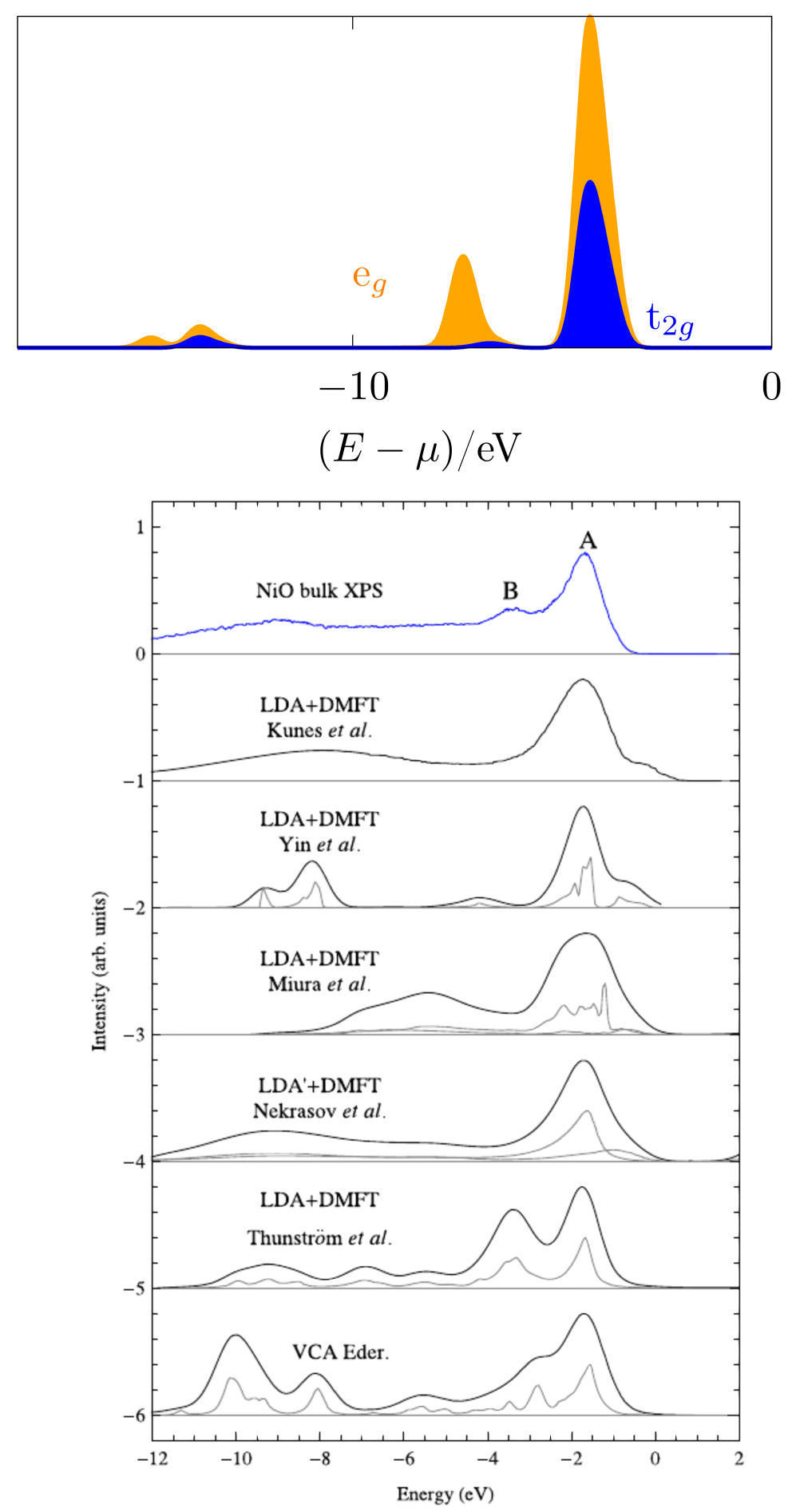

Figure 11.26.: Local density of states of the nickel 3d-states for the nonmagnetic ground state of $\mathrm{NiO}$ in the real-space-decomposition based DF+RDMF approach with $N N=1, M=1$ and $M_{\mathrm{CI}}=16384$ compared to DFT+DMFT results of Kunes et al. [Kuneš et al., 2007a,b], Yin et al. [Yin et al., 2008], Miura et al. [Miura and Fujiwara, 2008], Nekrasov et al. [Nekrasov et al., 2012], Thunström et al. [Thunström et al., 2012] and results from the variational cluster approximation (VCA) of Eder [Eder, 2007, 2008, 2015]. The bottom figure from [Kuo et al., 2017]. Black lines with $1 \mathrm{eV}$ broadening, grey lines show results given originally in the references. 



\section{Summary and conclusion}

One key result of this thesis is the development and implementation of an approach that combines density functionals and density-matrix functionals with a real-space based decomposition of the electron-electron interaction. The strongly correlated orbitals are treated with the density-matrix functional that allows a more natural description of electronic correlations because it is based one the one-particle reduced density matrix rather than the electron density. The approach is based on reduced density-matrix functional theory and does not suffer from a double-counting problem. We show that it is not necessary to introduce an effective Coulomb interaction in this approach as long as the local one-particle states for the evaluation of the density-matrix functional are chosen appropriately.

We have implemented the real-space-decomposition based DF+RDMF approach within the projector augmented-wave method in the CP-PAW code. We use a parametrized local approximation for the density functional and available pair-distributions functions of this density functional for the evaluation of the double-counting contribution. The density-matrix functional of the local interactions is approximated by a sum local densitymatrix functionals with the local approximation proposed by Blöchl et al. [Blöchl et al., 2011]. The resulting local density-matrix functionals have a small number of interacting one-particle states and a large number of non-interacting one-particle states. We have proposed the adaptive cluster approximation (ACA) to systematically approximate these local density-matrix functional by density-matrix functionals with a much smaller number of non-interacting one-particle states. Applications of the ACA to single-impurity Anderson models and Hubbard rings show a rapid convergence with the number of effective bath levels. We have also shown that the transformation of the one-particle basis introduced within the adaptive cluster approximation can be used to drastically reduce the bond dimensions of matrix product states for ground-state calculations and time evolutions of single-impurity Anderson models and quantum dots. In contrast to the natural orbital basis, the matrix elements of the interaction Hamiltonian are not affected by this transformation and, hence, the bond dimension of the MPO of the Hamiltonian grows only moderately due to the transformation.

We propose several new parametrizations of many-particle wave functions and numerical minimization schemes for the evaluation of the density-matrix functional from Levy's constrained minimization problem. Evaluating the density-matrix functional directly from its basic definition has the advantage that the density-matrix functional is a guaranteed upper bound of the exact function and can be systematically improved towards the exact result. We have shown that the Powell-Hestenes augmented Lagrangian is a universally applicable and stable minimization algorithm for the solution of the constrained minimization problem. It does not suffer from the Maratos effect and allows us to use parametrizations of many-particle wave functions that do not necessarily fulfill all constraints exactly.

We started with a parametrization of the many-particle wave function with a configuration-interaction-like approach in terms of Slater determinants. We showed that 


\section{Summary and conclusion}

a one-particle basis of natural orbitals or partial natural orbitals together with a selection criterion of Slater determinants based on the augmented Lagrangian leads to a rapid convergence to the exact result with the number of Slater determinants. We have proposed an impurity-bath separation ansatz based on the Schmidt decomposition where the impurity wave functions are parametrized as full many-particle wave functions and the bath wave functions are parametrized by a few Slater determinants. This ansatz leads to an efficient density-matrix functional for single-impurity Anderson models, but fails lattice models.

Matrix product states are in the same spirit as the impurity-bath separation ansatz in the sense that a Schmidt decomposition of the many-particle wave function is used, but can represent any many-particle state. We propose an evaluation of the density-matrix functional with matrix product states in a DMRG-like iterative solution of the constrained minimization problem. We show that the proposed DMRG-like algorithm converges in a similar number of sweeps than a conventional DMRG total-energy minimization.

The use of the augmented Lagrangian also allows us to employ parametrization of the many-particle wave function for which expectation values cannot be evaluated in a closed form in practice. We explore the evaluation of the density-matrix functional with Gutzwiller- and Jastrow-correlated wave functions in a quantum Monte Carlo based procedure and show that the density-matrix functional can be evaluated efficiently with such correlated wave functions.

Finally, we propose a computational protocol to evaluate the density-matrix functional on existing and near-term gate-based quantum computers with the help of hardwareefficient trial states. As an example, we attempt to evaluate the density-matrix functional for the half-filled Hubbard dimer on a 5-qubit quantum computer made available by IBM. We optimize the hardware-efficient trail states for this quantum-computer architecture and show that a parametrization of a hardware-efficient trail state obtained on a classical computer leads to qualitatively correct results on the quantum computer. The deviations are traced back to systematic deviations in the applications of two-qubit gates to the quantum state. The small runtime available for our experimentation on the quantum computer prevented the execution of the full constrained minimization problem. However, we conclude that the systematic deviations in the gate applications are naturally circumvented if all minimization steps are executed on the quantum computer.

Apart from wave-function based approaches, we have propose the evaluation of the density-matrix functional in a two-particle reduced density-matrix functional theory-like approach, where the two-particle reduced density matrix is constrained by a subset of the N-representability conditions. This approach is promising because it leads to a lower bound of the density-matrix functional. Combined with a wave-function based approach, that is a guaranteed upper bound, it is possible to give bounds to the exact density-matrix functional, and the difference between the lower and the upper bound could serve as a practical diagnostic for the accuracy of the approximations.

We apply the real-space-decomposition based DF+RDMF approach with the adaptive cluster approximation and the configuration-interaction-like approach to the non-spinpolarized ground state of the hydrogen molecule. We show that the dissociation limit of the hydrogen molecule is correctly described with the proposed approach and, thus, the symmetric-breaking-dilemma solved without the need to reinterpret the broken spinstate in spin-polarized density-functional theory. The real-space-decomposition based $\mathrm{DF}+\mathrm{RDMF}$ approach predicts the nonmagnetic state of the transition-metal oxide $\mathrm{NiO}$ as an insulator, unlike the $\mathrm{DFT}+\mathrm{U}$ method or local hybrid functionals, in agreement 
with experimental results. The band gap is estimated to $6 \mathrm{eV}$ and slightly overestimated compared to the experimental result of about $4 \mathrm{eV}$. We suspect the reason for the overestimation of the band gap in the adaptive cluster approximation because in the presented calculations only the first level effect bath was used. The inclusion of higher level effective baths is currently not possible in a reasonable computation time with the configurationinteraction-like evaluation of the density-matrix functional. However, we expect this to be possible with the proposed matrix product state-based approach or the proposed evaluation of the density-matrix functional with Gutzwiller-Jastor-correlated wave functions. The implementations for these methods are currently only at a proof-of-concept level. We have proposed several directions in which these implementations can be improved towards viable candidates for calculations of real materials.

Even though the spectral function is not directly accessible in reduced density-matrix functional theory and, thus, also not in the real-space-decomposition based DF+RDMF approach, we have proposed a method to approximate the local spectral function of the strongly correlated orbitals. Results for $\mathrm{NiO}$ qualitatively agree with experimental XPS results and DFT+DMFT results. In contrast to DFT $+\mathrm{U}$ results for the spectral function, the spectral function in our newly proposed approach also describes the satellite structures at binding energies of about $10 \mathrm{eV}$. The proper description of strong local electronic correlations with the proposed real-space-decomposition based DF $+\mathrm{RDMF}$ approach and the formulation of the approach in terms of an total-energy expression presents the foundation for performing efficient atomic structure relaxations, investigations of structural stability and ab-initio molecular dynamics simulations for correlated materials. 



\section{A. Supplemental information for quantum computer calculations}

\section{A.1. Hardware-efficient trial state}

The quantum circuit for the preparation of the modified hardware-efficient trial state shown in Figure 9.26 including the values of the variational parameters from optimization on a classical computer is

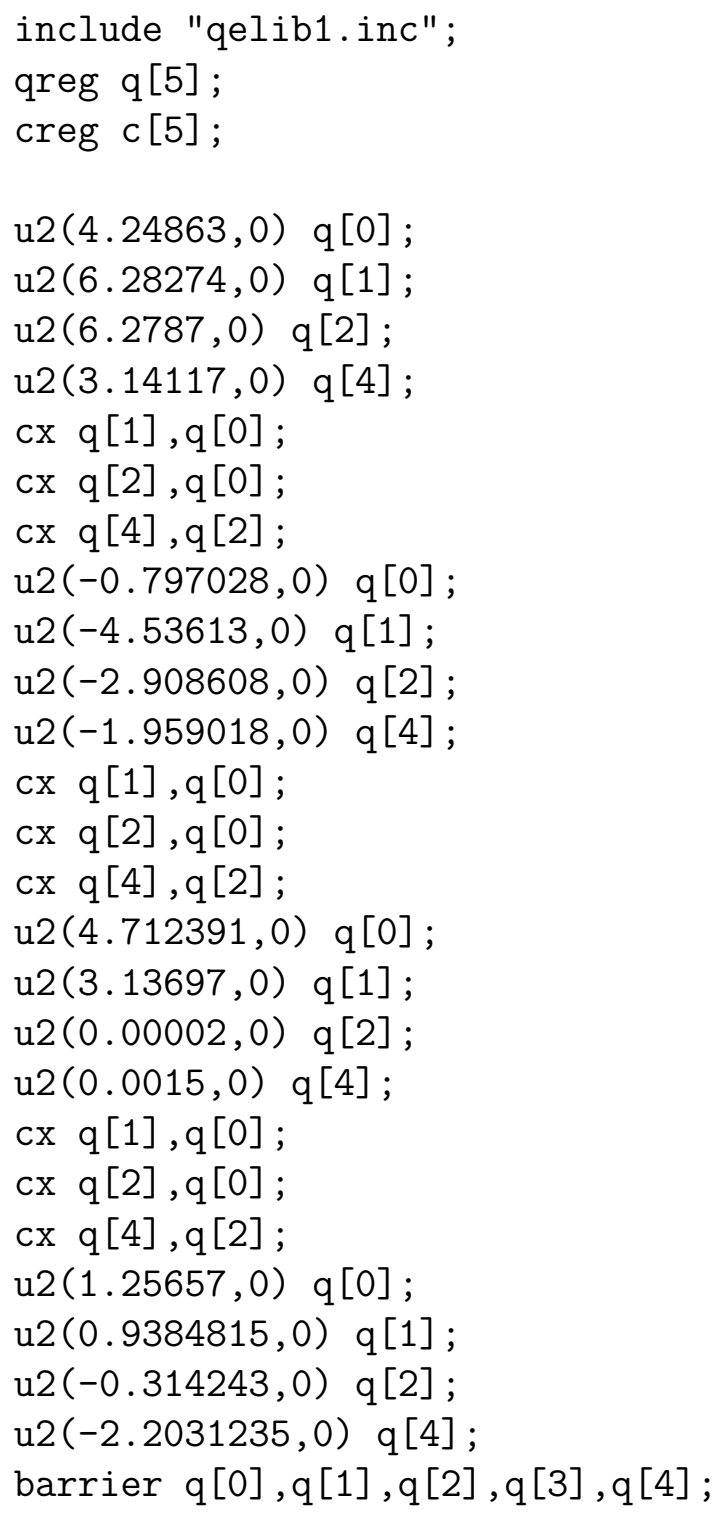




\section{A.2. Measurements}

Tables A.1-A.3 compare the exact expectation values for the state prepared by the circuit in section A.1 of multi-qubit observables to Monte Carlo (MC) simulations and the results obtained on the IBM Q5 Teneriffe (ibmqx4) quantum computer. The exact results were obtained from the simulation of the quantum gates as unitary matrices on a classical computer. The Monte Carlo results are from the Monte Carlo simulator included in the IBM Q experience framework. The Monte Carlo simulator can be selected there as an alternative backend. It takes the same input as the real quantum computer and returns results in the same format as the quantum computer. Thus, it allows us to easily check the correctness of the circuit layouts because it implements the same restrictions that the real quantum computer has. Additionally, it allows the verification of the results from the quantum computer. The calculations on the quantum computer were performed in the same calibration cycle of the device between 5th of July 2018 10:00 PM and 6th of July 2018 3:00 AM. The daily calibration changes the device specification slightly. Each expectation value is calculated from 8192 executions and measurements. 


\begin{tabular}{|c|c|c|c|c|c|}
\hline quantity & measur & rement circuit & exact value & MC-simulator & IBM Q5 \\
\hline$\left\langle\sigma_{z} \otimes \mathbb{1} \otimes \mathbb{1} \otimes \mathbb{1}\right\rangle$ & $\begin{array}{l}\left|q_{0}\right\rangle- \\
\left|q_{1}\right\rangle\end{array} \mid \begin{array}{l}|\Psi(\vec{u})\rangle \\
\left|q_{2}\right\rangle \\
\left|q_{3}\right\rangle\end{array}$ & $\begin{array}{l}\Delta= \\
\\
\end{array}$ & $-2.798 \cdot 10^{-6}$ & $4.1 \cdot 10^{-3}$ & 0.262 \\
\hline$\left\langle\mathbb{1} \otimes \sigma_{z} \otimes \mathbb{1} \otimes \mathbb{1}\right\rangle$ & $\begin{array}{l}\left|q_{0}\right\rangle- \\
\left|q_{1}\right\rangle-f_{|\Psi(\vec{u})\rangle} \mid \\
\left|q_{2}\right\rangle \\
\left|q_{3}\right\rangle\end{array}$ & $\sqrt{\Phi=}$ & $1.452 \cdot 10^{-6}$ & $1.4 \cdot 10^{-2}$ & -0.040 \\
\hline$\left\langle\mathbb{1} \otimes \mathbb{1} \otimes \sigma_{z} \otimes \mathbb{1}\right\rangle$ & $\begin{array}{l}\left|q_{0}\right\rangle- \\
\left|q_{1}\right\rangle \\
\left|q_{2}\right\rangle \\
\left|q_{3}\right\rangle\end{array}-|\Psi(\vec{u})\rangle \mid$ & $\sqrt{\Phi=}$ & $2.782 \cdot 10^{-6}$ & $-8.8 \cdot 10^{-3}$ & -0.048 \\
\hline$\left\langle\mathbb{1} \otimes \mathbb{1} \otimes \mathbb{1} \otimes \sigma_{z}\right\rangle$ & $\begin{array}{l}\left|q_{0}\right\rangle- \\
\left|q_{1}\right\rangle\end{array} \mid$\begin{tabular}{r|}
$|\Psi(\vec{u})\rangle$ \\
$\left|q_{2}\right\rangle$ \\
$\left|q_{3}\right\rangle$
\end{tabular} & $-\phi=$ & $-5.989 \cdot 10^{-6}$ & $-1.4 \cdot 10^{-3}$ & -0.095 \\
\hline$\left\langle\sigma_{z} \otimes \sigma_{z} \otimes \mathbb{1} \otimes \mathbb{1}\right\rangle$ & 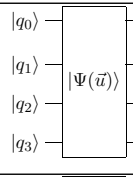 & $+D^{-1}$ & -0.4473 & -0.455 & -0.102 \\
\hline$\left\langle\mathbb{1} \otimes \mathbb{1} \otimes \sigma_{z} \otimes \sigma_{z}\right\rangle$ & $\left.\begin{array}{l}\left|q_{0}\right\rangle- \\
\left|q_{1}\right\rangle \\
\left|q_{2}\right\rangle \\
\left|q_{3}\right\rangle\end{array}-\mid \Psi(\vec{u}\rangle\right) \mid$ & $\bar{\square}$ & -0.4473 & -0.438 & -0.237 \\
\hline$\left\langle\sigma_{x} \otimes \sigma_{x} \otimes \mathbb{1} \otimes \mathbb{1}\right\rangle$ & $\begin{array}{l}\left|q_{0}\right\rangle- \\
\left|q_{1}\right\rangle \\
\left|q_{2}\right\rangle \\
\left|q_{3}\right\rangle\end{array}-{ }_{|\Psi(\vec{u})\rangle} \mid$ & $\begin{array}{l}-S^{\dagger}-(H-\square= \\
-S^{\dagger}-\sqrt{H}\end{array}$ & $3.721 \cdot 10^{-8}$ & $7.3 \cdot 10^{-3}$ & 0.082 \\
\hline$\left\langle\mathbb{1} \otimes \mathbb{1} \otimes \sigma_{x} \otimes \sigma_{x}\right\rangle$ & $\left.\begin{array}{l}\left|q_{0}\right\rangle- \\
\left|q_{1}\right\rangle \\
\left|q_{2}\right\rangle \\
\left|q_{3}\right\rangle\end{array}-\mid \Psi(\vec{u}\rangle\right) \mid$ & 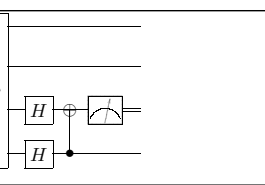 & $-2.839 \cdot 10^{-8}$ & $-7.3 \cdot 10^{-4}$ & 0.045 \\
\hline$\left\langle\sigma_{y} \otimes \sigma_{y} \otimes \mathbb{1} \otimes \mathbb{1}\right\rangle$ & $\begin{array}{l}\left|q_{0}\right\rangle- \\
\left|q_{1}\right\rangle \\
\left|q_{2}\right\rangle \\
\left|q_{3}\right\rangle\end{array}-|\Psi(\vec{u})\rangle$ & $\begin{array}{l}S^{S^{\dagger}}-\sqrt{H}-\Phi D^{-} \\
-S^{\dagger}-\sqrt{H} \cdot\end{array}$ & $3.397 \cdot 10^{-7}$ & -0.023 & 0.123 \\
\hline$\left\langle\mathbb{1} \otimes \mathbb{1} \otimes \sigma_{y} \otimes \sigma_{y}\right\rangle$ & 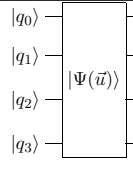 & $\sqrt{\sqrt{S^{t}-\sqrt[H]{S^{t}-\sqrt{H}} \cdot \Phi^{-}}}$ & $-4.439 \cdot 10^{-7}$ & -0.0015 & 0.039 \\
\hline
\end{tabular}

Table A.1.: List of measurements for the hardware-efficient trail state $|\Psi(\vec{u})\rangle$ from appendix A.1. The third column shows the expectation value calculated classically by simulating the quantum gates. The fourth column gives the results from a Monte Carlo based simulation of the quantum circuit that is included in IBM QISKit with 8192 shots. The last column shows the results from the quantum computer IBM Q5 Teneriffe with 8192 executions of the quantum program. 
A. Supplemental information for quantum computer calculations

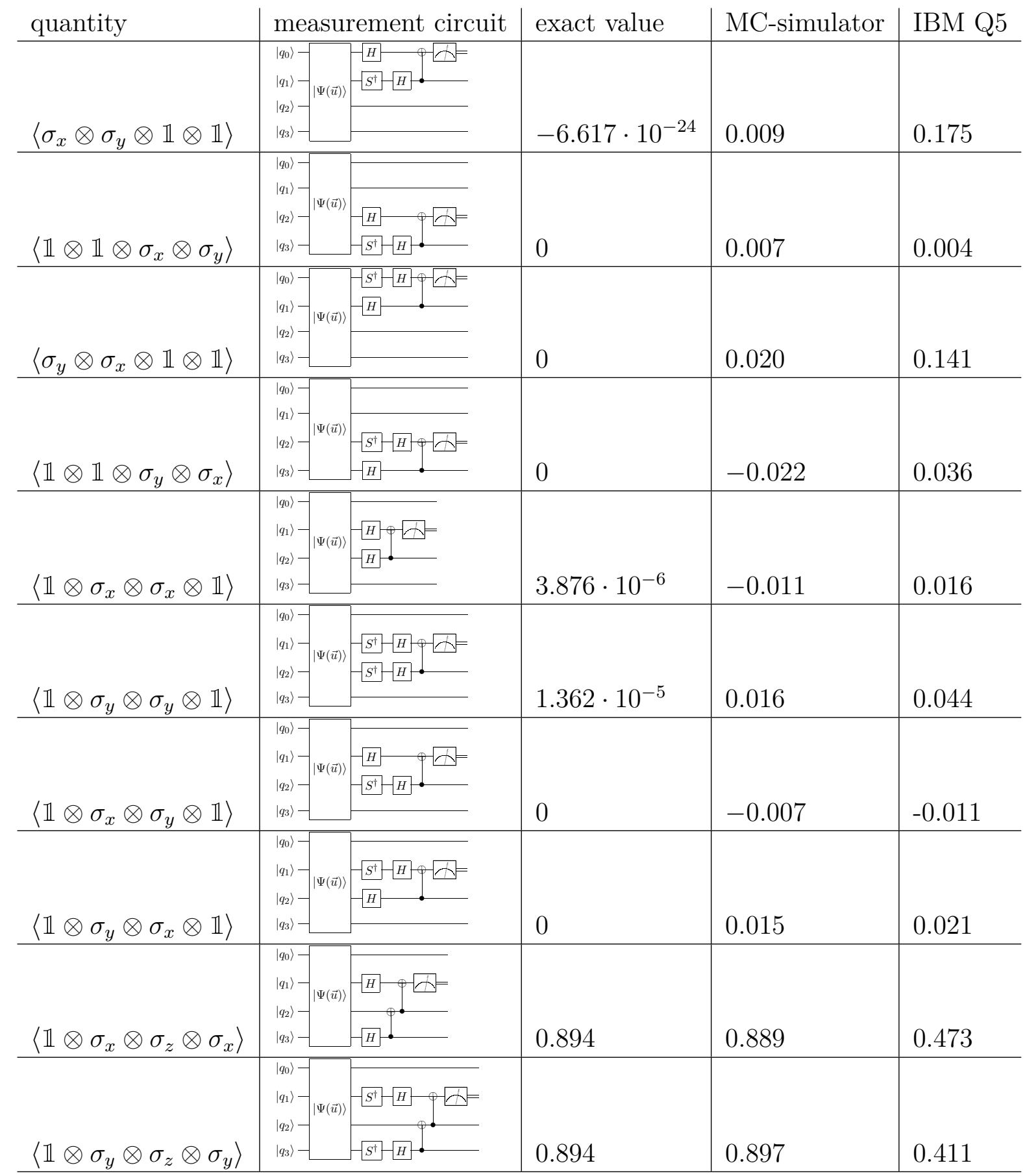

Table A.2.: This table continues Table A.1. List of measurements for the hardwareefficient trail state $|\Psi(\vec{u})\rangle$ from appendix A.1. The third column shows the expectation value calculated classically by simulating the quantum gates. The fourth column gives the results from a Monte Carlo based simulation of the quantum circuit that is included in IBM QISKit with 8192 shots. The last column shows the results from the quantum computer IBM Q5 Teneriffe with 8192 executions of the quantum program. 


\begin{tabular}{|c|c|c|c|c|c|c|}
\hline quantity & me & asui & rement circuit & exact value & MC-simulator & IBM Q5 \\
\hline$\left\langle\mathbb{1} \otimes \sigma_{x} \otimes \sigma_{z} \otimes \sigma_{y}\right\rangle$ & & & 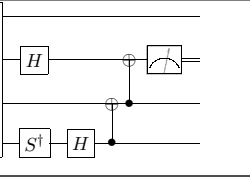 & 0 & -0.025 & $0-0.0002$ \\
\hline$\left\langle\mathbb{1} \otimes \sigma_{y} \otimes \sigma_{z} \otimes \sigma_{x}\right\rangle$ & $\begin{array}{l}\left|q_{0}\right\rangle- \\
\left|q_{1}\right\rangle- \\
\left|q_{2}\right\rangle \\
\left|q_{3}\right\rangle\end{array}$ & $|\Psi(\vec{u})\rangle$ & 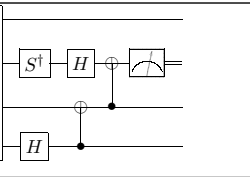 & 0 & 0.013 & 0.046 \\
\hline$\left\langle\sigma_{x} \otimes \sigma_{z} \otimes \sigma_{x} \otimes \mathbb{1}\right\rangle$ & $\begin{array}{l}\left|q_{0}\right\rangle- \\
\left|q_{1}\right\rangle- \\
\left|q_{2}\right\rangle- \\
\left|q_{3}\right\rangle-\end{array}$ & $|\Psi(\vec{u})\rangle$ & 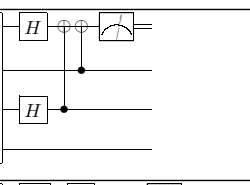 & 0.894 & 0.894 & 0.468 \\
\hline$\left\langle\sigma_{y} \otimes \sigma_{z} \otimes \sigma_{y} \otimes \mathbb{1}\right\rangle$ & $\begin{array}{l}\left|q_{0}\right\rangle- \\
\left|q_{1}\right\rangle- \\
\left|q_{2}\right\rangle- \\
\left|q_{3}\right\rangle- \\
\end{array}$ & $|\Psi(\vec{u})\rangle$ & 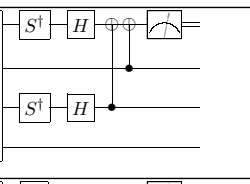 & 0.894 & 0.903 & 0.461 \\
\hline$\left\langle\sigma_{x} \otimes \sigma_{z} \otimes \sigma_{y} \otimes \mathbb{1}\right\rangle$ & $\begin{array}{l}\left|q_{0}\right\rangle- \\
\left|q_{1}\right\rangle- \\
\left|q_{2}\right\rangle \\
\left|q_{3}\right\rangle \\
\end{array}$ & $|\Psi(\vec{u})\rangle$ & 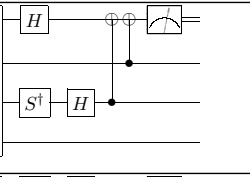 & 0 & -0.0005 & 0.141 \\
\hline$\left\langle\sigma_{y} \otimes \sigma_{z} \otimes \sigma_{x} \otimes \mathbb{1}\right\rangle$ & $\begin{array}{l}\left|q_{0}\right\rangle- \\
\left|q_{1}\right\rangle- \\
\left|q_{2}\right\rangle- \\
\left|q_{3}\right\rangle- \\
\end{array}$ & $|\Psi(\vec{u})\rangle$ & 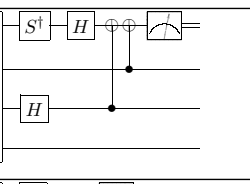 & 0 & -0.005 & 0.138 \\
\hline$\left\langle\sigma_{x} \otimes \sigma_{z} \otimes \sigma_{z} \otimes \sigma_{x}\right\rangle$ & $\begin{array}{l}\left|q_{0}\right\rangle- \\
\left|q_{1}\right\rangle- \\
\left|q_{2}\right\rangle- \\
\left|q_{3}\right\rangle \\
\end{array}$ & $|\Psi(\vec{u})\rangle$ & 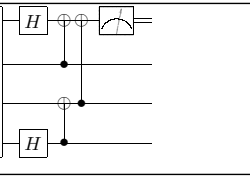 & $-2.960 \cdot 10^{-6}$ & -0.001 & 0.212 \\
\hline$\left\langle\sigma_{y} \otimes \sigma_{z} \otimes \sigma_{z} \otimes \sigma_{y}\right\rangle$ & $\begin{array}{l}\left|q_{0}\right\rangle- \\
\left|q_{1}\right\rangle- \\
\left|q_{2}\right\rangle- \\
\left|q_{3}\right\rangle-\end{array}$ & $|\Psi(\vec{u})\rangle$ & 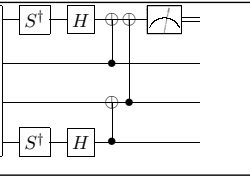 & $-1.341 \cdot 10^{-5}$ & 0.001 & 0.185 \\
\hline$\left\langle\sigma_{x} \otimes \sigma_{z} \otimes \sigma_{z} \otimes \sigma_{y}\right\rangle$ & $\begin{array}{l}\left|q_{0}\right\rangle- \\
\left|q_{1}\right\rangle- \\
\left|q_{2}\right\rangle- \\
\left|q_{3}\right\rangle\end{array}$ & $|\Psi(\vec{u})\rangle$ & 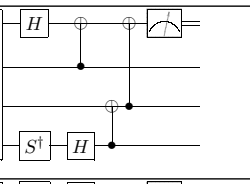 & 0 & 0.013 & 0.205 \\
\hline$\left\langle\sigma_{y} \otimes \sigma_{z} \otimes \sigma_{z} \otimes \sigma_{x}\right\rangle$ & $\begin{array}{l}\left|q_{0}\right\rangle- \\
\left|q_{1}\right\rangle- \\
\left|q_{2}\right\rangle- \\
\left|q_{3}\right\rangle-\end{array}$ & $|\Psi(\vec{u})\rangle$ & 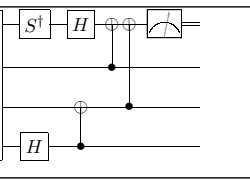 & 0 & -0.013 & 0.349 \\
\hline
\end{tabular}

Table A.3.: This table continues Tables A.1 and A.2. List of measurements for the hardware-efficient trail state $|\Psi(\vec{u})\rangle$ from appendix A.1. The third column shows the expectation value calculated classically by simulating the quantum gates. The fourth column gives the results from a Monte Carlo based simulation of the quantum circuit that is included in IBM QISKit with 8192 shots. The last column shows the results from the quantum computer IBM Q5 Teneriffe with 8192 executions of the quantum program. 



\section{B. Proofs related to the density-matrix functional}

\section{B.1. Separation property}

This proof was developed by the author and has been published in [Schade and Blöchl, 2018]. We show in the following that the density-matrix functional for an interaction of the form

$$
\hat{W}=\frac{1}{2} \sum_{\alpha, \beta, \gamma, \delta \leq N_{\mathrm{int}}} U_{\alpha, \beta, \gamma, \delta} \hat{c}_{\alpha}^{\dagger} \hat{c}_{\beta}^{\dagger} \hat{c}_{\delta} \hat{c}_{\gamma}
$$

for a block-diagonal one-particle reduced density matrix

$$
\rho^{(1)}=\left(\begin{array}{cc}
\rho_{\text {imp }}^{(1)} & 0 \\
0 & \rho_{\text {rest }}^{(1)}
\end{array}\right)
$$

with $\rho_{\text {imp }}^{(1)} \in \mathbb{C}^{N_{\text {imp }} \times N_{\text {imp }}}$ and $N_{\text {imp }} \geq N_{\text {int }}$ is the sum of the density-matrix functional of the interacting block and an entropy contribution from the non-interacting block. That is, the relation

$$
F_{\beta}^{\hat{W}}\left[\left(\begin{array}{cc}
\rho_{\text {imp }}^{(1)} & 0 \\
0 & \rho_{\text {rest }}^{(1)}
\end{array}\right)\right]=F_{\beta}^{\hat{W}}\left[\rho_{\text {imp }}^{(1)}\right]+F_{\beta}^{\hat{0}}\left[\rho_{\text {rest }}^{(1)}\right],
$$

holds. We use here the property that the grand potential is concave with the matrix elements of the one-particle Hamiltonian $h$ as discussed in section 2.4 and that the grand potential is an extensive quantity,

$$
\Omega_{\beta, \mu}^{\hat{W}}\left[\left(\begin{array}{cc}
h_{\mathrm{imp}} & 0 \\
0 & h_{\text {rest }}
\end{array}\right)\right]=\Omega_{\beta, \mu}^{\hat{W}}\left[h_{\mathrm{imp}}\right]+\Omega_{\beta, \mu}^{\hat{0}}\left[h_{\text {rest }}\right] .
$$

This can be shown by using that $\hat{h}_{\mathrm{imp}}+\hat{W}$ and $\hat{h}_{\text {rest }}$ commute. The derivatives of the grand potential with respect to the matrix elements $h$ of the one-particle Hamiltonian $\hat{h}$ are given by the thermal expectation values of the one-particle density-matrix operator as

$$
\frac{\partial \Omega_{\beta, \mu}^{\hat{W}}[h]}{\partial h_{i, j}}=\frac{\operatorname{Tr}\left(e^{-\beta(\hat{h}+\hat{W}-\mu \hat{N})} \hat{c}_{i}^{\dagger} \hat{c}_{j}\right)}{\operatorname{Tr} e^{-\beta(\hat{h}+\hat{W}-\mu \hat{N})}} .
$$

We can apply the general relation Eq. (B.5) to the situation of a one-particle Hamiltonian with block-diagonal matrix elements

$$
h=\left(\begin{array}{cc}
h_{\mathrm{imp}} & 0 \\
0 & h_{\mathrm{rest}}
\end{array}\right) .
$$


Then the Hamiltonian $\hat{h}_{\text {imp }}+\hat{W}$, which only acts on the impurity states, commutates with the one-particle Hamiltonian $\hat{h}_{\text {rest }}$ of the rest. The eigenstates $\left|\Psi_{a, b}\right\rangle$ of the full system,

$$
\begin{aligned}
\left(\hat{h}_{\text {imp }}+\hat{W}+\hat{h}_{\text {rest }}\right)\left|\Psi_{a, b}\right\rangle & =\epsilon_{a, b}\left|\Psi_{a, b}\right\rangle, \\
\hat{N}\left|\Psi_{a, b}\right\rangle & =n_{a, b}\left|\Psi_{a, b}\right\rangle,
\end{aligned}
$$

can be written as product states of the eigenstates of the impurity Hamiltonian and eigenstates of the rest Hamiltonian,

$$
\begin{aligned}
\left(\hat{h}_{\mathrm{imp}}+\hat{W}\right)\left|\Psi_{\mathrm{imp}, a}\right\rangle & =\epsilon_{\mathrm{imp}, a}\left|\Psi_{\mathrm{imp}, a}\right\rangle \\
\hat{N}\left|\Psi_{\mathrm{imp}, a}\right\rangle & =n_{\mathrm{imp}, a}\left|\Psi_{\mathrm{imp}, a}\right\rangle \\
\hat{h}_{\mathrm{rest}}\left|\Psi_{\mathrm{rest}, b}\right\rangle & =\epsilon_{\mathrm{rest}, b}\left|\Psi_{\mathrm{rest}, b}\right\rangle \\
\hat{N}\left|\Psi_{\text {rest }, b}\right\rangle & =n_{\mathrm{rest}, b}\left|\Psi_{\mathrm{rest}, b}\right\rangle
\end{aligned}
$$

as $\left|\Psi_{a, b}\right\rangle=\left|\Psi_{\text {imp }, a}\right\rangle \otimes\left|\Psi_{\text {rest }, b}\right\rangle$. Expectation values of the one-particle reduced densitymatrix operator between impurity and rest states vanish for such product states,

$$
\left\langle\Psi_{a, b}\left|\hat{c}_{\mathrm{imp}}^{\dagger} \hat{c}_{\text {rest }}\right| \Psi_{a, b}\right\rangle=\left\langle\Psi_{\mathrm{imp}, a}\left|\hat{c}_{\mathrm{imp}}^{\dagger}\right| \Psi_{\mathrm{imp}, a}\right\rangle \cdot\left\langle\Psi_{\text {rest }, b}\left|\hat{c}_{\text {rest }}\right| \Psi_{\text {rest }, b}\right\rangle=0 .
$$

The matrix elements vanish because the eigenstates $\left|\Psi_{\text {imp }, a}\right\rangle$ and $\left|\Psi_{\text {rest }, b}\right\rangle$ are also eigenstates of the operator $\hat{N}$ of the total particle number. Consequently also the expectation values

$$
\frac{\operatorname{Tr}\left(e^{-\beta(\hat{h}+\hat{W}-\mu \hat{N})} \hat{c}_{\mathrm{imp}}^{\dagger} \hat{c}_{\text {rest }}\right)}{\operatorname{Tr} e^{-\beta(\hat{h}+\hat{W}-\mu \hat{N})}}=\sum_{a, b} \frac{e^{-\beta\left(\epsilon_{a, b}-\mu n_{a, b}\right)}}{\sum_{a^{\prime}, b^{\prime}} e^{-\beta\left(\epsilon_{a^{\prime}, b^{\prime}}-\mu n_{a^{\prime}, b^{\prime}}\right)}}\left\langle\Psi_{a, b}\left|\hat{c}_{\mathrm{imp}}^{\dagger} \hat{c}_{\text {rest }}\right| \Psi_{a, b}\right\rangle
$$

vanish in the thermal ground-state ensemble. Thus, for a vanishing impurity-rest Hamiltonian the derivatives of the grand potential with respect to the matrix elements of the impurity-rest Hamiltonian vanish, i.e.,

$$
\left.\frac{\partial \Omega_{\beta, \mu}^{\hat{W}}\left[\left(\begin{array}{cc}
h_{\text {imp }} & h_{\text {imp,rest }} \\
h_{\text {imp,rest }} & h_{\text {rest }}
\end{array}\right)\right]}{\partial\left(h_{\text {imp,rest }}\right)_{i, j}}\right|_{h_{\text {imp rest }}=0}=0 \forall i, j .
$$

This means that $h_{\mathrm{imp}, \mathrm{rest}}=0$ is a stationary point of the maximization problem

$$
\max _{h_{\text {imp,rest }}} \Omega_{\beta, \mu}^{\hat{W}}\left[\left(\begin{array}{cc}
h_{\text {imp }} & h_{\text {imp,rest }} \\
h_{\text {imp,rest }}^{\dagger} & h_{\text {rest }}
\end{array}\right)\right] .
$$

Combined with the concavity of the grand potential with respect to the matrix elements of the one-particle Hamiltonian this shows that $h_{\mathrm{imp} \text {,rest }}=0$ is a maximizer of the problem in Eq. (B.16).

For the main part of the proof, that is to show Eq. (B.3), we use that the density-matrix functional is the Legendre-Fenchel transform of the grand potential [Lieb, 1983b] $\Omega_{\beta, \mu}^{\hat{W}}[h]$ with respect to the one-particle Hamiltonian

$$
F_{\beta}^{\hat{W}}\left[\rho^{(1)}\right]=\max _{h}\left\{\Omega_{\beta, \mu=0}^{\hat{W}}[h]-\operatorname{Tr}\left(\rho^{(1)} h\right)\right\} .
$$


For the block-diagonal density matrix in Eq. (B.3), the density-matrix functional is

$$
\begin{aligned}
& F_{\beta}^{\hat{W}}\left[\left(\begin{array}{cc}
\rho^{(1)}{ }_{\text {imp }} & 0 \\
0 & \rho^{(1)}{ }_{\text {rest }}
\end{array}\right)\right]=\max _{h_{\text {imp }}, h_{\text {rest }}, h_{\text {imp }, \text { rest }}, \mu}\left\{\Omega_{\beta, \mu}^{\hat{W}}\left[\left(\begin{array}{cc}
h_{\text {imp }} & h_{\text {imp }, \text { rest }} \\
h_{\text {imp,rest }}^{\dagger} & h_{\text {rest }}
\end{array}\right)\right]\right. \\
& \left.-\operatorname{Tr}\left(\rho_{\text {imp }}^{(1)} h_{\text {imp }}\right)-\operatorname{Tr}\left(\rho_{\text {rest }}^{(1)} h_{\text {rest }}\right)\right\} \text {. }
\end{aligned}
$$

The maximization over $h_{\text {imp,rest }}$ can be performed with the help of Eq. (B.16), which yields together with Eq. (B.4) the decomposition

$$
\begin{aligned}
F_{\beta}^{\hat{W}}\left[\left(\begin{array}{cc}
\rho^{(1)}{ }_{\text {imp }} & 0 \\
0 & \rho_{\text {rest }}^{(1)}
\end{array}\right)\right] & =\max _{h_{\text {imp }}, h_{\text {rest }}}\left\{\Omega_{\beta, \mu=0}^{\hat{W}}\left[\left(\begin{array}{cc}
h_{\text {imp }} & 0 \\
0 & h_{\text {rest }}
\end{array}\right)\right]\right. \\
& \left.-\operatorname{Tr}\left(\rho^{(1)}{ }_{\text {imp }} h_{\text {imp }}\right)-\operatorname{Tr}\left(\rho_{\text {rest }}^{(1)} h_{\text {rest }}\right)\right\} \\
& =\max _{h_{\text {imp }}}\left\{\Omega_{\beta, \mu=0}^{\hat{W}}\left[h_{\text {imp }}\right]-\operatorname{Tr}\left(\rho_{\text {imp }}^{(1)} h_{\text {imp }}\right)\right\} \\
& +\max _{h_{\text {rest }}}\left\{\Omega_{\beta, \mu=0}^{\hat{0}}\left[h_{\text {rest }}\right]-\operatorname{Tr}\left(\rho_{\text {rest }}^{(1)} h_{\text {rest }}\right)\right\} \\
& =F_{\beta}^{\hat{W}}\left[\rho_{\text {imp }}^{(1)}\right]+F_{\beta}^{\hat{0}}\left[\rho_{\text {rest }}^{(1)}\right] .
\end{aligned}
$$

This concludes the proof. The non-interacting density-matrix functional $F_{\beta}^{\hat{0}}\left[\rho^{(1)}{ }_{\text {rest }}\right]$ only contains an entropy contribution

$$
F_{\beta}^{\hat{0}}\left[\rho_{\text {rest }}^{(1)}\right]=\frac{1}{\beta} \operatorname{Tr}\left[\rho_{\text {rest }}^{(1)} \ln \left(\rho_{\text {rest }}^{(1)}\right)+\left(1-\rho_{\text {rest }}^{(1)}\right) \ln \left(1-\rho_{\text {rest }}^{(1)}\right)\right]
$$

and vanishes in the zero-temperature limit.

\section{B.2. Proof of existence of banded form}

We show here that for any hermitian matrix

$$
A=\left(\begin{array}{cc}
A_{\text {imp,imp }} & A_{\text {imp,bath }} \\
A_{\text {imp,bath }}^{\dagger} & A_{\text {bath,bath }}
\end{array}\right) \in \mathbb{C}^{N_{\chi} \times N_{\chi}}
$$

with $A_{\text {imp,imp }} \in \mathbb{C}^{N_{\text {imp }} \times N_{\text {imp }}}$ and $N_{\chi}=N_{\text {imp }}+N_{\text {bath }}$ there exists a unitary transformation $U$ of the form

$$
U=\left(\begin{array}{cc}
\mathbb{1} & 0 \\
0 & U_{\text {bath,bath }}
\end{array}\right)
$$


with $U \in U\left(N_{\chi}\right), U_{\text {bath,bath }} \in U\left(N_{\text {bath }}\right)$ such that the transformed matrix $\tilde{A}=U^{\dagger} A U$ has the form

$$
\tilde{A}=\left(\begin{array}{cccccc}
A_{\text {imp }, \text { imp }} & \tilde{A}_{\text {imp,bath }_{1}} & 0 & \ldots & & \\
\tilde{A}_{\text {imp,bath }}^{\dagger} & \tilde{A}_{\text {bath }_{1}, \text { bath }_{1}} & \tilde{A}_{\text {bath }_{1}, \text { bath }_{2}} & 0 & \ldots & \\
0 & \tilde{A}_{\text {bath }_{1}, \text { bath }_{2}} & \tilde{A}_{\text {bath }_{2}, \mathrm{bath}_{2}} & \tilde{A}_{\mathrm{bath}_{2}, \mathrm{bath}_{3}} & 0 & \ldots \\
\vdots & 0 & \tilde{A}_{\mathrm{bath}_{2}, \mathrm{bath}_{3}}^{\dagger} & \tilde{A}_{\mathrm{bath}_{3}, \mathrm{bath}_{3}} & \tilde{A}_{\mathrm{bath}_{3}, \mathrm{bath}_{4}} & \ddots \\
& \vdots & 0 & \tilde{A}_{\mathrm{bath}_{3}, \mathrm{bath}_{4}}^{\dagger} & \tilde{A}_{\mathrm{bath}_{4}, \mathrm{bath}_{4}} & \ddots \\
& & \vdots & \ddots & \ddots & \ddots
\end{array}\right)
$$

We furthermore show that the size of the $i$-th level effective bath, that is, the dimension of the matrix $\tilde{A}_{\text {bath }_{i} \text {,bath }_{i}}$ is at most $N_{\text {imp }}$. The proof proceeds in two steps: at first we show how one transformation step can be constructed and later how several transformations steps can be used to construct the banded form in Eq. (B.25). This proof was developed by the author and has been published in [Schade and Blöchl, 2018].

\section{B.2.1. One transformation step}

To illustrate the single transformation step, we rewrite the matrix $A$ as

$$
\begin{aligned}
& A=\left(\begin{array}{ll}
A_{\text {imp,imp }} & A_{\text {imp,bath }} \\
A_{\text {imp,bath }}^{\dagger} & A_{\text {bath,bath }}
\end{array}\right)
\end{aligned}
$$

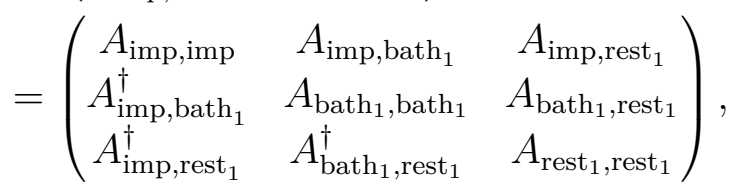

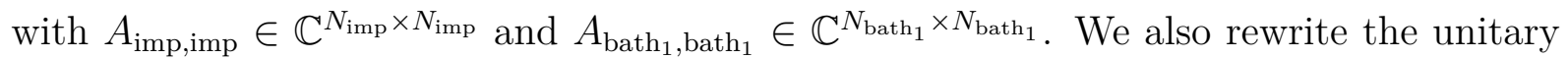
transform $U \in U\left(N_{\chi}\right)$ as

$$
\begin{aligned}
U & =\left(\begin{array}{cc}
1 & 0 \\
0 & U_{\text {bath }}
\end{array}\right) \\
& =\left(\begin{array}{ccc}
1_{N_{\text {imp }}} & 0 & 0 \\
0 & U_{\text {bath }_{1}, \text { bath }_{1}} & U_{\text {bath }_{1}, \text { rest }_{1}} \\
0 & U_{\text {rest }_{1}, \text { bath }_{1}} & U_{\text {rest }_{1}, \text { rest }_{1}}
\end{array}\right)
\end{aligned}
$$

with $U_{\text {bath }_{1}, \text { bath }_{1}} \in \mathbb{C}^{N_{\text {bath }_{1}} \times N_{\text {bath }_{1}}}$ and $U_{\text {rest }_{1}, \text { rest }_{1}} \in \mathbb{C}^{N_{\chi}-N_{\text {imp }}-N_{\text {bath }_{1}} \times N_{\chi}-N_{\text {imp }}-N_{\text {bath }_{1}}}$. The transformed matrix $A$ is then

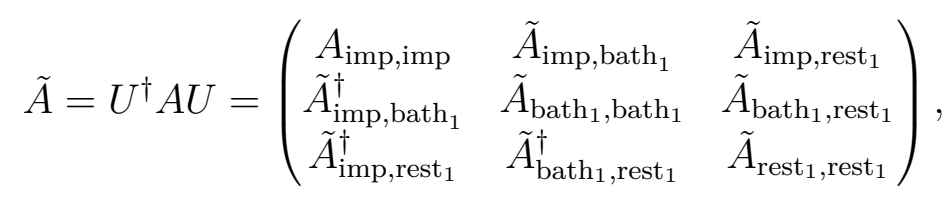

with

$$
\tilde{A}_{\text {imp,rest }}=A_{\text {imp, bath }} U_{\text {bath }_{1}, \text { rest }_{1}}+A_{\text {imp,rest } 1} U_{\text {rest }_{1}, \text { rest }_{1}} \text {. }
$$

Thus, unitary matrix $U_{\text {bath }}$ is defined implicitly by the condition

$$
\tilde{A}_{\text {imp,rest }_{1}}=0=A_{\text {imp, bath }} U_{\text {bath }_{1}, \text { rest }_{1}}+A_{\text {imp,rest }_{1}} U_{\text {rest }_{1} \text { rest }_{1}} .
$$


If we write $U_{\text {bath }}$ as

$$
U_{\text {bath }}=\left(\begin{array}{llll}
\vec{u}_{1} & \vec{u}_{2} & \ldots & \vec{u}_{N_{\chi}-N_{\text {imp }}}
\end{array}\right)
$$

$U \in U\left(N_{\chi}-N_{\text {imp }}\right)$ is equivalent to the orthonormality of the vectors $\vec{u}_{i}$, i.e. $\vec{u}_{i}^{\dagger} \cdot \vec{u}_{j}=\delta_{i, j}$. We have

$$
\left(\begin{array}{c}
U_{\text {bath }_{1}, \text { rest }_{1}} \\
U_{\text {rest }_{1}, \text { rest }_{1}}
\end{array}\right)=\left(\begin{array}{lll}
\vec{u}_{N_{\text {bath }_{1}+1}} & \ldots & \vec{u}_{N_{\chi}-N_{\text {imp }}}
\end{array}\right) .
$$

On the other hand, we can define

$$
\left(\begin{array}{c}
A_{\text {imp }, \text { bath }_{1}}^{T} \\
A_{\text {imp,rest }}^{T}
\end{array}\right)=\left(\begin{array}{lll}
\vec{a}_{1} & \ldots & \vec{a}_{N_{\text {imp }}}
\end{array}\right)
$$

and the condition in Eq. (B.32) can be written as the biorthogonality condition

$$
\vec{u}_{i}^{\dagger} \cdot \vec{a}_{j}=0 \forall i \in\left\{N_{\mathrm{bath}_{1}}+1, \ldots, N_{\chi}-N_{\mathrm{imp}}\right\}, j \in\left\{1, \ldots, N_{\mathrm{imp}}\right\} .
$$

Practically, we determine the vectors $\vec{u}_{i}$ by first choosing random vectors $\overrightarrow{\vec{u}}_{i}$ and then orthonormalize them with a modified Gram-Schmidt procedure such that they fulfill the condition in Eq. (B.36) and are orthonormal among each other. The rank of $\widetilde{A}_{\text {imp,bath }}$ is identical to the rank of

$$
\bar{A}=\left(\begin{array}{c}
A_{\mathrm{imp}_{\text {,bath }}}^{T} \\
A_{\mathrm{imp}_{\text {rest }}}^{T}
\end{array}\right)=\left(\begin{array}{lll}
\vec{a}_{1} & \ldots & \vec{a}_{\mathrm{N}_{\mathrm{imp}}}
\end{array}\right) .
$$

Thus, the rank of $\bar{A}$ is a lower bound for the size of the first-level effective bath $N_{\text {bath }}$. Because of $\bar{A} \in \mathbb{C}^{N_{\chi}-N_{\text {imp }}-N_{\text {bath }_{1}} \times N_{\text {imp }}}$ the rank of $\bar{A}$ is at most $N_{\text {imp }}$ and, hence, $N_{\text {bath }}$ is as most $N_{\text {imp }}$. In practice we choose $N_{\text {bath }_{1}}=N_{\text {imp }}$, because the detection of rank deficiency is numerically challenging.

After one transformation step, we have a matrix with the shape

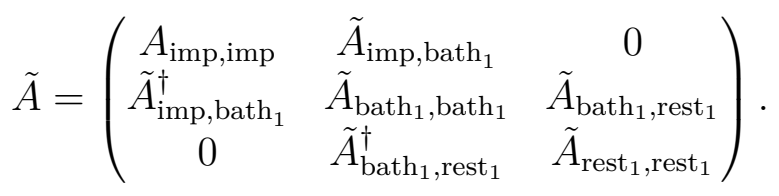

The construction of the unitary transformation only includes simple vector operations and all derivatives

$$
\frac{\partial \tilde{A}_{\alpha, \beta}}{\partial A_{i, j}}
$$

that are required to map the derivatives of the density-matrix functional with respect to the transformed one-particle reduced density matrix to derivatives with respect to the original one-particle reduced density matrix, can be calculated easily. 


\section{B.2.2. Iterated transformation}

We now apply the construction of the previous section iteratively. For that purpose, we consider the submatrix

$$
\tilde{A}_{\text {bath }_{1} \cup \mathrm{rest}_{1}}=\left(\begin{array}{cc}
\tilde{A}_{\mathrm{bath}_{1}, \text { bath }_{1}} & \tilde{A}_{\text {bath }_{1}, \text { rest }_{1}} \\
\tilde{A}_{\text {bath }_{1}, \text { rest }_{1}} & \tilde{A}_{\text {rest }_{1}, \text { rest }_{1}}
\end{array}\right)
$$

of $\tilde{A}$ in Eq. (B.38). Then we divide the block rest ${ }_{1}$ so that $\tilde{A}_{\text {bath }_{1} \cup \mathrm{Urst}_{1}}$ obtains the form

$$
\tilde{A}_{\text {bath }_{1} \cup \text { rest }_{1}}=\left(\begin{array}{ccc}
\tilde{A}_{\text {bath }_{1}, \text { bath }_{1}} & \tilde{A}_{\text {bath }_{1}, \text { bath }_{2}}^{\prime} & \tilde{A}_{\text {bath }_{1}, \text { rest }_{2}}^{\prime} \\
\left(\tilde{A}_{\text {bath }_{1}, \text { bath }_{2}}\right)^{\dagger} & \tilde{A}_{\text {bath }_{2}, \text { bath }_{2}}^{\prime} & \tilde{A}_{\text {bath }_{2}, \text { rest }_{2}} \\
\left(\tilde{A}_{\text {bath }_{1}, \text { rest }_{2}}^{\prime}\right)^{\dagger} & \left(\tilde{A}_{\text {bath }_{2}, \text { rest }_{2}}^{\prime}\right)^{\dagger} & \tilde{A}_{\text {rest }_{2}, \text { rest }_{2}}^{\prime}
\end{array}\right)
$$

with

$$
N_{\mathrm{bath}_{2}}=\operatorname{rk}\left(\tilde{A}_{\mathrm{bath}_{1}, \mathrm{rest}_{1}}\right) \leq N_{\mathrm{bath}_{1}} \leq N_{\mathrm{imp}}
$$

The construction discussed in the previous section can now be applied to $\tilde{A}_{\text {bath }_{1} \cup \text { rest }}$ and gives the unitary transformation $U_{2}$ such that

$$
U_{2}^{\dagger} \tilde{A}_{\text {bath }_{1} \cup \text { rest }_{1}} U_{2}=\left(\begin{array}{ccc}
\tilde{A}_{\text {bath }_{1}, \text { bath }_{1}} & \tilde{A}_{\text {bath }_{1}, \text { bath }_{2}} & 0 \\
\tilde{A}_{\text {bath }_{1}, \text { bath }_{2}} & \tilde{A}_{\text {bath }_{2}, \text { bath }_{2}} & \tilde{A}_{\text {bath }_{2}, \text { rest }_{2}} \\
0 & \tilde{A}_{\text {bath }_{2}, \text { rest }_{2}} & \tilde{A}_{\text {rest }_{2}, \text { rest }_{2}}
\end{array}\right) .
$$

Thus, after two transformation steps the transformed matrix has the shape

$$
\begin{aligned}
\tilde{A} & =\left(\begin{array}{cc}
\mathbb{1} & 0 \\
0 & U_{2}^{\dagger}
\end{array}\right) U^{\dagger} A U\left(\begin{array}{cc}
\mathbb{1} & 0 \\
0 & U_{2}
\end{array}\right) \\
& =\left(\begin{array}{cccc}
A_{\text {imp,imp }} & \tilde{A}_{\text {imp, bath }_{1}} & 0 & 0 \\
\tilde{A}_{\text {imp,bath }_{1}}^{\dagger} & \tilde{A}_{\text {bath }_{1}, \text { bath }_{1}} & \tilde{A}_{\text {bath }_{1}, \text { bath }_{2}} & 0 \\
0 & \tilde{A}_{\text {bath }_{1}, \text { bath }_{2}} & \tilde{A}_{\text {bath }_{2}, \text { bath }_{2}} & \tilde{A}_{\text {bath }_{2}, \text { rest }_{2}} \\
0 & 0 & \tilde{A}_{\text {bath }_{2}, \text { rest }_{2}} & \tilde{A}_{\text {rest }_{2}, \text { rest }_{2}}
\end{array}\right)
\end{aligned}
$$

with

$$
N_{\text {imp }} \geq N_{\text {bath }_{1}} \geq N_{\text {bath }_{2}} .
$$

After iterating the scheme sufficiently often we arrive at the banded form $\tilde{A}=U^{\dagger} A U$

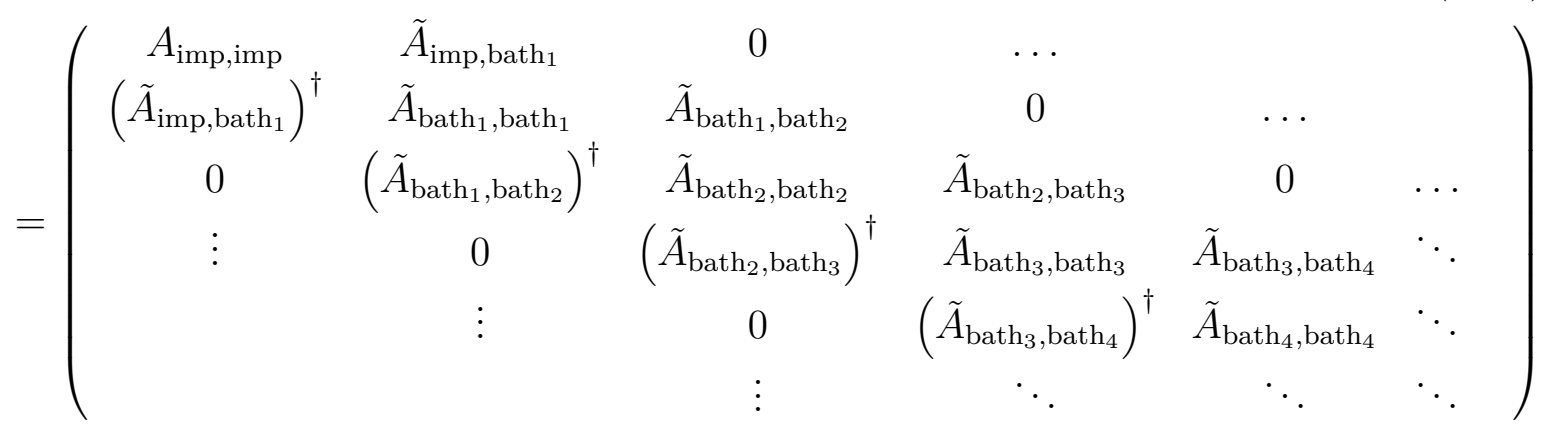

with

$$
N_{\text {imp }} \geq N_{\text {bath }_{1}} \geq N_{\text {bath }_{2}} \geq \ldots
$$




\section{B.3. Proof of relation of eigenvalue spectrum and block-diagonality}

The following proof was developed by the author and has been published in [Schade and Blöchl, 2018]. In this section, we prove a relation of the submatrix $A_{\text {bath,bath }}$ of a hermitian matrix

$$
A=\left(\begin{array}{ll}
A_{\text {imp,imp }} & A_{\text {imp,bath }} \\
A_{\text {imp,bath }}^{\dagger} & A_{\text {bath,bath }}
\end{array}\right)
$$

to the block-diagonality of the transformed matrix $\tilde{A}$ that is the result of the transformation proposed in section B.2. As in section B.2 we have $A_{\text {imp,imp }} \in \mathbb{C}^{N_{\text {imp }} \times N_{\text {imp }}}$, $A_{\text {bath,bath }} \in \mathbb{C}^{N_{\text {bath }} \times N_{\text {bath }}}$ and $N_{\chi}=N_{\text {imp }}+N_{\text {bath. }}$. We first transform $A$ with a unitary matrix

$$
U_{\lambda}=\left(\begin{array}{cc}
\mathbb{1} & 0 \\
0 & u_{\lambda}
\end{array}\right) \in U\left(N_{\chi}\right)
$$

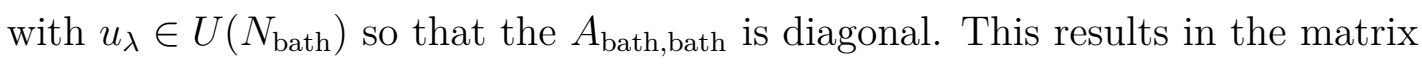

$$
A^{\prime}=U_{\lambda}^{\dagger} U_{\lambda}=\left(\begin{array}{cc}
A_{\text {imp,imp }} & A_{\text {imp,bath }}^{\prime} \\
\left(A_{\text {imp }, \text { bath }}^{\prime}\right)^{\dagger} & \operatorname{diag}\left(\lambda_{1}, \ldots, \lambda_{N_{\text {bath }}}\right)
\end{array}\right),
$$

where $\operatorname{diag}\left(\lambda_{1}, \ldots, \lambda_{N_{\text {bath }}}\right)$ denotes the diagonal matrix with the ordered eigenvalues $\lambda_{i}$ of $A_{\text {bath,bath }}$ on the diagonal. We assume that the eigenvalue spectrum $\lambda_{1}, \ldots, \lambda_{N_{\text {bath }}}$ consists of $M$ distinct values $\Lambda_{1}, \ldots, \Lambda_{M}$ with the degeneracies $n_{1}, \ldots, n_{M}$. Now we choose some $m \in\{1, \ldots, M\}$ and only consider the eigenvalue $\Lambda_{m}$. With respect to this eigenvalue, the matrix $A^{\prime}$ has the shape

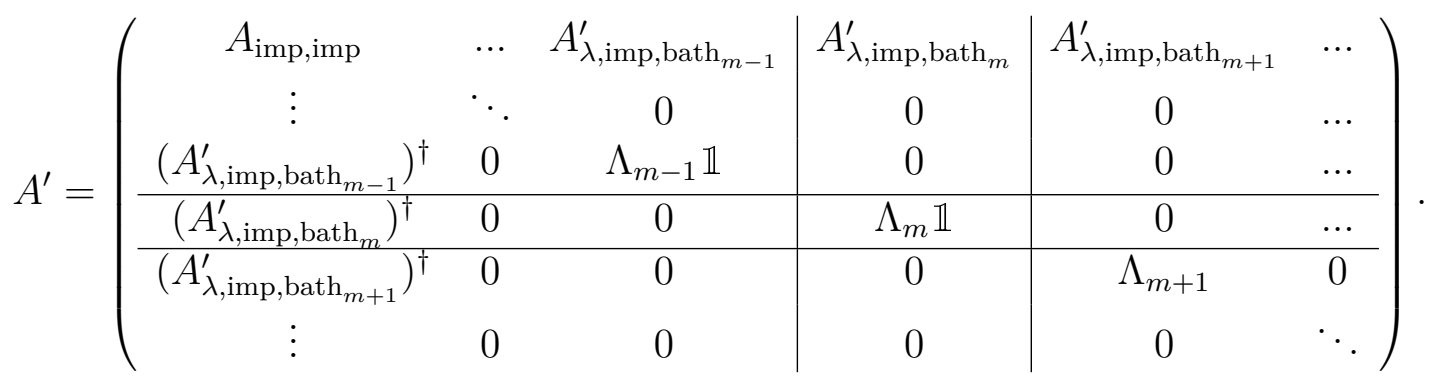

We can now introduce the unitary transform $U_{m}^{\prime}$ that transform $A^{\prime}$ to

$$
\begin{aligned}
& A_{m}^{\prime}=\left(U_{m}^{\prime}\right)^{\dagger} A^{\prime} U_{m}^{\prime}
\end{aligned}
$$

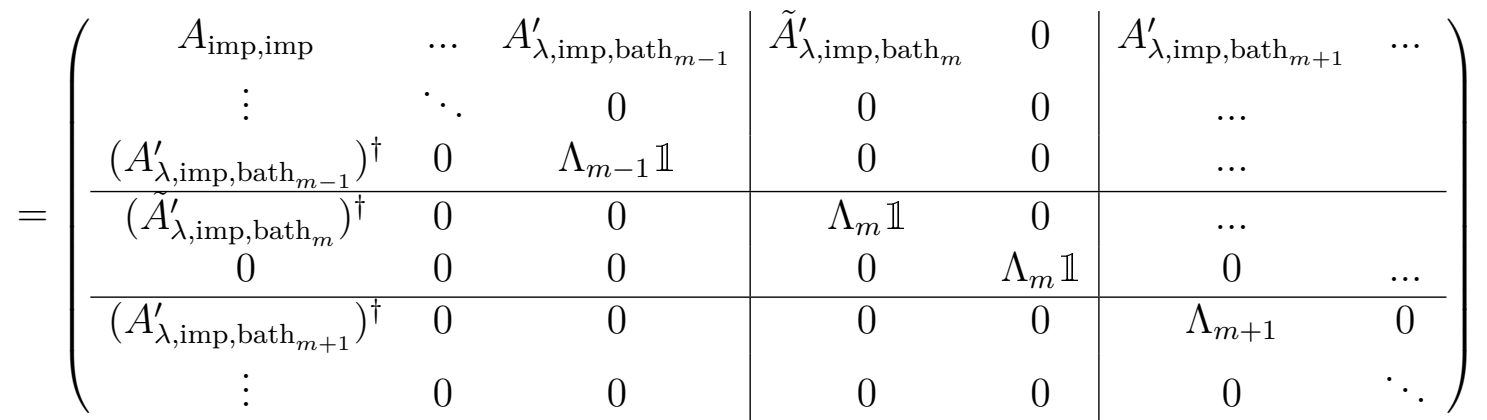


That means, that $U_{m}^{\prime}$ is chosen such that

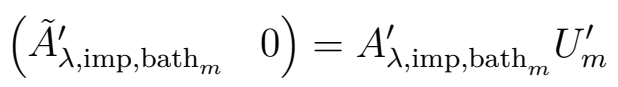

and $\tilde{A}_{\lambda, \text { imp bath }_{m}}^{\prime}$ has the least possible number of non-zero columns $\tilde{n}_{m}$. We have $\tilde{n}_{m}=$ $r k\left(A_{\lambda, \text { imp }_{\text {bath }}}^{\prime}\right)$ and hence the cases

1. $n_{m} \leq N_{\text {imp }}$, then $\tilde{n}_{m} \leq n_{m}$ or

2. $n_{m}>N_{\text {imp }}$, then $\tilde{n}_{m} \leq N_{\text {imp }}$.

We can construct such an $U_{m}^{\prime}$ for all $m \in\{1, \ldots M\}$ and reorder the columns and rows such that we obtain the transformed matrix

$$
\tilde{A}^{\prime}=\left(\begin{array}{cccc|ccc}
A_{\text {imp,imp }} & \tilde{A}_{\lambda, \text { imp,bath }}^{\prime} & \ldots & \tilde{A}_{\lambda, \text { imp, bath }}^{\prime} & 0 & \ldots & 0 \\
\left(\tilde{A}_{\lambda, \text { imp,bath }}^{\prime}\right)^{\dagger} & \Lambda_{1} & 0 & \ldots & & & \\
\vdots & 0 & \ddots & 0 & \ldots & & \\
\left(\tilde{A}_{\lambda, \text { imp, bath }_{M}}^{\prime}\right)^{\dagger} & \vdots & 0 & \Lambda_{M} & 0 & \ldots & \\
\hline 0 & & \vdots & 0 & \Lambda_{1} \mathbb{1} & 0 & \ldots \\
\vdots & & & \vdots & 0 & \ddots & 0 \\
0 & & & & \vdots & 0 & \Lambda_{M} \mathbb{1}
\end{array}\right) .
$$

The matrix $\tilde{A}^{\prime}$ is block-diagonal with the first block of the size

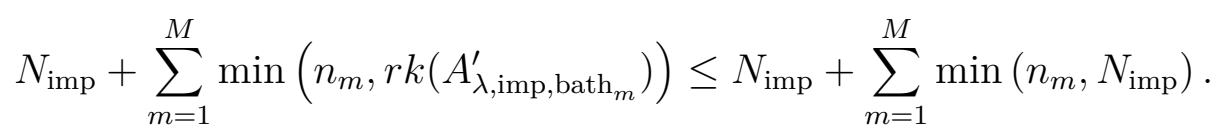

This concludes the proof. 


\section{Construction of an initial Cl-state for the density-matrix functional}

This section describes the analytic construction of Walther et al. [Walther, 2011] for a normalized many-particle wave function $|\Psi\rangle$ that corresponds to a given one-particle reduced density matrix $\rho^{(1)}$. This state can then be used as a starting point in a constrained minimization of the density-matrix functional. For this purpose, we first choose the oneparticle basis of the natural orbitals. The one-particle reduced density matrix is diagonal in this basis, i.e.,

$$
\tilde{\rho}^{(1)}=\operatorname{diag}\left(f_{1}, \ldots, f_{N_{\chi}}\right) .
$$

$N_{\chi}$ denotes the dimension of the one-particle basis and the occupations are in ascending order, i.e. $f_{i} \leq f_{i+1}$. Once we have constructed a many-particle wave function $|\tilde{\Psi}\rangle$ that fulfills this diagonal one-particle reduced density matrix $\tilde{\rho}^{(1)}$, we can transform the oneparticle basis back to the original basis and obtain the many-particle wave function $|\Psi\rangle$ that fulfills the one-particle-reduced density matrix $\rho^{(1)}$.

The many-particle wave function $|\tilde{\Psi}\rangle$ is constructed as

$$
|\tilde{\Psi}\rangle=\sum_{i=0}^{N_{\chi}} c_{i}|\underbrace{1 . .1}_{i \text { times }} 0 . .0\rangle .
$$

This many-particle wave function has the one-particle reduced density matrix

$$
\tilde{\rho}_{\alpha, \beta}^{(1)}=\delta_{\alpha, \beta} \sum_{i=\beta}^{N_{\chi}}\left|c_{i}\right|^{2} .
$$

Thus, the amplitudes $c_{i}$ have to fulfill

$$
f_{\beta}=\sum_{i=\beta}^{N_{\chi}}\left|c_{i}\right|^{2}
$$

and can be chosen as

$$
\left|c_{i}\right|^{2}=f_{i}-f_{i+1} \text { for } 0<i<N_{\chi}
$$

The coefficient of the vacuum state $|0 \ldots 0\rangle$ is

$$
\left|c_{0}\right|^{2}=1-f_{1}
$$

and the coefficient for the completely filled Slater determinant $|1 \ldots 1\rangle$ is

$$
\left|c_{N_{\chi}}\right|^{2}=f_{N_{\chi}}
$$





\section{References}

On the theory of superconductivity: the one-dimensional case. Proceedings of the Royal Society of London A: Mathematical, Physical and Engineering Sciences, 223(1154): 296-305, 1954. ISSN 0080-4630. doi: 10.1098/rspa.1954.0116. URL http://rspa. royalsocietypublishing.org/content/223/1154/296.

Carlo Adamo and Vincenzo Barone. Toward reliable density functional methods without adjustable parameters: The pbe0 model. The Journal of Chemical Physics, 110(13): 6158-6170, 1999. doi: 10.1063/1.478522. URL https://doi.org/10.1063/1.478522.

Peter Ågren. Charging effects in small capacitance Josephson junction circuits. PhD thesis, KTH, Physics, 2002. URL http://www.diva-portal.org/smash/get/diva2: 9216/FULLTEXT01.pdf. NR 20140805.

Tameem Albash and Daniel A. Lidar. Adiabatic quantum computation. Rev. Mod. Phys., 90:015002, Jan 2018. doi: 10.1103/RevModPhys.90.015002. URL https://link.aps . org/doi/10.1103/RevModPhys.90.015002.

Murat Altunbulak and Alexander Klyachko. The pauli principle revisited. Communications in Mathematical Physics, 282(2):287-322, Sep 2008a. ISSN 1432-0916. doi: 10.1007/s00220-008-0552-z. URL https://doi .org/10.1007/s00220-008-0552-z.

Murat Altunbulak and Alexander Klyachko. The pauli principle revisited. Communications in Mathematical Physics, 282(2):287-322, 2008b.

P. W. Anderson. Absence of diffusion in certain random lattices. Phys. Rev., 109:14921505, Mar 1958. doi: 10.1103/PhysRev.109.1492. URL https://link.aps.org/doi/ 10.1103/PhysRev.109.1492.

P.W. Anderson. Localized magnetic states in metals. Phys. Rev., 124:41, 1961.

V. I. Anisimov, I. V. Solovyev, M. A. Korotin, M. T. Czyżyk, and G. A. Sawatzky. Densityfunctional theory and nio photoemission spectra. Phys. Rev. B, 48:16929-16934, Dec 1993. doi: 10.1103/PhysRevB.48.16929. URL https://link.aps.org/doi/10.1103/ PhysRevB .48.16929.

V I Anisimov, A I Poteryaev, M A Korotin, A O Anokhin, and G Kotliar. First-principles calculations of the electronic structure and spectra of strongly correlated systems: dynamical mean-field theory. Journal of Physics: Condensed Matter, 9(35):7359, 1997a. URL http://stacks . iop.org/0953-8984/9/i=35/a=010.

Vladimir I. Anisimov, Jan Zaanen, and Ole K. Andersen. Band theory and mott insulators: Hubbard u instead of stoner i. Phys. Rev. B, 44:943-954, Jul 1991. doi: 10.1103/ PhysRevB.44.943. URL https://link.aps.org/doi/10.1103/PhysRevB.44.943. 
Vladimir I Anisimov, F Aryasetiawan, and A I Lichtenstein. First-principles calculations of the electronic structure and spectra of strongly correlated systems: the lda $+u$ method. Journal of Physics: Condensed Matter, 9(4):767, 1997b. URL http: //stacks . iop. org/0953-8984/9/i=4/a=002.

B. Apolloni, C. Carvalho, and D. de Falco. Quantum stochastic optimization. Stochastic Processes and their Applications, 33(2):233 - 244, 1989. ISSN 0304-4149. doi: https://doi.org/10.1016/0304-4149(89)90040-9. URL http://www.sciencedirect. com/science/article/pii/0304414989900409.

Alán Aspuru-Guzik, Anthony Dutoi, Peter Love, and Martin Head-Gordon. Simulated quantum computation of molecular energies. Science (New York, N.Y.), 309:1704-7, 102005.

Paul W. Ayers and Weitao Yang. Legendre-transform functionals for spin-densityfunctional theory. The Journal of Chemical Physics, 124(22):224108, 2006. doi: 10.1063/1.2200884. URL https://doi .org/10.1063/1.2200884.

Hilke Bahmann and Matthias Ernzerhof. Generalized-gradient exchange-correlation hole obtained from a correlation factor ansatz. The Journal of Chemical Physics, 128(23): 234104, 2008. doi: 10.1063/1.2937447. URL https://doi.org/10.1063/1.2937447.

Nikolai B. Balabanov and Kirk A. Peterson. Systematically convergent basis sets for transition metals. i. all-electron correlation consistent basis sets for the $3 \mathrm{~d}$ elements sc-zn. The Journal of Chemical Physics, 123(6):064107, 2005. doi: 10.1063/1.1998907. URL https://doi.org/10.1063/1.1998907.

Tim Baldsiefen. Reduced Density Matrix Functional Theory at Finite Temperature. PhD thesis, Freie Universität Berlin, 2012.

Tim Baldsiefen, Attila Cangi, and E. K. U. Gross. Reduced-density-matrix-functional theory at finite temperature: Theoretical foundations. Phys. Rev. A, 92:052514, Nov 2015. doi: 10.1103/PhysRevA.92.052514. URL http://link.aps.org/doi/10.1103/ PhysRevA.92.052514.

Tim Baldsiefen, Attila Cangi, F. G. Eich, and E. K. U. Gross. Exchange-correlation approximations for reduced-density-matrix-functional theory at finite temperature: Capturing magnetic phase transitions in the homogeneous electron gas. Phys. Rev. A, 96: 062508, Dec 2017. doi: 10.1103/PhysRevA.96.062508. URL https://link.aps.org/ doi/10.1103/PhysRevA.96.062508.

J. Bardeen, L. N. Cooper, and J. R. Schrieffer. Theory of superconductivity. Phys. Rev., 108:1175-1204, Dec 1957. doi: 10.1103/PhysRev.108.1175. URL https://link.aps . org/doi/10.1103/PhysRev.108.1175.

Adriano Barenco, Charles H. Bennett, Richard Cleve, David P. DiVincenzo, Norman Margolus, Peter Shor, Tycho Sleator, John A. Smolin, and Harald Weinfurter. Elementary gates for quantum computation. Phys. Rev. A, 52:3457-3467, Nov 1995. doi: 10.1103/ PhysRevA.52.3457. URL https://link.aps.org/doi/10.1103/PhysRevA.52.3457. 
R. Barends, J. Kelly, A. Megrant, D. Sank, E. Jeffrey, Y. Chen, Y. Yin, B. Chiaro, J. Mutus, C. Neill, P. O'Malley, P. Roushan, J. Wenner, T. C. White, A. N. Cleland, and John M. Martinis. Coherent josephson qubit suitable for scalable quantum integrated circuits. Phys. Rev. Lett., 111:080502, Aug 2013. doi: 10.1103/PhysRevLett.111.080502. URL https://link.aps.org/doi/10.1103/PhysRevLett.111.080502.

P. K. Barkoutsos, J. F. Gonthier, I. Sokolov, N. Moll, G. Salis, A. Fuhrer, M. Ganzhorn, D. J. Egger, M. Troyer, A. Mezzacapo, S. Filipp, and I. Tavernelli. Quantum algorithms for electronic structure calculations: particle/hole Hamiltonian and optimized wavefunction expansions. ArXiv e-prints, May 2018.

L. C. Bartel and B. Morosin. Exchange striction in nio. Phys. Rev. B, 3:1039-1043, Feb 1971. doi: 10.1103/PhysRevB.3.1039. URL https://link.aps.org/doi/10.1103/ PhysRevB . 3.1039.

Rodney J. Bartlett and Monika Musiał. Coupled-cluster theory in quantum chemistry. Rev. Mod. Phys., 79:291-352, Feb 2007. doi: 10.1103/RevModPhys.79.291. URL https://link.aps.org/doi/10.1103/RevModPhys.79.291.

A. D. Becke. A multicenter numerical integration scheme for polyatomic molecules. The Journal of Chemical Physics, 88(4):2547-2553, 1988. doi: 10.1063/1.454033. URL https://doi.org/10.1063/1.454033.

C. L. Benavides-Riveros and J. C. Várilly. Testing one-body density functionals on a solvable model. EPJ D, 66(10):274, 2012. ISSN 1434-6079. doi: 10.1140/epjd/e201230442-4. URL http://dx.doi.org/10.1140/epjd/e2012-30442-4.

Jarle Berntsen, Terje O. Espelid, and Alan Genz. An adaptive algorithm for the approximate calculation of multiple integrals. ACM Trans. Math. Softw., 17(4):437451, December 1991. ISSN 0098-3500. doi: 10.1145/210232.210233. URL http: //doi.acm.org/10.1145/210232.210233.

D.P. Bertsekas. Nonlinear programming. Athena Scientific optimization and computation series. Athena Scientific, 1999. ISBN 9781886529007.

H. Bethe. Zur theorie der metalle. Zeitschrift für Physik, 71(3):205-226. ISSN 0044-3328. doi: 10.1007/BF01341708. URL http://dx.doi.org/10.1007/BF01341708.

Silke Biermann. Dynamical screening effects in correlated electron materials - a progress report on combined many-body perturbation and dynamical mean field theory: ' $\mathrm{gw}$ + dmft'. Journal of Physics: Condensed Matter, 26(17):173202, 2014. URL http: //stacks.iop.org/0953-8984/26/i=17/a=173202.

K. Binder, K. Binder, D.M. Ceperley, J.P. Hansen, M.H. Kalos, D.P. Landau, D. Levesque, H. Müller-Krumbhaar, D. Stauffer, and J.J. Weis. Monte Carlo Methods in Statistical Physics. Topics in Current Physics. Springer Berlin Heidelberg, 2012. ISBN 9783642828034 . 
Alexandre Blais, Jay Gambetta, A. Wallraff, D. I. Schuster, S. M. Girvin, M. H. Devoret, and R. J. Schoelkopf. Quantum-information processing with circuit quantum electrodynamics. Phys. Rev. A, 75:032329, Mar 2007. doi: 10.1103/PhysRevA.75.032329. URL https://link.aps.org/doi/10.1103/PhysRevA.75.032329.

P. E. Blöchl. Projector augmented-wave method. Phys. Rev. B, 50:17953-17979, Dec 1994. doi: 10.1103/PhysRevB.50.17953. URL https://link.aps.org/doi/10.1103/ PhysRevB.50.17953.

P. E. Blöchl, C. J. Först, and J. Schimpl. The Projector Augmented Wave Method: abinitio molecular dynamics with full wave functions. eprint arXiv:cond-mat/0201015, January 2002.

P.E. Blöchl. Private communication. 2013.

Peter E. Blöchl, O. Jepsen, and O. K. Andersen. Improved tetrahedron method for brillouin-zone integrations. Phys. Rev. B, 49:16223-16233, Jun 1994 . doi: 10.1103/PhysRevB.49.16223. URL https://link.aps.org/doi/10.1103/PhysRevB. 49. 16223 .

Peter E. Blöchl, Christian F. J. Walther, and Thomas Pruschke. Method to include explicit correlations into density-functional calculations based on density-matrix functional theory. Phys. Rev. B, 84:205101, Nov 2011. doi: 10.1103/PhysRevB.84.205101. URL http://link.aps.org/doi/10.1103/PhysRevB.84.205101.

Peter E. Blöchl, Thomas Pruschke, and Michael Potthoff. Density-matrix functionals from green's functions. Phys. Rev. B, 88:205139, Nov 2013. doi: 10.1103/PhysRevB. 88.205139. URL http://link.aps.org/doi/10.1103/PhysRevB .88.205139.

P. E. Blöchl. Electrostatic decoupling of periodic images of plane-wave-expanded densities and derived atomic point charges. The Journal of Chemical Physics, 103(17):7422-7428, 1995. doi: 10.1063/1.470314. URL https://doi.org/10.1063/1.470314.

George H. Booth, Alex J. W. Thom, and Ali Alavi. Fermion monte carlo without fixed nodes: A game of life, death, and annihilation in slater determinant space. The Journal of Chemical Physics, 131(5):054106, 2009. doi: 10.1063/1.3193710. URL https://aip. scitation.org/doi/abs/10.1063/1.3193710.

$\mathrm{R}$ E Borland and K Dennis. The conditions on the one-matrix for three-body fermion wavefunctions with one-rank equal to six. Journal of Physics B: Atomic and Molecular Physics, 5(1):7, 1972. URL http://stacks.iop.org/0022-3700/5/i=1/a=009.

M. Born and V. Fock. Beweis des adiabatensatzes. Zeitschrift für Physik, 51(3):165-180, Mar 1928. ISSN 0044-3328. doi: 10.1007/BF01343193. URL https://doi.org/10. 1007/BF01343193.

M. Born and K. Huang. Dynamical Theory of Crystal Lattices. International series of monographs on physics. Clarendon Press, 1954. 
M. Born and R. Oppenheimer. Zur quantentheorie der molekeln. Annalen der Physik, 389 (20):457-484, 1927. doi: 10.1002/andp.19273892002. URL https://onlinelibrary . wiley.com/doi/abs/10.1002/andp. 19273892002.

Bouchaud, J.P., Georges, A., and Lhuillier, C. Pair wave functions for strongly correlated fermions and their determinantal representation. J. Phys. France, 49(4):553559, 1988. doi: 10.1051/jphys:01988004904055300. URL https://doi.org/10.1051/ jphys:01988004904055300.

V Bouchiat, D Vion, P Joyez, D Esteve, and M H Devoret. Quantum coherence with a single cooper pair. Physica Scripta, 1998(T76):165, 1998. URL http://stacks.iop. org $/ 1402-4896 / 1998 / i=T 76 / a=024$.

B.H. Brandow. Electronic structure of mott insulators. Advances in Physics, 26(5): 651-808, 1977. doi: 10.1080/00018737700101443. URL https://doi.org/10.1080/ 00018737700101443.

Sergey B. Bravyi and Alexei Yu. Kitaev. Fermionic quantum computation. Annals of Physics, 298(1):210 - 226, 2002. ISSN 0003-4916. doi: https://doi.org/10. 1006/aphy.2002.6254. URL http://www.sciencedirect.com/science/article/pii/ S0003491602962548.

G. Breit. The effect of retardation on the interaction of two electrons. Phys. Rev., 34: 553-573, Aug 1929. doi: 10.1103/PhysRev.34.553. URL https://link.aps.org/doi/ 10.1103/PhysRev.34.553.

C. G. Broyden. The convergence of a class of double-rank minimization algorithms 1 . general considerations. IMA Journal of Applied Mathematics, 6(1):76-90, 1970. doi: 10.1093/imamat/6.1.76.

M. A. Buijse and E. J. Baerands. An approximate exchange-correlation hole density as a functional of the natural orbitals. Mol. Phys., 100(4):401-421, 2002. doi: 10.1080/ 00268970110070243 . URL http://dx.doi.org/10.1080/00268970110070243.

J. Bünemann, W. Weber, and F. Gebhard. Multiband gutzwiller wave functions for general on-site interactions. Phys. Rev. B, 57:6896-6916, Mar 1998. doi: 10.1103/ PhysRevB.57.6896. URL https://link.aps.org/doi/10.1103/PhysRevB.57.6896.

Jörg Bünemann, Thorben Linneweber, Ute Löw, Frithjof B. Anders, and Florian Gebhard. Interplay of coulomb interaction and spin-orbit coupling. Phys. Rev. B, 94:035116, Jul 2016. doi: 10.1103/PhysRevB.94.035116. URL https://link.aps.org/doi/10.1103/ PhysRevB.94.035116.

Samuel Burer and Renato DC Monteiro. A nonlinear programming algorithm for solving semidefinite programs via low-rank factorization. Mathematical Programming, 95(2): 329-357, 2003.

K. Burke, J. C. Smith, P. E. Grabowski, and A. Pribram-Jones. Exact conditions on the temperature dependence of density functionals. Phys. Rev. B, 93:195132, May 2016. doi: 10.1103/PhysRevB.93.195132. URL https://link.aps.org/doi/10.1103/ PhysRevB.93.195132. 
Kieron Burke and John P. Perdew. Real-space analysis of the exchange-correlation energy. Int. J. Quantum Chem., 56(4):199-210, 1995. ISSN 1097-461X. doi: 10.1002/qua. 560560403. URL http://dx.doi.org/10.1002/qua.560560403.

C. A. Büsser, G. B. Martins, and A. E. Feiguin. Lanczos transformation for quantum impurity problems in $d$-dimensional lattices: Application to graphene nanoribbons. Phys. Rev. B, 88:245113, Dec 2013. doi: 10.1103/PhysRevB.88.245113. URL http: //link.aps.org/doi/10.1103/PhysRevB.88.245113.

Jörg Bünemann, Thorben Linneweber, and Florian Gebhard. Approximation schemes for the study of multi-band gutzwiller wave functions. physica status solidi (b), 254(1): 1600166, 2017. doi: 10.1002/pssb.201600166. URL https://onlinelibrary.wiley . com/doi/abs/10.1002/pssb. 201600166.

Manuela Capello, Federico Becca, Michele Fabrizio, Sandro Sorella, and Erio Tosatti. Variational description of mott insulators. Phys. Rev. Lett., 94:026406, Jan 2005. doi: 10.1103/PhysRevLett.94.026406. URL https://link.aps.org/doi/10.1103/ PhysRevLett.94.026406.

R. Car and M. Parrinello. Unified approach for molecular dynamics and density-functional theory. Phys. Rev. Lett., 55:2471-2474, Nov 1985. doi: 10.1103/PhysRevLett.55.2471. URL https://link.aps.org/doi/10.1103/PhysRevLett.55.2471.

B. C. Carlson and Joseph M. Keller. Eigenvalues of density matrices. Phys. Rev., 121: 659-661, Feb 1961. doi: 10.1103/PhysRev.121.659. URL http://link.aps.org/doi/ 10.1103/PhysRev.121.659.

D. Ceperley, G. V. Chester, and M. H. Kalos. Monte carlo simulation of a many-fermion study. Phys. Rev. B, 16:3081-3099, Oct 1977. doi: 10.1103/PhysRevB.16.3081. URL https://link.aps.org/doi/10.1103/PhysRevB.16.3081.

D. M. Ceperley and B. J. Alder. Ground state of the electron gas by a stochastic method. Phys. Rev. Lett., 45:566-569, Aug 1980. doi: 10.1103/PhysRevLett.45.566. URL https: //link.aps.org/doi/10.1103/PhysRevLett.45.566.

Romit Chakraborty and David A. Mazziotti. Generalized pauli conditions on the spectra of one-electron reduced density matrices of atoms and molecules. Phys. Rev. A, 89: 042505, Apr 2014. doi: 10.1103/PhysRevA.89.042505. URL https://link.aps.org/ doi/10.1103/PhysRevA.89.042505.

Garnet Kin-Lic Chan and Sandeep Sharma. The density matrix renormalization group in quantum chemistry. Annual Review of Physical Chemistry, 62(1):465-481, 2011. doi: 10. 1146/annurev-physchem-032210-103338. URL https://doi.org/10.1146/annurevphyschem-032210-103338. PMID: 21219144.

Ch Chang, M Pelissier, and Ph Durand. Regular two-component pauli-like effective hamiltonians in dirac theory. Physica Scripta, 34(5):394, 1986. URL http://stacks.iop. org $/ 1402-4896 / 34 / i=5 / a=007$. 
J. M. Chow, L. DiCarlo, J. M. Gambetta, F. Motzoi, L. Frunzio, S. M. Girvin, and R. J. Schoelkopf. Optimized driving of superconducting artificial atoms for improved singlequbit gates. Phys. Rev. A, 82:040305, Oct 2010. doi: 10.1103/PhysRevA.82.040305. URL https://link.aps.org/doi/10.1103/PhysRevA.82.040305.

Jerry M. Chow, A. D. Córcoles, Jay M. Gambetta, Chad Rigetti, B. R. Johnson, John A. Smolin, J. R. Rozen, George A. Keefe, Mary B. Rothwell, Mark B. Ketchen, and M. Steffen. Simple all-microwave entangling gate for fixed-frequency superconducting qubits. Phys. Rev. Lett., 107:080502, Aug 2011. doi: 10.1103/PhysRevLett.107.080502. URL https://link.aps.org/doi/10.1103/PhysRevLett.107.080502.

Jerzy Cioslowski. New constraints upon the electron-electron repulsion energy functional of the one-electron reduced density matrix. J. Chem. Phys., 123(16):164106, 2005. doi: http://dx.doi.org/10.1063/1.2074527. URL http://scitation.aip.org/ content/aip/journal/jcp/123/16/10.1063/1.2074527.

Jerzy Cioslowski and Katarzyna Pernal. Constraints upon natural spin orbital functionals imposed by properties of a homogeneous electron gas. J. Chem. Phys., 111(8):33963400, 1999. doi: http://dx.doi.org/10.1063/1.479623. URL http://scitation.aip. org/content/aip/journal/jcp/111/8/10.1063/1.479623.

Jerzy Cioslowski and Katarzyna Pernal. Density matrix functional theory of weak intermolecular interactions. J. Chem. Phys., 116(12):4802-4807, 2002. doi: http://dx.doi. org/10.1063/1.1446028. URL http://scitation.aip.org/content/aip/journal/ jcp/116/12/10.1063/1.1446028.

Jerzy Cioslowski, Katarzyna Pernal, and Paul Ziesche. Systematic construction of approximate one-matrix functionals for the electron-electron repulsion energy. J. Chem. Phys., 117(21):9560-9566, 2002. doi: http://dx.doi.org/10.1063/1.1516804. URL http: //scitation.aip.org/content/aip/journal/jcp/117/21/10.1063/1.1516804.

John W. Clark and Eugene Feenberg. Simplified treatment for strong short-range repulsions in n-particle systems. i. general theory. Phys. Rev., 113:388-399, Jan 1959. doi: 10.1103/PhysRev.113.388. URL https://link.aps.org/doi/10.1103/PhysRev . 113.388.

R. Cleve, A. Ekert, C. Macchiavello, and M. Mosca. Quantum algorithms revisited. Proceedings of the Royal Society of London A: Mathematical, Physical and Engineering Sciences, 454(1969):339-354, 1998. ISSN 1364-5021. doi: 10.1098/rspa.1998.0164. URL http://rspa.royalsocietypublishing.org/content/454/1969/339.

Matteo Cococcioni. The lda $+\mathrm{u}$ approach: A simple hubbard correction for correlated ground states. In Eva Pavarini, Erik Koch, Frithjof Anders, and Mark Jarrell, editors, Correlated Electrons: From Models to Materials, page 450 p., Jülich, Sep 2012. Forschungszentrum Jülich Zentralbibliothek, Verlag. ISBN 978-3-89336-796-2. URL http://hdl. handle.net/2128/4611.

F. Coester. Bound states of a many-particle system. Nuclear Physics, 7:421 - 424, 1958. ISSN 0029-5582. doi: https://doi.org/10.1016/0029-5582(58)90280-3. URL http: //www.sciencedirect.com/science/article/pii/0029558258902803. 
F. Coester and H. Kümmel. Short-range correlations in nuclear wave functions. Nuclear Physics, 17:477 - 485, 1960. ISSN 0029-5582. doi: https://doi.org/10.1016/ 0029-5582(60)90140-1. URL http://www.sciencedirect.com/science/article/ pii/0029558260901401.

Aron J. Cohen, Paula Mori-Sánchez, and Weitao Yang. Fractional spins and static correlation error in density functional theory. J. Chem. Phys., 129(12):121104, 2008a. doi: http://dx.doi.org/10.1063/1.2987202. URL http://scitation.aip.org/content/aip/journal/jcp/129/12/10.1063/1. 2987202 ; jsessionid=qP59vXHZGlmRN3B86tZJ_Sfn.x-aip-live-06.

Aron J. Cohen, Paula Mori-Sánchez, and Weitao Yang. Insights into current limitations of density functional theory. Science, 321(5890):792-794, 2008b. ISSN 0036-8075. doi: 10.1126/science.1158722. URL http://science.sciencemag.org/content/321/ $5890 / 792$.

Aron J. Cohen, Paula Mori-Sánchez, and Weitao Yang. Challenges for density functional theory. Chem. Rev., 112(1):289-320, 2012. doi: 10.1021/cr200107z. URL http://dx . doi.org/10.1021/cr200107z. PMID: 22191548.

Leon Cohen and C. Frishberg. Hierarchy equations for reduced density matrices. Phys. Rev. A, 13:927-930, Mar 1976. doi: 10.1103/PhysRevA.13.927. URL https://link. aps.org/doi/10.1103/PhysRevA.13.927.

A. J. Coleman. Structure of fermion density matrices. Rev. Mod. Phys., 35:668-686, Jul 1963. doi: 10.1103/RevModPhys.35.668. URL https://link.aps.org/doi/10.1103/ RevModPhys.35.668.

A.J. Coleman and V.I. Yukalov. Reduced Density Matrices: Coulson's Challenge. Lecture Notes in Chemistry. Springer Berlin Heidelberg, 2000. ISBN 9783540671480.

Bridgette Cooper and Peter J. Knowles. Benchmark studies of variational, unitary and extended coupled cluster methods. The Journal of Chemical Physics, 133(23):234102, 2010. doi: 10.1063/1.3520564. URL https://doi.org/10.1063/1.3520564.

Prof. C.A. Coulson and Miss I. Fischer. Xxxiv. notes on the molecular orbital treatment of the hydrogen molecule. The London, Edinburgh, and Dublin Philosophical Magazine and Journal of Science, 40(303):386-393, 1949. doi: 10.1080/14786444908521726. URL https://doi.org/10.1080/14786444908521726.

A. W. Cross, L. S. Bishop, J. A. Smolin, and J. M. Gambetta. Open Quantum Assembly Language. ArXiv e-prints, July 2017.

Gábor Csányi and T. A. Arias. Tensor product expansions for correlation in quantum many-body systems. Phys. Rev. B, 61:7348-7352, Mar 2000. doi: 10.1103/PhysRevB. 61.7348. URL http://link.aps.org/doi/10.1103/PhysRevB.61.7348.

Andrea Dal Corso. Projector augmented-wave method: Application to relativistic spindensity functional theory. Phys. Rev. B, 82:075116, Aug 2010. doi: 10.1103/PhysRevB. 82.075116. URL http://link.aps.org/doi/10.1103/PhysRevB.82.075116. 
Ernest R. Davidson. Properties and uses of natural orbitals. Rev. Mod. Phys., 44:451-464, Jul 1972. doi: 10.1103/RevModPhys.44.451. URL https://link.aps.org/doi/10 . 1103/RevModPhys . 44.451.

J H de Boer and E J W Verwey. Semi-conductors with partially and with completely filled $3 \mathrm{~d}$-lattice bands. Proceedings of the Physical Society, 49(4S):59, 1937. URL http://stacks. iop.org/0959-5309/49/i=4S/a=307.

A. Eugene DePrince. Variational optimization of the two-electron reduced-density matrix under pure-state n-representability conditions. The Journal of Chemical Physics, 145(16):164109, 2016. doi: 10.1063/1.4965888. URL https://doi.org/10.1063/1. 4965888.

David Deutsch. Quantum theory, the church-turing principle and the universal quantum computer. Proceedings of the Royal Society of London A: Mathematical, Physical and Engineering Sciences, 400(1818):97-117, 1985. ISSN 0080-4630. doi: 10.1098/rspa.1985. 0070. URL http://rspa.royalsocietypublishing.org/content/400/1818/97.

S. Di Sabatino, J. A. Berger, L. Reining, and P. Romaniello. Reduced density-matrix functional theory: Correlation and spectroscopy. J. Chem. Phys., 143(2):024108, 2015. doi: http://dx.doi.org/10.1063/1.4926327. URL http://scitation.aip.org/ content/aip/journal/jcp/143/2/10.1063/1.4926327.

Stefano Di Sabatino, J. A. Berger, Lucia Reining, and Pina Romaniello. Photoemission spectra from reduced density matrices: The band gap in strongly correlated systems. Phys. Rev. B, 94:155141, Oct 2016. doi: 10.1103/PhysRevB.94.155141. URL http: //link.aps.org/doi/10.1103/PhysRevB.94.155141.

P. A. M. Dirac. The quantum theory of the electron. Proceedings of the Royal Society of London. Series A, 117(778):610-624, 1928a. doi: 10.1098/rspa.1928.0023. URL http://rspa.royalsocietypublishing.org/content/117/778/610. short.

P. A. M. Dirac. The quantum theory of the electron. part ii. Proceedings of the Royal Society of London. Series A, 118(779):351-361, 1928b. doi: 10.1098/rspa.1928.0056. URL http://rspa.royalsocietypublishing. org/content/118/779/351. short.

David P. DiVincenzo. The physical implementation of quantum computation. Fortschritte der Physik, 48(9-11):771-783, 2000. doi: 10.1002/1521-3978(200009)48: 9/11<771::AID-PROP771>3.0.CO;2-E. URL https://onlinelibrary.wiley . com/doi/abs/10.1002/1521-3978\%28200009\%2948\%3A9/11\%3C771\%3A\%3AAIDPROP771\%3E3.0. CO\%3B2-E.

S. L. Dudarev, G. A. Botton, S. Y. Savrasov, C. J. Humphreys, and A. P. Sutton. Electronenergy-loss spectra and the structural stability of nickel oxide: An lsda+u study. Phys. Rev. B, 57:1505-1509, Jan 1998. doi: 10.1103/PhysRevB.57.1505. URL https://link. aps.org/doi/10.1103/PhysRevB.57.1505.

Thom H. Dunning. Gaussian basis sets for use in correlated molecular calculations. i. the atoms boron through neon and hydrogen. J. Chem. Phys., 90(2):1007-1023, 1989a. doi: 10.1063/1.456153. URL http://dx.doi.org/10.1063/1.456153. 
Thom H. Dunning. Gaussian basis sets for use in correlated molecular calculations. i. the atoms boron through neon and hydrogen. The Journal of Chemical Physics, 90(2): 1007-1023, 1989b. doi: 10.1063/1.456153. URL https://doi.org/10.1063/1.456153.

R. Eder. From cluster to solid: Variational cluster approximation applied to NiO. Phys. Rev. B, 76:241103, Dec 2007. doi: 10.1103/PhysRevB.76.241103. URL https://link. aps.org/doi/10.1103/PhysRevB .76.241103.

R. Eder. Correlated band structure of nio, coo, and mno by variational cluster approximation. Phys. Rev. B, 78:115111, Sep 2008. doi: 10.1103/PhysRevB.78.115111. URL https://link.aps.org/doi/10.1103/PhysRevB.78.115111.

R. Eder. Electronic structure of nio: Antiferromagnetic transition and photoelectron spectra in the ordered phase. Phys. Rev. B, 91:245146, Jun 2015. doi: 10.1103/PhysRevB. 91.245146. URL https://link.aps.org/doi/10.1103/PhysRevB.91.245146.

J. Eisert. Entanglement and tensor network states. ArXiv e-prints, August 2013.

E. Engel and R.M. Dreizler. Density Functional Theory: An Advanced Course. Theoretical and Mathematical Physics. Springer Berlin Heidelberg, 2011. ISBN 9783642140907.

E. Engel, A. Facco Bonetti, S. Keller, I. Andrejkovics, and R. M. Dreizler. Relativistic optimized-potential method: Exact transverse exchange and møller-plesset-based correlation potential. Phys. Rev. A, 58:964-992, Aug 1998. doi: 10.1103/PhysRevA.58.964. URL https://link.aps.org/doi/10.1103/PhysRevA.58.964.

R. M. Erdahl. Representability. International Journal of Quantum Chemistry, 13(6): 697-718, 1978. doi: 10.1002/qua.560130603. URL https://onlinelibrary.wiley. com/doi/abs/10.1002/qua. 560130603.

R.M. Erdahl. Two algorithms for the lower bound method of reduced density matrix theory. Reports on Mathematical Physics, 15(2):147 - 162, 1979. ISSN 0034-4877. doi: https://doi.org/10.1016/0034-4877(79)90015-6. URL http://www.sciencedirect. com/science/article/pii/0034487779900156.

R.M. Erdahl and V.H. Smith. Density Matrices and Density Functionals: Proceedings of the A. John Coleman Symposium. 1987. ISBN 9789027724779.

H. Eschrig. The Fundamentals of Density Functional Theory. Teubner Texte zur Physik. Vieweg+Teubner Verlag, 2003. ISBN 9783322976208.

S. Faas, J.G. Snijders, J.H. van Lenthe, E. van Lenthe, and E.J. Baerends. The zora formalism applied to the dirac-fock equation. Chemical Physics Letters, 246(6):632 640, 1995. ISSN 0009-2614. doi: 10.1016/0009-2614(95)01156-0. URL http://www . sciencedirect.com/science/article/pii/0009261495011560.

M. Fannes, B. Nachtergaele, and R. F. Werner. Finitely correlated states on quantum spin chains. Communications in Mathematical Physics, 144(3):443-490, Mar 1992. ISSN 1432-0916. doi: 10.1007/BF02099178. URL https://doi .org/10.1007/BF02099178. 
David Feller. The role of databases in support of computational chemistry calculations. Journal of Computational Chemistry, 17(13):1571-1586, 1996. doi: 10.1002/(SICI)1096-987X(199610)17:13<1571::AID-JCC9>3.0.CO;2P. URL https://onlinelibrary.wiley.com/doi/abs/10.1002/\%28SICI\%291096987X\%28199610\%2917\%3A13\%3C1571\%3A\%3AAID-JCC9\%3E3 . 0. CO\%3B2-P.

W. Fenchel. On conjugate convex functions. Canad. Jour. Math., 1:73-77, 1949. URL https://ci.nii.ac.jp/naid/10009393911/en/.

E. Fertitta, B. Paulus, G. Barcza, and Ö. Legeza. Investigation of metal-insulator-like transition through the ab initio density matrix renormalization group approach. Phys. Rev. B, 90:245129, Dec 2014. doi: 10.1103/PhysRevB.90.245129. URL https://link. aps.org/doi/10.1103/PhysRevB.90.245129.

R. Fletcher. A new approach to variable metric algorithms. The Computer Journal, 13 (3):317-322, 1970. doi: 10.1093/comjnl/13.3.317.

V. Fock. Konfigurationsraum und zweite quantelung. Zeitschrift für Physik, 75(9):622647, Sep 1932. ISSN 0044-3328. doi: 10.1007/BF01344458. URL https://doi.org/ 10.1007/BF01344458.

W. M. C. Foulkes, L. Mitas, R. J. Needs, and G. Rajagopal. Quantum monte carlo simulations of solids. Rev. Mod. Phys., 73:33-83, Jan 2001. doi: 10.1103/RevModPhys. 73.33. URL https://link.aps.org/doi/10.1103/RevModPhys.73.33.

Rupert L. Frank, Elliott H. Lieb, Robert Seiringer, and Heinz Siedentop. Müller's exchange-correlation energy in density-matrix-functional theory. Phys. Rev. A, 76: 052517, Nov 2007. doi: 10.1103/PhysRevA.76.052517. URL http://link.aps.org/ doi/10.1103/PhysRevA.76.052517.

Edward Fredkin and Tommaso Toffoli. Conservative logic. International Journal of Theoretical Physics, 21(3):219-253, Apr 1982. ISSN 1572-9575. doi: 10.1007/BF01857727. URL https://doi.org/10.1007/BF01857727.

Atsushi Fujimori and Fujio Minami. Valence-band photoemission and optical absorption in nickel compounds. Phys. Rev. B, 30:957-971, Jul 1984. doi: 10.1103/PhysRevB.30. 957. URL https://link.aps.org/doi/10.1103/PhysRevB.30.957.

Mituhiro Fukuda, Bastiaan J Braams, Maho Nakata, Michael L Overton, Jerome K Percus, Makoto Yamashita, and Zhengji Zhao. Large-scale semidefinite programs in electronic structure calculation. Mathematical programming, 109(2-3):553-580, 2007.

Daniel Gabay and Bertrand Mercier. A dual algorithm for the solution of nonlinear variational problems via finite element approximation. Computers and Mathematics with Applications, 2(1):17 - 40, 1976. ISSN 0898-1221. doi: https://doi. org/10.1016/0898-1221(76)90003-1. URL http://www. sciencedirect. com/science/ article/pii/0898122176900031.

Jay M. Gambetta, A. D. Córcoles, S. T. Merkel, B. R. Johnson, John A. Smolin, Jerry M. Chow, Colm A. Ryan, Chad Rigetti, S. Poletto, Thomas A. Ohki, Mark B. Ketchen, and 
M. Steffen. Characterization of addressability by simultaneous randomized benchmarking. Phys. Rev. Lett., 109:240504, Dec 2012. doi: 10.1103/PhysRevLett.109.240504. URL https://link.aps.org/doi/10.1103/PhysRevLett.109.240504.

Claude Garrod and Jerome K. Percus. Reduction of the nâ€particle variational problem. Journal of Mathematical Physics, 5(12):1756-1776, 1964. doi: 10.1063/1.1704098. URL https://doi.org/10.1063/1.1704098.

Alejandro J. Garza and Gustavo E. Scuseria. Predicting band gaps with hybrid density functionals. The Journal of Physical Chemistry Letters, 7(20):4165-4170, 2016. doi: 10. 1021/acs.jpclett.6b01807. URL https://doi.org/10.1021/acs.jpclett.6b01807.

F. Gebhard. The Mott Metal-Insulator Transition: Models and Methods. Springer Tracts in Modern Physics. Springer Berlin Heidelberg, 2003. ISBN 9783540148586.

Florian Gebhard. Gutzwiller correlated wave functions in finite dimensions d: A systematic expansion in 1/d. Phys. Rev. B, 41:9452-9473, May 1990. doi: 10.1103/PhysRevB. 41.9452. URL https://link.aps.org/doi/10.1103/PhysRevB. 41.9452.

K. Geerlings, Z. Leghtas, I. M. Pop, S. Shankar, L. Frunzio, R. J. Schoelkopf, M. Mirrahimi, and M. H. Devoret. Demonstrating a driven reset protocol for a superconducting qubit. Phys. Rev. Lett., 110:120501, Mar 2013. doi: 10.1103/PhysRevLett.110.120501. URL https://link.aps.org/doi/10.1103/PhysRevLett.110.120501.

Antoine Georges and Gabriel Kotliar. Hubbard model in infinite dimensions. Phys. Rev. B, 45:6479-6483, Mar 1992. doi: 10.1103/PhysRevB.45.6479. URL http://link.aps . org/doi/10.1103/PhysRevB.45.6479.

Semyon Aranovich Gershgorin. über die abgrenzung der eigenwerte einer matrix. Bulletin de l'Académie des Sciences de l'URSS. Classe des sciences mathématiques, (6):749-754, 1931.

K.J.H. Giesbertz. Are natural orbitals useful for generating an efficient expansion of the wave function? Chemical Physics Letters, 591:220 - 226, 2014. ISSN 0009-2614. doi: https://doi.org/10.1016/j.cplett.2013.11.038. URL http://www.sciencedirect.com/ science/article/pii/S0009261413014516.

T. L. Gilbert. Hohenberg-kohn theorem for nonlocal external potentials. Phys. Rev. B, 12:2111-2120, Sep 1975a. doi: 10.1103/PhysRevB.12.2111. URL http://link.aps . org/doi/10.1103/PhysRevB.12.2111.

T. L. Gilbert. Hohenberg-kohn theorem for nonlocal external potentials. Phys. Rev. B, 12:2111, 1975b. doi: 10.1103/PhysRevB.12.2111. URL http://link.aps.org/doi/ 10.1103/PhysRevB.12.2111.

Donald Goldfarb. A family of variable-metric methods derived by variational means. Math. Comp., 24:23-26, 1970.

R.C Grimm and R.G Storer. Monte-carlo solution of schrödinger's equation. Journal of Computational Physics, 7(1):134 - 156, 1971. ISSN 0021-9991. doi: https://doi. org/10.1016/0021-9991(71)90054-4. URL http://www. sciencedirect.com/science/ article/pii/0021999171900544. 
J. Gubernatis, N. Kawashima, and P. Werner. Quantum Monte Carlo Methods: Algorithms for Lattice Models. Cambridge University Press, 2016. ISBN 9781316483121.

O. Gunnarsson and B. I. Lundqvist. Exchange and correlation in atoms, molecules, and solids by the spin-density-functional formalism. Phys. Rev. B, 13:4274-4298, May 1976. doi: 10.1103/PhysRevB.13.4274. URL https://link.aps.org/doi/10.1103/ PhysRevB.13.4274.

Martin C. Gutzwiller. Effect of correlation on the ferromagnetism of transition metals. Phys. Rev. Lett., 10:159-162, Mar 1963a. doi: 10.1103/PhysRevLett.10.159. URL https://link.aps.org/doi/10.1103/PhysRevLett.10.159.

Martin C. Gutzwiller. Effect of correlation on the ferromagnetism of transition metals. Phys. Rev. Lett., 10(5):159, 1963b. doi: 10.1103/PhysRevLett.10.159.

Martin C. Gutzwiller. Correlation of electrons in a narrow $s$ band. Phys. Rev., 137: A1726-A1735, Mar 1965. doi: 10.1103/PhysRev.137.A1726. URL https://link.aps . org/doi/10.1103/PhysRev.137.A1726.

Jeff R. Hammond and David A. Mazziotti. Variational two-electron reduced-densitymatrix theory: Partial 3-positivity conditions for $n$-representability. Phys. Rev. A, 71: 062503, Jun 2005. doi: 10.1103/PhysRevA.71.062503. URL https://link.aps.org/ doi/10.1103/PhysRevA.71.062503.

John E. Harriman. Geometry of density matrices. ii. reduced density matrices and $n$ representability. Phys. Rev. A, 17:1257-1268, Apr 1978. doi: 10.1103/PhysRevA.17. 1257. URL https://link.aps.org/doi/10.1103/PhysRevA.17.1257.

John E. Harriman. Orthonormal orbitals for the representation of an arbitrary density. Phys. Rev. A, 24:680-682, Aug 1981. doi: 10.1103/PhysRevA.24.680. URL https: //link.aps.org/doi/10.1103/PhysRevA.24.680.

J Harris and R O Jones. The surface energy of a bounded electron gas. Journal of Physics F: Metal Physics, 4(8):1170, 1974. URL http://stacks.iop.org/0305-4608/4/i=8/ $\mathrm{a}=013$.

W. Keith Hastings. Monte carlo sampling methods using markov chains and their applications. Biometrika, 57(1):97-109, 1970. URL http://dx.doi.org/.

M. W. Haverkort, M. Zwierzycki, and O. K. Andersen. Multiplet ligand-field theory using wannier orbitals. Phys. Rev. B, 85:165113, Apr 2012. doi: 10.1103/PhysRevB. 85.165113. URL https://link.aps.org/doi/10.1103/PhysRevB.85.165113.

N. Helbig, N. N. Lathiotakis, M. Albrecht, and E. K. U. Gross. Discontinuity of the chemical potential in reduced-density-matrix-functional theory. EPL, 77(6):67003, 2007. URL http://stacks .iop.org/0295-5075/77/i=6/a=67003.

N. Helbig, N. N. Lathiotakis, and E. K. U. Gross. Discontinuity of the chemical potential in reduced-density-matrix-functional theory for open-shell systems. Phys. Rev. A, 79: 022504, Feb 2009. doi: 10.1103/PhysRevA.79.022504. URL http://link.aps.org/ doi/10.1103/PhysRevA.79.022504. 
N. Helbig, G. Theodorakopoulos, and N. N. Lathiotakis. Fractional spin in reduced density-matrix functional theory. J. Chem. Phys., 135(5):054109, 2011. doi: http: //dx.doi.org/10.1063/1.3615955. URL http://scitation.aip.org/content/aip/ journal/jcp/135/5/10.1063/1.3615955.

Nicole Helbig. Orbital Functionals in Density-Matrix- and Current-Density-Functional Theory. PhD thesis, Freie Universität Berlin, 2006.

K. Held. Electronic structure calculations using dynamical mean field theory. Advances in Physics, 56(6):829-926, 2007. doi: 10.1080/00018730701619647. URL https://doi . org/10.1080/00018730701619647.

Magnus R. Hestenes. Multiplier and gradient methods. J. Optim. Theory Appl., 4(5): 303-320, 1969. ISSN 1573-2878. doi: 10.1007/BF00927673. URL http://dx.doi .org/ 10.1007/BF00927673.

Jochen Heyd, Gustavo E. Scuseria, and Matthias Ernzerhof. Hybrid functionals based on a screened coulomb potential. The Journal of Chemical Physics, 118(18):8207-8215, 2003. doi: 10.1063/1.1564060. URL https://doi.org/10.1063/1.1564060.

Jochen Heyd, Gustavo E. Scuseria, and Matthias Ernzerhof. Erratum: "hybrid functionals based on a screened coulomb potential" [j. chem. phys. 118, 8207 (2003)]. The Journal of Chemical Physics, 124(21):219906, 2006. doi: 10.1063/1.2204597. URL https: //doi.org/10.1063/1.2204597.

P. Hohenberg and W. Kohn. Inhomogeneous electron gas. Phys. Rev., 136:B864-B871, Nov 1964. doi: 10.1103/PhysRev.136.B864. URL https://link.aps.org/doi/10. 1103/PhysRev.136. B864.

A. Holas and R. Balawender. Comment on "legendre-transform functionals for spindensity-functional theory" [j. chem. phys. 124, 224108 (2006)]. The Journal of Chemical Physics, 125(24):247101, 2006. doi: 10.1063/1.2423002. URL https://doi.org/10. 1063/1.2423002.

Joshua W. Hollett and Peter M. W. Gill. The two faces of static correlation. The Journal of Chemical Physics, 134(11):114111, 2011. doi: 10.1063/1.3570574. URL https: //doi.org/10.1063/1.3570574.

J. Hubbard. Electron correlations in narrow energy bands. Proc. R. Soc. Lond. A, 276: 238, 1963. ISSN 0080-4630. doi: 10.1098/rspa.1963.0204.

S. Hüfner and T. Riesterer. Electronic structure of nio. Phys. Rev. B, 33:7267-7269, May 1986. doi: 10.1103/PhysRevB.33.7267. URL https://link.aps.org/doi/10.1103/ PhysRevB. 33.7267.

J Hugel and M Kamal. The energy diagram of nio within an lcao-lsda $+\mathrm{u}$ approach. Journal of Physics: Condensed Matter, 9(3):647, 1997. URL http://stacks.iop. org $/ 0953-8984 / 9 / i=3 / a=005$. 
S. Hüfner, J. Osterwalder, T. Riesterer, and F. Hulliger. Photoemission and inverse photoemission spectroscopy of nio. Solid State Communications, 52(9):793 - 796, 1984. ISSN 0038-1098. doi: https://doi.org/10.1016/0038-1098(84)90007-3. URL http:// www.sciencedirect.com/science/article/pii/0038109884900073.

R. C. Jaklevic, John Lambe, A. H. Silver, and J. E. Mercereau. Quantum interference effects in josephson tunneling. Phys. Rev. Lett., 12:159-160, Feb 1964. doi: 10.1103/ PhysRevLett.12.159. URL https://link.aps.org/doi/10.1103/PhysRevLett.12. 159.

J. F. Janak. Proof that $\frac{\partial e}{\partial n_{i}}=\epsilon$ in density-functional theory. Phys. Rev. B, 18:7165-7168, Dec 1978. doi: 10.1103/PhysRevB.18.7165. URL https://link.aps.org/doi/10. 1103/PhysRevB.18.7165.

Robert Jastrow. Many-body problem with strong forces. Phys. Rev., 98:1479-1484, Jun 1955. doi: 10.1103/PhysRev.98.1479. URL https://link.aps.org/doi/10.1103/ PhysRev.98.1479.

M. A. Jenkins. Algorithm 493: Zeros of a real polynomial [c2]. ACM Trans. Math. Softw., 1(2):178-189, June 1975. ISSN 0098-3500. doi: 10.1145/355637.355643. URL http://doi.acm.org/10.1145/355637.355643.

M. A. Jenkins and J. F. Traub. A three-stage variable-shift iteration for polynomial zeros and its relation to generalized rayleigh iteration. Numerische Mathematik, 14 (3):252-263, Feb 1970. ISSN 0945-3245. doi: 10.1007/BF02163334. URL https: //doi.org/10.1007/BF02163334.

F. Jollet, G. Jomard, B. Amadon, J. P. Crocombette, and D. Torumba. Hybrid functional for correlated electrons in the projector augmented-wave formalism: Study of multiple minima for actinide oxides. Phys. Rev. B, 80:235109, Dec 2009. doi: 10.1103/PhysRevB. 80.235109. URL https://link.aps.org/doi/10.1103/PhysRevB.80. 235109.

R. O. Jones. Density functional theory: Its origins, rise to prominence, and future. Rev. Mod. Phys., 87:897-923, Aug 2015. doi: 10.1103/RevModPhys.87.897. URL http://link.aps.org/doi/10.1103/RevModPhys.87.897.

R. O. Jones and O. Gunnarsson. The density functional formalism, its applications and prospects. Rev. Mod. Phys., 61:689-746, Jul 1989. doi: 10.1103/RevModPhys.61.689. URL https://link.aps.org/doi/10.1103/RevModPhys.61.689.

P. Jordan and E. P. Wigner. Über das Paulische Äquivalenzverbot, pages 109-129. Springer Berlin Heidelberg, Berlin, Heidelberg, 1993. ISBN 978-3-662-02781-3. doi: 10.1007/9783-662-02781-3_9. URL https://doi.org/10.1007/978-3-662-02781-3_9.

Ebad Kamil, Thomas Pruschke, and Peter E. Blöchl. Resolvent approach for the evaluation of the reduced density-matrix functional of correlated electron systems. AIP Conference Proceedings, 1550(1):253-257, 2013. doi: 10.1063/1.4818405. URL https://aip.scitation.org/doi/abs/10.1063/1.4818405. 
Ebad Kamil, Robert Schade, Thomas Pruschke, and Peter E. Blöchl. Reduced densitymatrix functionals applied to the hubbard dimer. Phys. Rev. B, 93:085141, Feb 2016. doi: 10.1103/PhysRevB.93.085141. URL https://link.aps .org/doi/10.1103/ PhysRevB.93.085141.

J. Kanamori. Electron correlation and ferromagnetism of transition metals. Prog. Theor. Phys., 30:275, 1963. doi: doi:10.1143/PTP.30.275. URL http://ptp. oxfordjournals . org/content/30/3/275.

Abhinav Kandala, Antonio Mezzacapo, Kristan Temme, Maika Takita, Markus Brink, Jerry M. Chow, and Jay M. Gambetta. Hardware-efficient variational quantum eigensolver for small molecules and quantum magnets. Nature, 549:242 EP -, Sep 2017. URL http://dx.doi.org/10.1038/nature23879.

Alexander A Klyachko. Quantum marginal problem and n-representability. Journal of Physics: Conference Series, 36(1):72, 2006. URL http://stacks.iop.org/1742$6596 / 36 / i=1 / a=014$.

Erik Koch. The lanczos method. In Eva Pavarini, Erik Koch, Dieter Vollhardt, and Alexander Lichtenstein, editors, The LDA+DMFT approach to strongly correlated materials, page 450 p., Jülich, Sep 2011. Forschungszentrum Jülich Zentralbibliothek, Verlag. ISBN 978-3-89336-734-4. URL http://hdl .handle.net/2128/7348.

Jens Koch, Terri M. Yu, Jay Gambetta, A. A. Houck, D. I. Schuster, J. Majer, Alexandre Blais, M. H. Devoret, S. M. Girvin, and R. J. Schoelkopf. Charge-insensitive qubit design derived from the cooper pair box. Phys. Rev. A, 76:042319, Oct 2007. doi: 10.1103/PhysRevA.76.042319. URL https://link.aps .org/doi/10.1103/ PhysRevA.76.042319.

W. Kohn. Nobel lecture: Electronic structure of matter-wave functions and density functionals. Rev. Mod. Phys., 71:1253-1266, Oct 1999. doi: 10.1103/RevModPhys.71. 1253. URL https://link.aps.org/doi/10.1103/RevModPhys.71.1253.

W. Kohn and L. J. Sham. Self-consistent equations including exchange and correlation effects. Phys. Rev., 140:A1133-A1138, Nov 1965. doi: 10.1103/PhysRev.140.A1133. URL https://link.aps.org/doi/10.1103/PhysRev.140.A1133.

Christian Kollmar and Bernd A. Hess. The structure of the second-order reduced density matrix in density matrix functional theory and its construction from formal criteria. J. Chem. Phys., 120(7):3158-3171, 2004. doi: http://dx.doi.org/10.1063/1. 1634554. URL http://scitation.aip.org/content/aip/journal/jcp/120/7/10. 1063/1.1634554.

Jun Kondo. Resistance minimum in dilute magnetic alloys. Progress of Theoretical Physics, 32(1):37-49, 1964. doi: 10.1143/PTP.32.37.

G. Kotliar, S. Y. Savrasov, K. Haule, V. S. Oudovenko, O. Parcollet, and C. A. Marianetti. Electronic structure calculations with dynamical mean-field theory. Rev. Mod. Phys., 78:865-951, Aug 2006. doi: 10.1103/RevModPhys.78.865. URL http://link.aps . org/doi/10.1103/RevModPhys.78.865. 
Gabriel Kotliar. Electronic structure of correlated materials: Slave-boson methods and dynamical mean-field theory. In Eva Pavarini, Erik Koch, Dieter Vollhardt, and Alexander Lichtenstein, editors, DMFT at 25: Infinite Dimensions, 2014.

C. Krumnow, L. Veis, Ö. Legeza, and J. Eisert. Fermionic orbital optimization in tensor network states. Phys. Rev. Lett., 117:210402, Nov 2016. doi: 10.1103/PhysRevLett. 117.210402. URL https://link.aps.org/doi/10.1103/PhysRevLett.117.210402.

Hans Kummer. N-representability problem for reduced density matrices. Journal of Mathematical Physics, 8(10):2063-2081, 1967. doi: 10.1063/1.1705122. URL https: //doi.org/10.1063/1.1705122.

J. Kuneš, V. I. Anisimov, A. V. Lukoyanov, and D. Vollhardt. Local correlations and hole doping in nio: A dynamical mean-field study. Phys. Rev. B, 75:165115, Apr 2007a. doi: 10.1103/PhysRevB.75.165115. URL https://link.aps.org/doi/10. 1103/PhysRevB.75.165115.

J. Kuneš, V. I. Anisimov, S. L. Skornyakov, A. V. Lukoyanov, and D. Vollhardt. Nio: Correlated band structure of a charge-transfer insulator. Phys. Rev. Lett., 99:156404, Oct 2007b. doi: 10.1103/PhysRevLett.99.156404. URL https://link.aps.org/doi/ 10.1103/PhysRevLett.99.156404.

C. Y. Kuo, T. Haupricht, J. Weinen, Hua Wu, K. D. Tsuei, M. W. Haverkort, A. Tanaka, and L. H. Tjeng. Challenges from experiment: electronic structure of nio. The European Physical Journal Special Topics, 226(11):2445-2456, Jul 2017. ISSN 1951-6401. doi: 10.1140/epjst/e2017-70061-7. URL https ://doi .org/10.1140/epjst/e2017-700617 .

Yuki Kurashige. Multireference electron correlation methods with density matrix renormalisation group reference functions. Molecular Physics, 112(11):1485-1494, 2014. doi: 10.1080/00268976.2013.843730. URL https://doi.org/10.1080/00268976. 2013.843730 .

Werner Kutzelnigg. Die lösung des quantenmechanischen zwei-elektronenproblems durch unmittelbare bestimmung der natürlichen einelektronenfunktionen. Theoretica chimica acta, 1(4):327-342, 1963. ISSN 1432-2234. doi: 10.1007/BF00528764. URL http: //dx.doi.org/10.1007/BF00528764.

P. Lafarge, P. Joyez, D. Esteve, C. Urbina, and M. H. Devoret. Two-electron quantization of the charge on a superconductor. Nature, 365:422 EP -, Sep 1993. URL http: //dx.doi.org/10.1038/365422a0.

D. C. Langreth and J. P. Perdew. The exchange-correlation energy of a metallic surface. Solid State Communications, 17:1425-1429, December 1975. doi: 10.1016/00381098(75)90618-3.

David C. Langreth and John P. Perdew. Exchange-correlation energy of a metallic surface: Wave-vector analysis. Phys. Rev. B, 15:2884-2901, Mar 1977. doi: 10.1103/PhysRevB. 15.2884. URL http://link.aps.org/doi/10.1103/PhysRevB.15.2884. 
B. P. Lanyon, J. D. Whitfield, G. G. Gillett, M. E. Goggin, M. P. Almeida, I. Kassal, J. D. Biamonte, M. Mohseni, B. J. Powell, M. Barbieri, A. Aspuru-Guzik, and A. G. White. Towards quantum chemistry on a quantum computer. Nature Chemistry, 2:106 EP -, Jan 2010. URL http://dx.doi.org/10.1038/nchem.483. Article.

N. N. Lathiotakis and Miguel A. L. Marques. Benchmark calculations for reduced densitymatrix functional theory. J. Chem. Phys., 128(18):184103, 2008. doi: http://dx.doi.org/ 10.1063/1.2899328. URL http://scitation.aip.org/content/aip/journal/jcp/ 128/18/10.1063/1.2899328.

N. N. Lathiotakis, N. Helbig, and E. K. U. Gross. Performance of one-body reduced density-matrix functionals for the homogeneous electron gas. Phys. Rev. B, 75:195120, May 2007. doi: 10.1103/PhysRevB.75.195120. URL http://link.aps.org/doi/10. 1103/PhysRevB.75.195120.

N. N. Lathiotakis, N. I. Gidopoulos, and N. Helbig. Size consistency of explicit functionals of the natural orbitals in reduced density matrix functional theory. J. Chem. Phys., 132 (8):084105, 2010. doi: http://dx.doi.org/10.1063/1.3324699. URL http://scitation. aip.org/content/aip/journal/jcp/132/8/10.1063/1.3324699.

Vyacheslav Ivanovich Lebedev and DN Laikov. A quadrature formula for the sphere of the 131st algebraic order of accuracy. In Doklady Mathematics, volume 59, pages 477-481. Pleiades Publishing, Ltd., 1999.

A.M. Legendre. Mémoire sur l'intégration de quelques équations aux différences partielles: lû le Ier septembre 178\%. Mémoires de l'Académie Royale de Sciences. Imprimerie royale, 1787.

Ö. Legeza and J. Sólyom. Optimizing the density-matrix renormalization group method using quantum information entropy. Phys. Rev. B, 68:195116, Nov 2003. doi: 10. 1103/PhysRevB.68.195116. URL https://link.aps.org/doi/10.1103/PhysRevB. 68.195116.

Mel Levy. Universal variational functionals of electron densities, first order density matrixes and natural spin-orbitals and solution of the v-representability problem. Proc. Nat'l Acad. Sci. USA, 76:6062, 1979. URL http://www.pnas.org/content/76/12/ 6062. abstract.

Mel Levy and John P. Perdew. Hellmann-feynman, virial, and scaling requisites for the exact universal density functionals. shape of the correlation potential and diamagnetic susceptibility for atoms. Phys. Rev. A, 32:2010-2021, Oct 1985. doi: 10.1103/ PhysRevA.32.2010. URL https://link.aps.org/doi/10.1103/PhysRevA.32.2010.

C.-K. Li, R. Roberts, and X. Yin. Decomposition of Unitary Matrices and Quantum Gates. International Journal of Quantum Information, 11:1350015, May 2013. doi: 10.1142/S0219749913500159.

David A. Liberman. Slater transition-state band-structure calculations. Phys. Rev. B, 62: 6851-6853, Sep 2000. doi: 10.1103/PhysRevB.62.6851. URL http://link.aps.org/ doi/10.1103/PhysRevB.62.6851. 
A. I. Lichtenstein and M. I. Katsnelson. Ab initio calculations of quasiparticle band structure in correlated systems: Lda++ approach. Phys. Rev. B, 57:6884-6895, Mar 1998. doi: 10.1103/PhysRevB.57.6884. URL https://link.aps.org/doi/10.1103/ PhysRevB.57.6884.

Elliot H. Lieb. Variational principle for many-fermion systems. Phys. Rev. Lett., 46:457459, Feb 1981a. doi: 10.1103/PhysRevLett.46.457. URL http://link.aps.org/doi/ 10.1103/PhysRevLett. 46.457.

Elliott H. Lieb. Variational principle for many-fermion systems. Phys. Rev. Lett., 47: 69-69, Jul 1981b. doi: 10.1103/PhysRevLett.47.69. URL http://link.aps.org/doi/ 10.1103/PhysRevLett.47.69.

Elliott H. Lieb. Density functionals for coulomb systems. Int. J. Quantum Chem., 24(3): 243-277, 1983a. ISSN 1097-461X. doi: 10.1002/qua.560240302. URL http://dx.doi. org/10.1002/qua. 560240302 .

Elliott H. Lieb. Density functionals for coulomb systems. Int. J. Quantum Chem., 24(3): 243-277, 1983b. ISSN 1097-461X. doi: 10.1002/qua.560240302. URL http://dx.doi . org/10.1002/qua. 560240302 .

Elliott H. Lieb and F. Y. Wu. Absence of mott transition in an exact solution of the short-range, one-band model in one dimension. Phys. Rev. Lett., 20:1445-1448, Jun 1968. doi: 10.1103/PhysRevLett.20.1445. URL http://link.aps.org/doi/10.1103/ PhysRevLett . 20.1445.

A. I. Liechtenstein, V. I. Anisimov, and J. Zaanen. Density-functional theory and strong interactions: Orbital ordering in mott-hubbard insulators. Phys. Rev. B, 52:R5467R5470, Aug 1995. doi: 10.1103/PhysRevB.52.R5467. URL https://link.aps.org/ doi/10.1103/PhysRevB.52.R5467.

Chungwei Lin and Alexander A. Demkov. Efficient variational approach to the impurity problem and its application to the dynamical mean-field theory. Phys. Rev. B, 88: 035123, Jul 2013. doi: 10.1103/PhysRevB.88.035123. URL http://link.aps.org/ doi/10.1103/PhysRevB.88.035123.

Yi-Kai Liu, Matthias Christandl, and F. Verstraete. Quantum computational complexity of the $n$-representability problem: Qma complete. Phys. Rev. Lett., 98:110503, Mar 2007. doi: 10.1103/PhysRevLett.98.110503. URL https://link.aps.org/doi/10 . 1103/PhysRevLett.98.110503.

Seth Lloyd. Universal quantum simulators. Science, 273(5278):1073-1078, 1996. ISSN 00368075, 10959203. URL http://www.jstor.org/stable/2899535.

E. Y. Loh, J. E. Gubernatis, R. T. Scalettar, S. R. White, D. J. Scalapino, and R. L. Sugar. Sign problem in the numerical simulation of many-electron systems. Phys. Rev. B, 41:9301-9307, May 1990. doi: 10.1103/PhysRevB.41.9301. URL https://link. aps.org/doi/10.1103/PhysRevB.41.9301. 
S.G. Louie and M.L. Cohen. Conceptual Foundations of Materials: A Standard Model for Ground- and Excited-State Properties. Contemporary Concepts of Condensed Matter Science. Elsevier Science, 2006. ISBN 9780080464572.

Per-Olov Löwdin. A Quantum Mechanical Calculation of the Cohesive Energy, the Interionic Distance, and the Elastic Contants of Some Ionic Crystals. Almqvist \& Wiksells, 1948.

Per-Olov Löwdin. Quantum theory of many-particle systems. i. physical interpretations by means of density matrices, natural spin-orbitals, and convergence problems in the method of configurational interaction. Phys. Rev., 97:1474, 1955. doi: 10.1103/ PhysRev.97.1474. URL http://link.aps.org/doi/10.1103/PhysRev.97.1474.

Per-Olov Löwdin and Harrison Shull. Natural orbitals in the quantum theory of twoelectron systems. Phys. Rev., 101:1730-1739, Mar 1956. doi: 10.1103/PhysRev.101. 1730. URL http://link.aps.org/doi/10.1103/PhysRev.101.1730.

Y. Lu, M. Höppner, O. Gunnarsson, and M. W. Haverkort. Efficient real-frequency solver for dynamical mean-field theory. Phys. Rev. B, 90:085102, Aug 2014. doi: 10. 1103/PhysRevB.90.085102. URL http://link.aps.org/doi/10.1103/PhysRevB. 90. 085102.

Yingjin Ma and Haibo Ma. Assessment of various natural orbitals as the basis of large active space density-matrix renormalization group calculations. The Journal of Chemical Physics, 138(22):224105, 2013. doi: 10.1063/1.4809682. URL https: //doi.org/10.1063/1.4809682.

G.D. Mahan. Many-Particle Physics. Physics of Solids and Liquids. Springer US, 2013. ISBN 9781475757149.

Stefan Maintz, Volker L. Deringer, Andrei L. Tchougréeff, and Richard Dronskowski. Analytic projection from plane-wave and paw wavefunctions and application to chemicalbonding analysis in solids. Journal of Computational Chemistry, 34(29):2557-2567, 2013. doi: 10.1002/jcc.23424. URL https://onlinelibrary.wiley.com/doi/abs/ $10.1002 / j c c .23424$.

J. Malick, J. Povh, F. Rendl, and A. Wiegele. Regularization methods for semidefinite programming. SIAM Journal on Optimization, 20(1):336-356, 2009. doi: 10.1137/ 070704575. URL https://doi.org/10.1137/070704575.

Nicholas Maratos. Exact penalty function algorithms for finite dimensional and control optimization problems. PhD thesis, 1978.

Igor L. Markov and Mehdi Saeedi. Constant-optimized quantum circuits for modular multiplication and exponentiation. Quantum Info. Comput., 12(5-6):361-394, May 2012. ISSN 1533-7146. URL http://dl.acm.org/citation. cfm?id=2230996.2230997.

Konrad H. Marti and Markus Reiher. New electron correlation theories for transition metal chemistry. Phys. Chem. Chem. Phys., 13:6750-6759, 2011. doi: 10.1039/ C0CP01883J. URL http://dx.doi.org/10.1039/C0CP01883J. 
Takeo Matsubara. A new approach to quantum-statistical mechanics. Progress of Theoretical Physics, 14(4):351-378, 1955. doi: 10.1143/PTP.14.351. URL http: //dx.doi.org/10.1143/PTP.14.351.

David A. Mazziotti. Variational minimization of atomic and molecular ground-state energies via the two-particle reduced density matrix. Phys. Rev. A, 65:062511, Jun 2002. doi: 10.1103/PhysRevA.65.062511. URL https://link.aps.org/doi/10.1103/ PhysRevA.65.062511.

David A. Mazziotti. Exactness of wave functions from two-body exponential transformations in many-body quantum theory. Phys. Rev. A, 69:012507, Jan 2004a. doi: 10. 1103/PhysRevA.69.012507. URL https://link.aps.org/doi/10.1103/PhysRevA. 69.012507.

David A. Mazziotti. Realization of quantum chemistry without wave functions through first-order semidefinite programming. Phys. Rev. Lett., 93:213001, Nov 2004b. doi: 10.1103/PhysRevLett.93.213001. URL https://link.aps.org/doi/10.1103/ PhysRevLett.93.213001.

David A. Mazziotti. Large-scale semidefinite programming for many-electron quantum mechanics. Phys. Rev. Lett., 106:083001, Feb 2011. doi: 10.1103/PhysRevLett.106. 083001. URL https://link.aps.org/doi/10.1103/PhysRevLett.106.083001.

David A. Mazziotti. Structure of fermionic density matrices: Complete $n$-representability conditions. Phys. Rev. Lett., 108:263002, Jun 2012. doi: 10.1103/PhysRevLett.108. 263002. URL https://link.aps.org/doi/10.1103/PhysRevLett.108.263002.

David A. Mazziotti and Robert M. Erdahl. Uncertainty relations and reduced density matrices: Mapping many-body quantum mechanics onto four particles. Phys. Rev. A, 63:042113, Mar 2001. doi: 10.1103/PhysRevA.63.042113. URL https://link.aps. org/doi/10.1103/PhysRevA.63.042113.

Jarrod R McClean, Jonathan Romero, Ryan Babbush, and Alán Aspuru-Guzik. The theory of variational hybrid quantum-classical algorithms. New Journal of Physics, 18 (2):023023, 2016. URL http://stacks.iop.org/1367-2630/18/i=2/a=023023.

A.D. McLachlan. A variational solution of the time-dependent schrodinger equation. Molecular Physics, 8(1):39-44, 1964. doi: 10.1080/00268976400100041. URL https: //doi.org/10.1080/00268976400100041.

W. L. McMillan. Ground state of liquid he ${ }^{4}$. Phys. Rev., 138:A442-A451, Apr 1965. doi: 10.1103/PhysRev.138.A442. URL https://link.aps.org/doi/10.1103/PhysRev . 138. A442.

R. McWeeny. Some recent advances in density matrix theory. Rev. Mod. Phys., 32:335369, Apr 1960. doi: 10.1103/RevModPhys.32.335. URL https://link.aps.org/doi/ 10.1103/RevModPhys.32.335.

N. David Mermin. Thermal properties of the inhomogeneous electron gas. Phys. Rev., 137:A1441-A1443, Mar 1965. doi: 10.1103/PhysRev.137.A1441. URL https://link. aps.org/doi/10.1103/PhysRev.137.A1441. 
Nicholas Metropolis, Arianna W. Rosenbluth, Marshall N. Rosenbluth, Augusta H. Teller, and Edward Teller. Equation of state calculations by fast computing machines. The Journal of Chemical Physics, 21(6):1087-1092, 1953. doi: 10.1063/1.1699114. URL https://doi .org/10.1063/1.1699114.

Kristel Michielsen, Madita Nocon, Dennis Willsch, Fengping Jin, Thomas Lippert, and Hans De Raedt. Benchmarking gate-based quantum computers. Computer Physics Communications, 220:44 - 55, 2017. ISSN 0010-4655. doi: https://doi.org/ 10.1016/j.cpc.2017.06.011. URL http://www. sciencedirect.com/science/article/ pii/S0010465517301935.

T. Misawa, S. Morita, K. Yoshimi, M. Kawamura, Y. Motoyama, K. Ido, T. Ohgoe, M. Imada, and T. Kato. mVMC - Open-source software for many-variable variational Monte Carlo method. ArXiv e-prints, November 2017.

Oki Miura and Takeo Fujiwara. Electronic structure and effects of dynamical electron correlation in ferromagnetic bcc fe, fcc ni, and antiferromagnetic nio. Phys. Rev. B, 77: 195124, May 2008. doi: 10.1103/PhysRevB.77.195124. URL https://link.aps.org/ doi/10.1103/PhysRevB.77.195124.

N. Moll, P. Barkoutsos, L. S. Bishop, J. M. Chow, A. Cross, D. J. Egger, S. Filipp, A. Fuhrer, J. M. Gambetta, M. Ganzhorn, A. Kandala, A. Mezzacapo, P. Müller, W. Riess, G. Salis, J. Smolin, I. Tavernelli, and K. Temme. Quantum optimization using variational algorithms on near-term quantum devices. ArXiv e-prints, October 2017.

Paula Mori-Sánchez, Aron J. Cohen, and Weitao Yang. Localization and delocalization errors in density functional theory and implications for band-gap prediction. Phys. Rev. Lett., 100:146401, Apr 2008. doi: 10.1103/PhysRevLett.100.146401. URL http: //link.aps.org/doi/10.1103/PhysRevLett.100.146401.

N F Mott. The basis of the electron theory of metals, with special reference to the transition metals. Proceedings of the Physical Society. Section A, 62(7):416, 1949. URL http: //stacks. iop.org/0370-1298/62/i=7/a=303.

N F Mott and R Peierls. Discussion of the paper by de boer and verwey. Proceedings of the Physical Society, 49(4S):72, 1937. URL http://stacks.iop.org/0959-5309/49/ $i=4 \mathrm{~S} / \mathrm{a}=308$.

A.M.K. Müller. Explicit approximate relation between reduced two- and one-particle density matrices. Phys. Lett. A, 105(9):446 - 452, 1984. ISSN 0375-9601. doi: http: //dx.doi.org/10.1016/0375-9601(84)91034-X. URL http://www.sciencedirect.com/ science/article/pii/037596018491034X.

C W Müller. Sufficient conditions for pure state $\mathrm{n}$-representability. Journal of Physics A: Mathematical and General, 32(22):4139, 1999. URL http://stacks.iop.org/0305$4470 / 32 / i=22 / a=314$.

Maho Nakata, Hiroshi Nakatsuji, Masahiro Ehara, Mitsuhiro Fukuda, Kazuhide Nakata, and Katsuki Fujisawa. Variational calculations of fermion second-order reduced density 
matrices by semidefinite programming algorithm. The Journal of Chemical Physics, 114(19):8282-8292, 2001. doi: 10.1063/1.1360199. URL https://doi.org/10.1063/ 1.1360199 .

Frank Neese. The orca program system. Wiley Interdiscip. Rev. Comput. Mol. Sci., 2(1): 73-78, 2012. ISSN 1759-0884. doi: 10.1002/wcms.81. URL http://dx.doi.org/10. $1002 /$ wcms .81.

I. A. Nekrasov, V. S. Pavlov, and M. V. Sadovskii. Consistent lda' + dmft -an unambiguous way to avoid double counting problem: Nio test. JETP Letters, 95 (11):581-585, Aug 2012. ISSN 1090-6487. doi: 10.1134/S0021364012110070. URL https ://doi .org/10.1134/S0021364012110070.

J. von Neumann. Wahrscheinlichkeitstheoretischer aufbau der quantenmechanik. Nachrichten von der Gesellschaft der Wissenschaften zu Göttingen, MathematischPhysikalische Klasse, 1927:245-272, 1927. URL http://eudml.org/doc/59230.

M.A. Nielsen and I.L. Chuang. Quantum Computation and Quantum Information: 10th Anniversary Edition. Cambridge University Press, 2010. ISBN 9781139495486.

J. Nocedal and S. Wright. Numerical Optimization. Springer Series in Operations Research and Financial Engineering. Springer New York, 2006. ISBN 9780387400655.

Marcel Nooijen. Can the eigenstates of a many-body hamiltonian be represented exactly using a general two-body cluster expansion? Phys. Rev. Lett., 84:2108-2111, Mar 2000. doi: 10.1103/PhysRevLett.84.2108. URL https://link.aps.org/doi/10. 1103/PhysRevLett.84.2108.

P. Novák, J. Kuneš, L. Chaput, and W. E. Pickett. Exact exchange for correlated electrons. physica status solidi (b), 243(3):563-572. doi: 10.1002/pssb.200541371. URL https: //onlinelibrary.wiley.com/doi/abs/10.1002/pssb. 200541371.

Tohru Ogawa, Kunihiko Kanda, and Takeo Matsubara. Gutzwiller approximation for antiferromagnetism in hubbard model *). Progress of Theoretical Physics, 53(3):614633, 1975. doi: 10.1143/PTP.53.614. URL http://dx.doi.org/10.1143/PTP.53.614.

T. Oguchi, K. Terakura, and A. R. Williams. Band theory of the magnetic interaction in mno, mns, and nio. Phys. Rev. B, 28:6443-6452, Dec 1983. doi: 10.1103/PhysRevB. 28.6443. URL https://link.aps.org/doi/10.1103/PhysRevB. 28.6443.

P. J. J. O’Malley, R. Babbush, I. D. Kivlichan, J. Romero, J. R. McClean, R. Barends, J. Kelly, P. Roushan, A. Tranter, N. Ding, B. Campbell, Y. Chen, Z. Chen, B. Chiaro, A. Dunsworth, A. G. Fowler, E. Jeffrey, E. Lucero, A. Megrant, J. Y. Mutus, M. Neeley, C. Neill, C. Quintana, D. Sank, A. Vainsencher, J. Wenner, T. C. White, P. V. Coveney, P. J. Love, H. Neven, A. Aspuru-Guzik, and J. M. Martinis. Scalable quantum simulation of molecular energies. Phys. Rev. X, 6:031007, Jul 2016. doi: 10.1103/PhysRevX. 6.031007. URL https://link.aps.org/doi/10.1103/PhysRevX.6.031007.

Stellan Östlund and Stefan Rommer. Thermodynamic limit of density matrix renormalization. Phys. Rev. Lett., 75:3537-3540, Nov 1995. doi: 10.1103/PhysRevLett.75.3537. URL https://link.aps.org/doi/10.1103/PhysRevLett.75.3537. 
Rudolph Pariser and Robert G. Parr. A semi-empirical theory of the electronic spectra and electronic structure of complex unsaturated molecules. i. The Journal of Chemical Physics, 21(3):466-471, 1953a. doi: http://dx.doi.org/10.1063/1.1698929. URL http: //scitation.aip.org/content/aip/journal/jcp/21/3/10.1063/1.1698929.

Rudolph Pariser and Robert G. Parr. A semi-empirical theory of the electronic spectra and electronic structure of complex unsaturated molecules. ii. The Journal of Chemical Physics, 21(5):767-776, 1953b. doi: http://dx.doi.org/10.1063/1.1699030. URL http: //scitation.aip.org/content/aip/journal/jcp/21/5/10.1063/1.1699030.

Robert M. Parrish, Lori A. Burns, Daniel G. A. Smith, Andrew C. Simmonett, A. Eugene DePrince, Edward G. Hohenstein, Uğur Bozkaya, Alexander Yu. Sokolov, Roberto Di Remigio, Ryan M. Richard, Jérôme F. Gonthier, Andrew M. James, Harley R. McAlexander, Ashutosh Kumar, Masaaki Saitow, Xiao Wang, Benjamin P. Pritchard, Prakash Verma, Henry F. Schaefer, Konrad Patkowski, Rollin A. King, Edward F. Valeev, Francesco A. Evangelista, Justin M. Turney, T. Daniel Crawford, and C. David Sherrill. Psi4 1.1: An open-source electronic structure program emphasizing automation, advanced libraries, and interoperability. Journal of Chemical Theory and Computation, 13(7):3185-3197, 2017. doi: 10.1021/acs.jctc.7b00174. URL https: //doi.org/10.1021/acs.jctc.7b00174. PMID: 28489372.

Eva Pavarini. The lda+dmft approach. In Eva Pavarini, Erik Koch, Dieter Vollhardt, and Alexander Lichtenstein, editors, The LDA+DMFT approach to strongly correlated materials, page 450 p., Jl̈ich, Sep 2011. Forschungszentrum Jülich Zentralbibliothek, Verlag. ISBN 978-3-89336-734-4. URL http://hdl.handle.net/2128/7348.

R.E. Peierls, S.R.E.P. Rudolf Ernst Peierls, R.S. Peierls, and Oxford University Press. Quantum Theory of Solids. International series of monographs on physics. Clarendon Press, 1955. ISBN 9780198507819.

J. K. Percus. The role of model systems in the few-body reduction of the n-fermion problem. International Journal of Quantum Chemistry, 13(1):89-124, 1978. doi: 10. 1002/qua.560130108. URL https://onlinelibrary.wiley.com/doi/abs/10.1002/ qua. 560130108.

J. P. Perdew and Alex Zunger. Self-interaction correction to density-functional approximations for many-electron systems. Phys. Rev. B, 23:5048-5079, May 1981. doi: 10.1103/ PhysRevB.23.5048. URL https://link.aps.org/doi/10.1103/PhysRevB. 23. 5048.

John P. Perdew. Density functional theory and the band gap problem. International Journal of Quantum Chemistry, 28(S19):497-523. doi: 10.1002/qua.560280846. URL https://onlinelibrary.wiley.com/doi/abs/10.1002/qua.560280846.

John P. Perdew and Mel Levy. Physical content of the exact kohn-sham orbital energies: Band gaps and derivative discontinuities. Phys. Rev. Lett., 51:1884-1887, Nov 1983. doi: 10.1103/PhysRevLett.51.1884. URL https://link.aps.org/doi/ 10.1103/PhysRevLett.51.1884.

John P. Perdew and Yue Wang. Accurate and simple analytic representation of the electron-gas correlation energy. Phys. Rev. B, 45:13244-13249, Jun 1992a. doi: 10. 
1103/PhysRevB.45.13244. URL https://link.aps.org/doi/10.1103/PhysRevB. 45. 13244.

John P. Perdew and Yue Wang. Pair-distribution function and its coupling-constant average for the spin-polarized electron gas. Phys. Rev. B, 46:12947-12954, Nov 1992b. doi: 10.1103/PhysRevB.46.12947. URL https://link.aps.org/doi/10.1103/PhysRevB . 46. 12947.

John P. Perdew, Robert G. Parr, Mel Levy, and Jose L. Balduz. Density-functional theory for fractional particle number: Derivative discontinuities of the energy. Phys. Rev. Lett., 49:1691-1694, Dec 1982. doi: 10.1103/PhysRevLett.49.1691. URL http: //link.aps.org/doi/10.1103/PhysRevLett.49.1691.

John P. Perdew, Andreas Savin, and Kieron Burke. Escaping the symmetry dilemma through a pair-density interpretation of spin-density functional theory. Phys. Rev. A, 51:4531-4541, Jun 1995. doi: 10.1103/PhysRevA.51.4531. URL https://link.aps . org/doi/10.1103/PhysRevA.51.4531.

John P. Perdew, Kieron Burke, and Matthias Ernzerhof. Generalized gradient approximation made simple. Phys. Rev. Lett., 77:3865-3868, Oct 1996a. doi: 10.1103/ PhysRevLett.77.3865. URL http://link.aps.org/doi/10.1103/PhysRevLett.77. 3865.

John P. Perdew, Kieron Burke, and Yue Wang. Generalized gradient approximation for the exchange-correlation hole of a many-electron system. Phys. Rev. B, 54:16533-16539, Dec 1996b. doi: 10.1103/PhysRevB.54.16533. URL https://link.aps.org/doi/10. 1103/PhysRevB.54.16533.

John P. Perdew, Matthias Ernzerhof, and Kieron Burke. Rationale for mixing exact exchange with density functional approximations. The Journal of Chemical Physics, 105(22):9982-9985, 1996c. doi: 10.1063/1.472933. URL https://doi.org/10.1063/ 1.472933 .

Katarzyna Pernal. Long-range density-matrix-functional theory: Application to a modified homogeneous electron gas. Phys. Rev. A, 81:052511, May 2010. doi: 10.1103/PhysRevA.81.052511. URL http://link.aps.org/doi/10.1103/PhysRevA . 81.052511.

Katarzyna Pernal and Klaas J. H. Giesbertz. Reduced density matrix functional theory (rdmft) and linear response time-dependent rdmft (td-rdmft). Top. Curr. Chem., 368: 125-183, 2016.

Alberto Peruzzo, Jarrod McClean, Peter Shadbolt, Man-Hong Yung, Xiao-Qi Zhou, Peter J. Love, Alán Aspuru-Guzik, and Jeremy L. O'Brien. A variational eigenvalue solver on a photonic quantum processor. Nature Communications, 5:4213 EP -, Jul 2014. URL http://dx.doi.org/10.1038/ncomms5213. Article.

Kirk A. Peterson and Thom H. Dunning. Accurate correlation consistent basis sets for molecular core-valence correlation effects: The second row atoms al-ar, and the first row atoms b-ne revisited. The Journal of Chemical Physics, 117(23):10548-10560, 2002. doi: 10.1063/1.1520138. URL https://doi.org/10.1063/1.1520138. 
Piotr Piecuch, Karol Kowalski, Peng-Dong Fan, and Krzysztof Jedziniak. Exactness of two-body cluster expansions in many-body quantum theory. Phys. Rev. Lett., 90: 113001, Mar 2003. doi: 10.1103/PhysRevLett.90.113001. URL https://link.aps. org/doi/10.1103/PhysRevLett.90.113001.

S. Pittalis, C. R. Proetto, A. Floris, A. Sanna, C. Bersier, K. Burke, and E. K. U. Gross. Exact conditions in finite-temperature density-functional theory. Phys. Rev. Lett., 107: 163001, Oct 2011. doi: 10.1103/PhysRevLett.107.163001. URL https://link.aps . org/doi/10.1103/PhysRevLett.107.163001.

J. A. Pople. Electron interaction in unsaturated hydrocarbons. Trans. Faraday Soc., 49: 1375-1385, 1953. doi: 10.1039/TF9534901375. URL http://dx.doi.org/10.1039/ TF9534901375.

MJD Powell. A method for nonlinear constraints in minimization problems in optimization, r. Academic Press, NY, pages 283-298, 1969.

Th. Pruschke, D. L. Cox, and M. Jarrell. Hubbard model at infinite dimensions: Thermodynamic and transport properties. Phys. Rev. B, 47:3553-3565, Feb 1993. doi: 10.1103/ PhysRevB.47.3553. URL https://link.aps.org/doi/10.1103/PhysRevB.47.3553.

Pekka Pyykko. Relativistic effects in structural chemistry. Chemical Reviews, 88(3):563594, 1988. doi: 10.1021/cr00085a006. URL https://doi.org/10.1021/cr00085a006.

Pekka Pyykko and Jean Paul Desclaux. Relativity and the periodic system of elements. Accounts of Chemical Research, 12(8):276-281, 1979. doi: 10.1021/ar50140a002. URL https://doi.org/10.1021/ar50140a002.

Pekka Pyykkö. Relativistic effects in chemistry: More common than you thought. Annual Review of Physical Chemistry, 63(1):45-64, 2012. doi: 10.1146/annurevphyschem-032511-143755. URL https://doi.org/10.1146/annurev-physchem032511-143755. PMID: 22404585.

X. Ren, I. Leonov, G. Keller, M. Kollar, I. Nekrasov, and D. Vollhardt. LDA + DMFT computation of the electronic spectrum of nio. Phys. Rev. B, 74:195114, Nov 2006. doi: 10.1103/PhysRevB.74.195114. URL https://link.aps.org/doi/10.1103/ PhysRevB.74.195114.

Raffaele Resta. The insulating state of matter: A geometrical theory. In Eva Pavarini, Erik Koch, Richard Martin, and Richard Scalettar, editors, The Physics of Correlated Insulators, Metals, and Superconductors, page 450 p., Jülich, Sep 2017. Forschungszentrum Jülich Zentralbibliothek, Verlag. ISBN 978-3-95806-224-5. URL http://hdl. handle.net/2128/15283.

Chad Rigetti, Jay M. Gambetta, Stefano Poletto, B. L. T. Plourde, Jerry M. Chow, A. D. Córcoles, John A. Smolin, Seth T. Merkel, J. R. Rozen, George A. Keefe, Mary B. Rothwell, Mark B. Ketchen, and M. Steffen. Superconducting qubit in a waveguide cavity with a coherence time approaching $0.1 \mathrm{~ms}$. Phys. Rev. B, 86:100506, Sep 2012. doi: 10.1103/PhysRevB.86.100506. URL https://link.aps.org/doi/10. 1103/PhysRevB.86.100506. 
R. Rodríguez-Guzmán, K. W. Schmid, Carlos A. Jiménez-Hoyos, and Gustavo E. Scuseria. Symmetry-projected variational approach for ground and excited states of the two-dimensional hubbard model. Phys. Rev. B, 85:245130, Jun 2012. doi: 10. 1103/PhysRevB.85.245130. URL https://link.aps.org/doi/10.1103/PhysRevB. 85.245130 .

R. Rodríguez-Guzmán, Carlos A. Jiménez-Hoyos, R. Schutski, and Gustavo E. Scuseria. Multireference symmetry-projected variational approaches for ground and excited states of the one-dimensional hubbard model. Phys. Rev. B, 87:235129, Jun 2013. doi: 10.1103/PhysRevB.87.235129. URL https://link.aps.org/doi/10.1103/ PhysRevB.87.235129.

Daniel R. Rohr and Katarzyna Pernal. Open-shell reduced density matrix functional theory. J. Chem. Phys., 135(7):074104, 2011. doi: http://dx.doi.org/10.1063/1. 3624609. URL http://scitation.aip.org/content/aip/journal/jcp/135/7/10. 1063/1.3624609.

Daniel R. Rohr, Julien Toulouse, and Katarzyna Pernal. Combining density-functional theory and density-matrix-functional theory. Phys. Rev. A, 82:052502, Nov 2010. doi: 10.1103/PhysRevA.82.052502. URL http://link.aps.org/doi/10.1103/PhysRevA. 82.052502.

Stefan Rommer and Stellan Östlund. Class of ansatz wave functions for one-dimensional spin systems and their relation to the density matrix renormalization group. Phys. Rev. B, 55:2164-2181, Jan 1997. doi: 10.1103/PhysRevB.55.2164. URL https ://link . aps . org/doi/10.1103/PhysRevB.55.2164.

W. L. Roth. Magnetic structures of mno, feo, coo, and nio. Phys. Rev., 110:1333-1341, Jun 1958a. doi: 10.1103/PhysRev.110.1333. URL https://link.aps.org/doi/10 . 1103/PhysRev.110.1333.

W. L. Roth. Multispin axis structures for antiferromagnets. Phys. Rev., 111:772-781, Aug 1958b. doi: 10.1103/PhysRev.111.772. URL https://link.aps.org/doi/10.1103/ PhysRev.111.772.

Jean-Paul Ryckaert, Giovanni Ciccotti, and Herman J. C. Berendsen. Numerical integration of cartesian equations of motion of a system with constraints: molecular dynamics of alkenes. J. Comput. Phys., 23:327, 1977. doi: doi:10.1016/0021-9991(77)90098-5.

Anders W. Sandvik and Juhani Kurkijärvi. Quantum monte carlo simulation method for spin systems. Phys. Rev. B, 43:5950-5961, Mar 1991. doi: 10.1103/PhysRevB.43.5950. URL https://link.aps.org/doi/10.1103/PhysRevB.43.5950.

A. Savin. A combined density functional and configuration interaction method. Int. J. Quantum Chem., 34(S22):59-69, 1988. ISSN 1097-461X. doi: 10.1002/qua.560340811. URL http://dx.doi.org/10.1002/qua.560340811.

Andreas Savin. On degeneracy, Near-degeneracy and Density Functional Theory, pages 327-357. Theoretical and Computational Chemistry. Elsevier Science, 1996. ISBN 9780080540399 . 
G. A. Sawatzky and J. W. Allen. Magnitude and origin of the band gap in nio. Phys. Rev. Lett., 53:2339-2342, Dec 1984. doi: 10.1103/PhysRevLett.53.2339. URL https: //link.aps.org/doi/10.1103/PhysRevLett.53.2339.

Douglas J. Scalapino, Steven R. White, and Shoucheng Zhang. Insulator, metal, or superconductor: The criteria. Phys. Rev. B, 47:7995-8007, Apr 1993. doi: 10.1103/ PhysRevB.47.7995. URL https://link.aps.org/doi/10.1103/PhysRevB.47.7995.

Richard Scalettar. Insulator, metal, or superconductor: The criteria. In Eva Pavarini, Erik Koch, Richard Martin, and Richard Scalettar, editors, The Physics of Correlated Insulators, Metals, and Superconductors, page 450 p., Jülich, Sep 2017. Forschungszentrum Jülich Zentralbibliothek, Verlag. ISBN 978-3-95806-224-5. URL http://hdl. handle.net/2128/15283.

R. Schade and P. E. Blöchl. Adaptive cluster approximation for reduced density-matrix functional theory. ArXiv e-prints 1612.06692v1, December 2016. URL https://arxiv . org/abs/1612.06692v1.

Robert Schade. Relativistic effects in the PAW method beyond the scalar approximation. Master's thesis, Georg-August Univeristy Göttingen, 2012. URL https : //www2 .pt . tuclausthal.de/atp/members/rschade/Master.pdf.

Robert Schade and Peter E. Blöchl. Adaptive cluster approximation for reduced densitymatrix functional theory. Phys. Rev. B, 97:245131, Jun 2018. doi: 10.1103/PhysRevB. 97.245131. URL https://link.aps.org/doi/10.1103/PhysRevB.97.245131.

Robert Schade, Ebad Kamil, and Peter Blöchl. Reduced density-matrix functionals from many-particle theory. The European Physical Journal Special Topics, 226(11):26772692, Jul 2017. ISSN 1951-6401. doi: 10.1140/epjst/e2017-70046-0. URL https: //doi.org/10.1140/epjst/e2017-70046-0.

K. W. Schmid. On the use of general symmetry-projected Hartree-Fock-Bogoliubov configurations in variational approaches to the nuclear many-body problem. Progress in Particle and Nuclear Physics, 52:565-633, April 2004. doi: 10.1016/j.ppnp.2004.02.001.

K. W. Schmid, T. Dahm, J. Margueron, and H. Müther. Symmetry-projected variational approach to the one-dimensional hubbard model. Phys. Rev. B, 72:085116, Aug 2005. doi: 10.1103/PhysRevB.72.085116. URL https://link.aps.org/doi/10.1103/ PhysRevB.72.085116.

U. Schollwöck. The density-matrix renormalization group. Rev. Mod. Phys., 77:259-315, Apr 2005. doi: 10.1103/RevModPhys.77.259. URL https://link.aps.org/doi/10. 1103/RevModPhys.77.259.

Ulrich Schollwöck. The density-matrix renormalization group in the age of matrix product states. Annals of Physics, 326(1):96 - 192, 2011. ISSN 0003-4916. doi: https:// doi.org/10.1016/j.aop.2010.09.012. URL http://www.sciencedirect.com/science/ article/pii/S0003491610001752. January 2011 Special Issue. 
E. Schrödinger. An undulatory theory of the mechanics of atoms and molecules. Phys. Rev., 28:1049-1070, Dec 1926. doi: 10.1103/PhysRev.28.1049. URL https://link. aps.org/doi/10.1103/PhysRev.28.1049.

N. Schuch. Condensed Matter Applications of Entanglement Theory. ArXiv e-prints, June 2013.

Norbert Schuch and Frank Verstraete. Computational complexity of interacting electrons and fundamental limitations of density functional theory. Nature Physics, 5:732 EP -, Aug 2009. URL http://dx.doi.org/10.1038/nphys1370.

Karen L. Schuchardt, Brett T. Didier, Todd Elsethagen, Lisong Sun, Vidhya Gurumoorthi, Jared Chase, Jun Li, and Theresa L. Windus. Basis set exchange: A community database for computational sciences. Journal of Chemical Information and Modeling, 47(3):1045-1052, 2007. doi: 10.1021/ci600510j. URL https://doi.org/10.1021/ ci600510j. PMID: 17428029.

M. Schüler, M. Rösner, T. O. Wehling, A. I. Lichtenstein, and M. I. Katsnelson. Optimal hubbard models for materials with nonlocal coulomb interactions: Graphene, silicene, and benzene. Phys. Rev. Lett., 111:036601, Jul 2013. doi: 10.1103/PhysRevLett.111. 036601. URL https://link.aps.org/doi/10.1103/PhysRevLett.111.036601.

M. Schüler, C. Renk, and T. O. Wehling. Variational exact diagonalization method for anderson impurity models. Phys. Rev. B, 91:235142, Jun 2015. doi: 10.1103/PhysRevB. 91.235142. URL http://link.aps.org/doi/10.1103/PhysRevB.91.235142.

T. M. Schuler, D. L. Ederer, S. Itza-Ortiz, G. T. Woods, T. A. Callcott, and J. C. Woicik. Character of the insulating state in nio: A mixture of charge-transfer and mott-hubbard character. Phys. Rev. B, 71:115113, Mar 2005. doi: 10.1103/PhysRevB.71.115113. URL https://link.aps.org/doi/10.1103/PhysRevB.71.115113.

H. Schweitzer and G. Czycholl. Resistivity and thermopower of heavy-fermion systems. Phys. Rev. Lett., 67:3724-3727, Dec 1991. doi: 10.1103/PhysRevLett.67.3724. URL https://link.aps.org/doi/10.1103/PhysRevLett.67.3724.

P. Schwerdtfeger. Relativistic Electronic Structure Theory - Fundamentals. Theoretical and Computational Chemistry. Elsevier Science, 2002. ISBN 9780080540467.

P. Schwerdtfeger. Relativistic Electronic Structure Theory: Part 2. Applications. Theoretical and Computational Chemistry. Elsevier Science, 2004. ISBN 9780080540474.

Gustavo E. Scuseria, Carlos A. Jiménez-Hoyos, Thomas M. Henderson, Kousik Samanta, and Jason K. Ellis. Projected quasiparticle theory for molecular electronic structure. The Journal of Chemical Physics, 135(12):124108, 2011. doi: 10.1063/1.3643338. URL https://doi.org/10.1063/1.3643338.

L. J. Sham and M. Schlüter. Density-functional theory of the energy gap. Phys. Rev. Lett., 51:1888-1891, Nov 1983. doi: 10.1103/PhysRevLett.51.1888. URL https:// link.aps.org/doi/10.1103/PhysRevLett.51.1888. 
D. F. Shanno. Conditioning of quasi-newton methods for function minimization. Math. Comp., 24:647-656, 1970.

S. Sharma, J. K. Dewhurst, N. N. Lathiotakis, and E. K. U. Gross. Reduced density matrix functional for many-electron systems. Phys. Rev. B, 78:201103, Nov 2008. doi: 10.1103/PhysRevB.78.201103. URL http://link.aps.org/doi/10.1103/PhysRevB. 78.201103.

S. Sharma, J. K. Dewhurst, S. Shallcross, and E. K. U. Gross. Spectral density and metalinsulator phase transition in mott insulators within reduced density matrix functional theory. Phys. Rev. Lett., 110:116403, Mar 2013. doi: 10.1103/PhysRevLett.110.116403. URL http://link.aps.org/doi/10.1103/PhysRevLett.110.116403.

ISAIAH SHAVITT. The history and evolution of configuration interaction. Molecular Physics, 94(1):3-17, 1998. doi: 10.1080/002689798168303. URL https://www. tandfonline.com/doi/abs/10.1080/002689798168303.

Y Shinohara, S Sharma, J K Dewhurst, S Shallcross, N N Lathiotakis, and E K U Gross. Doping induced metal-insulator phase transition in nio - a reduced density matrix functional theory perspective. New J. Phys., 17(9):093038, 2015a. URL http: //stacks.iop.org/1367-2630/17/i=9/a=093038.

Y. Shinohara, S. Sharma, S. Shallcross, N. N. Lathiotakis, and E. K. U. Gross. Spectrum for nonmagnetic mott insulators from power functional within reduced density matrix functional theory. J. Chem. Theory Comput., 11(10):4895-4899, 2015b. doi: 10.1021/ acs.jctc.5b00661. URL http://dx.doi.org/10.1021/acs.jctc.5b00661. PMID: 26574277.

Tomonori Shirakawa and Seiji Yunoki. Block lanczos density-matrix renormalization group method for general anderson impurity models: Application to magnetic impurity problems in graphene. Phys. Rev. B, 90:195109, Nov 2014. doi: 10.1103/PhysRevB.90. 195109. URL http://link.aps.org/doi/10.1103/PhysRevB.90.195109.

P. Shor. Polynomial-time algorithms for prime factorization and discrete logarithms on a quantum computer. SIAM Journal on Computing, 26(5):1484-1509, 1997. doi: 10. 1137/S0097539795293172. URL https://doi.org/10.1137/S0097539795293172.

J. C. Slater. The theory of complex spectra. Phys. Rev., 34:1293-1322, Nov 1929. doi: 10.1103/PhysRev.34.1293. URL https://link.aps.org/doi/10.1103/PhysRev.34. 1293.

J. C. Slater. A simplification of the hartree-fock method. Phys. Rev., 81:385-390, Feb 1951. doi: 10.1103/PhysRev.81.385. URL http://link.aps.org/doi/10.1103/PhysRev . 81.385.

John C. Slater. Statistical exchange-correlation in the self-consistent field. volume 6 of Advances in Quantum Chemistry, pages 1 - 92. Academic Press, 1972. doi: https://doi.org/10.1016/S0065-3276(08)60541-9. URL http://www.sciencedirect. com/science/article/pii/S0065327608605419. 
I. V. Solovyev, P. H. Dederichs, and V. I. Anisimov. Corrected atomic limit in the local-density approximation and the electronic structure of d impurities in rb. Phys. Rev. B, 50:16861-16871, Dec 1994. doi: 10.1103/PhysRevB.50.16861. URL https: //link.aps.org/doi/10.1103/PhysRevB.50.16861.

Laurent Sorber, Marc Van Barel, and Lieven De Lathauwer. Unconstrained optimization of real functions in complex variables. SIAM Journal on Optimization, 22(3):879-898, 2012. doi: 10.1137/110832124. URL https://doi.org/10.1137/110832124.

Sandro Sorella. Generalized lanczos algorithm for variational quantum monte carlo. Phys. Rev. B, 64:024512, Jun 2001. doi: 10.1103/PhysRevB.64.024512. URL https://link. aps.org/doi/10.1103/PhysRevB.64.024512.

Sandro Sorella, Michele Casula, and Dario Rocca. Weak binding between two aromatic rings: Feeling the van der waals attraction by quantum monte carlo methods. The Journal of Chemical Physics, 127(1):014105, 2007. doi: 10.1063/1.2746035. URL https://doi.org/10.1063/1.2746035.

J. C. Spall. A stochastic approximation technique for generating maximum likelihood parameter estimates. In 1987 American Control Conference, pages 1161-1167, June 1987. doi: 10.23919/ACC.1987.4789489.

J. C. Spall. Multivariate stochastic approximation using a simultaneous perturbation gradient approximation. IEEE Transactions on Automatic Control, 37(3):332-341, Mar 1992. ISSN 0018-9286. doi: 10.1109/9.119632.

Hermann Stoll and Andreas Savin. Density Functionals for Correlation Energies of Atoms and Molecules, pages 177-207. Springer US, Boston, MA, 1985. ISBN 978-1-4757-08189.

Qiming Sun. Libcint: An efficient general integral library for gaussian basis functions. Journal of Computational Chemistry, 36:1664-1671, 2015. doi: 10.1002/jcc.23981. URL http://dx.doi.org/10.1002/jcc. 23981.

Qiming Sun, Timothy C. Berkelbach, Nick S. Blunt, George H. Booth, Sheng Guo, Zhendong Li, Junzi Liu, James D. McClain, Elvira R. Sayfutyarova, Sandeep Sharma, Sebastian Wouters, and Garnet Kin-Lic Chan. Pyscf: the python-based simulations of chemistry framework. Wiley Interdisciplinary Reviews: Computational Molecular Science, 8(1):e1340. doi: 10.1002/wcms.1340. URL https://onlinelibrary.wiley.com/ doi/abs/10.1002/wcms . 1340.

Andrew G. Taube and Rodney J. Bartlett. New perspectives on unitary coupled-cluster theory. International Journal of Quantum Chemistry, 106(15):3393-3401, 2006. doi: 10. 1002/qua.21198. URL https://onlinelibrary.wiley.com/doi/abs/10.1002/qua. 21198.

B. Thaller. The Dirac equation. Texts and monographs in physics. Springer-Verlag, 1992. ISBN 9780387548838. 
Iris Theophilou, Nektarios N. Lathiotakis, Miguel A. L. Marques, and Nicole Helbig. Generalized pauli constraints in reduced density matrix functional theory. The Journal of Chemical Physics, 142(15):154108, 2015. doi: 10.1063/1.4918346. URL https: //doi.org/10.1063/1.4918346.

Patrik Thunström, Igor Di Marco, and Olle Eriksson. Electronic entanglement in late transition metal oxides. Phys. Rev. Lett., 109:186401, Oct 2012. doi: 10.1103/PhysRevLett. 109.186401. URL https://link.aps.org/doi/10.1103/PhysRevLett.109.186401.

O. Tjernberg, S. Söderholm, G. Chiaia, R. Girard, U. O. Karlsson, H. Nylén, and I. Lindau. Influence of magnetic ordering on the nio valence band. Phys. Rev. B, 54:1024510248, Oct 1996. doi: 10.1103/PhysRevB.54.10245. URL https://link.aps.org/ doi/10.1103/PhysRevB.54.10245.

Julien Toulouse, Iann C. Gerber, Georg Jansen, Andreas Savin, and János G. Ángyán. Adiabatic-connection fluctuation-dissipation density-functional theory based on range separation. Phys. Rev. Lett., 102:096404, Mar 2009. doi: 10.1103/PhysRevLett.102. 096404. URL https://link.aps.org/doi/10.1103/PhysRevLett.102.096404.

W. Töws and G. M. Pastor. Lattice density functional theory of the single-impurity anderson model: Development and applications. Phys. Rev. B, 83:235101, Jun 2011. doi: 10.1103/PhysRevB.83.235101. URL http://link.aps.org/doi/10.1103/PhysRevB. 83. 235101.

W. Töws and G. M. Pastor. Spin-polarized density-matrix functional theory of the singleimpurity anderson model. Phys. Rev. B, 86:245123, Dec 2012. doi: 10.1103/PhysRevB. 86.245123. URL http://link.aps.org/doi/10.1103/PhysRevB.86.245123.

Fabien Tran, Peter Blaha, Karlheinz Schwarz, and Pavel Novák. Hybrid exchangecorrelation energy functionals for strongly correlated electrons: Applications to transition-metal monoxides. Phys. Rev. B, 74:155108, Oct 2006. doi: 10. 1103/PhysRevB.74.155108. URL https://link.aps.org/doi/10.1103/PhysRevB. 74.155108.

J. F. Traub. A class of globally convergent iteration functions for the solution of polynomial equations. Mathematics of Computation, 20(93):113-138, 1966. ISSN 00255718, 10886842. URL http://www.jstor.org/stable/2004275.

Oliver Treutler and Reinhart Ahlrichs. Efficient molecular numerical integration schemes. The Journal of Chemical Physics, 102(1):346-354, 1995. doi: 10.1063/1.469408. URL https://doi.org/10.1063/1.469408.

Matthias Troyer and Uwe-Jens Wiese. Computational complexity and fundamental limitations to fermionic quantum monte carlo simulations. Phys. Rev. Lett., 94:170201, May 2005. doi: 10.1103/PhysRevLett.94.170201. URL https://link.aps.org/doi/ 10.1103/PhysRevLett.94.170201.

M. T. Tuominen, J. M. Hergenrother, T. S. Tighe, and M. Tinkham. Experimental evidence for parity-based 2e periodicity in a superconducting single-electron tunneling transistor. Phys. Rev. Lett., 69:1997-2000, Sep 1992. doi: 10.1103/PhysRevLett.69. 1997. URL https://link.aps.org/doi/10.1103/PhysRevLett.69.1997. 
A. M. Turing. On computable numbers, with an application to the entscheidungsproblem. Proceedings of the London Mathematical Society, s2-42(1):230-265, 1936. doi: 10.1112/ plms/s2-42.1.230. URL https://londmathsoc.onlinelibrary.wiley.com/doi/abs/ $10.1112 / \mathrm{plms} / \mathrm{s} 2-42.1 .230$.

Yutaka Utsuno, Noritaka Shimizu, Takaharu Otsuka, and Takashi Abe. Efficient computation of hamiltonian matrix elements between non-orthogonal slater determinants. Computer Physics Communications, 184(1):102 - 108, 2013. ISSN 0010-4655. doi: https://doi.org/10.1016/j.cpc.2012.09.002. URL http://www.sciencedirect. com/science/article/pii/S0010465512002883.

C. Valdemoro. Approximating the second-order reduced density matrix in terms of the first-order one. Phys. Rev. A, 45:4462-4467, Apr 1992. doi: 10.1103/PhysRevA.45.4462. URL http://link.aps.org/doi/10.1103/PhysRevA.45.4462.

Steven M. Valone. Consequences of extending 1-matrix energy functionals from purestate representable to all ensemble representable 1 matrices. The Journal of Chemical Physics, 73(3):1344-1349, 1980. doi: http://dx.doi.org/10.1063/1.440249. URL http: //scitation.aip.org/content/aip/journal/jcp/73/3/10.1063/1.440249.

J. van Elp, H. Eskes, P. Kuiper, and G. A. Sawatzky. Electronic structure of li-doped nio. Phys. Rev. B, 45:1612-1622, Jan 1992. doi: 10.1103/PhysRevB.45.1612. URL https://link.aps.org/doi/10.1103/PhysRevB.45.1612.

E. van Lenthe, E. J. Baerends, and J. G. Snijders. Relativistic regular two-component hamiltonians. 99(6):4597-4610, 1993. ISSN 00219606. doi: DOI:10.1063/1.466059. URL http://dx.doi.org/doi/10.1063/1.466059.

E. van Lenthe, E. J. Baerends, and J. G. Snijders. Relativistic total energy using regular approximations. 101(11):9783-9792, 1994. ISSN 00219606. doi: DOI:10.1063/1.467943. URL http://dx.doi.org/doi/10.1063/1.467943.

E. van Lenthe, J. G. Snijders, and E. J. Baerends. The zero-order regular approximation for relativistic effects: The effect of spin-orbit coupling in closed shell molecules. The Journal of Chemical Physics, 105(15):6505-6516, 1996. doi: 10.1063/1.472460. URL http://link.aip.org/link/?JCP/105/6505/1.

L. Vandenberghe and S. Boyd. Semidefinite programming. SIAM Review, 38(1):49-95, 1996. doi: 10.1137/1038003. URL https://doi.org/10.1137/1038003.

Loup Verlet. Computer "experiments" on classical fluids. i. thermodynamical properties of lennard-jones molecules. Phys. Rev., 159:98-103, Jul 1967. doi: 10.1103/PhysRev. 159.98. URL https://link.aps.org/doi/10.1103/PhysRev.159.98.

Brecht Verstichel. Variational determination of the two-particle density matrix as a quantum many-body technique. PhD thesis, Universiteit Gent, 2012. URL https: //arxiv.org/abs/1203.5659.

Brecht Verstichel, Helen van Aggelen, Dimitri Van Neck, Paul W. Ayers, and Patrick Bultinck. Variational density matrix optimization using semidefinite programming. 
Computer Physics Communications, 182(9):2025 - 2028, 2011. ISSN 0010-4655. doi: https://doi.org/10.1016/j.cpc.2010.12.034. URL http://www.sciencedirect. com/science/article/pii/S0010465510005278. Computer Physics Communications Special Edition for Conference on Computational Physics Trondheim, Norway, June 23-26, 2010.

Brecht Verstichel, Helen van Aggelen, Ward Poelmans, and Dimitri Van Neck. Variational two-particle density matrix calculation for the hubbard model below half filling using spin-adapted lifting conditions. Phys. Rev. Lett., 108:213001, May 2012. doi: 10.1103/ PhysRevLett.108.213001. URL https://link.aps.org/doi/10.1103/PhysRevLett. 108.213001.

Brecht Verstichel, Helen van Aggelen, Ward Poelmans, Sebastian Wouters, and Dimitri Van Neck. Extensive v2dm study of the one-dimensional hubbard model for large lattice sizes: Exploiting translational invariance and parity. Computational and Theoretical Chemistry, 1003:12 - 21, 2013. ISSN 2210-271X. doi: https://doi.org/10.1016/j. comptc.2012.09.014. URL http://www.sciencedirect.com/science/article/pii/ S2210271X1200480X. Reduced Density Matrices: A Simpler Approach to ManyElectron Problems?

F. Verstraete and J. I. Cirac. Matrix product states represent ground states faithfully. Phys. Rev. B, 73:094423, Mar 2006. doi: 10.1103/PhysRevB.73.094423. URL https: //link.aps.org/doi/10.1103/PhysRevB.73.094423.

F. Verstraete, J. J. García-Ripoll, and J. I. Cirac. Matrix product density operators: Simulation of finite-temperature and dissipative systems. Phys. Rev. Lett., 93:207204, Nov 2004. doi: 10.1103/PhysRevLett.93.207204. URL https://link.aps.org/doi/ 10.1103/PhysRevLett.93.207204.

F. Verstraete, V. Murg, and J.I. Cirac. Matrix product states, projected entangled pair states, and variational renormalization group methods for quantum spin systems. Advances in Physics, 57(2):143-224, 2008. doi: 10.1080/14789940801912366. URL https://doi .org/10.1080/14789940801912366.

D. Vollhardt. Dynamical mean-field theory for correlated electrons. Annalen der Physik, 524(1):1-19, 2011. doi: 10.1002/andp.201100250. URL https://onlinelibrary . wiley.com/doi/abs/10.1002/andp. 201100250.

Dieter Vollhardt. Normal ${ }^{3}$ He: an almost localized fermi liquid. Rev. Mod. Phys., 56: 99-120, Jan 1984. doi: 10.1103/RevModPhys.56.99. URL https://link.aps.org/ doi/10.1103/RevModPhys.56.99.

S. H. Vosko, L. Wilk, and M. Nusair. Accurate spin-dependent electron liquid correlation energies for local spin density calculations: a critical analysis. Canadian Journal of Physics, 58(8):1200-1211, 1980. doi: 10.1139/p80-159. URL https://doi.org/10. 1139/p80-159.

Christian Walther. Beschreibung stark korrelierter Systeme mit Dichtematrixmethoden. PhD thesis, Technische Universität Clausthal, 2011. URL https://d-nb.info/ $1019261358 / 34$. 
Y. Wang, Y. Li, Z.-q. Yin, and B. Zeng. 16-qubit IBM universal quantum computer can be fully entangled. ArXiv e-prints, January 2018.

G. Wendin and V. S. Shumeiko. Superconducting Quantum Circuits, Qubits and Computing. eprint arXiv:cond-mat/0508729, August 2005.

Steven R. White. Density matrix formulation for quantum renormalization groups. Phys. Rev. Lett., 69:2863-2866, Nov 1992. doi: 10.1103/PhysRevLett.69.2863. URL https: //link.aps.org/doi/10.1103/PhysRevLett.69.2863.

Steven R. White. Density-matrix algorithms for quantum renormalization groups. Phys. Rev. B, 48:10345-10356, Oct 1993. doi: 10.1103/PhysRevB.48.10345. URL https: //link.aps.org/doi/10.1103/PhysRevB.48.10345.

James Daniel Whitfield, Norbert Schuch, and Frank Verstraete. The Computational Complexity of Density Functional Theory, pages 245-260. Springer International Publishing, Cham, 2014. ISBN 978-3-319-06379-9. doi: 10.1007/978-3-319-06379-9_14. URL https://doi.org/10.1007/978-3-319-06379-9_14.

D. Willsch, M. Nocon, F. Jin, H. De Raedt, and K. Michielsen. Gate-error analysis in simulations of quantum computers with transmon qubits. Phys. Rev. A, 96:062302, Dec 2017. doi: 10.1103/PhysRevA.96.062302. URL https://link.aps.org/doi/10. 1103/PhysRevA.96.062302.

Eiso Yamaka and Kenichi Sawamoto. Electrical conductivity of nio near the curie temperature. Phys. Rev., 112:1861-1862, Dec 1958. doi: 10.1103/PhysRev.112.1861. URL https://link.aps.org/doi/10.1103/PhysRev.112.1861.

Weitao Yang. Generalized adiabatic connection in density functional theory. The Journal of Chemical Physics, 109(23):10107-10110, 1998. doi: 10.1063/1.477701. URL https: //doi.org/10.1063/1.477701.

Weitao Yang, Yingkai Zhang, and Paul W. Ayers. Degenerate ground states and a fractional number of electrons in density and reduced density matrix functional theory. Phys. Rev. Lett., 84:5172-5175, May 2000. doi: 10.1103/PhysRevLett.84.5172. URL http://link.aps.org/doi/10.1103/PhysRevLett.84.5172.

A. Chi-Chih Yao. Quantum circuit complexity. In Proceedings of 1993 IEEE 34th Annual Foundations of Computer Science, pages 352-361, Nov 1993. doi: 10.1109/SFCS.1993. 366852 .

Koji Yasuda. Correlation energy functional in the density-matrix functional theory. Phys. Rev. A, 63:032517, Feb 2001. doi: 10.1103/PhysRevA.63.032517. URL http://link. aps.org/doi/10.1103/PhysRevA.63.032517.

Quan Yin, Alexey Gordienko, Xiangang Wan, and Sergey Y. Savrasov. Calculated momentum dependence of zhang-rice states in transition metal oxides. Phys. Rev. Lett., 100:066406, Feb 2008. doi: 10.1103/PhysRevLett.100.066406. URL https: //link.aps.org/doi/10.1103/PhysRevLett.100.066406. 
J. Zaanen, G. A. Sawatzky, and J. W. Allen. Band gaps and electronic structure of transition-metal compounds. Phys. Rev. Lett., 55:418-421, Jul 1985. doi: 10.1103/ PhysRevLett.55.418. URL https://link.aps.org/doi/10.1103/PhysRevLett.55. 418.

Michael P. Zaletel, Roger S. K. Mong, Christoph Karrasch, Joel E. Moore, and Frank Pollmann. Time-evolving a matrix product state with long-ranged interactions. Phys. Rev. B, 91:165112, Apr 2015. doi: 10.1103/PhysRevB.91.165112. URL https://link . aps .org/doi/10.1103/PhysRevB.91.165112.

Zhengji Zhao, Bastiaan J. Braams, Mituhiro Fukuda, Michael L. Overton, and Jerome K. Percus. The reduced density matrix method for electronic structure calculations and the role of three-index representability conditions. The Journal of Chemical Physics, 120(5):2095-2104, 2004. doi: 10.1063/1.1636721. URL https://doi.org/10.1063/1. 1636721.

Michael Zwolak and Guifré Vidal. Mixed-state dynamics in one-dimensional quantum lattice systems: A time-dependent superoperator renormalization algorithm. Phys. Rev. Lett., 93:207205, Nov 2004. doi: 10.1103/PhysRevLett.93.207205. URL https: //link.aps.org/doi/10.1103/PhysRevLett.93.207205.

Jiř́i Č́žzek. On the correlation problem in atomic and molecular systems. calculation of wavefunction components in ursell-type expansion using quantum-field theoretical methods. The Journal of Chemical Physics, 45(11):4256-4266, 1966. doi: 10.1063/1. 1727484. URL https://doi.org/10.1063/1.1727484. 
E. Curriculum vitae 


\section{Curriculum Vitae}

Robert Schade, Am Ehrenhain 9 - 38678 Clausthal-Zellerfeld

[+491623028115 • $\square$ robert.schade@tu-clausthal.de

( www2.pt.tu-clausthal.de/atp/rschade.html

PGP-Schlüsselkennung d2adeaa75d658aa3

\section{Personal data}

Name: Robert Schade

Place of birth: Mühlhausen/Germany

Nationality: German

Date of birth: 13.01 .1988

\section{Ph.D. studies}

New methods for the ab-initio simulation of correlated systems

Advisors: Peter E. Blöchl, Salvatore R. Manmana

scientific employee

Institute of Theoretical Physics, Clausthal University of Technology
Göttingen/TU Clausthal

2013-probably. Dec. 2018

Clausthal University of Technology 01.01.2013-31.12.2018

\section{Education}

Master of Science (physics)

Georg-August University Göttingen

final grade: very good $(1,0)$ with distinction

2010-2012

master's thesis

Relativistic effects in the PAW method beyond the scalar approximation, grade 1,0

2012

https://www2.pt.tu-clausthal.de/atp/members/rschade/Master.pdf

Berliner-Ungewitter-price

for a distinguished master's thesis

https://www. uni-goettingen.de/de/preise/45092.html

Bachelor of Science (physics)

final grade: very good $(1,4)$ with distinction

Georg-August University Göttingen

2007-2010

bachelor's thesis

Modeling a molecular magnet, grade 1,0

https://www2.pt.tu-clausthal.de/atp/members/rschade/Bachelor .pdf

stipend of the German National Academic Foundation

full stipend

2007-2012

university-entrance diploma

final grade: 1,0

Käthe-Kollwitz-Gymnasium, Lengenfeld u. St. 1998-2006

\section{Languages}

German: mother tongue

English: business fluent 


\section{Abilities}

Programming

$\mathrm{C}++$ 98/03: very good (some features from $\mathrm{C}++11$ and newer)

Fortran 90/95: very good (some features from Fortran 2003 and newer)

Julia: very good

QASM: good

Python: good

Assembler: basic

Java: basic

Additional: MPI (very good), OpenMP (very good), HDF5 (very good)

\section{Publications}

ORCID ID: 0000-0002-6268-5397

Title: Adaptive cluster approximation for reduced density-matrix functional theory

Authors: Robert Schade, Peter E. Blöchl

Journal: Phys. Rev. B 97, 245131

Link: https://journals.aps.org/prb/abstract/10.1103/PhysRevB.97.245131

Title: Reduced density-matrix functionals from many-particle theory

Authors: Robert Schade, Ebad Kamil, Peter E. Blöchl

Journal: Eur. Phys. J. Spec. Top. (2017) 226: 2677

Link: https://dx.doi.org/10.1140/epjst/e2017-70046-0

Title: Reduced density-matrix functionals applied to the Hubbard dimer

Autors: Ebad Kamil, Robert Schade, Thomas Pruschke, Peter E. Blöchl

Journal: Phys. Rev. B 93, 085141

Link: http://journals .aps .org/prb/abstract/10.1103/PhysRevB.93.085141

\section{Experience in university education (selection)}

Event: International hands-on course on first principles calculations, Göttingen 2014/15/16/17/18

Role: organizer and setup of computer exercises

Lecture: Advanced topics of programming for numerical physics

Role: lecturer

Semester: winter term 2015/2016 and winter term 2016/17

Content: parallelization (OpenMP, MPI), hardware-performance counter, performance modelling 


\section{Organized workshops and conferences}

Title: International hands-on course on first principles calculations, September 2018, Göttingen

Role: technical organizer

Type: two-week summer school with lectures, tutorials, indivdualized projects and poster session

Titel: International hands-on course on first principles calculations, 2014/2015/2016/2017, Göttingen

Role: main organizer

Typ: one-week summer school with lectures, tutorials and poster session

\section{Attended conferences and workshops (selection)}

Title: International Workshop on "New challenges in Reduced Density Matrix Functional Theory: Symmetries, time-evolution and entanglement", Lausanne 2017

Organizer: CECAM, Psi-k

Link: https ://www.cecam.org/workshop-0-1448.html

Title: 3nd International Workshop "Dynamical Mean-Field Approach for Strongly Correlated Materials", Dresden 2017

Organizer: DFG research group 1346: Dynamical Mean-Field Approach with Predictive Power for Strongly Correlated Materials

Link: http://www2.cpfs .mpg.de/dmft2017dd/

Title: Total Energy and Force Methods, Luxemburg 2016

Organizer: Psi-k, ESF, University Luxemburg

Link: http://totalenergy.uni.lu/

Title: Perspectives of many-particle methods: total energy, spectroscopy and time-dependent dynamics, Bremen 2015

Organizer: CECAM, Tim Wehling, Thomas Frauenheim, Andreas Savin, Johannes Lischner, Thorsten Klüner

Link: https://www.cecam.org/workshop-1210.html

Title: 2nd International Workshop "Dynamical Mean-Field Approach for Strongly Correlated Materials", Dresden 2015

Organizer: DFG research group 1346: Dynamical Mean-Field Approach with Predictive Power for Strongly Correlated Materials

Link: http://www2.cpfs.mpg.de/dmft2015dd/

Title: Node-level Performance Engineering, Stuttgart 2014

Organizer: HLRS Stuttgart, Georg Hager, Jan Treibig

Link: https://blogs.fau.de/hager/talks/nlpe 